\title{
Lebensstilbasierte Kundensegmentierung im Blumen- und Pflanzeneinzelhandel
}

\section{Analyse und strategische Optionen}

\author{
Dissertation \\ zur Erlangung des Doktorgrades \\ der Fakultät für Agrarwissenschaften \\ der Georg-August-Universität Göttingen
}

vorgelegt von

Stefan von Péterffy

geboren in Offenburg

Göttingen, im Dezember 2015 
D 7

1. Referentin/Referent: Prof. Dr. U. Enneking

2. Korreferentin/Korreferent: Prof. Dr. A. Spiller

Tag der mündlichen Prüfung: 11. Februar 2016 
Für Katharina - in Liebe 



\section{Danksagung}

An dieser Stelle möchte ich mich ganz herzlich bei denjenigen Personen bedanken, die durch ihre Unterstützung zum Gelingen dieser Arbeit beigetragen haben.

Mein ganz besonderer Dank gilt hierbei Prof. Dr. Enneking - einerseits für die Betreuung meiner Dissertation, andererseits für seine vielen guten, nicht nur fachlichen Ratschläge. Für seine Geduld, den stets motivierenden Gedankenaustausch und die immer freundschaftliche Zusammenarbeit danke ich ihm von ganzem Herzen.

Weiterhin gilt mein Dank Prof. Dr. Spiller für die Erstellung des Zweitgutachtens und Prof. Dr. Theuvsen, der sich als Drittprüfer für die Disputation zur Verfügung gestellt hat.

Göttingen, im Februar 2016

Stefan von Péterffy 



\section{Inhaltsverzeichnis}

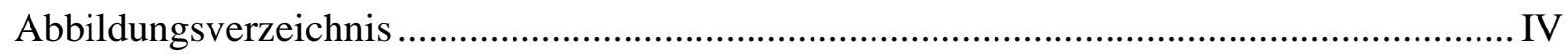

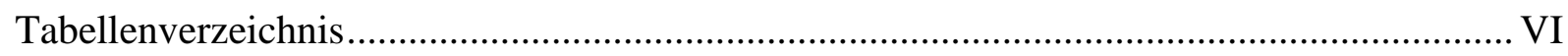

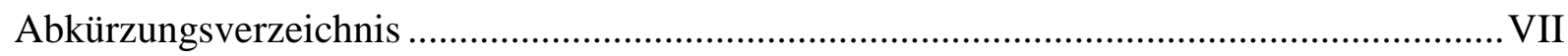

1 Aktuelle Situation und Problemstellung ............................................................................ 1

2 Das Angebot an Blumen und Pflanzen im Einzelhandel............................................... 6

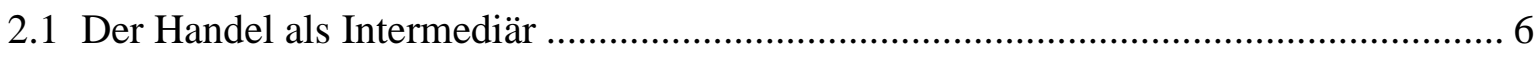

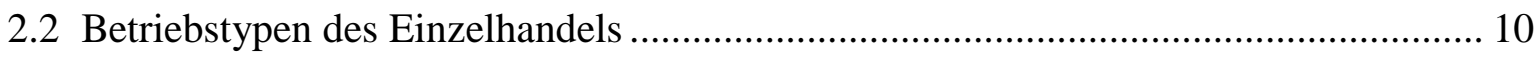

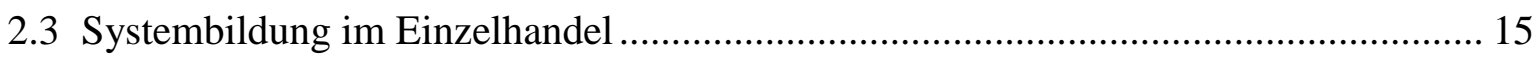

2.4 Der Markt für Blumen und Pflanzen ................................................................... 18

2.4.1 Das Angebot für Blumen und Pflanzen ......................................................... 20

2.4.2 Bestandteile des Angebots an Blumen und Pflanzen......................................... 23

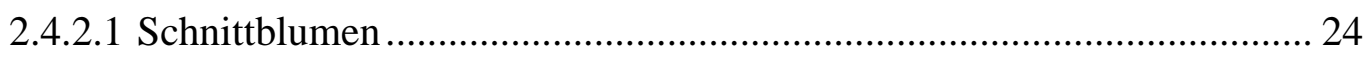

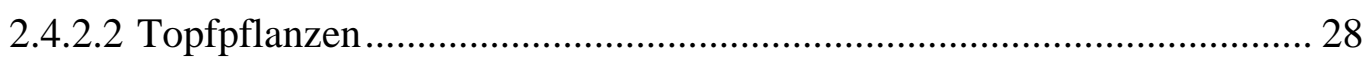

2.4.2.2.1 Zimmerpflanzen............................................................... 30

2.4.2.2.2 Beet- und Balkonpflanzen .................................................... 34

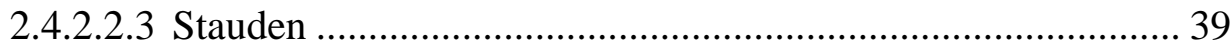

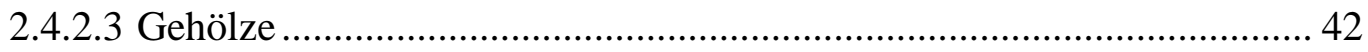

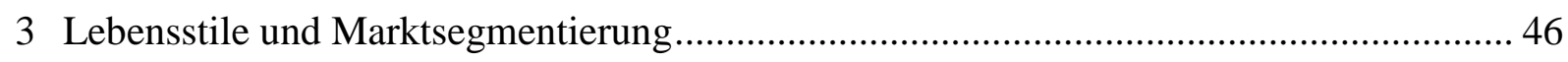

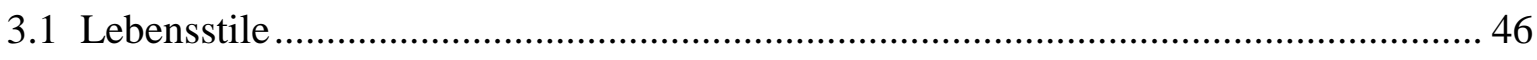

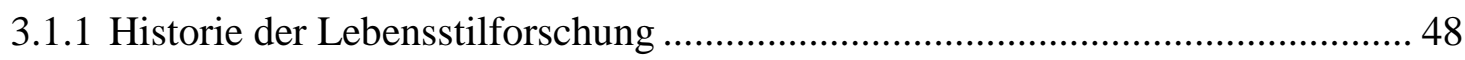

3.1.2 Grundsteine der Lebensstilforschung …................................................... 52

3.1.3 Lebensstile und Konsumentenverhalten ......................................................... 55

3.1.4 Aktuelle Bedeutung der Lebensstilforschung ............................................... 56

3.1.5 Lebensstile in der empirischen Forschung .............................................. 58

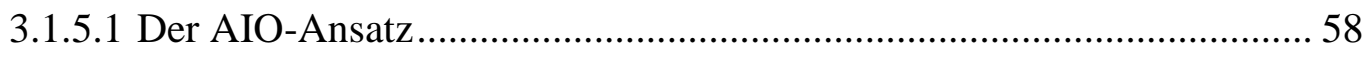

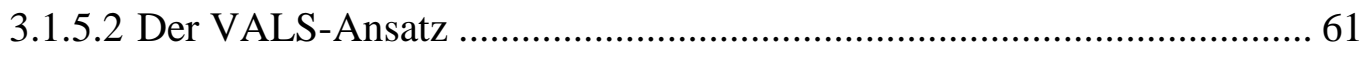

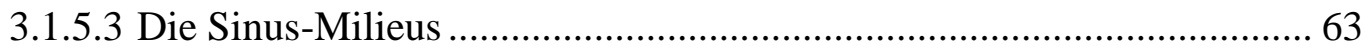

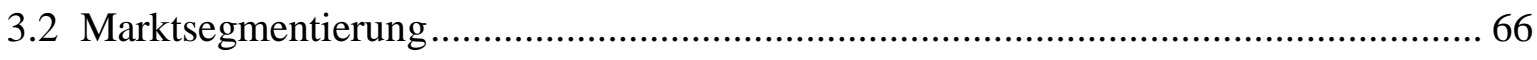

3.2.1 Markterfassung und Marktbearbeitung - ein einheitlicher Ansatz .................... 68

3.2.2 Kriterien der Marktsegmentierung .............................................................. 71

3.2.2.1 Demographische Segmentierungskriterien ......................................... 74

3.2.2.2 Soziologische Kriterien ................................................................... 78 


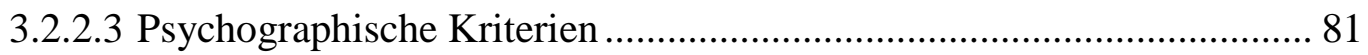

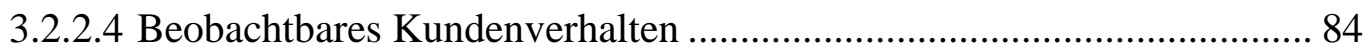

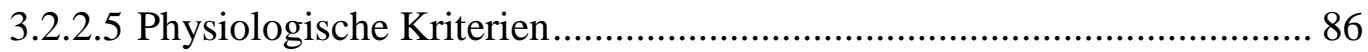

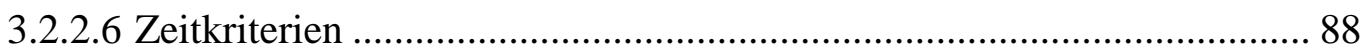

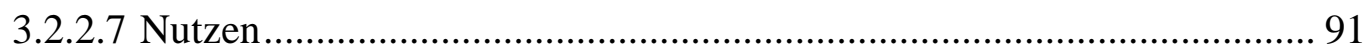

3.2.3 Identifizierung der relevanten Segmente ...................................................... 92

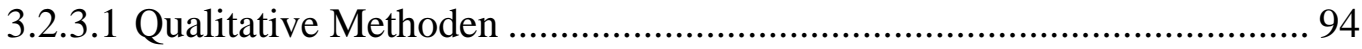

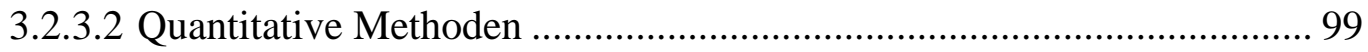

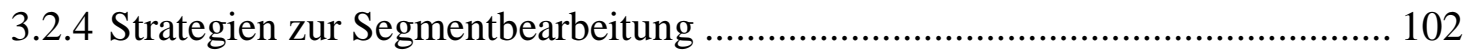

3.3 Lebensstile als Grundlage der Marktsegmentierung ................................................ 105

4 Der Einsatz segmentspezifischer Marketinginstrumente ............................................ 109

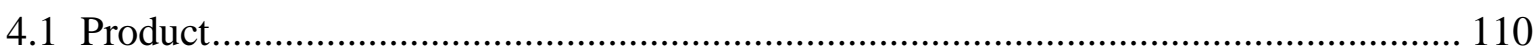

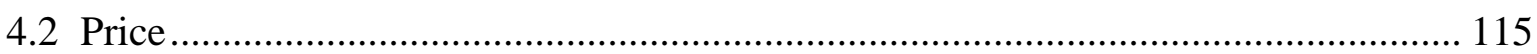

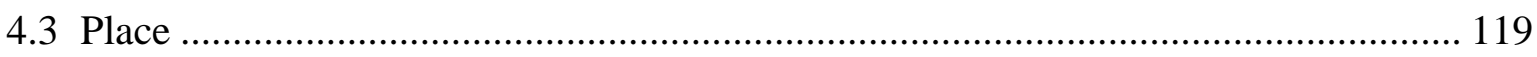

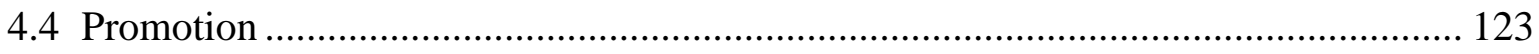

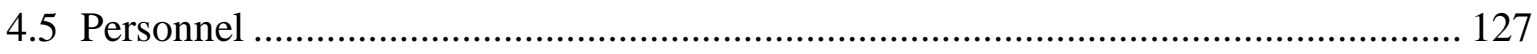

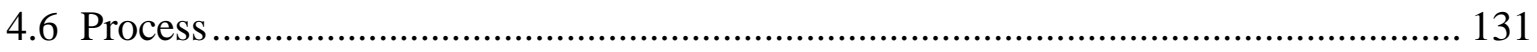

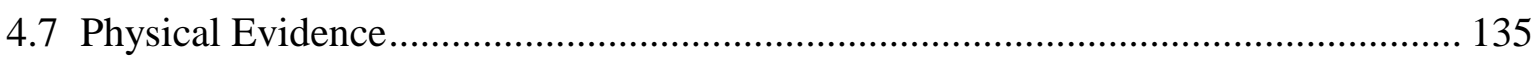

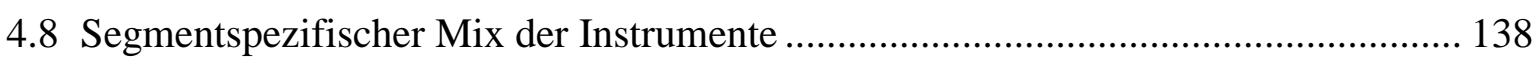

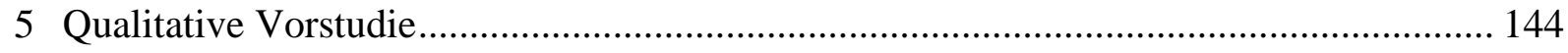

5.1 Methoden der qualitativen Untersuchung …......................................................... 146

5.1.1 Theorie zum explorativen Interview …...................................................... 147

5.1.2 Theorie zu den interne Faktoren .................................................................. 149

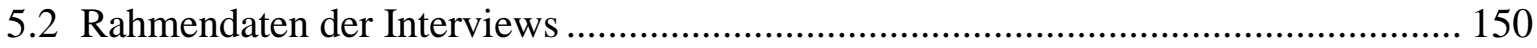

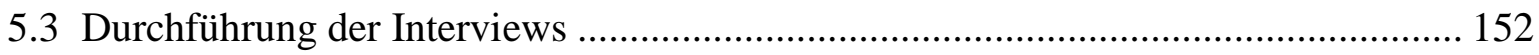

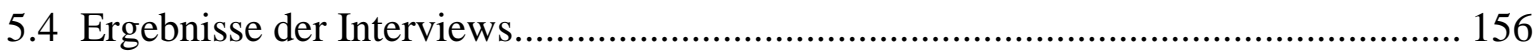

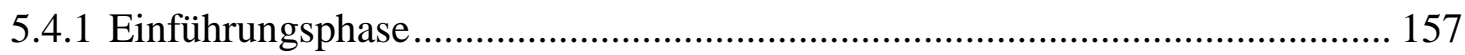

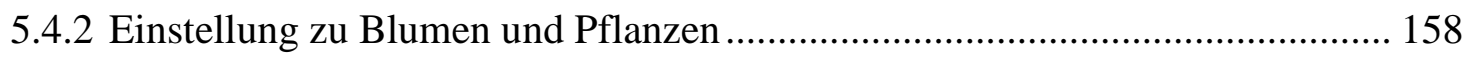

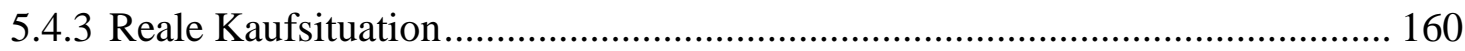

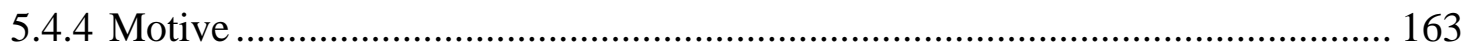

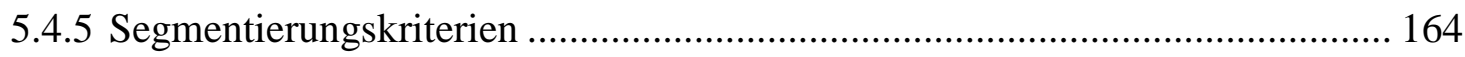

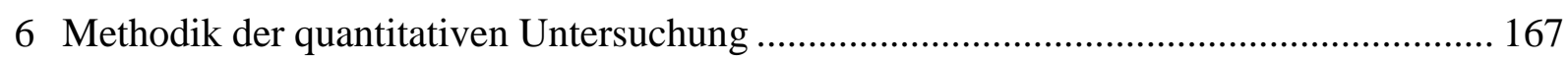

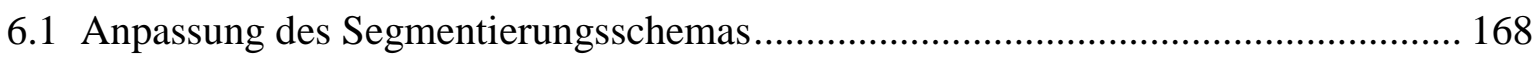

6.2 Umsetzung der Forschungsfragen in den Fragekatalog ......................................... 171 


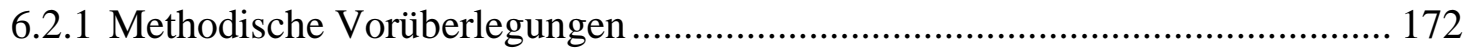

6.2.2 Operationalisierung der sozialökonomischen Situation.................................. 174

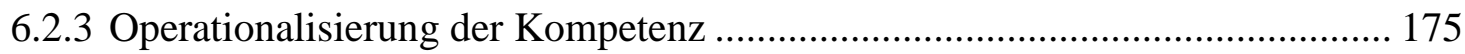

6.2.4 Operationalisierung der Performanz ….......................................................... 177

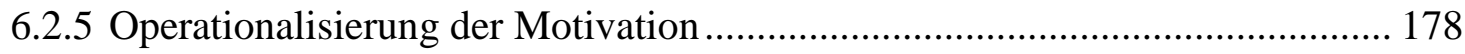

6.2.6 Operationalisierung des beobachtbaren Kaufverhaltens................................. 179

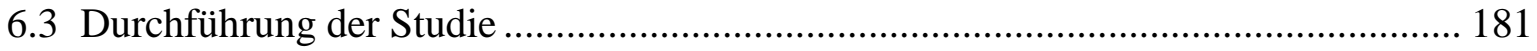

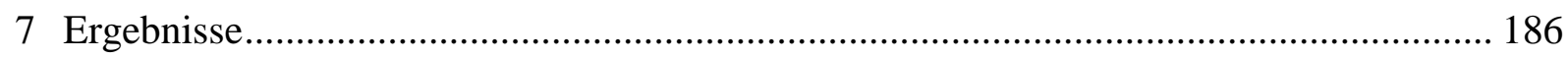

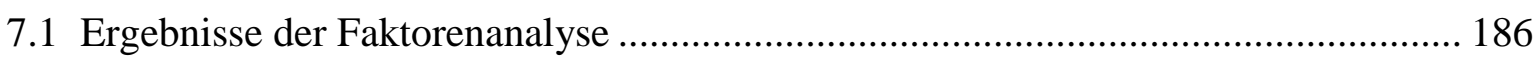

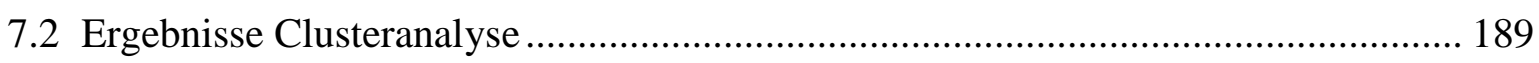

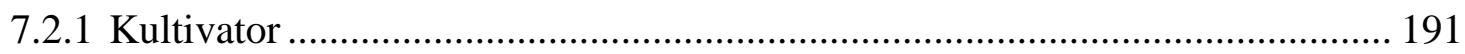

7.2.2 Durchschnittlicher Gartenfreund................................................................ 192

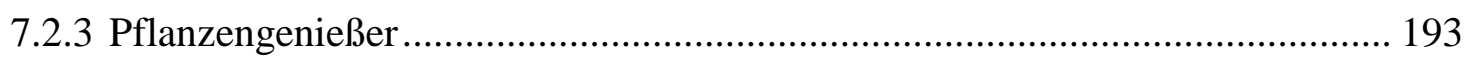

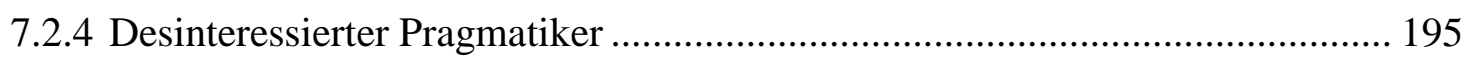

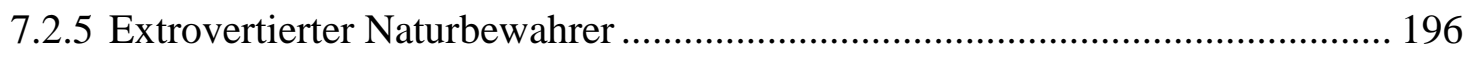

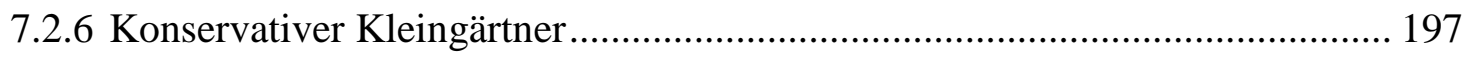

7.3 Clusterzuordnung unter Berücksichtigung der Lebensstildimensionen ..................... 199

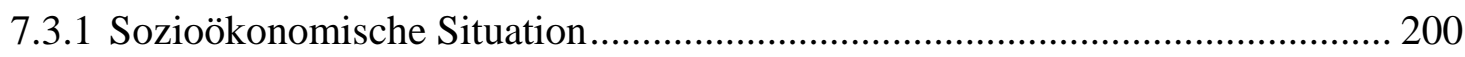

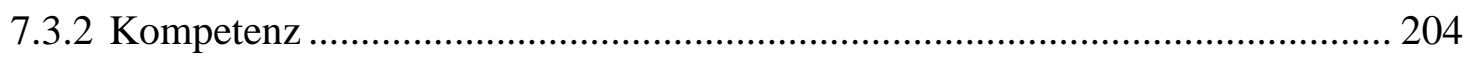

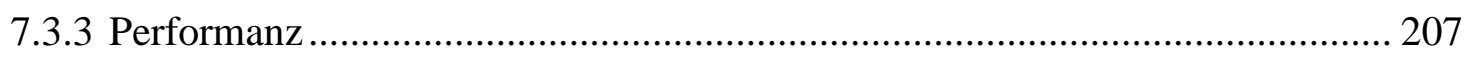

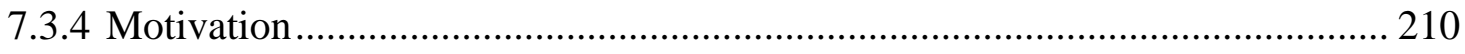

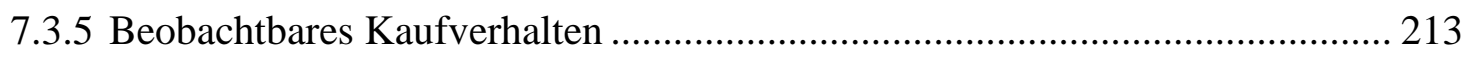

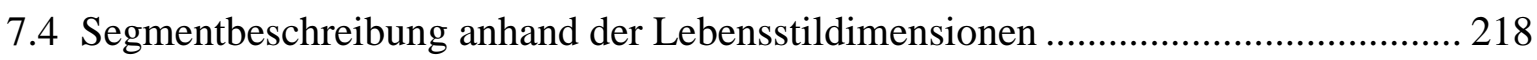

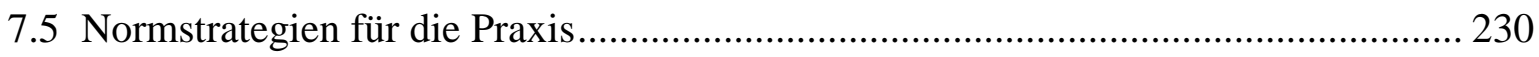

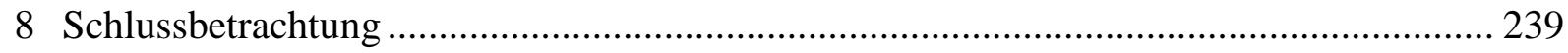

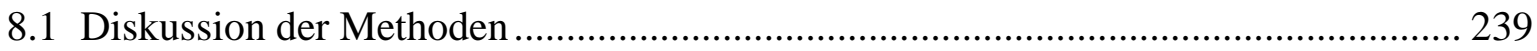

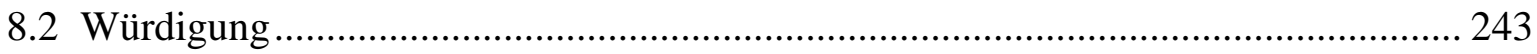

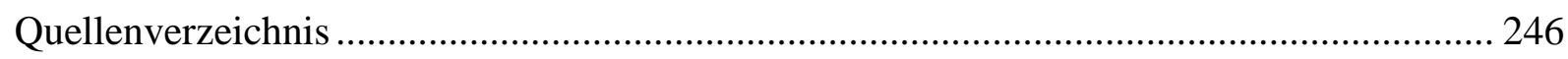

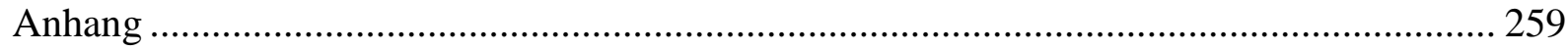




\section{Abbildungsverzeichnis}

Abbildung 1: Schematische Darstellung der Arbeit ........................................................... 4

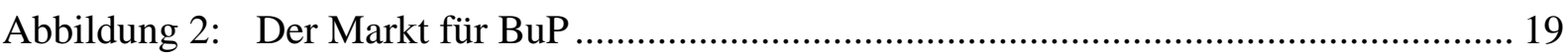

Abbildung 3: Entwicklung Gesamtumsatz Blumen und Pflanzen zu

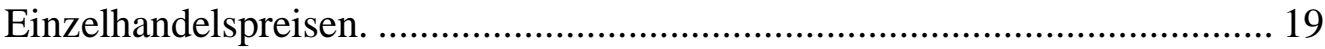

Abbildung 4: Marktstruktur bei Blumen und Pflanzen...................................................... 22

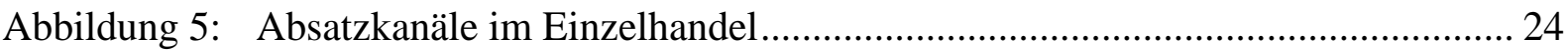

Abbildung 6: Umsatzentwicklung Schnittblumen ......................................................... 25

Abbildung 7: Marktanteile Einkaufsstätten Schnittblumen............................................... 26

Abbildung 8: Ranking Umsatzanteile Schnittblumen 2008............................................ 27

Abbildung 9: Verwendungszweck Schnittblumenprodukte 2007 ...................................... 28

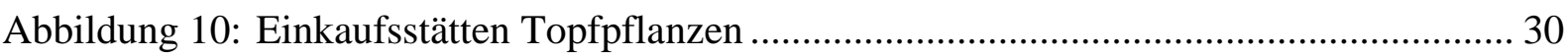

Abbildung 11: Umsatzentwicklung Topfpflanzen ............................................................ 31

Abbildung 12: Umsatzentwicklung blühende Zimmerpflanzen .......................................... 32

Abbildung 13: Umsatzanteile blühende Zimmerpflanzen 2008 in Prozent............................ 32

Abbildung 14 Marktentwicklung grüne Zimmerpflanzen ................................................ 33

Abbildung 15 Umsatzanteile grüne Zimmerpflanzen 2008 in Prozent ................................ 34

Abbildung 16: Umsatzentwicklung Beet- und Balkonpflanzen in Mio. €............................. 35

Abbildung 17: Saisonverlauf Beet- und Balkonpflanzen ................................................... 36

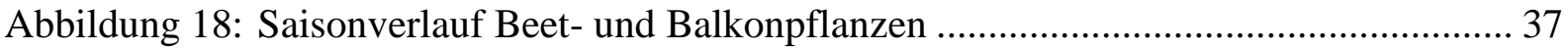

Abbildung 19: Einkaufsstätten Beet- und Balkonpflanzen................................................... 38

Abbildung 20: Umsatzanteile Beet- und Balkonpflanzen 2008 in Prozent ............................ 39

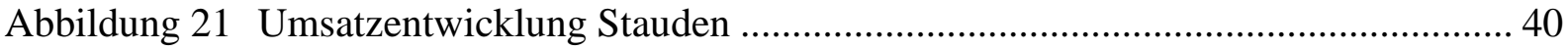

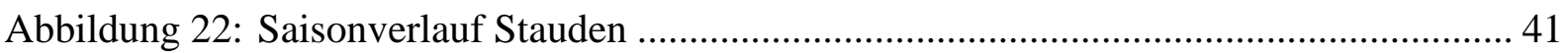

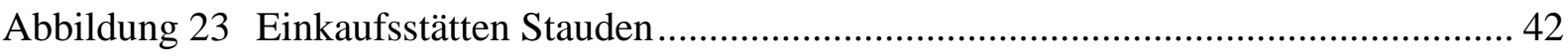

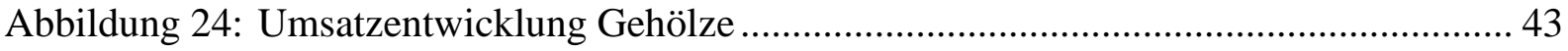

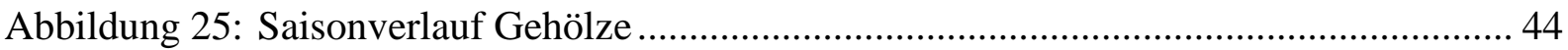

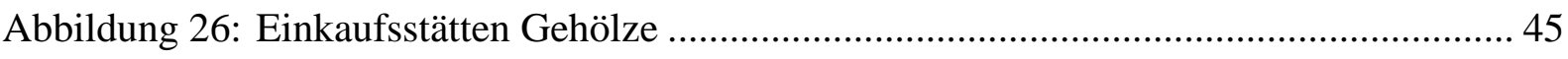

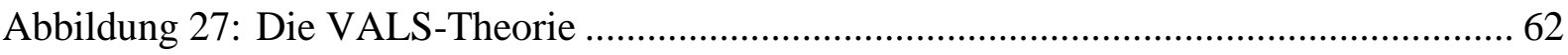

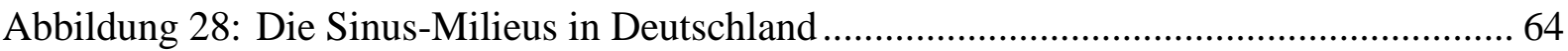

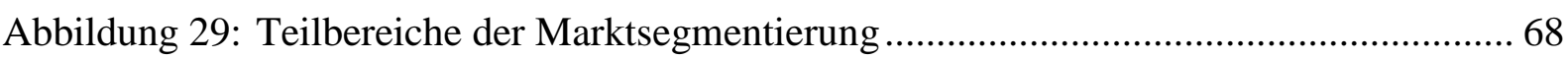




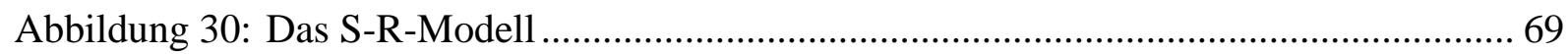

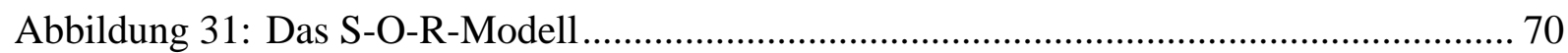

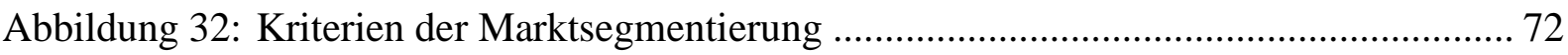

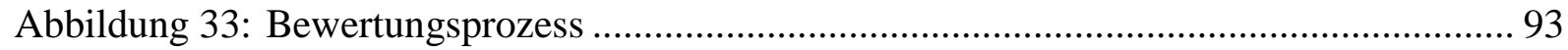

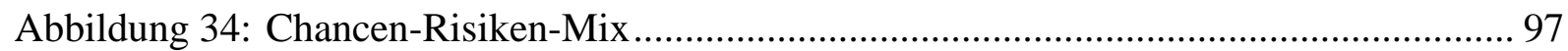

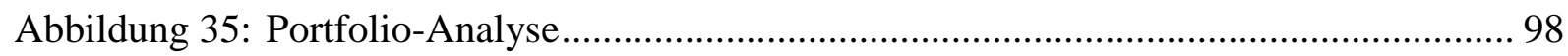

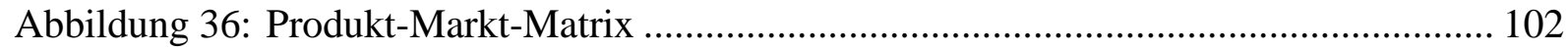

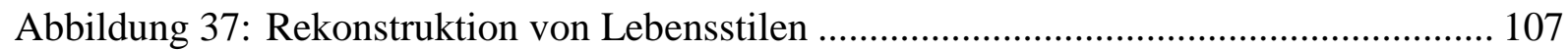

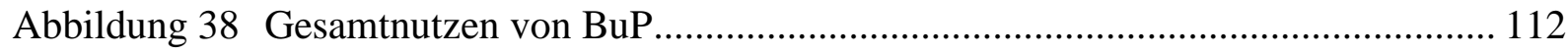

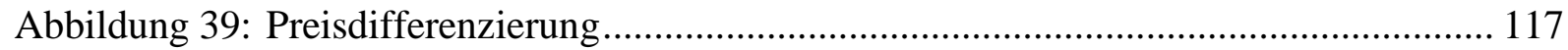

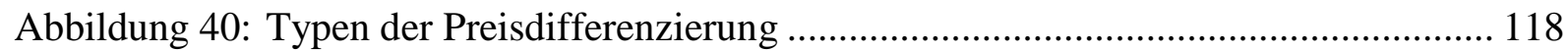

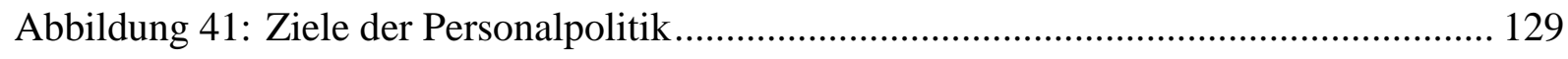

Abbildung 42: Prozess- und Organisationsentwicklung................................................... 135

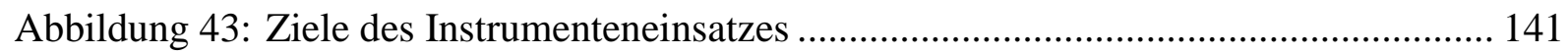

Abbildung 44: Segmentspezifische Instrumente …....................................................... 142

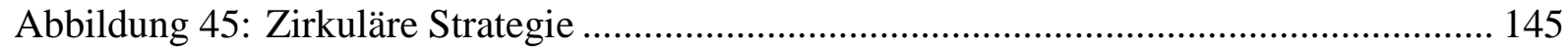

Abbildung 46: Dimensionen von Lebensstil-Konzeptionen .............................................. 169

Abbildung 47: Dimensionen von Lebensstil-Konzeptionen bezogen auf BuP .................... 171 


\section{Tabellenverzeichnis}

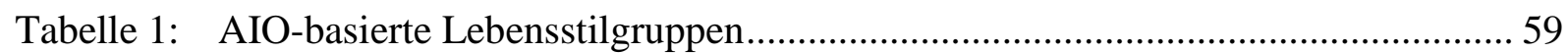

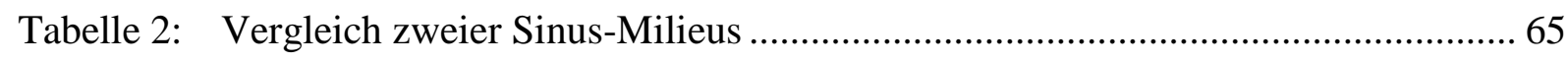

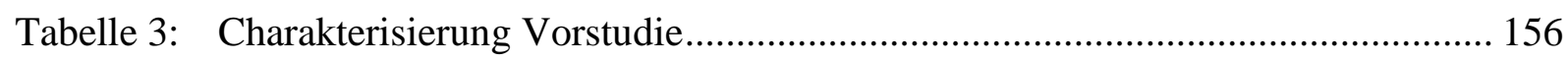

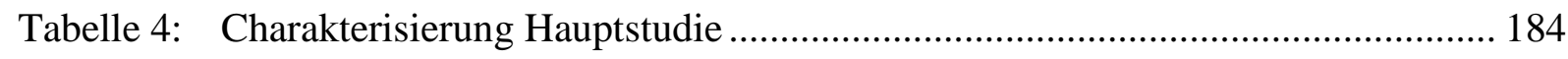

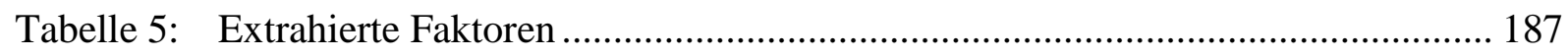

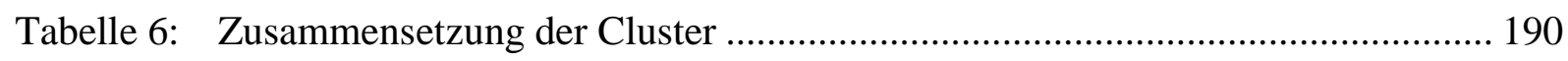

Tabelle 7: Stärke der Einflüsse der Motivfaktoren auf die gefundenen Cluster ................. 191

Tabelle 8: Einflussvariablen sozioökonomische Dimension............................................. 201

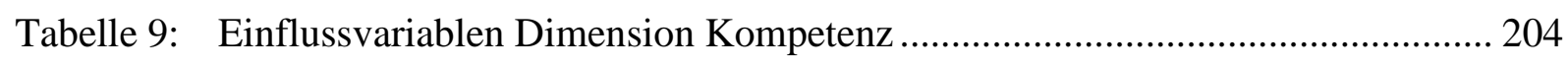

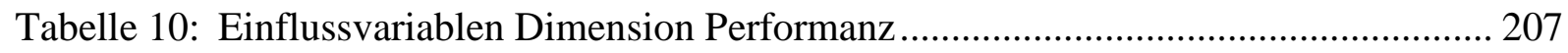

Tabelle 11: Einflussvariablen Dimension Motivation........................................................... 211

Tabelle 12: Einflussvariablen Dimension Beobachtbares Kaufverhalten ............................ 214

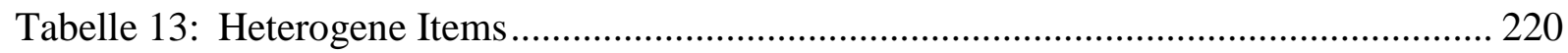

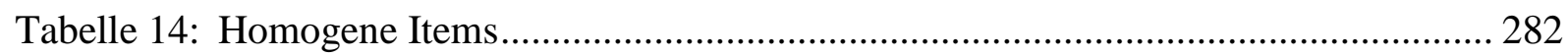




\section{Abkürzungsverzeichnis}

$\begin{array}{ll}\text { BuP } & \text { Blumen und Pflanzen } \\ \text { DIY } & \text { „Do It Yourself“ (= Baumärkte) } \\ \text { Ga-La-Bau } & \text { Garten- und Landschaftsbau } \\ \text { LEH } & \text { Lebensmitteleinzelhandel } \\ \text { SVG } & \text { Selbstversorgungsgrad } \\ \text { VBN } & \text { Vereinigte Blumenversteigerungszentralen der Niederlanden }\end{array}$





\section{Aktuelle Situation und Problemstellung}

Die aktuelle Situation auf dem Markt für Blumen und Pflanzen (BuP) ist geprägt von den Charakteristika eines reifen bzw. gesättigten Marktes. Zusätzlich hat die stets fortschreitende Internationalisierung der Wirtschaft der letzten Jahre auch vor der Blumen- und Pflanzenbranche nicht Halt gemacht und die dortigen Marktstrukturen grundlegend verändert bzw. in Bewegung gebracht. Als Ergebnis dieses Wandlungsprozesses kommt es für die gesamte Blumen- und Pflanzenbranche zu einem erhöhten Wettbewerb um die Kunden.

Der gesättigte Markt von BuP zeichnet sich durch nur noch sehr kleine Marktwachstumsraten aus. Die Umsätze von Blumen und Pflanzen in Deutschland sind während der letzten Jahre zwar kontinuierlich, doch nur noch sehr langsam gestiegen. Lag das Marktvolumen im Jahr 2000 noch bei ca. 8,3 Milliarden Euro (alle Preise in Einzelhandelspreisen), so stieg es bis 2008 um 0,66 Milliarden Euro auf 8,96 Milliarden Euro. Dies entspricht zwar einem Marktwachstum von ca. $8 \%$ innerhalb von acht Jahren, doch im gleichen Zeitraum sind parallel die allgemeinen Preissteigerungsraten in Deutschland gestiegen - und zwar weitaus stärker als die Umsatzsteigerungsraten für Blumen und Pflanzen. Lag der sogenannte Verbraucherpreisindex zur Mitte des Jahres 2000 noch bei 92,8 Punkten (Basisjahr 2005 = 100 Punkte), erreichte er Mitte 2008 bereits 107,3 Punkte. Dies entspricht für den Zeitraum 2000-2008 einer allgemeinen Preissteigerungsrate in Höhe von ca. 16 \% (Statistisches Bundesamt 2009).

Zusätzlich zum höheren Anstieg des allgemeinen Verbraucherpreisindexes kommt allerdings erschwerend hinzu, dass gerade die Preise der wichtigsten Betriebsmittel für die Produktion von Blumen und Pflanzen während des Zeitraums 2000 bis 2008 noch stärker als der Preisindex angestiegen sind, nämlich um durchschnittlich $22 \%$ (Lange 2009, S. 45). Bringt man diese Zahlenreihe in Zusammenhang, zeigt sich, dass die allgemeinen Kostensteigerungen entlang der Wertschöpfungskette für Blumen und Pflanzen bei Weitem nicht an die Konsumenten weitergegeben werden konnten. Dies ging und geht zu Lasten der Margen aller Marktteilnehmer, die in der Wertschöpfungskette dem Endkunden gegenübertreten.

Die Möglichkeit, dass unter den gegebenen Umständen die Margen konstant blieben, weil sich das Marktvolumen auf Grund der geringeren Absatzmengen verkleinert hat oder weil sich die relativ geringen Umsatzzuwächse durch entsprechende Kosteneinsparungen egalisiert haben, wurde wissenschaftlich bisher nicht untersucht. Erfahrungsbedingt lässt sich dies aber mit hoher Wahrscheinlichkeit ausschließen. 
Zusätzlich zu dem beschriebenen Problem der akut fehlenden Marktdynamik steht die BuPBranche auch vor einer langfristigen Herausforderung. Im Bereich des gesamten Blumen- und Pflanzeneinzelhandels sind ältere Personen die mit Abstand wichtigste Kundengruppe des Teilbereichs ,,privater Verbrauch“, der ca. $77 \%$ des Gesamtmarktes für Blumen und Pflanzen ausmacht. Etwa die Hälfte der Umsätze des privaten Verbrauchs wird von Kunden getätigt, die 60 Jahre oder älter sind, während auf die Gruppe der 50- bis 59-Jährigen ein Anteil von $22 \%$ des privaten Verbrauchs entfällt. Somit sorgen Personen ab 50 Jahren für etwa drei Viertel aller privaten Blumen- und Pflanzenumsätze. Zum Vergleich: Die Gruppe aller Personen unter 35 Jahren hat im Rahmen des privaten Verbrauchs nur einen Umsatzanteil in Höhe von $9 \%$ (Behr/Niehues 2009, S. 96). Es ist für die BuP-Branche auf lange Sicht somit von existenzieller Bedeutung, schon heute Angebote zu entwickeln, welche die derzeit jüngere Käuferschicht anspricht und so langfristig an das Thema Blumen und Pflanzen heranführt und auch bindet. Geschieht dies nicht, werden die jetzt noch relativ konstanten Umsätze der Einzelhändler zusammen mit den aktuell älteren Kunden im Laufe der Zeit verschwinden (Altmann 2010).

Neben den beiden eben angesprochenen Schwierigkeiten der BuP-Branche leiden die Händler aktuell auch an dem Problem der Veränderung der Nachfrage: Die Umsätze der Branche für Blumen und Pflanzen sind insgesamt gesehen zwar keinen großen Schwankungen unterworfen, doch branchenintern haben sich während der letzten Jahre die Umsatzanteile der einzelnen Warengruppen massiv verschoben. Beispielsweise haben Topfpflanzen in den Jahren 2000 bis 2008 ihren Anteil am Gesamtmarkt für Blumen und Pflanzen von $42 \%$ um 6 Prozentpunkte auf $48 \%$ steigern können. Parallel dazu verloren Schnittblumen an Bedeutung: Hatten sie im Jahr 2000 noch einen Anteil von knapp $41 \%$ am Gesamtmarkt für Blumen und Pflanzen, so fiel dieser bis 2008 auf gut $35 \%$, was einem Rückgang von ebenfalls 6 Prozentpunkten entspricht (Behr/Niehues 2009, S. 89f.).

Mit der Bedeutung der einzelnen Warengruppen ist auch die Bedeutung der einzelnen Einkaufsstätten innerhalb der grünen Branche vom Wandel betroffen: Wurden beispielsweise im Jahr 2000 absolut noch etwa 3,01 Milliarden Euro mit Topfpflanzen im Facheinzelhandel umgesetzt, so waren dies 2008 nur noch ca. 2,71 Milliarden Euro. Obwohl während dieses Zeitraums in Deutschland insgesamt $6 \%$ mehr Topfpflanzen verkauft wurden, hat der Facheinzelhandel bei Topfpflanzen somit Umsatzeinbußen in Höhe von $10 \% \mathrm{zu}$ beklagen (Behr/Niehues 2009, S. 92f.). Im Umkehrschluss bedeutet dies, dass sämtliche Umsatzzu- 
wächse bei Topfpflanzen außerhalb des Facheinzelhandels generiert wurden, also beispielsweise in Discountern, Baumärkten oder im Lebensmitteleinzelhandel.

Alle genannten Probleme der Branche führen zu der grundlegenden Frage, was der Kunde von $\mathrm{BuP}$ überhaupt erwartet, was er hier im Fachhandel sucht und sich wünscht. Wäre dies bekannt, und sei es nur in Ansätzen, wäre dies ein zwar nur erster, aber dennoch großer Schritt in die richtige Richtung. Die Anzahl der sich eröffnenden Handlungsoptionen für die Marktangebotsseite wäre enorm und könnten dem weiteren, erfolgreichen Fortbestand der Branche dienlich sein.

Die vorliegende Arbeit widmet sich dieser Problematik und hat zum Ziel, eine lebensstilbasierte Kundensegmentierung im Blumen- und Pflanzeneinzelhandel zu erstellen. Da diesbezüglich kaum auf bereits vorhandene Forschungsarbeiten zurückgegriffen werden kann, müssen grundsätzliche Theorien diskutiert und miteinander in Verbindung gebracht werden. So sind einerseits die aktuellen Gegebenheiten des Marktes für BuP zu bestimmen, sozialwissenschaftliche Theorien über Lebensstile zu diskutieren und die Theorien des Marketings, insbesondere die der Marktsegmentierung und die der zur Verfügung stehenden Marketinginstrumente, zu berücksichtigen. Basierend auf diesen Theorien soll ein interdisziplinäres Modell entwickelt werden, das die Kaufmotive der Konsumenten für BuP erforscht. Ziel ist es, Rückschlüsse über das Zustandekommen von Kaufentscheidungen zu erhalten, was es im Umkehrschluss ermöglicht, Handlungsempfehlungen für die Marktangebotsseite abzuleiten. Abbildung 1 zeigt den schematischen Aufbau und die verschiedenen Schwerpunkte der vorliegenden Arbeit, die primär aus einem theoretischen und einem empirischen Teil besteht.

Das folgende Kapitel zwei dient der Beschreibung der Gegebenheiten auf dem Markt für Blumen und Pflanzen. Es zeigt neben den Entwicklungen der letzten Jahre auch die aktuellen Probleme der Branche und den entsprechenden Handlungsdruck auf. Kapitel drei und vier stellen den theoretischen Schwerpunkt dieser Arbeit dar. Alle dortigen Erläuterungen haben zum Ziel, ein besseres Verständnis davon zu entwickeln, wie eine erfolgreiche Segmentierung von BuP-Kunden anhand von Lebensstilen ausgestaltet sein muss. Dabei gilt es, die grundlegenden Theorien dieser Arbeit detailliert darzulegen. Zum einen wurden über die entsprechenden Zusammenhänge im Hinblick auf BuP bislang nur sehr wenige wissenschaftliche Arbeiten verfasst, auf die zurückgegriffen werden könnte, andererseits fließen diese Theorien in der vorliegenden Arbeit in die Modellierung eines 5-Dimensionen-Schemas nach Lüdtke ein. Das 5-Dimensionen-Schema stellt dabei den Übergang von der Theorie zur Empirie dar auf ihm basieren sämtliche Überlegungen, die im empirischen Teil zum Einsatz kommen. 


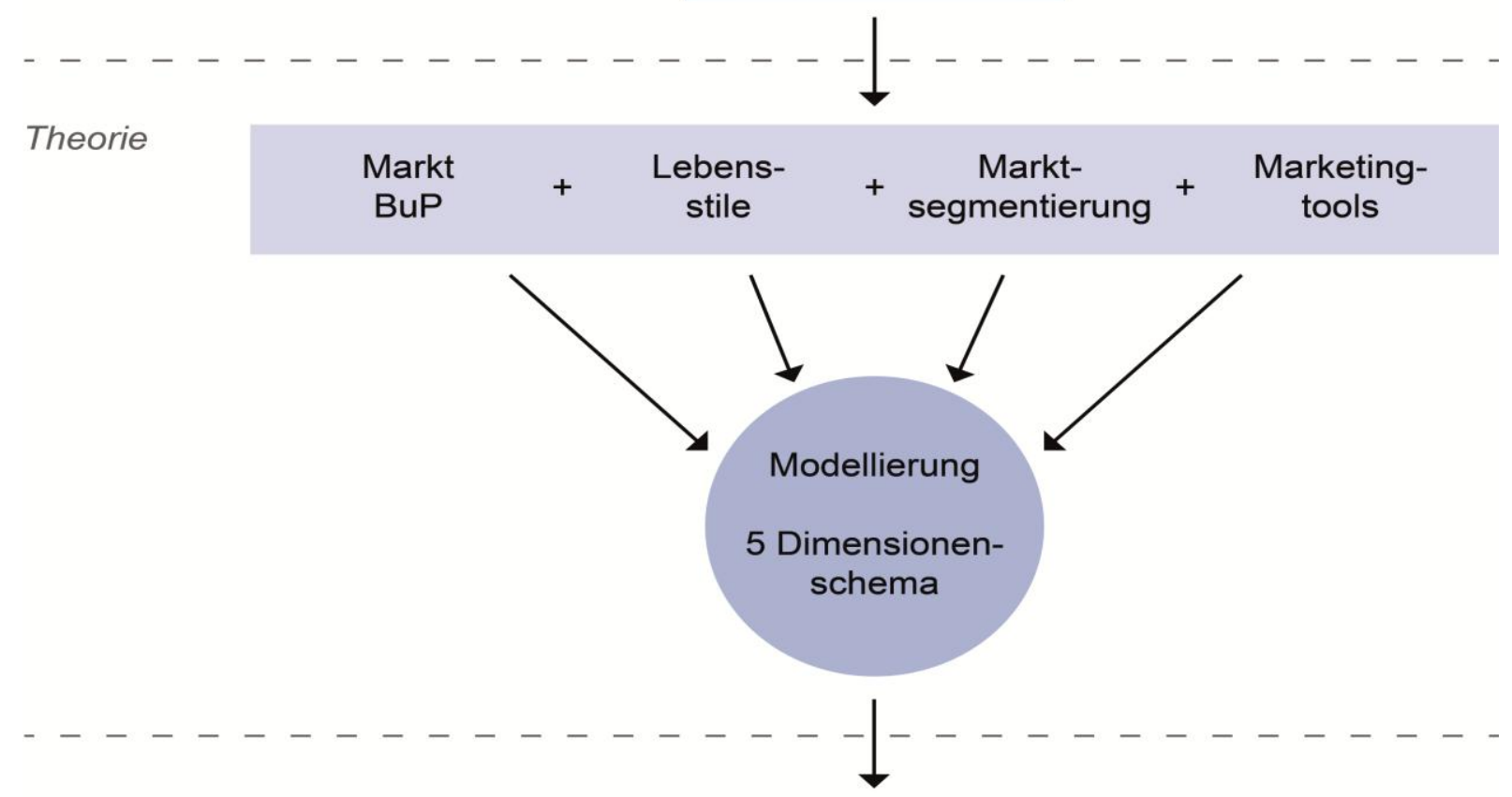

Empirie

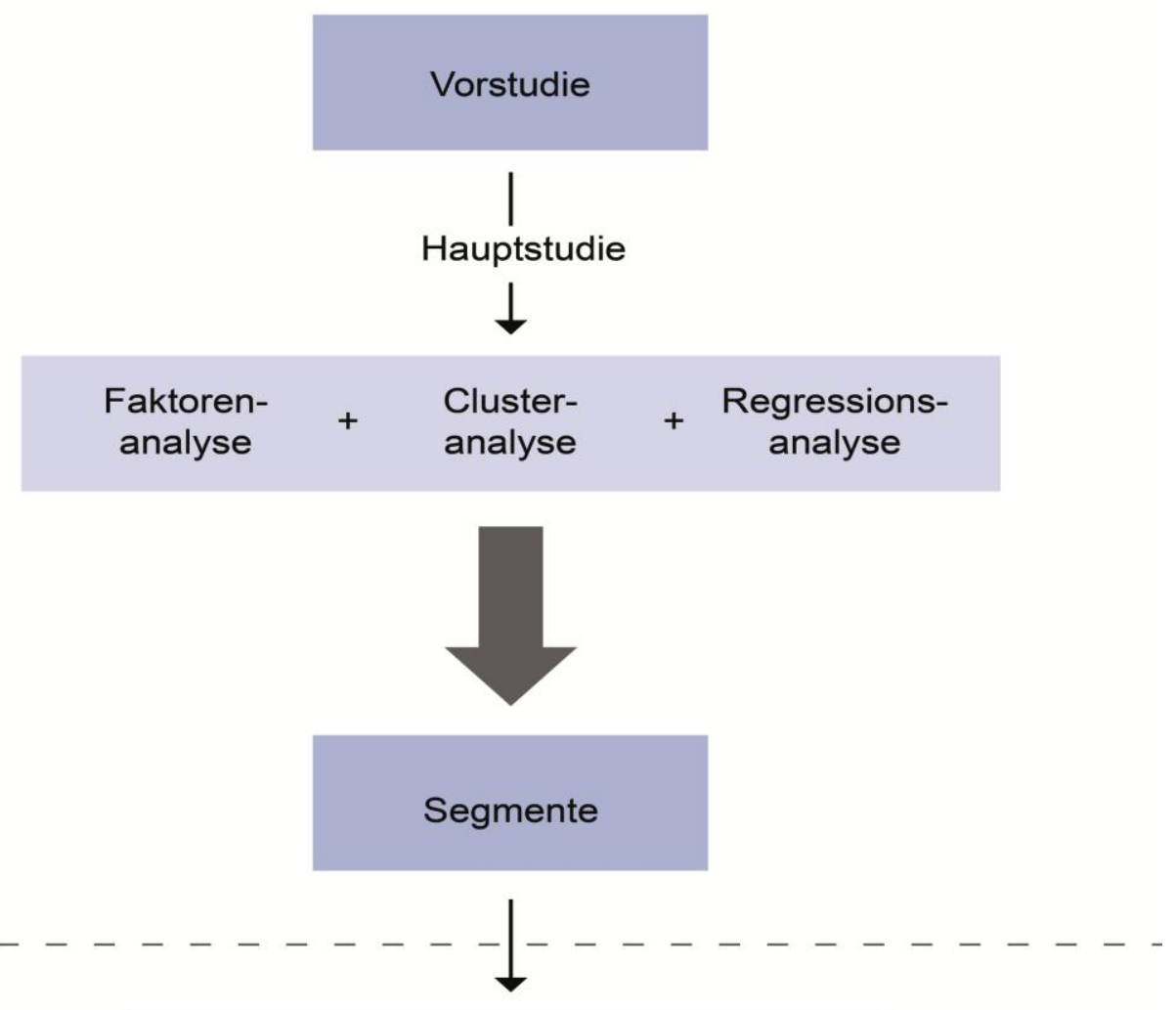

Schlussfolgerung

Implikationen für Wissenschaft und Praxis

Abbildung 1: Schematische Darstellung der Arbeit

Quelle: Eigene Darstellung 
Kapitel fünf betrachtet die durchgeführte qualitative Vorstudie. Diese hat zum Ziel, neben den theoriebasierten Erkenntnissen über den Markt für BuP auch konkrete Ansichten aus Konsumentenperspektive kennenzulernen. Kapitel sechs und sieben beschäftigen sich mit der Methodik und der Durchführung der quantitativen Hauptstudie. Dabei werden erstmals Konsumenten von BuP anhand ihrer Kaufmotive in verschiedene Gruppen bzw. Cluster eingeteilt und motivbasiert beschrieben. Weiterhin werden die Cluster nach den „clusterbildenden“ Variablen auch anhand der „clustererklärenden“ Variablen beschrieben, was deren Charakteristik komplettiert. Durch die detaillierte Beschreibung der Cluster können diese sehr gut vom Marketing bearbeitet werden. Daher werden die Cluster nach ihrer vollständigen Beschreibung als Segmente benannt. Im Rahmen der Hauptstudie kommen die Methoden der Faktorenanalyse, der Clusteranalyse und der multinominalen logistischen Regressionsanalyse zum Einsatz. Mit der folgenden Darstellung der einzelnen motivbasierten Segmente endet der empirische Teil dieser Arbeit. In Kapitel acht finden abschließende Betrachtungen über diese Arbeit statt. Diese teilen sich auf in eine rückblickende, wissenschaftliche Diskussion der in dieser Arbeit zum Einsatz gekommenen Methoden und in Handlungsoptionen für die Praxis, die auf den gewonnenen Erkenntnissen dieser Arbeit beruhen. 


\section{Das Angebot an Blumen und Pflanzen im Einzelhandel}

In diesem Kapitel soll ein Überblick darüber gegeben werden, wie sich das Angebot von Blumen und Pflanzen im deutschen Einzelhandel zusammensetzt. Hierzu wird in einem ersten Schritt zuerst der Handel bzw. der Einzelhandel auf theoretischer Basis näher betrachtet, um danach den Einzelhandel für Blumen und Pflanzen entsprechend fundierter vorstellen zu können.

\subsection{Der Handel als Intermediär}

Handelsbetriebe dienen als Bindeglied zwischen produzierenden und konsumierenden Wirtschaftsobjekten. Ihre primäre Aufgabe ist es, Ungleichheiten räumlicher, zeitlicher, qualitativer und quantitativer Art zwischen den beiden Marktseiten auszugleichen. Man spricht hierbei auch von den sogenannten „transpositorischen Grundfunktionen“ des Handels. Handelsbetriebe können an jeder Stelle zwischen Produktion und Konsumtion zwischengeschaltet sein. Sie können sich eng an die Produktionsseite anlehnen, wie der in der Landwirtschaft häufig verbreitete „Aufkaufhandel“", oder sich zwischen zwei Betrieben positionieren, die auf verschiedenen Verarbeitungsstufen an dem gleichen Produkt arbeiten („Werkstoffhandel“), oder als Handelsbetrieb fungieren, der sehr nahe an der Verbraucherseite angesiedelt ist und Fertigerzeugnisse vom Produzenten übernimmt und diese den Endverbrauchern zugänglich macht („Produktions- oder Konsumwarenhandelsbetrieb“).

Aus den transpositorischen Grundfunktionen des Handels ergeben sich weitere, untergeordnete Handelsfunktionen, wie die Sachgüterumgruppierungsfunktion, die Bedarfsanpassungsfunktion, die Marktausgleichsfunktion und die Sachgüteraufbereitungsfunktion. Die Sachgütergruppierungsfunktion lässt sich weiterhin unterteilen in die Gruppierungen nach dem Sortiment und die Gruppierung nach der Quantität. Sie sorgt somit dafür, dass aus produktionslastigen Erzeugnissen ein in Art und Menge verbraucherfreundliches Ge- und Verbrauchsgut wird. Die Bedarfsanpassungsfunktion lässt sich ebenfalls weiter in die Überbrückungs- und die Sicherungsfunktion unterteilen. Durch die Kombination einer eingekauften Ware mit einer handelstypischen Leistung werden räumliche, zeitliche oder auch finanzielle Diskrepanzen überbrückt. Typische Beispiele hierfür sind entsprechende Transport-, Lagerungs- oder Finanzierungsfunktionen des Handels. Zur Sicherungsfunktion des Handels zählen sowohl sämtliche Maßnahmen zur Wahrung der Qualität des gehandelten Gutes (Objektsicherung) als auch die Absicherung der Lieferanten vor Absatz- und Entgeltrisiken sowie 
die Absicherung der Konsumenten vor Fehlkäufen durch Beratung und Umtauschleistung (Subjektsicherung). Die Marktausgleichsfunktion sorgt, wie der Name bereits sagt, dafür, dass die angebotene Ware auch einen Käufer findet. Um Angebot und Nachfrage in Ausgleich zu bringen, zählen zur Marktausgleichsfunktion Aktivitäten wie Marktuntersuchung, Marktbeeinflussung, Umsatzakquisition und Umsatzabwicklung. Die Sachgüteraufbereitungsfunktion schließlich umfasst Maßnahmen, welche die bestehende Ware (geringfügig) umwandeln. $\mathrm{Zu}$ ihr gehören Sortierung, Mischung, Manipulation, Montage und Wartung von Waren (Berekoven 1995, S. 2 ff.; Barth 1999, S. 27 ff.).

Durch die Kombination der verschiedenen Funktionen des Handels erwirtschaften Handelsbetriebe ihre Wertschöpfung. Dabei wird ihre Leistung dem Sektor der Dienstleistungen zugerechnet, da im Handelsbetrieb keinerlei transformatorische Prozesse ablaufen, wobei die Maßnahmen der Sachgüteraufbereitungsfunktion aufgrund ihrer Geringfügigkeit ihrem Ursprung, der Distributionsfunktion, zugeordnet werden. Die Wertschöpfung der Handelsbetriebe besteht also darin, fremderstellte Sachleistungen mit selbst erbrachten Dienstleistungen zu kombinieren. Dadurch werden die wenig verbraucherfreundlichen Industrieprodukte zu wertvolleren, verwendungsreifen Ge- und Verbrauchsgütern (Barth 1999, S. 2).

Basierend auf den Grundfunktionen des Handels haben sich vielerlei spezielle Handelsbetriebsformen entwickelt, die man neben ihrer Verrichtung bzw. ihrer Funktion auch anhand ihrer Strukturmerkmale und anhand ihrer Faktorkombination voneinander unterscheiden kann. Zu den Strukturmerkmalen eines Handelsbetriebes zählt die Wahl der Betriebsform, des Warenkreises, des Umsatzverfahrens und des Standortes. Mit den getroffenen Entscheidungen, die die Struktur des Betriebs betreffen, steckt dieser gleichzeitig sein gesamtes formales Dasein ab. Daher sind die Entscheidungen, die hier getroffen werden, von langfristiger und strategischer Bedeutung, da sie nicht kurzfristig veränderbar sind.

Durch die Entscheidung, wo sich der Handelsbetrieb auf der Wertschöpfungskette zwischen Produzent und Konsument positioniert, entscheidet er sich indirekt auch für seine Betriebsform. Man unterscheidet hierbei zwischen Großhandelsbetrieben und Einzelhandelsbetrieben. Großhandelsbetriebe verkaufen ihre Ware nicht an private Letztverwender, sondern ausschließlich an Wiederverkäufer, gewerbliche Nutzer und Großabnehmer. Einzelhandelsbetriebe hingegen verkaufen ihre Waren an den privaten Letztverwender in haushaltstypischen Mengen. 
Der Warenkreis, den ein Handelsbetrieb führt, entspricht seinem angebotenen Sortiment. Er ist Ergebnis einer Auswahl an verschiedenen, zur Verfügung stehenden Waren, die einerseits den Anforderungen der Kunden und andererseits den Anforderungen des Betriebes gerecht werden müssen. Grundsätzlich kann ein breites oder ein tiefes Sortiment angestrebt werden: Ein breites Sortiment sorgt dafür, dass viele verschiedene Waren angeboten werden, wie zum Beispiel in einem Vollsortimenter im Lebensmitteleinzelhandel; ein tiefes Sortiment hingegen sorgt dafür, dass viele alternative Waren angeboten werden, beispielsweise in einem auf Kaffeemaschinen spezialisierten Geschäft.

Die Wahl des Umsatzverfahrens entspricht der Festlegung auf ein System zur Bearbeitung des Absatzmarktes und somit indirekt auch der Festlegung einer bestimmten Form der Akquise. Als mögliche Techniken zur Marktbearbeitung bzw. zur Akquise seien hier folgende Umsatzverfahren erwähnt:

- Verkäufer/-in

- teilweise Selbstbedienung

- Selbstbedienung

- Automatenverkauf

- Katalogverkauf

- Verkauf über elektronische Medien.

Das gewählte Umsatzverfahren bestimmt die Art der Akquise und somit indirekt auch den zu Grunde liegenden Betriebstyp.

Mit der Wahl des (interlokalen) Standortes entscheidet sich das Handelsunternehmen für einen geographischen Ort, an dem es seine Leistungen erbringen und anbieten will. Diese Entscheidung wird unter Abwägung von Absatz- und Kostenorientierung getroffen. Absatzorientierung liegt dann vor, wenn der Handelsbetrieb die räumliche Nähe zu seinen Abnehmern sucht, beispielsweise in einem Einkaufszentrum oder in der Fußgängerzone. Typischerweise folgen bei der Standortwahl besonders Facheinzelhandelsgeschäfte der Absatzorientierung. Ihr gegenüber steht die Kostenorientierung, die dann vorliegt, wenn ein Handelsbetrieb eine möglichst kostengünstige Fläche sucht, um so die Raumkosten möglichst gering zu halten. Dies trifft meist auf Handelsbetriebe zu, die einen großen Flächenbedarf haben, beispielsweise Großhändler mit entsprechenden Lagerflächen (Barth 1991, S. 735 ff.; Barth 1999, S. 44 ff.; Seyffert 1972, S. 146 ff.). 
Die Faktorkombination eines Handelsbetriebs setzt sich zusammen aus den Einzelfaktoren Arbeit, Ware und Betriebsmittel.

Als Teil des Dienstleistungssektors spielt auch im Handelsbetrieb die menschliche Arbeit eine ausgesprochen wichtige Rolle, stellt sie doch erst sicher, dass industrielle Sachleistungen für den Ge- oder Verbraucher in der passenden Ausgestaltung verfügbar sind. Durch die Leistungserbringung der Mitarbeiter ergibt sich für den Handelsbetrieb die Möglichkeit, sich von anderen Handelsbetrieben abzugrenzen und somit eine Unique Selling Proposition einzunehmen. Entsprechend der hohen Bedeutung des Humankapitals für einen Handelsbetrieb sind allerdings auch dessen Kosten von Gewicht: Im Jahr 2006 betrugen beispielsweise die Personalkosten (ggf. einschließlich des kalkulatorischen Unternehmerlohns) im Einzelhandel mit Uhren und Schmuck 24,8\%, im Lebensmitteleinzelhandel 13,1\%, im Textileinzelhandel 23,1 \% und im Möbeleinzelhandel 16,4\% des getätigten Umsatzes inklusive Umsatzsteuer. Die großen Unterschiede der Personalkostenquote liegen zum einen darin begründet, dass manche Branchen beratungsintensiver sind als andere, zum anderen haben sie damit zu tun, dass manche Branchen in ihrem Umsatzverfahren den Anteil der Selbstbedienung bzw. der teilweisen Selbstbedienung deutlich steigern konnten (Institut für Handelsforschung 2008).

Waren erfahren im Handelsbetrieb keinerlei Transformationen. Wie bereits beschrieben, werden die gehandelten Waren ausschließlich mit den transpositorischen Grundfunktionen des Handels „veredelt“ und erlangen so von der ab Werk gegebenen Sacheignung die für den Verbraucher entscheidende Sach- und Verwendungseignung. Die zusätzlich geschaffene Verwendungseignung stellt somit die vom Handelsbetrieb generierte Wertschöpfung dar und lässt sich monetär messen als Differenz zwischen Erlös des Handelsbetriebes und dessen Wareneinsatz. Man spricht hierbei auch vom sogenannten Rohertrag oder von der Handelsspanne. Die Handelsspanne stellt demnach das Entgelt für die vom Handelsbetrieb durchgeführten Distributionsaufgaben dar. Je nach den zu erbringenden Handelsleistungen und der Umschlagsgeschwindigkeit der gehandelten Artikel unterscheiden sich wiederum die Handelsspannen. So betrugen sie im Jahre 2011 beispielsweise im Einzelhandel mit Textilien 44,8 \%, im Einzelhandel mit Schuhen und Lederwaren 44,8 \%, im Einzelhandel mit Möbeln 39,7 \%, im Einzelhandel mit Brennstoffen $12 \%$ und im Einzelhandel mit Blumen, Pflanzen und zoologischem Bedarf 42,5 \% des jeweiligen Nettoumsatzes. Die Zahlen der Handelsspannen zeigen indirekt auch die Quoten des Wareneinsatzes der verschiedenen Branchen. Gerade bei Handelsbetrieben ist dieser besonders hoch und typischerweise der größte Kostenblock (IBH Retail Consults 2010; Barth 1999, S. 65 ff.). 
Betriebsmittel eines Handelsbetriebes sind alle Sachgegenstände, die abgesehen von der Handelsware zur Erbringung der Handelsleistung erforderlich sind. Hierzu zählen beispielsweise der Fuhrpark, technische Hilfsgeräte wie Hubwagen oder Einwickelautomaten, Regale bzw. die Einrichtung, Kassensysteme und auch die Räumlichkeiten, in denen die Handelsleistung erbracht wird. Da die Raumkosten neben den Personalkosen meist die zweitgrößten Betriebskosten eines Handelsbetriebes darstellen, sind sie für die ökonomische Bewertung eines Handelsbetriebes von besonderer Bedeutung. Neben dem bereits angesprochenen Problem der interlokalen Standortwahl (Absatz- oder Kostenorientierung) muss eine geeignete Entscheidung über den intralokalen Standort getroffen werden. Darunter wird die räumliche Anordnung der im Handelsbetrieb eingesetzten Produktionsverfahren verstanden. Dies liegt darin begründet, dass der Raum sowohl die Präsentations- und Kontaktfunktion als auch die Lager- und Verwaltungsfunktion erfüllen muss. Eine erfolgreiche intralokale Standortwahl spiegelt sich in diversen Raumleistungskennzahlen wider, beispielsweise im erzielten Umsatz pro Quadratmeter benötigten Raumes. Dieser lag im Jahr 2006 beispielsweise für den deutschen Einzelhandel allgemein bei $3.300 € / \mathrm{m}^{2}$, im deutschen Textileinzelhandel im Jahr 2004 bei $2.000 € / \mathrm{m}^{2}$, im Lebensmitteleinzelhandel im Jahr 1995 bei $4.850 € / \mathrm{m}^{2}$ und im Jahr 2005 bei $4.350 € / \mathrm{m}^{2}$ (Pirner 2010, S. 66; Lerchenmüller 2003, S. 85; Kreimer/Gerling 2010, S. 19; Reuter/Blee 2006; EHI Retail Institut e. V. 2010).

Letztendlich zeigt sich die jeweilige Erscheinungsform eines Handelsbetriebes im dreidimensionalen Raum zwischen den Verrichtungsarten, den Strukturmerkmalen und den Faktorkombinationen (Barth 1999, S. 25).

\subsection{Betriebstypen des Einzelhandels}

In Kapitel 2.1 wurde bereits im Rahmen der Strukturmerkmale auf die verschiedenen Betriebsformen von Handelsbetrieben eingegangen. Diese Betriebsformen wurden je nach Lage auf der Distributionskette zwischen Produzent und Konsument in Groß- und Einzelhandelsbetriebe unterteilt. Da allerdings auch innerhalb der gleichen Betriebsform viele verschiedene Erscheinungstypen von Handelsbetrieben zu beobachten sind, ist es sinnvoll, sie weiter in verschiedene „Betriebstypen“ zu untergliedern. Dabei wird in diesem Kapitel thematisch bedingt ausschließlich auf die Betriebsform des Einzelhandels näher eingegangen (weiterführende Detaillierungen über die Betriebsform Großhandel finden sich bei Barth oder Lerchmüller). 
Um die Einzelhandelsbetriebe weiter zu unterteilen, werden sie anhand verschiedener Ausprägungen differenziert. Diese Ausprägungen sind sowohl langfristige installierte Kombinationen von Strukturmerkmalen als auch taktisch variierbare Instrumentalmerkmale. Sie setzen sich nach Barth folgendermaßen zusammen:

I. Langfristige Strukturmerkmale

1. Betriebsform

2. Warenkreis

3. Umsatzverfahren

4. Standort

5. Verkaufsfläche

II. Kurzfristige Instrumentalmerkmale

1. Preispolitik aktiv oder passiv

2. Qualitätspolitik hohes, mittleres oder niedriges Niveau

3. Dienstleistungspolitik hohes oder niedriges Angebot

4. Distributionspolitik Monosystem oder Polysystem.

Für die Betrachtung des Einzelhandels ist die Unterteilung der Distributionspolitik in Monound Polysystem besonders geeignet, da sich dadurch auch Filialbetriebe systematisch erfassen lassen. Dennoch muss kritisch beachtet werden, dass eine solche Typologie im Laufe der Zeit immer wieder den Gegebenheiten der realen Handelswelt angepasst werden muss. Durch den fortwährenden Wandel der Konsumentenwünsche und -ansprüche sind die Einzelhändler stets gezwungen, sowohl ihre Instrumentalmerkmale als auch ihre Strukturmerkmale neu zu überdenken und gegebenenfalls neu zu installieren (Barth 1999, S. 81 ff.).

Anhand des obigen Schemas lassen sich gemäß dem Ausschuss für Begriffsdefinitionen aus der Handels- und Absatzwirtschaft des Instituts für Handelsforschung in Köln etwa 40 verschiedene Typen an Einzelhandelsbetrieben identifizieren (Institut für Handelsforschung 1995). Im Folgenden werden nur die drei Typen näher betrachtet, die im Kontext dieser Arbeit als am interessantesten erscheinen. Sie sind die bedienungsorientierten Einzelhändler, die discountierenden Einzelhändler und der Versandhandel. 


\section{Bedienungsorientierte Einzelhändler}

$\mathrm{Zu}$ den bedienungsorientierten Betriebstypen des Einzelhandels gehören die Fachgeschäfte, die Spezialgeschäfte, die Warenhäuser, die Kaufhäuser und die neu aufkommenden Convenience Stores.

Fachgeschäfte sind Einzelhandelsgeschäfte einer bestimmten Branche, beispielsweise Textil-, Möbel- oder Schmuckwarengeschäfte. Sie führen ein sowohl breites als auch tiefes Sortiment und bieten umfangreiche Dienstleistungen an, zu denen auch eine intensive Kundenberatung gehört. Um eine intensive Kunden- und Verkaufsberatung sicherstellen zu können, ist in Fachgeschäften entsprechend geschultes und ausgebildetes Personal erforderlich, was wiederum zu einer hohen Personalaufwandsquote führt. Zusätzlich dazu führen Fachgeschäfte neben den gängigsten Artikeln auch Artikel mit geringerer Umschlagshäufigkeit, um sich so von Nicht-Fachgeschäften abzuheben. Dies führt ebenfalls zu höheren Kosten (Lager, Finanzierung, Pflege, Schwund, Verderb), welche die umsatzstarken Artikel zum größten Teil kompensieren müssen. Das typische Fachgeschäft zeichnet sich somit auch dadurch aus, dass es Mischkalkulation und eine passive Preispolitik betreibt.

Spezialgeschäfte sind Einzelhandelsgeschäfte, die nur einen Teil des Angebots eines Fachgeschäftes anbieten, beispielsweise ein Spezialgeschäft nur für Hemden oder nur für Sofas. Aufgrund des noch tieferen Sortiments und des ausgeweiteten Dienstleistungsangebots im Vergleich zum Fachgeschäft sind hier entsprechend noch besser ausgebildete und geschulte Mitarbeiter notwendig. In gleicher Weise verhält es sich mit den Kosten, die für die noch geringere Umschlagshäufigkeit der Spezialartikel anfallen. Entsprechend den hohen Kosten sind Spezialgeschäfte meist in hohen Preisregionen angesiedelt.

Warenhäuser sind großflächige Einzelhandelsbetriebe, die den Ansatz verfolgen, den Kunden „alles unter einem Dach“ zu bieten. Warenhäuser sind in verschiedene Abteilungen untergliedert, die den wichtigsten Branchen der Konsumgüter entsprechen, beispielsweise Sport-, Haushalts-, Lebensmittel- oder Elektronikabteilung. Neben dem One-Stop-Shopping bieten Warenhäuser ein sowohl breites als auch tiefes Sortiment. Abhängig vom Standort des Warenhauses variieren die Preisniveaus. Je nach Kaufkraft des relevanten Marktes wird dies entweder direkt oder indirekt über das Qualitätsniveau den Marktgegebenheiten angepasst.

Kaufhäuser ähneln den Warenhäusern in ihrer Größe und Organisation, weisen allerdings eine größere Branchenhomogenität auf als die Warenhäuser. In Kaufhäusern ist meist nur eine einzige Branche vertreten, beispielsweise die Branche für Bekleidung. 
Die in Deutschland neu aufkommenden Convenience Stores sind besonders bei Berufstätigen und jüngeren Käufern beliebt. Der Convenience Store zeichnet sich dadurch aus, dass er kleinflächig ist und ein breites und flaches Sortiment an Konsumgütern des täglichen Bedarfs mit hoher Umschlagshäufigkeit führt. Typisches Beispiel für Convenience Stores sind die Shops an Tankstellen oder die 7-Eleven Shops aus dem englischsprachigen Raum. Convenience Stores stellen die Einkaufsbequemlichkeit für den Kunden heraus, weniger eine aktive und aggressive Preispolitik. Voraussetzung für das Funktionieren eines Convenience Stores ist eine sehr hohe Kundenfrequenz. Diese sorgt dafür, dass trotz der kleinen Summen pro Einkauf eine hohe Umschlagshäufigkeit der Waren erreicht wird und somit die Kosten niedrig bleiben (Barth 1999, S. 86 ff.).

\section{Discountierende Einzelhandelsbetriebe}

Discountierende Einzelhandelsbetriebe zeichnen sich durch eine Kombination verschiedener Schwerpunkte aus: Sie führen ein Sortiment, das ausschließlich auf eine hohe Umschlagshäufigkeit ausgerichtet ist, bieten nahezu keinerlei Dienstleistungen an, verlangen einen hohen Grad an Selbstbedienung und geben die dadurch niedrig gehaltenen Kosten durch eine sehr aggressive Niedrigpreispolitik an die Kunden weiter. Durch den hohen Grad der Selbstbedienung ist im discountierenden Einzelhandel der Kunde selbst zum „Mitarbeiter“ des Geschäfts geworden, da er selbst viele Aufgaben übernimmt, die in einem bedienungsorientierten Einzelhandelsbetrieb eine angestellte Person erledigt, wie beispielsweise die Beratung oder das Vergleichen von Produkten. Der Produktionsfaktor Kunde wird im discountierenden Betrieb durch niedrige Preise entlohnt. $\mathrm{Zu}$ den wichtigsten discountierenden Einzelhandelsbetrieben zählen der Supermarkt, der Verbrauchermarkt, und der Fachmarkt.

Ein Supermarkt führt hauptsächlich Nahrungs- und Genussmittel sowie Gebrauchsgüter des täglichen Bedarfs. Dabei setzen Supermärkte als Nahversorger auf die Frische ihrer Produkte. Teilweise führen Supermärkte auch zeitlich befristete Aktionen mit Non-Food-Artikeln des nicht täglichen Bedarfs durch, beispielsweise mit PCs, Fahrkarten für die Deutsche Bahn oder auch mit bestimmten Möbelstücken. Die Verkaufsfläche eines Supermarktes beträgt zwischen $100 \mathrm{~m}^{2}$ und $1.000 \mathrm{~m}^{2}$, als Standort sind sie meist in oder zumindest in der Nähe guter Wohnlagen zu finden. Typische Beispiele sind Edeka, Rewe, Aldi oder Lidl.

Der Verbrauchermarkt entspricht im Kern dem Supermarkt, allerdings bieten Verbrauchermärkte einen größeren Warenkreis als Supermärkte und benötigen daher auch mehr Fläche. 
Verbrauchermärkte haben eine Verkaufsfläche von mindestens $1.000 \mathrm{~m}^{2}$, daher sind sie wegen der Raumkosten oftmals in preisgünstigen Lagen des Stadtrandes oder ,auf der grünen Wiese“ zu finden. Aufgrund der erschwerten Erreichbarkeit der Verbrauchermärkte bieten sie im Gegenzug ihren Kunden eine Vielzahl an kostenfreien Stellplätzen. Typische Beispiele hierfür sind E-Center, Kaufland oder Real.

Der Fachmarkt ist ein Einzelhandelsgeschäft, in dem ein weites, bedarfsorientiertes Sortiment mit einem vergleichsweise hohen Servicegrad kombiniert wird. Fachmärkte führen hauptsächlich Non-Food-Sortimente und setzen dabei ebenfalls auf das Prinzip der Selbstbedienung, wobei dem Kunden im Bedarfsfall eine qualifizierte Beratung kostenfrei zur Verfügung steht. Das widerspricht zwar dem kostenminimierenden Ansatz der discountierenden Betriebe, dennoch sind Fachmärkte tendenziell eher diesen als den Fachgeschäften zuzuordnen. Typische Beispiele für Fachmärkte sind Fressnapf, Media Markt, Saturn oder Charles Vögele Moden (Barth 1999, S. 88 ff.).

\section{Versandhandel}

Der Versandhandel unterscheidet sich sehr stark von den beiden bisher vorgestellten Betriebstypen. Während sowohl beim bedienungsorientierten als auch beim discountierenden Einzelhandel der direkte Kundenkontakt gegeben war, ist dies hier nicht mehr der Fall: Der Versandhandel tritt nur noch indirekt mit seinen Kunden in Kontakt und betreibt Distanzhandel. An die Stelle des physischen Ladengeschäfts tritt beim Versandhandel der Katalog, der die angebotenen Waren beschreibt und anbietet. Dabei kann der Katalog sowohl klassisch in gedruckter Form als auch elektronisch im Internet zur Verfügung gestellt werden. Der Versandhandel bietet somit seinen Kunden den Vorteil, nicht an Ladenöffnungszeiten gebunden zu sein und jederzeit an jedem Ort zur Verfügung zu stehen. Kauf- bzw. Bestellvorgänge können vom Kunden telefonisch, per Post oder via Datenübertragung ausgelöst werden. Im letzteren Fall spricht man von eBusiness oder eCommerce, also dem webbasierten Einkauf von Waren. Im Versandhandel unterscheidet man zwischen dem Spezialversandhandel mit Waren aus einer Branche und einem tiefen Sortiment und dem Universalversandhandel, der ein breites Sortiment aus vielen verschiedenen Branchen führt.

Betriebswirtschaftlich zeichnet sich der Versandhandel dadurch aus, dass er die Standortauswahl allein von den anfallenden Kosten abhängig machen kann und der „Störfaktor“ Kunde innerhalb des Versandhandelsbetriebes von den Informations- und Warenströmen ferngehal- 
ten werden kann. Entsprechend hoch ist der Automatisierungsgrad im Versandhandel, was wiederum zu einer günstigen Kostenstruktur der Versandhändler führt. Beispiele für Spezialversandhändler sind Conrad Electronic oder Sportscheck, für Universalversandhändler der Otto-Versand oder der ehemalige Quelle-Versand.

\subsection{Systembildung im Einzelhandel}

Der hohe Wettbewerbsdruck auf den gesättigten Märkten verlangt von jeglichem Distributionssystem bzw. jedem Einzelhändler, nicht nur möglichst effizient, sondern zudem auch innovativ zu sein. Infolgedessen entstehen immer mehr bzw. immer größere Verbunde von Einzelhändlern, sogenannte Handelssysteme, die von einer zentralen Stelle aus koordiniert und organisiert werden.

Die Zentralen der Verbundsysteme des Einzelhandels folgen dabei dem ökonomischen Gedanken der Größenvorteile, der sogenannten Skalenerträge, die den einzelnen Betrieben des Verbunds jeweils Vorteile sowohl auf der Beschaffungs- als auch auf der Absatzseite sichern sollen. Neben dem Ausnutzen der Größenvorteile der gebündelten Marktmacht auf Beschaffungs- und Absatzseite führen Handelssysteme einerseits auch zu Standards bei innerbetrieblichen Arbeits- und Verwaltungsabläufen, was wiederum zu einzelbetrieblichen Kostensenkungen führt. Andererseits lassen sich in Handelssystemen auch die Kosten absatzpolitischer Instrumente besser verteilen, da diese nur einmal geplant werden und jedes Mitglied des Verbundes diese nutzen kann oder ggf. sogar nutzen muss. Im Folgenden werden die drei gängigsten Handelssysteme in Deutschland vorgestellt: das Filialsystem, das Franchisesystem und das Verbundsystem.

Filialsysteme sind grundsätzlich für alle Betriebstypen des Einzelhandels möglich. Typischerweise sind Filialsysteme hierarchisch strukturiert und bestehen aus der übergeordneten Organisationseinheit „Zentrale“ und den einzelnen Filialen, die anhand einer zentral durchgeführten Standortplanung geographisch an unterschiedlichen Standorten angesiedelt und von der Zentrale aus einheitlich geführt und gesteuert werden. Filialsysteme kombinieren somit eine zentrale Organisation mit einem dezentralen Absatz. Indem in Filialsystemen die Zentrale den einzelnen Filialen alle wichtigen Entscheidungen abnimmt, besteht die Möglichkeit, systemweit geltende Standards zu setzen und dadurch besonders große Skalenerträge zu erzielen. Dies gilt sowohl auf der Beschaffungs- und Absatzseite als auch bei den innerbetrieblichen Abläufen. Nachteilig an einer strikten Gleichschaltung aller Filialen wirkt sich allerdings 
aus, dass es nicht möglich ist, durch eine individuelle Filialsteuerung auf besondere Gegebenheiten des relevanten Marktes einer jeden einzelnen Filiale einzugehen.

Grundsätzlich bestehen für ein Filialsystem zwei Arten von Distributionsmöglichkeiten: die Monosystempolitik oder die Polysystempolitik. Monosystem bedeutet, dass das Filialnetz ausschließlich aus einem einheitlichen Betriebstyp besteht und in jeder Filiale stets das gleiche Sortiment geführt wird. Durch die Festlegung auf nur eine, zudem einheitlich geführte Vertriebsschiene bestehen für Monosystemunternehmen lediglich begrenzte Möglichkeiten für Wachstum und Marktdurchdringung. Typisches Beispiel für ein Monosystemunternehmen ist ALDI. Polysystemunternehmen setzen hingegen in ihrer Strategie für Wachstum und Marktdurchdringung auf mehrere, unterschiedliche Vertriebsschienen. Entsprechend können die verschiedenen Vertriebsschienen entweder in einer einzigen Branche angesiedelt sind, z. B. Lidl und Kaufland im Lebensmitteleinzelhandel, oder in mehreren Branchen mit jeweils einem einzigen Betriebstyp, z. B. die zum Tengelmann-Konzern gehörenden Filialisten Obi, KIK und Kaiser's, oder in mehreren Branchen mit mehreren Betriebstypen pro Branche, wie beispielsweise die zur Metro AG gehörenden Elektro-Filialisten Media Markt und Saturn und die ebenfalls zur Metro AG gehörenden Lebensmitteleinzelhandelsfilialisten real und extra (wobei extra im Jahr 2008 an REWE verkauft wurde). Ziel der Polysystempolitik ist es, in jeder Branche verschiedene Kundensegmente anzusprechen und somit eine größere Marktdurchdringung zu erreichen. Dabei hängen die generierbaren Skalenerträge der Polysystempolitik sehr stark davon ab, inwieweit die Warenkreise der einzelnen Vertriebsschienen deckungsgleich sind (Barth 1999, S. 100 ff.).

Bei Franchisesystemen erlaubt ein Franchisegeber einem Franchisenehmer die Nutzung seines bestehenden Geschäftskonzeptes gegen ein bestimmtes Entgelt. Dabei bleiben beide Seiten als rechtlich selbständige Unternehmen bestehen. Franchisesysteme sind die am weitesten verbreitete Form der Kooperationsmöglichkeiten auf unterschiedlichen Ebenen der Handelsstufen (vertikale Kooperation). Man spricht dabei auch vom System der „Vertragshändler““. Franchisesysteme bestechen einerseits dadurch, dass sich alle Franchisenehmer sowohl nach innen als auch nach außen einheitlich darstellen (z. B. einheitliches Marketing und einheitliche Preise), und andererseits dadurch, dass sich alle Franchisenehmer ins gleiche Distributionssystem eingliedern, das von Franchisesystem-Fremden nicht genutzt werden darf. Durch die standardisierte Form der einzelnen Franchise-Betriebsstätten können in jeglicher Hinsicht große Skalenerträge generiert werden. 
Die Zentrale des Franchisesystems übernimmt dabei die Führungsposition des Systems und erledigt für die Franchisenehmer die organisatorischen und administrativen Aufgaben, so dass sich diese besser auf ihre wesentlichen Aufgaben konzentrieren können. Im Gegenzug dafür erhält die Zentrale von allen Franchisenehmern Warenbelieferungsverträge, die somit bei der Zentrale die geballte Nachfrage aller einzelnen Franchisenehmer bündeln. Typische Beispiele für Franchise-Systeme sind McDonald's aus der Systemgastronomie, Accor aus der Hotelbranche oder Fressnapf aus der Branche der Tiernahrung (Barth 1999, S. 103 f.).

Verbundsysteme sind Zusammenschlüsse kleinerer und mittlerer selbständiger Einzelhändler zu einer Gruppe, um gemeinsam den großen Filialunternehmen mit ihren Größenvorteilen entgegenwirken zu können. Verbundsysteme haben aufgrund ihrer Zusammensetzung stets mit dem Problem zu kämpfen, dass die Interessen des Verbundes, die hauptsächlich in der Generierung von Größenvorteilen bestehen, teilweise den Interessen einzelner Verbundmitglieder entgegengesetzt sind, beispielsweise bei der Sortimentsgestaltung. Der Funktionskopf eines Verbundes muss dabei stets das Wohl des gesamten Verbundes im Blick haben, da meist nur das Überleben des Verbundes auch das Überleben der einzelnen kleinen und mittleren Mitglieder sicherstellt. Verbundsysteme werden unterschieden nach „horizontalem“ und „vertikalem“ Verbund.

Horizontale Verbundsysteme basieren auf Kooperationen von verschiedenen, selbständigen Einzelhändlern der gleichen Branche, die das Ziel verfolgen, durch die Bündelung ihrer Nachfrage günstigere Beschaffungswege zu erreichen. Neben dem Ziel der besseren Einkaufskonditionen kann der horizontale Verbund allerdings auch das Ziel der Sortimentsbündelung verfolgen. Durch die zwischenbetriebliche Kooperation von Einzelhändlern aus verschiedenen Branchen entsteht mit dem Verbund in den Augen des Kunden ein großer, „monolithischer“ Anbieter mit sowohl tiefem als auch breitem Sortiment. Sind alle Kooperationspartner des Verbundes am gleichen Standort, so bietet der Verbund den Kunden die Möglichkeit des „One-Stop-Shoppings“.

Vertikale Verbundsysteme sind Verbundsysteme, die sich über mehrere Stufen der Handelsebene erstrecken. Je nachdem ob der Einzel- oder der Großhandel die Bemühungen zur Kooperation anstößt, spricht man von Vorwärts- oder Rückwärtsintegration. Vertikale Verbundsysteme sollen den eigenständigen Groß- und Einzelhändlern ebenfalls die Größenvorteile entlang der Wertschöpfungskette verschaffen, die große Filialunternehmen schon haben. Vertikale Verbundsysteme können nochmals unterteilt werden in Einkaufsverbände und in freiwillige Ketten. 
Die Gründung von Einkaufsverbänden basierte auf der Idee, durch eine Bündelung der Nachfrage kleinen und mittleren Einzelhändlern bessere Konditionen im Einkauf zu verschaffen. Heutzutage sind aus vielen dieser Einkaufsverbände oder Einkaufsgenossenschaften Handelskonzerne geworden, die neben dem Einkauf für ihre „Mitglieder“ noch viele weitere verbandsrelevante Aufgaben wahrnehmen, wie beispielsweise Marktuntersuchungen, Standortanalysen, gemeinsame Werbung oder das Entwickeln von eigenen Handelsmarken. Typisches Beispiel hierfür ist die Gruppe EDEKA, deren Name für „Einkaufsgenossenschaft deutscher Kolonialwarenhändler“" steht.

Freiwillige Ketten oder ,freiwillige Filialbetriebe“ sind im Gegensatz zu Einkaufsverbänden nicht von Einzelhändlern, sondern von Großhändlern ins Leben gerufen worden, die eine Vorwärtsintegration durchführen wollten. Ziel dieser Vorwärtsintegration war es wiederum, die Größenvorteile der großen Filialbetriebe durch den freiwilligen Zusammenschluss zu kopieren. Dass die einzelnen Mitglieder der freiwilligen Ketten dabei selbständige Unternehmer sind und bleiben, ist Vor- und Nachteil zugleich: Einerseits sind haftbare Unternehmer meist motivierter als angestellte Filial- oder Marktleiter, andererseits besteht für den Verbund der freiwilligen Ketten dadurch die Gefahr, dass sich die gemeinsamen Interessen des Verbundes und einzelne Interessen von Verbundmitgliedern konträr zueinander verhalten.

\subsection{Der Markt für Blumen und Pflanzen}

Der Gesamtmarkt für Blumen und Pflanzen setzt sich aus folgenden drei Teilbereichen zusammen: Schnittblumen, Topfpflanzen, und Gehölze, wobei zu den genannten Teilbereichen noch weitere Untergruppen zählen, wie Abbildung 2 verdeutlicht.

Die aktuelle Situation auf dem Markt für Blumen und Pflanzen in Deutschland ist allgemein von den Charakteristika eines gesättigten Marktes geprägt. Dies zeigt sich am deutlichsten an den Umsatzzahlen der Branche: Die Entwicklung des Gesamtmarktumsatzes (Summe des privaten und institutionellen Verbrauchs, bewertet zu Einzelhandelspreisen) für Zierpflanzen, worunter im weiteren Verlauf dieser Arbeit die Summe aller oben genannten Teilbereiche verstanden werden soll, zeigt sich sehr schleppend und uneinheitlich (vgl. dazu unten Abbildung 3). 


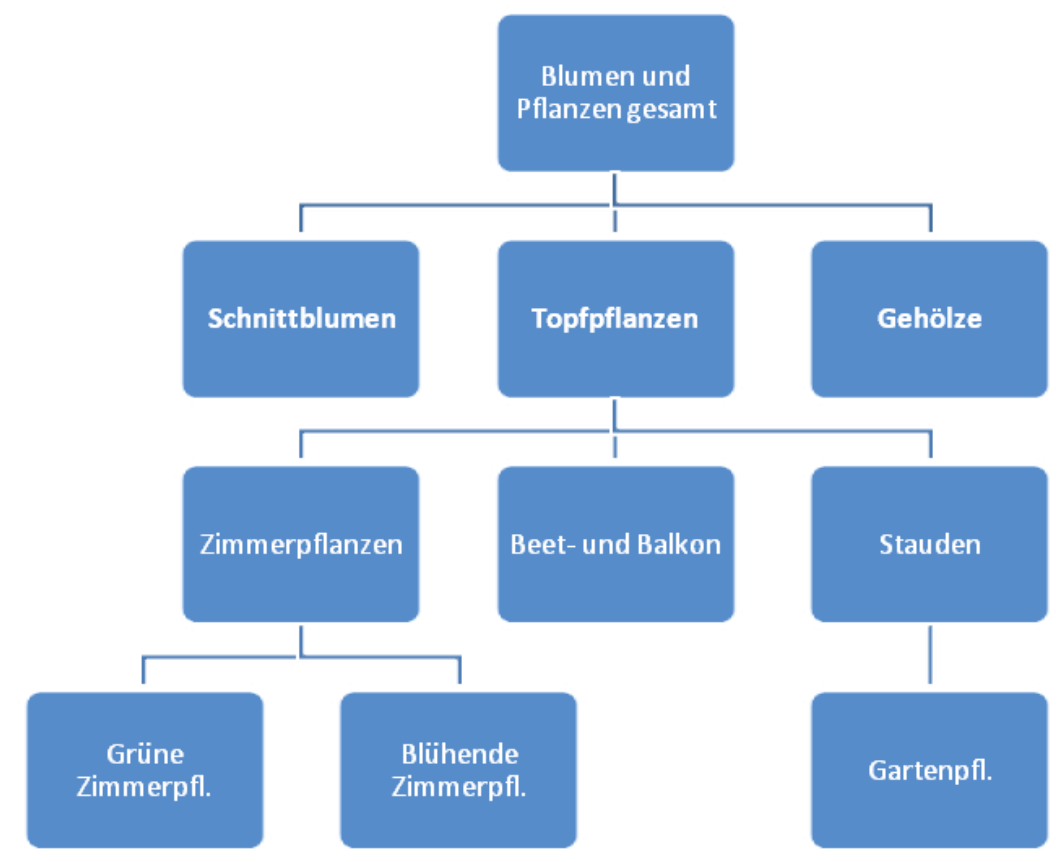

Abbildung 2: Der Markt für BuP

Quelle: Eigene Darstellung

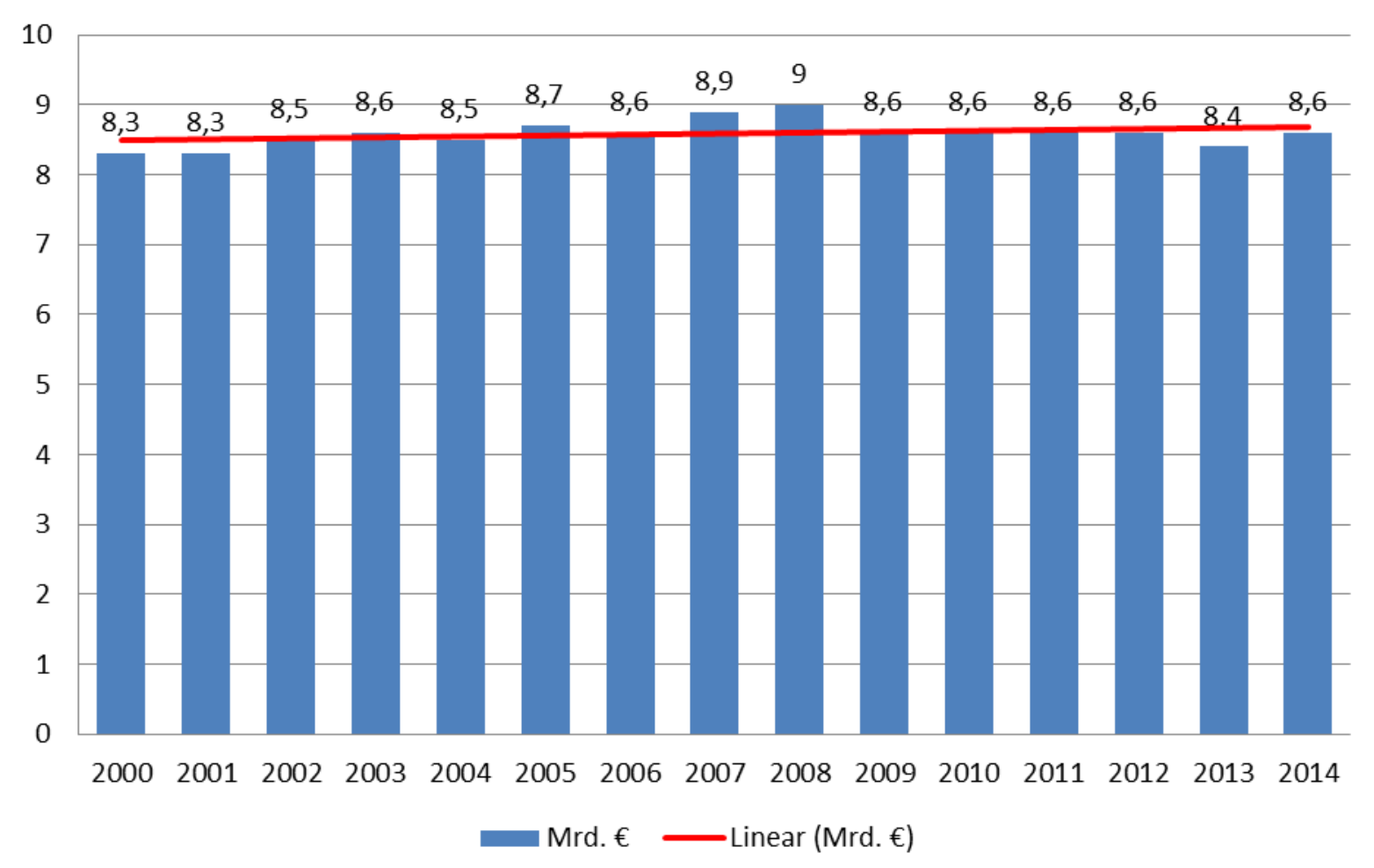

Abbildung 3: Entwicklung Gesamtumsatz Blumen und Pflanzen zu Einzelhandelspreisen.

Quelle: Eigene Darstellung nach Angaben von Behr/Niehues 2009, S. 89; Behr 2010; Behr 2015; Altmann 2010, S. 3; Bednarsky 2010; Lange 2009, S. 43; Statistisches Bundesamt 2015 
Zwischen den Jahren 2000 bis 2014 entwickelte sich der Markt zwar sehr langsam, aber dennoch positiv. Im Schnitt betrug das Marktwachstum während dieser Periode etwa $+0,75 \%$ pro Jahr. Im Jahr 2009 allerdings fiel der Umsatz (alle Zahlen zu Einzelhandelspreisen), primär durch die Weltwirtschafts- und Finanzkrise bedingt, von 8,96 Mrd. Euro in 2008 auf 8,57 Mrd. Euro, was einem prozentualen Rückgang von ca. 4,4 \% entspricht. Im Jahr 2014 wurde ein hochgerechneter Branchenumsatz von ca. 8,6 Mrd. Euro erreicht (Stand: Prognose April 2015), was in etwa dem Stand von 2009 entspricht. De facto hat sich die Blumen-und Pflanzenbranche seit dem Umsatzeinbruch im Jahr 2008/2009 nicht mehr weiterentwickelt. Sie steckt in einer Phase der Stagnation, wie die rot eingefärbte Trendlinie in Abbildung 3 zeigt.

Während die BuP-Branche stagniert, hat sich ihre Umwelt dynamisch weiterentwickelt, so auch der sogenannte Verbraucherpreisindex. Die Statistik des Statistischen Bundesamtes sagt aus, dass der Index zur Mitte des Jahres 2000 noch bei 85,7 Punkten lag (Basisjahr $2010=$ 100 Punkte) und zur Mitte des Jahres 2014 bei 106,6 Punkten. Dies entspricht einer allgemeinen Preissteigerungsrate von ca. 21,8 \% während dieses Zeitraums. Wäre der Markt für Blumen und Pflanzen proportional zu den Preissteigerungen gewachsen, müsste er für das Jahr 2014 einen Gesamtumsatz in Höhe von ca. 10,1 Mrd. Euro aufweisen. Der Vergleich von Branchenwachstum und Preisindexsteigerung legt ein reales Branchenschrumpfen offen. Rechnet man den Branchenumsatz auf die Einwohner Deutschlands um, so lagen die ProKopf-Ausgaben für Zierpflanzen im Jahr 2009 bzw. 2014 bei 104,00 Euro pro Person (Behr/Niehues 2009, S. 89; Behr 2010; Behr 2015; Altmann 2010, S. 3; Bednarsky 2010; Lange 2009, S. 43; Statistisches Bundesamt 2015).

\subsubsection{Das Angebot für Blumen und Pflanzen}

Das inländische Angebot an Zierpflanzen stammt nur zu einem bestimmten Teil aus inländischer Produktion. Dabei variiert der „Selbstversorgungsgrad“ in Deutschland zwischen den einzelnen Zierpflanzengruppen sehr stark.

In Deutschland existieren etwa 9.500 Gartenbaubetriebe, die Zierpflanzen produzieren, wovon ca. $62 \%$ reine Zierpflanzenbetriebe sind. Dabei bewirtschaften alle Gartenbaubetriebe zusammen etwa 7.150 Hektar Produktionsfläche, die sich zu etwa zwei Dritteln im Freiland und zu ca. einem Drittel unter Glas befinden. 
Anhand der Daten zu den Jahresdurchschnittspreisen der deutschen Landgard und der VBN (Vereinigte Blumenversteigerungszentralen der Niederlanden) ergab sich im Jahr 2005 ein Wert der Zierpflanzenproduktion in Deutschland in Höhe von ca. 1,9 Mrd. Euro zu Erzeugerpreisen. Der Produktionswert von Schnittblumen lag 2005 bei 239 Mio. Euro, der von Topfpflanzen (inkl. Stauden, aber ohne Gehölze) bei ca. 1,05 Mrd. Euro, Beet- und Balkonpflanzen lagen wertmäßig bei 560 Mio. Euro und Zimmerpflanzen bei 320 Mio. Euro.

Wertmäßig erreichte die deutsche Blumen- und Pflanzenbranche damit im Jahr 2008 einen Selbstversorgungsgrad in Höhe von ca. 50 \%. Der Selbstversorgungsgrad (SVG) setzt die inländisch produzierten Werte ins Verhältnis zum Wert aller angebotenen Waren und berechnet sich nach der Formel

$$
\mathrm{SVG}=(\mathrm{IP} / \mathrm{IP}+(\mathrm{I}-\mathrm{E})) \times 100
$$

wobei IP für die inländische Produktion zu Erzeugerpreisen, I für die Importe und E für die Exporte steht.

Auf die einzelnen Zierpflanzenarten bezogen variiert der SVG in Deutschland allerdings sehr stark. Er liegt bei $84 \%$ für Stauden, bei $76 \%$ für Beet- und Balkonpflanzen, bei $61 \%$ für Topfpflanzen, bei $41 \%$ für Zimmerpflanzen und bei $19 \%$ für Schnittblumen. Der Rest stammt aus Importen. Der Wert der Importe an Zierpflanzen lag im Jahr 2008 bei 2,31 Mrd. Euro, wovon ca. $48 \%$ auf Schnittblumen, $36 \%$ auf Topfpflanzen und $10 \%$ auf Gehölze entfielen. Mit großem Abstand wichtigstes Lieferland nach Deutschland sind die Niederlande. Das Nachbarland nimmt sowohl bei Topfpflanzen als auch bei Schnittblumen die mit Abstand wichtigste Position ein. Bei Topfpflanzen folgen Dänemark, Italien, Belgien und Spanien, bei Schnittblumen Italien, Kenia und Ecuador (o. V. 2010; Behr/Niehues 2009, S. 81 ff.).

Durch die Kombination von inländischen Produkten und zugekauften Waren aus dem Ausland ergibt sich eine wertmäßige Verteilung der einzelnen Produktgruppen am Gesamtbranchenumsatz der jeweiligen Jahre, wie sie Abbildung 4 verdeutlicht. Dabei liegt dem Jahr 2000 ein Umsatz von 8,3 Mrd. Euro zu Grunde und dem Jahr 2008 ein Umsatz von 8,96 Mrd. Euro, jeweils zu Einzelhandelspreisen. Es fällt auf, dass zwischen den Jahren 2000 und 2008 die Warengruppe der Schnittblumen große Verluste erleiden musste, während Stauden und blühende Zimmerpflanzen sehr große bis große Zuwächse erzielen konnten. Da Stauden am Gesamtmarktvolumen allerdings nur einen kleinen Teil ausmachen, sind die größten absolut in Euro gemessenen Verschiebungen im Sortiment die von Schnittblumen zu blühenden Zimmerpflanzen. 


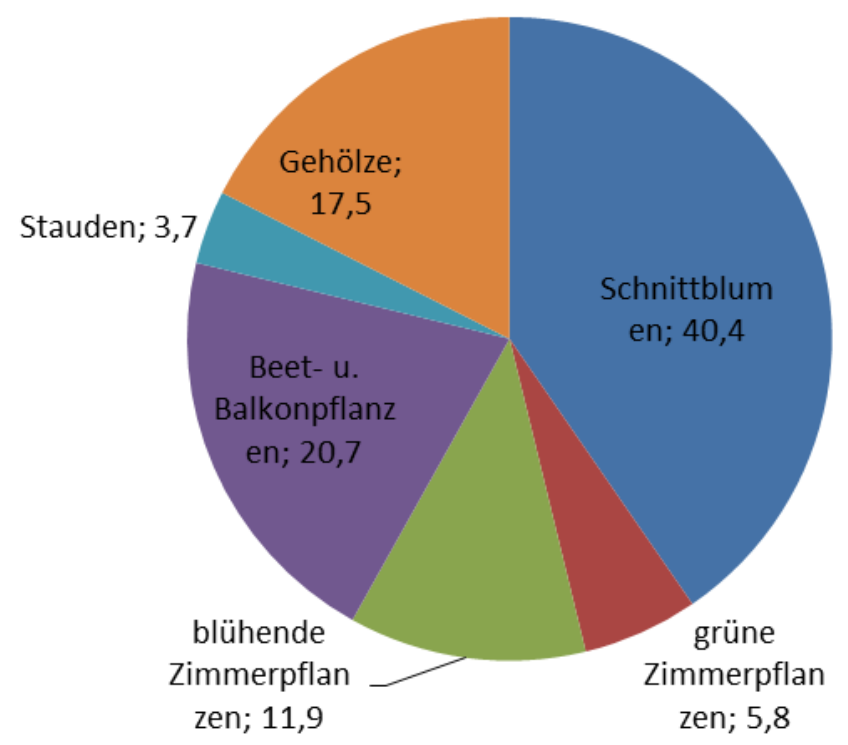

Umsatzverteilung 2008

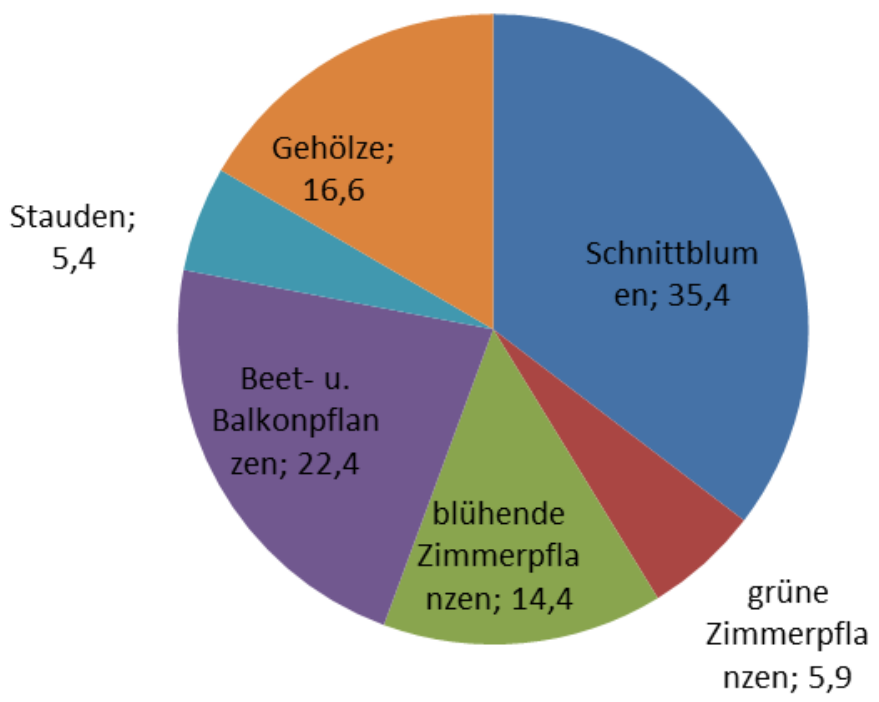

\section{Abbildung 4: Marktstruktur bei Blumen und Pflanzen}

Quelle: Eigene Darstellung nach Angaben von Behr/Niehues 2009, S. 86 ff.; Behr 2010, S. 1-10

Der Wert der Exporte deutscher Zierpflanzen ins Ausland ist während der Jahre 2000 bis 2008 zwar stark gestiegen, bewegt sich aber aktuell dennoch auf sehr niedrigem Niveau. Im Jahr 2008 betrug der Exportwert etwa 600 Mio. Euro, wobei sich dieser primär aus den Produktgruppen Topfpflanzen (ca. 371 Mio. Euro) und Gehölzen (ca. 150 Mio. Euro) zusammen- 
setzte. Wichtigste Absatzmärkte für deutsche Exporte im Zierpflanzenbereich waren dabei die Niederlande (27\%), Österreich (15\%) und die Schweiz (10 \%) (Behr/Niehues 2009, S. 86 ff.; Behr 2010, S. 1-10).

\subsubsection{Bestandteile des Angebots an Blumen und Pflanzen}

Die Distributionsstruktur für Zierpflanzen ist sehr vielfältig. Bezogen auf die Fragestellung dieser Arbeit werden im Folgenden allerdings nur Vermarktungswege auf Einzelhandelsbasis betrachtet.

Nach den Erläuterungen aus Kapitel 2.2 bestehen bedienungsorientierte Betriebstypen des Einzelhandels aus Fachgeschäften, Spezialgeschäften und Warenhäusern. Im Folgenden soll diese Dreiteilung weiter vereinfacht werden und die Absatzwege für Blumen und Pflanzen mit Fachhandel und Nicht-Fachhandel bezeichnet werden.

Der Bereich des Fachhandels setzt sich aus folgenden Geschäften des Blumen- und Pflanzeneinzelhandels zusammen: Garten- und Landschaftsbau, Blumenfachgeschäft, Gärtnerei/ Baumschule, Gartencenter und Wochenmarkt. Der Bereich des Nicht-Fachhandels setzt sich aus den Geschäften des Lebensmitteleinzelhandel (LEH), der Discounter, der Versandhäuser, der Verbrauchermärkte und der Baumärkte (DIY) zusammen. Weiterhin zählen allerdings noch Kauf- und Warenhäuser, Tankstellen und Online-Shops zur Gruppe des Nicht-Fachhandels. Aufgrund ihrer jeweils relativ geringen Bedeutung innerhalb der Nicht-Facheinzelhandelsgruppe werden Letztere allerdings zur Gruppe „Andere“ zusammengefasst.

Abbildung 5 zeigt den Anteil der einzelnen Geschäftstypen am Gesamtumsatz der Branche. Dabei ist zu beachten, dass hier lediglich der private und nicht der private und institutionelle Bereich erfasst ist. Neben den Umsatzanteilen unterscheiden sich die Einzelhändler untereinander auch anhand ihres Sortiments. So werden manche Produkte in bestimmten Geschäften besonders häufig gekauft, was eine detailliertere Betrachtung der Umsatzverteilung der Branche erfordert. 


\section{Private Einkaufsstätten von Zierpflanzen}

in Prozent des Gesamtumsatzes

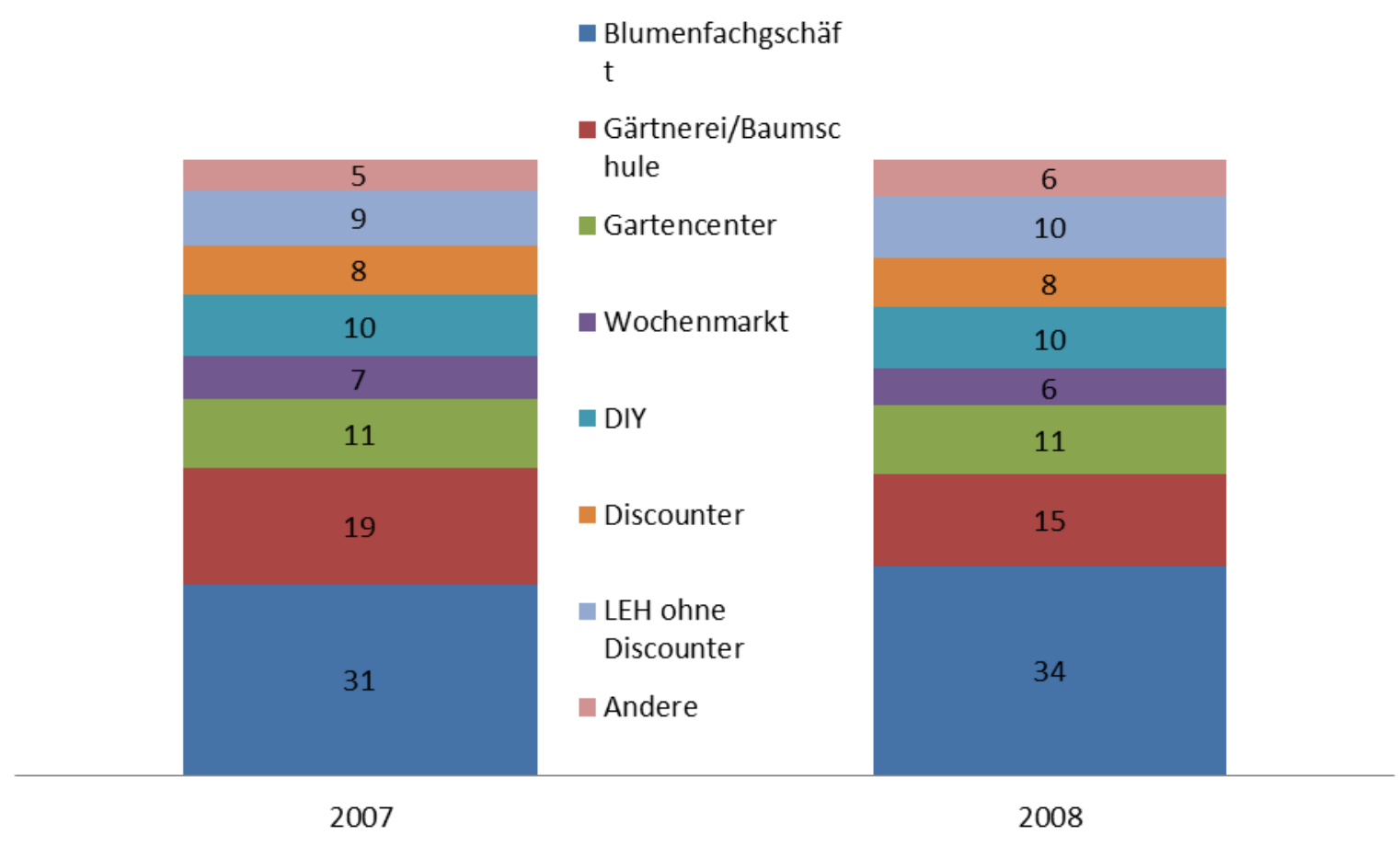

Abbildung 5: Absatzkanäle im Einzelhandel

Quelle: Eigene Darstellung nach Angaben von Behr/Niehues 2009, S. 90

\subsubsection{Schnittblumen}

Als „Schnittblumen“ werden im Folgenden einzeln verkaufte Schnittblumen, zu einem gemischten Strauß floristisch arrangierte Schnittblumen, zu einem sogenannten „Monostrauß“ gebündelte Schnittblumen und Arrangements bzw. Gestecke aus Schnittblumen verstanden. Weiterhin sollen unter Schnittblumen in dieser Arbeit nur frische - im Sinne von nicht durch Trocknung haltbar gemachte - Blumen verstanden werden.

Schnittblumen haben während der letzten Jahre immer mehr an Bedeutung verloren. Dies zeigt sich sehr deutlich daran, dass während der Jahre 2002 bis 2008 kein Umsatzwachstum generiert werden konnte, während der Gesamtumsatz für Blumen und Pflanzen zugelegt hat. 2002 betrug der Schnittblumenumsatz branchenweit 3,175 Mrd. Euro zu Einzelhandelspreisen - genau wie in 2008 auch, was einem Pro-Kopf-Verbrauch in Höhe von 39,00 Euro pro Jahr für Schnittblumen entspricht. Der Umsatz mit Schnittblumen war zeitweise gar rückläufig und erreichte in den Jahren 2003 und 2004 mit jeweils 3,1 Mrd. Euro seinen Tiefpunkt während 
der obigen Zeitspanne. Verbunden mit der Berücksichtigung der Entwicklung des in Kapitel 2.4 erwähnten Preisindexes ergibt sich für den Bereich der Schnittblumen zwischen 2002 und 2008 gar ein realer Umsatzrückgang. Abbildung 6 zeigt diese Entwicklung, wobei die ausgewiesenen Umsätze sowohl den privaten als auch den institutionellen Verbrauch mit einschließen.

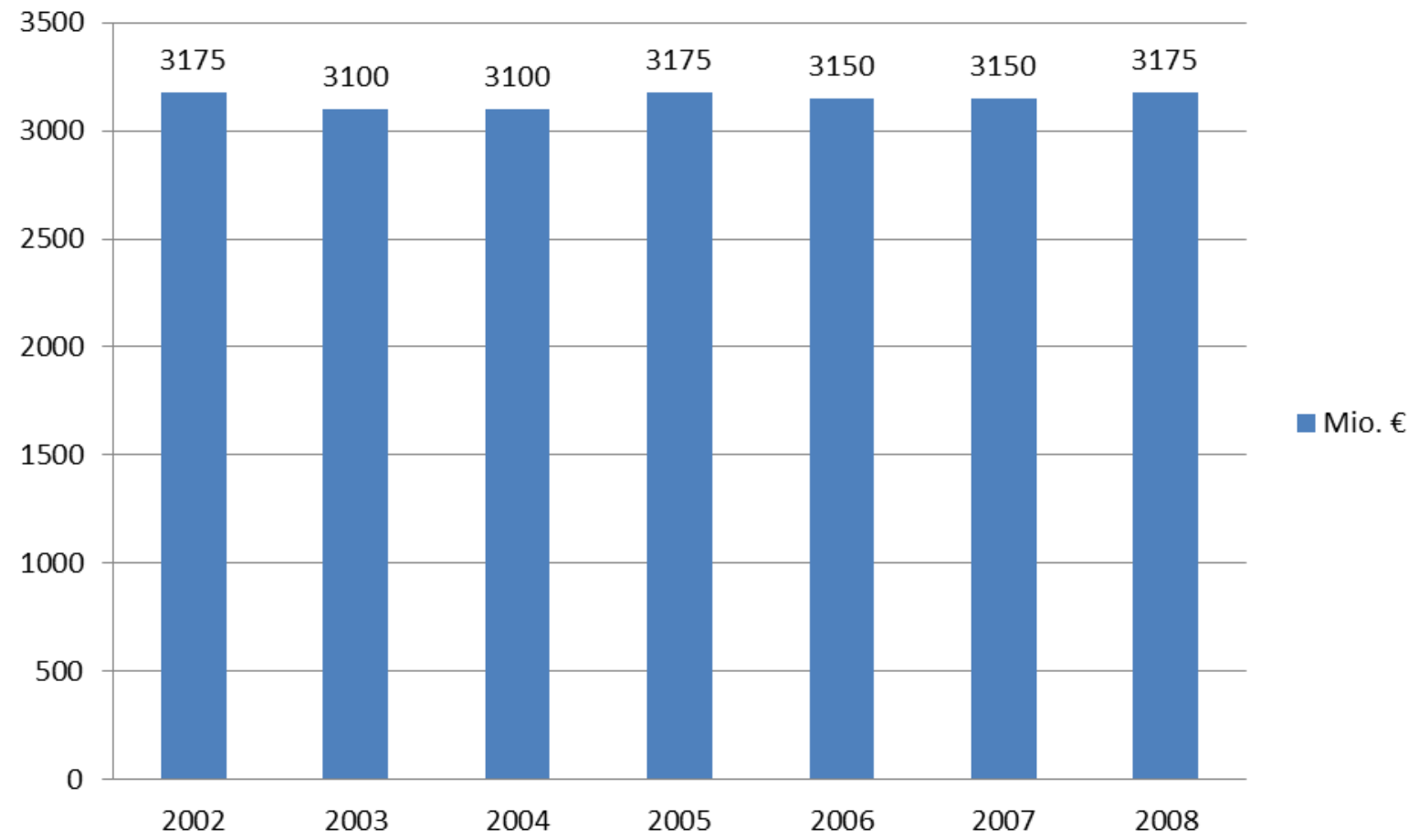

Abbildung 6: Umsatzentwicklung Schnittblumen

Quelle: Eigene Darstellung nach Angaben von Behr 2011, S. 9

Schnittblumen werden ,traditionell“ in einem sehr hohen Wertanteil im Facheinzelhandel gekauft, wobei hier besonders auffällig ist, dass der Betriebstyp des Blumenfachgeschäftes in den Jahren 2000 bis 2008 etwa $9 \%$ an Umsatz mit Schnittblumen eingebüßt hat. Dennoch sind die Blumenfachgeschäfte auch 2008 noch die mit Abstand wichtigste Einkaufsstätte für Blumen, da sie alleine für ca. $59 \%$ des Gesamtumsatzes mit Schnittblumen verantwortlich sind. Gärtnereien kamen in 2008 auf einen Schnittblumenanteil von 11 \%, Wochenmärkte auf $8 \%$ und Gartencenter auf $3 \%$ Marktanteil des Gesamtumsatzes bei Schnittblumen.

Obwohl die Blumenfachgeschäfte zwar nach wie vor noch unangefochten an der Spitze liegen, haben die Geschäfte des LEH in den Jahren 2000 bis 2008 stark aufgeholt: Lagen sie im Jahr 2000 mit $9 \%$ Umsatzanteil noch auf dem dritten Platz hinter den Blumenfachgeschäften 
und den Gärtnereien, haben die LEH-Unternehmen im Jahr 2008 bereits die Gärtnereien überholt und belegen mit $14 \%$ Umsatzanteil den zweiten Platz. Graphisch werden diese Änderungen in Abbildung 7 verdeutlicht; die prozentualen Anteile der einzelnen Verkaufsstätten beziehen sich auf den branchenweiten Schnittblumenumsatz in Höhe von 3,175 Mrd. Euro im Jahr 2008 (Behr/Niehues 2009, S. 90 f.). Während Baumärkte in der branchenweiten Übersicht noch eine wichtige Rolle gespielt haben, sind sie beim Absatz von Schnittblumen nahe$\mathrm{zu}$ nicht von Bedeutung.

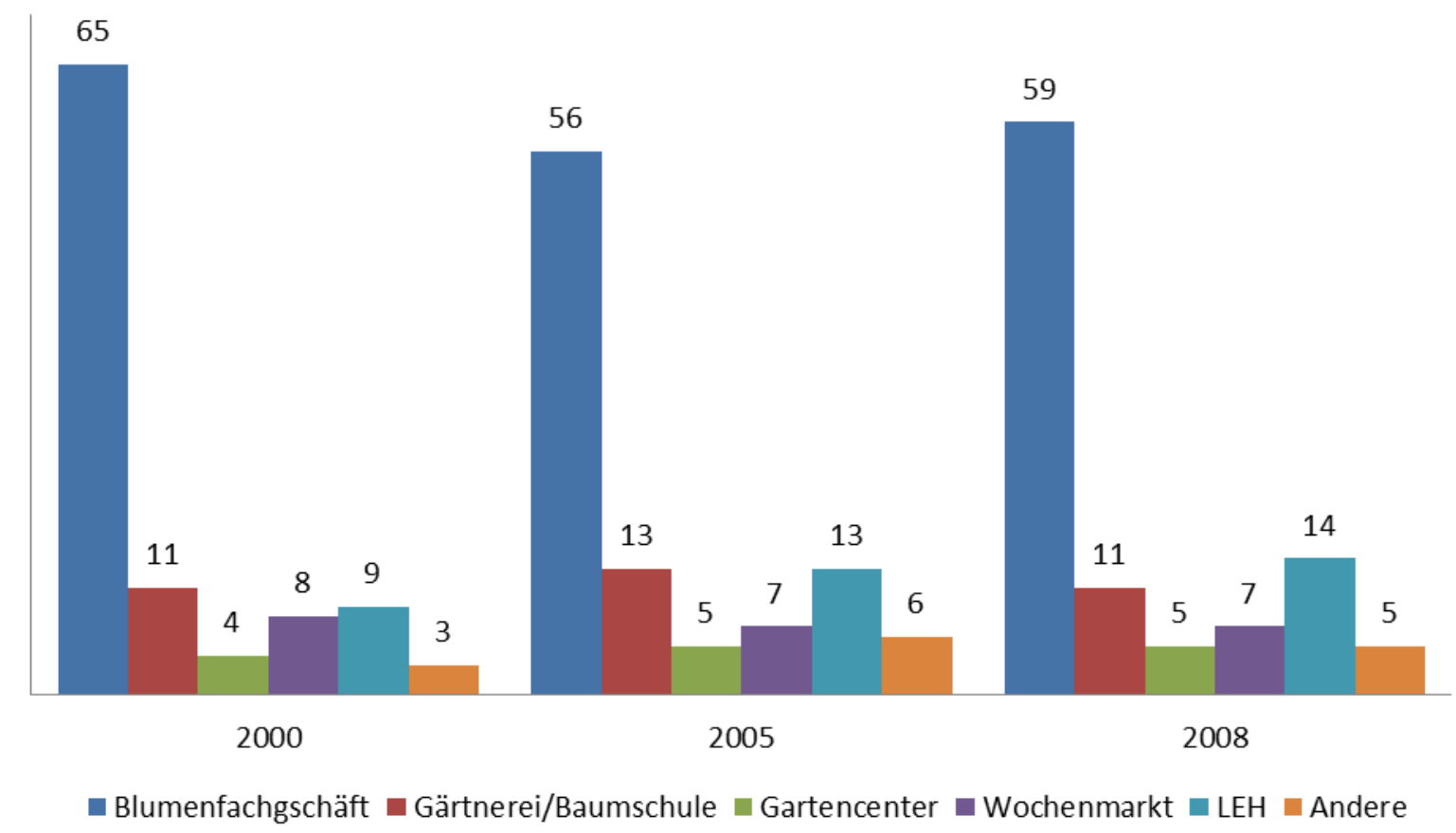

Abbildung 7: Marktanteile Einkaufsstätten Schnittblumen

Quelle: Eigene Darstellung nach Angaben von Behr/Niehues 2009, S. 90 f.

Das mit weitem Abstand wichtigste Verkaufsprodukt im Bereich der Schnittblumen sind Rosen. Sie allein tragen im Jahr 2008 mit ca. 37 \% zum gesamten Schnittblumenumsatz (also sowohl privater als auch institutioneller Umsatz) bei. Mit weitem Abstand folgen Tulpen (11\%) und Chrysanthemen (8\%). Abbildung 8 zeigt die Umsatzanteile der zehn wichtigsten Produktgruppen im Schnittblumenbereich im Jahre 2008 im deutschen Einzelhandel. 


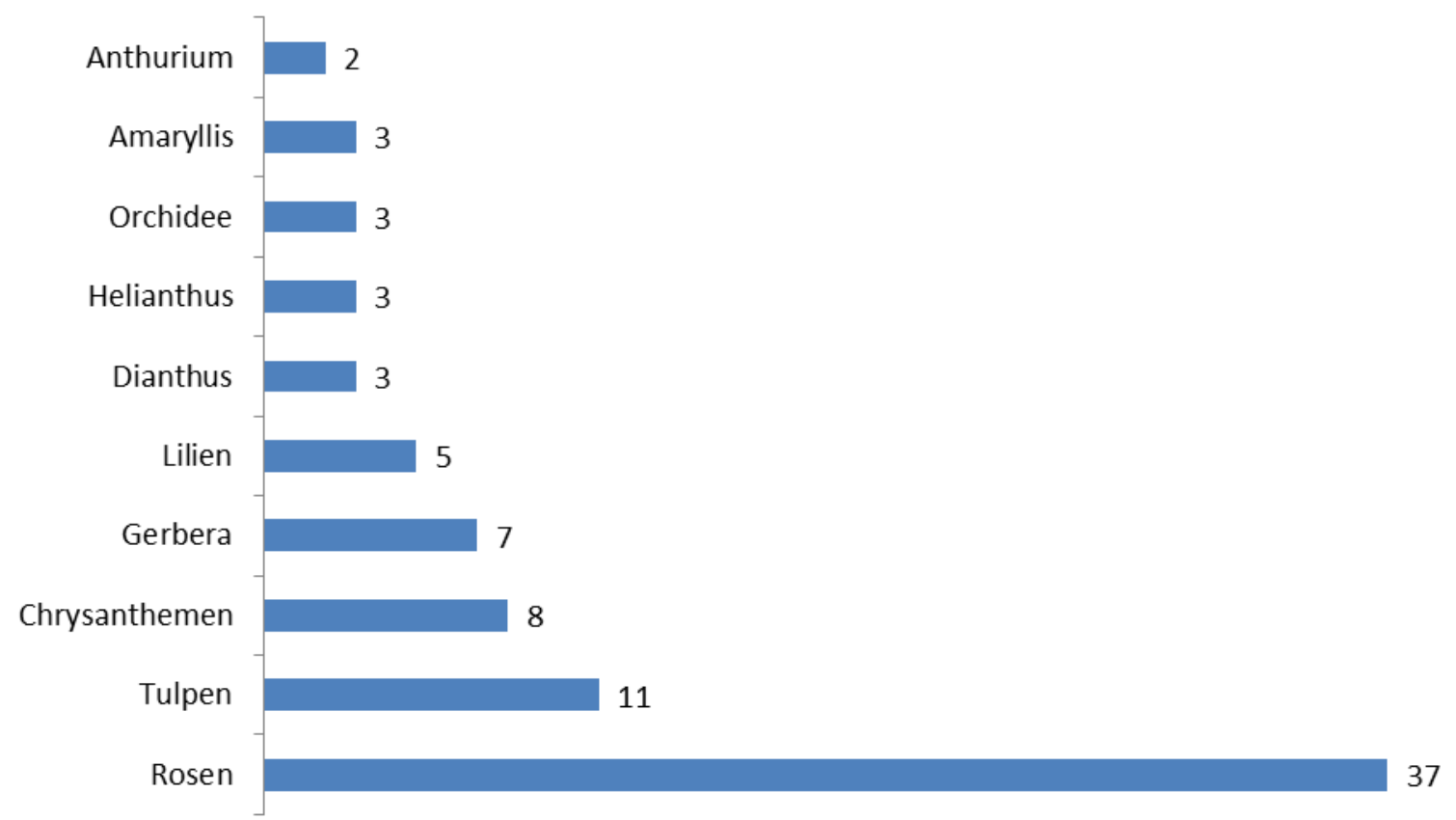

Abbildung 8: Ranking Umsatzanteile Schnittblumen 2008

Quelle: Eigene Darstellung nach Angaben von Behr/Niehues 2009, S. 93

Die Verwendung von Schnittblumen durch die Verbraucher lässt sich grundsätzlich in zwei Weisen unterscheiden: Einerseits werden Schnittblumen als Geschenk gekauft, andererseits für den Eigenbedarf. Als Geschenkempfänger sind Freunde bzw. Bekannte und Familienangehörige die beiden wichtigsten Gruppen. Die Verwendungsmöglichkeit des Eigenbedarfs an Schnittblumen lässt sich unterteilen in Eigenbedarf für Eigenheim, als Grab- und Trauerschmuck und für sonstige eigene Verwendungen.

Im Jahr 2007 teilten sich die privaten Ausgaben für Schnittblumen wie folgt auf. Während Monosträuße wertmäßig zu 50 \% für den Eigenbedarf gekauft wurden, waren dies bei gemischten, floristisch arrangierten Sträußen lediglich ca. $25 \%$. Bei Gestecken aus Schnittblumen sind ca. $42 \%$ der Ausgaben für den Eigenbedarf (Niehues 2009, S. 33). Abbildung 9 verdeutlicht die Verteilung der Ausgaben nach ihrem Zweck und zeigt, wie sehr Schnittblumen als Geschenk geschätzt werden. Dabei beziehen sich die Angaben nur auf den privaten Konsum. 


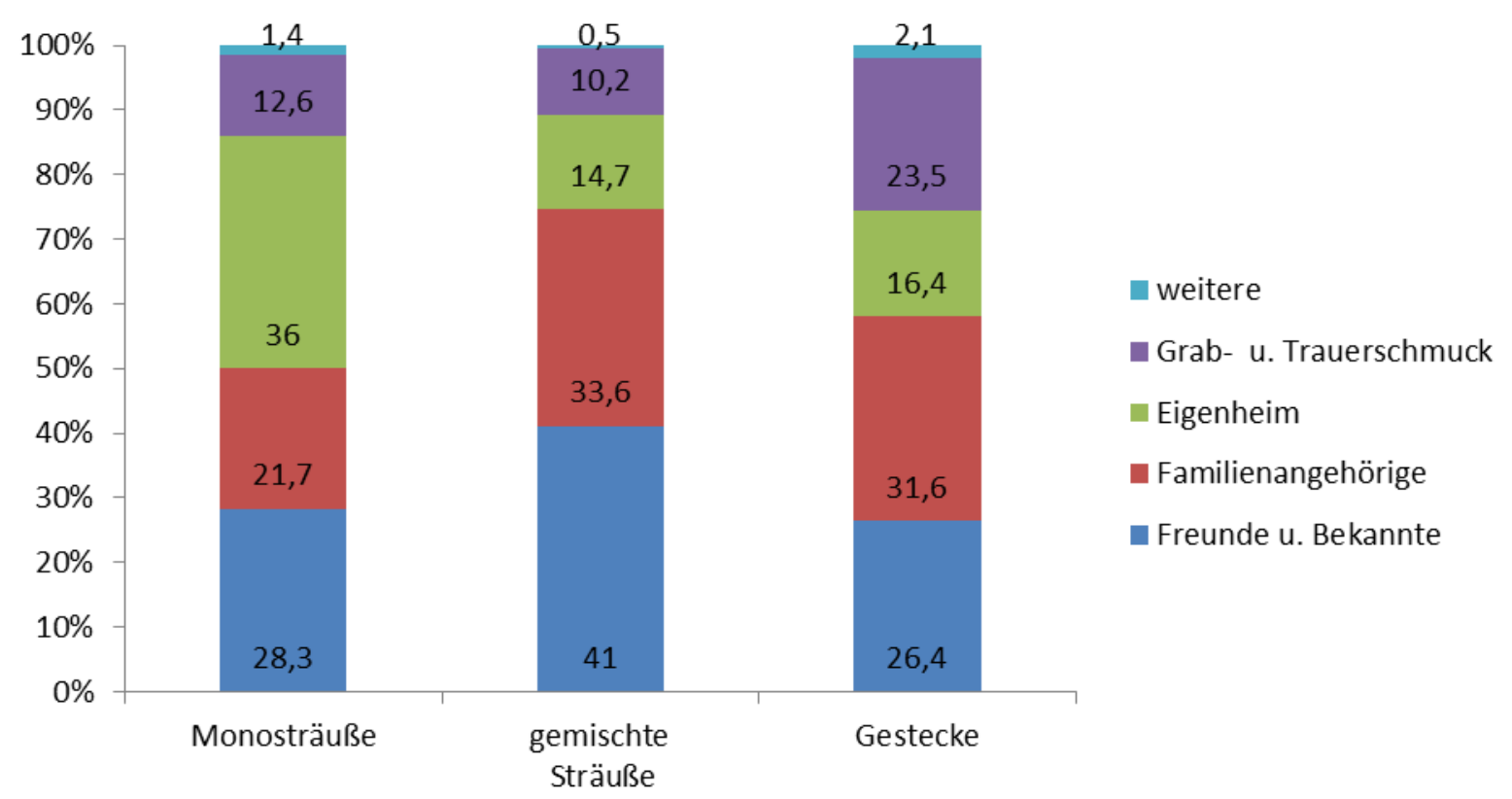

Abbildung 9: Verwendungszweck Schnittblumenprodukte 2007

Quelle: Eigene Darstellung nach Angaben von Niehues 2009, S. 33

\subsubsection{Topfpflanzen}

Topfpflanzen bestehen, wie bereits Abbildung 2 gezeigt hat, aus den Untergruppen Zimmerpflanzen (blühend und grün), Beet- und Balkonpflanzen sowie Stauden. Da Topfpflanzen im Gesamtmarkt für Blumen und Pflanzen mit ca. 46 \% Umsatzanteil im Jahr 2008 den mit Abstand größten Umsatzanteil für sich beanspruchen, werden die einzelnen Untergruppen der Topfpflanzen im weiteren Verlauf separat vorgestellt.

Wie Schnittblumen werden Topfpflanzen an vielen verschiedenen Orten des Einzelhandels zum Kauf angeboten, sowohl im Facheinzelhandel als auch im Nicht-Facheinzelhandel. Zu Ersterem zählen hier der Garten- und Landschaftsbau, das Blumenfachgeschäft, die Gärtnerei bzw. die Baumschule und der Verkaufsstand auf dem Wochenmarkt. Zur Gruppe des NichtFacheinzelhandels zählen der Lebensmitteleinzelhandel (LEH), Baumärkte (DIY), Kauf- und Warenhäuser, Möbelfachgeschäfte, Tankstellen und Online-Shops. Aufgrund ihrer jeweils recht geringen Bedeutung sind die letzten vier Einkaufsstätten des Nicht-Facheinzelhandels zur Gruppe „Andere“ zusammengefasst.

Im Jahr 2008 erreichte der Facheinzelhandel bei Topfpflanzen einen Marktanteil von etwa $63 \%$. Im Jahr 2000 lag sein Marktanteil noch bei ca. 71\%, so dass der Facheinzelhandel wäh- 
rend dieser Zeitspanne, in welcher Topfpflanzen insgesamt ein Wachstum von ca. 11,5\% verzeichnen konnten, ca. $11 \%$ seiner Marktposition von 2000 eingebüßt hat.

Großer Gewinner der Umsatzverteilung bei Topfpflanzen ist neben der Gruppe „Andere“ der LEH. Von 2000 bis 2008 konnte er seinen Marktanteil von 14 auf 17 \% erweitern, was einem Wachstum von ca. $21 \%$ über acht Jahre entspricht. Somit hat der LEH im Laufe der Jahre seinen stärksten Umsatzanteil innerhalb des Marktes für Blumen und Pflanzen im Bereich Topfpflanzen erreicht: Knapp 60 \% des Gesamtumsatzes mit Blumen und Pflanzen macht ein typisches LEH-Geschäft mit Topfpflanzen. Die Gruppe „Andere“ konnte ihren Marktanteil im Laufe der Jahre noch stärker als der LEH ausbauen, was allerdings auch daran liegt, dass das Ausgangsniveau in 2000 recht gering war. Dennoch darf nicht unbeachtet bleiben, dass „Andere“ mittlerweile $9 \%$ Marktanteil bei Topfpflanzen haben, was hauptsächlich an den Möbelhäusern und den Online-Shops liegt. Möbelhäuser sind innerhalb der Gruppe Topfpflanzen bei der Untergruppe „grüne Zimmerpflanzen“ stark vertreten (ca. 5 \% Marktanteil), was darauf zurückzuführen ist, dass die dort angebotenen Pflanzen als eine Art Einrichtungsgegenstand gesehen werden.

Baumärkte haben während der Jahre 2000 bis 2008 ihren Anteil von 12 auf $14 \%$ steigern können, was einem Wachstum von ca. $17 \%$ entspricht, das ebenfalls deutlich über dem Marktwachstum von Topfpflanzen liegt. In Baumärkten ist die Bedeutung der Topfpflanzen bezogen auf den Gesamtumsatz mit Blumen und Pflanzen noch bedeutender als im LEH - ca. $75 \%$ des Gesamtumsatzes mit Blumen und Pflanzen entfallen in Baumärkten auf Topfpflanzen.

Wie bereits erwähnt, sind die anteiligen Marktgewinne der Nicht-Facheinzelhändler die anteiligen Marktverluste der Facheinzelhändler. Dabei konnten das Gartencenter und der Gartenund Landschaftsbau zumindest ihre Position aus dem Jahr 2000 ins Jahr 2008 retten. Das Gartencenter hält in 2008 genauso wie noch in 2000 einen Umsatzanteil von 17 \%, der Gartenund Landschaftsbau trägt nach wie vor $2 \%$ zum Umsatz bei Topfpflanzen bei. Gärtnereien bzw. Baumschulen sind zwar auch im Jahr 2008 noch die wichtigste Einkaufsquelle für Topfpflanzen, ihr Marktanteil ist jedoch im Laufe der Jahre von $24 \%$ in 2000 auf $21 \%$ in 2008 gesunken, was einem Rückgang um ca. $13 \%$ entspricht. Blumenfachgeschäfte verloren seit 2000 etwa vier Prozentpunkte an Marktanteil, was in ihrem Fall einen relativen Marktanteilsrückgang in Höhe von ca. 17 \% bedeutet (Behr/Niehues 2009, S. 91 ff.; Behr 2010; Behr 2015; Altmann 2010, S. 3; Bednarsky 2010; Lange 2009; Niehues 2009). 
Wie bereits erwähnt, haben Topfpflanzen während der letzten Jahre ein besonders hohes Marktwachstum erfahren. Gerade vor diesem Hintergrund scheinen die hier aufgezeigten Entwicklungen besonders interessant, da der Gesamtmarkt für Blumen und Pflanzen zwar auch Verschiebungen von Umsatzanteilen aufzeigt, jedoch nicht in solch einem dynamischen Ausmaß. Abbildung 10 stellt die obigen Ausführungen nochmals graphisch dar.

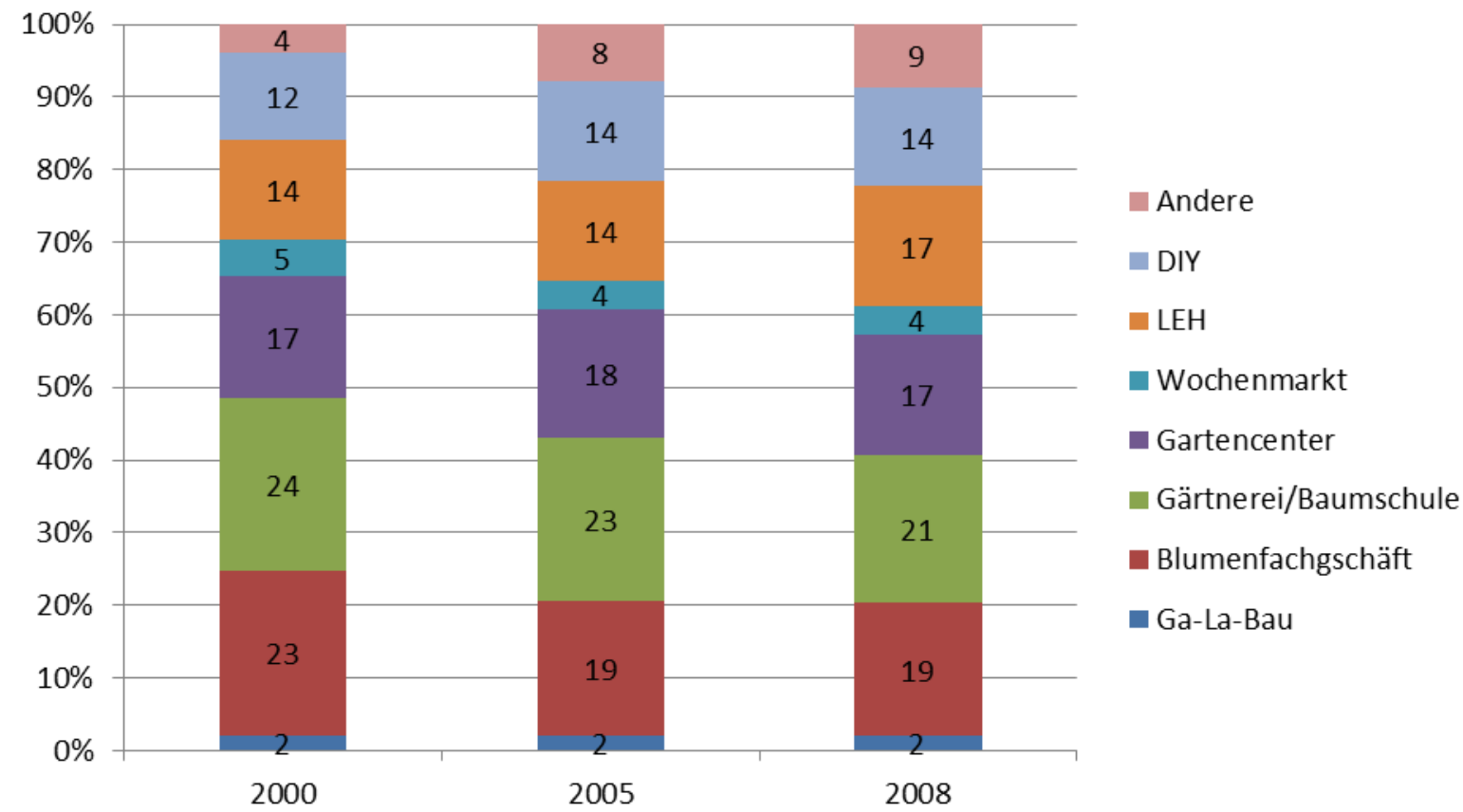

Abbildung 10: Einkaufsstätten Topfpflanzen

Quelle: Eigene Darstellung nach Angaben von Behr/Niehues 2009, S. 91 ff.; Behr 2010; Behr 2015; Altmann 2010, S. 3; Bednarsky 2010; Lange 2009; Niehues 2009

\subsection{Zimmerpflanzen}

Zimmerpflanzen untergliedern sich in blühende und grüne Zimmerpflanzen und haben während der letzten Jahre stark an Bedeutung auf dem deutschen Blumen- und Pflanzenmarkt gewonnen.

Zwischen den Jahren 2002 und 2008 ist der Gesamtmarkt für Zimmerpflanzen um 14,5\% gewachsen, was einer durchschnittlichen Marktentwicklung von ca. 2,4 \% pro Jahr entspricht. In absoluten Beträgen bedeutet dies, dass im Jahr 2002 1,58 Mrd. Euro von privaten und institutionellen Käufern für Zimmerpflanzen ausgegeben wurden, während es im Jahr 2008 bereits 1,81 Mrd. Euro waren (Niehues 2009, S. 35). Abbildung 11 verdeutlicht diesen Aufwärtstrend. 


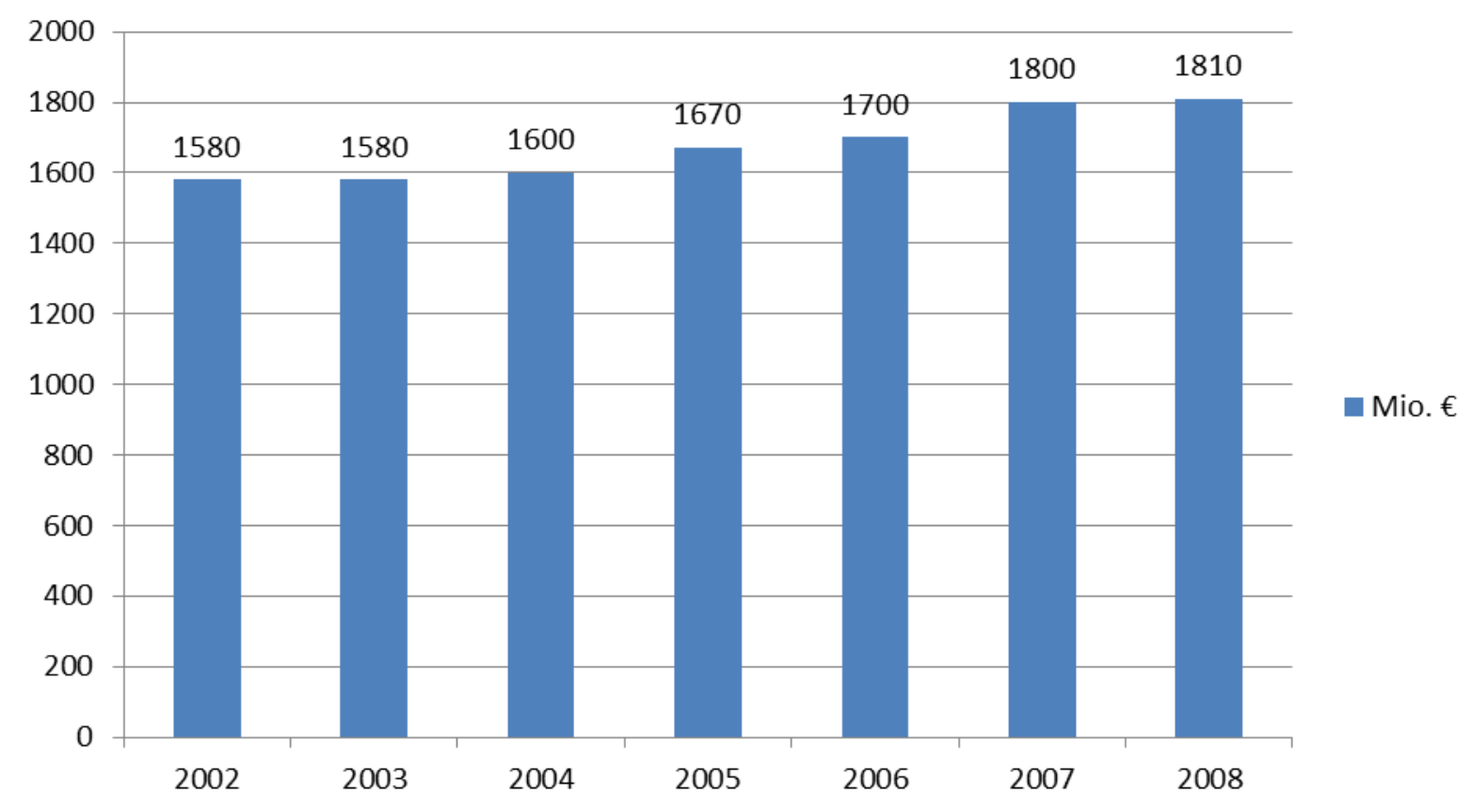

Abbildung 11: Umsatzentwicklung Topfpflanzen

Quelle: Eigene Darstellung nach Angaben von Niehues 2009, S. 35

\section{Blühende Zimmerpflanzen}

Blühende Zimmerpflanzen haben während der Jahre 2000 bis 2008 in absoluten Zahlen den größten Marktzuwachs verzeichnen können. Ihr Anteil am Gesamtumsatz stieg von 0,988 Mrd. Euro in 2000 auf 1,29 Mrd. Euro in 2008, was einer Steigerung von ca. $31 \%$ bzw. 0,302 Mrd. Euro entspricht. In den Jahren 2002 bis 2008 wuchs der Umsatz mit blühenden Zimmerpflanzen um durchschnittlich 3,3\% pro Jahr. Dies macht wiederum deutlich, dass blühende Zimmerpflanzen eine weitaus höhere Marktdynamik aufwiesen als die Branche allgemein (Niehues 2009, S. 36) (vgl. hierzu die Abbildungen 3, 4 und 12).

Unter den blühenden Topfpflanzen nehmen Alpenveilchen von der abgesetzten Menge her den wichtigsten Platz ein - keine andere Zimmerpflanze wurde in Deutschland öfter verkauft. Auf Platz zwei folgt der Weihnachtsstern, obwohl er in relevanten Mengen nur zwischen November und Dezember im Einzelhandel verkauft wird. Bezogen auf die monetären Umsatzanteile der einzelnen Pflanzen haben allerdings Orchideen ganz klar die Spitzenposition unter den blühenden Zimmerpflanzen inne. Sie sind für ca. $24 \%$ des Umsatzes verantwortlich. Die mengenmäßig überlegenen Alpenveilchen und Weihnachtssterne folgen auf Platz zwei und drei, generieren allerdings jeweils nur $8 \%$ des Gesamtumsatzes (Behr/Niehues 2009, S. 94). 
Abbildung 13 zeigt die zehn umsatzstärksten blühenden Topfpflanzen in Deutschland im Jahr 2008, wobei ein Gesamtumsatz in Höhe von 1,29 Mrd. Euro zu Grunde liegt.

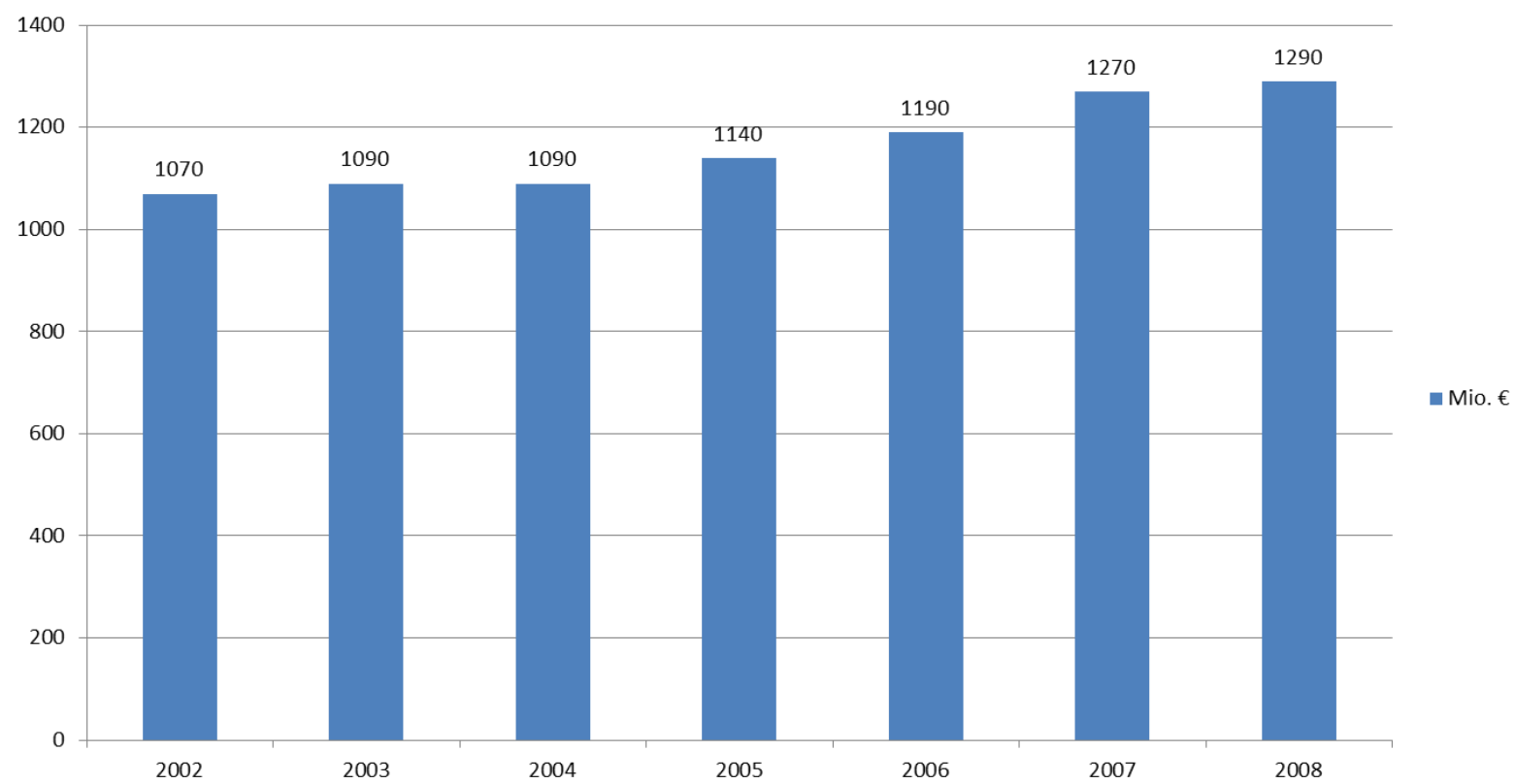

Abbildung 12: Umsatzentwicklung blühende Zimmerpflanzen

Quelle: Eigene Darstellung nach Angaben von Niehues 2009, S. 36

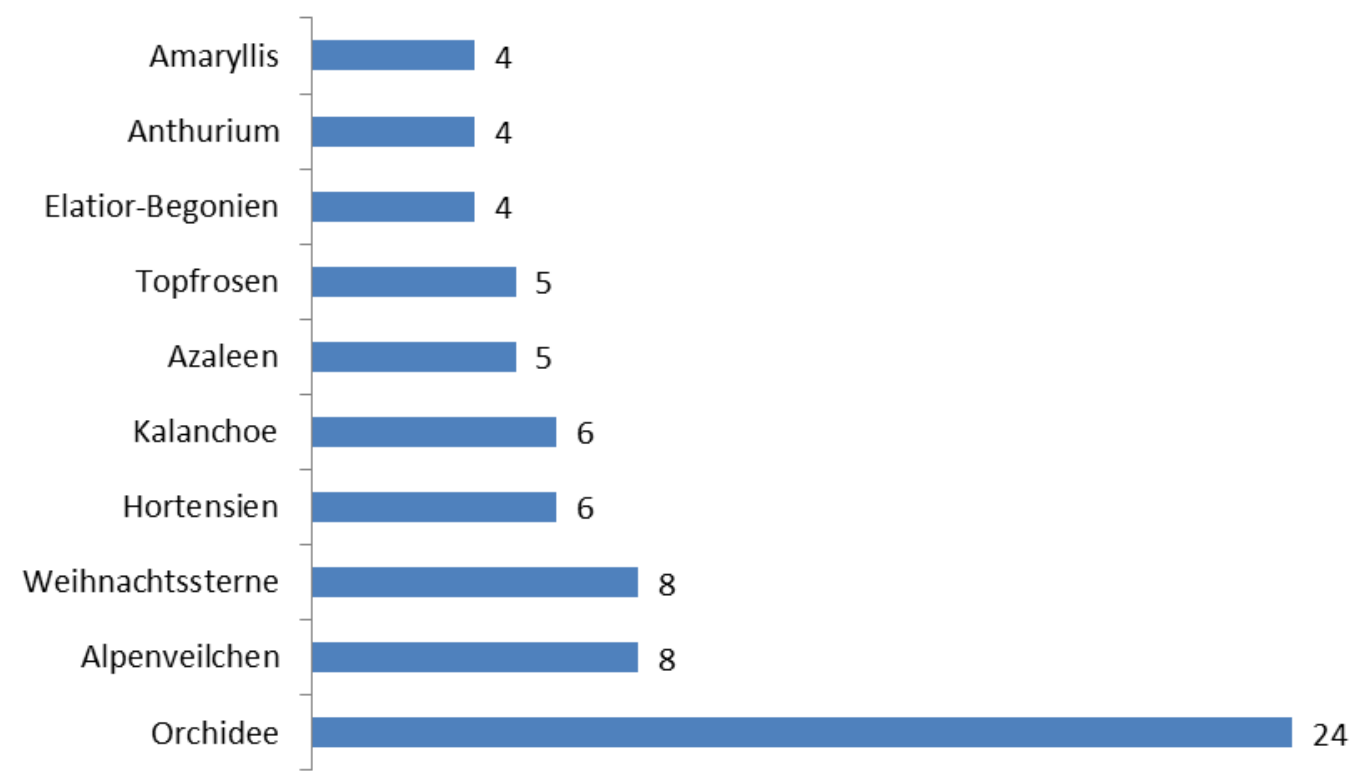

Abbildung 13: Umsatzanteile blühende Zimmerpflanzen 2008 in Prozent

Quelle: Eigene Darstellung nach Angaben von Behr/Niehues 2009, S. 94 


\section{Grüne Zimmerpflanzen}

Der Markt für grüne Zimmerpflanzen hat sich de facto von 2002 bis 2008 kaum verändert, die Schwankungen bewegen sich zwischen dem Tiefstwert von 490 Mio. Euro im Jahr 2003 und dem Höchstwert von 530 Mio. Euro in den Jahren 2005 und 2007. Für das Jahr 2008 wurde ein Rückgang des Marktvolumens um rund 10 Mio. auf 520 Mio. Euro festgestellt. Insgesamt kann für die Jahre 2002 bis 2008 jedoch ein Zuwachs von knapp $2 \%$ festgestellt werden, was einem durchschnittlichen Marktwachstum von ca. 0,3\% pro Jahr entspricht (Niehues 2009, S. 37). Abbildung 14 zeigt die Entwicklung auf. Deutlich wird, dass die grünen Zimmerpflanzen zwar eine stabile Marktposition besetzen, bezogen auf die allgemeine Preisentwicklung über die Jahre hinweg aber reale Verluste erfahren haben.

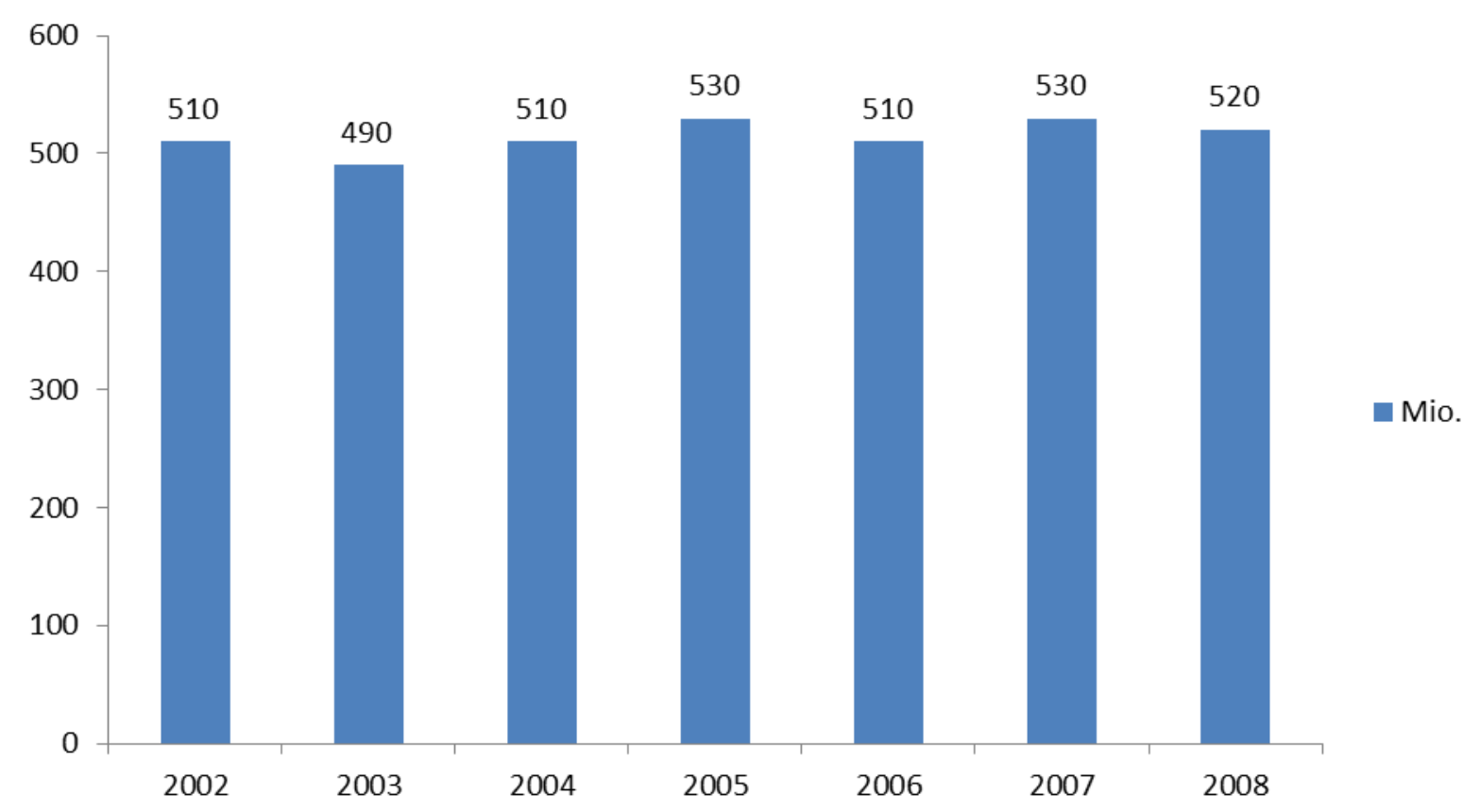

Abbildung 14 Marktentwicklung grüne Zimmerpflanzen

Quelle: Eigene Darstellung nach Angaben von Niehues 2009, S. 37

Eine nicht eindeutige Trennung einzelner Positionen bei Grünpflanzen macht eine Erhebung bzw. die Vergleichbarkeit einzelner Pflanzen schwierig, so dass Veränderungen zu vorherigen Erhebungen dieser Art vorsichtig zu interpretieren sind. Für das Jahr 2008 wurden zu der Gruppe der Zimmerpalmen die Phoenix-Palme und das Palmfarn gerechnet, außerdem wurde die neue Gruppe Kakteen geschaffen und Spatiphyllum bzw. „Einblatt“ nun als blühende Zimmerpflanze eingeordnet. Ausgehend von dieser neuen Klassifizierung nehmen Zimmer- 
palmen mit einem wertmäßigen Anteil von $10 \%$ aller Grünpflanzenumsätze im Jahr 2008 die oberste Position ein, gefolgt von verschiedenen Efeuarten, die einen Umsatzanteil von $9 \%$ ausmachen. An dritter und vierter Stelle kommen mit je rund $6 \%$ Drachenbaum- und FicusArten. Zwischen vier und zwei Prozent erreichen auf den Plätzen fünf bis zehn die Kakteen (4\%), die Yucca-Palmen, Farne und der Glücksklee (je $3 \%$ ) sowie Zamioculcas und Elefantenfuß (je $2 \%$ ) (Behr/Niehues 2009, S. 95). Die Verteilung der Umsatzanteile im Bereich grüne Zimmerpflanzen wird in Abbildung 15 visualisiert.

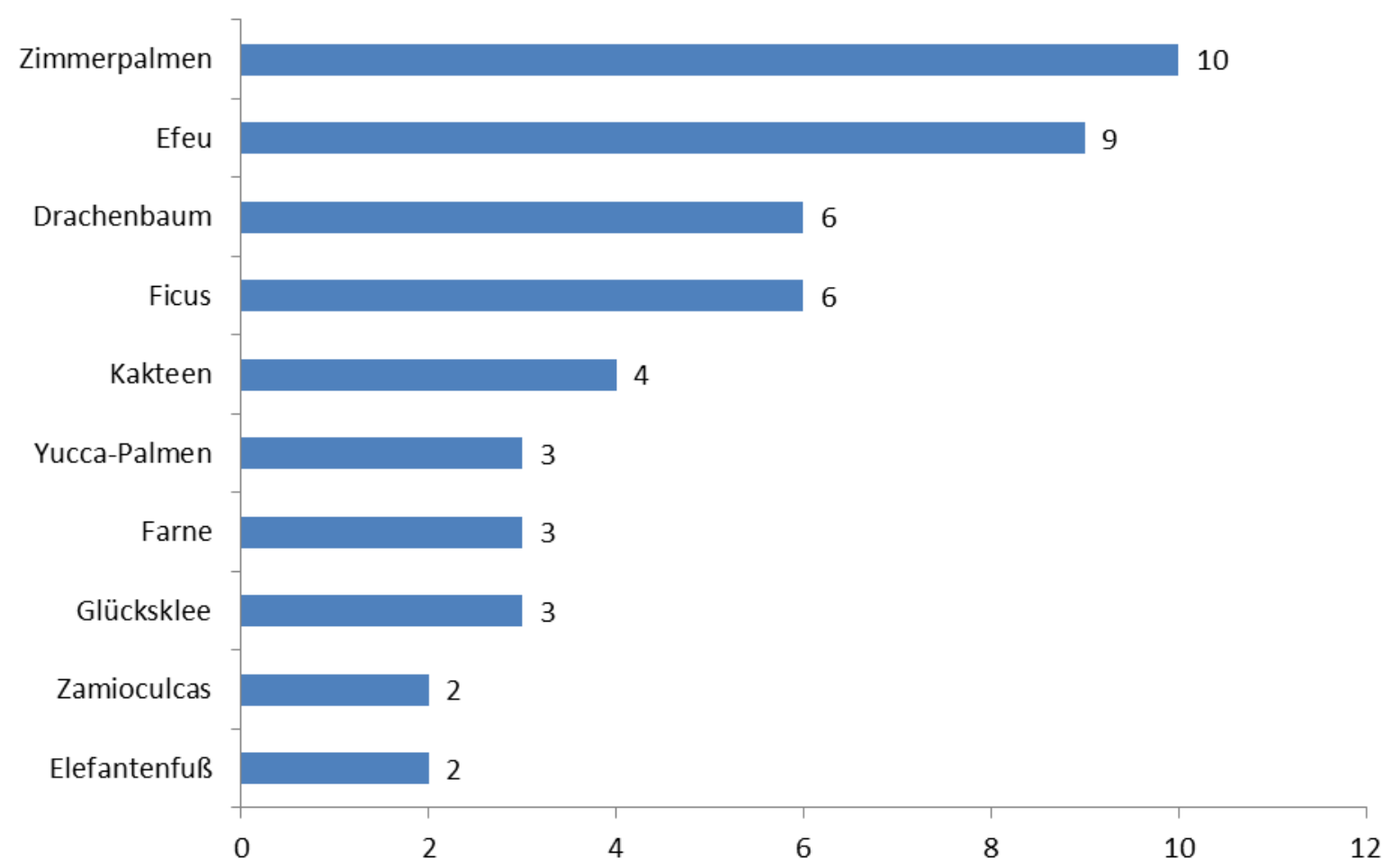

Abbildung 15 Umsatzanteile grüne Zimmerpflanzen 2008 in Prozent

Quelle: Eigene Darstellung nach Angaben von Behr/Niehues 2009, S. 95

\subsection{Beet- und Balkonpflanzen}

Beet- und Balkonpflanzen gehören ebenso wie Gehölze und Zimmerpflanzen zur Gruppe der Topfpflanzen. Wie der Name bereits sagt, sind Beet- und Balkonpflanzen für das Freiland gedacht und dementsprechend auch mehr als Zimmerpflanzen von der Wetterlage abhängig. „Wie das Wetter, so der Umsatz“ oder „Wetter schlägt Konjunktur“ sind typische Sprichwörter für die Verkaufsbedingungen von Beet- und Balkonpflanzen und heben die Abhängigkeit der Ware für Beet und Balkon vom Wetter hervor. 
Wie Abbildung 4 verdeutlicht, stellten Beet- und Balkonpflanzen im Jahr 2008 mit 22,4 \% Umsatzanteil die bedeutendste Gruppe aller Topfpflanzengruppen. Der Umsatz mit Beet- und Balkonpflanzen zu Einzelhandelspreisen hat sich zwischen den Jahren 2002 und 2010 um insgesamt knapp $7 \%$ vergrößert, was einem durchschnittlichen jährlichen Marktwachstum von ca. $0,9 \%$ entspricht. Dabei muss allerdings berücksichtigt werden, dass die Umsatzentwicklung während dieses Zeitraums nicht einheitlich verlaufen ist. Stieg zwischen 2002 und 2008 der Umsatz von 1,83 Mrd. Euro auf 2,01 Mrd. Euro an, so fiel er in 2009, bedingt durch die Wirtschaftskrise, wieder auf 1,955 Mrd. Euro. Für 2010 wurde ebenfalls ein Umsatz in Höhe von 1,955 Mrd. Euro erwartet (Stand September 2010) (Niehues 2009, S. 45; Behr 2010). Abbildung 16 zeigt diese Entwicklung auf.

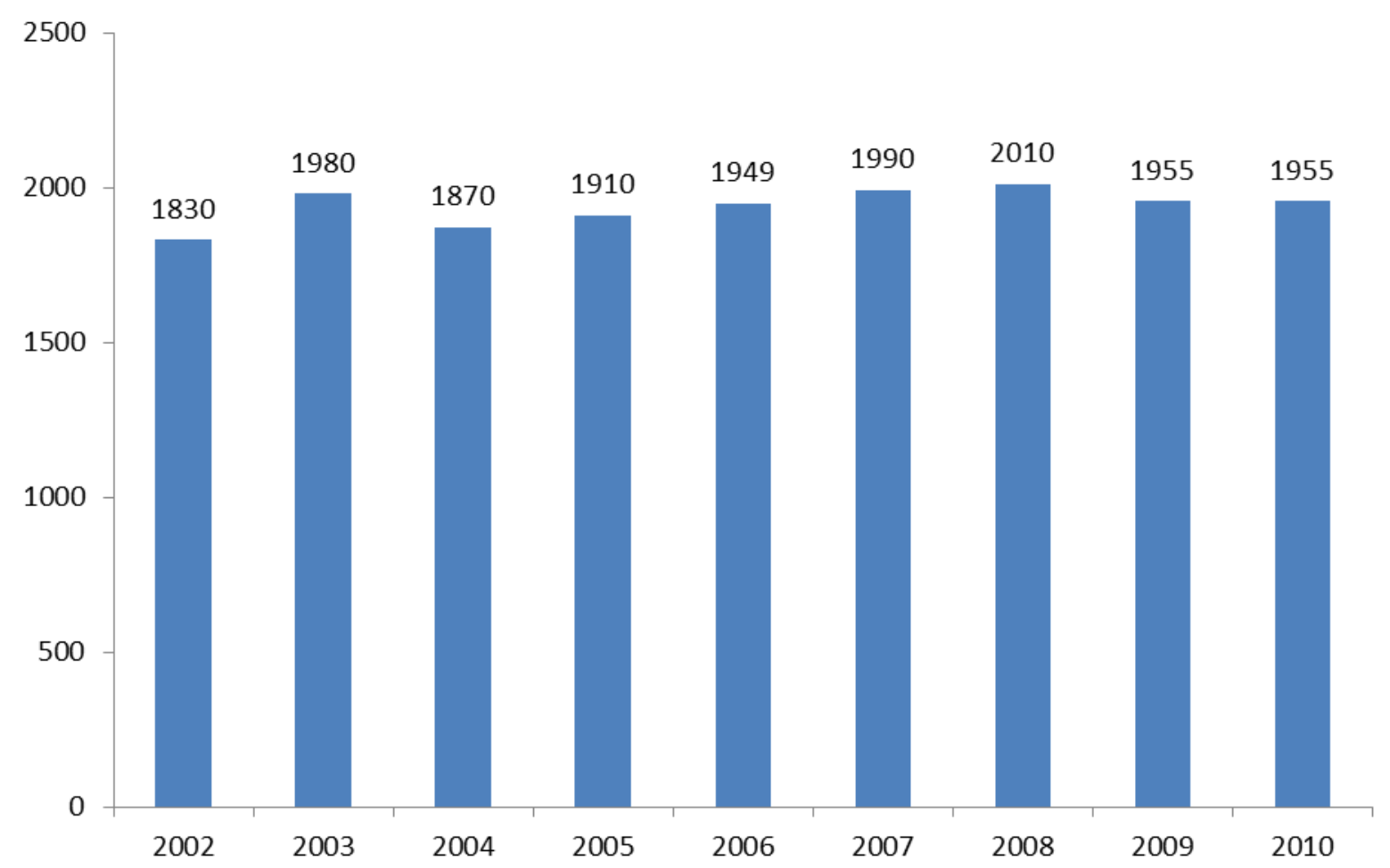

Abbildung 16: Umsatzentwicklung Beet- und Balkonpflanzen in Mio. $€$

Quelle: Eigene Darstellung in Anlehnung an Niehues 2009, S. 45; Behr 2010

Da das Wetter für den Verkauf von Beet- und Balkonpflanzen eine sehr wichtige Rolle spielt, beginnt der Verkauf für Freilandpflanzen mit den ersten frostfreien Tagen des Jahres ,aufzutauen“ und findet seinen Umsatzhöhepunkt meist Mitte Mai. Mit zunehmend sommerlichen Temperaturen und spätestens mit dem Einsetzen des Sommerurlaubs fallen die Umsätze für Beet- und Balkonpflanzen wieder sehr stark. Je nach Wetterlage beginnt etwa ab September 
der Verkauf für Beet- und Balkonpflanzen der „Herbstsaison“. Die Umsätze steigern sich mit fortschreitendem Datum und knicken mit beginnendem Frost bzw. Anfang November wieder abrupt ein. Dies liegt daran, dass in diesen Tagen die meisten Trauertage wie Allerheiligen oder der Volkstrauertag liegen und zu diesen Ereignissen vielfach die Gräber und Beete neu und winterfest angepflanzt werden. Die Herbstsaison für Beet- und Balkonpflanzen ist nicht so umsatzstark wie die Frühjahressaison. Während der letzten Jahre hat sich eine Umsatzverteilung eingependelt, in der während der ersten Jahreshälfte gut drei Viertel des Jahresumsatzes von Beet- und Balkonpflanzen getätigt werden (Niehues 2009, S. 55). Die Abbildungen 17 und 18 machen deutlich, wie unterschiedlich die einzelne Monatsumsätze wetterbedingt ausfallen können und wie sich die Jahresumsätze während der Jahre 2008 bis 2010 zusammengesetzt haben.

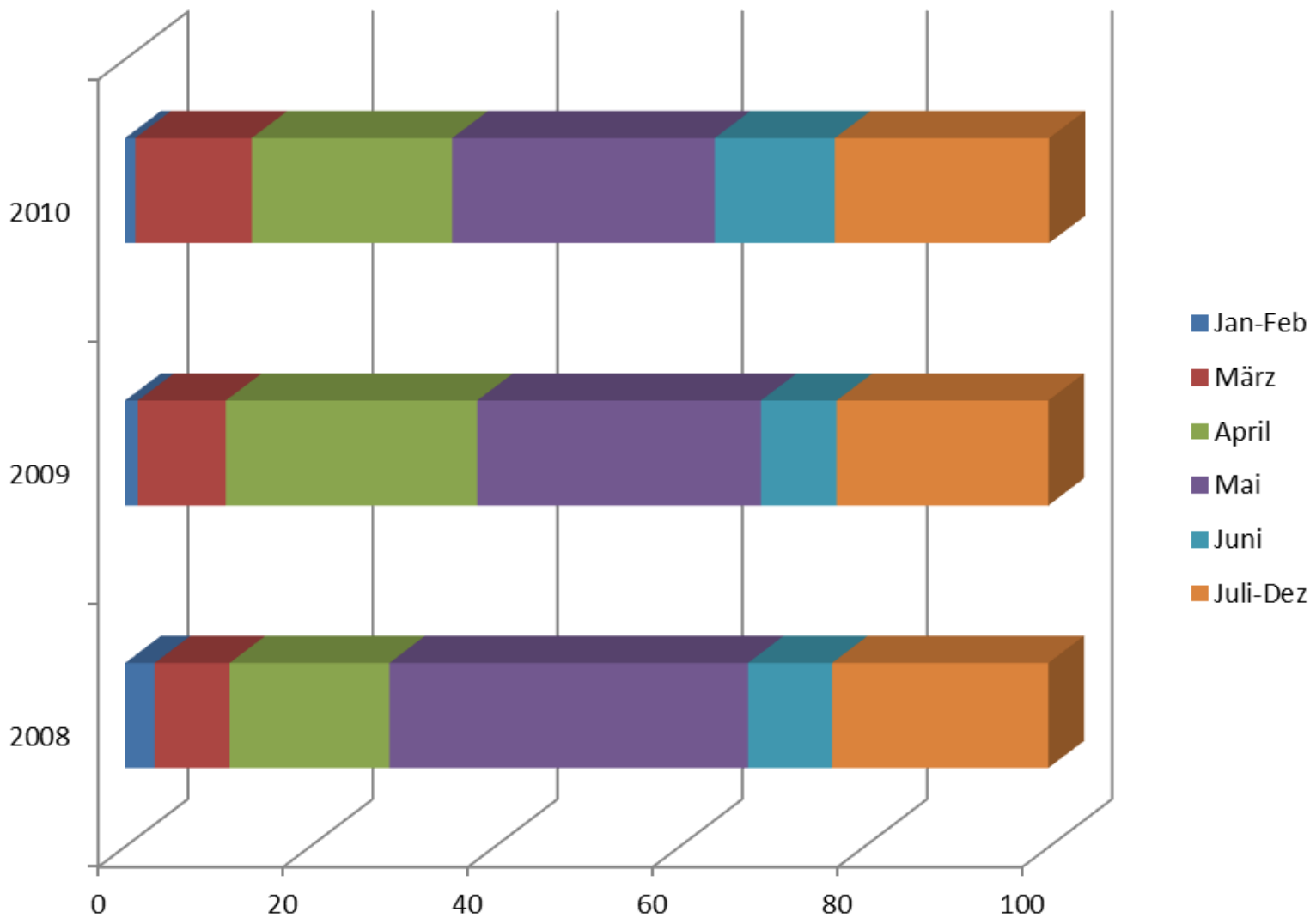

Abbildung 17: Saisonverlauf Beet- und Balkonpflanzen

Quelle: Eigene Darstellung nach Angaben von Niehues 2009, S. 55 


\section{Erlöse pro Monat}

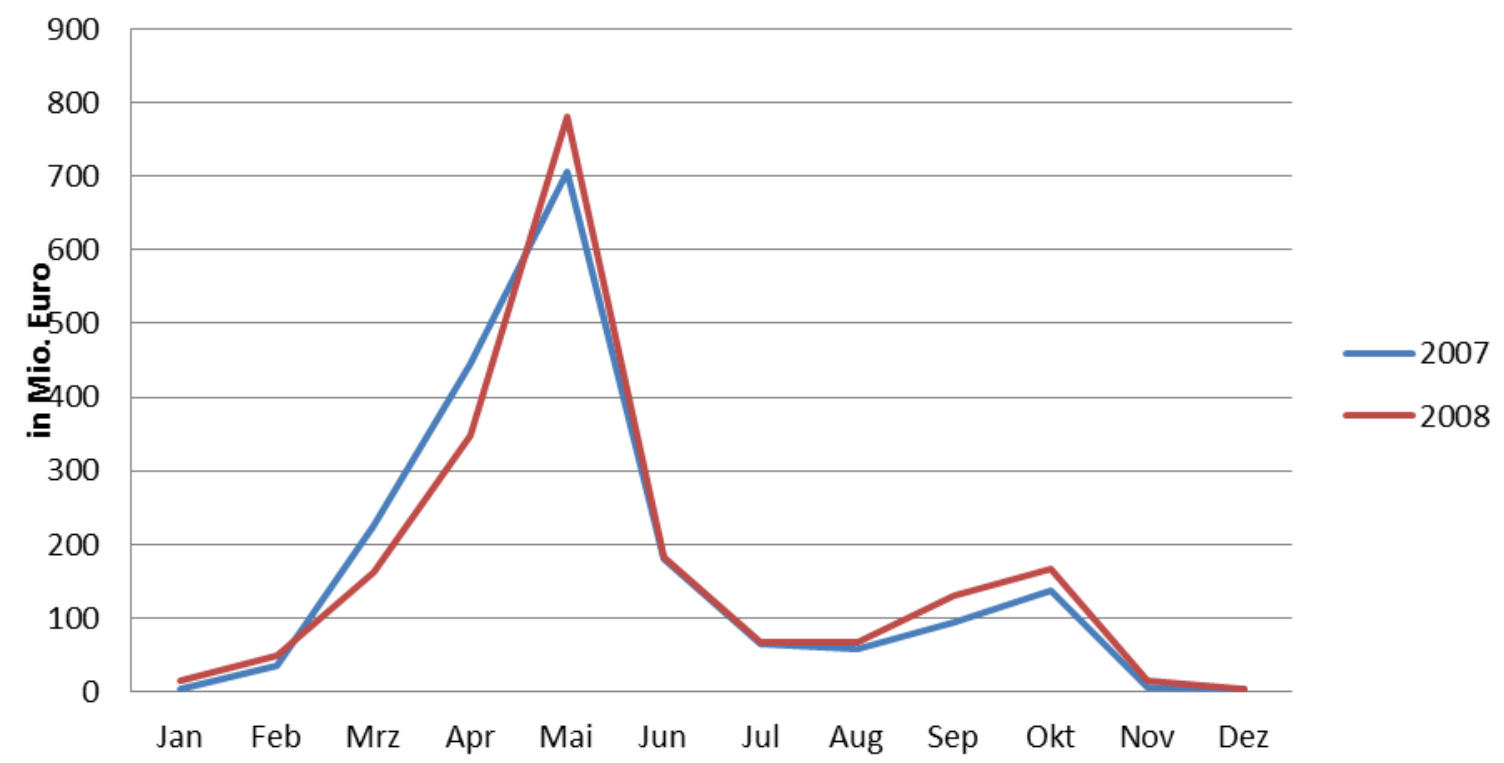

Abbildung 18: Saisonverlauf Beet- und Balkonpflanzen

Quelle: Eigene Darstellung nach Angaben von Niehues 2009, S. 55

Was die Einkaufsstätten von Beet- und Balkonpflanzen betrifft, zeigt sich ein differenzierteres Einkaufsverhalten als bei Topfpflanzen allgemein. Der Facheinzelhandel erlöste im Jahr 2008 ca. $57 \%$ des gesamten Jahresumsatzes mit Beet- und Balkonpflanzen, während die Facheinzelhandelsquote bei Topfpflanzen bei $60 \%$ lag. Innerhalb des Facheinzelhandels hatten die Gärtnereien bzw. Baumschulen im Jahre 2008 mit 19,2 \% den größten Umsatzanteil bei Beetund Balkonpflanzen. Nahezu gleichauf liegen die Baumärkte (DIY) mit 19,1\%. An dritter Stelle folgen die Gartencenter mit 16,5 \% Umsatzanteil. Im Vergleich mit den erreichten Umsatzanteilen des Jahres 2007 zeigt sich allerdings, dass Gärtnereien und Baumschulen die größten Verluste hinnehmen mussten. Da ihr Umsatzanteil 2007 noch bei ca. 23,2 \% lag, haben sie in 2008 ca. 17,2 \% ihres Anteils verloren. Gewinner dieser Zeitperiode sind besonders die Verbrauchermärkte und die Discounter, die ihren Anteil von 5,2 auf 5,8\% bzw. von 8,0 auf 9,0 \% steigern konnten, was einem Zuwachs von 11,5 bzw. 11,3\% entspricht. Baumärkte legten zwischen 2007 und 2008 ebenfalls stark zu. Sie steigerten ihren Umsatzanteil von 17,6 auf 19,1\%, was einem Wachstum von 8,5\% entspricht (Behr/Niehues 2009, S. 93 f; Niehues 2009, S. 53). Abbildung 19 zeigt diese Entwicklung für alle Einkaufsstätten. 


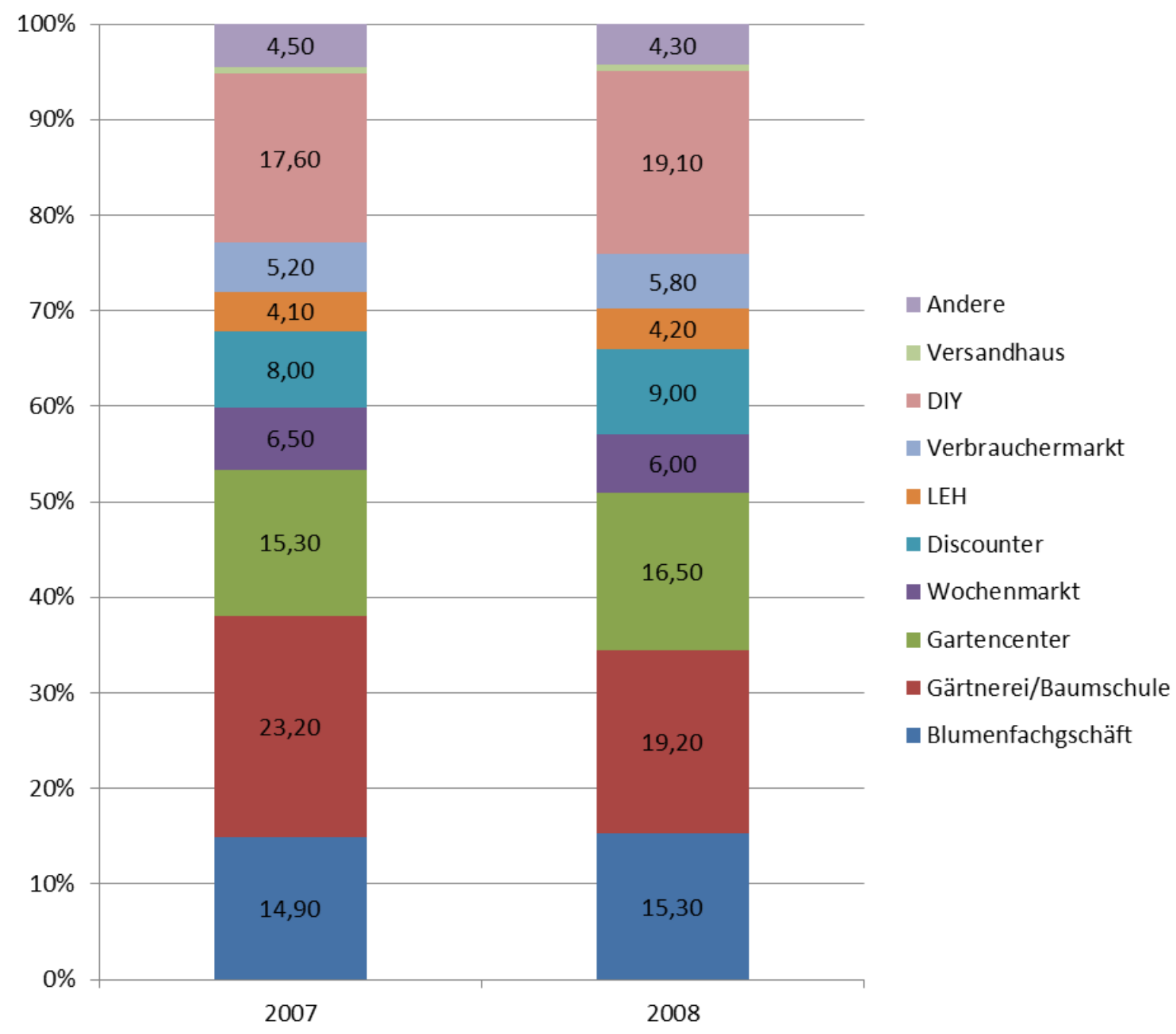

Abbildung 19: Einkaufsstätten Beet- und Balkonpflanzen

Quelle: Eigene Darstellung nach Angaben von Behr/Niehues 2009, S. 93 f; Niehues 2009, S. 53

Die für den Verkauf von Beet- und Balkonpflanzen mit Abstand wichtigste Pflanze ist die Geranie (Pelargonium). Sie wird hauptsächlich im April und im Mai verkauft und trägt mit ca. $14 \%$ zum Jahresumsatz aller Beet- und Balkonpflanzen bei. Auf Platz zwei folgen mit einem Umsatzanteil von $8 \%$ Stiefmütterchen. Diese werden sowohl im Frühjahr als auch im Herbst verkauft. Platz drei belegen Callunen bzw. Sommerheide-Pflanzen. Unter den Pflanzen der Herbstsaison sind sie diejenigen mit dem höchsten Umsatzanteil (Behr/Niehues 2009, S. 94). Abbildung 20 zeigt die Umsatzanteile für die zehn umsatzstärksten Beet- und Balkonpflanzen des Jahres 2008. 


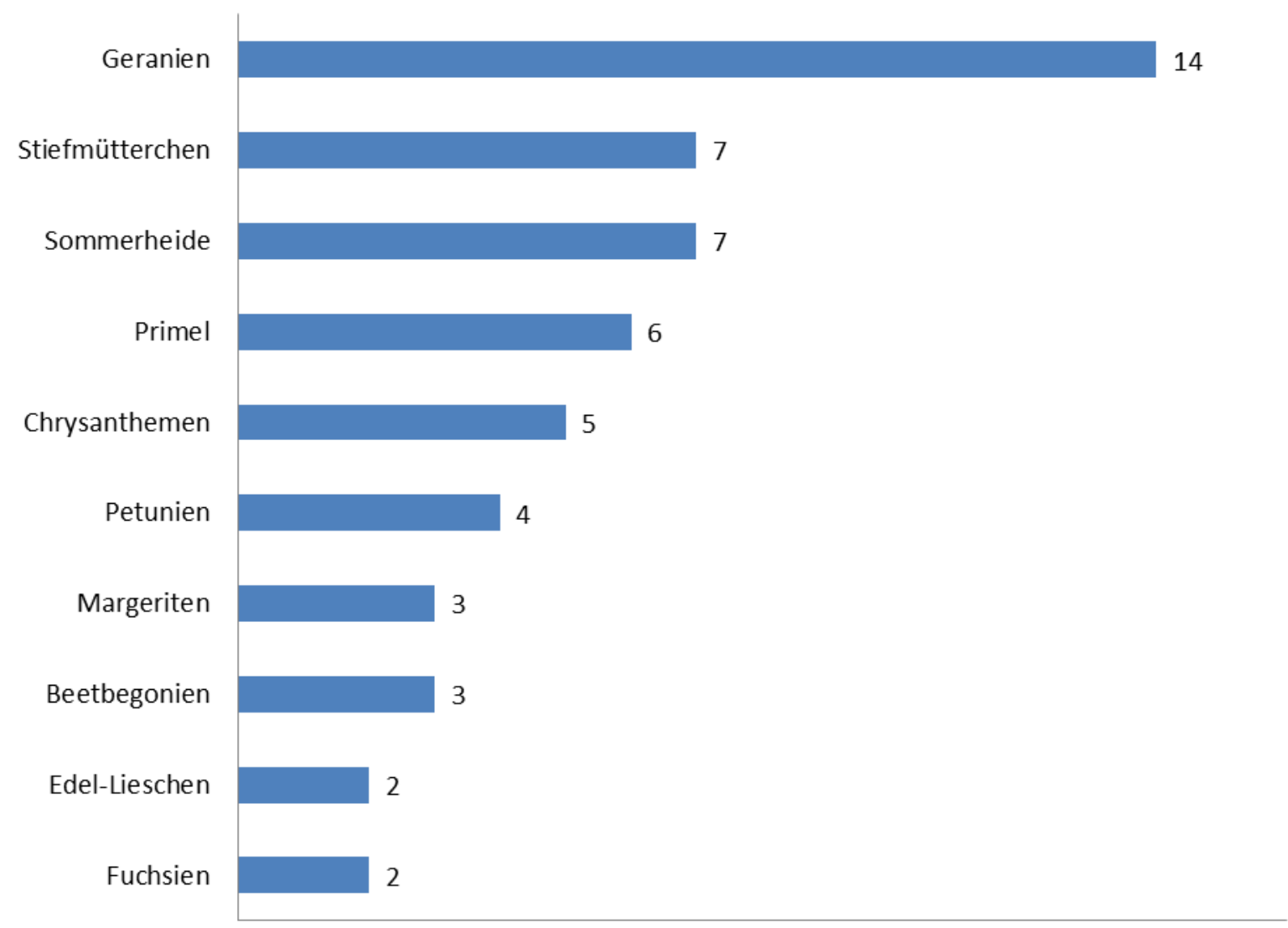

Abbildung 20: Umsatzanteile Beet- und Balkonpflanzen 2008 in Prozent

Quelle: Eigene Darstellung nach Angaben von Behr/Niehues 2009, S. 94

\subsection{Stauden}

Stauden gehören wie Zimmerpflanzen sowie Beet- und Balkonpflanzen zu den Topfpflanzen. Eine exakte Trennung zwischen Beet- und Balkonpflanzen und Stauden ist in manchen Bereichen schwierig, da es eine Definitionsfrage ist, was zu den Stauden gezählt wird und was nicht. Im weiteren Verlauf dieser Arbeit sollen Stauden als mehrjährige Freilandpflanzen verstanden werden, deren oberirdische Pflanzteile nach jeder Vegetationsperiode absterben und in jeder Folgeperiode wieder erneut austreiben. Der Corpus der Staude verholzt im Laufe der Jahre nicht, sondern bleibt weich. Typische und bekannte Beispiele für Stauden sind Gartenlilien, Christrosen oder Lavendel.

Wie in den Abbildungen 4 und 21 zu sehen ist, erfreuen sich Stauden während der letzten Jahre immer größerer Beliebtheit. Wurden im Jahr 2002 in Deutschland noch ca. 307 Mio. Euro zu Einzelhandelspreisen für Stauden ausgegeben, waren dies im Jahr 2008 bereits ca. 480 Mio. Euro (Behr/Niehues 2009, S. 95; Niehues 2009, S. 47). Dies bedeutet eine Umsatz- 
steigerung von ca. $57 \%$ über acht Jahre, was einem durchschnittlichen Wachstum von ca. 7,1 \% pro Jahr entspricht. Unter dem Gesichtspunkt der Zuwachsraten der letzten Jahre sind Stauden die beliebteste aller in Kapitel 2.4.1 vorgestellten Zierpflanzengruppen.

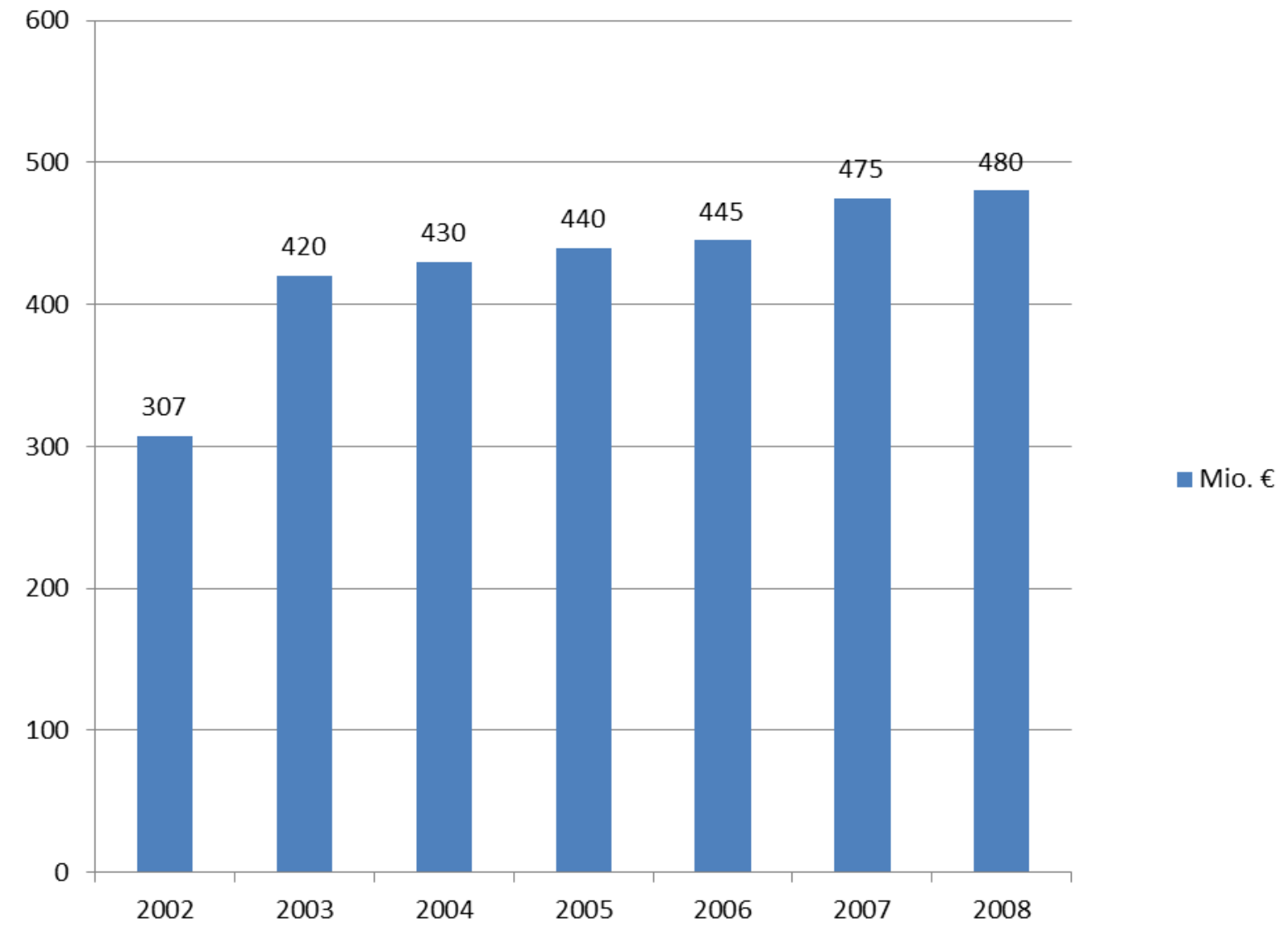

\section{Abbildung 21 Umsatzentwicklung Stauden}

Quelle: Eigene Darstellung nach Angaben von Behr/Niehues 2009, S. 95; Niehues 2009, S. 47

Als Freilandpflanzen ähneln die hauptsächlichen Verkaufszeiten von Stauden denen der Beetund Balkonpflanzen. Mit Frühlingsbeginn steigen die Umsätze stark an und finden ihren Höhepunkt im Mai. Danach fallen sie wieder bis auf den Verkaufsmonat September, der für die Herbstsaison steht (Niehues 2009, S. 55). Abbildung 22 verdeutlicht den stark saisonal verlaufenden Umsatz. 


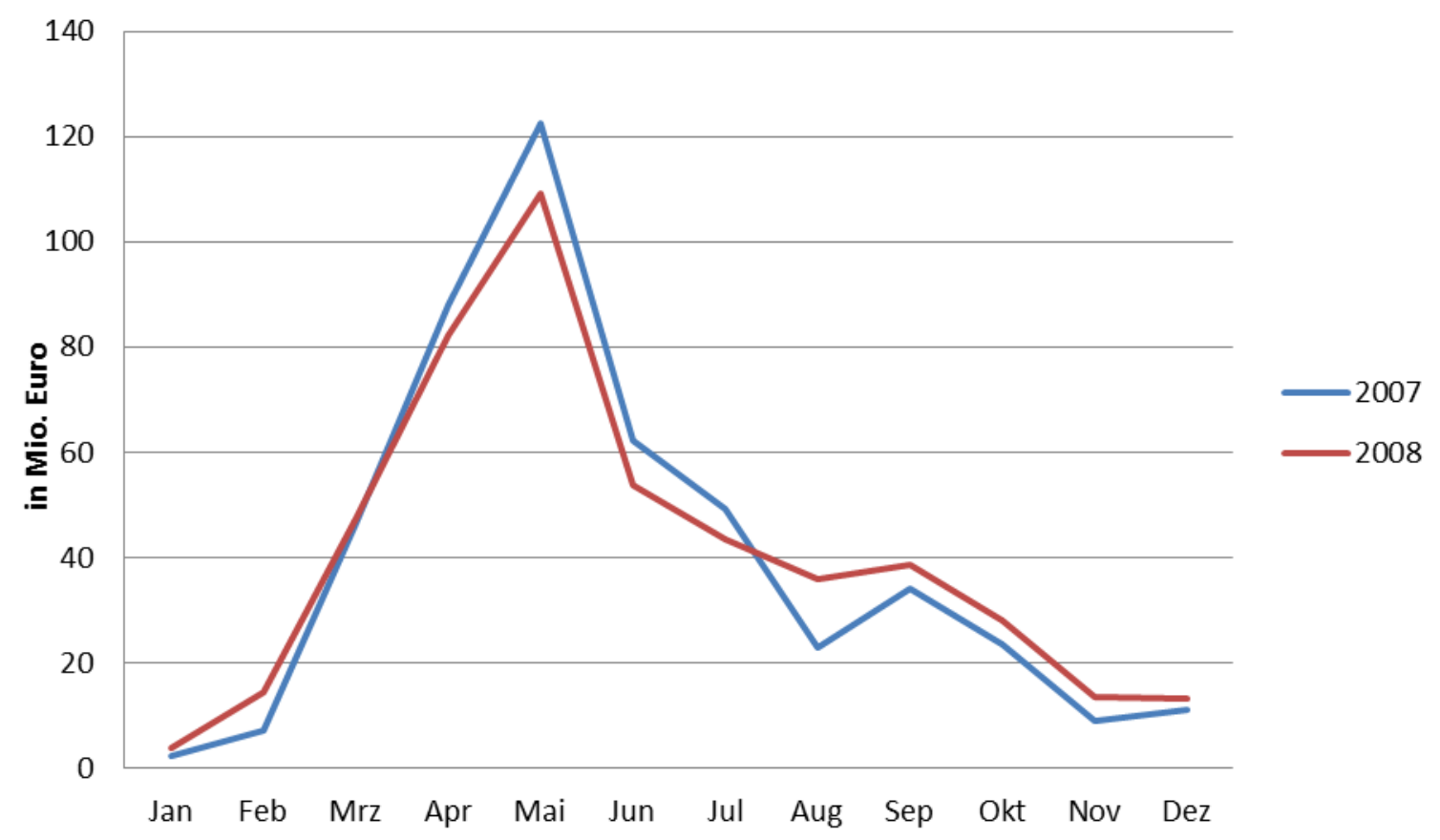

Abbildung 22: Saisonverlauf Stauden

Quelle: Eigene Darstellung nach Angaben von Niehues 2009, S. 55

Bei den Einkaufsstätten für Stauden zeigt sich, dass zwischen den Jahren 2007 und 2008 viel Bewegung zu verzeichnen war. Der Facheinzelhandel konnte im Bereich Stauden seinen Umsatzanteil von 53,5 auf 56,7 \% steigern, was einem Wachstum von ca. $6 \%$ entspricht. Innerhalb des Facheinzelhandels sind wiederum Gärtnereien und Baumschulen die größten Verlierer. Ihr Anteil am Staudenumsatz hat sich zwischen 2007 und 2008 um ca. 26,2 \% verringert. Trugen Gärtnereien und Baumschulen im Jahr 2007 noch mit ca. 21,4 \% zum Gesamtumsatz mit Stauden bei, so waren dies 2008 nur noch ca. 15,8 \%. Alle anderen Geschäftstypen des Facheinzelhandels konnten ihren Umsatzanteil hingegen steigern: das Blumenfachgeschäft von 10,6 auf 13,6 \%, das Gartencenter von 17,8 auf 23,0 \% und der Wochenmarkt von 3,7 auf 4,3\%. Mit 23,0\% stellten im Jahr 2008 Gartencenter die Gruppe mit dem höchsten Umsatzanteil. 2007 waren dies mit 21,4 \% noch die Gärtnereien und Baumschulen. Enorme anteilige Verluste beim Staudenumsatz mussten auch der LEH und das Versandhaus hinnehmen. Im LEH ging der Anteil von 4,7 auf 2,7\% zurück und im Versandhaus von 6,0 auf 2,4\%. Die bei Beet- und Balkonpflanzen noch dynamischste Gruppe der Discounter konnte auch bei den Stauden ihren Umsatzanteil steigern, von 8,4\% in 2007 auf 9,9\% in 2008 (Niehues 2009, S. 53). Abbildung 23 zeigt die Umsatzverteilungen der einzelnen Verkaufsstätten. 


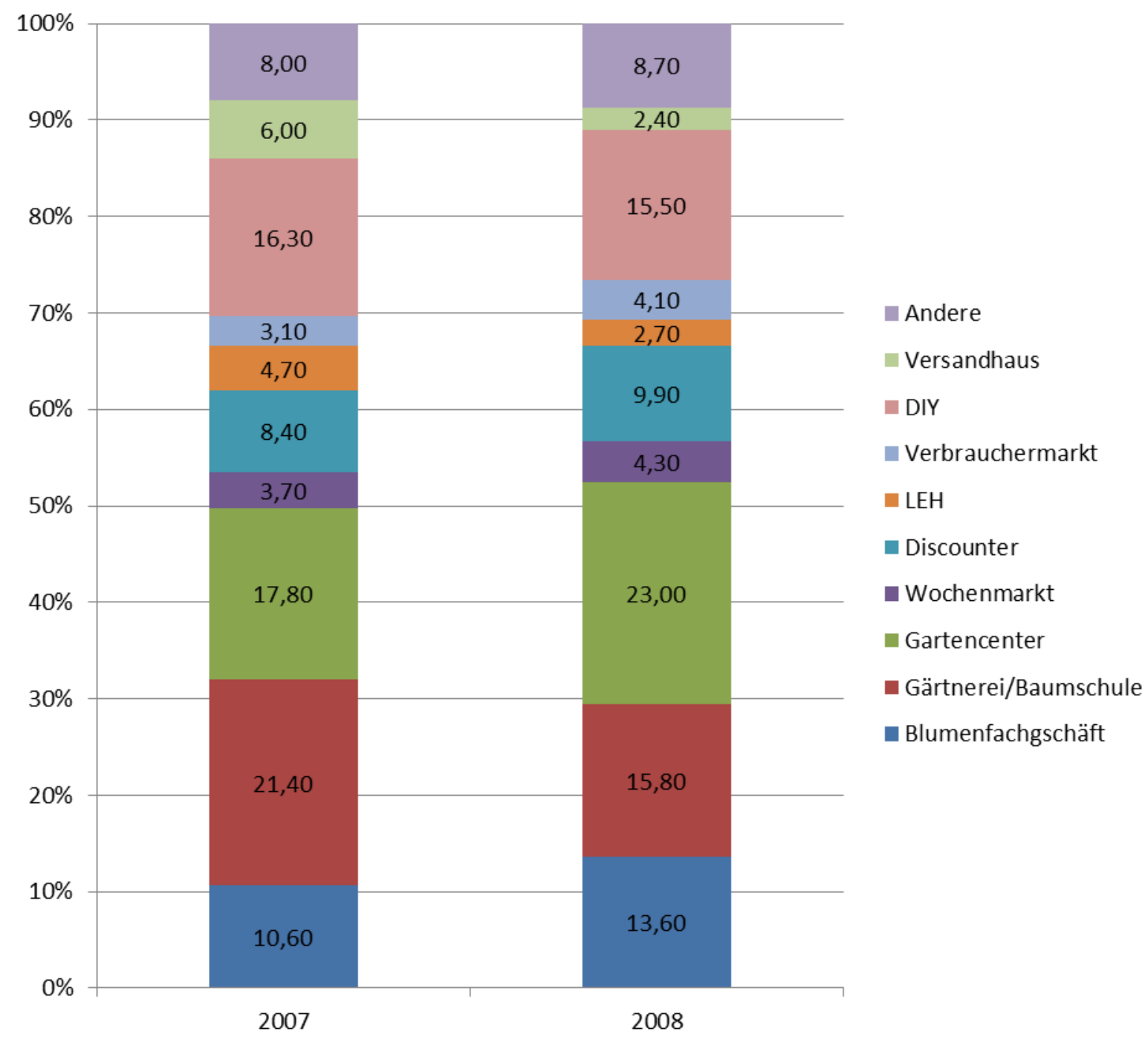

Abbildung 23 Einkaufsstätten Stauden

Quelle: Eigene Darstellung nach Angaben von Niehues 2009, S. 53

\subsubsection{Gehölze}

Neben Topfpflanzen und Schnittblumen unterteilen sich Zierpflanzen zudem in die Gruppe der „Gehölze“ (siehe Abbildung 2). Der branchenweite Umsatz mit Gehölzen, zu denen sowohl Obst- und Ziergehölze als auch Forstpflanzen zählen, belief sich im Jahr 2008 auf ca. 1,485 Mrd. Euro zu Einzelhandelspreisen. Erfasst ist hierbei der Gesamtmarkt, also sowohl private als auch institutionelle Käufer. Nicht berücksichtigt sind allerdings die Käufe von Waldbesitzern und Obst- und Weinbaubetrieben. Insgesamt hat sich der Markt für Gehölze die letzten Jahre über sehr schlecht entwickelt: Die Umsätze lagen im Jahr 2002 noch bei ca. 1,5 Mrd. Euro, was bis 2008 einem durchschnittlichen jährlichen Rückgang von ca. 0,2 \% 
entspricht. Im Jahr 2003 erreichte der Umsatz mit Gehölzen mit ca. 1,55 Mrd. Euro seinen Höchststand, in 2006 mit ca. 1,39 Mrd. Euro seinen Tiefpunkt (Niehues 2009, S. 48). Abbildung 24 zeigt die Entwicklung der einzelnen Jahre von 2002 bis 2008.

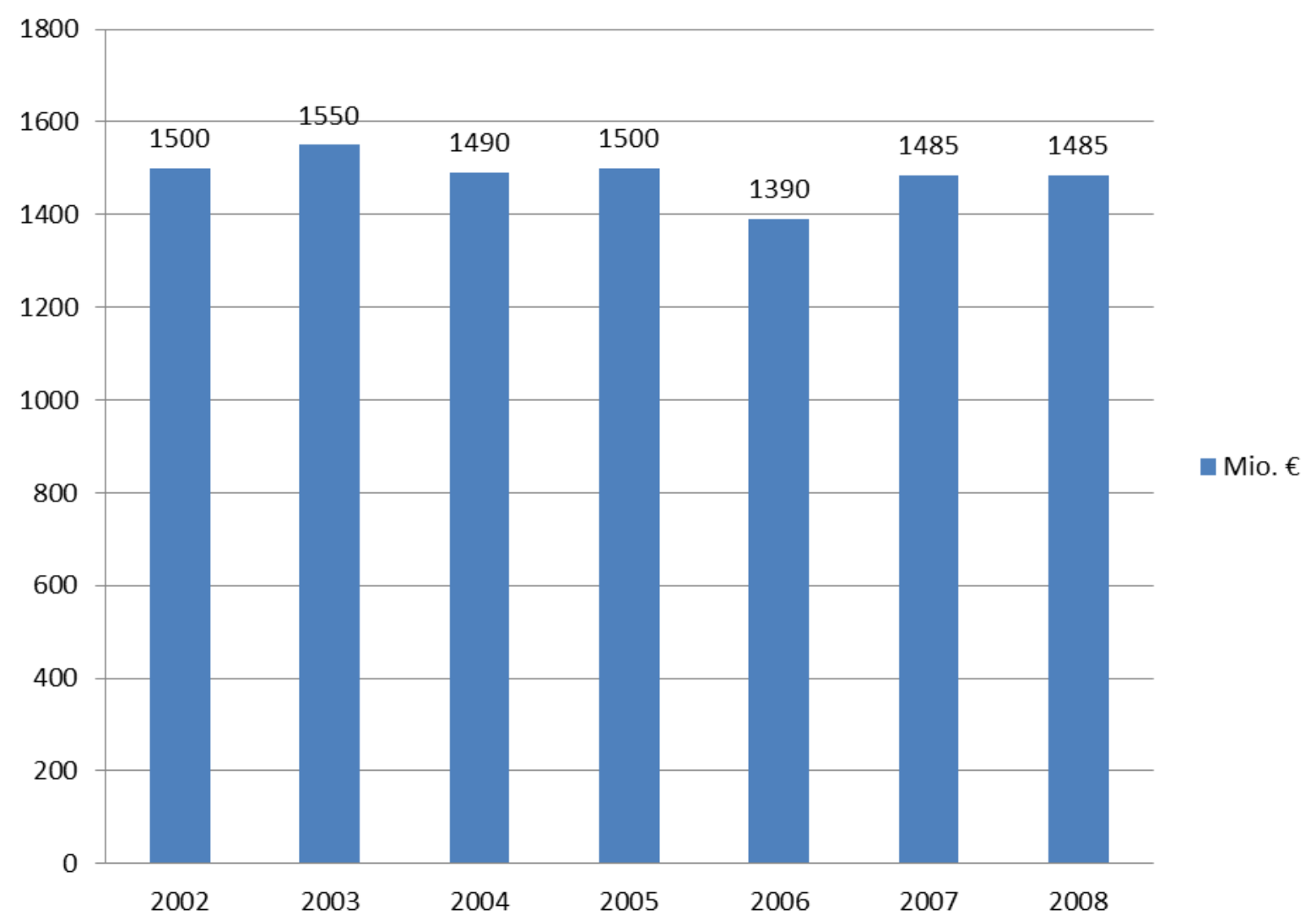

Abbildung 24: Umsatzentwicklung Gehölze

Quelle: Eigene Darstellung nach Angaben von Niehues 2009, S. 48

Da Gehölze ebenfalls für das Freiland gekauft werden, unterliegen auch sie primär den Bedingungen der natürlichen Pflanzzeit. Eine Ausnahme bilden dabei die Monate November und Dezember. Obwohl diese aufgrund ihrer Temperaturen nicht zur Verkaufssaison für Freilandpflanzen zählen, verzeichnen Gehölze während dieser Monate steigende Umsätze. Dies liegt daran, dass auch abgeschnittene Gehölzäste zu den Gehölzen gezählt werden und diese zur Weihnachtszeit als Dekoration in sehr großer Stückzahl verkauft werden. Ansonsten ergibt sich auch für Gehölze das typische Bild der Umsatzverläufe für Freilandpflanzen: Während der ersten Wintermonate sind die Umsätze nahezu gleich null, mit dem Auftauen des Bodens ziehen die Umsätze an und erreichen ihren Höhepunkt im April oder Mai. Nach der Talsohle im Hochsommer nehmen die Umsätze zur Herbstsaison im September und 
Oktober nochmals zu. Die untypischen steigenden Umsätze zum Ende des Jahres basieren, wie bereits erwähnt, auf der Umfunktionierung der Gehölze von einer Freilandpflanze zu weihnachtlichem Dekorationsmaterial (Niehues 2009, S. 55). Abbildung 25 zeichnet den Verlauf der Erlöse für die Jahre 2007 und 2008 nach.

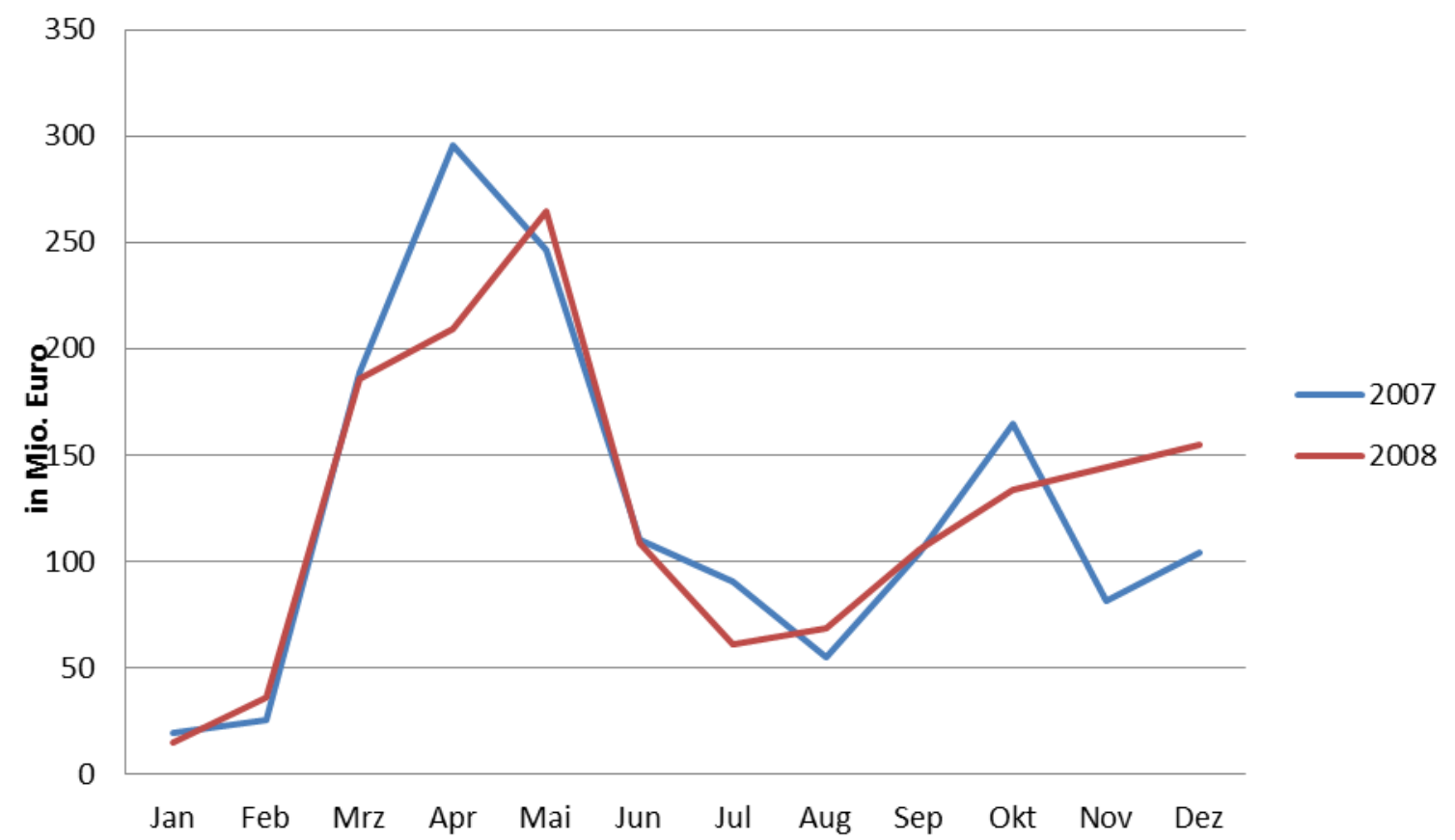

Abbildung 25: Saisonverlauf Gehölze

Quelle: Eigene Darstellung nach Angaben von Niehues 2009, S. 55

Bei den Einkaufsstätten für Gehölze spielt der Facheinzelhandel die wichtigste Rolle. 2008 hatte er einen Marktanteil am Umsatz von ca. $77 \%$. Zu den Geschäftstypen des Facheinzelhandels zählen hierbei der Garten- und Landschaftsbau (Ga-La-Bau), die Blumenfachgeschäfte, die Gärtnereien bzw. Baumschulen, die Gartencenter und die Erlöse auf dem Wochenmarkt. Gehölze unterscheiden sich in der Art ihres Verkaufs sehr von anderen Topfpflanzen, was sich auch in den Marktanteilen widerspiegelt. Verglichen mit den Topfpflanzen und Schnittblumen ist die Anzahl der Käufer von Gehölzen sehr viel kleiner, ebenso die Frequenz der Käufe. Gehölze werden zudem nicht jedes Jahr neu gepflanzt, sondern sind im Normalfall auf eine langfristige Nutzungsdauer ausgelegt. Dementsprechend sind Kunden für Gehölze meist sehr stark an Beratung interessiert, was dem Facheinzelhandel entgegenkommt.

Als wichtigste Einkaufsstätte für Gehölze konnten Einzelhandelsgärtnereien bzw. Einzelhandelsbaumschulen ihren Marktanteil von $37 \%$ in 2007 auf $38 \%$ in 2008 steigern. Mit einem 
Marktanteil von $20 \%$ folgt auf dem zweiten Platz der Garten- und Landschaftsbau vor den Gartencentern auf Rang drei mit $14 \%$ Umsatzanteil. Unter den Nicht-Facheinzelhändlern spielen die Baumärkte die wichtigste Rolle. Mit $13 \%$ Marktanteil liegen sie nahezu gleichauf mit den Gartencentern. Der LEH erreicht bei Gehölzen lediglich 5 \% Marktanteil, „Andere“ ebenfalls nur $5 \%$, wobei „Andere“ sich zusammensetzt aus Kauf- und Warenhäusern, Tankstellen und dem Internet-Einkauf (Niehues 2009, S. 53). Abbildung 26 zeigt die Marktanteile der Einkaufsstätten im Vergleich.

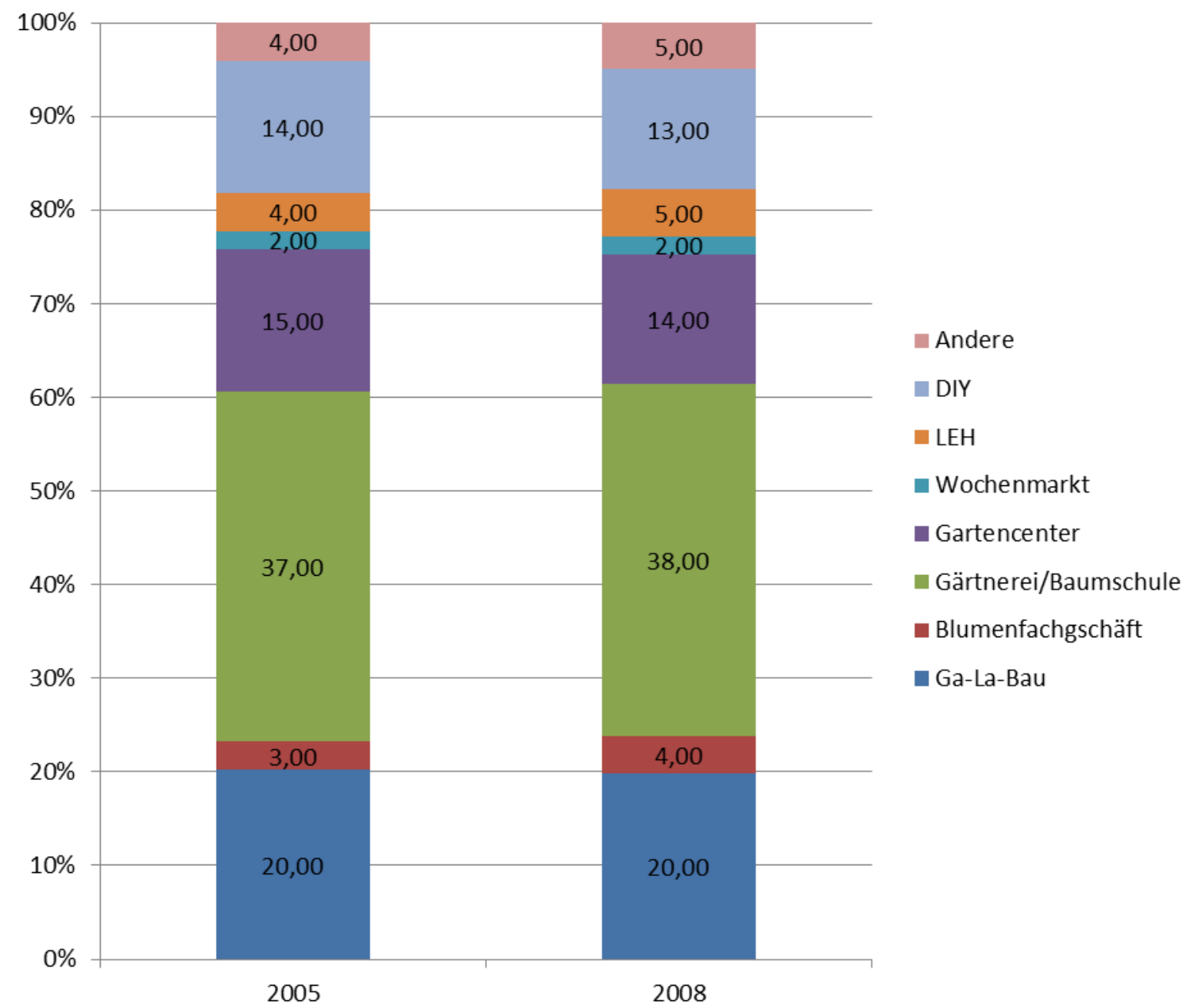

Abbildung 26: Einkaufsstätten Gehölze

Quelle: Eigene Darstellung nach Angaben von Niehues 2009, S. 53 


\section{Lebensstile und Marktsegmentierung}

\subsection{Lebensstile}

Die Begriffe „Lebensstil“, „Lebensführung“, „Milieu“ und „Lifestyle“ tauchen in verschiedenen Forschungsbereichen immer häufiger auf, werden aber oft nur unscharf bzw. nicht ausreichend differenziert. Es gilt daher zu klären, inwiefern diese Begriffe als Synonym gebraucht werden können bzw. welche Merkmale bedeutungsunterscheidend sind, so dass eine ausreichende Abgrenzung erfolgen werden kann, die für den weiteren Verlauf dieser Arbeit gültig ist.

Das Wortfragment „Stil“ hat im Laufe der Zeit vielerlei semantische Anpassungen erfahren. Es wurde bis zum 17. Jahrhundert hauptsächlich nur auf die angewandte Sprache und Schrift bezogen, teilweise auch noch im Bereich der Kunst gebraucht. Erst im 18. Jahrhundert erweiterte sich dieser Bezugsrahmen und es entstand der sogenannte „stilistische Pluralismus“ - ein erstes Verständnis von Stil, das dem heutigen Verständnis von Lebensstil ähnelt. In einem erweiterten Verständnis eignet sich der Stil somit einerseits zur Integration des Einzelnen und andererseits auch zur Abgrenzung von Anderen (Burzan 2005, S. 97 f.). Heutzutage wird der Stil einer Person sowohl über zur Schau gestellte Äußerlichkeiten wie beispielsweise Schmuck, Kleidung, Frisur etc. als auch über individuelle Wertschätzungen wie beispielsweise Lebenseinstellungen, Genüsse etc. interpretiert. Man spricht hierbei auch von der „Zeichen-“ und der „Bedeutungsebene“ (Schulze 2005, S. 103).

Der Wissenschaftsbereich der Soziologie hat daraus zunächst den Begriff des „Lebensstils“ bzw. „die Disziplin der Lebensstilforschung“ abgeleitet, welcher umgangssprachlich verstanden wird als Lebensstil einer Person, die alltäglichen Lebensgewohnheiten nachgeht, welche wiederum die betrachtete Person ausmachen (Freter 2008, S. 68). Dabei kann es beispielsweise darum gehen, welche Musik eine Person gerne hört, welche Sportart sie betreibt, wie sie wohnt, wie sie sich kleidet, welche Werte sie vertritt, welche Einstellungen sie hat. Aus der Summe dieser einzelnen Entscheidungen, die eine Person trifft und nach außen zeigt, entwickelt sich ihr individueller Stil - ihr Lebensstil. Allerdings existiert im Bereich der Forschung bis heute keine allgemeine Definition von „Lebensstil“, obwohl er vielfach beschrieben worden ist. Teilweise sind diese Definitionen uneinheitlich, manche sogar gegensätzlich (Enneking/Franz 2005, S. 4). Im Folgenden werden einige angeführt, um einerseits die Vielfältigkeit und andererseits die Abweichungen zu verdeutlichen. 
So verstand Lüdtke im Jahre 1990 unter Lebensstil eine „unverwechselbare Struktur und Form der Lebensorganisation eines privaten Haushalts bzw. der in ihm lebenden Individuen“ (Lüdtke 1990, S. 434). Georg beschrieb 1998 Lebensstile als „relativ stabile, ganzheitliche und routinisierte Muster der Organisation von expressiv-ästhetischen Wahlprozessen“ (Georg 1998, S. 98). Demgegenüber formulierte Reusswig im Jahr 2002 folgende Definition: „Lebensstile [sind für uns] gruppenspezifische Formen der individuellen Lebensführung und -deutung. Sie beschreiben typische Muster der Organisation des Alltags, der Identitätsbildung, der Sinngebung (oder des Sinnbastelns), der Kommunikation und der Stilierung(sversuche) des Selbst““(Reusswig 2002, S. 158).

Ebenso gibt es unterschiedliche Definitionen des Begriffs „Lebensführung“. Behringer versteht ihn wie folgt: „Basis von Lebensführung sind die alltäglich erbrachten praktischen Tätigkeiten in den jeweiligen Lebensbereichen. Für die Frage der Lebensführung interessiert aber vor allem, wie diese Tätigkeiten von den Personen im Hinblick auf ihre zeitliche, sachliche, räumliche, soziale, mediale, sinnhafte und emotionale Dimension aktiv reguliert und strukturiert werden“" (Behringer 1998, S. 41). Hingegen erscheint Scholl und Hage Lebensführung ,als alltäglicher Prozess, in dem sich ein Mensch mit den ihm begegnenden Verhaltenszumutungen (als Berufstätige, als Ehefrau, als Mutter usw.) im Rahmen bestimmter Gegebenheiten (Wohnverhältnisse, Haushaltseinkommen usw.) auseinandersetzt, sie in Einklang miteinander sowie mit seinen eigenen Interessen zu bringen versucht und dabei in spezifischer Weise auf sein soziales und räumliches Umfeld wie Familienangehörige, Arbeitsstätte, Nachbarn, Nutzung von Verkehrsmitteln usw. einwirkt“" (Scholl/Hage 2004, S. 14).

Die Heterogenität des Verständnisses von Lebensstilen, wie sie die verschiedenen Definitionen deutlich machen, liegt darin begründet, dass Lebensstilansätze thematisch meist modular aufgebaut sind und jeder Ansatz von Inhalt und Anzahl der Module unterschiedlich ausfällt (Enneking/Franz 2005, S. 2). Zudem zeigt sich hier, dass die Begriffe Lebensstil und Lebensführung in der Literatur nicht exakt voneinander abgegrenzt werden. Teilweise werden sie synonym verwendet oder gar „einbettend“, wie beispielsweise in Ansätzen, die den Lebensstil als einen bestimmten Teil der gesamten Lebensführung sehen (Otte 2008, S. 14 f.; Freter 2008, S. 68 f.). Konsens besteht immerhin dahingehend, dass sich die beiden Begriffe in ihrer Bedeutung zumindest sehr ähnlich sind, weshalb sie im weiteren Verlauf der Arbeit als gleich betrachtet werden. Diese Vorgehensweise folgt dem Gedanken Hartmanns, der eine Trennung der beiden Begriffe letztlich für „unproduktiv“ hält (Hartmann 1999, S. 46). 
Ähnlich verhält es sich in der Literatur mit den Begriffen Lebensstil und Lifestyle, die oftmals als gleichbedeutend verstanden werden. Im weiteren Verlauf dieser Arbeit sollen diese beiden Begriffe allerdings nicht synonym verwendet werden. Vielmehr wird der Lifestyle einer Person hier als Teil des entsprechenden Lebensstils der Person verstanden. Im Gegensatz zum Lebensstil ist der Lifestyle nur von zeitlich begrenzter Dauer und primär nach außen gerichtet. Er orientiert sich oftmals an jenen gesellschaftlichen Strömungen, die gerade in Mode sind (Richter 2005, S. 115 f.).

Der Begriff „Milieu“ wird in der Literatur ebenfalls nicht einheitlich verwendet. Dabei benutzte bereits der französische Soziologe Comte (1798-1857) den Begriff in seinen Arbeiten. Die deutsche Übersetzung des ursprünglich französischen Wortes bedeutet so viel wie ,in der Mitte“ oder „Umwelt“. Das Individuum ist demnach „geprägt von seiner sozialen Umgebung“ (Hradil 1987, S. 165). Diaz-Bone versteht unter Milieus Kollektive von Lebensstilen, die sich im „sozialen Raum“ befinden (Diaz-Bone 2010, S. 30). In Anlehnung an diese Konzeption werden Milieus in der vorliegenden Arbeit mit Lebensstilkollektiven bzw. -typen gleichgesetzt.

\subsubsection{Historie der Lebensstilforschung}

\section{Gesellschaftliche Ungleichheit}

Ausgangspunkt der heutigen Lebensstilforschung sind frühere Arbeiten zur gesellschaftlichen Ungleichheit, die charakteristisch für soziale Systeme ist. Die Individuen der Gesellschaft haben ungleiche Chancen und Berechtigungen, die unterschiedlichen gesellschaftlichen Ebenen zu betreten bzw. zu erreichen. Stellvertretend für diese Individuen mit unterschiedlichen Rechten wird die Gesellschaft in Schichten und Klassen untereilt. Dabei haben wichtige Autoren zur Thematik der Klassen und Schichten bereits Ansätze von Lebensstilen beschrieben, ohne dabei das Wort „Lebensstil“ explizit zu nennen. Im Folgenden finden sich die Kernaussagen zweier wichtiger Arbeiten zur sozialen Ungleichheit und deren logische Verknüpfung zur aktuellen Lebensstilforschung.

Der deutsche Gesellschaftstheoretiker Karl Marx (1818-1883) beschrieb, dass die Allokation von Ressourcen und der Zugang zu Institutionen für Individuen von deren jeweiliger sozialer Klasse abhängig seien. Diese Klassen der Gesellschaft unterscheiden sich nach Marx einerseits durch die Ausprägungen des Eigentums und andererseits durch „Empfindungen, Illusionen, Denkweisen und Lebensanschauungen“ (zitiert bei Burzan 2005, S. 16). Nach Marx ist 
die Klassenzugehörigkeit der Individuen Grund der Ungleichheit einer Gesellschaft. Die Zuordnung von Personen zu bestimmten Gesellschaftsgruppen bzw. Klassen ist für Marx auch anhand von äußeren Erkennungsmerkmalen bzw. äußeren Zeichen möglich, beispielsweise anhand der Kleider, welche die Personen tragen (Georg 1998, S. 11). Zwar gebraucht Marx nicht den Begriff „Lebensstil“, jedoch sind Ansätze davon zu erkennen, wenn er beispielsweise von der Abgrenzung der Klassenzugehörigen über deren Kleidungsstil oder der Abgrenzung der Klassen an sich spricht.

Eine weitere Grundlage für den modernen Begriff des Lebensstils stammt von Georg Simmel (1858-1918), der erstmals von einem „Stil des Lebens“ sprach. Simmel sah jegliche Art von Stil als Distanzierung an. Im zufolge soll der Stil des Lebens sowohl individualisieren als auch differenzieren. Er sieht dabei den Stil des Lebens als völlig individuell geprägt an. Der Stil und die Harmonie des Einzelnen mit seiner sozialen Lebenswelt stehen dabei im Fokus der Betrachtung (Simmel o. J., S. 304 ff.).

Sowohl nach Marx als auch nach Simmel spielt die Ungleichheit der Menschen bzw. die Ungleichheit der Ressourcenausstattung der Individuen mit Ressourcen wie beispielsweise Geld, Macht oder Bildung eine wichtige Rolle für das Verständnis des Begriffs „Lebensstil“. Für beide ist diese Ungleichheit ausschlaggebend für den Lebensstil eines jeden Einzelnen. Des Weiteren sind in beiden Ansätzen bereits weitere Überlegungen zu erkennen, die bis auf das heutige Verständnis von Lebensstil ausstrahlen. So sehen beide Autoren den Lebensstil einerseits als ein Instrument der Integration und andererseits als ein Mittel der Differenzierung durch Stilisierung.

\section{Lebensstile als Weiterentwicklung des Klassen- und Schichtenmodells}

Das eben vorgestellte hierarchische Gesellschaftsmodell nach Klassen und Schichten war in den Sozialwissenschaften in Deutschland bis Mitte/Ende der 1980er Jahre Standard. Erst danach entstanden weitere Konzepte von Gesellschaftsmodellen wie beispielsweise das Modell des „Lebensstils“ oder das „Milieu“-Konzept. Beide Konzepte galten zunächst als Ergänzung des bestehenden Modells, lösten dieses im Laufe der Zeit jedoch mehr und mehr ab. Das reine Klassen- und Schichtenmodell wirkte zunehmend veraltet und wurde bis dahin nur aufgrund mangelnder, besserer Alternativen am Leben erhalten (Beck 1986, S. 121). Mit den neuen Ansätzen wurden die Individuen einer Gesellschaft nun erstmals nicht mehr nur anhand einiger weniger Merkmale „vertikal“ voneinander abgegrenzt. Vielmehr entwickelten sich soziale 
Gruppen mit sowohl ethischen als auch ästhetischen Ähnlichkeiten, denen bestimmte Interessen in ihrem Alltag gemeinsam waren (Diaz-Bone 2004, S. 2).

Als Ursprung des Lebensstilkonzepts gelten drei fundamentale Bewegungen aus den 1970er und 1980er Jahren, die die Gesellschaft damals veränderten: erstens die zunehmende Individualisierung der Lebensführung, zweitens die Pluralisierung der Lebenslagen und drittens der Wertewandel der Gesellschaft in den vorangehenden Jahren.

Die Individualisierung der Lebensführung lässt sich nach Hradil auf die neu aufgekommene Wahlfreiheit von Lebensweisen zurückführen (Hradil 1999, S. 56). Beck sieht in diesem Zusammenhang die Individualisierung als typisch für eine moderne Gesellschaft an. Des Weiteren ist sie Zeichen eines subjektiven Bewusstseins (Beck 1986, S. 206 ff.). Otte vereint die beiden Ansätze von Lebensstil und Individualisierung und kommt zu dem Schluss, dass der Lebensstil eines Individuums letztendlich das „Produkt individueller Entscheidungen“ sei und dem Individuum zu sozialer Orientierung und Identität verhelfe (Otte 1998, S. 185 f.).

Der Grundgedanke, der hinter der Pluralisierung der Lebenslagen als Ursprung der Lebensstilforschung steht, ist, dass durch gesteigerten Wohlstand und ein gestiegenes Bildungsniveau der Bevölkerung viele neue Handlungsmöglichkeiten für die Individuen einer Gesellschaft entstehen. Diese neuen Wahlmöglichkeiten erlauben es, dass bei der Bewältigung des Alltags neue Stile entstehen (Hradil 1999, S. 431; Hölscher 1998, S. 110 f.).

Innerhalb einer Gesellschaft sind die „Werte“ mit ihrer Rangfolge stets fließend. Man spricht daher auch von einem kontinuierlichen Wertewandel. Richter sieht hier einen kontinuierlichen Übergang von materialistischen zu postmaterialistischen Werten. Dabei verbindet er mit dem Wandel zum Postmaterialismus eine „Ästhetisierung der Gesellschaft“, die einem neuen Lebensstil entspricht (Richter 2005, S. 98). Da Einstellungen zu Werten vielfältig sind, existieren auch verschiedene Arten von Lebensstilen.

Als Ergebnis des gesellschaftlichen Wandels in Deutschland wurden in den 1980er und 1990er Jahren neue Theorien zur sozialen Struktur der Bundesrepublik entwickelt. Viele davon basieren auf der Theorie der Lebensstilforschung. Zu den wichtigsten Arbeiten gehören hier sicherlich „Sozialstrukturanalyse in einer fortgeschrittenen Gesellschaft. Von Klassen und Schichten zu Lagen und Milieus“ von Stefan Hradil und „Die Erlebnisgesellschaft“ von Gerhardt Schulze, die beide Ende der 1980er Jahre erschienen. Neben diesen wegweisenden Arbeiten spielte für die zunehmende Relevanz des Konzepts der Lebensstile ebenso eine Rolle, dass es gerade in der deutschen Kultur verpönt ist, Menschen in Klassen und Schichten zu 
unterteilen (Vester et al. 2001, S. 168). Der nächste Abschnitt zeigt auf, in welcher Art und Form der Ansatz der Lebensstile heute präsent ist und welche Bedeutung er für den weiteren Verlauf dieser Arbeit hat.

\section{Lebensstil heute}

Die Soziologie versucht die Frage zu beantworten, inwieweit ein Lebensstil das Ergebnis einer spezifischen Zugehörigkeit zu einer Klasse, Schicht oder Gruppe sein kann (Reeb 1998, S. 5). Die Soziologie an sich wird dabei als Ursprung der Lebensstilforschung angesehen. Allerdings ist das Thema heute auch in der Psychologie und der Ökonomie stark verankert. In der Psychologie wird aktuell der Frage nachgegangen, inwieweit erkannte Muster der Persönlichkeit und der Motivation einer Person Einfluss auf deren Lebensstil haben können. In der Ökonomie werden die ,theoretischen“ Ergebnisse der beiden anderen Wissenschaftsdisziplinen aufgegriffen und zusammengeführt. Basierend auf den psychologischen und den soziologischen Erkenntnissen entsteht dabei eine kombinierte, nun ökonomische Sicht auf den Lebensstil.

Unternehmen aller Art versuchen Märkte bzw. Kunden anhand deren vermuteter Lebensstile zu gruppieren, um so den Markt zielgerichteter bzw. zielgruppengenauer bearbeiten zu können. Neben den dadurch erhofften Mehrerlösen und parallelen Streuverlustreduktionen bei der Kundenansprache dient eine Aufteilung des Marktes anhand von Lebensstilen auch dazu, die Reaktion der einzelnen Gruppen auf eine unternehmerische Aktion im Vorfeld besser abschätzen zu können. Die „Vorhersage“ von Reaktionen auf der Nachfrageseite soll die Sicherheit eines Marktauf- bzw. -eintritts erhöhen bzw. deren Risiko mindern (Reeb 1998, S. 6).

Es hat sich bisher gezeigt, dass das Verständnis von Lebensstil sehr heterogen geprägt ist und Lebensstile auch in äußerst verschiedenen Wissenschaftsbereichen thematisiert werden. Obwohl dies eine gemeinsame und „verbindliche“ Definition des Begriffs Lebensstil nicht einfacher macht, soll nun anhand von Gemeinsamkeiten der verschiedenen Ansätze genau dies versucht werden.

Grundsätzlich lassen sich vier verschiedene Grundpfeiler der verschiedenen Definitionen ausmachen: Freiwilligkeit, Charakter, Ganzheitlichkeit sowie Stilisierungschancen und neigungen. Freiwilligkeit steht für die individuelle Wahlmöglichkeit eines Individuums, was seinen Lebensstil betrifft. So kann es durchaus sein, dass Personen trotz gleicher bzw. zumindest sehr ähnlicher Lebensumstände überaus verschiedene Lebensstile führen. Der Charakter 
einer Lebensstilführung sorgt für die Unverwechselbarkeit und Identifizierbarkeit einer Person. Daraus ergibt sich sowohl Erkennbarkeit innerhalb einer Gruppe als auch Abgrenzung zwischen verschiedenen Gruppen. Ganzheitlichkeit wiederum steht für den Gedanken, dass alle sozialen Gegebenheiten den Lebensstil beeinflussen. Der Aspekt der Stilisierungschancen und -neigungen schließlich beschreibt die Tatsache, dass erst durch die neu entstandene Pluralisierung der Lebenslagen bzw. die neue Pluralisierung der Werte die Wahlmöglichkeit hinsichtlich der Lebensführung gegeben ist.

Basierend auf diesen vier Säulen und den Gedanken von Klocke und Lück bzw. Müller folgend soll das Verständnis von Lebensstil für den weiteren Verlauf dieser Arbeit festgelegt werden als der individuelle Umgang mit sozialen, kulturellen und materiellen Ressourcen, wobei der Grad des Umgangs frei wählbar ist (Klocke/Luck 2001, S. 12 ff.; Müller 1992, S. 374 ff.).

\subsubsection{Grundsteine der Lebensstilforschung}

Nachdem nun die historische Entwicklung der Lebensstilforschung aufgezeigt wurde, soll dieses Kapitel die wichtigsten Autoren bzw. Beiträge zur Lebensstilforschung vorstellen. Es gibt zahlreiche Veröffentlichungen zu diesem Thema, so dass hier nur eine eng begrenzte Auswahl an Autoren und Arbeiten vorgestellt werden kann. Diese Auswahl basiert auf der Einschätzung des Autors darüber, inwieweit die Beiträge Einfluss auf das weitere Vorgehen der vorliegenden Forschungsarbeit haben.

\section{„Die feinen Unterschiede“- Pierre Bourdieu}

Pierre Felix Bourdieu (1930-2002) war französischer Soziologe und veröffentlichte im Jahr 1979 sein Hauptwerk „Die feinen Unterschiede. Kritik der gesellschaftlichen Urteilskraft“. Es gilt als Basis der aktuellen Interpretation von Lebensstilen und wird bis heute in vielen aktuellen Arbeiten zitiert. Zu Beginn der 1980er Jahre wurde Bourdieu auch in Deutschland immer populärer. Hartmann bezeichnete die zunehmende Verbreitung der Bourdieu'schen Werke als Beginn der deutschen Lebensstilforschung. Dabei sollte allerdings beachtet werden, dass Bourdieu zur damaligen Zeit ein typisches gesellschaftliches Modell mit Klassen entwickelte, das er jedoch um Komponenten des Lebensstils erweiterte (Hartmann 1999, S. 90; Georg 1998, S. 64). 
Inhaltlich differenziert Bourdieu drei Arten von Ressourcen, spezieller: drei Arten von Kapital, die er in der Gesellschaft ungleich verteilt sieht: das ökonomische, das kulturelle und das soziale Kapital (Nolte 2005, S. 26). Das ökonomische Kapital besteht aus finanziellem Vermögen und dem, was in Finanzen umgewandelt werden kann. Typischerweise fallen das Einkommen, Bestandsvermögen und Eigentum an hochwertigen Gütern darunter. Das kulturelle Kapital gliedert sich nach Bourdieu in drei Arten: erstens in ,,inkorporiertes kulturelles Kapital“, beispielsweise Wissen und Fertigkeiten, zweitens in „objektiviertes kulturelles Kapital“, beispielsweise hochwertige, besondere Gegenstände, und drittens in ,institutionalisiertes kulturelles Kapital“, beispielsweise allgemein anerkannte Bildungsabschlüsse oder Ähnliches. Das soziale Kapital schließlich repräsentiert die sozialen Beziehungen, in denen sich das Individuum aufhält bzw. bewegt. Basierend auf der unterschiedlichen Verteilung der Ressourcen bzw. der verschiedenen Formen des Kapitals sieht Bourdieu die Gesellschaft nach wie vor als Klassengesellschaft im Sinne von Karl Marx - Lebensstile interpretiert Bourdieu als Reaktion des Individuums auf seine spezifische „Klassenlage“ (Müller 1992, S. 281).

Ein weiterer, zentraler Punkt ist nach Bourdieu der sogenannte „Habitus“ einer Person. Automatisch und unbewusst entwickelt er sich im Laufe der Lebenszeit. Dabei wird seine Ausprägung stark durch die Gegebenheiten der Klassenlage bestimmt. Er stellt somit das Produkt aus ökonomischem und kulturellem Kapital dar. Der ausgebildete Habitus sorgt dafür, dass Personen in bestimmten - sowohl alltäglichen als auch nicht alltäglichen - Lebenssituationen eine bestimmte Handlungsweise an den Tag legen. Somit führt er indirekt dazu, dass sich die meisten Personen in der gleichen Situation anders verhalten und somit die Personen unterschiedliche Lebensstile führen. Nach Bourdieu sind also die verschiedenen Ausprägungen des Habitus verschiedene Lebensstile (Hradil 1999, S. 138 ff.; Blasius/Winkler 1998, S. 74).

Wie beschrieben wird die Ungleichheit der Klassen nach Bourdieus Idee durch verschiedene Lebensstile sichtbar. Dabei deuten Statussymbole auf einen bestimmten Lebensstil hin, beispielsweise Autos, Uhren, Kleidung oder Freizeitbeschäftigungen. Nach Bourdieu sind diese Statussymbole bzw. alle Objekte, die einen bestimmten Lebensstil symbolisieren bzw. symbolisieren sollen, Distinktionen. Sie sind somit als äußerlich sichtbare Merkmale eines Lebensstils anzusehen (Krais/Gebauer 2002, S. 24; Wahl 2000, S. 63). 


\section{„Die Erlebnisgesellschaft. Kultursoziologie der Gegenwart“ - Gerhard Schulze}

Schulze veröffentlichte sein oben genanntes Werk 1992. Darin beschreibt er, dass die Menschen einer modernen Gesellschaft das Erlebnis des Konsums dem Nutzen des Konsums immer mehr vorziehen. Dies basiert darauf, dass sich die Gesellschaft bzw. jeder Einzelne einer Gesellschaft Glück und auch ein ereignisreiches Leben wünscht. Durch das verstärkte Streben nach dem Erlebnis entstehen durch die entsprechenden Handlungen bestimmte Stile, die sich Schulze zufolge in sogenannten ,sozialen Milieus“ ausdrücken. Schulze ersetzt dabei nicht die gesellschaftlichen Klassen und Schichten durch die Milieus. Er sieht jedoch die Milieus als so zentral an, dass er Klassen und Schichten lediglich als eine Besonderheit innerhalb der Milieustruktur versteht (Hellmann 2003, S. 410).

Unter Berücksichtigung der alltagstypischen Handlungsweisen, des Alters und der Bildung von Personen der Gesellschaft identifiziert Schulze fünf Gruppen bzw. fünf Milieus, in welche die Personen einer Gesellschaft eingeteilt werden können.

1. Das Niveaumilieu strebt nach Rang.

2. Das Integrationsmilieu strebt nach Komfort.

3. Das Harmoniemilieu strebt nach Geborgenheit.

4. Das Selbstverwirklichungsmilieu strebt nach Selbstverwirklichung.

5. Das Unterhaltungsmilieu strebt nach Stimulation.

Hierbei gilt es zu betonen, dass die Grenzen zwischen den einzelnen Gruppen nicht „,scharf“ sind. Vielmehr sind die Grenzen als Bereiche zu verstehen, in denen auch Überschneidungen zwischen einzelnen Gruppen möglich sind (Schulze 2000, S. 163 ff., 384). Dies erhöht die Realitätsnähe des Schulze'schen Milieumodells erheblich. Kritisch anzumerken bleibt, dass eine Aufteilung einer Gesellschaft in nur fünf Gruppen bzw. Milieus aus ökonomischer Sicht zu grob ist (Diaz-Bone 2004, S. 8).

\section{„Lebensstilorientierte Marketing-Theorie“- Thomas Banning}

Aus ökonomischer Sicht geschrieben ist, wie der Titel bereits zu erkennen gibt, Thomas Bannings Werk aus dem Jahre 1987. Banning erkennt zwei Arten von Lebensstilen - einerseits den realisierten Lebensstil und andererseits den gewünschten Lebensstil einer Person. Beide sind nur in seltenen Fällen deckungsgleich. Gründe, warum die beiden Lebensstile auseinanderfallen, sieht Banning unter anderem in den finanziellen und temporären Restriktionen der Personen. Allgemein spricht Banning dabei von „situativen Einflüssen“, die das Ausei- 
nanderdriften der beiden Lebensstilarten begründen. Durch die permanente Veränderung des Alltags und auch der eigenen Idealvorstellungen ändern sich auch permanent die Lebensstile. Banning zufolge sind Lebensstile also dynamischer Natur. Gerade aus ökonomischer Sicht ist dieser Ansatz daher interessant, weil durch die Aufteilung in den realisierten und den gewünschten Lebensstil ein Ansatzpunkt für die Imagebildung entsteht. Hilft ein Produkt in den Augen des Konsumenten, einen gewissen erwünschten Lebensstil zu erreichen bzw. zu repräsentieren, kann dies die Kaufentscheidung des Konsumenten positiv beeinflussen (Kotler et al. 2006, S. 343 f.; Banning 1987, S. 88 ff., 182; Kozlova 2008, S. 42 ff.).

\subsubsection{Lebensstile und Konsumentenverhalten}

Wie bereits festgestellt, gibt es verschiedene Ansätze zu dem Konzept des Lebensstils. In dem folgenden Abschnitt sollen diese Unterschiede jedoch ausgeblendet werden, um den Fokus der Betrachtung darauf zu richten, wie Lebensstile überhaupt das Verhalten von Konsumenten beeinflussen. Schon in Kapitel 3.1.1 wurde beschrieben, dass die moderne Gesellschaft auf drei umfassenden Veränderungen aus den 1970er und 1980er Jahren basiert: erstens der zunehmenden Individualisierung der Lebensführung, zweitens der Pluralisierung der Lebenslagen und drittens dem vollzogenen Wertewandel der Gesellschaft. Da alle drei dieser Bewegungen parallel verliefen bzw. immer noch verlaufen, ist es nahezu unmöglich, sie unabhängig voneinander zu betrachten - die einzelnen Strömungen bedingen sich wechselseitig zu stark.

Sowohl die zunehmende Individualisierung der Lebensführung als auch die Pluralisierung der Lebenslagen sorgen bis heute dafür, dass Distinktionen im Sinne Bourdieus großen Schwankungen in der Wertschätzung unterworfen sind. Beispielsweise war es in den Nachkriegsjahren geradezu eine Besonderheit, in Haushalten eine Waschmaschine oder gar einen eigenen Pkw vorzufinden. Heutzutage besteht die Besonderheit vielmehr darin, in einem Haushalt keine Waschmaschine oder keinen Pkw vorzufinden. Ähnlich wie den beiden exemplarisch genannten geht es auch vielen anderen Gütern, die früher als Statussymbole galten. Durch ihre Verbreitung in allen Gesellschaftsbereichen verlieren sie ihre besondere Stellung und werden so im Laufe der Zeit immer mehr zu gewöhnlichen Gütern. Daher ändert sich auch die Kaufentscheidung, die Konsumenten zu treffen haben. Nach der Frage, ob sie überhaupt das (gewöhnliche) Gut kaufen sollen, gilt es die Frage zu beantworten, in welcher Ausstattung es gekauft werden soll. Während früher also der Besitz eines Gutes eine Distinktion darstellte, 
ist es heutzutage eher die Ausstattung und die Art und Weise der Nutzung des ehemaligen Statussymbols (Müller-Schneider 1994, S. 78 ff.; Hölscher 1998, S. 102).

Parallel zum „Verwässern“ der Statussymbole hat sich im gleichen Zeitraum die gewünschte Wirkungsrichtung des Konsums geändert. Bis Mitte der 1960er Jahre verfolgten Konsumenten hauptsächlich die nach außen gerichtete Konsumwirkung. Die Wahl des Konsumgutes orientierte sich an Restriktionen oder Regeln innerhalb der Gruppe bzw. der Schicht, zu der man gehörte (oder gehören wollte). Seither hat sich die Wirkungsrichtung des Konsums geändert und verläuft zunehmend von außen nach innen. Der Demonstrativkonsum nimmt ab, während der innenorientierte Konsum zunimmt. Für Hölscher ist diese Tendenz darin begründet, dass in der Gesellschaft die Wünsche nach einer Ästhetisierung des Alltags und die Erlebnisorientierung immer mehr zunehmen. Dabei wird beim Erreichen dieser Wünsche immer weniger auf gesellschaftliche Spielregeln geachtet. Eine salopp formulierte Begründung für das Außerachtlassen von gesellschaftlichen Rahmenbedingungen könnte sein: „Weil es mir gefällt‘“ (Hölscher 1998, S. 103 f.; Schulze 2000, S. 427 ff.).

Parallel zur geänderten Wirkungsrichtung des Konsums erkennt Stihler eine Zunahme des Strebens nach Erlebnisorientierung und Hedonismus, wobei Letzterer in diesem Kontext als Konsum von besonders erlebnisreichen Produkten verstanden wird. Gerade beim Verbrauch dieser hedonistischen Güter ist zu beobachten, dass der Konsum nahezu keinen abnehmenden Grenznutzen für die Konsumenten aufweist. Sie haben nach Stihler diesbezüglich einen ,endlosen Hang“ zum Konsum (Stihler 2000, S. 172; Wiswede 2000, S. 59).

\subsubsection{Aktuelle Bedeutung der Lebensstilforschung}

Abwechslung im Leben stellt für nahezu alle Personen aller Altersgruppen ein erstrebenswertes Ziel dar - was sich auch darin widerspiegelt, dass der Konsum einer Person ständigen Veränderungen unterworfen ist. Mit der Änderung des individuellen Konsummusters ändert sich auch der aktuelle Lebensstil einer Person. Als Folge dieser Zunahme an individuellen Entscheidungen von Individuen entsteht somit eine weitere Pluralisierung der Lebensstile (Papastefanou 2007, S. 238 ff.; Beck 1986, S. 121 ff.; Beck 1994, S. 14).

Durch die Vielzahl der möglichen Konsumentscheidungen in Verbindung mit dem Trend zur Individualisierung entwickelt sich der Wunsch des Einzelnen, einen möglichst besonderen und dabei möglichst hohen Lebens- und Konsumstil zu pflegen. Da dieses Verlangen des Einzelnen allerdings durch seine Budgetrestriktion nicht unbegrenzt zu befriedigen ist, gilt es für 
jeden, beim Konsum ,individuelle Akzente“ zu setzen. Dabei müssen aus finanziellen Gründen teilweise auch Güter gegeneinander abgewogen werden, die alle besonders gut in das Lebensstilbild der Person passen würden, beispielsweise eine wertvolle Uhr und eine mondäne Urlaubsreise. Durch das gegenseitige Abwägen der Güter versucht der Verbraucher im Rahmen seines Budgets den maximalen Grad an Individualisierung zu erreichen, teilweise auch dadurch, dass er auf teure Produkte aus anderen Lebens- bzw. Konsumbereichen ganz oder zumindest nahezu ganz verzichtet. Das Ausrichten des Handels an diesem sogenannten ,partikularen Luxus“ erschwert eine Einschätzung des Konsumentenprofils erheblich. Diese neue Art der Konsumenten passt nicht mehr in vorgefertigte Schubladen, die allesamt zu bestimmten Lebensstilmustern gehören (Wiswede 2000, S. 60; Koschnick 2006, S. 89; Steimer 2006, S. 18). Es ist am reinen Einkaufsverhalten einer Person also immer weniger zu erkennen, ob beispielweise jemand aus wirklicher Notwendigkeit im Discounter einkauft oder ob er dies tut, weil er schon einen Großteil seines Einkommens für eine teure Wohnung ausgibt.

Für Konsumenten dieser Art hat sich der Begriff des „hybriden Verbrauchers“ verbreitet. Hybrid bedeutet so viel wie „Mischung aus zweierlei“, hier also, dass eine Person innerhalb eines Marktes zwar meist einer bestimmten Zielgruppe zugehört, die Zielgruppe der Person von Markt zu Markt jedoch verschieden ist. Die Konsumstile der Individuen verändern sich demnach zwischen den Märkten - so wie bei einer Person, die einerseits beim Einkauf von Lebensmitteln sehr preissensibel ist und andererseits in Fragen der Wohnung gerne bereit ist, mehr Geld auszugeben.

Die bisherigen Ausführungen zu Lebensstil und Konsum dürften deutlich gemacht haben, dass das soziale Gefüge, in dem sich eine Person befindet, deren Konsumentscheidungen beeinflusst - und die Konsumentscheidungen wiederum den Lebensstil (Wiswede 2000, S. 51). Neben dem Verwässern von Statussymbolen ist zudem die geänderte Wirkungsrichtung des Konsums von entscheidender Bedeutung für die aktuelle Lebensstilforschung. Auf Grund ihrer Geeignetheit, Menschen mit gleichen bzw. mit ähnlichen Einstellungen und Überzeugungen zu identifizieren, werden die einzelnen Komponenten des Konstrukts „Lebensstil“ immer häufiger auch als Kriterien für eine marketingorientierte Segmentierung der Konsumenten verwendet. Darüber hinaus bieten die Lebensstilkriterien weitere Vorteile, die eine Konsumentensegmentierung anhand von Lebensstilen zusätzlich interessant macht (siehe dazu detailliertere Ausführungen in Kapitel 3.2.2.3). 


\subsubsection{Lebensstile in der empirischen Forschung}

Wie bereits erwähnt, sind Lebensstile neben dem sozialen und psychologischen Bereich auch Teil der aktuellen ökonomischen Forschung. Ein besonderer Schwerpunkt liegt dabei auf Erklärungsmodellen für Käuferverhalten und auf der Marktsegmentierung, also der Zusammenfassung verschiedener individueller Verbrauchertypen zu Gruppen, an denen sich das Angebot von Unternehmen orientiert. Im Folgenden werden einige ausgewählte Modelle vorgestellt, die auf der Basis der Lebensstilforschung Individuen zu großen Gruppen zusammenfassen. Dazu wurden von den Marktforschungsunternehmen bzw. den Instituten Eigenschaften und Attribute, die für die jeweilige Gruppe typisch sind, gesammelt und den Befragten ,angeboten“. Aufgrund der sich dabei abzeichnenden Verhaltens- bzw. Entscheidungsmuster wurden die Individuen in bestimmte Lebensstilgruppen bzw. Milieus eingeteilt. Eine solche Zuordnung erfolgt jeweils aufgrund von Beobachtungen in Experimenten oder anhand von Befragungen (Koschnick 2006, S. 48, 55; Hartmann 1999, S. 50).

\subsubsection{Der AIO-Ansatz}

Der AIO-Ansatz basiert auf den drei Parametern ,activities“, „interests“ und „opinions“, also Aktivitäten, Interessen und Meinungen. Der Ansatz stellt das erste quantitative Modell über Lebensstile dar, da es über die reine Erhebung von Strukturdaten im Sinne von soziodemographischen Daten hinausgeht. Die erkannten Gruppen des AIO-Ansatzes basieren auf dem Abfragen von etwa 500 Eigenschaften bzw. Variablen. Entwickelt wurde das Modell von Wells und Tigert im Jahre 1971 (Wells/Tigert 1971, S. 27 ff.; Hamm 2003, S. 89; Kroeber-Riel/ Weinberg 2003, S. 560).

Basierend auf Wells' und Tigerts Arbeit erstellt die US-amerikanische Agentur Conrad \& Burnett seit Mitte der 1970er Jahre Lebensstilgruppierungen. Dabei wurde der ursprüngliche Ansatz in der Art weiterentwickelt, dass die vielen abgefragten Variablen bzw. Items in aktive und passive Variablen unterschieden werden. Die aktiven Variablen dienen dabei der Bildung der Gruppen bzw. Typen, die passiven Variablen der Erklärung und Beschreibung dieser Gruppen. Insgesamt wurden zwölf verschiedene Arten von Lebensstilgruppen erkannt, wobei diese nochmals in den Gruppen „modern“, „gehoben“ und „traditionell“ zusammengefasst wurden. Conrad \& Burnett hat in Deutschland bis heute fünf entsprechende Umfragen durchgeführt. Die letzte dieser Studie fand allerdings im Jahre 1990 statt, und da sich die Alltagswelt und somit auch die Einstellungen der Personen seither stark verändert haben, ist davon 
auszugehen, dass die damals identifizierten Gruppen bzw. Typen heute bei Weitem nicht mehr repräsentativ sind (Drieseberg 1995, S. 149; Hartmann 1999, S. 53 ff.).

Als Beispiel sei hier auf eine neuseeländische Untersuchung verwiesen, um zu zeigen, wie sich der jeweilige Lebensstil einer Person auf deren Verhalten im Hinblick auf Museums- und Galeriebesuche auswirkt. Dazu wurden 3.773 von Neuseeländern beantwortete Fragebögen ausgewertet, mit denen die AIO-Ausprägungen der Befragten über Zustimmung oder Ablehnung bestimmter Aussagen wie beispielsweise „Ich interessiere mich für Fußball“ erfasst wurden. Insgesamt wurden auf diese Weise mit den Fragebögen 171 Variablen von den Probanden abgefragt. Mittels statistischer Auswertungsprogramme wurden die Testpersonen in sieben Gruppen eingeteilt, die sich in ihrem Lebensstil sehr ähnlich sind. Anhand der durchschnittlichen Museums- und Galeriebesuche der jeweiligen Gruppe wurde letztendlich eine Reihenfolge darüber erarbeitet, welche der sieben Lebensstilgruppen die größte Attraktivität als Zielgruppe für Museen und Galerien darstellt.

Tabelle 1 veranschaulicht das Ergebnis der obigen Umfrage in kompakter Form. Sie zeigt die sieben erkannten Lebensstilgruppen in den drei AIO-Bereichen und deren jeweilige Repräsentanz in der Grundgesamtheit auf. Die Lebensstilgruppen tragen allesamt erklärende Namen. Zusätzlich werden in den einzelnen Feldern beispielhafte Aktivitäten, Interessen und Meinungen der entsprechenden Gruppe aufgeführt.

Die Gruppe der „educated liberals“ stellte sich in der Untersuchung letztendlich als diejenige Gruppe heraus, die die meisten Museums- und Galerienbesuche vorzuweisen hatte. Sie wurde somit als erste Zielgruppe für Marketingaktivitäten von Museen und Galerien erkannt. Ihnen stehen die Gruppen der „conservative quiet lifers“ und der ,accepting mid-lifers“ gegenüber, die am wenigsten ins Museum gehen bzw. einem Museumsbesuch gegenüber am meisten abgeneigt sind (Freter 2008, S. 136).

Tabelle 1: AIO-basierte Lebensstilgruppen

\begin{tabular}{|l|l|}
\hline \multicolumn{2}{|l|}{ Activities / Aktivitäten } \\
\hline Active "family values" people (15,5\%) & - Geringer Radio- u. TV-Konsum \\
\hline \multirow{2}{*}{ Conservative quiet lifers $(13,5 \%)$} & $\begin{array}{l}\text { - Passive, häusliche Aktivit.: Kreuzworträtsel, Gartenarbeit, TV u. Ra- } \\
\text { dio }\end{array}$ \\
\hline & - Kaum soziale Aktivitäten, z. B. Museum, Galerien, Kino \\
\hline Educated liberales $(9,7 \%)$ & - Reisen, Abenteuerlust, exotisches Essen \\
& - Kulturelle Aktivitäten: Bücherei, Theater, Museum \\
& - Viel-Leser: Bücher u. Zeitschriften \\
& - Geringer Radio- u. TV-Konsum \\
\hline
\end{tabular}




\begin{tabular}{|c|c|}
\hline Accepting mid-lifers $(17,1 \%)$ & $\begin{array}{l}\text { - Bevorzugen es, Aktivitäten alleine durchzuführen } \\
\text { - Beobachten statt selbst teilzunehmen }\end{array}$ \\
\hline Success-driven extroverts $(16,4 \%)$ & $\begin{array}{l}\text { - Sport- und Entertainment-Aktivitäten, Kino u. Theater } \\
\text { - Geringer Radio- u. TV-Konsum, hoher Anteil von Sport u. Komö- } \\
\text { dien }\end{array}$ \\
\hline Pragmatic strugglers $(14,7 \%)$ & $\begin{array}{ll}\text { - Hören oft Radio } \\
\text { - Schauen gerne Spielfilme und Serien im TV }\end{array}$ \\
\hline Social strivers $(13 \%)$ & $\begin{array}{l}\text { - Hoher Radio- u. TV-Konsum } \\
\text { - Lesen gerne Zeitschriften }\end{array}$ \\
\hline & Interests / Interessen \\
\hline Active "family values" people $(15,5 \%)$ & - Familie + Kinder \\
\hline Conservative quiet lifers $(13,5 \%)$ & $\begin{array}{l}\text { - Kein Interesse an Geselligkeit } \\
\text { - Wenig Interesse, enge Freundschaften zu schließen }\end{array}$ \\
\hline Educated liberales $(9,7 \%)$ & $\begin{array}{l}\text { - Umfangreiche Interessen } \\
\text { - An sozialen Aspekten interessiert } \\
\text { - Suchen Abwechslung } \\
\text { - Suchen intellektuelle Stimulation }\end{array}$ \\
\hline Accepting mid-lifers $(17,1 \%)$ & - Kein Interesse an Geselligkeit \\
\hline Success-driven extroverts $(16,4 \%)$ & $\begin{array}{l}\text { - Selbstverwirklichung } \\
\text { - Erfolg } \\
\text { - Unabhängigkeit } \\
\text { - Ihre Erscheinung u. ihr Image sind ihnen sehr wichtig } \\
\text { - Führungsansprüche }\end{array}$ \\
\hline Pragmatic strugglers $(14,7 \%)$ & $\begin{array}{l}\text { - Interessieren sich für praktische Dinge rund ums Haus } \\
\text { - Fokus auf die Familie }\end{array}$ \\
\hline Social strivers $(13 \%)$ & $\begin{array}{l}\text { - Meinungen u. Bestätigungen anderer sind wichtig } \\
\text { - Familie }\end{array}$ \\
\hline \multicolumn{2}{|r|}{ Opinions / Meinungen } \\
\hline Active "family values" people $(15,5 \%)$ & $\begin{array}{l}\text { - Traditionelle soziale, moralische u. religiöse Prinzipien } \\
\text { - Kirche u. Gemeinde } \\
\text { - Optimistische Grundhaltung }\end{array}$ \\
\hline Conservative quiet lifers $(13,5 \%)$ & $\begin{array}{l}\text { - Arbeiten lieber alleine } \\
\text { - Glauben an Einfachheit } \\
\text { - Kirche } \\
\text { - Konservative Grundhaltung }\end{array}$ \\
\hline Educated liberales $(9,7 \%)$ & $\begin{array}{l}\text { - Zuversichtliche u. optimistische Grundhaltung } \\
\text { - Progressiv u. egalitär }\end{array}$ \\
\hline Accepting mid-lifers $(17,1 \%)$ & $\begin{array}{l}\text { - Akzeptieren ihr Leben und die Gesellschaft } \\
\text { - Lehnen Veränderungen ab, Status quo gilt als besser } \\
\text { - Starke politische Meinung }\end{array}$ \\
\hline Success-driven extroverts $(16,4 \%)$ & - Glauben die Kontrolle über ihr Leben und ihre Zukunft zu haben \\
\hline Pragmatic strugglers $(14,7 \%)$ & $\begin{array}{l}\text { - Glauben an Natürlichkeit u. Einfachheit } \\
\text { - Konservative politische Grundhaltung }\end{array}$ \\
\hline Social strivers $(13 \%)$ & $\begin{array}{l}\text { - „Leben ist ein Kampf“ } \\
\text { - Konformisten } \\
\text { - Pessimistische, konservative u. vorsichtige Grundhaltung } \\
\text { - Kirche u. Gemeinde }\end{array}$ \\
\hline
\end{tabular}

Quelle: Eigene Darstellung in Anlehnung an Freter 2008, S. 137 


\subsubsection{Der VALS-Ansatz}

VALS dient als Abkürzung für „Value and Lifestyle“ und ist ein Ansatz zur Typologisierung von Individuen, die auf einer schriftlichen, ca. 800 Variablen umfassenden Befragung von Personen basiert. Die VALS-Theorie wurde erstmals 1983 unter dem Namen „The Nine American Lifestyles. Who we are and here we're going“" von Mitchell veröffentlicht (Mitchell 1983). Mitchell entwickelt darin aus den beantworteten Fragebögen neun verschiedene Lebensstilansätze, wobei er sich bei deren Entwicklung wiederum stark an zwei weitere Theorien anlehnt: einerseits an die Bedürfnispyramide von Maslow und andererseits an die sozialen Charaktertypen von Riesman.

Die Bedürfnispyramide von Maslow ist horizontal in fünf verschiedene Bereiche aufgeteilt, wobei das Fundament der Pyramide die Grundbedürfnisse eines Menschen darstellen, beispielsweise „Hunger“. Die Spitze der Pyramide stellt die letzte Stufe der menschlichen Bedürfnisse dar, „Selbstverwirklichung“. Nach Maslow arbeiten sich die Menschen in der Pyramide von unten nach oben, wobei keine Stufe übersprungen werden kann. Beispielhaft bedeutet dies, dass zuerst die Grundbedürfnisse wie Hunger oder Durst befriedigt sein müssen, bevor sich jemand nach einer höheren Stufe der Pyramide, beispielsweise sozialer Anerkennung, sehnt.

Nach Riesman besteht das menschliche Verhalten aus drei verschiedenen Idealtypen: dem innengeleiteten, dem traditionellen und dem außengeleiteten Typus. Der innengeleitete Typus entwickelt seine Orientierung bereits in der Phase der Sozialisation. Dennoch gilt die dort entwickelte Orientierung lebenslang. Der traditionsgeleitete Typus wird von festen kulturellen Gewohnheiten und Verhaltensformen bestimmt. Der außengeleitete Typus stellt den Gegenpol zum innengeleiteten Typus dar. Hier stehen Beziehungen zu anderen Menschen sowie der eigene Status im Fokus (Michman 1991, S. 185 f.; Hartmann 1999, S. 58). Riesman sieht dabei zweierlei Möglichkeiten, wie sich Menschen nach der Phase der Sozialisierung weiterentwickeln können: entweder primär innengeleitet oder primär außengeleitet.

In Anlehnung in die Bedürfnispyramide von Maslow und an die beiden verschiedenen Möglichkeiten zur Weiterentwicklung der Persönlichkeit nach Riesman entwickelt Mitchell eine Theorie, der zufolge es zweierlei Möglichkeiten gibt, um von der niedrigsten Bedürfnisstufe der „Survivors“ nach ganz oben auf die Stufe der „Integrated“ zu gelangen. Welchen der beiden Wege nach oben Individuen in der Entwicklung ihrer Persönlichkeit wählen, hängt davon ab, ob bei ihnen der innen- oder der außengeleitete Typus dominant ausgebildet ist. Mitchell 
spricht dabei vom ,inner-directed way“ und vom „outer-directed way“. „Inner-directed people“ sind in ihrer Persönlichkeit eher introvertiert als Personen, die den „outer-directed way“ bestreiten. Auf dem Pfad des ,,inner-directed way“ dominieren individuelle Werte, Bedürfnisse und Moralvorstellungen die Persönlichkeitsentwicklung. Weiterhin stehen Personen des „inner-directed way“ Versuchen von externen Persönlichkeitsbeeinflussungen sehr skeptisch gegenüber. Personen, die den „outer-directed way“ gehen, orientieren sich stärker an ihrer Umwelt. Erfolg und Anerkennung von außen steht für diese Personengruppe im Blickfeld. „Outer-directed people“ sind in ihrer Art eher extrovertiert als Personen des „inner-directed way“ (Mitchell 1983, S. 33 ff.).

Mitchell teilt die neun von ihm erkannten Personengruppen letztendlich in vier verschiedene Bereiche ein: „inner-directed“, „outer-directed“, „need-driven“ und ,integrated“. Abbildung 27 veranschaulicht die VALS-Typologie. Dabei lässt sich auch die Kombination aus den beiden oben erwähnten Theoremen gut erkennen.

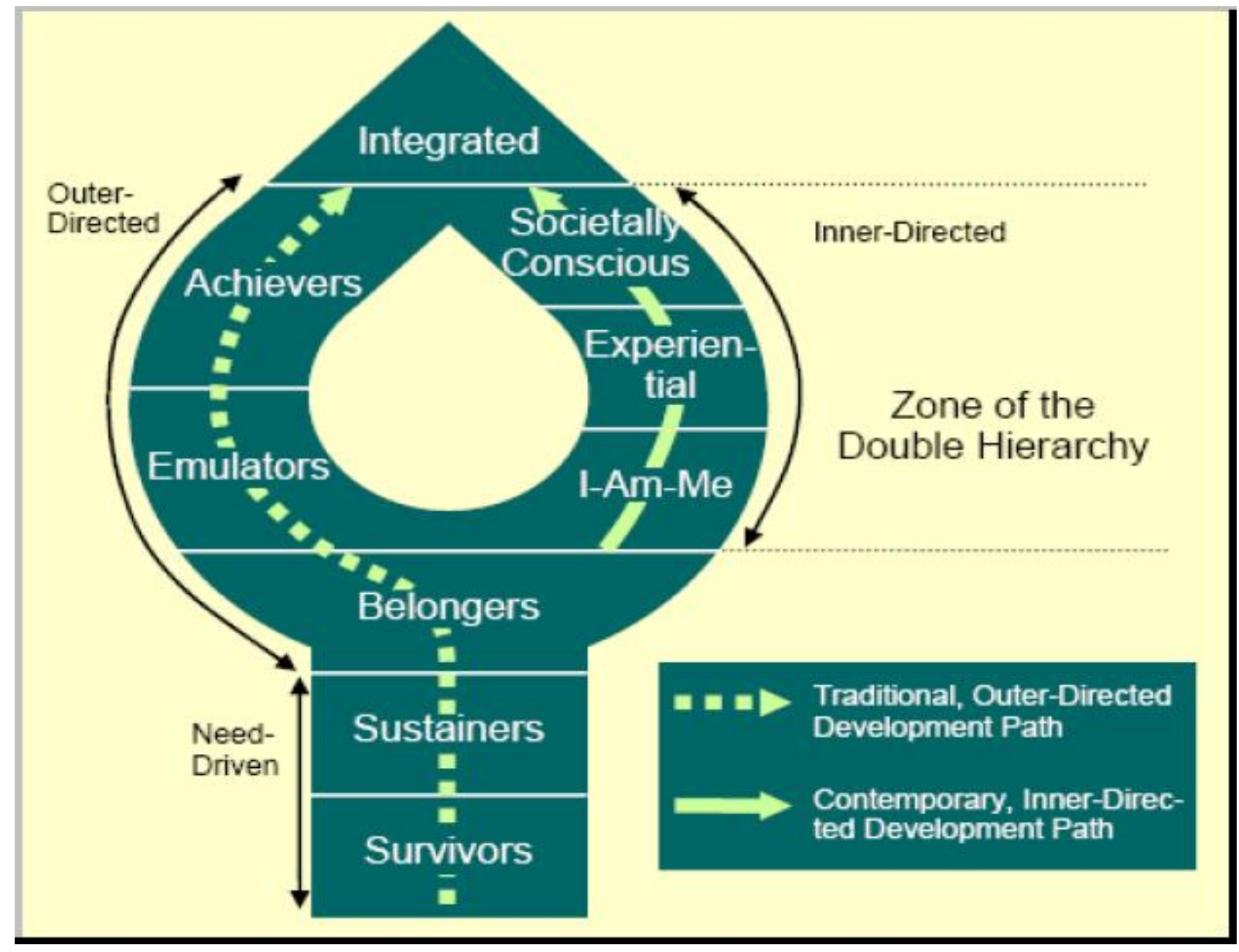

Abbildung 27: Die VALS-Theorie

Quelle: Mitchell 1983, S. 32 
Die hier vorgestellte, ursprüngliche VALS-Theorie wurde mehrfach aktualisiert. Aktuell ist VALS 3 die neueste Version. Im Gegensatz zur Ursprungsversion richten die überarbeiteten Versionen den Fokus ihrer Betrachtung noch stärker auf psychologische Merkmale.

\subsubsection{Die Sinus-Milieus}

Seit Ende der 1970er Jahre wird vom Institut „SINUS Sociovision GmbH“ in Heidelberg regelmäßig eine Lebensstiltypologie veröffentlicht, in der die gefundenen Gruppierungen als „Milieus“ bezeichnet werden. Das Ziel dieser Einteilung besteht laut SINUS Sociovision darin, eine Typologie zu formulieren, die alle Bereiche des Lebens berücksichtigt und keine Facette unbeachtet lässt. Die Sinus-Milieus sind Gruppierungen von Menschen, die eine ähnliche Auffassung des Lebens und ähnliche Lebensweisen aufweisen. Die ursprüngliche Typologie basierte auf etwa 1.000 durchgeführten qualitativen Interviews. Seit den 1980er Jahren wird mit Hilfe eines standardisierten, 45 Fragen umfassenden Fragesets der sogenannte „Milieu-Indikator“ einer Person entwickelt. Die Fragen geben Aufschluss über zahlreiche Bestandteile des Lebensstils, beispielsweise über Einstellungen, Werte, Konsum oder Arbeit. Zusätzlich zu den Fragen werden soziodemographische Daten als passive Variablen abgefragt. Die Einteilung der Personen in Gruppen bzw. in Milieus erfolgt auf der Grundlage einer durchgeführten Clusteranalyse (Diaz-Bone 2004, S. 5; SINU07, S. 9; Freter 2008, S 22; Hartmann 1999, S. 70f.).

Aus dieser Analyse ergeben sich Milieus, die in einem zweidimensionalen Koordinatensystem dargestellt werden, bei dem auf der Abszisse die Grundorientierung und auf der Ordinate die soziale Lage abgetragen sind. Die Abszisse wird auch als „Wertachse“ bezeichnet, die Ordinate auch als „Schichtachse“. Beide Achsen sind dreistufig unterteilt, so dass sich im zweidimensionalen Raum des Systems eine 3x3-Matrix ergibt, in welche die gefundenen Milieus eingetragen werden. Dabei stellt laut SINUS Sociovision die Schichtachse lediglich eine deskriptive Größe dar; sie beruht auf den passiven Variablen. Die Wertachse hingegen basiert auf Wertorientierungen und Lebensstilen, also den aktiven Variablen. Sie dient als konstituierende Größe.

Das Sinus-Modell unterscheidet zehn verschiedene Milieus; die prozentualen Anteile beziehen sich dabei auf die Gesamtbevölkerung ab 14 Jahren in Deutschland. Die Überlappungen der einzelnen Milieus zeigen eine gewisse Unschärfe des Modells, die vom Institut allerdings gewollt ist. SINUS sieht diese Unschärfe als Zugeständnis an die „Alltagswirklichkeit“ der 
Menschen an. Abbildung 28 veranschaulicht die Beschreibung, während Tabelle 2 exemplarisch zwei Milieus und deren Beschreibung einander gegenüberstellt. Die exakte Vorgehensweise des SINUS-Institutes ist nicht veröffentlicht, was wissenschaftlich betrachtet ein „Black-Box-Problem“ darstellt. Wie die Milieus genau gebildet und voneinander abgegrenzt werden, ist exklusives Wissen des SINUS-Institutes und Teil von dessen Beratungsexpertise. Eine vollständige wissenschaftliche Beurteilung der Sinus-Milieus ist somit nicht möglich (Ascheberg 2010, S. 3; Diaz-Bone 2004, S. 10; Sinus Sociovision 2010a; Sinus Sociovision 2010b, S. 2).

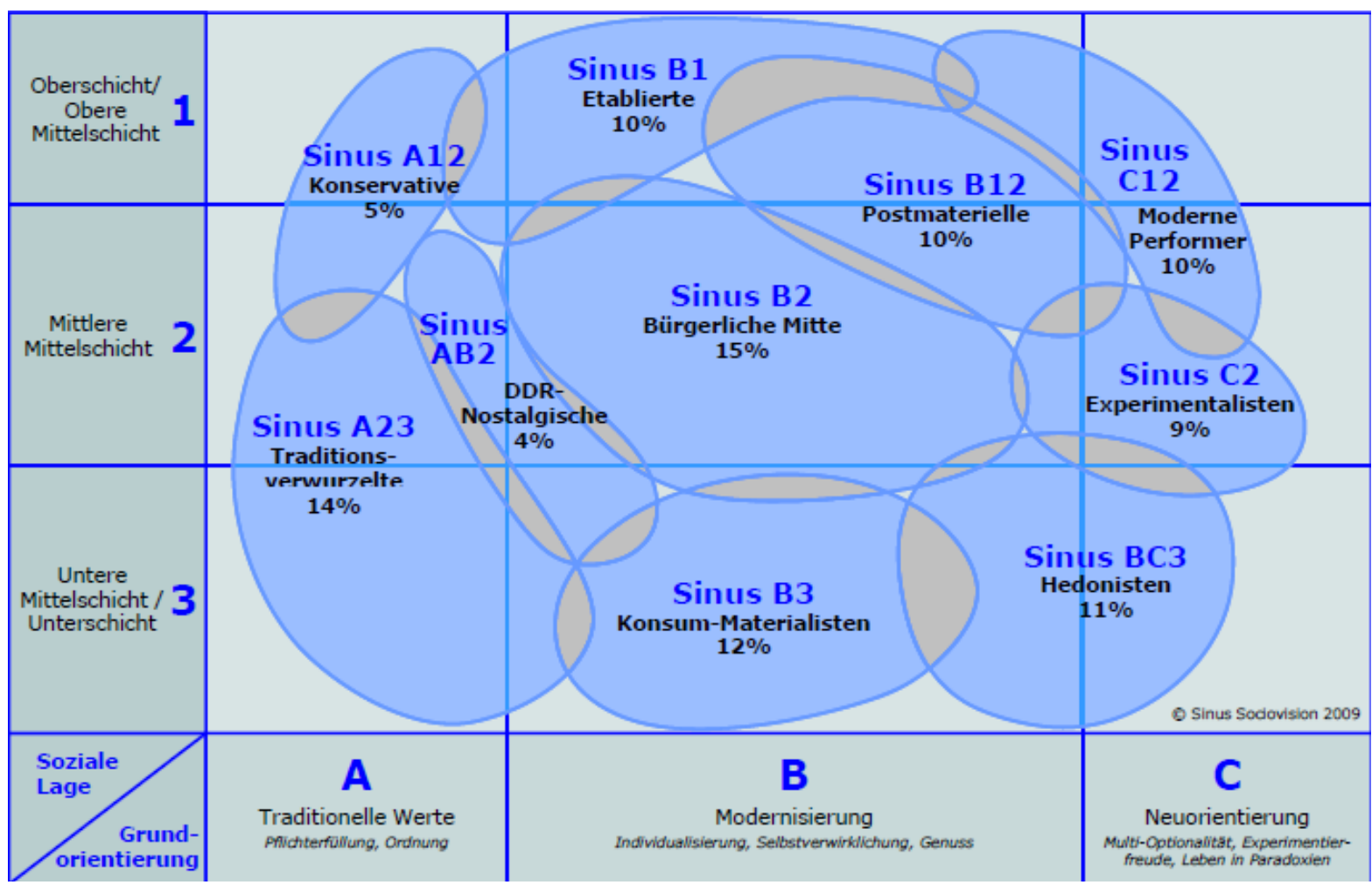

Abbildung 28: Die Sinus-Milieus in Deutschland

Quelle: Sinus Sociovision GmbH 2010b

Wie bereits erwähnt, wird das Modell der Sinus-Milieus regelmäßig aktualisiert - nach Angaben des Institutes jährlich - und dabei auch an die „Rahmenbedingungen der Gesellschaft“ angepasst. So ist es auch zu erklären, dass während der 1990er Jahre zwei verschiedene Modelle veröffentlicht wurden - eines für Ost- und eines für Westdeutschland. Die gemeinsame Darstellung in einem Modell wäre zur damaligen Zeit noch nicht sinnvoll gewesen, da die gesellschaftlichen Unterschiede zwischen Ost und West noch zu groß waren. Seit dem Jahr 2001 gibt es nur noch ein gesamtdeutsches Modell, in dem nur noch das „DDR-nostalgische“ Mi- 
lieu aus dem Ost-Modell übrig geblieben ist. Die Typologie von 2001 mit insgesamt zehn verschiedenen Milieus und deren Grundstruktur im Koordinatensystem ist noch heute gültig. Geändert haben sich lediglich die verhältnismäßigen Anteile der Milieuzugehörigen an der Gesamtbevölkerung (Becker 2006, S. 263).

Tabelle 2: Vergleich zweier Sinus-Milieus

\begin{tabular}{|c|c|}
\hline Traditionsverwurzelte & Postmaterielle \\
\hline $\begin{array}{l}\text { Die Sicherheit und Ordnung liebende Kriegsgeneration: } \\
\text { verwurzelt in der Welt des Kleinbürgertums bzw. in der } \\
\text { traditionellen Arbeiterkultur. }\end{array}$ & $\begin{array}{l}\text { Das aufgeklärte Nach-68er-Milieu: liberale Grund- } \\
\text { haltung, postmaterielle Werte und intellektuelle } \\
\text { Interessen. }\end{array}$ \\
\hline Lebenswelt: & Lebenswelt: \\
\hline $\begin{array}{l}\text { - Die Traditionsverwurzelten sind die sicherheits- und } \\
\text { ordnungsliebende Kriegs- und Nachkriegsgeneration. } \\
\text { Ihre Wurzeln haben sie entweder in der kleinbürgerli- } \\
\text { chen Welt oder in der traditionellen Arbeiterkultur. Sie } \\
\text { verstehen sich als die Bewahrer der traditionellen Wer- } \\
\text { te Pflichterfüllung, Disziplin, Bescheidenheit und Mo- } \\
\text { ral. } \\
\text { - Inzwischen sind die meisten Rentner oder Pensionäre } \\
\text { nach einem Arbeitsleben in der unteren Mitte der Ge- } \\
\text { sellschaft (kleine Angestellte und Beamte, Arbeiter u. } \\
\text { Bauern). } \\
\text { - Sie setzen fort, was sie auch früher gelebt haben: die } \\
\text { Pflege von Familien- und Nachbarschaftsbanden, die } \\
\text { Suche nach Anerkennung im unmittelbaren sozialen } \\
\text { Umfeld. Sich selbst in den Mittelpunkt zu stellen, Tra- } \\
\text { ditionelles und Bewährtes aufzugeben, kommt für sie } \\
\text { nicht in Frage. } \\
\text { - Ihre Interessen kreisen eng um die eigenen vier Wände, } \\
\text { die Familie und vor allem um die eigene Gesundheit. } \\
\text { Fernsehen, Basteln, Gartenarbeit, manchmal auch Aus- } \\
\text { flüge und Kaffeefahrten füllen die freie Zeit. Nach ei- } \\
\text { nem arbeitsreichen Leben genießen sie es, ,sich auszu- } \\
\text { ruhen“. } \\
\text { Die Traditionsverwurzelten sind sehr zurückhaltende } \\
\text { Konsumenten. Ein Leben lang haben sie gespart und nur } \\
\text { „Sinnvolles“ und Notwendiges angeschafft. Auch heute } \\
\text { halten sie ihr Geld zusammen und sind für sich selbst } \\
\text { sehr sparsam. Kinder und Enkelkinder unterstützen sie } \\
\text { dagegen gerne. }\end{array}$ & $\begin{array}{l}\text { - Die Postmateriellen sind überwiegend hochge- } \\
\text { bildet, kosmopolitisch und tolerant. Gewöhnt, in } \\
\text { globalen Zusammenhängen zu denken, setzen } \\
\text { sie sich kritisch mit den Auswirkungen von } \\
\text { Übertechnisierung und Globalisierung auseinan- } \\
\text { der. Höchster Wert ist die Lebensqualität des } \\
\text { Einzelnen. } \\
\text { - Sie haben großes Vertrauen in ihre eigenen be- } \\
\text { ruflichen Fähigkeiten und gehen souverän mit } \\
\text { beruflichen Herausforderungen um. Sie wollen } \\
\text { Erfolg im Beruf - aber nicht um jeden Preis. } \\
\text { - Ihre postmateriellen Ansprüche richten sich auf } \\
\text { die Entfaltung ihrer individuellen Bedürfnisse } \\
\text { und Neigungen, auf das Schaffen von Freiräu- } \\
\text { men für sich und auf mehr Zeitsouveränität. } \\
\text { - In hohem Maße sind sie interessiert an Literatur, } \\
\text { Kunst und Kultur. Weiterbildung ist ein lebens- } \\
\text { längliches Thema, weil sie sich mehr über Intel- } \\
\text { lekt und Kreativität definieren als über Besitz } \\
\text { und Konsum. } \\
\text { Ihr Lebensstil ist umwelt- und gesundheitsbewusst } \\
\text { (Balance zwischen Körper, Geist und Seele). Sie } \\
\text { schätzen subtile Genüsse, die durchaus einen ho- } \\
\text { hen Preis haben dürfen. Überflüssigen Konsum } \\
\text { lehnen sie aber ab. Nach dem Motto „Weniger ist } \\
\text { mehr“ kaufen sie selektiv und mit hoher Kenner- } \\
\text { schaft. }\end{array}$ \\
\hline Soziale Lage: & Soziale Lage: \\
\hline $\begin{array}{l}\text { - Altersschwerpunkt ist die Kries- und Nachkriegsgene- } \\
\text { ration (65 und älter); entsprechend hoher Frauenanteil } \\
\text { - Überwiegend Hauptschule mit abgeschlossener Berufs- } \\
\text { ausbildung } \\
\text { - Hoher Anteil von Rentnern und Pensionären; früher: } \\
\text { kleine Angestellte u. Beamte, Arbeiter, Facharbeiter } \\
\text { und Bauern. } \\
\text { Meist kleine bis mittlere Einkommen }\end{array}$ & $\begin{array}{l}\text { - Breites Altersspektrum - von Anfang } 20 \text { bis zur } \\
\text { Generation der ,jungen Alten“; häufig größere } \\
\text { Haushalte mit Kindern } \\
\text { - Hohe bis höchste Formalbildung (Abitur, Studi- } \\
\text { um) } \\
\text { - Qualifizierte und leitende Angestellte und Be- } \\
\text { amte, Freiberufler, Studenten } \\
\text { Hohes Einkommensniveau }\end{array}$ \\
\hline
\end{tabular}

Quelle: Eigene Darstellung auf der Grundlage von Sinus Sociovision 2010a; Freter 2008, S. 123 
Trotz des „Black-Box-Problems“ hat sich der Ansatz der Sinus-Milieus in Deutschland durchgesetzt. Es gilt als einflussreichstes Marktforschungsmodell für Lebensstile und wird teilweise auch in der wissenschaftlichen Literatur benutzt. Anhand der detaillierten Beschreibungen der Milieumerkmale (siehe hierzu beispielhaft Tabelle 2) sind auch segmentspezifische Einstellungen gegenüber bestimmten Produkten, Marken oder Ähnlichem ermittelbar (Meffert 2000, S. 200).

\subsection{Marktsegmentierung}

Mit Beginn der Nachkriegszeit erlebte die deutsche Wirtschaft eine gewaltige Phase des Aufschwungs, die getragen war von der hohen Nachfrage nach Gütern nahezu aller Art. Typisch für diese Zeit war, dass der limitierende Faktor des Marktes die Produktion war, die Nachfrage die Kapazität der produzierenden Unternehmen also überstieg. In einer solchen Situation liegt die gesamte Marktmacht bei den Unternehmen, da sich deren Verkäufer unter solchen Bedingungen ihre Kunden aussuchen können. Man spricht dabei von einem sogenannten „Verkäufermarkt“.

Heute herrscht eine völlig veränderte Marktsituation: Die Phase der Marktsättigung ist längst erreicht. Die Wachstumsraten der Nachfrage nach Gütern nahezu aller Art haben sich deutlich abgeschwächt, und aus den Wachstumsmärkten von einst sind Verdrängungsmärkte geworden. Zusätzlich suchen infolge der Globalisierung immer mehr Unternehmen Zugang zu bereits bestehenden Märkten, was den Wettbewerb weiter anheizt. Heutzutage übersteigt auf nahezu allen Märkten das Angebot die Nachfrage, so dass die Marktmacht inzwischen bei den Konsumenten liegt. Es herrscht ein „Käufermarkt“‘.

Um als Unternehmen auf einem Käufermarkt langfristig überleben zu können, ist es nötig, sich die Gunst der Käufer zu sichern - da es allein diese sind, die mit Kauf oder Nichtkauf über die Zukunft eines Unternehmens entscheiden. Ausgangspunkt eines modernen Unternehmens muss also stets der Kunde sein. Dies betrifft nicht nur den Vertrieb oder andere Unternehmensbereiche, die mit Kunden in direktem Kontakt stehen. Vielmehr muss heutzutage das gesamte Unternehmen kundenorientiert arbeiten. Neben den Anpassungen der formalen Unternehmensstrukturen bedeutet dies einen gleichzeitigen Wechsel des unternehmerischen Selbstverständnisses und auch der Unternehmenskultur. Diese Entwicklung brachte und bringt auch heute noch viele Unternehmen in Schwierigkeiten, da der Wandel der Marktbedingungen weder verlangsamt noch gestoppt werden kann. Einen wichtigen Schritt in Rich- 
tung Kundenorientierung stellt die strategische Marketingmaßnahme der Marktsegmentierung dar.

Marktsegmentierung steht für die Aufteilung des gesamten Marktes in verschiedene Gruppen anhand zuvor festgelegter Kriterien. Per Definition versteht man darunter eine Marketingstrategie, die einen heterogenen Gesamtmarkt anhand geeigneter Segmentierungskriterien in homogene Teilmärkte aufteilt und diese Teilmärkte segmentspezifisch bearbeitet (Freter 2008, S. 25). Basis aller Überlegungen zur Marktsegmentierung ist der Gedanke, dass nicht alle Käufer gleich sind, sich die Käufer also in ihren Wünschen und Erwartungen an ein Produkt unterscheiden. Durch eine Marktsegmentierung sollen innerhalb der gesamten Käufergruppe einzelne Gruppen von Käufern identifiziert werden, die in sich möglichst homogen und untereinander möglichst heterogen sind. Die scharfe Abgrenzung der einzelnen Käufergruppen soll es ermöglichen, die Instrumente des Marketings zielgenauer und somit effektiver einzusetzen, was letztendlich hilft, den Unternehmenserfolg zu sichern (Kotler et al. 2007a, S. 357).

Basis der Marktsegmentierung ist der gesamte Markt, weshalb dieser in verschiedene Anspruchs- bzw. Interessengruppen des Unternehmens unterteilt wird, was der Idee des Stakeholder-Value-Ansatzes folgt. $\mathrm{Zu}$ diesen Gruppen zählen beispielsweise die Eigentümer des Unternehmens, die Lieferanten, die Geldgeber, die Mitarbeiter, die Lieferanten oder auch die Kunden des Unternehmens (zum Stakeholder-Value-Ansatz vgl. Freeman, „Strategic Management: A Stakeholder Approach“). Alle Stakeholder eines Unternehmens haben ihre eigenen Interessen an der Unternehmung und vertreten diese auch im Rahmen ihrer Möglichkeiten. Eine ganzheitliche Unternehmensstrategie muss alle Interessengruppen berücksichtigen und deren teilweise auch gegensätzliche Interessen vertreten. Da sich die vorliegende Arbeit auf die Segmentierung von Kunden im Einzelhandel beschränkt, wird im weiteren Verlauf jedoch lediglich die Interessensgruppe der Kunden näher betrachtet. Des Weiteren soll gelten, dass unter „Kunden“ in dieser Arbeit nur Kunden auf Endabnehmerebene verstanden werden. Die Kunden der Handelsebene werden in dieser Arbeit, thematisch bedingt, nicht näher betrachtet.

Im weiteren Verlauf des Kapitels werden die einzelnen Schritte der Marktsegmentierung vorgestellt und näher erläutert, ebenso die anzuwenden Werkzeuge zur Sicherung der Segmentierung und die unternehmerischen Potenziale, die anhand einer Segmentierung gehoben werden können. 


\subsubsection{Markterfassung und Marktbearbeitung - ein einheitlicher Ansatz}

Der Begriff „Marktsegmentierung“ wurde bereits definiert und schließt die ,,segmentspezifische Bearbeitung“ der erkannten Nachfragegruppen mit ein. Teilweise wird unter Marktsegmentierung allerdings nur die Aufteilung eines heterogenen Marktes in homogene Teilmärkte, also lediglich die Abgrenzung, nicht aber die Bearbeitung der erkannten Teilgruppen verstanden. Um diese unterschiedlichen Verständnisse von Marktsegmentierung zu vermeiden, wird sie zunächst mit „Marktsegmentierung im engeren Sinne“ und mit „Marktsegmentierung im weiteren Sinne“ näher spezifiziert. Marktsegmentierung im engeren Sinne gruppiert den Markt bzw. erfasst diesen in einem ersten Schritt. Man spricht daher auch von „Markterfassung“. Da dazu im Vorfeld die notwendigen Informationen erhoben bzw. vorliegen müssen, ist auch von der „Informationsseite“, auf welcher die Markterfassung stattfindet, die Rede. Marktsegmentierung im weiteren Sinne baut auf der Markterfassung auf und beschreibt, wie die dort erkannten Segmente bewertet und dementsprechend von dem Unternehmen bearbeitet werden können. Man spricht daher von der „Aktionsseite“ bzw. von der Seite der „Marktbearbeitung“. Die Aufspaltung, genauer die Zusammensetzung der „Marktsegmentierung“ zeigt Abbildung 29.

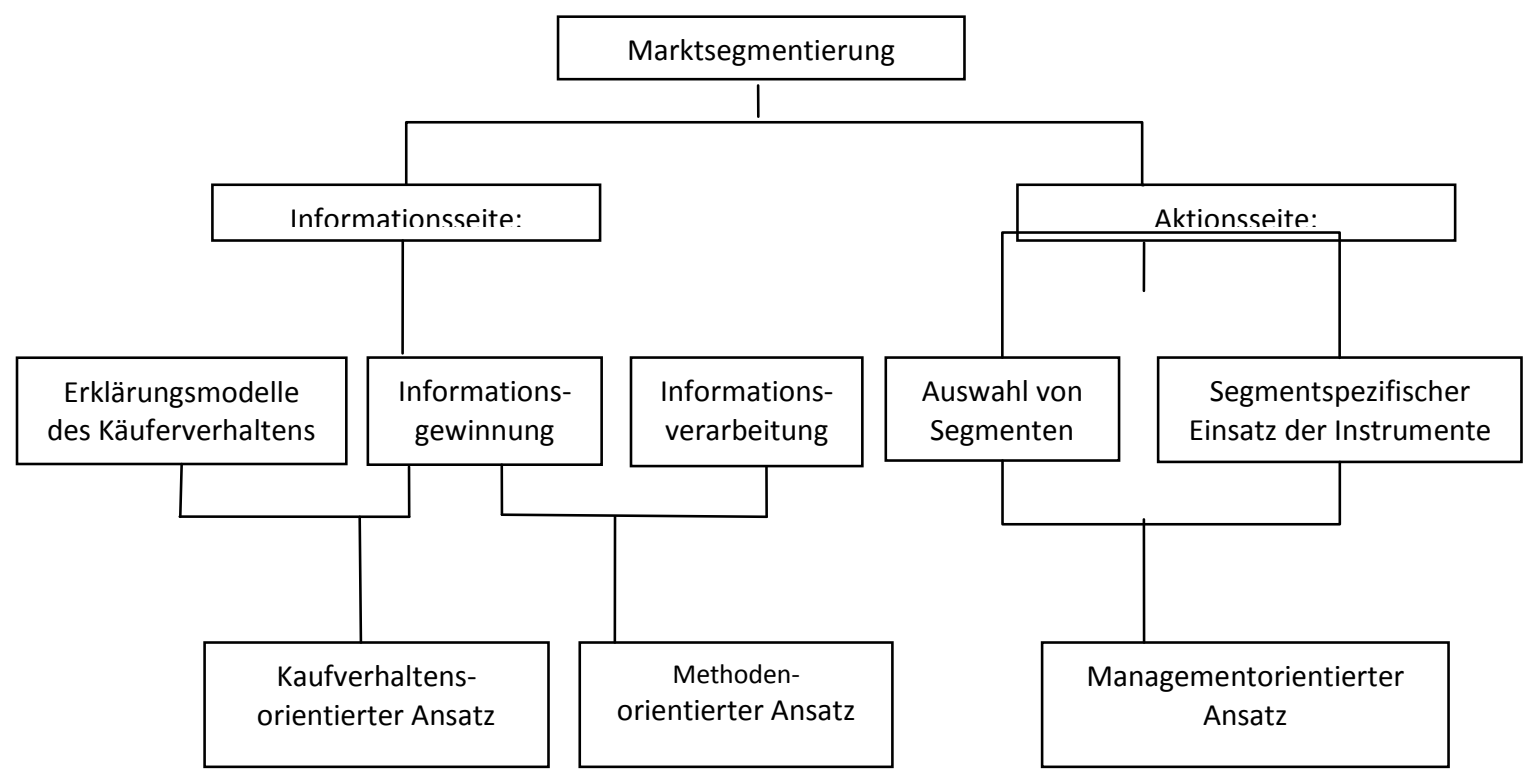

\section{Abbildung 29: Teilbereiche der Marktsegmentierung}

Quelle: Freter 2008, S. 27 
Wie bereits zu Beginn dieses Kapitels definiert, wird Marktsegmentierung in dieser Arbeit ausschließlich „,im weiteren Sinne“ verstanden.

\section{Käuferverhalten auf Markterfassungsseite}

Basis der Markterfassung bzw. der Informationsseite der Marktsegmentierung stellt das Verhalten der einzelnen Käufer dar, die es zu segmentieren gilt. Erst mit Kenntnis der Faktoren, die zu einem bestimmten Käuferverhalten führen, können am Ende betriebswirtschaftliche Instrumente eingesetzt werden, die die Entscheidungsfindung der Konsumenten in die gewünschte Richtung lenken (Homburg/Krohmer 2006, S. 27).

Im Folgenden werden daher zwei Modellgruppierungen zur Erklärung von Käuferverhaltensmustern aufgezeigt, die sich in „echte Verhaltensmodelle“ und in „Black-Box-Modelle“ unterteilen lassen. Black-Box-Modelle beziehen als Erklärung des Konsumentenverhaltens ausschließlich beobachtbare Größen in das Modell mit ein. Diese sind die Modell-Inputs (die Stimuli der Konsumenten) und die Modell-Outputs (die Reaktionen der Konsumenten). Man spricht daher auch vom Bereich der S-R-Modelle, die im Gebiet des „Behaviorismus“ angesiedelt sind. Die Transformationsprozesse innerhalb des Modells werden nicht thematisiert bzw. erklärt; sie finden unsichtbar in einer Black Box statt, wie sie in Abbildung 30 veranschaulicht sind.

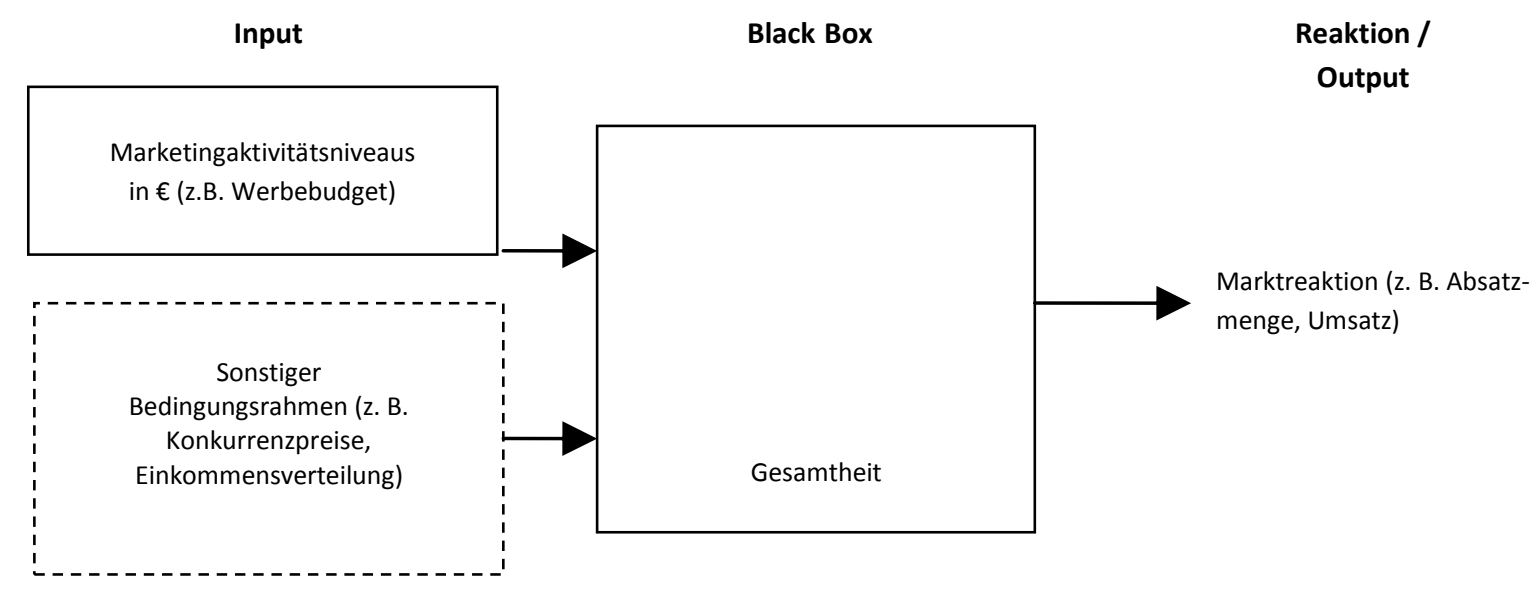

Abbildung 30: Das S-R-Modell

Quelle: Freter 2008, S. 66 f. 
Da S-R-Modelle die Transformationsprozesse innerhalb der Black Box ausblenden, geben sie letztendlich nur Reaktionsfunktionen der Gesamtheit an potenziellen Käufern auf deren Stimuli wieder - auf das Modell bezogen also nur mathematische Beziehungen über den $\mathrm{Zu}$ sammenhang von Inputveränderungen zu Outputveränderungen. Da für ein kundenorientiertes Angebot allerdings die Frage „Wie und warum reagiert der Konsument auf einen Stimulus?“ von großer Bedeutung ist, haben sich bei den modernen Erklärungen zum Konsumentenverhalten die echten Verhaltensmodelle durchgesetzt (Kuß/Tomczak 2004, S. 2 f.).

Echte Verhaltensmodelle gehören dem Bereich des „Neobehaviorismus“ an. In ihnen werden als Erklärung des Verhaltens auch nicht beobachtbare Größen zugelassen, also Zustände oder Aktionen, die sich im Inneren des Konsumenten abspielen, beispielsweise Motive oder Einstellungen. Diese sogenannten ,intervenierenden Variablen“ sollen die Black Box der behavioristischen Modelle mit Informationen und Daten füllen, um so die Ursachen des beobachteten Verhaltens besser erklären zu können (siehe Abbildung 31). Da diese Modelle neben den Inputs und Outputs auch die Vorgänge im Organismus des Käufers mit in Betracht ziehen, gehören die neobehavioristischen Käuferverhaltensmodelle zu den sogenannten S-O-R-Modellen.

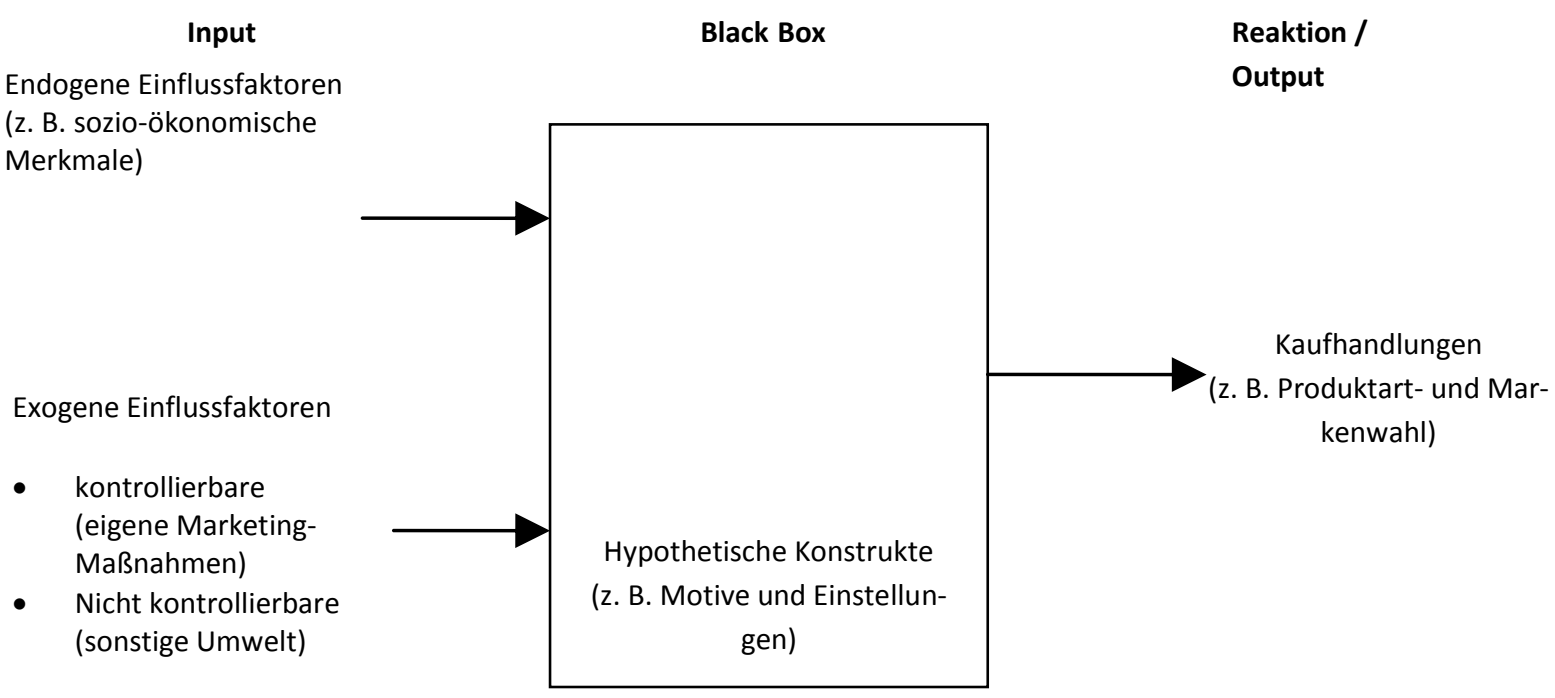

Abbildung 31: Das S-O-R-Modell

Quelle: Freter 2008, S. 67 
Im Gegensatz zu den Black-Box-Modellen betrachten S-O-R-Modelle neben den intervenierenden Variablen (auch: „hypothetische Konstrukte“) gleichzeitig mehrere Inputs, die sowohl exogener als auch endogener Art sein können. Exogene Inputs sind äußere Einflüsse, die auf den Organismus bei dessen Kaufentscheidungsprozess einwirken, also sowohl die angewandten Marketinginstrumente des Unternehmens, die auf den Konsumenten einwirken sollen, als auch weitere Umwelteinflüsse, die vom Unternehmen nicht beeinflusst werden können, wie beispielsweise Angebote von Wettbewerbern, die ebenfalls auf den Konsumenten einwirken. Endogene Inputs sind individuelle Merkmale des Konsumenten, die bei dessen Kaufentscheidung von Bedeutung sind, beispielsweise soziale Lage, Geschlecht, Alter oder auch individuelle Merkmale psychographischer Natur, wie sie den Lebensstilen zu Grunde liegen, beispielsweise innere Werte oder persönliche Einstellungen (siehe hierzu auch Kapitel 3.1.2).

Die Eigenschaft der S-O-R-Modelle, hypothetische Konstrukte im Modell zuzulassen, wirkt sich auch auf die weiteren Schritte der Marktsegmentierung aus. Durch das Berücksichtigen von Faktoren, die das Verhalten der Konsumenten (mit-)bestimmen, ergeben sich durch die Zusammensetzung dieser Faktoren bereits Kriterien bzw. Anhaltspunkte für Kriterien, nach denen die Käuferschicht in einem weiteren Schritt segmentiert werden kann (Foscht/Swoboda 2007, S. 25; Meffert 2000, S. 99 f.).

\subsubsection{Kriterien der Marktsegmentierung}

Um die gesamte Gruppe der potenziellen Käuferschaft in einzelne Segmente untergliedern zu können, ist es nötig, Merkmale bzw. Kriterien anzusetzen, nach denen sich die Individuen der Gruppe unterscheiden und anhand derer im Folgenden die einzelnen Segmente gebildet werden können. Da hierfür eine Vielzahl an sortierenden Merkmalen möglich ist, werden diese zur besseren Übersicht in übergeordneten Gruppen zusammengefasst und im Anschluss separat vorgestellt. Nach der empirischen Untersuchung dieser Arbeit werden die hier vorgestellten Segmentierungskriterien wieder thematisch aufgegriffen, um erneut zu überprüfen, inwiefern sie für die Blumen- und Pflanzenbranche geeignet sind. Einen Überblick über die verschiedenen Segmentierungskriterien gibt Abbildung 32. 


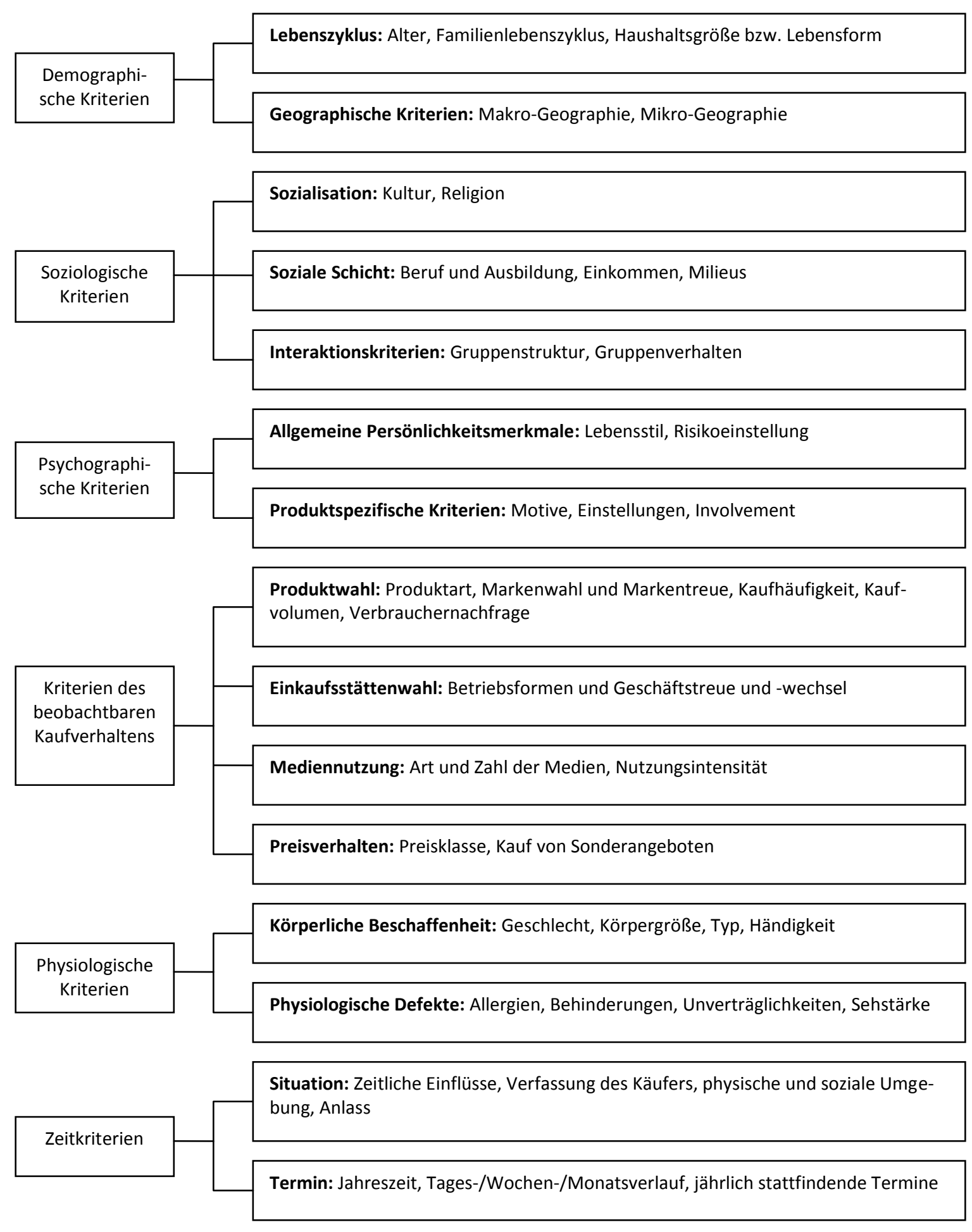

\section{Abbildung 32: Kriterien der Marktsegmentierung}

Quelle: Freter 2008, S. 93

Da die Auswahl der Segmentierungskriterien neben der möglichst exakten Markterfassung von großer Bedeutung für die spätere Bearbeitung der einzelnen Segmente ist, muss deren Auswahl gründlich überdacht sein. Als Entscheidungshilfe werden daher im Folgenden die 
vorgestellten Kriterien einzeln nach einem 7-Punkte-Schema bewertet, das sich wie folgt zusammensetzt:

1. Kaufverhaltensrelevanz

2. Ansprechbarkeit

3. Zugänglichkeit

4. Messbarkeit

5. Zeitliche Stabilität

6. Wirtschaftlichkeit

7. Trennschärfe

Unter Bezugnahme auf relevante Beiträge (Meffert/Bruhn 2006, S. 153 ff.; Meffert 2000, S. 186 f.; Wedel/Kamakura 2003, S. 4 f.; Homburg/Krohmer 2006, S. 485 f.; Freter 2008, S. 90 ff.) werden diese Punkte wie folgt verstanden:

Kaufverhaltensrelevanz bedeutet, dass die gebildeten Segmente in sich möglichst homogen sein sollten, also die Individuen des Segments möglichst gleiche Kaufverhaltensmuster aufweisen. Die Segmente untereinander sollten jedoch möglichst unterschiedlich bzw. heterogen sein. Je mehr diese Bedingung erfüllt ist, desto einfacher bzw. gezielter gestaltet sich der spätere segmentspezifische Einsatz von Marketinginstrumenten.

Ansprechbarkeit der Segmentierungskriterien beschreibt die Möglichkeit, wie die getroffene Auswahl der Segmentierungskriterien auch als Ansatzpunkte zur späteren segmentspezifischen Marktbearbeitung benutzt werden kann. Je ausgeprägter die Ansprechbarkeit der Kriterien ist, desto einfacher gestaltet sich die richtige Wahl von Marketinginstrumenten während der späteren Phase der Marktbearbeitung.

Die anhand der Segmentierungskriterien gebildeten Segmente sollten eine möglichst hohe Zugänglichkeit aufweisen, d. h., die einzelnen Individuen einer Gruppe sollten also möglichst bekannt sein, um so die Marketinginstrumente möglichst ohne Streuverlust einsetzen zu können.

Die Messbarkeit der Kriterien ist von großer Bedeutung bei der praktischen Umsetzung und Anwendung der Marktsegmentierung (und somit auch beim Kriterium der Wirtschaftlichkeit der Kriterien). Je eindeutiger die Kriterien bewertet bzw. gemessen werden können, desto größer ist ihre Operationalität für den gesamten Prozess der Marktsegmentierung.

Da der Prozess der Marktsegmentierung lange dauern bzw. der Zeitraum, bis er sich „rechnet“, sehr groß sein kann, ist es wichtig, dass die ausgewählten Kriterien eine gewisse Be- 
ständigkeit zeigen und sich nicht in kurzen Zeitabständen immer wieder verändern. Die Kriterien sollten also eine möglichst hohe zeitliche Stabilität aufweisen.

Die Kriterien, anhand welcher die Marktsegmente gebildet werden, sollen helfen, Segmente $\mathrm{zu}$ identifizieren, deren Bearbeitung eine besonders hohe Wirtschaftlichkeit eignet. Da die Marktsegmentierung zuerst Kosten verursacht, bevor eventuell höhere Erlöse erzielt werden können, ist dieser Punkt von entscheidender Bedeutung. Die Kriterien müssen darauf ausgerichtet sein, die wirtschaftlich lohnenden Segmente zu identifizieren, da sich sonst kein Unternehmen für eine Marktsegmentierung entscheiden würde.

Die gewählten Segmentierungskriterien sollten zudem eine möglichst hohe Trennschärfe der identifizierten Segmente unterstützen, also die Möglichkeit, die Segmente möglichst exakt voneinander zu trennen.

\subsubsection{Demographische Segmentierungskriterien}

Wie in Abbildung 32 dargestellt, gliedert sich die Gruppe der demographischen Kriterien auf in die Kriterien des Lebenszyklus und die der geographischen Kriterien. Demographische Kriterien sind die bekanntesten und auch am weitesten verbreiteten Kriterien zur Marktsegmentierung.

Die Kriterien des Lebenszyklus sind vielfältig. Zu ihnen gehören sämtliche Ausprägungen eines Individuums, die eng mit dem „Aufenthaltsort“ im Lebenszyklus zu tun haben, beispielsweise das Alter, das Einkommen, die Haushaltsgröße oder der Familienlebenszyklus.

Das Alter ist ein sehr einfaches, weit verbreitetes und auch sehr aussagekräftiges Segmentierungskriterium: In allen Altersklassen unterscheiden sich die Bedürfnisse sehr stark. Studenten suchen andere Wohnungen als junge Familienväter, Teenager lesen andere Zeitschriften als Senioren und Kleinkinder brauchen andere Nahrung als Erwachsene. Dabei gilt es allerdings zu beachten, dass die Segmentierung anhand der Zugehörigkeit zu einer bestimmten Altersgruppe bei einem entsprechend großen Markt nicht ausreichend sein könnte, da sich das Segment „20-30 Jahre“ für eine segmentspezifische Bearbeitung eventuell als zu groß erweist. Eine solche Segmentierung beispielsweise für alle Einwohner der Bundesrepublik Deutschland wäre zu weit gefasst. Man müsste das Segment „20-30 Jahre“ weiter, nach anderen Kriterien segmentieren, beispielsweise dem Geschlecht. Des Weiteren gilt es zu beachten, dass eine Segmentierung anhand des Alters zwar von Dauer sein kann, die Personen, die das jeweilige Segment bilden, allerdings altersbedingt immer wechseln. 
Neben dem Alter spielt auch die äußere Form, wie man sein Leben führt, eine wichtige Rolle für die demographischen Segmentierungskriterien. Eine einfache und weit verbreitete Form der Erfassung der Lebensumstände von Personen ist die der Haushaltsgröße, in der sich die Personen befinden. Folgt man der Einteilung des Statistischen Bundesamtes, müssen vier unterschiedliche Haushaltstypen voneinander differenziert werden:

1. Haushalte mit Paaren, ohne Kinder

2. Haushalte mit Paaren, mit Kindern

3. Haushalte mit Alleinstehenden, ohne Kinder

4. Haushalte mit Alleinstehenden, mit Kindern.

Die Unterteilung aller Haushalte in die verschiedenen Gruppen von Haushalten ist ein entscheidender Schritt zur Marktsegmentierung, lässt sich doch anhand dieser Unterscheidung ablesen, wie die Bewohner der einzelnen Haushalte miteinander in Verbindung stehen. Dass Unternehmen diese Art der Segmentierung berücksichtigen und auch aktiv betreiben, zeigt sich beispielsweise daran, dass Unternehmen ihr Angebot der steigenden Anzahl von Haushalten mit Alleinstehenden ohne Kinder („Single-Haushalte“) anpassen, beispielsweise durch ein vermehrtes Angebot an kleineren Packungsgrößen bei Lebensmitteln.

Durch die recht exakte Erfassung der Entwicklung der verschiedenen Haushaltstypen lassen sich auch langfristige Trends ablesen bzw. erahnen. Langfristig steigt die Anzahl der Haushalte mit kinderlosen Paaren, die Anzahl der Haushalte der Paare mit Kindern fällt, die Anzahl der Haushalte der Alleinerziehenden steigt und die Anzahl der Haushalte der kinderlosen Alleinstehenden steigt ebenfalls. Somit sind bereits die Segmente erkannt, deren Bedeutung weiter zunehmen wird. Entsprechende Anpassungen des Angebotes sind daher zu erwarten (Freter 2008, S. 105).

Neben den Kriterien des Lebenszyklus werden die demographischen Kriterien auch durch die geographischen Kriterien determiniert. Geographische Kriterien können einerseits als sehr grobes Segmentierungskriterium benutzt werden, beispielsweise für eine Segmentierung anhand des Staates, in dem man wohnt, andererseits aber auch als recht feines Segmentierungskriterium, beispielsweise bei einer Segmentierung anhand eines Stadtviertels oder anhand der Straße, in der man wohnt. Um die Art der geographischen Daten besser zu beschreiben, unterscheidet man daher zwischen der groben Makro-Geographie und der feineren Mikro-Geographie. 
Die makro-geographische Segmentierung kann global gesehen werden oder auch in kleineren Kreisen erfolgen, beispielsweise innerhalb Deutschlands auf Ebene der Bundesländer. Bezogen auf das Beispiel Deutschland spricht man so lange noch von makro-geographischer Segmentierung, bis Gemeinden als Differenzierungskriterium erreicht sind. Bei allen kleineren Kriterien, also von Stadtteilen bis hin zum einzelnen Gemeindebewohner, spricht man von mikro-geographischen Segmentierungskriterien (Meffert 2000, S. 189). Dass als makrogeographische Kriterien dabei nicht nur offizielle Grenzen benutzt werden können, zeigt sich bei den sogenannten Nielsen-Gebieten. Diese orientieren sich lediglich an den Grenzen der Bundesländer, teilen das Gebiet der Bundesrepublik Deutschland allerdings letztendlich nach „,neuen Grenzen“ auf, die sich auch an wirtschaftlichen Daten orientieren (Nielsen Company 2011).

Mikro-geographische Segmentierungskriterien beziehen sich demgegenüber auf recht kleine Gebiete oder Plätze innerhalb einer Gemeinde. Grundlegender Gedanke dabei ist, dass innerhalb dieses kleinen Gebietes, bezogen auf ihre Lebensart, sehr ähnliche Personen wohnen. Begründen lässt sich dieser Gedanke nach Holland mit der Idee „Gleich und gleich gesellt sich gern“. Der Idee Hollands folgend, ähneln sich innerhalb eines mikro-geographischen Gebietes also nicht nur der geographische Ort, an dem sich die Personen befinden, sondern auch ihre weiteren soziodemographischen Kriterien, wie Einkommen oder Alter. Dazu ist zu erwarten, dass sich innerhalb des mikro-geographischen Gebietes auch die Lebensstile der einzelnen Personen sehr ähneln (Holland 2004, S. 81; Meffert 2000, S. 191).

Um eine Marktsegmentierung anhand der geographischen Kriterien vorzunehmen, ist im Vorfeld zu beachten, dass makro-geographische Daten recht problemlos bzw. recht kostengünstig $\mathrm{zu}$ beschaffen sind, da diese bereits vorliegen - beispielsweise beim Statistischen Bundesamt. Mikro-geographische Daten hingegen liegen aufgrund ihres Detaillierungsgrades meist nicht vor und müssen daher meist erst teuer erhoben oder, falls möglich, gekauft werden. Die höheren Kosten einer geographisch feinen Segmentierung lassen sich allerdings dadurch kompensieren, dass die Ergebnisse der Segmentierung genauer sind. Somit lassen sich Entscheidungen finden, die genau zu dem identifizierten Gebiet passen und somit einen großen Zuspruch der Bewohner bzw. der Angesprochenen erfahren.

Um die demographischen Segmentierungskriterien zu bewerten, wird im Folgenden das oben erwähnte 7-Punkte-Schema angewandt (Karasu 2010, S. 34 ff.; Rennhak/Kesting 2008, S. 17 f.; Freter 2008, S. 112 f.). 
Demographische Kriterien sind in Bezug auf die Kaufverhaltensrelevanz ausschließlich deskriptiv. Sie geben keine Erklärung über die Gründe des Verhaltens. Auf mikro-geographischer Ebene allerdings geben sie Hinweise auf regionale Gepflogenheit. Fazit: kaum Kaufverhaltensrelevanz.

Die Ansprechbarkeit für den späteren Einsatz von segmentspezifischen Marketinginstrumenten ist hier bedingt gegeben. Das Instrument Werbung kann über ein Medium eingesetzt werden, das die gleiche Zielgruppe hat. Auch kann der Einsatz der Werbebotschaft anhand der mikro-geographischen Daten variiert werden. Fazit: gegebene Ansprechbarkeit.

Die Zugänglichkeit der Segmente ist bei den demographischen Kriterien gegeben. Dies liegt daran, dass gerade der Zugang über Werbung nahezu problemlos gesichert ist. Dabei kann wiederum die Werbebotschaft auch geographisch variiert werden. Auch sind bei gegebener mikro-geographischer Segmentierung direkte und persönliche Ansprachen möglich. Fazit: hohe Zugänglichkeit.

Die Messbarkeit der (makro-)demographischen Daten ist leicht. Auch können Unternehmen diesbezüglich sehr oft auf bereits bestehendes Datenmaterial zugreifen. Probleme bereiten allerdings die mikro-geographischen Daten. Sie müssen meist extra erhoben werden, was hohe Kosten verursacht. Fazit: gegebene Messbarkeit.

Trotz der Bewegungen innerhalb der Segmente weisen die gebildeten Segmente eine lange Lebensdauer auf. Auch in mikro-geographischer Sicht ändern sich die Segmente nicht sehr schnell, es gilt die Annahme von Holland. Eventuell doch vonstattengehende Veränderungen passieren nur sehr langsam und sind auch sehr gut prognostizierbar. Fazit: hohe zeitliche Stabilität.

(Makro-)demographische Daten liegen in den meisten Fällen bereits vor. Eine eigene Erhebung ist daher meist nicht notwendig. Dies ist für alle Unternehmen ein wichtiger Punkt, da eine Primärdatenerhebung meist mit hohen Kosten verbunden ist. Bei mikro-geographischen Daten ist hingegen eine Primärdatenerhebung meist notwendig. Ein Kosten-Nutzen-Vergleich im Vorfeld erleichtert die Entscheidung. Fazit: hohe Wirtschaftlichkeit.

Die Trennschärfe ist bei demographischen Kriterien nur schwach ausgeprägt. Bei den geographischen Kriterien ist das Ausmaß der Trennschärfe größer: Je nach geographischem Gebiet können sich bestimmte Konsumgewohnheiten entwickelt haben, die zur Segmenttrennung geeignet sind. Fazit: gegebene Trennschärfe. 


\subsubsection{Soziologische Kriterien}

Die soziologischen Kriterien werden sehr erfolgreich von kommerziellen Anbietern zur Marktsegmentierung herangezogen, so dass sie eine aktuelle Relevanz aufweisen. Aufgrund ihrer Komplexität werden die soziologischen Kriterien unterteilt in „Sozialisation“, „soziale Schichten“ und „Interaktionskriterien“.

Unter Sozialisation fasst man die Eigenschaften und Verhaltensweisen von Menschen zusammen, die nicht in ihrem Wesen begründet sind, sondern erst durch die Prägung der Gesellschaft Form annehmen, beispielsweise durch Kultur und Religion. So geht man in Deutschland von einer Sozialisation aus, die christlich-abendländisch geprägt ist. Durch die meist lebenslange Zugehörigkeit der Menschen zu ,ihrer“ Sozialisation ist diese bei der Marktsegmentierung nach soziologischen Kriterien eine entsprechend langlebige und jährlich wiederkehrende Komponente, die von der Marktangebotsseite entsprechend stark beachtet wird. Dies zeigt sich zum Beispiel daran, dass das Angebot von Tannenbäumen zur Weihnachtszeit in Ländern mit christlicher Sozialisation signifikant ansteigt.

Durch die zunehmende Migration muss die bisher primär nationale Sozialisation heutzutage aber auch im interkulturellen Zusammenhang gesehen werden, bei dem die Grenzen einzelner Kulturen nach und nach ineinander übergehen, wie beispielsweise das Interesse am amerikanischen Halloweenfest in Deutschland zeigt. Ein Brauch aus den USA schwappt nach Deutschland über und schafft einen neuen Markt z. B. für entsprechende Kostüme. Ebenso entstehen durch Migration Subkulturen innerhalb einer bestehenden Sozialisation, die sich durch eigene Religionsfeste und Wertevorstellungen abgrenzen. Dadurch entstehen neue Absatzmärkte mit entsprechend neuen Prägungen, wie beispielsweise jene muslimischer Art innerhalb des christlich abendländisch geprägten Deutschlands.

$\mathrm{Zu}$ den soziologischen Kriterien gehören auch die sozialen Schichten. Darunter versteht man homogene, relativ dauerhafte Gesellschaftsgruppen, die sich durch Wertvorstellungen, Interessen, Lebensstile und Verhaltensmuster, basierend auf unterschiedlichen Berufen, Einkommen, Vermögen und Abstammung, voneinander abgrenzen. Die sozialen Schichten lassen sich in Unter-, Mittel- und Oberschicht gliedern, wobei jede dieser Gruppen nochmals in jeweils eine untere und eine obere Schicht unterteilt wird. So ergibt sich beim Ansatz der sozialen Schichten eine Klassifikation von insgesamt sechs Gruppen (Meffert 2000, S. 194; Kroeber-Riel/Weinberg 2003, S. 564 ff.). 
Die Unterscheidungsmerkmale der sozialen Schichten lassen sich auch als Indikatoren zur Bestimmung des Konsumentenverhaltens benutzten. So gibt z. B. die Art des Berufs einen Hinweis auf die Höhe des Einkommens, das letztendlich als freies Einkommen zum Konsum genutzt werden kann. Die Wahl des Berufs hat aber auch Auswirkungen auf das Umfeld, das soziale Ansehen und den Lebensstil. Darauf aufbauend ist das Einkommen ein häufig benutzter Indikator zur Segmentierung, da er Auskunft über die finanzielle Kaufkraft einer Person gibt. Es gilt: je höher das Einkommen, desto geringer ist der Anteil, der für die Befriedigung der Grundbedürfnisse ausgegeben wird. Damit geht mit steigendem Einkommen eine Veränderung des Verbrauchsmusters hin zu Luxusgütern einher.

Zur Einteilung der sozialen Schichten gehören ebenso die Milieus, die Sub- bzw. Teilkulturen innerhalb einer Gesellschaft darstellen. Es gibt verschiedene Modelle, welche die Milieulandschaft einer Gesellschaft differenzieren. Grundlage der Unterscheidungen sind z. B. beim Sinus-Milieu die soziale Lage und die Grundorientierung (traditionell, modern, neu) oder bei den Erlebnismilieus nach Schulze die Bildung und das Alter.

Basierend auf diesen Kriterien lässt sich eine Gesellschaft in soziale Schichten einteilen, die theoretisch Aufschluss über das Käuferverhalten geben, wie z. B. der Kauf einer teuren Luxusarmbanduhr durch eine Person aus der Oberschicht. Jedoch zeigt sich in der Realität immer mehr ein hybrides Kaufverhalten im Sinne eines Wechsels zwischen Preisbereitschaft und Sparorientierung. So ist ein Porsche fahrender Aldi-Käufer nicht auszuschließen, also ein Konsument, der einerseits bereit ist, viel Geld für einen Sportwagen auszugeben, andererseits aber auf preiswerte Lebensmittel achtet.

Interaktionskriterien stellen den dritten Pfeiler der soziologischen Kriterien dar. Sie beruhen auf aktuellen Trends und Entwicklungen, welche die Bedeutung der Gruppe bzw. die Bedeutung des sozialen Umfelds einer Person als mitentscheidenden Faktor zur Kaufentscheidung herausstellt (Weißbacher 2006, S. 1 ff.). Wäre es demnach möglich, Informationen über die Mitgliedsstruktur der Gruppe, ihre Präferenzen oder ihr Kauf- bzw. Entscheidungsverhalten zu erhalten, könnten spezifische Gruppenangebote erstellt werden. Um eine solche Gruppenanalyse durchzuführen, müssen nach Voeth zwei Bereiche unterschieden werden: die Gruppenstruktur und das Gruppenverhalten.

Die Gruppenstrukturanalyse umfasst die Aspekte, die sich gegenseitig bedingen: allgemeine Merkmale der Gruppe, Bedarf, Organisation und Rollen. Unter allgemeinen Merkmalen werden Gruppengröße, Herkunftsland, Existenzdauer, aber auch das Konsumentenverhalten in 
Verbindung mit Alter und Einkommen zusammengefasst. So unterscheidet sich z. B. eine Schulklasse von einer Stammtischgruppe. Bedarf als weiterer Aspekt hängt eng mit den allgemeinen Merkmalen einer Gruppe zusammen, da sich der Bedarf am Alter, Einkommen etc. orientiert. Ebenso spielt die Organisation der Gruppe eine Rolle, da entscheidend ist, inwieweit der Beschaffungs- und Nachfrageprozess formal geregelt ist und wer der entsprechende Ansprechpartner ist. Auf der anderen Seite sind aber auch die informellen Rollen innerhalb einer Gruppe von Bedeutung, da sich die übrigen Gruppenteilnehmer an der Rolle des Entscheiders oder Beeinflussers orientieren.

Das Gruppenverhalten, das durch die Gruppenstruktur beeinflusst wird, lässt sich in die voneinander abhängigen Aspekte Informationsbeschaffung, Kommunikation und Entscheidungsfindung unterteilen. Zum einen lässt sich ein unterschiedliches Bedürfnis bezüglich der Informationen feststellen, so dass sich in dieser Hinsicht unterschiedliche Nachfragegruppen bilden. Zum anderen ist das Kommunikationsverhalten der Gruppenteilnehmer untereinander für ein Unternehmen von Bedeutung, da es den Informationsaustausch bestimmt. Zuletzt gehört die Entscheidungsfindung als weiterer Aspekt zum Gruppenverhalten. Hier geht man davon aus, dass eine individuelle Kaufentscheidung der Gruppenentscheidung untergeordnet wird, so dass mit entsprechenden Marketingmaßnahmen bestimmte Gruppen direkt angesprochen werden können (Voeth 2003, S. 305).

Abschließend sollen nun die soziologischen Kriterien zur Marktsegmentierung nach dem 7-Punkte-Schema und unter Berücksichtigung der relevanten Autoren (Stuhldreier 2002, S. 43 ff.; Freter 2008, S. 133 f.) bewertet werden:

Soziologische Kriterien sind in Bezug auf das Kaufverhalten als sehr nützlich einzuschätzen, insofern die entsprechenden Daten ermittelt werden können. Erschwert wird der Einsatz der soziologischen Kriterien zur Ermittlung des Kaufverhaltens allerdings durch die zunehmende Unschärfe der Gruppen bzw. Kulturen. Fazit: hohe Kaufverhaltensrelevanz.

Die Aussagefähigkeit der soziologischen Kriterien ist bedingt gegeben. Erfolgsfördernde Faktoren sind eine tatsächlich vorhandene Kaufkraft einer Gruppe und die zur jeweiligen Käufergruppe passende Form der Ansprache. Fazit: mittlere Ansprechbarkeit.

Die Zugänglichkeit der Segmente ist bei den soziologischen Kriterien ebenfalls bedingt gegeben. Zum einen lassen sich Zugehörigkeitsmerkmale wie Einkommen und Bildung zwar feststellen, zum anderen wird die Zuteilung durch die hybriden Formen jedoch enorm erschwert.

Fazit: mittlere Zugänglichkeit. 
Die Messbarkeit der soziologischen Kriterien ist gegeben, sofern diese sich an der religiösen Zugehörigkeit, dem Einkommen oder dem Beruf orientieren. Die Entscheidungsfindung einer Gruppe ist allerdings von verschiedenen Faktoren beeinflusst, so dass hier Werte nur sehr schwer ermittelbar sind. Fazit: mittlere Messbarkeit.

Die zeitliche Stabilität ist sowohl bei der Sozialisation als auch bei den sozialen Schichten gegeben. Ein plötzlicher sozialer Ab- oder Aufstieg ist zwar möglich, aber nicht die Regel. Die Gruppenzugehörigkeit einer einzelnen Person kann nicht als Konstante angesehen werden, dafür aber das (Weiter-)Bestehen einer Gruppe mit denselben Interessen. Fazit: hohe zeitliche Stabilität.

Die hohe Wirtschaftlichkeit begründet sich in der Größe des Segments. So werden große Käuferschaften ermittelt, die eine gemeinsame Kultur, ein ähnliches Einkommen oder die gleichen Interessen haben. Fazit: hohe Wirtschaftlichkeit.

Die einzelnen Gruppen, die unter den soziologischen Kriterien benannt wurden, weisen nur eine geringe Trennschärfe auf. Es kann leicht zu Überschneidungen kommen, z. B. bei der Milieu-Zuordnung aufgrund der Preissensibilität eines Käufers. Fazit: geringe Trennschärfe.

\subsubsection{Psychographische Kriterien}

Eine weitere Komponente innerhalb der Marktsegmentierungsmerkmale stellen die psychographischen Kriterien dar, die über Bedürfnisse, Motivationen, Wünsche etc. einer Person Auskunft geben. Diese Kriterien lassen sich in allgemeine Persönlichkeitsmerkmale und in produktspezifische Kriterien unterteilen (Reinelt/Fauconnier 2005, S. 40 ff.).

$\mathrm{Zu}$ den allgemeinen Persönlichkeitsmerkmalen gehören unter anderem die beiden Aspekte Lebensstil und Risikoeinstellung. Das Segmentierungsmerkmal Lebensstil kann beispielsweise anhand des bereits vorgestellten AIO-Ansatzes, bei dem die Aktivitätsbereitschaft, das Interesse und die Meinung einzelner Bevölkerungsgruppen klassifiziert wird, näher bestimmt werden. So weisen z. B. die konservativen, ruhigen Personen ein sehr reduziertes Radio- und TV-Konsumverhalten auf, identifizieren sich mit den Werten Familie und Kinder und vertreten traditionelle soziale, moralische und religiöse Prinzipien. Werden die Persönlichkeitsmerkmale berücksichtigt, können verschiedene Segmente abgegrenzt werden, deren Bearbeitung rentabel ist, da viele Menschen ihren Lebensstil durch bestimmte Produkte auszudrücken versuchen. 
Unter Risikoeinstellung fasst man verschiedene Arten von Risiken, die bei jeder Kaufentscheidung entstehen können. So muss sich jeder Käufer vor dem Kauf zunächst über seine persönlichen Zweifel hinwegsetzen, ob z. B. das Produkt seinen Preis wert ist oder ob mit dem Kauf ein Verlust der sozialen Anerkennung einhergeht. Werden bestimmte Bedenken vom Hersteller bereits antizipiert, so kann dieser bewusst durch Marketingstrategien dagegen angehen. Beispielsweise kann ein Autobauer mit besonderer Sicherheit werben und dem Sicherheitsrisiko im Straßenverkehr entgegenwirken. Auf der anderen Seite reagiert der Markt aber auch auf risikofreudige Menschen, z. B. mit der Möglichkeit eines „Sneak Preview“ im Kino, bei dem der gezeigte Film unbekannt ist (Foscht/Swoboda 2007, S. 83 f.; Solomon et. al. 2006, S. 271).

Im Rahmen der psychographischen Kriterien werden im Folgenden die produktspezifischen Merkmale anhand der Variablen Motive, Einstellungen und Involvement untersucht. Unter einem Motiv versteht man aktivierende und handlungssteuernde Antriebskräfte des menschlichen Verhaltens. Um daraus einen Nutzen zur Marktsegmentierung ziehen zu können, ist es erforderlich, zwischen Motiven, denen entsprochen werden kann, und solchen, denen nicht entsprochen werden kann, zu unterscheiden. Ein stillbares Bedürfnis, wie Hunger etwa, ist im Moment der Nahrungsaufnahme befriedigt. Jedoch spielen bei der Kaufentscheidung noch weitere Motive eine Rolle, z. B. das Verlangen nach Neuheiten und Abwechslung oder das Motiv der Selbstverwirklichung. So kommt beim Kauf eines Produkts stets ein ganzes Bündel verschiedener Motive zum Tragen (Kroeber-Riel/Weinberg 2003, S. 148 f.; Raab/Unger 2005, S. 200).

Ein weiteres produktspezifisches Kriterium sind Einstellungen, die als eine psychische Tendenz zu verstehen sind und auf Erfahrungen beruhen. Sie drücken sich im Verhalten gegenüber einem Gegenstand aus, z. B. gegenüber Produkten, Marken oder Geschäftstypen. Daraus lässt sich die Hypothese ableiten, dass sich die Wahrscheinlichkeit einer Kaufentscheidung erhöht, je positiver die Einstellung des Konsumenten gegenüber dem jeweiligen Produkt ist. Diese Hypothese lässt sich allerdings nicht generell bestätigen, denn die Relevanz der Einstellung hängt auch von situationsspezifischen Variablen und weiteren unabhängigen Einflussfaktoren ab, so dass Einstellungen nur im komplexen Zusammenhang als Argument zur Kaufentscheidung betrachtet werden kann (Müller-Hagedorn/Schuckel 2003, S. 104).

Das Kriterium Involvement bezeichnet die subjektive Wichtigkeit eines Produktes für den Käufer. Das Ausmaß der Wichtigkeit geht somit vom Käufer selbst aus und nicht vom Produkt, so dass die Käufertypen als wählerisch, interessiert oder faul klassifiziert werden. So 
muss der Markt für jeden dieser Käufertypen entsprechende Angebote präsentieren, z. B. einen kompetenten Berater für den wählerischen Kunden oder ein gutes Preis-Leistungs-Verhältnis für den in der Sache eher desinteressierten Kunden (Meffert 2000, S. 112).

Insgesamt können die psychographischen Kriterien wie folgt beurteilt werden (Diez 2006, S. 36; Heise 1997, S. 320 f.; Reeb 1998, S. 72; Banning 1987, S. 45 f.; Karasu 2010, S. 37; Freter 2008, S. 144 ff.):

Das Kaufverhalten wird durch die psychographischen Kriterien wie Lebensstil, Einstellungen und Motive stark beeinflusst, da das Verhalten und die Persönlichkeit der Zielgruppe genau beschrieben werden können. Jedoch hängen Relevanz und Dominanz stets von weiteren Faktoren ab. So ist z. B. die Relevanz des Motivs wiederum von der jeweiligen Produktart abhängig. Fazit: Kaufverhaltensrelevanz mittel bis hoch.

Die Aussagefähigkeit der Motive und Einstellungen sind sehr hoch, da sie Ansatzpunkte für den Einsatz von Marketinginstrumenten sein können. So lassen sich Werbebotschaften und Werbemittel je nach Bedarf variieren. Fazit: Ansprechbarkeit hoch.

Die Erhebung von Daten im Bereich der psychographischen Kriterien lässt sich nur schwer durchführen. Für eine effektive Marktsegmentierung muss hierbei produktspezifisch vorgegangen werden. Fazit: Zugänglichkeit gering bis mittel.

Um eine Aussage über psychographische Kriterien zu machen, werden Verfahren benötigt, die von Experten durchzuführen sind. Diese Verfahren sind meist aufwendige Experimente, die auch eine finanzielle Belastung darstellen. Zudem lassen entsprechende Erhebungen keine Aussage darüber zu, welche unbewussten Motive oder Einstellungen die betreffenden Personen zu einem Kauf bewegen, so dass es stets eine unbekannte Grauzone gibt. Fazit: Messbarkeit gering bis mittel.

Als relativ stabil kann die Segmentaufteilung durch psychographische Kriterien gelten. Werte, Meinungen und wiederkehrende Motive (z. B. Hunger zwischen 12 und 14 Uhr) geben stabile Komponenten an, auf die sich der Markt einstellen kann, z. B. mit einem Mittagstisch. Fazit:

\section{Zeitliche Stabilität hoch.}

Die Messung der entsprechenden Daten erfordert einen sehr hohen finanziellen Aufwand, vor allem da die Erhebungen stets aktualisiert werden müssen, um auf Veränderungen reagieren zu können. Produktspezifische Anpassungen anhand der psychographischen Kriterien ermöglichen jedoch eine gezielte Segmentierung, die eine hohe Wirtschaftlichkeit erzielt. Fazit: Wirtschaftlichkeit mittel bis hoch. 
Konstrukte wie Lebensstil, Motiv oder Einstellung weisen keine hohe Trennschärfe auf. Alle drei Komponenten bezeichnen eine Gesinnung bzw. innere Haltung und können nicht eindeutig voneinander differenziert werden. Fazit: Trennschärfe gering.

\subsubsection{Beobachtbares Kundenverhalten}

Die Kriterien des beobachtbaren Konsumentenverhaltens verweisen nicht auf die Faktoren, die eine Kaufentscheidung beeinflussen, sondern auf die Ergebnisse von Kaufentscheidungsprozessen. Diese lassen sich in vier Bereiche unterteilen: „Produktwahl“, „Einkaufsstättenwahl“, „Mediennutzung“ und „Preisverhalten“ (Meffert 2000, S. 208).

In Bezug auf die Produktwahl muss zunächst die Gesamtheit der Konsumenten in Käufer und Nichtkäufer unterschieden werden. Des Weiteren lassen sich darunter in Abhängigkeit von der Markentreue unterschiedliche Charaktere finden. Konsumenten, die eine hohe Markentreue zeigen, werden in der Regel auch in Zukunft die gleichen Marken kaufen, wechselbereite Käufer hingegen lassen sich von innovativen Ideen oder Sonderangeboten beeinflussen. Ein weiterer Aspekt ist die Verbrauchsintensivität, die von intensivem bis gar keinem Verbrauch reichen kann. Besonders bedeutend sind die Produkte mit hohem Verbrauch, da davon die Kaufhäufigkeit abzulesen ist. Ebenso spielt das Kaufvolumen eine Rolle, d. h., ob Kunden Produkte auf Vorrat oder nur nach konkretem Bedarf einkaufen. Je nach Verhaltensmuster sprechen diese Kunden Mengenrabatte oder kurzzeitige Sonderangebote an (Kotler et al. 2007a, S. 379 f.). Zudem kann die Verbundnachfrage ein Bestandteil der Produktwahl sein. Diese bezeichnet Güter, die durch den Kauf eines Produktes erforderlich sind, wie z. B. Computerzubehör beim Kauf eines PCs. So liegt es nahe, die Produkte passend zu präsentieren, damit der Kunde alle erforderlichen Produkte in Reichweite hat.

Das beobachtbare Kundenverhalten zeigt sich auch bei der Einkaufsstättenwahl, die Auskunft darüber gibt, wo Konsumenten bestimmte Produkte erwerben, in welcher Intensität sie dies tun und welche Struktur der Käuferschaft in den einzelnen Geschäften vorherherrscht. Dieses beobachtbare Konsumentenverhalten hängt in erster Linie davon ab, ob ein bestimmtes Produkt in einem Geschäft angeboten wird oder nicht. Die Produktverfügbarkeit wird primär durch den Handel bzw. durch den gewählten Distributionskanal bestimmt. Die Entscheidung über die Art des Kooperationspartners im Handel entscheidet somit über den Ort der Einkaufsstätte und ist somit für Unternehmen von sehr großer strategischer Bedeutung. 
Ein weiterer Aspekt, der das beobachtbare Konsumentenverhalten charakterisiert, ist das Kommunikationsverhalten, da das Nutzungsverhalten vergleichbar ist mit der Medienwahl. Hierbei ist zu beobachten, dass die Nutzerschaft von Fernsehen und Internet erheblich zunimmt, während der Konsum von Zeitschriften und Hörfunk abnimmt (Köcher 2006, S. $15 \mathrm{ff})$.

Schließlich lässt sich das Konsumentenverhalten am Preis einzelner Güter aufzeigen. Dies zeigt zum einen die Tatsache, dass $65 \%$ der deutschen Bevölkerung auf Sonderangebote achten, und zum anderen die unterschiedlichen Preisklassen, in denen ein Produkt angeboten wird. Somit kann jeder Konsument gemäß seinem Einkommen das entsprechende Produkt erwerben. Allerdings ist hier wiederum ein hybrides Kaufverhalten zu bedenken, bei dem nicht vom Einkommen auf die Preissensibilität geschlossen werden kann (Barth 2003, S. 60).

Abschließend können die Kriterien des beobachtbaren Konsumentenverhaltens wie folgt bewertet werden (Karasu 2010, S. 38; Rennhak/Kesting 2008, S. 13; Diez 2006, S. 31 ff.; Freter 2008, S. 162 f.):

Die Relevanz des Kriteriums wird als mittel eingestuft. Zum einen kann die Verhaltensprägung eines Konsumenten von Bedeutung sein. So wird ein Konsument sein Merkmal der Markentreue für längere Zeit beibehalten und die Produkte kaufen, die es in der Einkaufstätte gibt. Zum anderen kann von der Mediennutzung nur eine sehr geringe Kaufverhaltensrelevanz abgeleitet werden. Fazit: Kaufverhaltensrelevanz mittel.

Das beobachtbare Konsumentenverhalten zeigt verschiedene Möglichkeiten auf, die genannten Merkmale als Marketingstrategie zu benutzen. So kann der Markt in der Form darauf reagieren, dass er z. B. für Vielkäufer besondere Mengenrabatte anbietet oder für preissensible Menschen spezielle Sonderangebote. Fazit: Ansprechbarkeit hoch.

Die Zugänglichkeit dieses Kriteriums ist nur gering, da aufgrund der Produktmenge schnell die Grenzen der Befragung erreicht sind. Eine Möglichkeit, zu ausgewählten Produkten Zugang zu bekommen, ist der Zugriff auf bestehende Datenbanken, die den bisherigen Kauf ähnlicher Produkte dokumentieren. Fazit: Zugänglichkeit gering.

Das Konsumentenverhalten lässt sich verhältnismäßig leicht messen, da hierzu bereits statistische Materialien vorliegen, z. B. von Werbungtreibenden, die die Erhebung in Auftrag geben. Fazit: Messbarkeit hoch. 
Die zeitliche Stabilität lässt sich schwer generalisieren. So gibt es zeitlich stabile Käufer oder Nichtkäufer, aber ebenso hybride Käufer, deren Kaufverhalten nicht vorhersagbar ist. Fazit: zeitliche Stabilität mittel.

Die Wirtschaftlichkeit ist besonders im Bereich der kommunikativen Verhaltensmerkmale gegeben. Bei den anderen Kriterien lohnt sich eine Erhebung nur in Zusammenhang mit anderen Kriterien, z. B. im sozioökonomischen Bereich. Fazit: Wirtschaftlichkeit hoch.

Die Trennschärfe ist bei diesem Kriterium nicht gegeben, da die Einteilung der Konsumenten produkt- und situationsabhängig ist. Vor allem das hybride Kaufverhalten verhindert eine klare Trennung. Fazit: Trennschärfe gering.

\subsubsection{Physiologische Kriterien}

Ein weiteres Kriterium zur Marktsegmentierung sind die physiologischen Merkmale, zu denen zum einen die körperliche Beschaffenheit gehört, zum anderen die physiologischen Defekte.

Um die körperliche Beschaffenheit als Segmentierungsmerkmal näher zu beschreiben, werden die vier Kriterien Geschlecht, Körpergröße, Haut- und Haartyp sowie Händigkeit berücksichtigt, an denen sich entsprechende Produktpaletten orientieren, so z. B. im Textil- oder Kosmetiksektor. In der Textilindustrie ist eine Einteilung in Käufer und Nichtkäufer nach den Kriterien Geschlecht und Körpergröße auf den ersten Blick ersichtlich, da bestimmte Produkte nur an ein bestimmtes Geschlecht mit einer bestimmten Körpergröße gerichtet sind. Ebenso ist die Einteilung der potenziellen Käufer in verschiedene Typen in der Kosmetikindustrie von großer Bedeutung. So lassen sich verschiedene Haut- oder Haartypen unterscheiden, mit deren Hilfe unterschiedliche Kunden angesprochen werden sollen. Mit der Identifizierung als Hauttyp 1 benötigt der Käufer z. B. Produkte, die für empfindliche Haut geeignet sind, um sich optimal zu pflegen. Daneben ist die Händigkeit ein Kriterium zur Marktsegmentierung, da sich manche Produkte auf Links- bzw. Rechtshänder spezialisieren. Dies zeigt sich z. B. an manchen Instrumenten oder Werkzeugen, die speziell auf die Händigkeit des Käufers eingestellt sind (Freter 2008, S. 166 ff.).

$\mathrm{Zu}$ den physiologischen Kriterien gehören neben der körperlichen Beschaffenheit auch die physiologischen Defekte. Darunter versteht man verschiedene Defekte wie Allergien, Behinderungen, Unverträglichkeiten oder eine Beeinträchtigung der Sehleistung. So wird durch eine Unverträglichkeit wie Diabetes, Laktoseintoleranz und Glutenunverträglichkeit ein neuer 
Markt geöffnet für diejenigen, die diesen Defekt aufweisen und auf spezielle Produkte angewiesen sind. Dieses Situation machen sich viele Supermärkte zu Nutze und nehmen in ihr Sortiment entsprechende Produkte auf, die früher nur im Reformhaus zu finden waren, wie z. B. zuckerfreie Schokolade.

Abschließend können die physiologischen Kriterien wie folgt bewertet werden (Freter 2008, S. $171 \mathrm{ff}$.$) :$

Bei der Betrachtung der physiologischen Kriterien lässt sich feststellen, dass die Kaufrelevanz sehr hoch ist. Käufer mit einer bestimmten körperlichen Beschaffenheit oder einem körperlichen Defekt sind gezwungen, bestimmte Produkte zu kaufen, um speziell auf ihre Bedürfnisse einzugehen. Fazit: hohe Kaufverhaltensrelevanz.

Der Aussagewert für den Einsatz dieses Kriteriums kann ebenfalls als hoch eingestuft werden, da sich die angebotenen Produkte an den Bedürfnissen des Kunden orientieren müssen. Besonders im Bereich der körperlichen Defekte lässt sich ein bisher noch nicht ausgeschöpftes Marktpotenzial feststellen. Fazit: hohe Ansprechbarkeit.

Die Erreichbarkeit ist als mittel einzustufen. Zum einen ist die Segmentierung nach dem Geschlecht zwar noch gegeben, aber bei den andern Kriterien zur körperlichen Beschaffenheit fast unmöglich. Zum anderen erfolgt ein großer Teil der Kommunikation zu körperlichen Defekten übers Internet bzw. über bestimmte Foren, auf denen Produkte empfohlen oder diskutiert werden. Damit ist die Erreichbarkeit vor allem für jüngere Menschen gegeben. Fazit: mittlere Zugänglichkeit.

Die Messbarkeit der physiologischen Kriterien ist als hoch anzusehen. Die Merkmale der körperlichen Beschaffenheit sind schnell ersichtlich. Schwieriger könnte es sich mit den physiologischen Defekten verhalten, da diese zunächst (ärztlich) festgestellt werden müssen. Fazit: mittlere bis hohe Messbarkeit.

Die zeitliche Stabilität ist vor allem für die Kriterien Geschlecht, Händigkeit und körperliche Defekte gegeben. Zeitliche Schwankungen unterworfen sind dagegen Kriterien wie Haut- und Haartyp oder Körpergröße. Fazit: mittlere bis hohe Zeitstabilität.

Die physiologischen Kriterien weisen eine sehr hohe Wirtschaftlichkeit auf. Vor allem bei Menschen mit körperlichen Defekten wie z. B. Diabetes besteht eine sehr hohe Bereitschaft, zu bestimmten Produktkategorien zu greifen. Fazit: hohe Wirtschaftlichkeit 
Die Trennschärfe lässt sich als mittel charakterisieren. Zum einen erlaubt der medizinische Fortschritt den Betroffenen immer mehr, sich „,normal“ zu ernähren, ohne auf ihre Unverträglichkeit zu achten. Zum anderen kommt es vor, dass sich Käufer ihres Segments noch nicht bewusst sind. So könnte z. B. die Kosmetikindustrie die männliche Zielgruppe mehr ansprechen und dadurch das Bewusstsein für eine bestimme Zugehörigkeit schärfen. Fazit: mittlere

\section{Trennschärfe.}

\subsubsection{Zeitkriterien}

Ein weiterer Ansatzpunkt, um das Kaufverhalten zu untersuchen, ist der Faktor Zeit. Hier geht man nicht davon aus, dass Konsumenten immer die gleichen Produkte kaufen, sondern die Kaufentscheidung maßgeblich von temporären und situativen Faktoren abhängt. So lassen sich die Zeitkriterien in die zwei Bereiche Situation und Termin einteilen, die im Folgenden näher betrachtet werden.

Beschreibt man die Situation als Faktor der Kaufentscheidung, müssen mehrere Aspekte berücksichtigt werden. Darunter fällt z. B. der zeitliche Einfluss einer Kaufentscheidung. Zeit als stets knappes Gut unserer schnelllebigen Zeit - bringt viele Käufer dazu, beispielsweise dasjenige Produkt zu wählen, das die größte Zeitersparnis mit sich bringt. Besonders in der Gastronomie wird dieser Umstand genutzt, wo viele Fast-Food-Ketten oder Zustellservices großen Erfolg haben. Auf der anderen Seite entstehen immer mehr Wellness-Oasen, die sich bewusst von der Hektik des Alltags abgrenzen (Solomon et. al. 2006, S. 310 ff.).

Ein weiterer Faktor ist die Verfassung des Käufers, die sich sowohl auf die Stimmung und das körperliche Empfinden als auch auf die zum gegebenen Zeitpunkt bestehenden Finanzmittel bezieht. Ein gut gelaunter Kunde ist schneller bereit, mehr Geld für einen eventuellen Spontankauf auszugeben. Firmen versuchen entsprechend, durch eine gute Atmosphäre wie z. B. freundliches Personal oder eine gute Einteilung der Geschäftsräume die Kaufentscheidung positiv zu beeinflussen. Ebenso macht sich die Wirtschaft grundlegende Emotionen wie Liebe und Trauer zu Nutze und bietet beispielsweise Plüschtiere an, die es einem Käufer ermöglichen sollen, seinem Partner sein Liebe zu zeigen, oder Trauerseminar, um zu lernen, mit einem Todesfall umzugehen (Silberer 1999, S. 133 f.).

$\mathrm{Zu}$ den situativen Faktoren gehört auch die physische Umgebung. Darunter fallen die Ladendekoration, Licht-, Geräusch- und Klimaverhältnisse sowie die Regalsortierung der Einkaufsstätte. Die Bedeutung zeigt sich in folgendem Beispiel: Ist die Musik in einem Geschäft zu 
laut oder die Klimaanlage zu stark eingestellt, so verärgert dieser Umstand potenzielle Käufer, da sie sich in dieser Situation unwohl und gestört fühlen, und dadurch kann einen Kauf verhindern. Andere Firmen reagieren auf die physische Umgebung, die nicht beeinflussbar ist, indem z. B. ein Betreiber eines Vergnügungsparks bei Regenwetter Regencapes anbietet, um den wettertrotzenden Kunden gerecht zu werden.

Ebenso spielt die soziale Umgebung eine wichtige Rolle, die sich auf die Anwesenheit von Begleitpersonen des Käufers bezieht. Entscheidend könnte z. B. die geschätzte Meinung eines Freundes oder eines fachkundigen Verkäufers sein. Damit gerät das Beratungsgespräch in den Fokus, das durch geschultes Personal geführt werden sollte. Eine besondere Konstellation bilden Mutter und Kind beim Einkauf, da die Mutter durch die Aufsichtspflicht des Kindes vom Einkauf abgelenkt ist. Immer mehr Firmen bieten deshalb besondere Leistungen an, wie z. B. extra breite Mutter-Kind-Parkplätze oder Kinderbetreuung für den Aufenthalt in der Einkaufsstätte, um die Bedingungen zu verbessern (Kuß/Tomczak 2004, S. 225).

Ein weiterer zu berücksichtigender Faktor ist der Anlass bzw. der Zweck des Kaufs. Je dringlicher ein Einkauf ist, desto eingeschränkter ist die Informations- und Auswahlentscheidung und umso höher die Bereitschaft des Kunden zu Kompromissen, z. B. in Bezug auf den Preis. So ist ein Kunde am 24. Dezember wahrscheinlich bereit, einen höheren Preis für ein Produkt zu bezahlen, da der Anlass eine hohe Dringlichkeit hat: Die Geschäfte schließen mittags, und abends müssen die Geschenke unter dem Baum liegen.

Neben den eben beschriebenen situativen Faktoren zählen Termine zu den Zeitkriterien. Sie beziehen sich im Gegensatz zu den situativen Faktoren auf regelmäßig stattfindende, längerfristige Begebenheiten. Als größte Komponente in diesem Zusammenhang können die Jahreszeiten genannt werden. Durch die unterschiedlichen Phasen des Jahres - Frühling, Sommer, Herbst und Winter - ändern sich die Bedürfnisse der Menschen. So steigt z. B. im Sommer die Nachfrage nach Grillkohle und Sonnencreme, während im Winter Heizlüfter und Tee nachgefragt werden. Für Unternehmen ist es demnach sehr rentabel, sich auf diese Bedürfnisse einzustellen, wie sich in der zunehmenden Spezifizierung der Lebensmittel zeigt: Im Sommer stehen in den Supermarktregalen Joghurt mit Limetten-, im Winter mit BratapfelGeschmack.

Aber auch der Tagesverlauf bestimmt das Konsumentenverhalten, denn abhängig von der Tageszeit werden unterschiedliche Lebensmittel konsumiert. So variiert z. B. der Kaffeekonsum über den Tag verteilt. Die Wirtschaft reagiert in der Weise darauf, dass es beispielsweise spe- 
zielle Frühaufsteher- oder Langschläfer-Angebote gibt oder die Kassen bzw. das Beratungspersonalteam unterschiedlich besetzt sind. Auch der Wochen- bzw. Monatsverlauf beeinflusst das Konsumentenverhalten. So verfügen die meisten Menschen an Sonntagen über besonders viel Zeit, so dass viele Sonntagszeitungen eine Möglichkeit bieten, die Zeit zu füllen. Zudem ist davon auszugehen, dass zu Beginn eines Monats die Kaufbereitschaft höher ist, da das Gehalt auf dem Konto gutgeschrieben wurde. Zuletzt gehören auch die jährlich wiederkehrenden Termine zu den Zeitkriterien. Die Konsumenten passen ihren Einkauf z. B. den christlichen Feiertagen wie Weihnachten und Ostern oder anderen Feiertagen wie Silvester an. Somit können sich die Anbieter schon frühzeitig auf die sichere Nachfrage der entsprechenden Produkte einstellen (Freter 2008, S. 183 f.).

Die Zeitkriterien können vor diesem Hintergrund wie folgt bewertet werden (Roslow et al. 2000, S. 1167 ff.; Freter 2008, S. 181 ff.):

Die Kaufverhaltensrelevanz ist als hoch anzusehen. Zum einen beeinflussen die situativen Faktoren zu einem großen Teil die Kaufentscheidung, zum anderen sind die festen Termine ebenfalls als sicherer Faktor zur Kaufentscheidung zu betrachten. Fazit: hohe Kaufverhaltensrelevanz.

Das Marketing kann aufgrund der Zeitkriterien ganz bewusst sein Angebot vermarkten und somit auf unterschiedliche Bedürfnisse der Menschen eingehen, indem z. B. Regencapes oder ein Langschläfer-Frühstück angeboten werden. Fazit: hohe Ansprechbarkeit.

Die Zugänglichkeit der Zeitkriterien ist nur bedingt gegeben, da immer noch der Konsument den Kauf bestimmt und nicht die passende Situation. Somit müssen die Zeitkriterien in Zusammenhang mit psychographischen und sozio-demographischen Kriterien gesehen werden. Fazit: mittlere Zugänglichkeit.

Die Messbarkeit der Zeitkriterien ist bei Betrachtung der Termine gegeben, da sich die Termine sehr genau vorhersagen lassen. In Bezug auf die situativen Faktoren müssen entsprechende Fokusgruppengespräche bzw. Marktforschungen betrieben werden. Fazit: mittlere bis hohe Messbarkeit.

Ebenso besteht eine hohe zeitliche Stabilität bei den fest vorgegebenen jährlichen Terminen. Auf der anderen Seite gilt dies für die situativen Faktoren nicht, da diese einmalig und vorübergehend sind. Fazit: mittlere Zeitstabilität. 
Der Nutzen, der sich aus den Erkenntnissen der Zeitkriterien ergibt, überwiegt in jedem Fall den Aufwand, um eine Situationsanalyse durchzuführen, so dass man von einer hohen Wirtschaftlichkeit sprechen kann. Fazit: hohe Wirtschaftlichkeit.

Bei Betrachtung der einzelnen Segmente in Bezug auf die Trennschärfe kann zusammenfassend festhalten werden, dass diese als hoch einzuschätzen ist: Entweder ein Konsument ist gut gelaunt oder er ist es nicht; entweder er hat Zeitdruck oder er hat keinen. Fazit: hohe Trennschärfe.

\subsubsection{Nutzen}

Als letztes Kriterium zur Marktsegmentierung wird hier der Nutzen angesprochen, der sich nach der Bedürfnisbefriedigung der Kunden richtet. An dieser Stelle sollen die Produkte danach segmentiert werden, wie sehr die Produkte in der Lage sind, den Kunden einen quantifizierbaren Nutzen zu stiften. Dabei ist zu beachten, dass das gleiche Produkt bei unterschiedlichen Personen einen unterschiedlichen Grad von Nutzen stiften kann (Weinstein 2004, S. 99). So kann z. B. ein Haarshampoo den Konsumenten einen unterschiedlichen Nutzen bieten. Es dient dazu, die Haare zu säubern, gut zu riechen oder glänzende Haare zu haben. Diese einzelnen Aspekte können für unterschiedliche Konsumenten von unterschiedlicher Wichtigkeit sein und damit einen unterschiedlichen Nutzen haben. Der Nutzenfaktor stellt somit ein sinnvolles Kriterium zur Marktsegmentierung dar.

Um den unterschiedlichen Nutzen eines Produktes festzustellen, können sogenannte ConjointAnalysen dienen. Hierbei wird der Proband gebeten, die Wichtigkeit einzelner Produktkomponenten bzw. -kriterien zur Gesamtnutzenbeurteilung von Produkten bzw. Leistungen zu benennen. Anschließend werden durch Clusteranalysen aus der Gesamtheit an Probanden Gruppen bestimmt, die sich in ihrem Wichtigkeitsempfinden einzelner Komponenten oder Kriterien ähneln, somit ähnliches Konsumentenverhalten aufweisen und dementsprechend zu einem Marktsegment zusammengefasst werden können.

Abschließend kann der Nutzen als Marktsegmentierungskriterium wie folgt beurteilt werden (Scheer 2008, S. 62; Diez 2006, S. 37 f.; Freter 2008, S. 188):

Die Kaufverhaltensrelevanz ist hoch, da im Zuge der Conjoint-Analyse die Probanden geistig eine Kauf- oder Nichtkaufsituation erleben. Fazit: hohe Kaufverhaltensrelevanz.

Infolge der Bewertung von Produktkomponenten bzw. Teilleistungen durch die Probanden weist die Segmentierung anhand des Nutzens eine sehr hohe Ansprechbarkeit auf. So lassen 
sich bestimmte Instrumente direkt anhand der Bewertung einsetzen, wie beispielsweise die Produktpolitik oder die Preispolitik. Fazit: hohe Ansprechbarkeit.

Die Zugänglichkeit zu den einzelnen Segmenten stellt sich als schwierig dar. Die unterschiedlichen individuellen Nutzen für ein ganz bestimmtes Produkt erlauben im Generellen keinen Rückschluss auf ein bestimmtes Verhalten beim Medienkonsum, was eine segmentspezifische Ansprache schwierig macht. Fazit: geringe Zugänglichkeit.

Zur Erfassung bzw. Bestimmung von Nutzensegmenten stehen neben den hier erwähnten Methoden noch diverse andere Verfahren zur Verfügung. Die Messbarkeit von Nutzensegmenten ist also gegeben. Fazit: hohe Messbarkeit.

Die Lebensdauer von Nutzensegmenten kann als hoch eingestuft werden. Dies liegt darin begründet, dass die Probanden im Vorfeld der eigentlichen Segmentierung Kaufoptionen gründlich gegeneinander abwägen und sich aus rationalen Gründen für die Bevorzugung eines bestimmten Produktes bzw. einer bestimmten Leistung entscheiden. Somit spielen sowohl spontane Impulskäufe als auch routiniert ablaufende Käufe nahezu keinerlei Rolle bei der Entscheidungsfindung der Probanden, womit letztendlich das gebildete Nutzensegment weniger „spontanen“ Launen der Probanden unterliegt. Fazit: hohe zeitliche Stabilität.

Der zeitliche Aufwand zur Durchführung einer Nutzenwertmessung ist relativ hoch, was auch die Wirtschaftlichkeit negativ beeinflusst. Im Gegenzug dazu ermöglicht die Nutzenwertanalyse aber wie gesehen eine hohe Zugänglichkeit, was im Folgenden den Markterfolg eines Produktes erhöht. Die Wirtschaftlichkeit der Nutzenwertmessung hängt davon ab, in welchem Verhältnis die beiden Seiten zueinander stehen. Fazit: mittlere Wirtschaftlichkeit.

Durch die exakte Quantifizierbarkeit der Ergebnisse der Nutzenmessung ist die Trennschärfe bei Nutzensegmenten gegeben. Fazit: hohe Trennschärfe.

\subsubsection{Identifizierung der relevanten Segmente}

Nachdem im vorangegangenen Abschnitt näher auf die einzelnen Kriterien einer Marktsegmentierung eingegangen worden ist, geht es im Folgenden um die Bewertung der jeweils gefundenen Segmente. Dem ökonomischen Grundgedanken folgend gilt es, diejenigen Segmente zu identifizieren, die dem Unternehmen die meisten Chancen zur Gewinnmaximierung bieten und dementsprechend bearbeitet werden sollten. Da die Festlegung auf Zielsegmente eine äußerst weitreichende unternehmerische Entscheidung ist, empfiehlt es sich, die Komplexität der Entscheidungsfindung zu reduzieren, indem man den Gesamtprozess der Entscheidungs- 
findung in mehrere, jeweils separat kontrollierbare Einzelschritte unterteilt (Keegan et al. 2002, S. 284 f.), wie es Abbildung 33 zeigt.

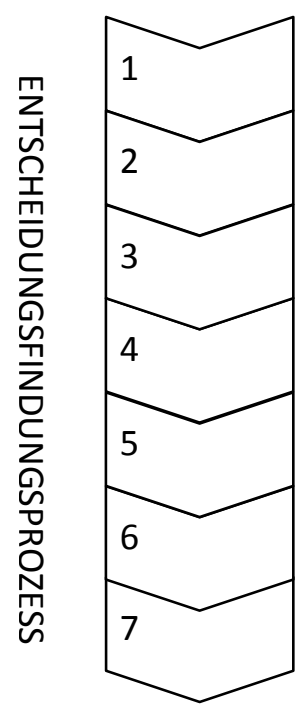

1 Bestimmung der Bewertungskriterien

2 Operationalisierung der Bewertungskriterien

3 Ggf. Gewichtung der Bewertungskriterien

4 Grobselektion

5 Feinselektion

6 Bestimmung der Anzahl zu bearbeitender Segmente

7 Kontrolle

\section{Abbildung 33: Bewertungsprozess}

Quelle: Eigene Darstellung in Anlehnung an Freter 2008, S. 219

Der Prozess zur Festlegung der ökonomisch sinnvollsten Segmente wird demnach in sieben Einzelschritte unterteilt, die letztendlich das Risiko einer Fehlentscheidung verringern sollen.

Um dem Ziel der Segmentauswahl näher zu kommen, werden in Schritt 1 Segmentbewertungskriterien festgelegt. Die letztendliche Auswahl dieser Kriterien unterliegt voll und ganz dem unternehmerischen Kontext und muss daher vom Anwender selbst getroffen werden. Es lassen sich dabei jedoch unternehmensabhängige und unternehmensunabhängige Bewertungskriterien unterscheiden. $\mathrm{Zu}$ den unternehmensunabhängigen Bewertungskriterien zählen beispielsweise:

- Segmentgröße

- Wachstumspotenzial

- Wettbewerbsintensität

- Erreichbarkeit

- Risiko 
$\mathrm{Zu}$ den dominierenden unternehmensabhängigen Bewertungskriterien zählen unter anderem:

- Benötigte Ressourcen zur Segmentbearbeitung

- Entstehende Kosten

- Erwartete Rentabilität

- Bestehende Markentreue der Kunden

- Marktreaktion auf eigenen Markteintritt

- Deckung mit den allgemeinen Unternehmenszielen und der Unternehmenskultur

Die Aufzählung erhebt keinen Anspruch auf Vollständigkeit, vielmehr variiert die Zusammenstellung der Kriterien von Unternehmen zu Unternehmen (Kotler et al. 2007b, S. 387; Keegan et al. 2002, S. 244 ff.).

In Schritt 2 werden die ausgewählten Kriterien „operationalisiert“. Das bedeutet, dass sie auf ihre Bearbeitungsmöglichkeit geprüft und gegebenenfalls messbar gemacht werden. Eine eventuelle Gewichtung einzelner Kriterien erfolgt in Schritt 3. Schritt 4 und Schritt 5 stehen für die eigentliche Auswahl der Segmente, wobei diese wiederum in eine Grob- und eine Feinselektion unterschieden werden kann und später detaillierter vorgestellt wird. Hierbei ist die zuvor getroffene Einteilung in unternehmensunabhängige und unternehmensabhängige Kriterien hilfreich. Die unternehmensunabhängigen Bewertungskriterien eignen sich sehr gut, um in der Grobselektion die gegebene Anzahl von Segmenten zu reduzieren. Die unternehmensabhängigen Kriterien dienen in einer weiteren Bewertungsrunde der Feinselektion. Diese erfordert den Einsatz spezieller Methoden, die im Anschluss näher erläutert werden. Schritt 6 greift das Ergebnis der Feinselektion auf und legt fest, wie viele und welche Segmente bearbeitet werden sollen. In Schritt 7 erfolgt eine abschließende Kontrolle (Keegan et al. 2002, S. 285; Freter 2008, S. 222).

Die Schritte vier und fünf des mehrstufigen Entscheidungsprozesses stehen für die eigentliche Auswahl der Segmente. Es kommt zunächst zu einer Grob- und anschließend zu einer Feinselektion. Die Methoden zur groben Segmentauswahl werden auch qualitative Methoden zur Segmentauswahl und -bewertung genannt, die Methoden zur Feinauswahl auch quantitative Methoden zur Segmentauswahl und -bewertung.

\subsubsection{Qualitative Methoden}

Qualitative Methoden eignen sich gut für die Grobselektion, insofern diese einer Eliminierung von Segmenten gleichkommt, die schon „im Voraus“ nicht für eine tiefer gehende Untersu- 
chung in Betracht kommen, beispielsweise aufgrund einer Nichtkompatibilität der Segmente mit den höheren Unternehmenszielen (Meffert 2000, S. 214). Nach Freter gehören zu den qualitativen Methoden (Freter 2008, S. 222):

- Checklistenverfahren

- Profilmethode

- Scoring-Modell

- Chancen-Risiken-Matrix

- Portfolioanalyse

Das Checklistenverfahren stellt das einfachste unter den fünf erwähnten Verfahren dar. Es prüft die einzelnen Segmente lediglich im Hinblick auf das Vorhandensein zuvor bestimmter Parameter. Sind diese zu einem bestimmten Grad nicht erfüllt, wird das entsprechende Segment eliminiert und nicht weiter untersucht. Beispielhaft sollen hier Segment A und Segment B dienen, wobei Segment B eine Segmentgröße von 1.000 potenziellen Nachfragern hat und Segment A nur 10 potenzielle Nachfrager. Wäre „Segmentgröße > 500 potenzielle Nachfrager" der einzige zu prüfende Punkt, müsste Segment A eliminiert werden. Eine Ausweitung der Checkliste auf alle gefundenen Segmente und alle relevanten Prüfkriterien entspricht jedoch eher der Realität als dieses recht einfache Beispiel, so dass letztendlich auch Segmente manche Voraussetzungen erfüllen können und manche nicht. Dementsprechend liegt es letztendlich wieder am Anwender, im Einzelfall zu entscheiden, ob ein Segment eliminiert wird. Alternativ kann der Anwender bereits im Vorfeld einen finalen Check definieren, beispielsweise „80\% aller Prüfkriterien erfüllt?“. Checklisten eignen sich für eine einfache und daher auch schnelle und kostengünstige Entscheidung über die Notwendigkeit einer weiterführenden Untersuchung eines Segments. Nachteilig sind Checklisten insofern, als einerseits die einzelnen Beurteilungskriterien nicht gewichtet sind und andererseits das Verfahren nur als Orientierungshilfe gewertet werden kann, da die Ergebnisse keine monetären Eigenschaften besitzen (Freter 2008, S. 224).

Die Profilmethode basiert grundsätzlich auf dem Verfahren der Checklisten, bietet aber den zusätzlichen Vorteil einer abgestuften Beurteilung, die es erlaubt, das Vorhandensein oder Nicht-Vorhandensein eines Prüfkriteriums nicht nur mit ja oder nein zu beantworten, sondern mit zusätzlichen Informationen zu versehen, wie z. B. „sehr stark vorhanden“ oder „stark vorhanden“. Zur besseren Operationalisierung werden die Prüfergebnisse meist mit Punkten versehen - je positiver ein Prüfkriterium bestätigt werden kann, desto mehr Punkte erhält es und umgekehrt. Setzt man die Ergebnisse der Profilmethode in eine Graphik um und verbindet die 
einzelnen segmentspezifischen Antworten, so ergibt sich pro Segment eine Profillinie, anhand derer sich die Stärken und Schwächen der einzelnen Segmente schnell ablesen und miteinander vergleichen lassen. Dadurch ist eine einfache, aber sehr wirkungsvolle analytische Grundlage zur Segmentauswahl geschaffen (Hungenberg 2004, S. 147 ff.). Nachteilig an der Profilmethode ist jedoch, dass die Vergabe der Punkte pro Prüfkriterium sehr subjektiv ist. Des Weiteren wird auch hier nicht zwischen der Relevanz der einzelnen Prüfkriterien unterschieden und die Segmente werden auch weiterhin nicht monetär bewertet (Freter 2008, S. 224).

Das Scoring-Modell setzt auf der Profilmethode auf und unterscheidet sich von diesem dadurch, wie das Scoring-Modell Gewichtungsfaktoren für die einzelnen Prüfkriterien zulässt, wobei die Summe der einzelnen Gewichtungsfaktoren 1 ergeben sollte. In der Handhabung werden zunächst wie bei der Profilmethode die einzelnen Antworten zu den jeweiligen Prüfkriterien gewichtet, beispielsweise mit 5 Punkten für „sehr stark vorhanden.“ Im Anschluss werden die vergebenen Punkte mit dem Gewichtungsfaktor des entsprechenden Prüfkriteriums multipliziert. Die Produkte der einzelnen Prüfkriterien werden letztendlich pro Segment addiert. Je höher die Summe der Punkte, desto attraktiver erscheint das Segment. Wie das Checklistenverfahren und die Profil-Methode ist das Scoring-Modell einfach zu handhaben und ermöglicht dennoch eine strukturierte Entscheidungsgrundlage. Nachteilig ist dabei allerdings wiederum die Subjektivität der Gewichtungsfaktoren und der Punktevergabe. Zusätzlich besteht die Möglichkeit, dass sich die Aussagefähigkeit der Summen verwässert, wenn es bei der Addition zu einer Kompensation hoher und niedriger Kriterienerfüllungsgrade kommt (Berndt 2010, S. 119).

Die Chancen-Risiken-Matrix trägt alle gefundenen Segmente in eine zweidimensionale Matrix ein, an deren Achsen einerseits Chancen und andererseits Risiken abgetragen sind. Als Chance könnten beispielsweise Marktwachstumspotenziale gelten, wenn abzusehen ist, dass ein Wettbewerber vom Markt verschwindet. Risiken könnten beispielsweise sein, dass ein Segment nur sehr finanzschwache potenzielle Kunden im Fokus hat. Abbildung 34 zeigt die Verteilung der drei beispielhaften Segmente S1, S2 und S3. Die Attraktivität eines Segments hängt bei der Chancen-Risiken-Matrix davon ab, wie weit das Segment vom Ursprung (Risiko hoch, Chancen gering) entfernt ist. Im abgebildeten Beispiel erscheint S2 als das attraktivste Segment, da die Chancen weitaus höher sind als bei S1 und das Risiko dabei nur minimal erhöht ist. Neben der leichten Verständlichkeit besteht ein weiterer Vorteil der ChancenRisiken-Matrix darin, dass auch die Risiken einer Segmentbearbeitung mit einfließen. Nachteilig ist hingegen, dass die Chancen-Risiken-Matrix an sich keinen Einblick mehr in die ein- 
zelnen Beurteilungen gewährt, anhand derer die Segmente bewertet worden sind. Des Weiteren ist eine Klassifizierung sämtlicher Beurteilungskriterien in Chance oder Risiko eventuell nicht immer möglich (Freter 2008, S. 228).

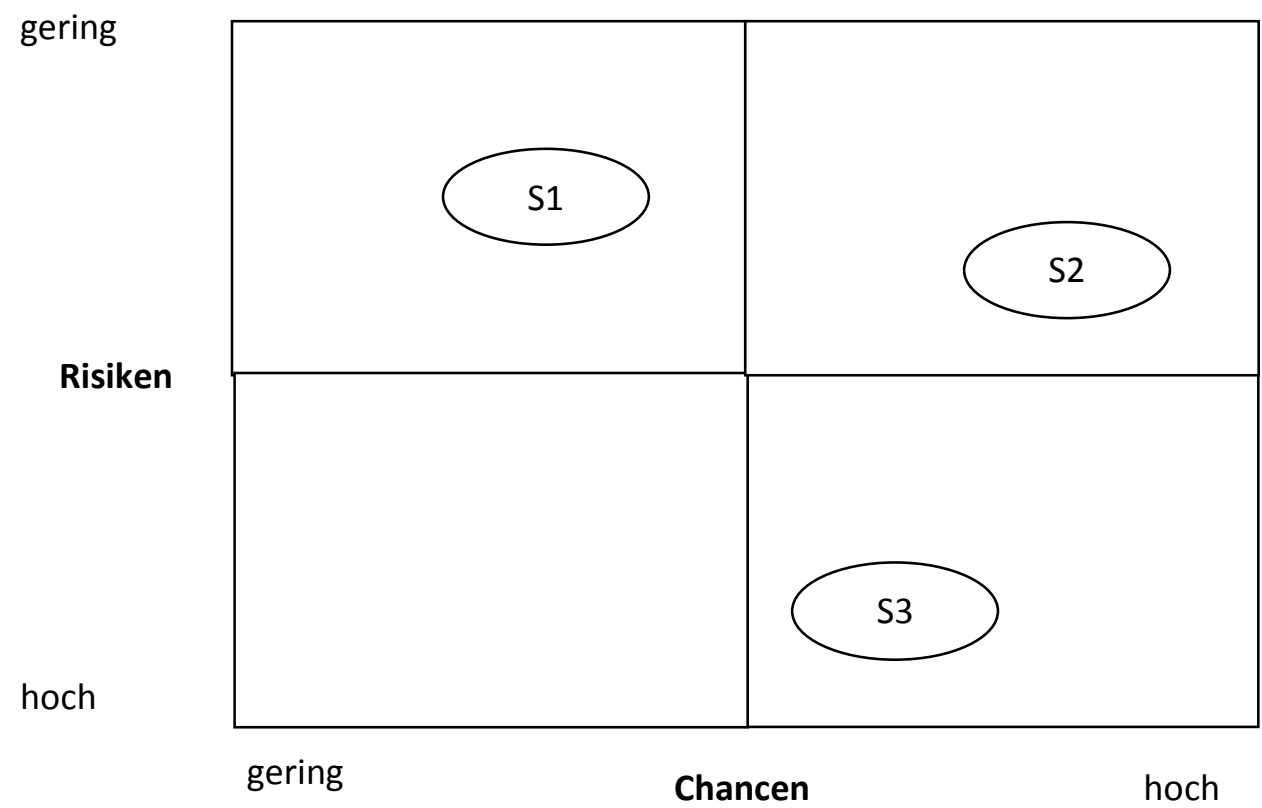

Abbildung 34: Chancen-Risiken-Mix

Quelle: Eigene Darstellung nach Freter 2008, S. 227

Die Portfolioanalyse ähnelt der Chancen-Risiken-Matrix in der Art, dass auch hier wiederum Segmente in einer Matrix abgetragen werden. Ursprünglich wurde die Portfolioanalyse für die strategische Bewertung von Geschäftsfeldern entwickelt, wobei sie sich auch auf die Bewertung von Segmenten übertragen lässt: In einem Koordinatensystem werden das vom Markt abhängige und somit nicht beeinflussbare Segmentwachstum an der Ordinate und der unternehmensbezogene, relative Marktanteil des Unternehmens an der Abszisse abgetragen, wobei beide Dimensionen mit „hoch“ und „niedrig“ bewertet sind. Es entstehen somit wiederum vier Quadranten im Koordinatensystem, die je nach Lage eine entsprechende Marktsituation darstellen. Abbildung 35 zeigt die vier Quadranten. „Fragezeichen“ steht dabei für eine Situation, in der das Unternehmen sich entscheiden muss, ob das Segment verstärkt bearbeitet werden soll oder ob es sich aus diesem Segment zurückzieht. Diese Unklarheit liegt daran, dass das Wachstum des Segments zwar hoch, der relative Marktanteil in diesem Segment allerdings gering ist. Es hängt also von der strategischen Entscheidung des Unternehmens ab, 
ob der Marktanteil in diesem attraktiven Segment als ausbaufähig angesehen wird oder ob das Segment aufgegeben wird, weil die Marktanteile bereits an Wettbewerber verteilt sind. „Stars“ sind Segmente mit hohem Wachstum und einem relativ hohen Marktanteil des Unternehmens im Segment. Aufgrund dieser sehr guten Ausgangslage muss aus ökonomischer Sicht in diese Segmente investiert werden. „Milchkühe“ stehen für die Situation, dass ein Segment nur ein niedriges Wachstum aufweist, ein Unternehmen aber in ihm einen relativ hohen Marktanteil besitzt. Bei dieser Konstellation empfiehlt es sich, keine Investitionen mehr in diesem Segment zu tätigen, sondern vielmehr die erreichte Marktdominanz kostengünstig auslaufen zu lassen und so in der Endlebensphase des Segments hohe Deckungsbeiträge zu realisieren. „Arme Hunde“ sind die unattraktivsten Segmente, da sie ein geringes Segmentwachstum aufweisen und nur ein geringer relativer Marktanteil des Unternehmens bei ihnen zu verzeichnen ist. Aus diesen Segmenten sollte sich das Unternehmen zurückziehen. Die Namensgebung der einzelnen Quadranten liegt an deren direkter Übersetzung aus dem Englischen. Ursprünglich entwickelt wurde die Portfolioanalyse von der Beratungsgesellschaft Boston Consulting Group.

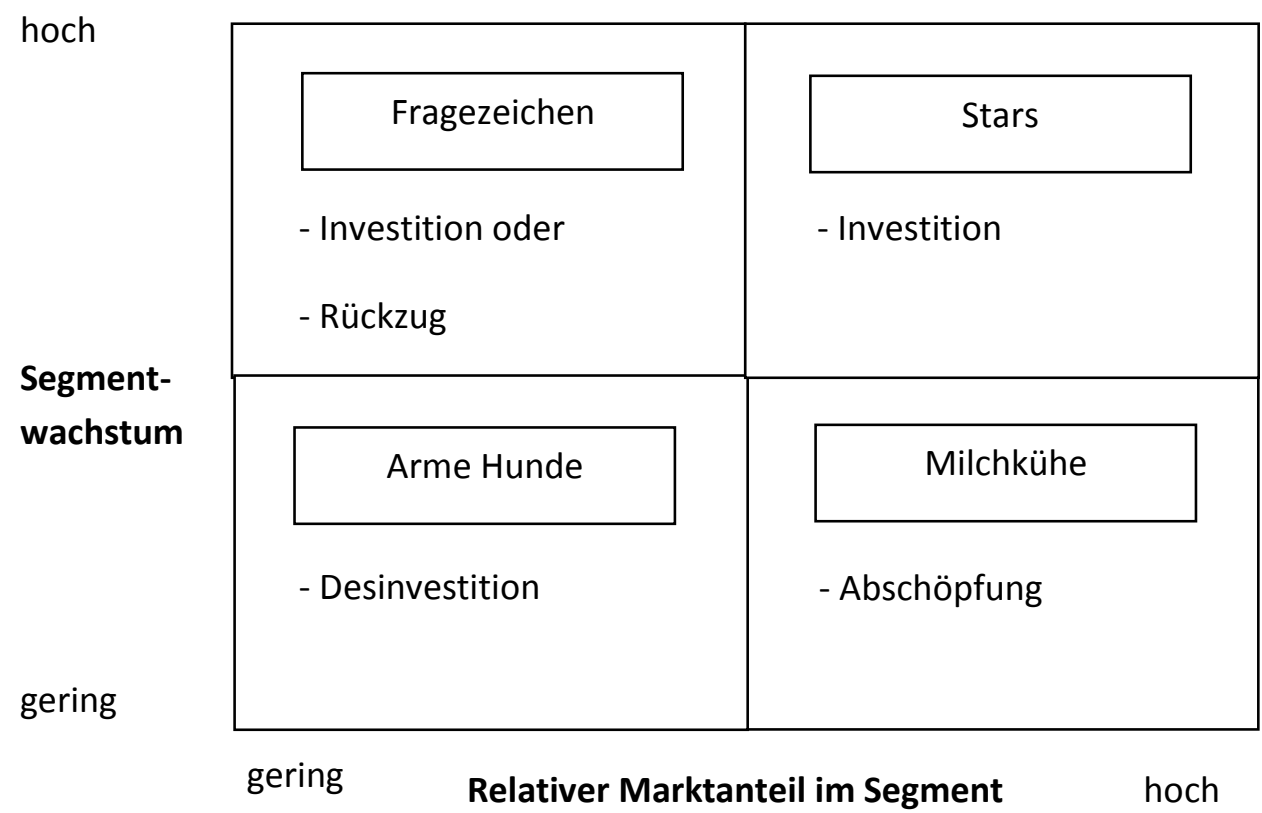

Abbildung 35: Portfolio-Analyse

Quelle: Freter 2008, S. 229 
Die Portfolioanalyse hilft nicht nur dabei, festzustellen, „wo“ sich ein Segment befindet, vielmehr gibt sie auch Vorschläge, ,wie“ diese Segmente zu bearbeiten sind. Dies ist möglich, weil die Attraktivität des Segments in Zusammenhang mit der unternehmensindividuellen Marktsituation betrachtet wird. Von Nachteil ist allerdings, dass der relative Marktanteil eines Unternehmens nur bekannt ist, wenn das Unternehmen sich bereits in diesem Segment engagiert. Für den Fall einer hypothetischen Überlegung, „ob“ sich das Unternehmen in diesem Segment überhaupt engagieren soll, müssen relative Marktanteile als potenzielle erreichbare Marktanteile geschätzt werden. Je nachdem, wo sich ein Segment nach Abschätzung der potenziell erreichbaren Marktanteile in der Matrix wiederfindet, sollte eine weitere eingehende Analyse erfolgen. „Arme Hunde“-Segmente sollte man dabei am wenigsten in Betracht ziehen, Segmente der anderen Quadranten kommen jedoch für feinere Analysen in Frage.

\subsubsection{Quantitative Methoden}

Die Feinselektion baut auf der Vorauswahl der Grobselektion auf. Die quantitativen Methoden der Feinselektion ermöglichen im Gegensatz zu den qualitativen Methoden der Grobselektion eine monetäre Bewertungsgrundlage zur Entscheidungsfindung. Aufgrund dieser viel genaueren Ergebnislage sind die quantitativen Methoden im Vergleich zu den qualitativen Methoden deutlich aufwendiger und werden deshalb nur bei Segmenten angewandt, die nicht schon durch das grobe Raster der Vorselektion durchfallen. Im Gegensatz zur Grobselektion werden bei den quantitativen Methoden der Feinselektion primär unternehmensabhängige Bewertungskriterien herangezogen. Dies hat allerdings zur Folge, dass bei der Überlegung, zukünftig eventuell in ein Segment „einzusteigen“, viele Parameter abgeschätzt werden müssen, wie beispielsweise die Frage nach den entstehenden Kosten, dem personellen Mehraufwand oder den zu erwartenden Erlösen. Soll ein Segment bewertet werden, das bereits bearbeitet wird, muss auf die unternehmensinternen Daten der Betriebsdatenerfassung zurückgegriffen werden. Nach Freter gehören zu den qualitativen Methoden:

- Gewinnrechnung

- Amortisationsrechnung

- Kapitalwertmethode

Die Gewinnanalyse ist eine Analysemethode, bei der die Kosten und die Erlöse der Bearbeitung eines Segments miteinander verglichen werden. Die Gewinnrechnung kann im Vorfeld einer Segmentbearbeitung stattfinden oder zur Beurteilung von Segmenten, die bereits vom 
Unternehmen bearbeitet werden. Zur Beurteilung von Segmenten, die eventuell bearbeitet werden sollen, wird die Break-even-Analyse verwendet. Diese setzt den erwarteten Umsatz mit den erwarteten Kosten ins Verhältnis und ermittelt so diejenige Menge, die mindestens verkauft werden muss, um positive Gewinne zu erwirtschaften. Falls das Unternehmen angesichts der zu Grunde liegenden Bedingungen erwartet, die kritische Menge zu erreichen, sollte das Segment bearbeitet werden. Ist hingegen der Absatz der kritischen Menge nicht zu erwarten, sollte das Unternehmen das Segment meiden. Die Break-even-Methode stellt ein sehr einfaches Verfahren dar, mit dem in monetären Einheiten gerechnet wird und mit dessen Hilfe sich anhand von erwarteten Ein- und Auszahlungsströmen eine Aussage treffen lässt, ob ein Segment bearbeitet werden soll oder nicht. Problematisch ist allerdings, dass bei der Breakeven-Analyse viele Parameter im Vorfeld abgeschätzt werden müssen, beispielsweise der erzielbare Marktpreis für das Produkt. Unterliegt das Produkt überdies dem typischen Verlauf des Produktlebenszyklus, verändern sich viele Parameter im Laufe der Produktlebenszeit. Die Break-even-Methode berïcksichtigt diese Dynamik nicht - ihr liegen statische Durchschnittswerte zu Grunde, was die Abbildung der realen, dynamischen Welt nur bedingt widerzuspiegeln vermag.

Zur Beurteilung von Segmenten, die bereits bearbeitet werden, nutzt die Gewinnrechnung die so genannte Absatzsegmentrechnung. Mit ihrer Hilfe lassen sich Gewinne und Verluste nicht nur ,pro Stück“ darstellen, sondern nach bestimmten Bezugsgrößen differenzieren, die individuell gewählt werden können. Typische Bezugsgrößen können dabei „pro Kunde“, „,pro Auftrag“ oder „pro Verkaufsgebiet“" sein. Durch diese Aufschlüsselung lassen sich die Bezugsgrößen in eine Reihenfolge bringen, die aussagt, welche Kunden, Aufträge oder Verkaufsgebiete verstärkt weiterbearbeitet werden sollen oder welche in Zukunft nicht mehr bearbeitet werden sollen. Durch diese selektive Absatzpolitik konzentriert sich das Unternehmen immer mehr auf die deckungsbeitragsstarken Bezugsgrößen, was letztendlich hilft, dem originären Unternehmensziel der Gewinnmaximierung sukzessive ein Stück näher zu kommen (Köhler 1993, S. 384). Die Absatzsegmentrechnung bezieht sich auf Geschehnisse der Vergangenheit, daher können die ihr zu Grunde liegenden Daten aus dem vorhandenen Datenmaterial des Unternehmens recht einfach übernommen werden. Des Weiteren erlaubt sie sehr konkrete Rückschlüsse auf den Erfolg oder Nichterfolg in bestimmten Segmenten. Als problematisch ist dabei allerdings zu erwähnen, dass das Verfahren für eine Prognose über Segmente nur bedingt geeignet ist, da sie zukünftige Entwicklungen wie beispielsweise Preisoder Absatzmengenänderungen nicht berücksichtigt. Werden in der Absatzsegmentrechnung 
geschätzte Werte für die Zukunft als Datengrundlage benutzt, lassen sich auch konkrete Aussagen über zukünftige Rentabilitäten von Segmenten machen - allerdings bei sehr hoher Abhängigkeit von der „Treffsicherheit“ der geschätzten zukünftigen Daten (Freter 2008, S. 236 ff.).

Anhand der Amortisationsrechnung lässt sich feststellen, wie lange ein Segment bearbeitet werden muss, um die zu Beginn entstehenden einmalig auftretenden Markteintrittskosten anhand zukünftiger Zahlungsüberschüsse wieder zu egalisieren. Beispielsweise möchte ein Blumenfachgeschäft seinen Kundenkreis erweitern und gezielt neue Käuferschichten ansprechen. Durch den erwarteten Mehrumsatz wäre aber auch ein neuer bzw. größerer Kühlraum nötig, der im Vorfeld angeschafft werden müsste. Liegt die „Lebensdauer“ des Segments über der Amortisationsdauer, sollte das Segment bearbeitet werden, da sich ab dem Ende der Amortisationszeit unter sonst gleich bleibenden Bedingungen Gewinne erzielen lassen.

Die Amortisationsrechnung erlaubt anhand periodengenauer (erwarteter) Zahlungsüberschüsse die Berücksichtigung von Preis- und Marktschwankungen, was dem Modell mehr Realitätsnähe verleiht. Des Weiteren können zukünftige Zahlungen auch anhand von Verzinsungen $\mathrm{zu}$ Barwerten umgerechnet werden (dynamische Amortisation). Gleichzeitig erweist sich dies auch wieder als Schwachpunkt, da exakte Zukunftswerte stets mit hoher Unsicherheit verbunden sind. Des Weiteren trifft die Amortisationsrechnung keinerlei Aussage darüber, wie hoch die zu erwartenden Gewinne nach Ablauf der Amortisationsdauer sind (Freter 2008, S. 239).

Die Kapitalwertmethode bezieht im Gegensatz zur Amortisationsmethode nicht nur Zahlungen bis zum Amortisationszeitpunkt in die Analyse mit ein, sondern sämtliche in Zukunft stattfindenden Zahlungen. Der Kapitalwert errechnet sich dabei aus den Anschaffungskosten zum jetzigen Zeitpunkt im Vergleich zu sämtlichen zu erwartenden Zahlungen, ebenfalls abgezinst auf den jetzigen Zeitpunkt. Dabei stellt dieser „Kalkulationszinsfuß“, zu dem die zukünftigen Zahlungen abgezinst werden, wiederum ein zusätzliches Problem dar, da auch er neben den zukünftigen Zahlungen ein weiterer Parameter ist, den es zu schätzen gilt und der in der Rechnung über mehrere Perioden hinweg als konstant auftritt. Vorteilhaft ist bei der Kapitalwertmethode allerdings, dass sämtliche zu erwartenden Zahlungen berücksichtigt und auf einen aktuellen Barwert diskontiert werden, was einen direkten Vergleich zwischen Zahlungseingängen und Zahlungsausgängen ermöglicht. Je weiter der Vorgriffshorizont der Kapitalwertmethode angesetzt wird, desto vorsichtiger müssen deren Ergebnisse interpretiert werden. Eine Möglichkeit, diese Unsicherheiten in die Berechnung einfließen zu lassen, stellen 
„Risikozuschläge“ bei Auszahlungen bzw. „Risikoabschläge“ bei Einzahlungen dar (Wöhe 2005, S. 620; Freter 2008, S. 240).

\subsubsection{Strategien zur Segmentbearbeitung}

Nachdem bisher sowohl beschrieben wurde, wie man Segmente findet, als auch, wie die attraktivsten aller gefundenen Segmente identifiziert werden können, widmet sich dieser Abschnitt der Frage, wie die attraktivsten Segmente bearbeitet werden sollten. Dazu wird auf die von Abell weiterentwickelte Ansoff'sche Produkt-Markt-Matrix zurückgegriffen, welche die verschiedenen möglichen Grundformen der segmentspezifischen Marktbearbeitung darstellt (Bea 2005, S. 142; Koch 2006, S. 77). Die Matrix setzt dabei die verschiedenen Produkte des Unternehmens und die verschiedenen Kunden (hier: Segmente) miteinander in Beziehung. Abbildung 35 zeigt eine Matrix, bei der von drei verschiedenen Produkten und drei verschiedenen Segmenten ausgegangen wurde.

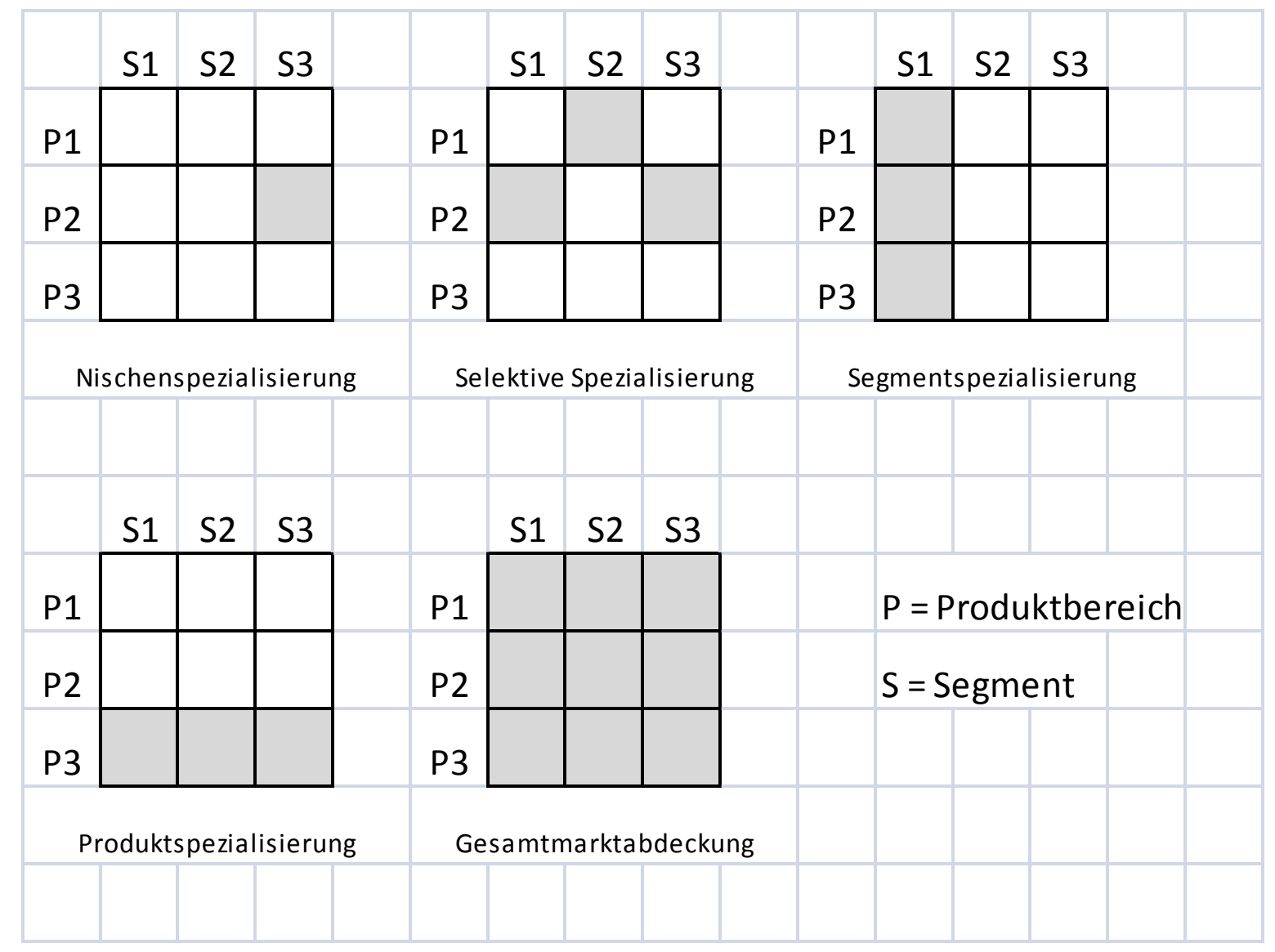

Abbildung 36: Produkt-Markt-Matrix

Quelle: Eigene Darstellung, in Anlehnung an Abell 1980, S. 192 ff.; Freter 2008, S. 250 
Ja nachdem, wie der Produktbereich mit dem Bereich der Segmente kombiniert wird, sind fünf verschiedene Produkt-Markt-Konstellationen denkbar (Kotler et al. 2007a, S. 88):

- Ein Produkt in einem Segment

- Mehrere Produkte in mehreren Segmenten

- Mehrere Produkte in einem Segment

- Ein Produkt in mehreren Segmenten

- Alle Produkte in allen Segmenten $\rightarrow$ Nischenspezialisierung

$\rightarrow$ Selektive Spezialisierung

$\rightarrow$ Segmentspezialisierung

$\rightarrow$ Produktspezialisierung

$\rightarrow$ Gesamtmarktabdeckung

Bei der Nischenspezialisierung wird nur ein einziges Segment des gesamten Marktes mit einem Produkt angesprochen. Kotler spricht dabei auch vom sogenannten „konzentrierten Marketing“ (Kotler et al. 2011, S. 456 ff.). Dabei ist das Segment der ausschlaggebende Faktor, was bedeutet, dass das Produkt an das Segment angepasst wird und nicht umgekehrt. Je nachdem, wie „breit“ der Begriff Segment im jeweiligen Kontext definiert wurde, muss unter „Produkt““ auch ein Bündel angebotener ähnlicher Produkte verstanden werden (Koch 2006, S. 80). Entscheidend ist dabei allerdings, dass in diesem Fall alle Produkte auf das Segment zugeschnitten wurden (Freter 2008, S. 251).

Eine selektive Spezialisierung liegt vor, wenn sich ein Unternehmen mit mehreren Produkten in verschiedenen ausgewählten Segmenten engagiert. Nach Kotler spricht man unter diesen Umständen von einer „selektiven Multi-Segment-Abdeckung“ (Kotler et al. 2007a, S. 88). Die Auswahl der Segmente erfolgt losgelöst voneinander: Pro Segment gibt es ein angebotenes Produkt bzw. ein extra zusammengestelltes Bündel an Produkten. Durch die Bearbeitung mehrere Segmente verlagert sich das unternehmerische Risiko auf mehrere, voneinander unabhängige Teile des Gesamtmarktes. Allerdings steigt mit der Anzahl der bearbeiteten Segmente und der Anzahl der angebotenen Produkte auch die Komplexität der Unternehmensführung (Freter 2008, S. 251).

Eine Segmentspezialisierung liegt vor, wenn alle Produkte des Unternehmens an nur einem einzigen Segment ausgerichtet sind. Anhand der vielen gesammelten Erfahrungswerte innerhalb des Segments kann das Unternehmen sehr gut auf die segmentspezifischen Bedürfnisse eingehen und hat so Vorteile gegenüber Unternehmen, denen zu diesem Segment weniger bis gar keine Erfahrungswerte zur Verfügung stehen. Für das erfahrene Unternehmen bietet sich eine Ausdehnung der angebotenen Produkte innerhalb des bestehenden Segments an, da somit die Erfahrungsvorteile weiterhin genutzt und auch weiterentwickelt werden können. Eine ge- 
wisse Gefahr besteht indes darin, dass das Unternehmen von nur einem einzigen Segment abhängt (Freter 2008, S. 252).

Bietet ein Unternehmen nur ein Produkt bzw. nur ein entsprechendes Bündel an Produkten in allen Segmenten an, so spricht man von Produktspezialisierung. Für das Unternehmen ergeben sich dadurch Vorteile über die Spezialisierungseffekte. Diese können entlang der gesamten Wertschöpfungskette entstehen, von der Produktion bis zum Vertrieb. Nachteilig erweist sich die Produktspezialisierung dahingehend, dass das gesamte Unternehmen vom Erfolg nur eines einzigen Produktes bzw. Produktbündels abhängig ist (Freter 2008, S. 251 f.).

Bietet ein Unternehmen alle seine Produkte in sämtlichen Marktsegmenten an, spricht man von Gesamtmarktabdeckung. Durch Gesamtmarktabdeckung ergeben sich für das Unternehmen sowohl produktionsbedingte Spezialisierungseffekte als auch die Vorteile der Segmentspezialisierung. Gleichzeitig findet eine breite Risikodiversifikation statt. Problematisch muss bei der Gesamtmarktabdeckung die Komplexität der Unternehmensführung gesehen werden. Ein Unternehmen, das erfolgreich eine Gesamtmarktabdeckung betreiben möchte, muss in jedem Teilbereich mindestens so gut sein wie ein Unternehmen, das sich auf einen einzigen Teilbereich spezialisiert hat.

Für die letztendliche Anzahl der Segmente und die damit verbundene Frage, wie die Segmente angesprochen werden sollten, ergeben sich, basierend auf obigen Überlegungen, wiederum zwei Möglichkeiten:

- Die als lohnend eingestuften Segmente lassen sich separat voneinander bearbeiten.

- Segmente, die zuvor als nicht lohnend eingestuft wurden, sind durch das Heben von Erfahrungs- und/oder Spezialisierungseffekten im Verbund mit anderen Segmenten oder Produkten doch lohnend.

Für den ersten Fall stellt die optimale Anzahl der zu bearbeitenden Segmente diejenige Anzahl dar, bei welcher der Deckungsbeitrag maximiert wird (Meffert 2000, S. 218). Im zweiten Fall spielen Abhängigkeiten und Verbundeffekte eine Rolle. Durch sie entstehen Veränderungen in der monetären Bewertung einzelner Bereiche, die zuvor nur isoliert voneinander betrachtet wurden. Soweit es die Komplexität zulässt, sollten solche Verbundeffekte, die auch negative Auswirkungen haben können, in den Entscheidungsprozess der Segmentauswahl mit einfließen, da sie mitunter sehr große Auswirkungen haben können (Kotler et al. 2007a, S. 390). 


\subsection{Lebensstile als Grundlage der Marktsegmentierung}

Wie in Abbildung 32 gezeigt, kann eine Segmentierung des Gesamtmarktes anhand von vielen verschiedenen Kriterien erfolgen. Neben der methodischen Geeignetheit der dazu in Frage kommenden Segmentierungskriterien (7-Punkte-Schema) ist weiterhin zu klären, welche inhaltlichen Segmentierungskriterien überhaupt zum Einsatz kommen, um den Gesamtmarkt in Segmente zu unterteilen. Die Auswahl der passenden Kriterien stellt dabei einen wesentlichen Schritt der ganzen Segmentierung dar, da die Qualität der späteren Segmentierung im Wesentlichen davon abhängt, wie gut die ausgewählten Segmentierungskriterien zur Trennung der Grundgesamtheit geeignet sind.

Für den weiteren Verlauf dieser Arbeit wird eine „lebensstilbasierte Kundensegmentierung“ angestrebt. Aus dem Arbeitstitel lässt sich ableiten, dass eine Segmentierung der Kunden der BuP-Branche angestrebt wird, die auf Kriterien der Lebensstilforschung basiert. Die Vermischung der Lebensstilforschung mit der Theorie des Marketings soll helfen, die beschriebenen Probleme der BuP-Branche zu verkleinern. Der Ansatz dieser Arbeit ist somit sowohl problemorientiert als auch interdisziplinär. Die Lebensstilforschung ist ein Ansatz der Verhaltensforschung und somit zumindest in Teilen ein Ansatz der Konsumentenforschung und daher auch des verhaltensorientierten Marketings. Eine Integration der Lebensstilforschung in das Feld des Marketings ist aus diesem Grund nur folgerichtig. Ziel des interdisziplinären Einsatzes der Lebensstilforschung im Marketing muss dabei sein, mit Hilfe der Theorien, Methoden und Erkenntnissen der Lebensstilforschung ein besseres Verständnis von dem Konsumentenverhalten zu erlangen (Banning 1987, S. 61 ff.).

Wie in diesem Kapitel bereits aufgezeigt, existieren diverse Untersuchungen oder Modelle im Bereich der Segmentierung, die auf einer Segmentierung anhand von Lebensstilkriterien beruhen. Da die Autoren dieser Modelle allerdings (wenn überhaupt) nur ihre Ergebnisse frei veröffentlichen, ist es nicht bekannt, mit welchen lebensstiltypischen Segmentierungsvariablen die einzelnen Untersuchungen gearbeitet haben. Es ist somit bei einer anstehenden Marktsegmentierung anhand von Lebensstilen in den allermeisten Fällen nicht möglich, auf bereits bestehende Modelle inhaltlich zurückzugreifen. Dies macht das Erstellen eines eigenen lebensstilbasierten Ansatzes erforderlich. Das entsprechende Vorgehen wird im Folgenden aufgezeigt, wobei sich die Ausführungen am „Schema der Rekonstruktion von Lebensstilen“

nach Lüdtke orientieren (Lüdtke 1989, S. 42 ff.), die wiederum sehr stark auf der Forschung Bourdieus beruhen. Zum besseren Verständnis dieser Rekonstruktion sei bereits an dieser 
Stelle auf Abbildung 37 verwiesen, welche die folgenden Ausführungen graphisch zusammenfasst.

Um Lebensstile von Probanden bestimmen zu können, werden in einem ersten Schritt Kenntnisse über mögliche Segmentierungskriterien, die überhaupt in Frage kommen, benötigt. Aus der Definition von Lebensstilen und der bereits geleisteten theoretischen Vorarbeit lassen sich vier theoretische Dimensionen der Lebensorganisation ableiten, die im Folgenden kurz erläutert werden.

Die sozioökonomische Situation setzt sich zusammen aus dem ökonomischen und dem sozialen Kapital nach Bourdieu in Verbindung mit der Arbeitsorganisation, der Haushaltsstruktur und der Wohnumwelt. Die sozioökonomische Situation beinhaltet ein Bündel verschiedener objektiver Ressourcen und Zwänge des Handelns, aus dem sich für jedes Individuum eine bestimmte individuelle Status-Rollen-Situation ergibt. Die steuernden Prozesse sind Allokation, Statuserwerb, Lebenslauf.

Unter Kompetenz versteht man das kulturelle Kapital im Sinne Bourdieus, also Wissen, Fähigkeiten und soziale Kompetenzen, wie beispielsweise Kommunikationsfähigkeiten oder auch die Fähigkeiten zur Konfliktverarbeitung, Kommunikation und Gestaltung sozialer Rollen. Die steuernden Prozesse sind Sozialisation und Bildung.

Unter Performanz versteht man die Gesamtheit der relevanten Handlungs- und Interaktionsäußerungen mit all ihren Ausprägungen, Begleitumständen und Ergebnissen. Dazu zählen auch Bourdieus Praktiken und Werke. Die steuernden Prozesse sind Handeln und Interaktion.

Unter Motivation versteht man verallgemeinert den Sinn des Handelns. Er speist aus inneren Handlungsdispositionen. Diese stehen für kulturell geformte Bedürfnisse, Einstellungen und Ziele. Die steuernden Prozesse sind Sozialisation, Entwicklung, Erfahrung.

Da die vier Dimensionen allesamt Teile des Konstrukts „Lebensstil“ sind, lassen sie sich nicht scharf voneinander abgrenzen und bedingen sich zudem untereinander. In Abbildung 37 werden diese gegenseitigen Wechselwirkungen durch die Pfeile zwischen den Dimensionen symbolisiert. Man spricht dabei auch von einem sogenannten „Fließgleichgewicht“ zwischen den Dimensionen.

Im zweiten Schritt zur Segmentierung anhand von Lebensstilen bedarf es der Extraktion von Lebensstildeskriptoren aus den vier Dimensionen. Die Deskriptoren bestehen beispielsweise aus beobachtbaren Eigenschaften der Personen und dienen als operationalisierende Variablen der Segmentierung. Basierend auf den bereits beschriebenen Theorien zur Lebensstilfor- 
schung eignen sich als operationale Variablen beispielsweise individuelle Verhaltensmuster bei Ausgaben finanzieller Art oder bei der Zeitverwendung für Aktivitäten und Ausstattungen in den Bereichen Ernährung, Kultur, Freizeit, Wohnung, Selbstdarstellung und Repräsentation. Des Weiteren kommen beispielsweise auch Wertbindungen, Geschmack, Selbstbild, Ziele und Perspektiven als operationale Variablen in Frage.
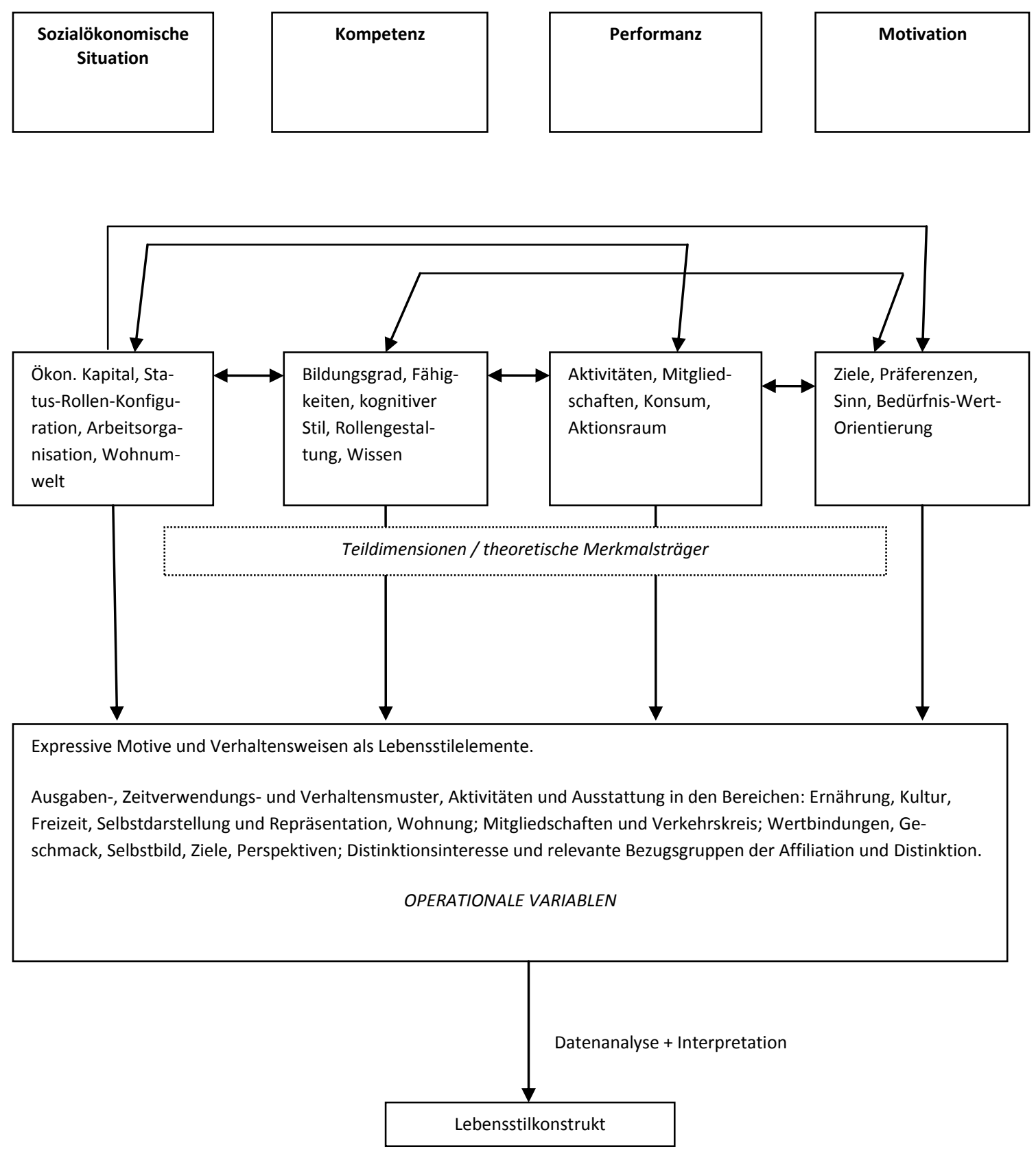

Abbildung 37: Rekonstruktion von Lebensstilen

Quelle: Eigene Darstellung in Anlehnung an Lüdtke 1989, S. 43 
In einem dritten Schritt werden die operationalen Variablen dahingehend geprüft, ob bzw. inwieweit sie auf ein Individuum zutreffen und inwiefern sie dabei typische empirische Muster bilden, die einem Individuum eindeutig zugeordnet werden können. Die einem Individuum zugeordneten Variablen ergeben in ihrer Summe ein Bündel von deskriptiven Eigenschaften, das in seiner Gesamtheit inhaltlich interpretiert werden muss. Das Ergebnis dieser Interpretation ergibt letztendlich das Konstrukt „Lebensstil“, welches zu dem jeweiligen Individuum passt und unter dem mehrere Individuen bei gleicher bis ähnlicher Lebensstilausprägung zusammengefasst werden können.

Basierend auf dem gezeigten Modell nach Lüdtke ist für den weiteren Verlauf der vorliegenden Arbeit eine Segmentierung nach Lebensstilen möglich. Dabei gilt es aber zu beachten, dass diese Segmentierung nicht produktspezifisch ist, was im eigentlichen Sinne der Marketingidee wäre. Um eine solche um produktspezifische Eigenschaften erweiterte Segmentierung durchzuführen, muss das bestehende Modell um produktspezifische Abfragen erweitert werden. Da für diese Arbeit eine solche Segmentierung spezifisch für Kunden des BuPMarktes angestrebt wird, eignet sich die gezeigte Methode in den Augen des Autors sehr gut, sofern es gelingt, sie im weiteren Verlauf um entsprechende produktspezifische Items zu erweitern. 


\section{Der Einsatz segmentspezifischer Marketinginstrumente}

Nachdem im vorangehenden Kapitel das Vorgehen einer typischen Marktsegmentierung aufgezeigt wurde, beschäftigt sich dieses Kapitel mit der Frage, wie die einzelnen Segmente zu bearbeiten sind. Da die einzelnen Segmente untereinander eine möglichst ausgeprägte Heterogenität aufweisen, sind für deren jeweilige Bearbeitung auch unterschiedliche Marketinginstrumente zu wählen. Im weiteren Verlauf dieses Kapitels werden daher die „4Ps“ der Marketingbereiche vorgestellt und gleichzeitig auf Gegebenheiten der Blumen- und Pflanzenbranche angepasst. Die 4Ps stehen dabei für die Marketingteilbereiche:

$\begin{array}{llll}- & \text { Product } & \rightarrow & \text { Leistungspolitik } \\ - & \text { Promotion } & \rightarrow & \text { Kommunikationspolitik } \\ - & \text { Place } \rightarrow & \text { Vertriebspolitik } \\ - & \text { Price } \rightarrow & \text { Preispolitik }\end{array}$

Da in dieser Arbeit der Markt für Blumen und Pflanzen im Fokus steht, wird eine Erweiterung des klassischen „4Ps“-Ansatzes vorgenommen. Im Endverkauf von Blumen und Pflanzen werden neben dem reinen Kaufgeschäft eines Konsumgutes parallel auch viele Service- und Dienstleistungen angeboten. Entsprechend folgt diese Arbeit der Erweiterung des MarketingMix um folgende Bereiche:

\begin{tabular}{lll} 
Personnel $\rightarrow$ & \multicolumn{2}{c}{ Personalpolitik } \\
Physical Facilities & $\rightarrow$ & Ausstattungspolitik \\
Process Management & $\rightarrow$ & Prozesspolitik.
\end{tabular}

Eine exakte Zuordnung des Bereichs „Blumen- und Pflanzen-Einzelhandel“ in den Bereich des Konsumgütermarketings oder in den Bereich des reinen Dienstleistungsmarketings ist in den Augen des Autors nicht möglich. Vielmehr stellt der Einzelhandel für BuP eine Mischform aus beiden Bereichen dar. Der hier verwendete erweiterte Ansatz entspricht daher den „7Ps“ des Dienstleistungsmarketings nach Meffert. Dabei spielen sowohl die vier klassischen Bereiche des Konsumgütermarketings eine Rolle als auch deren Erweiterung auf Dienstleistungen. Letztere ist erforderlich, da sich bei Dienstleistungsunternehmen die Anforderungen an das Marketing teilweise stark von den Marketinganforderungen an Sachgüterunternehmen unterscheiden (Meffert/Bruhn 2006, S. 387 ff.).

Auf der Suche nach dem Einsatz der richtigen Marketinginstrumente werden im Folgenden die jeweiligen Instrumente der sieben einzelnen Dienstleistungsmarketingbereiche vorgestellt 
und auf ihre Anwendbarkeit innerhalb der BuP-Branche bewertet. Dabei spielt auch der mögliche Grad der Fokussierung der einzelnen Instrumente in der BuP-Branche eine wichtige Rolle. Die gebildeten Segmente sollen möglichst gezielt angesprochen werden - Fehlstreuungen sind teuer und daher möglichst zu vermeiden. Wichtige Voraussetzung für den wirkungsvollen, segmentspezifischen Einsatz ist daher, dass die Zielsegmente gut erreichbar sind, sowohl in kommunikativer als auch in distributiver Hinsicht. Dabei gilt es jedoch, die Erreichbarkeit der Segmente nicht nur separat, sondern vor allem auch unter wirtschaftlichen Gesichtspunkten zu betrachten (Freter 2008, S. 293). Als angestrebtes Optimum soll daher derjenige Mix an Instrumenten gelten, der ein zuvor definiertes strategisches Unternehmensziel bestmöglich erreicht (Weis 2009, S. 89). Des Weiteren sei darauf verwiesen, dass eine jeweils losgelöste Betrachtung der einzelnen Ps nicht möglich ist. Jeder Bereich des Marketing-Mix steht in Wechselbeziehungen mit allen anderen. Dennoch werden im Folgenden die wichtigsten Eigenschaften der einzelnen Teilbereiche aus Übersichtsgründen separat vorgestellt.

\subsection{Product}

Die Produktpolitik ist der wichtigste Teilbereich des Marketing-Mix. Dies liegt einerseits daran, dass das Produkt an sich stets der eigentliche Grund ist, warum eine Verkäufer-KäuferBeziehung zu Stande kommt. Andererseits strahlen die in der Produktpolitik festgelegten Zieleigenschaften des Produkts auf alle anderen Marketingbereiche aus.

„Produktpolitik beschäftigt sich mit der Suche, Entwicklung und Gestaltung der von einem Unternehmen auf dem Markt anzubietenden Produkte bzw. Leistungen“ (Weis 2009, S. 240). Nach Weis umfasst die „Produktpolitik im engeren Sinne“ dabei:

- Qualität

- Allgemeiner Nutzen

- Grundnutzen

- Zusatznutzen

- Verpackung

- Produktimage

- Design

- Prestige

- Markenpolitik 
Im „weiteren Sinne“ zählt für Weis auch die „Programm- und Sortimentspolitik“ zum Bereich der Produktpolitik. Sie umfasst:

- Programmbreite

- Programmtiefe

- Programgestaltung

- Sortimentsbreite

- Sortimentstiefe

- Sortimentsgestaltung

- Produktelimination

- Diversifikation

Die „richtige“ Produktpolitik ist diejenige, die es schafft, dem Kunden den größten Gesamtnutzen zu stiften. Dieser setzt sich zusammen aus Grundnutzen und Zusatznutzen. Daher erfolgt die Festlegung des ,richtigen“ Produktes auch als zweistufiger Prozess, der sich aus der Kombination aus Grund- und Zusatznutzen zusammensetzt (Meffert/Bruhn 2006, S. 392). Abbildung 38 zeigt diese Zusammensetzung auf und verdeutlicht, wie sehr der Gesamtnutzen beim BuP-Kauf von Faktoren abhängt, die weit über das reine, physische Produkt hinausreichen.

Die Kernleistung eines Einzelhandelsgeschäfts für BuP besteht darin, dem Kunden durch den Verkauf von Blumen oder Pflanzen einen Grundnutzen zu stiften. Um sich dabei von der Konkurrenz abzugrenzen, ist es Aufgabe eines jeden Geschäfts, sein Angebot möglichst einzigartig darzustellen, um so bereits hier eine Unique Selling Proposition aufzubauen (vgl. hierzu Weis 2009, S. 471). Dabei gilt es allerdings zu beachten, dass gerade in einem Massenmarkt wie in der BuP-Branche ein Alleinstellungsmerkmal allein durch das Angebot von Zierpflanzen nahezu nicht erreicht werden kann. Für eine eindeutige Profilierung nur anhand des Grundnutzens ist das Produkt „Zierpflanze“ zu weit verbreitet bzw. bieten sich dem Konsumenten zu viele alternative Einkaufsstätten an. Aufgrund der vom Konsumenten wahrgenommenen Homogenität des Angebots bleibt den Einzelhändlern unter diesen Bedingungen letztendlich lediglich der Preis, um sich von den Wettbewerbern abzugrenzen. Um sich dem Kunden als einzigartig zu präsentieren und um der abfallenden Preisspirale zu entkommen, müssen die Einzelhändler daher eine Differenzierung außerhalb des Produktpreises anstreben (Meffert/Bruhn 2006, S. 393; Weis 2009, S. 243). 


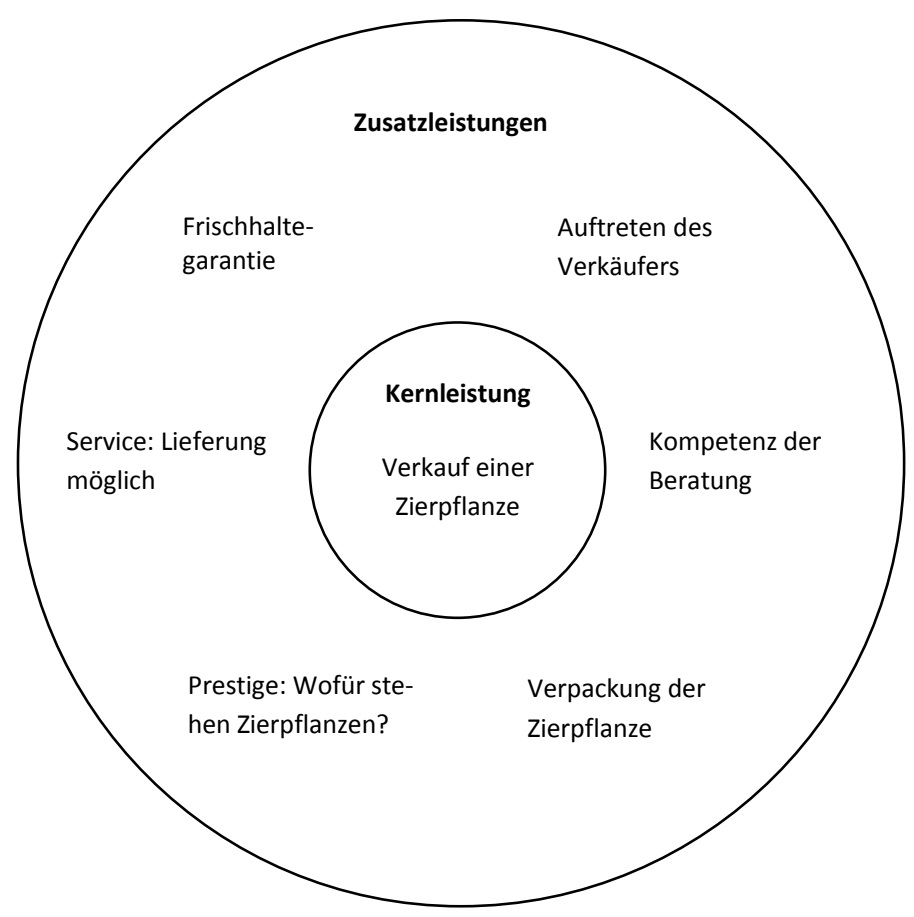

Abbildung 38 Gesamtnutzen von BuP

Quelle: Eigene Darstellung in Anlehnung an Meffert/Bruhn 2006, S. 392

Die Zusatzleistungen eines Produktes generieren den Zusatznutzen des Konsumenten. Dabei wird die Kernleistung um weitere Leistungen ergänzt, was das Kernprodukt in der Wahrnehmung des Konsumenten verändert und es dem Unternehmen ermöglicht, eine Stellung zu beziehen, die aus Kundensicht einzigartig ist. Ziel des Unternehmens sollte es dabei sein, mit seinen Zusatzleistungen dem Kunden ein abgerundetes Bündel an verschiedenen Leistungen in nur einem Produkt zu bieten (Bruhn 2004, S. 125 f.). Beispielhaft für die BuP-Branche sind hier Lieferservice, kompetente Beratung, Kundenfreundlichkeit, Herausstellung der symbolischen Bedeutung der Zierpflanze, Dekoration, Zubehör wie Grußkarten oder Übertöpfe, Frischhaltegarantie, Anpflanzservice für Balkonkästen, Überwinterungsservice für Großpflanzen, Pflanzendoktor, Einpackservice und vieles mehr. Betrachtet man die zahlreichen Möglichkeiten, wie der eigentliche Kauf einer Zierpflanze mit Dienstleistungen aufgewertet werden kann, zeigt sich, auf welch vielfältige Weise ein einfaches Standardverbrauchsgut zu etwas Individuellem aufgewertet werden kann.

Die Produkt- bzw. Leistungspolitik muss sich an den Kundenwünschen orientieren bzw. das angebotene Produkt muss in seiner Art einen möglichst großen Kundennutzen generieren. 
Dabei gilt es für das Unternehmen allerdings zusätzlich zu berücksichtigen, dass hier weitere Einflüsse eine Rolle spielen. Diese sind primär das bestehende Angebot der Wettbewerber, deren erwartete Reaktion auf das geänderte Marktangebot, der Erfolg der bisherigen eigenen Leistungen und die bisherige Zusammenstellung des eigenen Leistungsprogramms. Daher ist die Produkt- und Leistungspolitik nicht nur allein dem Kundennutzen unterzuordnen, vielmehr muss sie auch mit den strategischen Unternehmenszielen und der angestrebten Unternehmenskultur kompatibel sein. Hebel zur Anpassung der Produkt- und Leistungspolitik sind die Qualität oder der Preis der Leistung (Meffert/Bruhn 2006, S. 394).

Nach Weis zählen die oben „im weiteren Sinne“ genannten Punkte der Programm- und Sortimentspolitik zur übergeordneten Produktpolitik. Freter zufolge lassen sich diese Punkte zu zwei zentralen Aspekten bündeln: Produktdifferenzierung und Produktmodularisierung (Freter 2008, S. 302 ff.). Unter Produktdifferenzierung versteht man, wie eben an Beispielen der BuP-Branche gezeigt worden ist, den Versuch, das eigene Produkt von den Produkten der Wettbewerber abzugrenzen. Stehen in einem Unternehmen bereits mehrere Produkte zum Verkauf, beziehen sich diese Bemühungen auf das gesamte Produkt- bzw. Leistungsprogramm, welches das Unternehmen anbietet. Die Produktdifferenzierung an sich geschieht entweder durch die Innovation eines völlig neuen Produktes, durch Variation bzw. Modifikation der bestehenden objektiven Produkteigenschaften wie Größe, Farbe oder Form oder anhand einer Differenzierung durch zusätzliche produktbegleitende Services, die den Kundennutzen steigern. Man spricht dabei auch von sogenannten „Value-Added-Services“ (Meffert/ Bruhn 2006, S. 397; Haller 2007, S. 367 f.; Freter 2008, S. 302). Als Beispiele der Produktdifferenzierung im BuP-Einzelhandel können ein neu entwickelter „Wochenendstrauß“ (Innovation), das Verschönern von Blattgrün durch Besprühen mit „Blattglanz“ (Variation) oder das neue Angebot eines Pflanzen-Bringdienstes (Differenzierung) genannt werden.

Segmentspezifische Anpassungen des Produktes sind das Ziel einer jeden vorgelagerten Marktsegmentierung. Dies kann allerdings zu Komplexitätsproblemen führen, falls das Unternehmen zu viele spezielle Produkte oder Produktvariationen im Sinne der Produktdifferenzierung führt. Diese Probleme entstehen nicht nur auf Produktionsseite, sondern ziehen sich durch das gesamte Unternehmen hindurch. Die herkömmliche industrielle Fertigung nach den Gesetzen der Massenproduktion ist für die Variation des Kernproduktes nicht geeignet, während die individuelle Auftragsfertigung das genaue Gegenteil darstellt (Günther 2005, S. 11; Piller 2006, S. 176). Eine Möglichkeit, diesem Problem zu begegnen, ist die sogenannte Produktmodularisierung, ist doch eine Folge, die der modulare Aufbau des Produktprogramms 
ermöglicht, die kundenindividuelle Massenproduktion, auch „Mass Customization“ genannt. Mass Customization stellt eine Mischform aus Massenproduktion und individueller Auftragsfertigung dar und ermöglicht es, ,,jedem Kunden individualisierte Produkte und Dienstleistungen zu bieten“ (Weis 2009, S. 245) - jedoch zu Kosten, die mit den Kosten der Massenfertigung vergleichbar sind. Der Prozess des Customizings entsteht dadurch, dass sich der Kunde „sein“ Produkt aus den vielen Kombinationsmöglichkeiten der in Massenproduktion hergestellten Güter bzw. Module selbst modular zusammenstellt. Gehandhabt wird diese Art der Aufnahme der Kundenwünsche in den direkten Produktionsprozess primär durch webbasierte Kataloge, aus denen sich der Kunde nach dem Baukastenprinzip die einzelnen Module zusammenstellt, um so letztendlich seinen individuell zusammengestellten Produktionsauftrag an das Unternehmen zu übermitteln. Typisches Beispiel für ein Unternehmen, das Mass Customization betreibt, ist das Unternehmen DELL, das seine PCs ausschließlich in kundenindividueller Massenproduktion herstellt.

Neben der kundenindividuellen Massenproduktion erlaubt die Produktmodularisierung auch die sogenannte „Plattformstrategie“. Auch sie verbindet die Gesetze der Massenproduktion mit denen der Auftragsfertigung, allerdings in abgeschwächter Form. Die Plattformstrategie basiert auf der Idee, dass für mehrere Produktvarianten die gleiche Plattform als „Fundament“ benutzt wird. Auf die standardisierte Plattform werden dann die verschiedenen Module aufgesetzt, um so äußerlich unterschiedliche Produkte zu erhalten. Die Plattformstrategie bietet sich für Unternehmen an, die gleichzeitig mehrere Segmente mit segmentspezifischen Produkten bearbeiten. Durch die segmentübergreifende Standardisierung der Plattform ergeben sich, wiederum anhand der Gesetze der Massenproduktion, Kostenvorteile im Vergleich zu gänzlich modular aufgebauten Produkten. Die Strategie der Nutzung von Plattformen wird sehr stark in der Automobilindustrie angewandt. Verschiedene Modelle mit völlig unterschiedlichen technischen Parametern und Erscheinungsbildern basieren auf der gleichen Plattform bzw. dem gleichen Chassis, wie beispielsweise die Modelle „Golf IV“, „New Beatle“ und „Bora“ aus dem Hause Volkswagen (Wannenwetsch/Nicolai 2004, S. 121 ff.; Kotler et al. 2007a, S. 390).

Die Möglichkeit einer Produktmodularisierung innerhalb der BuP-Branche ist auf den ersten Blick nicht gleich erkennbar. Dennoch sind Ausprägungen vorhanden, wie beispielsweise vorgebundene Basis-Blumensträuße, die durch Personal anhand der geäußerten Kundenwünsche individuell ergänzt werden (Plattformstrategie), oder das vom Kunden selbst zusammen- 
gestellte Pflanzenarrangement, das aus Pflanze, Übertopf, Dekoration, Grußkarte und Verpackung besteht (Mass Customization).

\subsection{Price}

„Price“ bzw. die Preispolitik ist der zweite der vier klassischen Bereiche des Marketing-Mix. In der Literatur wird der Begriff der Kontrahierungspolitik teilweise als Überbegriff der Preispolitik angesehen und die Preispolitik neben der Rabattpolitik, den Liefer- und Zahlungsbedingungen und der Absatzfinanzierungspolitik nur als Teil der Kontrahierungspolitik aufgefasst (Weis 2009, S. 331). Da in den Augen des Autors allerdings die beiden zuletzt genannten Teilbereiche ebenfalls in den Bereich der (positiven oder negativen) Rabattpolitik fallen, soll im weiteren Verlauf dieser Arbeit der Ansicht Freters (2008, S. 325) gefolgt und der Begriff „Preispolitik“ als Überbegriff beibehalten werden.

Der Preis für ein Produkt oder eine Leistung stellt sowohl für den Käufer als auch für den Verkäufer ein ausgesprochen wichtiges Entscheidungskriterium dar. Auf Kundenseite beeinflusst der Preis in erster Linie die Entscheidung über Kauf oder Nichtkauf eines Produktes. Falls eine Kaufentscheidung getroffen wird, beeinflusst der Preis weiterhin die Wahl der Produktart, die Markenwahl, die mengenmäßige Größe des Einkaufs und die Frequenz des eventuellen Wiederkaufs. Aus Sicht des Verkäufers spielt der Preis in Kombination mit der abgesetzten Menge eine entscheidende Rolle, da er den Umsatz des Unternehmens determiniert (Freter 2008, S. 325). Aufgrund der immensen Wichtigkeit des Preises auf beiden Seiten ist die Preispolitik eines Unternehmens entscheidend für dessen Markterfolg und sollte deshalb auch mit angemessener Sorgfalt betrieben werden. Unter Preispolitik fallen alle Entscheidungen, die Einfluss auf die Preise und deren Durchsetzung haben. Dabei können sich diese Entscheidungen auf das gesamte Angebot eines Unternehmens, auf Teilbereiche oder auch nur auf einzelne Produkte oder Leistungen beziehen (Weis 2009, S. 333).

Die Festlegung von Preisen kann nicht losgelöst von weiteren Betrachtungen im Unternehmen und auch im Umfeld des Unternehmens erfolgen. Bevor Preise festgesetzt werden, ist anhand einer Situationsanalyse festzustellen, welchen preispolitischen Spielraum ein Unternehmen überhaupt hat. Dieser Spielraum kann von den eigenen finanziellen Mitteln abhängen, von der gegebenen Marktsituation oder auch von staatlichen Regulierungen wie beispielsweise bei Büchern. In einem zweiten Schritt müssen die Ziele definiert werden, die mit den Preisen innerhalb des gesetzten Rahmens erreicht werden sollen. Man unterscheidet dabei 
unternehmensgerichtete Ziele, beispielsweise eine Maximierung des Stückdeckungsbeitrages, oder marktgerichtete Ziele, beispielsweise ein möglichst hohes Marktwachstum. Sind mehrere Ziele definiert, die sich ganz oder teilweise konträr zueinander verhalten, sind die einzelnen Ziele mit Prioritäten zu versehen, um so das wichtigste Ziel nicht aus den Augen zu verlieren. In einem weiteren Schritt werden preispolitische Strategien festgelegt, wie das definierte Ziel erreicht werden kann. Man unterscheidet dabei Strategien bezüglich

- Preispositionierung,

- Preiswettbewerb,

- Produktlebenszyklus,

- Variabilität und

- Preisdifferenzierung (Meffert/Bruhn 2006, S. 553).

Unter Preispositionierung fallen die Strategien der Hoch-, Mittel- und Niedrigpreisstrategie. Preiswettbewerb steht für die Strategien der Preisführerschaft, des Preiskampfes und der Preisfolgerschaft. Die Produktlebenszyklusstrategie basiert auf dem Gedanken, dass ein Produkt oder eine Leistung je nach dessen Position im Produktlebenszyklus unterschiedliche Preissetzungsspielräume bietet. Abhängig davon, wie der Preis festgelegt wird, spricht man von einer Skimmingstrategie oder einer Penetrationsstrategie. Die Variabilität steht für die preispolitischen Strategien der Festpreisstrategie, der flexiblen Preisstrategie und der pulsierenden Preisstrategie. Eine Preisdifferenzierung liegt vor, wenn ein Unternehmen gleiche Produkte an unterschiedliche Kunden zu unterschiedlichen Preisen verkauft (Meffert/Bruhn 2006, S. 553). Da der letzte Punkt ein sehr wichtiges Instrument für die Marktbearbeitungsseite der Marktsegmentierung darstellt, wird die Preisdifferenzierung hier näher vorgestellt, um im Anschluss auf ihre Einsatzmöglichkeiten in der BuP-Branche einzugehen.

Grundsätzlich lässt sich das Werkzeug Preisdifferenzierung nochmals unterteilen in Preisdifferenzierung des ersten, des zweiten und des dritten Grades (Diller 2008, S. 228 f.). Wie bereits erwähnt, verkauft ein Unternehmen, das Preisdifferenzierung betreibt, das gleiche Produkt an verschiedene Kunden zu unterschiedlichen Preisen, wobei die meisten Unternehmen pro Preis eine Variation des Urproduktes verkaufen. Hierbei entstehen wiederum direkte Wechselbeziehungen zwischen den Kernbereichen des Marketing-Mix, genauer zwischen Preis- und Produktpolitik. Abbildung 39 zeigt vereinfacht die Potenziale, die eine Preisdifferenzierung bietet. 


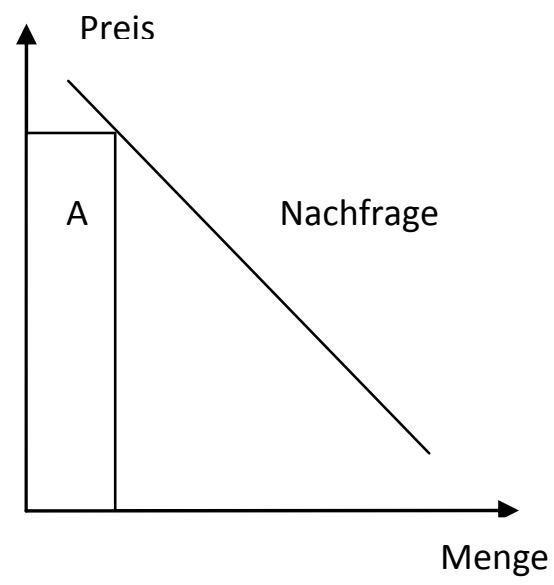

Situation 1: nur hochpreisiges Angebot. Erlöse $=\mathrm{A}$

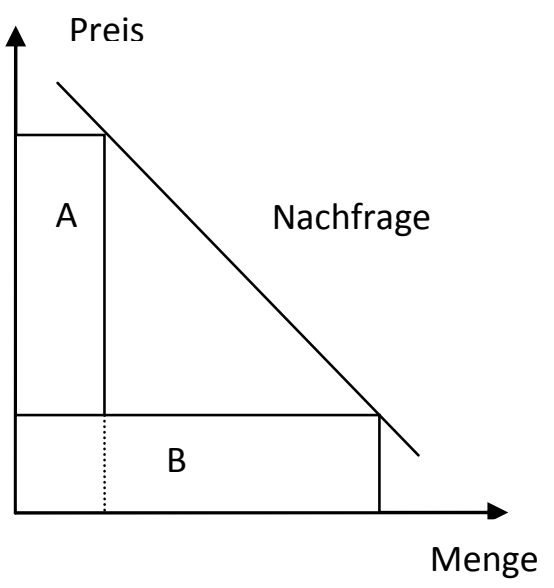

Situation 2: hoch- und niedrigpreisiges Angebot. Erlöse $=A+B$

\section{Abbildung 39: Preisdifferenzierung}

Quelle: Eigene Darstellung

Um eine Preisdifferenzierung zu erreichen, bieten sich dem Unternehmen verschiedene Möglichkeiten (Esch 2006, S. 318 ff.; Meffert/Bruhn 2006, S. 567 ff.; Freter 2008, S. 331 ff.):

1. Der Unternehmer setzt jeden einzelnen Preis so fest, dass jeder Kunde gezwungen ist, seine maximale Zahlungsbereitschaft anzubieten. Man spricht dabei auch von Preisindividualisierung oder Preisdifferenzierung ersten Grades.

Beispiel: individuelle Preisverhandlung, Auktionen, Börsen, Feilschen

2. Der Unternehmer bietet ein Produkt zu mehreren Preisen an. Die Kunden offenbaren durch die Wahl der Preiskategorie dem Unternehmer ihre Präferenzen. Sie betreiben dadurch Selbstselektion. Man spricht dabei von horizontaler Preisdifferenzierung bzw. Preisdifferenzierung zweiten Grades.

Beispiel: Bahnfahrt 1. oder 2. Klasse, Mengenrabatte, Preisnachlässe auf Produktkombinationen

3. Der Unternehmer segmentiert seine potenziellen Kunden im Vorfeld und fordert von jedem Kunden den Preis, den er für dessen Segment im Vorfeld als optimal festgesetzt hat. Arbitragen werden durch Segmentzugehörigkeitsprüfungen (z. B. Vorlage eines 
Ausweises) unterbunden. Man spricht hierbei von vertikaler Preisdifferenzierung oder Preisdifferenzierung dritten Grades.

Beispiel: Studenten- oder Seniorenrabatte, unterschiedliche Preise in Nord- und Süddeutschland, Preisnachlässe für Buchungen in der Nebensaison.

Abbildung 40 fasst diese Überlegungen zusammen.

\begin{tabular}{|c|c|c|}
\hline \multicolumn{3}{|c|}{ Typen der Preisdifferenzierung } \\
\hline $\begin{array}{c}\text { Preisdifferenzierung } \\
\text { 1. Grades }\end{array}$ & $\begin{array}{c}\text { Preisdifferenzierung } \\
\text { 2. Grades }\end{array}$ & $\begin{array}{c}\text { Preisdifferenzierung } \\
\text { 3. Grades }\end{array}$ \\
\hline $\begin{array}{l}\text { Preisindividuali- } \\
\text { sierung (Auktio- } \\
\text { nen, Verhand- } \\
\text { lungen) }\end{array}$ & $\begin{aligned}> & \text { Mehr-Personen- } \\
& \text { Preisbildungen } \\
> & \text { Leistungsbezogene } \\
> & \text { Mengenbezogene } \\
& \text { PDPD } \\
> & \text { Preisbündelung }\end{aligned}$ & $\begin{aligned}> & \text { Personenbezogene } \\
& \text { PD } \\
> & \text { Räumliche PD } \\
> & \text { Zeitliche PD }\end{aligned}$ \\
\hline \multicolumn{3}{|c|}{ Implementierungsformen der Preisdifferenzierung } \\
\hline
\end{tabular}

\section{Abbildung 40: Typen der Preisdifferenzierung}

Quelle: Freter 2008, S. 332

Übertragen auf die BuP-Branche ergeben sich diverse Möglichkeiten, eine Preisdifferenzierung vorzunehmen. Da der Einzelhandel von Blumen und Pflanzen en gros kein Markt für hochpreisige Artikel ist, kommt eine Preisindividualisierung nahezu nicht in Frage. Die entstehenden Transaktionskosten, jeden Preis mit jedem Kunden neu auszuhandeln, würden die eventuell zusätzlich generierten Erlöse bei Weitem übersteigen. Denkbar sind solche Preisverhandlungen lediglich bei einmaligen, sehr hohen Auftragswerten, beispielsweise beim Kauf eines sehr hochwertigen Bonsais oder wenn ein Kunde seinen Garten komplett neu anlegt und sämtliche Pflanzen an einem Ort kauft. Ähnlich verhält es sich mit der Organisation des Einzelhandelsverkaufs als Auktion oder Börse. Die Organisation einer Auktion für Privatkundschaft ist zu aufwendig im Vergleich zu den potenziellen Erlöszuwächsen. Weiterhin 
ist problematisch, dass alle Interessenten zur gleichen Zeit an der Auktion teilnehmen müssen, aber auch, dass die Produkte, die zum Verkauf gestellt werden, für den anonymen Markt produziert wurden, was letztendlich wieder negativen Einfluss auf die maximale Zahlungsbereitschaft der Auktionsteilnehmer haben kann.

Bessere Ansatzpunkte für die BuP-Branche bietet die Preisdifferenzierung zweiten Grades. Einige Möglichkeiten der Differenzierung sind:

- Qualitätsunterschiede der Produkte (z. B. langstielige Rosen und alternativ günstigere, kurzstielige Rosen)

- Optionale, kostenpflichtige Zusatzservices (z. B. „de Luxe Geschenkverpackung“ anstatt normales Einwickelpapier bzw. gängiger Folie)

- Mengenrabatte, Staffelpreise, Boni für besonders treue Kunden

- Preisbündelungen (z. B. komplett fertiggestelltes Blumenarrangement „Gute Besserung“) - die Summe aller Einzelteile des Arrangements (hier: Strauß, „Gute Besserung“-Karte, Verpackung, Schokolade) ist einzeln teurer, entweder finanziell oder organisatorisch

Die Preisdifferenzierung dritten Grades bietet ebenfalls interessante Möglichkeiten für die Einzelhändler der BuP-Branche, wie folgende Beispiele zeigen:

- Personelle Differenzierung: spezielle Preise für bestimmte Gruppen wie beispielsweise Schüler, Senioren oder alle Personen, die in einem bestimmten Monat geboren sind

- Räumliche Differenzierung: bei Filialbetrieben unterschiedliche Preise in den einzelnen Filialen

- Zeitliche Differenzierung: z. B. spezielle Preisgestaltung für Kunden, die innerhalb eines bestimmten Zeitfensters einkaufen, etwa „Early-Bird-Angebot“

\subsection{Place}

„Place“ bzw. zu Deutsch Distributionspolitik „,beschäftigt sich mit allen Entscheidungen und deren Realisierung, die im Zusammenhang mit dem Weg eines Produktes oder einer Leistung vom Produzenten zum Endverbraucher oder Verwender gefällt werden müssen“ (Weis 2009, S. 391). Dieser Definition folgend umfasst die Distributionspolitik neben den verschiedenen Absatzwegen (direkter und indirekter Absatz) auch die Bereiche der Logistik (Lager, Transport, Standort). Da sich die Problemstellung dieser Arbeit allerdings auf den Einzelhandel bezieht, wird im Folgenden der Fokus auf die Absatzwege gelegt. 
Der indirekte Absatzweg beinhaltet die Möglichkeiten der Distribution von Produkten über den Großhandel, Einzelhandel, Vertragshändler, Franchisepartner etc. (Weis 2009, S. 391). Er richtet sich daher an Unternehmen, die in der Wertschöpfungskette weiter vorne angesiedelt sind, in den meisten Fällen also an produzierende Unternehmen, die vor der Frage stehen, auf welchem Absatzkanal bzw. auf welchen Absatzkanälen sie ihr Produkt bzw. ihre Produkte am besten vermarkten können. Der Fragestellung dieser Arbeit folgend wird daher der indirekte Absatzweg nicht weiter thematisiert, vielmehr liegt der Schwerpunkt auf dem direkten Absatz. Dieser zeichnet sich dadurch aus, dass der „Hersteller seine Produkte unmittelbar an den Endverbraucher verkauft" und es dadurch eine direkte Verbindung zwischen Hersteller und Konsument gibt (Esch 2006, S. 327). In dem hier zu Grunde liegenden Fall nimmt der Einzelhändler den Platz des Herstellers ein. Dies liegt zum einen daran, dass der Einzelhändler in direkter Verbindung mit dem Konsumenten steht, zum anderen daran, dass der Einzelhändler für Blumen und Pflanzen zusätzlich auch Hersteller von Dienstleistungen ist.

Die konkreten Ziele der Distributionspolitik leiten sich aus der übergeordneten, unternehmensindividuellen Unternehmensstrategie ab. Da allerdings jedes Unternehmen das Ziel hat, sein eigenes Überleben am Markt zu sichern, sind bei jedem Einzelhändler die Ziele „Versorgungssicherheit in Qualität“ und „Versorgungssicherheit in Quantität“ gleich. Wären diese nicht erfüllt, entstünden für die Unternehmen Opportunitätskosten in Form von Fehlmengen bzw. in Form von potenziellem Umsatz, der nicht generiert werden kann (Meffert/Bruhn 2006, S. 595; Herrmann 1999, S. 861). Um diese Art von Kosten zu vermeiden, bietet sich nach Meffert (Meffert/Bruhn 2006, S. 596 ff.) folgender Maßnahmenkatalog an, der an die Gegebenheiten der BuP-Branche angepasst ist:

- Hohe Präsenz und Erreichbarkeit

Für die im Einzelhandelsgeschäft für BuP oft zu erbringenden Dienstleistungen ist ein simultanes Anwesendsein von Kunde und Verkäufer notwendig. Dementsprechend ist ein Standort zu wählen, der eine hohe Kundennähe aufweist sowie schnell und einfach zu erreichen ist. Dazu zählt auch, den Kunden genügend Stellplätze in möglichst direkter Nähe zur Einkaufsstätte zur Verfügung zu stellen.

- Integration des Kunden in den Erstellungsprozess

Da Zierpflanzen im Einzelhandel oft noch weiter nach individuellen Kundenwünschen veredelt werden, muss dem Kunden die Möglichkeit gegeben werden, beim Erstellungsprozess „,seines“ Produktes live dabei zu sein und ggf. noch kurzfristige Änderungswünsche mit einzubringen. Das Aufbinden von Sträußen oder floristische Arbeit 
im Allgemeinen in einem separaten, vom Kunden getrennten Binderaum erweist sich hierbei als kontraproduktiv. Um die Kunden die Wertigkeit der geleisteten Arbeit zu demonstrieren und um sie in den Erstellungsprozess mit einzubinden, bietet sich eine kombinierte Bedien- und Arbeitstheke an, bei welcher der Kunde „seinen Wunsch“ im Entstehen sehen kann.

\section{- Kurze Lieferzeit}

Da die Leistung im Einzelhandelsgeschäft während des direkten Kundenkontaktes erbracht wird, entstehen für den Kunden Wartezeiten, die es für ihn so angenehm wie möglich zu gestalten gilt - einerseits durch das Einbeziehen in den Leistungsprozess (siehe oben), andererseits durch konzentriertes und zügiges Arbeiten, so dass der Kunde sieht, dass seine Wartezeit nicht umsonst ist. Wartezeiten für die Kundschaft gilt es daher möglichst zu minimieren - auch aus dem Grund heraus, weil somit die Bedienquote einer Arbeitskraft pro Zeiteinheit erhöht wird. Kurze Lieferzeit bedeutet in diesem Falle auch, dass der Kunde die Möglichkeit einer schnellen Verbindung zum Einzelhandelsgeschäft hat, dabei also kein großer organisatorischer oder zeitlicher Aufwand als Kaufbarriere auftritt. Dies ergibt sich auch aus dem obigen Punkt der hohen Erreichbarkeit und Präsenz des Einzelhandelsgeschäfts.

- Hohe Lieferbereitschaft und Lieferzuverlässigkeit

Da ein Verkauf im Einzelhandel nur stattfinden kann, wenn Kunden vor Ort sind, kann der genaue Zeitpunkt der Warenabgabe nicht vom Unternehmen bestimmt werden. Daher ist die Bereitschaft zur Leistungserstellung während der Öffnungszeiten permanent aufrechtzuerhalten, was ein stets voll lieferbares Sortiment sowie genügend kompetentes Personal erfordert. Aufgrund der Verderblichkeit der Ware der BuP-Branche kann dies bei einer zu geringen Lagerumschlagshäufigkeit allerdings zu Problemen führen. Dies ist etwa dann der Fall, wenn die Zierpflanzen bereits zu lange im Geschäft stehen und nicht mehr frisch sind, wenn der Kunde sie kauft, dieser aber genau diese Frische und eine entsprechende Haltbarkeit erwartet. Das permanente Überwachen der angebotenen Qualität und das Aufrechterhalten der Frische des gesamten Sortiments ist somit ein entscheidender Faktor für den langfristigen Erfolg eines BuPEinzelhandels. Des Weiteren besteht in diesem Punkt ein Trade-off zwischen genügend kompetentem Personal, auf dessen Leistung sich der Kunde immer verlassen kann, und den dadurch entstehenden Personalkosten. Da diese im BuP-Einzelhandel nach dem Wareneinsatz den zweitgrößten Kostenblock darstellen, gilt es, diese stets so 
niedrig wie möglich zu halten, ohne dass dies allerdings langfristig auf Kosten der Lieferbereitschaft und -zuverlässigkeit gehen darf.

Sonderfälle der direkten Distribution stellen die Leistungserstellung beim Kunden, die Leistungserstellung an einem dritten Ort und der E-Commerce bzw. hier der Business-to-Consumer-Verkauf (B2C) dar (Meffert/Bruhn 2006, S. 603).

Die komplette Leistungserstellung beim Kunden ist für BuP-Einzelhandelsgeschäfte praktisch nicht handhabbar, da auch das Anbieten der gesamten physischen Auswahl an alternativen Produkten im Ladengeschäft zur Leistung gehört. Für Einzelhändler der BuP-Branche ist aufgrund ihrer aus Verkauf und Dienstleistung kombinierten Leistung jedoch eine Mischform denkbar, beispielsweise das Nach-Hause-Bringen der zuvor ausgesuchten Produkte, ein Beratungsgespräch vor Ort oder ein Ein- bzw. Bepflanzservice. Die Möglichkeiten der Durchführung der Leistung an einem dritten Ort entsprechen den eben genannten Möglichkeiten der Leistungserstellung beim Kunden, da es für den außer Haus agierenden BuP-Einzelhandelsmitarbeiter keinen Unterschied macht, ob die Teilleistung beim Kunden selbst oder an einem anderen, vom Kunden bestimmten Ort erbracht wird.

Der direkte Vertrieb von Zierpflanzen via Internet zeichnet sich durch Besonderheiten aus. Durch die Digitalisierung verwässern die typischen Strukturen der indirekten Distribution, so dass dadurch auch Produzenten oder primäre Großhändler problemlos direkt mit Konsumenten in Kontakt treten können und so zu Wettbewerbern der direkt vertreibenden Einzelhändler werden. Dabei darf allerdings nicht übersehen werden, dass auch Einzelhändler große Vorteile aus einer B2C-Beziehung ziehen können, beispielsweise über das Vorstellen des Unternehmens anhand der „digitalen Visitenkarte“ einer Homepage, das Anbieten und Erklären sämtlicher angebotenen Leistungen im Internet, das „digitale Schaufenster“ in Form eines Webshops oder bei bestehendem Datenmaterial die Verbesserung des Kundenbeziehungsmanagements. Gerade der kombinierte Leistungsprozess im BuP-Einzelhandel stellt beim B2C allerdings ein Problem dar, da eine eventuelle Veredelung des Kernproduktes individuell bestellt wird und somit nicht im Voraus im Webshop abbildbar ist. Als potenzielle Lösungsmöglichkeiten dieses Problems bieten sich Überlegungen $\mathrm{zu}$ einer Leistungsmodularisierung (Mass Customization) (vgl. Kapitel 4.1) oder einer teilweisen Leistungserstellung für den anonymen Onlinemarkt (vgl. Kapitel 4.2) an. 


\subsection{Promotion}

„Promotion“ bzw. zu Deutsch „Kommunikationspolitik“ wird auch als „Kundenkommunikation“ bezeichnet und steht für eine ,ganzheitliche, integrierte Marketingkommunikation über alle zur Verfügung stehenden Kommunikationskanäle, -instrumente und -aktivitäten“ (Weis 2009, S. 449). Als Teil des Marketing-Mix steht die Kommunikationspolitik somit für die segmentspezifische Ansprache von Käufern und potenziellen Kunden, wobei sie auch unternehmensintern ausgerichtet sein kann, beispielsweise für die Kommunikation mit den Mitarbeitern (Bruhn 2004, S. 199).

Wie bereits mehrfach beschrieben, führt die Mischform aus Konsumgut und Dienstleistung im BuP-Einzelhandel zu Besonderheiten. So ist hier die Kommunikationspolitik unter zweierlei Aspekten zu betrachten: einerseits als Instrument der eigentlichen Leistungserbringung (Beratung von Kunden), andererseits als Instrument der Kommunikationspolitik an sich (z. B. Werbeanzeigen). Da „Kundenberatung“ im Kontext dieser Arbeit primär bereits der Produktpolitik zugeschrieben wurde (vgl. Kapitel 4.1), sollen im weiteren Verlauf unter Kommunikationspolitik nur die folgenden Gruppen der Markt-, Kunden- und Mitarbeiterkommunikation verstanden werden (Bruhn 2005a, S. 328; Freter 2008, S. 315 ff.):

- Klassische Medien

- Direktwerbemedien

- Persönliche Kommunikation

- Verkaufsförderung

Im Vorfeld eines Einsatzes von kommunikationspolitischen Instrumenten bedarf es, basierend auf einer Marktsegmentierung, einer Festlegung von Zielgruppen, auf welche die Kommunikationswirkung zielt. Dabei gilt es parallel zu unterscheiden, ob die segmentspezifische Kundenansprache direkte ökonomische Ziele (z.B. Umsatzsteigerungen) oder auch Kommunikationsziele psychologischer Art verfolgt. Diese lassen sich nach Bruhn in kognitiv, affektiv und konativ orientierte Ziele unterscheiden (Bruhn 2005a, S. 138 ff.).

Kognitiv orientierte Ziele sind nach Meffert möglichst hohe Wirkungsgrade bei:

- Berührungs- und Kontakterfolg

- Aufmerksamkeitswirkung

- Erinnerungswirkung

- Informationsfunktion 
Berührungs- und Kontakterfolge betreffen die Genauigkeit, mit der die Kommunikation ihre Zielgruppe erreicht. Streuverluste sind teuer und daher so weit wie möglich zu vermeiden. Die Aufmerksamkeitswirkung zielt darauf ab, dass die Werbebotschaft beim Kunden bewusst wahrgenommen wird, was aufgrund der allgemeinen Werbeflut schwer zu erreichen ist. Die Erinnerungswirkung bezieht sich darauf, dass der Konsument die wahrgenommenen Informationen der Werbebotschaft - und sei es nur teilweise - speichert. Die Informationsfunktion besteht darin, dass der Empfänger der Werbebotschaft über den Umfang der angebotenen Produkte und Leistungen inhaltliche Informationen erhält (Meffert/Bruhn 2006, S. 481).

Affektiv orientierte Ziele sind:

- Gefühlswirkung

- Positive Hinstimmung

- Interessenweckung

- Imagewirkung

Durch die angestrebte Gefühlswirkung sollen beim Empfänger der Werbebotschaft grundsätzliche positive Emotionen ausgelöst werden. Die positive Hinstimmung soll das unbewusste Bedürfnis des Beworbenen konkretisieren und ihm anhand der Informationen rational erklären. Die Interessenweckung sorgt im Anschluss dafür, dass sich der Botschaftsempfänger für die angebotene Leistung aktiv interessiert und sie als potenziellen Kauf einstuft. Das Ziel der Imagewirkung beinhaltet das Schaffen eines positiven Bildes des Unternehmens in den Augen der Öffentlichkeit. Gerade bei Dienstleistungen bzw. dienstleistungsreichen Mischprodukten wie Blumen und Pflanzen ist dies ein wichtiger Punkt, da hierbei die Bewertung meist sehr subjektiv geprägt ist (Meffert/Bruhn 2006, S. 482).

Unter konativ orientierte Ziele fallen:

- Auslösung von Kaufhandlungen

- Beeinflussung des Informationsverhaltens

- Beeinflussung des Weiterempfehlungsverhaltens

Die Auslösung der zuvor ins Kalkül gezogenen Kaufhandlung soll den Beworbenen als Neukunden akquirieren oder als bestehenden Kunden an das Unternehmen binden. Die Beeinflussung des Informationsverhaltens des Kunden zielt darauf ab, ein ehrliches Kundenfeedback zu erhalten, um so die angebotenen Produkte und Leistungen kundengerechter gestalten zu können. Das Weiterempfehlungsverhalten des Kunden soll dahingehend beeinflusst werden, dass der Kunde das Unternehmen und dessen Leistungen weiterempfiehlt. Gerade im Bereich von 
Dienstleistungen sind positive Erfahrungsberichte von Vertrauten für potenzielle Kunden sehr wichtig, da es an objektiven Beurteilungskriterien für Dienstleistungen mangelt (Meffert/ Bruhn 2006, S. 482).

Neben der Festlegung der Ziele einer Werbebotschaft besteht eine weitere Fragestellung der Kommunikationspolitik darin, wie bzw. mit welchen Instrumenten sich die entsprechenden Zielgruppen möglichst exakt erreichen lassen, um so die einzelnen Segmente ,individuell“ ansprechen zu können. Im Folgenden werden daher Erklärungen und konkrete Werbeträger der oben genannten Kommunikationsmediengruppen vorgestellt. Grundsätzlich stellt sich dabei allerdings vor jeder Auswahl von Werbeträgern die Frage nach den Kosten und dem Nutzen, der damit generiert werden kann. Der Nutzen einer Werbebotschaft hängt maßgeblich von der qualitativen Art der Werbebotschaft ab. Um die Kosten der verschiedenen Werbeträger miteinander vergleichen zu können, wird in der Massenwerbung der sogenannte „Tausend-Kontakt-Preis“ berechnet. Er gibt die Kosten der Werbeaktivität pro 1.000 erreichter Personen in Geldeinheiten an. Sind die erreichten Personen nicht alle Angehörige des Zielsegments ist der Tausend-Kontakt-Preis durch den Anteil der Zielgruppe innerhalb der erreichten Gruppe zu dividieren. Es ergibt sich somit der zielgruppengewichtete Tausend-Kontakt-Preis in Geldeinheiten (Weis 2009, S. 484).

Klassische Medien stellen eine sehr wichtige Auswahl an Werbeträgern dar. Sie verfolgen die Beeinflussung der Umworbenen mittels des Einsatzes von Massenmedien, beispielsweise:

- Zeitschriften/Zeitungen $\rightarrow \quad$ Anzeigen, Beilagen

- Außenwerbung $\quad \rightarrow \quad$ Plakate, Leuchtwerbung

- TV, Radio, Kino $\quad \rightarrow \quad$ Spots, Teleshopping

In dieser Arbeit wird davon ausgegangen, dass klassische Medien nur in eine Richtung wirken und sich somit dem Empfänger der Werbebotschaft keine direkte Rückkopplungsmöglichkeit bietet. In der Literatur wird den klassischen Medien teilweise auch eine Möglichkeit zur „direct response“" zugeschrieben, beispielsweise anhand von Rückantwortkarten oder Coupons. Daher werden klassische Medien dort als Teil der Direktwerbemedien angesehen (vgl. Freter 2008, S. 315).

Direktwerbemedien bestehen aus physischen und aus digitalen Werbeträgern. Physische Medien sind:

- Adressierte Werbeträger $\quad \rightarrow \quad$ Kataloge, Produktproben, Kundenzeitung

- Unadressierte Werbeträger $\rightarrow$ Hauswurfsendungen, Handzettel 
Adressierte physische Werbeträger eignen sich sehr gut, um die Fehlstreuung einer Werbewirkung einzugrenzen, da sie eine sehr zielgruppenspezifische Ansprache ermöglichen. Mit der steigenden Flut an unadressierten Werbebotschaften steigen adressierte Werbebotschaften in ihrer Bedeutung, da sie den Endverbraucher auf persönlicher Ebene ansprechen (Holland 2004, S. 25; Wirtz 2005, S. 173). Unadressierte Werbeträger sprechen den Empfänger hingegen nicht persönlich an. Dennoch erfolgt ihre Verteilung nicht wahllos. Die Werbeträger werden meist aufgrund einer mikro-geographischen Segmentierung verteilt, beispielsweise nach Städten, Stadtvierteln oder einzelnen Straßen (Bruns 2007b, S. 185).

$\mathrm{Zu}$ den digitalen Instrumenten der Direktwerbemedien zählen:

- Telefon $\quad \rightarrow \quad$ aktiv, passiv

- Internet $\rightarrow$ Homepage, E-Mail

Telefonmarketing kann sowohl von Kundenseite als auch von Unternehmensseite aus initiiert sein. Ruft ein Unternehmen seine Zielpersonen von sich aus an, spricht man vom aktiven Telefonmarketing. Passives Telefonmarketing liegt vor, wenn Kunden das Unternehmen anrufen. In beiden Fällen findet jedoch ein persönlicher Informationsaustausch statt (Holland 2004, S. 30). Die Möglichkeiten des Internets bestehen primär aus dem Anbieten einer Homepage und der Kommunikationsmöglichkeit via E-Mail, wobei eine Homepage nicht nur als passive „digitale Visitenkarte“ gesehen werden darf. Vielmehr ermöglicht die „aktive Homepage“ auch das Ersetzen von persönlicher Beratung, das Sammeln von Kundendaten und die daraus folgende persönliche Kundenansprache, das In-Kontakt-Treten mit dem Unternehmen oder anderen Kunden via Chats oder Foren und den direkten Kauf von Produkten (E-Commerce) (Bruns 2007, S. 277 ff.). Basierend auf den gesammelten Kundendaten lassen sich die Onlinebesucher einer Homepage sehr gut segmentieren. Dies ermöglicht die Erstellung von sogenannten Verteilerlisten, die es erlauben, ausgewählten Segmenten E-Mails mit segmentspezifischen Informationen zu schicken. E-Mails, die anhand von segmentspezifischen Verteilerlisten versandt werden, sind eine preisgünstige Alternative zum adressierten physischen Werbebrief (Wirtz 2005, S. 178).

Die persönliche Kommunikation oder „Dialogkommunikation“ steht für den direkten Kontakt zwischen Käufer und Verkäufer. Wegen der fehlenden objektiven Leistungsbewertungskriterien spielt diese Art der Interaktion besonders im Bereich der Dienstleistungen eine wichtige Rolle. Die persönliche Kommunikation richtet sich exakt an einen (potenziellen) Kunden, auf dessen Wünsche und Probleme in einer wechselseitigen Kommunikation individuell einge- 
gangen werden kann. Eine Fehlstreuung ist daher nicht möglich. Im Rahmen der persönlichen Kommunikation kommt den kommunikativen Fähigkeiten des Verkäufers eine besondere Bedeutung zu (Meffert/Bruhn 2006, S. 486 f.; Freter 2008, S. 324).

Unter den Begriff der Verkaufsförderung fallen sämtliche zeitlich befristeten Maßnahmen mit Aktionscharakter, die eventuell bereits eingesetzte Marketinginstrumente unterstützen und den Absatz durch Anreize für (potenzielle) Kunden stimulieren. Typische Beispiele sind das Verteilen von Gratisproben oder das Anbieten eines Preisausschreibens (Esch 2006, S. 272 f.). Ein gezielter Einsatz von Instrumenten der Verkaufsförderung ist in seiner Zielgenauigkeit mit der von Massenmedien zu vergleichen, was einen hohen Grad an Fehlstreuung bedeutet.

Neben den bisher beschriebenen Unsicherheiten, die es bei der Auswahl des passenden Werbeinstruments zu berücksichtigen gilt, müssen zudem diverse Störfaktoren des Entscheidungsprozesses ins Kalkül mit einbezogen werden. Solche Störfaktoren können marktbezogen, kundenbezogen, konkurrenzbezogen, umfeldbezogen oder auch unternehmensbezogen sein. Aufgrund des Fokus dieser Arbeit sei zur weiteren Vertiefung des Aspekts der Störfaktoren innerhalb der Kommunikationssituation jedoch lediglich auf die weiterführende Literatur, beispielsweise von Bruhn (2005a), verwiesen.

\subsection{Personnel}

Neben den bereits beschriebenen vier klassischen Bestandteilen des Marketing-Mix wird in der neueren Literatur besonders im Dienstleistungsbereich der Marketing-Mix von 4Ps auf 7Ps erweitert. Personnel stellt dabei die erste der drei Erweiterungen dar.

„Personnel“ bzw. Personalpolitik beschäftigt sich mit allen Entscheidungen rund um den Pool an Mitarbeitern. Dazu gehören die Personalentwicklung, die Kommunikation mit dem Personal, die Kommunikation innerhalb des Personalpools und auch die Freisetzung von bestehendem Personal. Innerhalb des Unternehmens - und besonders im Dienstleistungsunternehmen - nimmt das Personal bzw. nehmen die Mitarbeiter eine besondere Stellung ein, da sie es sind, die das Unternehmen in den Augen der Kunden ausmachen und dieses ihnen gegenüber vertreten. Auf Grund dieser besonderen Bedeutung als Bindeglied zwischen Unternehmensseite und Kundenseite wird Personalpolitik als Instrument eingesetzt, um die operativen oder taktischen Ziele einer „Abteilung Personal“ in das strategische Ziel einer unternehmensweiten Kundenorientierung einzubinden. Meffert spricht dabei vom „Konzept des internen Marke- 
tings“ (Meffert/Bruhn 2006, S. 623), das es anzuwenden gilt. Internes Marketing zielt auf die unternehmensinternen Prozesse ab, die es anhand von Instrumenten des Marketing- und des Personalmanagements so zu verbessern gilt, dass durch „konsequente Kunden- und Mitarbeiterorientierung“ das Marketing als Leitgedanke in den Köpfen der Mitarbeiter verankert wird. Dadurch sollen die übergeordneten, marktgerichteten Ziele des Unternehmens wirkungsvoller erreicht werden (Michaelis 2009, S. 37; Bruhn 1999, S. 20).

Nach Bruhn besteht der Komplex des Beziehungskonstrukts zwischen Unternehmen, Mitarbeitern und Kunden aus drei verschiedenen Beziehungen, die für ein erfolgreiches Agieren am Markt jeweils parallel bearbeitet werden müssen (Bruhn 1999, S. 21):

1. Unternehmen-Kunde

Das Unternehmen muss nach außen kundenorientiert ausgerichtet sein.

2. Unternehmen-Mitarbeiter

Das Unternehmen muss sich an seinen internen Kunden bzw. an seinen Mitarbeitern orientieren (internes Marketing).

\section{Mitarbeiter-Kunde}

Der Mitarbeiter als Stellvertreter des Unternehmens muss bei jeder Leistungserstellung am Kunden individuell auf dessen Wünsche eingehen.

Durch die gleichzeitige Bearbeitung aller drei Beziehungen strebt das Unternehmen neben einer hohen Kundenzufriedenheit auch eine (vorgelagerte) Zufriedenheit der Mitarbeiter an. Die Ziele der Personalpolitik unterscheiden sich demnach sowohl in ihrer Wirkungsrichtung (intern, extern) als auch auf der zeitlichen Managementebene (taktisch, strategisch), an der sie ansetzen. Abbildung 41 fasst diese unterschiedlichen Ausrichtungen der Personalpolitik zusammen und erlaubt gleichzeitig eine Systematisierung der Ziele in vier verschiedene Gruppen.

Die internen taktischen Ziele der Personalpolitik versuchen den bestehenden Mitarbeitern die Werte des Unternehmens zu vermitteln, also dessen Strategie, Ziel und auch dessen Zweck. Dabei gilt es zu unterscheiden, dass den Mitarbeiter einerseits das eigene Unternehmen nähergebracht wird und sie dadurch einen Sinn für die Unternehmenskultur entwickeln und andererseits den Mitarbeitern die entsprechenden Fähigkeiten und Kompetenzen vermittelt werden, welche sie im Kundendialog anwenden können, wollen und sollen. Die taktisch-externe Zielsetzung setzt auf diesen angewandten Fähigkeiten der Mitarbeiter auf und hat eine positi- 
ve Wahrnehmung des Unternehmens durch Kundensicht zum Ziel. Wie bereits erwähnt, ist infolge des engen Kontakts zwischen Verkäufer und Käufer im BuP-Einzelhandel dieser Punkt von zentraler Bedeutung (vgl. zur ,persönlichen Kommunikation“ in Kapitel 4.4). Die internen strategischen Ziele der Personalpolitik basieren auf dem ganzheitlichen, unternehmensweiten Gedanken der Mitarbeiterzufriedenheit. Indem die Identifikation der Mitarbeiter mit dem Unternehmen gefördert wird, wird mittel- bis langfristig eine positive, kunden- und qualitätsorientierte Unternehmenskultur angestrebt. Die externen strategischen Ziele stellen die letztendlichen Ziele der Personalpolitik dar. Zu ihnen gehört das Binden der Bestandskunden an das Unternehmen, die Gewinnung von Neukunden sowie die Rückgewinnung verloren gegangener Kunden (Meffert/Bruhn 2006, S. 631 f.).

\begin{tabular}{|c|c|c|}
\hline Zielniveau & Intern & Extern \\
\hline $\begin{array}{l}\text { Taktisch: } \\
\text { Individual-Ebene }\end{array}$ & $\begin{array}{l}\text { Information über } \\
\text { die Implementierungsinhalte } \\
\text { • „Kennen“ } \\
\text { • „Verstehen“ } \\
\text { Know-how-Vermittlung und Motivation } \\
\text { - „Können“ } \\
\text { - „Wollen“ } \\
\text { • „Dürfen“ }\end{array}$ & $\begin{array}{l}\text { - Differenzierung gegenüber dem } \\
\text { Wettbewerb } \\
\text { - Kundenzufriedenheit }\end{array}$ \\
\hline $\begin{array}{l}\text { Strategisch: } \\
\text { Gesamtunter- } \\
\text { nehmensebene }\end{array}$ & $\begin{array}{ll}\text { - } & \text { Qualitätsbewusstsein } \\
\text { - } & \text { Kundenorientierung } \\
\text { - } & \text { Mitarbeiterzufriedenheit }\end{array}$ & $\begin{array}{ll}\text { - } & \text { Kundengewinnung } \\
\text { - } & \text { Kundenbindung } \\
\text { - } & \text { Kundenrückgewinnung }\end{array}$ \\
\hline
\end{tabular}

Abbildung 41: Ziele der Personalpolitik

Quelle: Eigene Darstellung nach Meffert/Bruhn 2006, S. 631

Die Instrumente, mit denen eine zielgerichtete Personalpolitik betrieben wird, unterscheiden sich darin, zu welchem Zeitpunkt sie angesetzt werden: Die Personalplanung findet im Vorfeld statt, während die operativen Veränderungen am bestehenden Mitarbeiterpool erfolgen. Die Instrumente werden im Folgenden kurz vorgestellt und nach Möglichkeit gleichzeitig im Kontext der BuP-Einzelhandelsbranche einsatzorientiert näher beschrieben. 
Während der Personalplanungsphase wird der Personal-Ist-Bestand ermittelt und mit dem Personal-Soll-Bestand verglichen. Sich ergebende Differenzen bilden die Basis für weiterreichende Überlegungen und Planungen bezüglich des bestehenden Mitarbeiterstamms. Der Soll-Zustand des Personals sagt aus, wie viele Mitarbeiter mit welcher Qualifikation an welchem Ort und zu welcher Zeit benötigt werden, um den Konsumenten das geplante Leistungsprogramm ohne Einschränkungen anbieten zu können. Dabei gilt es zu beachten, dass die Anforderungen an den Personal-Soll-Zustand einerseits quantitativer und andererseits qualitativer Art sind. Die quantitative Betrachtung erfasst sowohl im Ist- als auch im SollBereich die Anzahl der Mitarbeiter. Gerade im Einzelhandel für BuP ist dies im Soll-Bereich ein schwieriger Punkt, da der Geschäftsverlauf sowohl von zeitlichen als auch von natürlichen Nachfrageschwankungen geprägt ist. Zeitliche Schwankungen ergeben sich auf Grund des stark ausgeprägten Saisongeschäftes (vgl. Kapitel 2.4), natürliche Schwankungen wegen der Abhängigkeit vom Wetter. Zusätzlich zu den Nachfrageschwankungen treten in der dienstleistungsreichen BuP-Branche die Probleme auf, dass die Dauer der „Arbeit am Kunden“ nicht im Vorfeld abgeschätzt werden kann, da sie je nach individuellem Kundenwunsch unterschiedlich lange ausfällt und Nachfrageschwankungen im BuP-Einzelhandel auf Grund der Verderblichkeit der Produkte nur sehr kurzfristig mit einem Pufferlager ausgeglichen werden können.

Eine mögliche Maßnahme, um einer quantitativen Differenz zwischen personellem Ist- und Soll-Bestand eines BuP-Einzelhandels entgegenzuwirken, könnte beispielsweise eine Verkürzung der Leistungserstellung durch technische Unterstützung oder durch komprimiertere Beratung sein. Da der mögliche Technisierungsgrad innerhalb des BuP-Einzelhandels allerdings recht gering ist und die Beratung stets kundenorientiert erfolgen soll, sind Effizienzsteigerungen innerhalb des Mitarbeiterstamms zur Behebung von fehlenden Mitarbeitern zwar möglich, jedoch nur in begrenztem Umfang. Weitere Möglichkeiten, um mit einer zu hohen Nachfrage im Vergleich zum zur Verfügung stehenden Personal umzugehen, sind einerseits die Aufstockung des bestehenden Personals und andererseits die Belassung der bestehenden Personalstärke. Eine Aufstockung des Personals durch neue Festanstellungen ist in der BuPBranche aus ökonomischer Sicht schwierig, da sich die saisonalen Spitzen der Nachfrage nur auf einige wenige Wochen im Jahr beschränken. Daher bietet sich für BuP-Einzelhändler an, das bestehende Personal durch zeitlich begrenzte Aushilfen aufzustocken. Eine weitere Möglichkeit, zeitlich begrenzte Unterstützung zu erhalten, bieten Zeitarbeitsfirmen. Eine ökonomisch sinnvolle Alternative kann es aber auch sein, trotz erhöhter Nachfrage das bestehende 
Personal nicht aufzustocken: Obwohl nicht das ganze Umsatzpotenzial abgeschöpft wird, spart sich das Unternehmen einerseits Transaktionskosten und stellt andererseits somit gleichzeitig sicher, dass die bestehenden Personalressourcen dauerhaft optimal ausgenutzt werden.

Qualitative Anforderungen an das Personal stehen für die Eignung und die Fähigkeit der Mitarbeiter, die ihnen übertragenen Aufgaben in Sinne des Unternehmens fachlich fundiert zu erfüllen. $\mathrm{Zu}$ den qualitativen Merkmalen eines Mitarbeiters gehören die fachliche Kompetenz des Mitarbeiters (Wissen und Können) sowie seine persönlichen Merkmale, beispielsweise Kommunikationsfähigkeit, Sozialkompetenz, Vertrauenswürdigkeit und Verantwortungsbewusstsein. Da im Dienstleistungsbereich der Kundendialog eine zentrale Rolle spielt, sind die qualitativen Merkmale der Mitarbeiter im BuP-Einzelhandel von entscheidender Bedeutung. Mitarbeiter in ausreichender Zahl zu finden, stellt für den BuP-Einzelhandel somit weniger ein Problem dar, als qualitativ gut ausgebildete Mitarbeiter zu finden, die dazu bereit sind, sich nur zeitlich begrenzt einstellen zu lassen (nach Meffert/Bruhn 2006, S. 632 ff.).

Instrumente zur Veränderungen am bestehenden Personalstamm stellen die Variation des Personaleinsatzes, die Personalveränderung und die Mitarbeiterkommunikation dar. Der Einsatz des Personals und dessen Variation umschließt alle Maßnahmen im Hinblick auf die Arbeitsplatzorganisation, den Arbeitsplatz an sich und die Arbeitszeit. Um innerhalb dieses Rahmens von Mitarbeitern eine gute Leistungsqualität verlangen und auch erwarten zu können, benötigen diese eine entsprechende Handlungsfreiheit bzw. einen Handlungsspielraum, innerhalb dessen sie selbständig agieren können. Personalveränderung bedeutet eine quantitative oder qualitative Veränderung des bestehenden Personals, also die Neueinstellung von Mitarbeitern, eine Personalentwicklung im Sinne von Schulungen und/oder Weiterbildungen und die Personalfreisetzung. Unter Mitarbeiterkommunikation versteht man sämtliche Kommunikationsund Informationsflüsse innerhalb eines Unternehmens, die darauf abzielen, die Meinung, die Einstellung oder das Verhalten der Mitarbeiter mental zu beeinflussen (Bruhn 2005b, S. 1203; Meffert/Bruhn 2006, S. 643 ff.).

\subsection{Process}

„Process“ stellt die zweite Erweiterung des klassischen Marketing-Mix dar. Process steht für Prozessmanagement, also das Management der betrieblichen Abläufe, die zur Erstellung von Produkten und/oder Leistungen notwendig sind. Dabei gilt es zu beachten, dass Prozesse in einem bereits bestehenden Unternehmen nicht einfach „erfunden“ werden. Vielmehr hat sich 
im Laufe der Zeit über die gegebene Organisationsstruktur des (noch nicht prozessorientierten) Unternehmens eine Ablaufstruktur herausgebildet, anhand derer die betrieblichen Leistungen erbracht werden und die im Rahmen des Prozessmanagements auf ihre Geeignetheit hin geprüft wird.

Im einfachsten Falle bzw. im Falle eines kleinen Unternehmens basiert die Organisation auf einer oder zumindest wenigen Personen, beispielsweise dem Eigentümer einer inhabergeführten Einzelhandelsgärtnerei. Man spricht dabei von der „,personalen Organisation“. Sie zeichnet sich dadurch aus, dass eine bzw. wenige Personen den gesamten Überblick über sämtliche internen und externen Angelegenheiten des Unternehmens haben. Betriebliche Aktivitäten sind meist sehr kundenorientiert und basieren dabei auf persönlichen Anweisungen der Entscheidungsträger. Dieser Punkt der personalen Organisation stellt dessen größten Vorteil und gleichzeitig auch dessen größten Nachteil dar: Durch die Nähe des Entscheidungsträgers zu den Kunden und seinen Mitarbeitern kann die personale Organisation sehr schnell, sehr flexibel, sehr kundenfreundlich und sehr einfach vom Entscheidungsträger durch direkte Arbeitsanweisungen gesteuert werden. Der Nachteil ist die daraus resultierende Abhängigkeit der Organisation von diesem Entscheidungsträger. Um einen möglichen Ausfall zu kompensieren, müssen im Vorfeld Stellvertreter benannt werden, da jede aktive Organisation täglich neue Entscheidungsfragen aufwirft. Ein weiterer Nachteil der personalen Organisation besteht in ihrer Begrenztheit. Erreicht ein Unternehmen eine bestimmte Größe und eine bestimmte Zahl an Geschäftsbeziehungen jedweder Art, kann der Komplex der Unternehmung mit all ihren Verbindungen und Abhängigkeiten immer schwerer von einer Person überblickt werden. Bei Beibehaltung der personalen Organisation wird dies früher oder später zu Fehleinschätzungen bzw. Fehlentscheidungen des Entscheidungsträgers führen (Lenz 2005, S. 33; Stöger 2009, S. 35 f.).

Um dem letztgenannten Problem zu entgehen, ist es notwendig, dass sich eine größer und komplexer gewordene personal organisierte Unternehmung mit den eigenen Abläufen beschäftigt, diese beschreibt, bündelt und standardisiert, damit die Abläufe bzw. die Prozesse auch von anderen Personen ausgelöst und gesteuert werden können. Prozesse sind betriebliche Abläufe eines Unternehmens, um ein bestimmtes Vorhaben zu erreichen. Diese einzelnen, betrieblichen Schritte können innerhalb oder außerhalb eines Unternehmens erfolgen und wiederholen sich in regelmäßigen Abständen (Freud/Götzer 2008, S. 7 f.).

Um an obigem Beispiel festzuhalten, ist hier an eine inhabergeführte Einzelhandelsgärtnerei zu denken, die sich im Laufe der Zeit zu einem mittelgroßen Gartencenter entwickelt hat. Um 
die gestiegene Anzahl an Organisationsschwerpunkten, beispielsweise Einkauf, Verkauf und Personal, bewältigen zu können, werden im Gartencenter nun Abteilungen mit jeweiligen Abteilungsleitern gebildet, innerhalb derer die zugehörigen betrieblichen Abläufe bzw. Prozesse abgearbeitet werden, z. B. „Bestellung von Ware bei Lieferant A“. Da sich die Prozesse an den Funktionen ihrer zugehörigen Organisationseinheit orientieren spricht man hierbei auch von der sogenannten „funktionalen Organisation“ oder auch vom angewandten „Verrichtungsprinzip“. Das Ziel dieser Organisationsform besteht in der Dezentralisation der Entscheidungen, also darin, die Unternehmensführung auf mehrere Abteilungen zu verteilen, um so der Abhängigkeit der Unternehmung von nur einer Person zu entgehen. Die funktionsorientierte Organisation basiert dabei auf der Standardisierung der Prozesse, der klaren Zuweisung von Verantwortungsbereichen und einer hohen Transparenz, so dass auch die Sachbearbeiter innerhalb der einzelnen Abteilungen recht einfach austauschbar sind. Als Nachteil der Funktionsorientierung ist zu erwähnen, dass einzelne Abteilungen eventuell ein Eigenleben entwickeln können und so den Bezug zu ihrer Umwelt verlieren, beispielsweise wenn die Abteilung Einkauf zwar gute Preise aushandelt, die Ware aber zu ungünstigen Verkaufszeitpunkten einbestellt und so das gesamte Unternehmen Schaden davonträgt. Des Weiteren steigt mit der Anzahl der gebildeten Abteilungen die Anzahl der Abhängigkeiten und dadurch auch die Komplexität der Beziehungen untereinander, was eventuell Zuständigkeitsprobleme mit sich bringen kann oder gar bei manchem Mitarbeiter die „Nicht zuständig“-Einstellung fördert (Oelsnitz 2000, S. 58; Stöger 2009, S. 36).

Da die funktional gegliederte Organisation ab einer bestimmten Größe dazu führen kann, dass einzelne Abteilungen den Bezug zur Umwelt verlieren und damit den Weg zur weiteren marktgerechten Unternehmensentwicklung behindern, wird in einem weiteren Schritt der Markt dem funktional gegliederten Unternehmen wieder näher gebracht. Man spricht dabei auch von der zweidimensionalen „Matrix-Organisation“. Die Matrix-Organisation verbindet dabei die bestehenden Zuständigkeiten für Funktionen mit organisatorisch definierter Marktverantwortung. Durch die gleichzeitige Verwendung von zwei oder mehreren Autoritätslinien spricht man anstatt von der Matrix-Organisation auch von einer Gitternetzorganisation. Diese Bezeichnung stellt die bildliche Erweiterung zur eindimensionalen Organisation der personalen und funktionalen Organisation dar. Als Beispiel für die Matrix-Organisation können hier sehr große Unternehmen dienen, die ihre einzelnen Abteilungen zwar selbständig, aber dennoch dem Vorstand untergeordnet agieren lassen, beispielsweise die eigenständig agierenden „Länderabteilungen“ Landgard Suisse, Landgard Austria usw., die unter dem Dach der Hol- 
ding „Landgard“ koordiniert werden (Landgard 2011). Durch die teilweisen Doppelzuständigkeiten und die vielen Schnittstellen zwischen den einzelnen Elementen innerhalb der Matrix-Organisation kann es untereinander zu Informationsasymmetrien kommen, was letztendlich zu Situationen führen kann, in denen sich Abteilungen gegenseitig negativ beeinflussen. Elementen der Matrix-Organisation fehlt der Überblick über das „,große Ganze“, da diesen nur die übergeordnete Geschäftseinheit hat, hier: die Landgard Holding. Mit zunehmender Größe steigt neben dem Kommunikations- und Informationsbedarf auch die Komplexität der Beherrschbarkeit und Steuerung einer Unternehmung mit Gitternetzorganisation (Stöger 2009, S. 37; Oelsnitz 2000, S. 74 f.).

Aus dem Problem der hohen Transaktionskosten der Matrix-Organisation hat sich die „Organisation ergebnisverantwortlicher Einheiten“ entwickelt. Diese Organisationsform von Unternehmen steht für Organisationseinheiten, die allein für ihr Ergebnis verantwortlich sind und deren Schnittstellen zu anderen Organisationseinheiten des Unternehmens klar definiert sind. Dadurch werden sämtliche Abläufe in die alleinige Verantwortung der entsprechenden Organisationseinheit gelegt, was wiederum dazu beiträgt, die Transparenz der Abläufe zu erhöhen. Die Prozesse werden hierbei wieder an den Kunden ausgerichtet, einzelne Subprozesse, Kompetenzen und Verantwortungen dem kundenorientierten Prozess untergeordnet (Stöger 2009, S. 37).

Wie gezeigt, steigt mit der Entwicklung eines Unternehmens auch der Anspruch an dessen formale Strukturen. Die an sich sehr kundenorientierte Form der personalen Organisation kann durch zu viel Unternehmenswachstum Opfer ihres eigenen Erfolges werden. Unternehmensabläufe müssen daher mit dem Unternehmen wachsen. Das bedeutet, dass die Prozesse in regelmäßigen Abständen darauf zu prüfen sind, ob sie noch den strategischen Zielen der Unternehmung entsprechen. Abbildung 42 zeigt diesen Zusammenhang auf.

Für die typischen Ausprägungen der Branche des BuP-Einzelhandels scheint die personale Organisation die am weitesten verbreitete Organisationsform zu sein. Dies erlaubt es wie schon beschrieben, die Leistung sehr nahe am Kunden und an dessen Wünschen zu erstellen, bringt aber auch die einzelnen Unternehmen in die Schwierigkeiten gehemmter Expansionsmöglichkeiten und des Risikos eines Ausfalls der zentral agierenden Person. Besonders deutlich muss in diesem Zusammenhang darauf hingewiesen sein, dass für eine dauerhafte und erfolgreiche Unternehmensentwicklung nicht auf eine Erweiterung der personalen Organisationssteuerung verzichtet werden kann. Wünschenswert wäre dabei eine Organisation aus ergebnisorientierten Einheiten, realistisch muss für den Bereich der BuP-Branche allerdings da- 
von ausgegangen werden, dass für die meisten Einzelhandelsbetriebe eine funktionale Organisationsstruktur der naheliegendste Schritt ist (Lenz 2005, S. 30 f.).

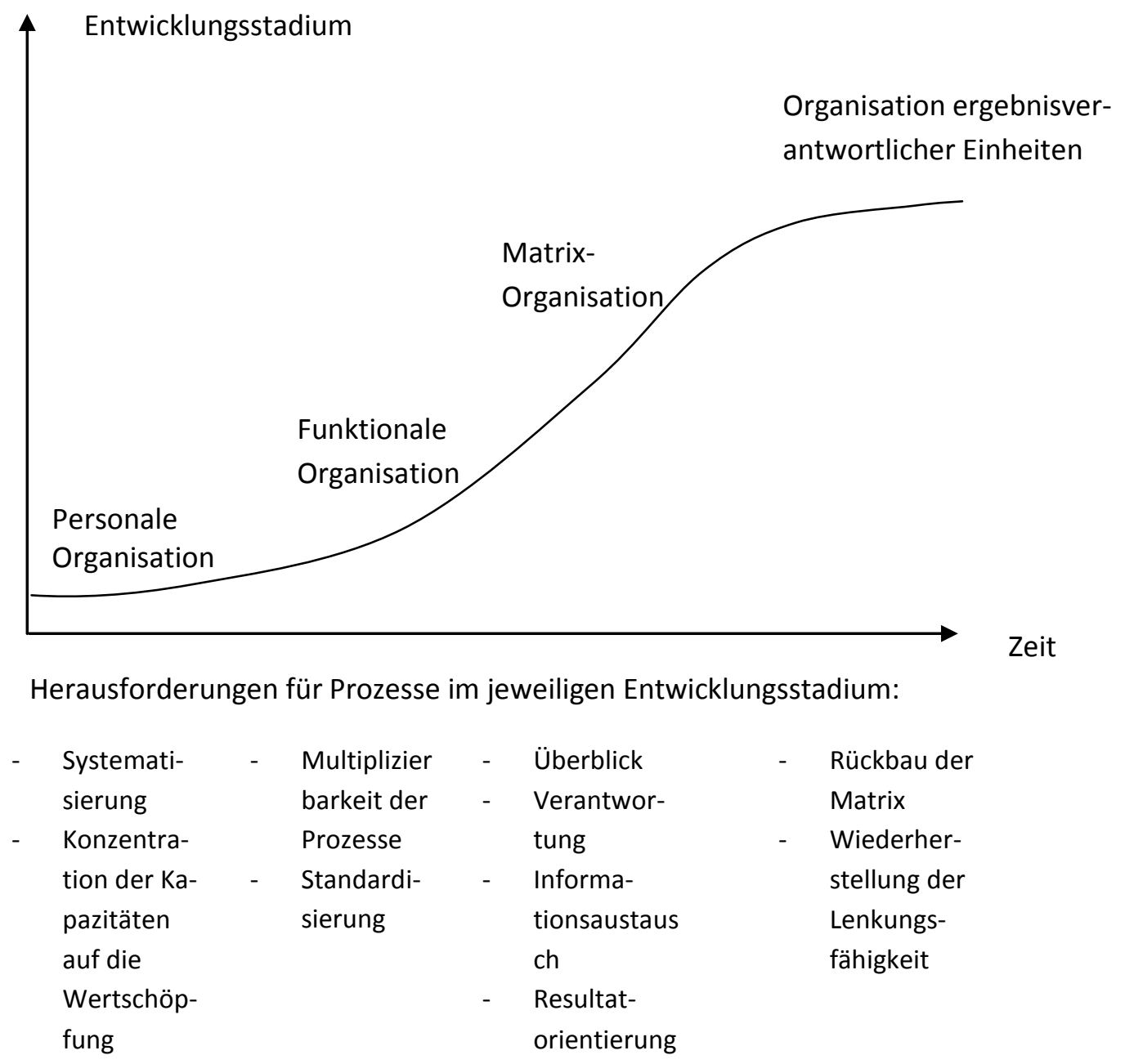

\section{Abbildung 42: Prozess- und Organisationsentwicklung}

Quelle: Stöger 2009, S. 38

\subsection{Physical Evidence}

„Physical Evidence“ stellt den letzten der drei Erweiterungspunkte des klassischen Marketing-Mix dar und steht für die Gestaltung des Verkaufsraumes und der darin präsentierten Waren. Durch den permanenten Geschmackswandel der Konsumenten und deren steigende Ansprüche ist die Gestaltung des Verkaufsraumes hinsichtlich Größe, Ausgestaltung und auch Standort immer wieder neu am Kunden auszurichten. Durch die passende Kombination der 
Stellschrauben der Verkaufsraumgestaltung ist es dem Einzelhandelsbetrieb möglich, Kunden und Interessenten die Attraktivität und die Leistungsfähigkeit der Verkaufsstelle aufzuzeigen. Parallel zur Schaffung einer geeigneten Laden- und Einkaufsatmosphäre müssen bei der Verkaufsraumgestaltung allerdings auch Nutzungsaspekte durch das Personal berücksichtigt werden.

Bei der Verkaufsraumgestaltung zur Schaffung einer geeigneten Einkaufsatmosphäre muss grundsätzlich immer beachtet werden, dass sich der Verkaufsraum seinem Standortumfeld bzw. seiner Zielgruppe in Form des passenden Sortiments und der entsprechenden Preispolitik anpasst (vgl. Kapitel 4.2 und 4.3). Zusätzlich kann die Wahrnehmung der vom Verkaufsraum ausgestrahlten Signale an die Kunden durch die Anordnung der Ladeneinrichtung, der Farbgebung, der Dekoration, der Beleuchtung, der Raumtemperatur, der Gerüche und auch der Geräusche des Verkaufsraumes verstärkt werden. In Summe all dieser großen und kleinen Variationsmöglichkeiten entsteht ein Gesamteindruck der Verkaufsstelle, der den Kunden ansprechen und dessen Interesse am Produkt wecken oder verstärken soll. Dabei ist in gestalterischer Sicht darauf zu achten, dass die Kunden und Interessenten keiner Reizüberflutung ausgesetzt werden. Im Zuge des sogenannten „Store Designs“ müssen vielmehr die einzelnen Atmosphäre-Bausteine so miteinander kombiniert werden, dass die Atmosphäre einerseits das Kaufpotenzial beim Kunden aktiviert und andererseits ein angenehmes Gefühl vermittelt, das zum Verweilen im Verkaufsraum einlädt (Kotler et al. 2006, S. 1150; Berekoven 1995, S. 276 ff.; Esch 2006, S. 74 ff.; Ahlert/Kenning 2007, S. 278). Über die Anordnung der Ladeneinrichtung lässt sich der Verkaufsraum in verschiedene Funktionszonen einteilen, beispielsweise in eine Verkehrsfläche für Kunden, eine Fläche für die Warenpräsentation oder den Kassenbereich. Dabei ist zu beachten, dass in jedem Verkaufsraum auf individuelle Gegebenheiten vor Ort eingegangen werden muss. Dies können einerseits bauliche Aspekte sein (die Raumaufteilung steht schon fest), andererseits auch Gegebenheiten, die von der Branche oder der erwarteten Kundschaft abhängig sind.

Neben den Aspekten der Schaffung einer kaufstimulierenden Atmosphäre muss bei der Gestaltung des Verkaufsraumes auch auf die Nutzungsaspekte des Raumes eingegangen werden, beispielsweise wie viel Raum als Nebenräume und wie viel Raum als Verkaufsraum benötigt bzw. benutzt wird. Typische Nebenräume eines Einzelhandelsgeschäftes sind das Lager, der Verwaltungsbereich, der Personalbereich, die sanitären Anlagen und der Servicebereich, wobei der Servicebereich auch als Teil des Verkaufsraumes angesehen werden kann, je nachdem, wie sehr der Service als Dienstleistung zum Verkauf dazugehört. Im Zuge des Flächen- 
managements gilt es, eine Aufteilung der gesamten zur Verfügung stehenden Fläche zu finden, die aus ökonomischen Gesichtspunkten die beste aller möglichen Lösungen darstellt. Hilfreich sind hierbei auch Kennzahlen, welche die erbrachten oder zu erbringenden Leistungen mit dem Einsatz des Verkaufsladens in Verbindung bringen, beispielsweise Umsatz pro Quadratmeter Verkaufsfläche oder Umsatz pro Quadratmeter Gesamtfläche (vgl. hierzu auch die „Raumleistungskennzahlen“ in Kapitel 2.1). Sind Kennzahlen für die Branche bekannt, so lassen sich daraus Rückschlüsse ziehen, beispielsweise ob die Verkaufsfläche im Verhältnis zum Branchendurchschnitt effektiv genutzt wird, oder sie lassen sich zu Überlegungen im Vorfeld heranziehen, um festzulegen, wie viel Umsatz bei gegebener Verkaufsfläche erreicht werden sollte (Bergener 2008, S. 89; Berekoven 1995, S. 283 ff.; Ahlert/Kenning 2007, S. 269 ff.).

Im Zuge der Einteilung der Verkaufsflächen stellt sich auch das Problem, welche Art der Waren wo im Laden präsentiert werden soll. Dies liegt daran, dass einzelne Bereiche eines Ladengeschäfts unterschiedliche Wertigkeiten im Sinne von Attraktivität für Kunden besitzen. Überlegungen der Warenplatzierung im Einzelhandel basieren daher auf Erkenntnissen über Kundenverhalten im Verkaufsraum. Bekannte Erkenntnisse, die auch in vielen Einzelhandelsgeschäften Auswirkungen auf die Verkaufsraumgestaltung haben, sind beispielsweise:

- Kunden halten sich im Raum rechts und laufen gegen den Uhrzeigersinn.

- Die Ladenmitte und die Ecken des Verkaufsraumes sind schwach frequentiert.

- Kunden richten ihren Blick meist nach rechts.

- Etagenwechsel werden umso unbeliebter, je weiter diese vom Eingang entfernt sind.

Basierend auf dem Kundenverhalten ergeben sich innerhalb des Verkaufsraumes sowohl hoch als auch niedrig frequentierte Bereiche. In den attraktiven bzw. hoch frequentierten Bereichen der Verkaufszone sind die Artikel des Kernsortiments und diejenigen Artikel zu platzieren, denen eine besondere Aufmerksamkeit gewidmet werden soll, beispielsweise Aktionsware. In den weniger frequentierten Bereichen sind entweder solche Artikel zu platzieren, die eine weniger hohe Umschlagshäufigkeit aufweisen und/oder auf Grund ihrer Spezifität größere Bemühungen des Kunden rechtfertigen, beispielsweise gefärbte Glasvasen oder Grußkarten (Esch 2006, S. 78 f.; Berekoven 1995, S. 289 f.).

Neben der Aufteilung der Grundfläche stellt sich die Frage nach der Aufteilung in der Höhe, also die Frage, ob mehrstufige Regale überhaupt benutzt werden und wenn ja, welche Artikel in welcher Höhe positioniert werden. Grundsätzlich gilt dabei, dass die optimale Höhe die 
Sicht- bzw. Griffhöhe des Kunden ist: „eye-level is buy-level““. Da die Verkaufsfläche aber auch begrenzt ist, werden oft mehrstufige Regale benutzt, was wiederum zu unterschiedlichen Wertigkeiten der Präsentationsebenen führt. Analog zur Kundenfrequenz im Verkaufsraum ergeben sich somit auch bei den verschiedenen Präsentationsebenen gute und weniger gute Ebenen für bestimmte Produkte. So sind Artikel, die gefördert werden sollen, in Sichthöhe zu platzieren, teure, qualitativ hochwertige und auch empfindliche Artikel gehören im Regal nach oben. Magnetartikel, große Artikel, Artikel mit viel Gewicht und auffällige Artikel werden im Regal in der unteren Hälfte platziert (Ahlert/Kenning 2007, S. 276; Berekoven 1995, S. 293 f.).

Bezogen auf die BuP-Branche ergeben sich unter dem Gesichtspunkt der Verkaufsraumgestaltung vielerlei Gestaltungsmöglichkeiten, die hier nicht alle erwähnt werden können. Festzuhalten bleibt allerdings, dass die Grundregeln der Raumgestaltung stets beachtet werden sollten und sich die restliche Anpassung des Raumes an den individuellen Gegebenheiten vor Ort orientierten sollte. Für weiterführende Informationen zur Layout-Gestaltung eines Einzelhandelsgeschäfts sei auf entsprechende Literatur zur Gestaltung von Ladenverkaufsräumen verwiesen.

\subsection{Segmentspezifischer Mix der Instrumente}

Wie in diesem Kapitel bisher gezeigt wurde, gibt es mehrere Teilbereiche des Marketings, die in einem letzten Schritt zu einem absatzpolitisch orientierten Mix kombiniert werden müssen. Dabei ist zu beachten, dass es den einen, „optimalen“ Mix nicht gibt. Vielmehr ist die letztendliche Kombination der einzelnen Instrumente im Hinblick auf ihre Intensität und ihre zeitliche Ausdehnung den strategischen Zielen der individuellen Unternehmung unterzuordnen. Jedes Unternehmen muss daher selbst entscheiden, in welcher Art die Instrumente miteinander kombiniert werden, um die entsprechende Zielgruppe bzw. die entsprechenden Zielgruppen zu erreichen und so den Absatz in die erwünschte Richtung zu lenken. Obwohl die einzelnen Instrumente des Marketing-Mix in dieser Arbeit separat vorgestellt wurden, ist ein einzelner, unabhängiger Einsatz eines einzigen Instrumentes nahezu nicht möglich. Einerseits sind die gegenseitigen Wechselwirkungen der Instrumente untereinander zu beachten, andererseits erfordert eine zielgerichtete, segmentspezifische Ansprache einen kombinierten Instrumenteneinsatz. 
Die Auswahl der einzelnen Marketinginstrumente für eine segmentspezifische Kundenansprache stellt für jedes Unternehmen eine große Herausforderung dar, wenn es darum geht, die betrieblichen Ressourcen entsprechend zuzuweisen. Dies liegt zum einen daran, dass es weder einen allgemein gültigen noch einen branchenspezifisch optimalen Instrumenten-Mix gibt, zum anderen daran, dass die erwähnten Interdependenzen zwischen den einzelnen Marketinginstrumenten in ihrer ganzen Komplexität nicht zu überschauen sind. Aufgrund dieser Komplexität, die dem unternehmensindividuellen Marketinginstrumente-Mix zur segmentspezifischen Kundenansprache zu Grunde liegt, lässt sich das Problem des individuell optimalen Marketing-Mix nur stufenweise lösen (Esch 2006, S. 184; Freter 2008, S. 351).

In einem ersten Schritt ist zu klären, welche Instrumente des Marketings dem betreffenden Unternehmen überhaupt zur Verfügung stehen. Bezogen auf Unternehmen der BuP-Einzelhandelsbranche waren dies die sieben Ps, die im bisherigen Verlauf dieses Kapitels vorgestellt wurden. Da die einzelnen Instrumente der sieben Ps in ihrer Wirkung allerdings stark von dem ihnen zu Grunde liegenden Markt abhängig sind, soll für den weiteren Verlauf dieser Erläuterungen festgelegt sein, dass es sich hier um den Markt des Blumen- und Pflanzeneinzelhandels handelt.

Bevor mit den Überlegungen zur Kombination der einzelnen Instrumente begonnen werden kann, muss festgelegt werden, in welcher Phase des sogenannten Produktlebenszyklus sich das Produkt bzw. die Leistung befindet, da die einzelnen Instrumente während der unterschiedlichen Phasen des Produktlebenszyklus unterschiedliche Wirkungen erzielen. Anhand des Produktlebenszyklus wird die Lebensdauer eines Produktes in verschiedenen Phasen dargestellt. Man unterscheidet die Phasen Einführung, Marktdurchdringung, Sättigung und Degeneration. Da das Produkt Zierpflanze bereits seit langem am Markt ist, nahezu „überall“ erhältlich und wenigen technischen Änderungen unterworfen ist, sind Zierpflanzen sehr viel weniger den Auswirkungen des Produktlebenszyklus unterworfen als typische industrielle Ge- oder Verbrauchsgüter wie beispielsweise CD-Player oder Autos. Vor diesem Hintergrund wird auf die Frage des phasenbezogenen Einsatzes der Marketinginstrumente im weiteren Verlauf nicht näher eingegangen. Vielmehr liegt der Fokus der Betrachtung auf der segmentspezifischen Kombination der Instrumente.

Die optimale Zusammenstellung der Marketing-Instrumente ist ein komplexes Problem. Neben den Interdependenzen zwischen den einzelnen Komponenten des Marketing-Mix sehen Kuß und Tomczak hier noch folgende weitere Probleme (Kuß/Tomczak 2002, S. 203 ff.): 
- Die mengenmäßig sehr große Anzahl der konkreten Instrumente der einzelnen Ps des Marketingmix ergeben eine Fülle an theoretischen Kombinationsmöglichkeiten, die nicht mehr alle einzeln näher untersucht werden können.

- Durch die permanente Veränderung bzw. Anpassung von Instrumenten entstehen permanent neue Varianten und Ausprägungen der Instrumente, was das Problem der vielen Kombinationsmöglichkeiten immer mehr verstärkt.

- Falls mehrere Personen für den komplexen Einsatz von Marketinginstrumenten verantwortlich sind, können zwischen ihnen Informationsasymmetrien entstehen, was eine zielgerichtete Kombination der Instrumente weiter erschwert.

Nachdem die einzelnen Aktionsfelder der sieben Ps und die grundsätzlichen Probleme der Instrumentenkombination bekannt sind, werden in einem zweiten Schritt die Ziele definiert, die mit dem Einsatz der Instrumente der einzelnen Marketingbereiche erreicht werden sollen. Dabei sind bei mehreren Zielen diese mit Prioritäten zu versehen und müssen sich die hier definierten Ziele an den im Vorfeld identifizierten Zielsegmenten orientieren (vgl. Kap. 3.2.4). Zu betonen ist hierbei, dass an dieser Stelle noch keine Auswahl konkreter Instrumente erfolgt, sondern lediglich das Ziel bzw. die Ziele formuliert werden. Abbildung 43 zeigt den Zusammenhang der möglichen Ziele mit ihren übergeordneten Marketingbereichen. Sie basiert dabei weitestgehend auf den bisher in Kapitel 4 entwickelten Zielen und ist daher auf die BuP-Branche angepasst.

Bei der Auswahl der Ziele und deren Gewichtung bzw. bei der „Definition des Kernanliegens“ ist wiederum das Problem der gegenseitigen Wechselbeziehungen zu beachten. Zusätzlich muss hierbei allerdings auch die zeitliche Komponente mit berücksichtigt werden, da es zum Erreichen der Ziele unterschiedlich lange dauern kann, bis die eingesetzten Instrumente wirken. Bei einem sehr langen Planungs- bzw. Vorgriffshorizont sind parallel zudem die externen Einflüsse des Marktgeschehens ins Kalkül mit einzubeziehen, da auch sie Einfluss auf das eigene Unternehmen haben, beispielsweise wenn direkte Wettbewerber in den Markt einoder aus diesem austreten. Weiterhin muss bei der Auswahl der Ziele des Marketing-Mix abgewogen werden, in welchem Verhältnis sich die Chancen und Risiken der einzelnen Zielverfolgung bewegen. Basierend auf diesen Überlegungen gilt es, die Budgetierung vorzunehmen, also die Zuteilung der betrieblichen Ressourcen jedweder Art zur Erreichung der einzelnen, gewichteten Ziele (Esch 2006, S. 186 ff.). 


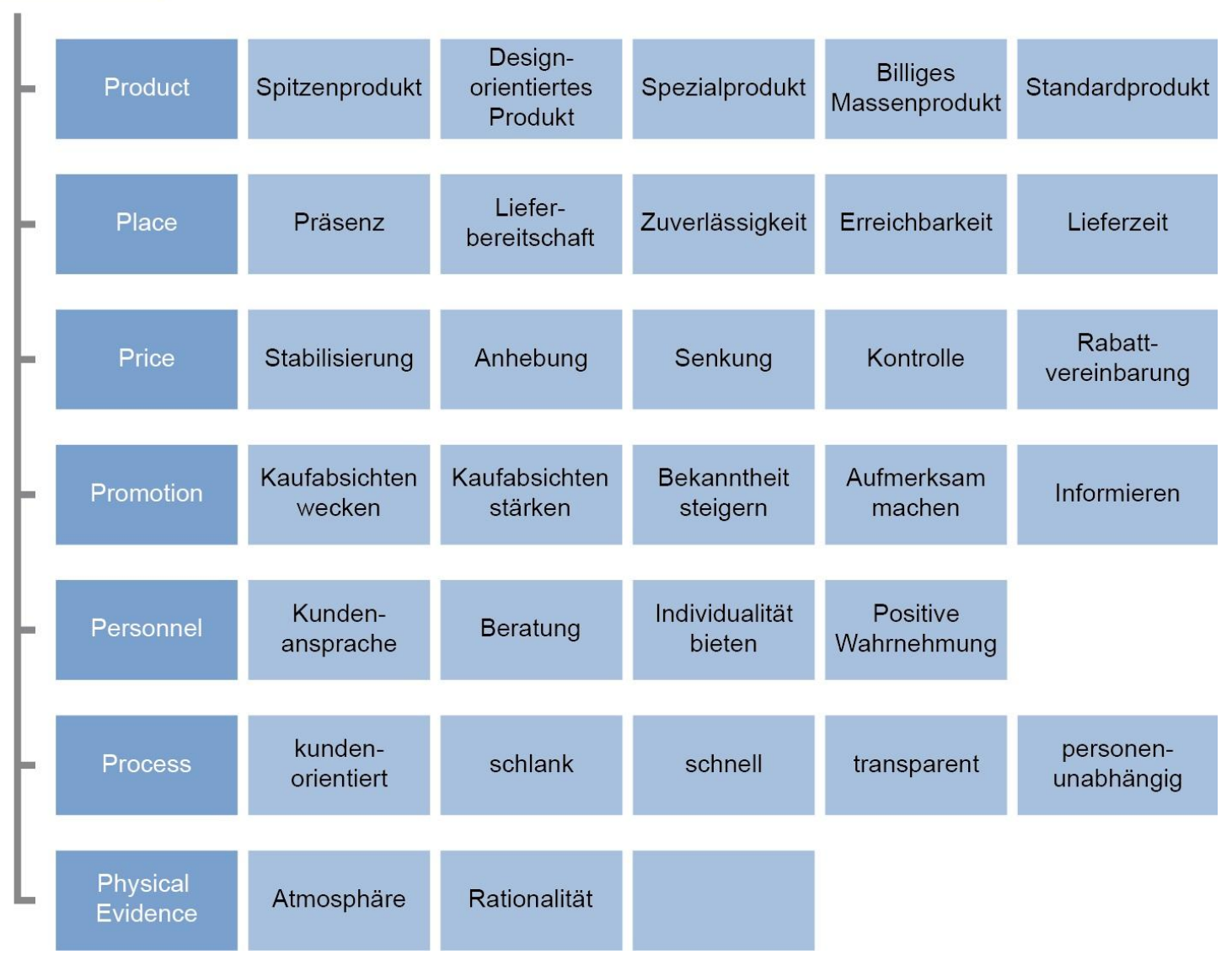

Abbildung 43: Ziele des Instrumenteneinsatzes

Quelle: Eigene Darstellung

Sind diese Überlegungen erwogen und die entsprechenden Entscheidungen getroffen worden, folgt in einem weiteren Schritt die instrumentale Planung zur Erreichung der definierten Ziele. Die Instrumente müssen also dazu geeignet sein, das definierte Bündel an „groben“ Zielen durch den Einsatz von Werkzeugen zu erreichen. Bezogen auf die bisherige Arbeit wird in diesem Schritt nun die Auswahl an denjenigen Instrumenten getätigt, die es erlauben, die aus strategischer Sicht attraktivsten Segmente segmentspezifisch anzusprechen. Abbildung 44 zeigt solche konkreten Instrumente in Bezug zu den bisherigen Überlegungen in dieser Arbeit. Sie erhebt keinen Anspruch auf Vollständigkeit, soll aber die wichtigsten Möglichkeiten darstellen. 


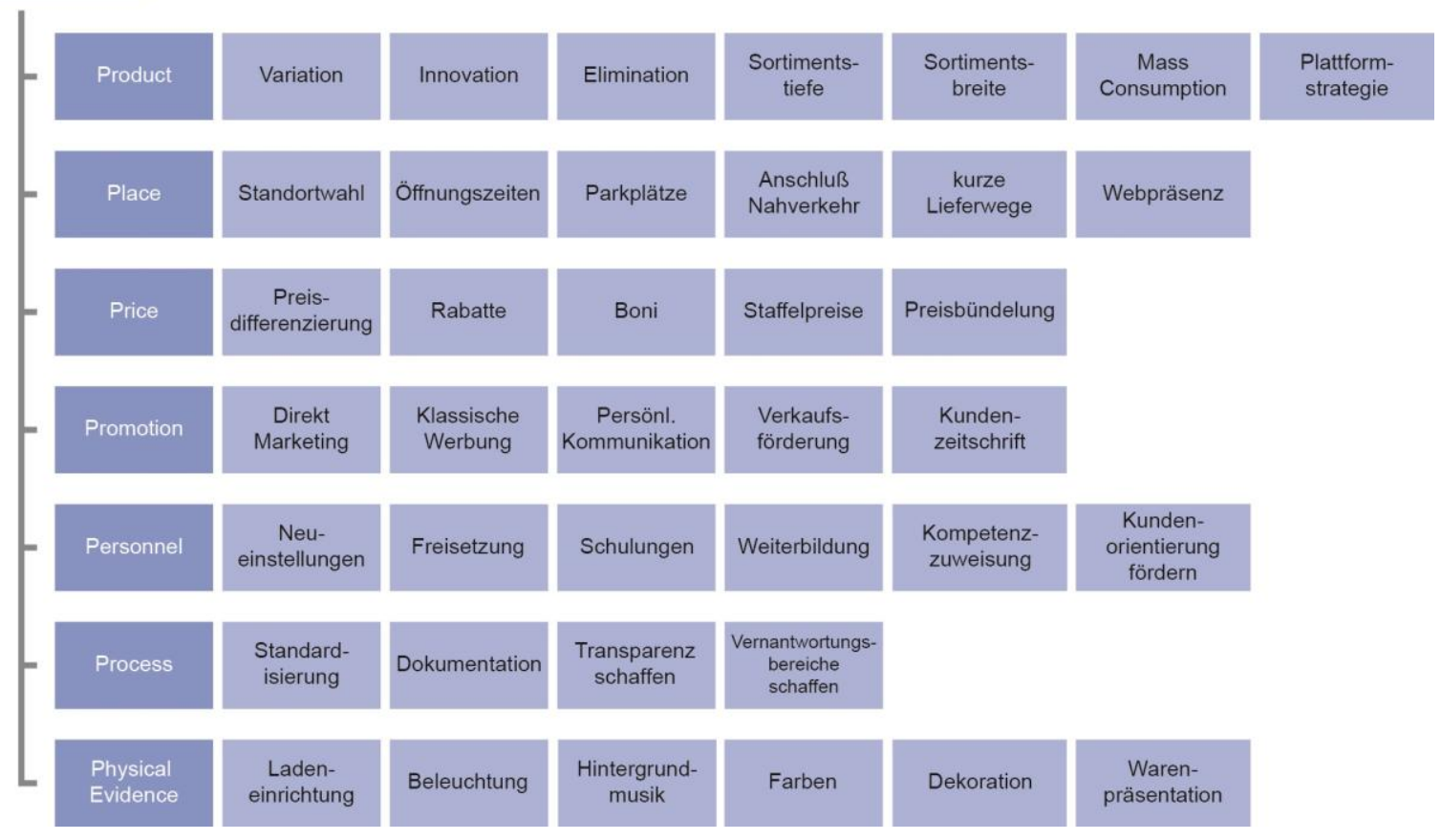

Abbildung 44: Segmentspezifische Instrumente

Quelle: Eigene Darstellung in Anlehnung an Esch 2006, S. 191

Sind die Instrumente ausgewählt, die zur segmentspezifischen Marktbearbeitung herangezogen werden, sind sie in einem letzten Schritt so zu konkretisieren, dass sie direkt umgesetzt werden können. Beispielsweise muss das Instrument Preisdifferenzierung dahingehend weiter konkretisiert werden, dass eine Entscheidung darüber getroffen wird, welche Art der Preisdifferenzierung eingesetzt (z. B. personelle Preisdifferenzierung) und wie diese sichergestellt werden soll (z. B. Ausweiskontrolle). Im Zuge dieses sogenannten Feintunings ist für jedes gewählte Instrument separat zu entscheiden, inwieweit es konkretisiert werden muss. Ziel der Detaillierung ist „lediglich“ das Ableiten der einzelnen Handlungsschritte, die letztendlich eine segmentspezifische Marktbearbeitung ermöglichen und die Wirkung der eingesetzten Werkzeuge auch sichern. Da sich die Umwelt eines Unternehmens in einem permanenten Wandel befindet, darf das Wort „sichern“ in diesem Zusammenhang nicht missinterpretiert werden: Die Sicherung der Werkzeuge bezieht sich auf deren Funktionsweise. Sichern im Sinne von möglichst langfristig einsetzbar machen ist in diesem Kontext zwar nicht falsch, dennoch muss der Prozess des Instrumenteneinsatzes regelmäßig kritisch hinterfragt und permanent an neue Umweltgegebenheiten angepasst werden. 
Welche Instrumente letztendlich für die Branche des BuP-Einzelhandels die vielversprechendsten sind, kann an dieser Stelle nicht beantwortet werden. Vielmehr ist der Instrumenteneinsatz abhängig von der gegebenen Markt- und Kundensituation. Die Instrumente dienen lediglich als Werkzeug dazu, das Unternehmen den externen Anforderungen immer besser anzupassen, um so stets aufs Neue das Überleben des Unternehmens am Markt zu sichern. 


\section{Qualitative Vorstudie}

Ausgehend von der bisher geleisteten theoretischen Vorarbeit liegt die Vermutung nahe, dass der Kaufentscheidungsprozess für BuP von zwei Einflussgruppen bestimmt wird: einerseits vom Umfeld, das durch die anbietenden Einzelhändler zur Verfügung gestellt wird, andererseits von den inneren Werten der Konsumenten.

Unter dem Umfeld, das Einzelhändler den Konsumenten zur Verfügung stellen, sind Faktoren zu verstehen, welche die Konsumenten nicht beeinflussen können und welche für sie als gegeben anzusehen sind. Darunter fallen beispielsweise die Öffnungszeiten, die Erreichbarkeit, die Tiefe und die Breite des Sortiments oder auch die Preispolitik, die der jeweilige Einzelhändler betreibt. Im weiteren Verlauf dieser Arbeit sollen diese verschiedenen Faktoren als aus Sicht des Konsumenten - „externe Faktoren“ bezeichnet werden. Die inneren Werte der Konsumenten werden im weiteren Verlauf dieser Arbeit hingegen als „interne Faktoren“ angesehen. Unter ihnen sind die innerlichen Einflüsse der Konsumenten zu verstehen, die ebenfalls die Kaufentscheidung beeinflussen. Zu den internen Faktoren zählen beispielsweise die Einstellungen der Konsumenten oder deren Werte und Vorstellungen. Für jeden Konsumenten gilt dabei, dass seine individuelle Kaufentscheidung eine Kombination aus seinen internen Faktoren und den ihn umgebenden externen Faktoren darstellt. Nur wenn beide Faktorengruppen miteinander harmonieren, ist eine positive Kaufentscheidung zu erwarten.

Unter Berücksichtigung der Fragestellung, die dieser Arbeit zu Grunde liegt, ergibt sich für die geplante empirische Untersuchung ein Ungleichgewicht bei den Voraussetzungen. Während die externen Faktoren im bisherigen Verlauf dieser Arbeit bereits beschrieben wurden, bietet die Literatur zusätzliches Informationsmaterial, auf das bei der Vorbereitung der empirischen Untersuchung gegebenenfalls zurückgegriffen werden kann. In der geplanten Konsumentenbefragung können den Probanden daher mit überschaubarem Aufwand entsprechende Informationen über die externen Faktoren gegeben werden. Im Gegensatz dazu gibt es in der wissenschaftlichen Literatur bisher nur sehr wenige Erkenntnisse darüber, wie die internen Faktoren die Kaufentscheidung eines Individuums in Bezug auf BuP beeinflussen könnten. Es ist also unklar, in welchem Zusammenhang die individuellen Einstellungen und Werte der Konsumenten mit dem Kaufentscheidungsprozess stehen. Da die internen Faktoren allerdings eine entscheidende Rolle für die geplante lebensstilbasierte Segmentierung der Konsumenten auf der Grundlage der empirischen Untersuchung spielen, sollten dafür im Vorfeld 
wenigstens begründete Annahmen und Erwartungen zu den möglichen Einflussfaktoren vorliegen.

Um diesem Mangel an Informationen im Vorfeld der empirischen Untersuchung zu begegnen, wurde im Rahmen dieser Arbeit, basierend auf der sogenannten „zirkulären Strategie“, eine qualitative Vorstudie durchgeführt. Die zirkuläre Strategie besagt, dass eine zuvor definierte Reihe an Forschungsschritten „mehrmals durchlaufen wird und der jeweils nächste Schritt von den Ergebnissen des jeweils vorherigen abhängt“" (Witt 2001, Abs. 15; Lamnek 2005, S. 194; Mayer 2006, S. 22f.). Abbildung 45 verdeutlicht den Ansatz der zirkulären Strategie (Witt 2001).

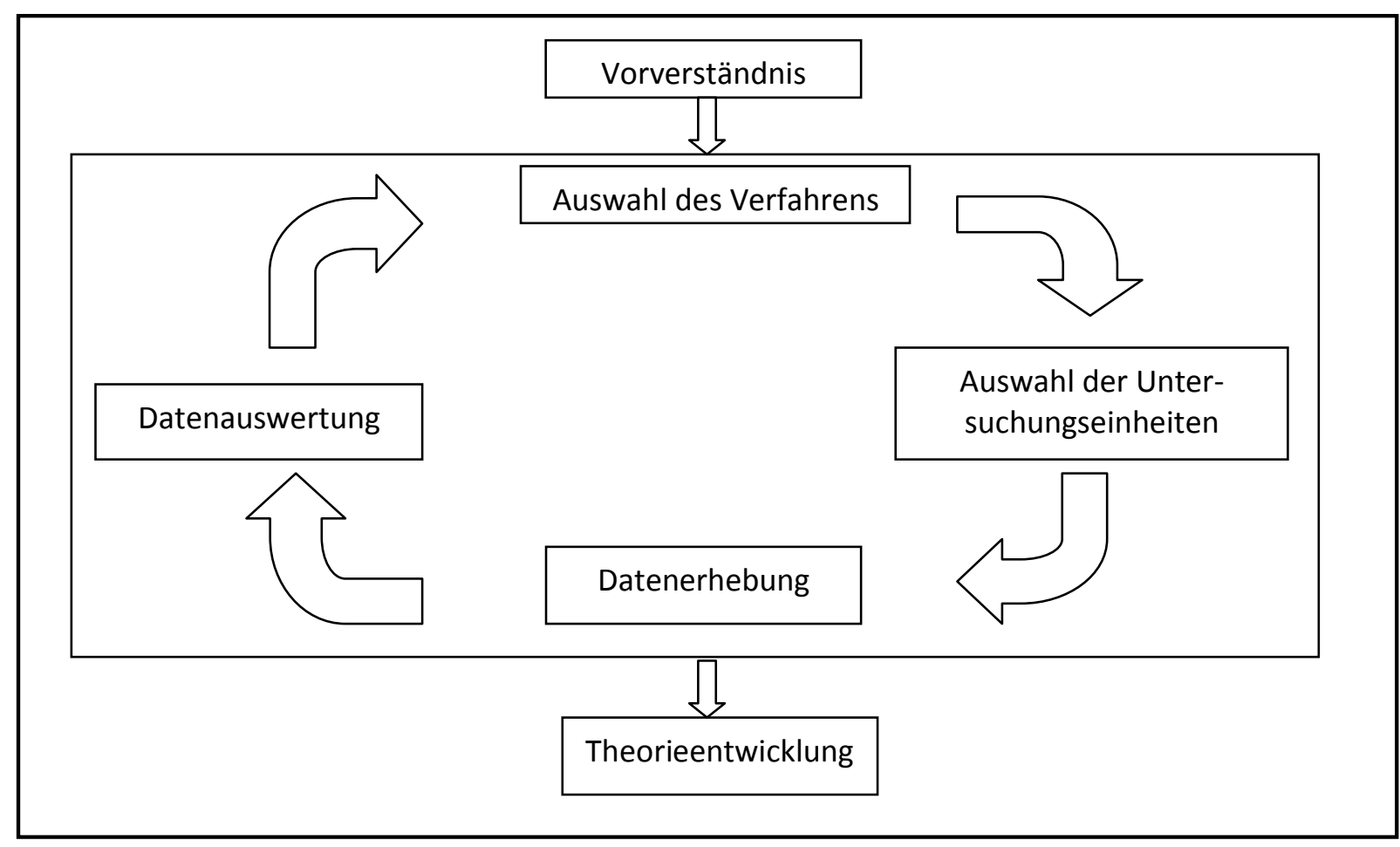

Abbildung 45: Zirkuläre Strategie

Quelle: Witt 2001

Die zirkuläre Strategie eignet sich nach Witt besonders für Situationen, in denen zu Beginn der Forschung nur ein vages Grundverständnis über den Forschungsgegenstand vorhanden ist. Basierend auf diesem Grundverständnis werden die einzelnen Schritte der zirkulären Strategie durchlaufen. Die Schritte sind wie folgt:

1. Auswahl des Verfahrens

2. Auswahl der Untersuchungseinheiten 


\section{Datenerhebung}

\section{Datenauswertung}

Dabei ist zu beachten, dass sich die einzelnen Schritte untereinander sowohl für das weitere Vorgehen als auch für die Konzeption der Untersuchung wechselseitig beeinflussen können, beispielsweise über eine auf Erkenntnissen der bisherigen Datenauswertung basierende Verbesserung der Fragestellung. Des Weiteren ist zu beachten, dass die zirkuläre Strategie nicht mit einmaligem Durchlaufen seinen Zweck erfüllt hat. Gerade das mehrmalige Durchlaufen des Regelkreises und die permanente Modifikation der einzelnen Schritte anhand der bereits erkannten Zusammenhänge festigen die Erkenntnisse, auf denen ein Theoriemodell aufgebaut werden kann. Ein bestimmtes Ende der Durchläufe ist nicht gegeben, vielmehr muss dies vom Anwender selbst entschieden werden. Allerdings ist von weiteren Kreisläufen abzusehen, wenn der zusätzlich generierte Informationsgewinn in keinem adäquaten Verhältnis mehr zum verursachten Aufwand steht.

\subsection{Methoden der qualitativen Untersuchung}

Im Rahmen dieser Arbeit wurde die qualitative Vorstudie zur empirischen Erhebung mit Hilfe von explorativen Einzelinterviews durchgeführt. Explorative Interviews, auch „Tiefeninterviews" genannt, sind besonders wertvoll, wenn das Forschungsvorhaben noch in der Anfangsphase steckt und wenn es dabei „um die Präzisierung von Entscheidungs- und Forschungsproblemen“" geht (Böhler 2004, S 87). Im Kontext der zirkulären Strategie entspricht die Entscheidung für diese Methodik dem Punkt „Auswahl des Verfahrens“, wobei die Interviewten die Untersuchungseinheiten darstellen und die Informationen, die während des Interviews gesammelt werden, der Datenerhebung entsprechen.

Die Durchführung der qualitativen Vorstudie soll im Vorfeld der empirischen Hauptstudie dieser Arbeit somit das Verständnis über das Denken und Fühlen der Probanden erhöhen. Des Weiteren dient die qualitative Vorstudie dazu, die internen Faktoren der Befragten, also deren Einstellungen, Werte, Erinnerungen und Erfahrungen, besser abschätzen zu können. Das explorative Interview besteht primär aus indirekten Fragen an den jeweiligen Probanden. So soll dessen erzählerisches Moment unterstrichen werden. Es dient wiederum dazu, möglichst viel über persönliche Meinung, Wissen, Informationen, Erfahrungen und Einstellungen des Befragten zu erfahren (Mayer 2006, S. 36 ff.; Berekoven 2006, S. 98; Weis 2008, S. 35 ff.). Das 
explorative Interview eignet sich somit sehr gut als Methode für die Vorstudie im Rahmen dieser Arbeit.

\subsubsection{Theorie zum explorativen Interview}

In der wissenschaftlichen Literatur werden mehrere Begriffe synonym für das explorative Interview gebraucht: „qualitatives (offenes) Interview“, „psychologische Exploration“ oder „Tiefeninterview“ (Salcher 1995, S. 31). Das Tiefeninterview wird nach Salcher definiert als „ein langes und intensives Gespräch zwischen Interviewer und Befragtem über vorgegebene Themen, das der Interviewer in weitgehend eigener Regie so zu steuern versucht, dass er möglichst alle relevanten Einstellungen und Meinungen der befragten Person zu diesem Thema erfährt, auch wenn es sich um Aspekte handelt, die der befragten Person bis zu diesem Zeitpunkt selbst nicht klar bewusst waren“ (Salcher 1995, S. 34).

Der Interviewer versucht daher das Tiefeninterview „zu steuern“. Dies kann prinzipiell nach zwei verschiedenen Methoden geschehen: anhand einer teil- oder anhand einer nicht standardisierten Befragung des Interviewpartners (Böhler 2004, S. 87). Dem Tiefeninterview liegt also kein starrer Fragekatalog zu Grunde, in dessen thematischem Korsett sich der Befragte eventuell eingeengt fühlen könnte. Vielmehr bedient sich das Tiefeninterview der Methode, dass lediglich ein Rahmenthema vorgegeben ist, worüber sich Interviewer und Interviewter frei unterhalten. Hat der Interviewer innerhalb des Rahmenthemas sämtliche Freiheiten zur Befragung, spricht man von einem „nicht standardisierten Interview“. Alternativ kann sich der Interviewer innerhalb des Rahmenthemas an einem grob strukturierten Frageschema orientieren. Dieses Schema, dem der Interviewer gegebenenfalls folgt, bezeichnet man als „Interviewleitfaden“. Der Leitfaden gibt dem Interviewer weder die Reihenfolge der Fragen noch deren exakte Ausformulierung vor, er dient lediglich als thematischer „roter Faden“, um das Interview zu führen. Kommt bei einem Tiefeninterview ein Leitfaden zum Einsatz, spricht man von einer „teilstandardisierten Befragung“ oder auch von einem „Leitfadeninterview“. Der Leitfaden zeichnet sich dadurch aus, dass seine Fragen offen formuliert sind. So soll die interviewte Person zum freien Erzählen animiert werden (Böhler 2004, S. 87; Mayer 2006, S. 36).

Bei der Durchführung von mehreren Interviews erhöht der konsequente Einsatz eines Leitfadens die Vergleichbarkeit der gewonnenen Informationen. Des Weiteren erhalten die erhobenen Daten durch den Leitfaden eine Struktur, was bei ihrer Auswertung und Interpretation 
hilfreich ist. Der Leitfaden dient dem Interviewer als Hilfestellung: Er soll gewährleisten, dass der Interviewer keine wesentlichen Dinge vergisst, die im Rahmen des Gesprächs zur Sprache kommen sollen. Im Rahmen des freien Gesprächs muss der Interviewer dabei selbst entscheiden, wann er näher ins Detail geht, den Befragten weitererzählen lässt, bei bestimmten Punkten nachfragt oder zum Leitfaden zurückkehrt, wenn der Befragte in themenfremde Bereiche abschweift (Flick 1999, S. 112; Friebertshäuser 1997, S. 367 f.; Mayer 2006, S. 36 f.).

Basierend auf den bisherigen Ausführungen scheint das explorative Interview für die Vorstudie zur hier anstehenden empirischen Forschungsfrage als geeignet. Im Folgenden werden die Vorteile des explorativen Interviews nochmals in kurzer Form dargestellt:

\section{Vorteile}

- Die einzelnen gesammelten Daten und Informationen sind bestimmten Personen direkt zuzuordnen.

- Der Verbraucher wird direkt befragt.

- Es besteht die Möglichkeit, dass Informationen über die Befragten zu Tage kommen, über die sich die Befragten selbst nicht im Klaren waren.

- Man erhält vollständige Argumentations- und somit auch Gedankengänge der Befragten.

- Der Interviewer wird durch seine Rolle als Fragensteller dazu „gezwungen“, das ihm bekannte Thema von „der anderen Seite“ aus zu betrachten.

Neben den aufgezählten Vorteilen gilt es allerdings auch einige Nachteile zu berïcksichtigen, die im Folgenden aufgelistet sind:

\section{Nachteile}

- Bei mehreren befragten Personen ergibt sich das Problem der repräsentativen Auswahl.

- Die Ergebnisse sind trotz Leitfaden nicht exakt vergleichbar und nicht exakt gleich strukturiert.

- Es bedeutet einen hohen Zeitaufwand und somit hohe finanzielle Kosten pro Interview.

- Die Auskunftspersonen müssen in der Lage sein, das zu artikulieren, was sie meinen.

- Es bestehen hohe psychologische Anforderungen an den Interviewer, was die Führung des Interviews betrifft.

- Der Interviewer muss sich mit der Materie auskennen. 
(Salcher 1995, S. 37 f.; Kuss 2010, S. 141)

\subsubsection{Theorie zu den interne Faktoren}

Wie bereits beschrieben, fließen neben externen Faktoren auch interne Faktoren der Individuen in den Kaufentscheidungsprozess ein. Im Folgenden werden diese auf theoretischer Basis näher betrachtet. Es sei aber bereits hier erwähnt, dass zwischen den internen Faktoren starke Wechselbeziehungen auftreten. Persönliche Einstellungen, Werte, Erinnerungen und gemachte Erfahrungen können nicht jeweils isoliert voneinander betrachtet werden.

Werte bzw. die persönliche Wertehaltung der Individuen spielen innerhalb der internen Faktoren eine entscheidende Rolle. Nach Schwartz versteht man unter Werten „Konzepte, welche die Art bestimmen, wie soziale Akteure ihre Aktionen auswählen, Menschen und Ereignisse bewerten und ihre Aktionen und Bewertungen erklären“ (Schwartz 1999, S. 24). Eigene, individuelle Werthaltungen speisen sich nach Schwartz einerseits aus den Werten der Kultur, welche die Individuen umgibt, andererseits aus einzelnen, persönlich gemachten Erfahrungen. Aus den ihnen innewohnenden Werten leiten Individuen Heuristiken im Sinne von Verhaltensrichtlinien bei Entscheidungsfindungsprozessen ab. Man spricht dabei auch von „Normen“. Da Personen innerhalb eines Kulturkreises naturgemäß unterschiedliche persönliche Erfahrungen während ihres Lebens gesammelt haben, unterscheiden sich die Werthaltungen der Individuen des gleichen Kulturkreises. Allerdings ist zu erwarten, dass sich die gemeinsam erfahrene Kultur zumindest als grober Wertekompass in allen entsprechenden Individuen wiederfindet. Neben dem kulturellen Umfeld einer Person spielt in gleicher Art und Weise auch deren soziales Umfeld bzw. soziales Gefüge eine wichtige Rolle bei der Entstehung von individuellen Werten. Dabei gilt es zu beachten, dass je engmaschiger das soziale Gefüge ist, das die Individuen umgibt, desto übereinstimmender wiederum deren Werte und Einstellungen sind (Schwartz 1999, S. 23-47; Scherer/Cho 2003, S. 261-267; Trommsdorff/Teichert 2011, S. 151 ff.).

Neben Werten spielen auch Einstellungen eine entscheidende Rolle bei den internen Faktoren. Unter „Einstellung“ versteht man nach Tromsdorff den ,Zustand einer gelernten und relativ dauerhaften Bereitschaft, in einer entsprechenden Situation gegenüber dem betreffenden Objekt regelmäßig mehr oder weniger stark positiv beziehungsweise negativ zu reagieren“ (Trommsdorff 1998, S. 142, 152). Dabei können die „Objekte“, zu denen Einstellungen bestehen, sowohl reale, physische Güter sein als auch Personen, geographische Orte oder Situa- 
tionen, in denen man sich befindet. Bei der Entstehung von Einstellungen gegenüber einem Objekt steht das betrachtete Objekt im Zentrum der Wahrnehmung des Betrachters, so wie es sich dem Betrachter in dessen objektiver und psychologischer Realität darstellt. Positive oder negative Einstellungen dem Objekt gegenüber entstehen dadurch, dass im Hinblick auf dieses Objekt ein Vergleich zwischen den objektiven und den psychologischen Inhalten des Objekts stattfindet. Je stärker die gebildeten Eigenschaftsdimensionen wie gut/schlecht, nachteilig/vorteilhaft, angenehm/unangenehm oder sympathisch/unsympathisch sind, desto stabiler erweisen sich die Einstellungen im Laufe der Zeit. Auch steigt mit der Stärke der Einstellung die Resistenz der Individuen gegenüber Überzeugungsversuchen von außen. Starke Einstellungen gehen somit einem offenkundigen Verhalten voraus (Ajzen 2001; Frewer et al. 1998, S. 15-30; Freter 2008, S. 72; Kroeber-Riel/Weinberg 1996, S. 196 ff.). Eine stark ausgeprägte Einstellung eines Individuums einem Objekt gegenüber ist besonders dann zu erwarten, wenn das Individuum sich bereits über einen längeren Zeitraum mit dem Objekt beschäftigt hat. Im Umkehrschluss sind Einstellungen von Individuen umso fragiler und damit umso formbarer, je mehr das entsprechende Objekt dem Individuum noch unbekannt ist (Fazio et al. 1982, S. 339-357; Tenbült et al. 2008, S. 622-635).

In Zusammenhang mit der hier zu Grunde liegenden Forschungsarbeit besteht die Relevanz von Einstellungen und Werten darin, dass sie dafür ausschlaggebend sind, wie BuP an sich eingeschätzt und wie deren einzelne Merkmalsausprägungen wahrgenommen werden. Eine möglichst genaue Erfassung der Einstellungen gegenüber BuP ist somit von entscheidendem Erfolg für die zu beantwortende Forschungsfrage dieser Arbeit.

\subsection{Rahmendaten der Interviews}

Zur Durchführung der qualitativen Vorstudie wurden insgesamt elf Tiefeninterviews durchgeführt. Diese fanden je nach Wunsch der Teilnehmer entweder bei ihnen zu Hause oder an einem Ort ihrer Wahl statt. Rekrutiert wurden die Teilnehmer sowohl über direkte wahllose Ansprache vor einem Baumarkt als auch über eine Zeitungsanzeige, die eine „Aufwandsentschädigung“ in Form eines Gutscheins über 10 Euro bei einem regionalen BuP-Einzelhändler anbot. Als Thema wurde in der Anzeige nur grob „Haus und Garten“ angegeben. Die Teilbezeichnung des Forschungsvorhabens „lebensstilbasierte Kundensegmentierung“ wurde bewusst nicht verwendet, um eine Selbstselektion der Teilnehmer im Vorfeld zu umgehen. Die Interviews fanden zwischen Dezember 2012 und Februar 2013 statt. Alle Interviews wurden dabei akustisch aufgezeichnet, wobei sich der Interviewer während des Interviews darüber 
hinaus noch Gesprächsnotizen machte. In einem zeitlich kurzen Abstand zu jedem einzelnen Interview wurde ein entsprechender Interviewbericht verfasst, da so noch möglichst viele Erinnerungen, Eindrücke und nonverbale Reaktionen der Probanden aus dem Gedächtnis des Interviewers berücksichtigt werden konnten. Sämtliche Berichte wurden am Ende der Interviewphase nochmals zu einem „großen“ Bericht verdichtet, so dass Gemeinsamkeiten oder auch Unterschiede der verschiedenen Interviewpartner deutlich wurden. Zu diesem Zweck wurde eine Tabelle erstellt, welche die Kernaussagen eines jeden Interviews zu jeder einzelnen Frage zusammenfasst. Auf diese Art entstand ein Fundus an Informationen, der hierarchisch aufgebaut war: von der einzelnen Antwort eines Probanden auf jede einzelne Frage über den einzelnen Interviewbericht bis zum letztendlich verdichteten Bericht aller Einzelberichte in Form einer großen Tabelle.

Eine separate, nachträgliche Abschrift der Wortlaute der Interviews der Vorstudie fand nicht statt. Dies liegt zum einen darin begründet, dass der Interviewer selbst die qualitativen Analysen der geführten Interviews vornahm. Dazu lagen ihm neben den selbst gesammelten Eindrücken ihre Gesprächsnotizen vor, die er während der Interviews gemacht hatte. Zusätzlich stand neben den einzelnen Interviewberichten auch noch die Tabelle mit den verdichteten Aussagen der Probanden bei der Analyse zu Verfügung. Zum anderen bestand für den Interviewer im Falle einer Unsicherheit jederzeit die Möglichkeit, anhand der Tonaufnahmen der entsprechenden Interviews den genauen Wortlaut noch einmal zu rezipieren. Zwar werden in der wissenschaftlichen Literatur zur Auswertung von Interviews Transkriptionen meist als nötig angesehen (Mayer 2006, S. 46 ff.; Mayring 1999, S. 68 ff.), eine Transkription der Interviews wurde im frühen Stadium der Forschungsfrage allerdings auch deshalb nicht für unbedingt erforderlich gehalten, weil dieses Vorgehen die Analyse nicht signifikant verbessert hätte. Ziel der Befragung war wie erwähnt primär eine lose Sammlung von Ideen, die es erlauben sollte, erste Erkenntnisse darüber zu gewinnen, wie das Themengebiet sich aus Konsumentensicht darstellt. „Qualitative analysis can range from summarizing the discussion, to identifying themes, to elaborate coding schemes. The type of analysis depends on how the resulting information is or be used" (Goldmann/McDonald 1987, in Fern 2001, S. 92; Gläser/Laudel 2006, S. 188 ff.).

Grundlage der Analyse der Interviews war ein Schema nach Goldmann und McDonald, die für die Analyse von qualitativen Interviews durch den Interviewer selbst folgende Punkte als wichtig erachten (Fern 2001, S. 92):

1. Reihenfolge, in welcher Themen diskutiert werden 
2. Intensität oder Stärke der (beobachteten) Gefühle

3. Gründe hinter den Gefühlen

4. Irreführung

5. Allgemeingültigkeit

Während jeder qualitativen Analyse eines Interviews der Vorstudie wurde dieses Schema beachtet.

\subsection{Durchführung der Interviews}

Den geführten Interviews der qualitativen Vorstudie lag wie beschrieben ein Leitfaden zu Grunde. Durch die thematische Vorstrukturierung der anstehenden Gespräche sollte sichergestellt werden, dass möglichst alle als relevant erachteten Aspekte des Realitätsausschnitts aus dem Bereich Blumen und Pflanzen angesprochen werden. „Der Leitfaden schneidet die interessanten Themen aus dem Horizont möglicher Gesprächsthemen heraus und dient dazu, das Interview auf diese Themen zu fokussieren“(Meuser/Nagel 1997, S. 488).

Der zum Einsatz gekommene Interviewleitfaden gliedert sich inhaltlich und thematisch in fünf verschiedene Phasen bzw. Bereiche auf:

1. Kennenlernen

2. Einstellung zu BuP

3. Reale Kaufsituationen

4. Motive

5. Segmentierungskriterien.

Die Interviews wurden mit Hilfe eines digitalen Audiorekorders aufgezeichnet. Außer diesem und einem Notizblock kamen keinerlei andere Hilfsmittel oder Materialien während der Interviews zum Einsatz. Nach drei geführten Interviews wurde der Leitfaden nochmals kritisch auf seine Geeignetheit überprüft. Da während der bis dato geführten Interviews allerdings keinerlei relevanten Beeinträchtigungen zum Tragen gekommen waren, wurde der Leitfaden in nur sehr geringem Ausmaß geändert. Die vorgenommenen Änderungen betrafen lediglich Details der Rechtschreibung, weshalb im Weiteren ausschließlich auf den finalen Leitfaden vom 30.01.2013 eingegangen wird. Dieser ist im Anhang einzusehen und wird im Folgenden näher vorgestellt. Die Reihenfolge orientiert sich dabei nicht an den Zeitpunkten, zu denen die einzelnen Themen zur Sprache kamen, sondern an der obigen Aufzählung der verschiedenen Themenbereiche des Leitfadens. 
Basierend auf der Tatsache, dass sich beim Aufeinandertreffen von Interviewer und Interviewtem zwei Personen gegenüberstehen, die sich fremd sind, ist es im allerersten Schritt notwendig, sich kennenzulernen bzw. sich erst einmal vorzustellen. Es erschien für den Interviewer sinnvoll, zuerst einmal einige Angaben zur eigenen Person zu machen (Name, Alter, Beruf, Wohnort), damit sich die Interviewten überzeugen konnten, dass der Interviewer nicht anonym bleiben möchte und auch aus der gleichen Region kommt. Somit sollte eine erste Gemeinsamkeit festgestellt werden. Dazu sei erwähnt, dass den Befragten während des Vorstellens das Informationsschreiben zum Durchlesen überreicht wurde, das im Anhang einsehbar ist (Deckblatt des Leitfadens). Dazu wurde den Befragten nochmals mündlich versichert, dass alle Daten und Informationen nur in anonymisierter Form verarbeitet werden. Trotz des an sich nicht sehr heiklen Themas des Interviews wurde dieser Punkt zu Beginn eines jeden Interviews offensiv von Seiten des Interviewers angesprochen - einerseits um eventuell doch vorhandene Sorgen diesbezüglich zu zerstreuen, andererseits um die Professionalität des geführten Interviews zu unterstreichen. Im Anschluss daran wurde nochmals das in der Zeitungsannonce angegebene Thema genannt („Haus und Garten“), um thematisch den ersten Schritt in Richtung Blumen und Pflanzen zu vollziehen.

Wenn das Interview bei den Befragten zu Hause stattfand, wurde versucht, gleich zu Beginn mögliche Anknüpfungspunkte aus deren Wohnumfeld zu erkennen, um einen direkten, persönlichen Bezug des Interviewten zum Thema zu schaffen, beispielsweise über den thematischen Brückenschlag: ,... will ich mich mit Ihnen heute über das Thema Haus und Garten unterhalten. Dabei besonders über Blumen und Pflanzen im oder um Ihr Haus herum. Und wie ich sehe, haben Sie in Ihrem Flur ja auch eine schöne Pflanze stehen. Wie lange haben Sie diese Pflanze denn schon?“ Durch die Ansprache der Befragten auf ein Thema aus ,ihrer Welt" in Verbindung mit einer leicht zu beantwortenden Frage sollte den Interviewten der Einstieg ins Erzählen möglichst leicht gemacht werden. Auch sollte so eine Signalwirkung erzeugt werden, die den Befragten zu Beginn erkennen ließ, dass das anstehende Interview in keiner Weise schwierig oder anstrengend werden könnte. Ziel des Vorstellens war es stets, eine angenehme, persönliche und ungezwungene Gesprächsatmosphäre aufzubauen.

Nach erfolgtem Einstieg in ein locker geführtes Gespräch über Blumen und Pflanzen diente der zweite Bereich des Leitfadens dazu, im Laufe des Gesprächs dem Thema „Einstellung der Interviewten zu Blumen und Pflanzen“ näherzukommen. Dabei wurde nach Möglichkeit mit der Frage „Welche Blumen oder Pflanzen haben Sie zur Zeit bei sich noch im Haus oder Garten?“ versucht, thematisch an die Einstiegsfrage anzuknüpfen. Sowohl diese Frage als auch 
die Fragen nach deren Pflege und die Frage nach der Freude an Blumen und Pflanzen sollten es dem Interviewer ermöglichen, neben den direkten Antworten auf die Fragen anhand der Wortwahl zu erkennen, wie hoch in etwa der Grad des Involvements der Befragten ist. Des Weiteren wurden die Interviewten nach ihren ersten Assoziationen in Zusammenhang mit Blumen und Pflanzen gefragt sowie nach den unterschiedlichen Präferenzen für Schnittblumen und Topfpflanzen (,Wieso erfreuen sich manche Menschen mehr an Schnittblumen als an Topfpflanzen und andere mehr an Topfpflanzen als an Schnittblumen?“). Der Grund für eine derart offen gestellte Frage nach den Assoziationen war, möglichst einen Eindruck davon zu bekommen, welche Werte, Überzeugungen oder Einstellungen für die Befragten mit Blumen und Pflanzen in Verbindung stehen. So sollte dem Interviewer ein Anhaltspunkt gegeben werden, wie die Befragten an sich auf Blumen und Pflanzen reagieren und welche Kriterien zur Beurteilung von Blumen und Pflanzen sie kennen und gegebenenfalls selbst anwenden. Weitere Fragen in diesem Bereich bezogen sich auf die Verwendung von Blumen und Pflanzen als Teil der Wohnungseinrichtung und als „Werkzeug“ zur Beeinflussung des Raumklimas. Des Weiteren folgten Fragen zur Abhängigkeit der Freizeitgestaltung vom Wetter und zur Gestaltung von Friedhofgräber. Die Intention hierbei war festzustellen, inwieweit das Thema „Blumen und Pflanzen“ für die Befragten vom Wetter abhängt und inwieweit die Arbeit damit als belastende oder erfreuliche Beschäftigung empfunden wird.

Im Themengebiet „Reale Kaufsituation“ wurde versucht, mit den Befragten deren letzte konkrete Kaufsituation einer Blume oder Pflanze zu rekonstruieren. Die Befragten wurden gebeten, detailliert von ihrem letzten Kauf zu erzählen („Erzählen Sie mir von Ihrem letzten Kauf. Wie lief der ab?“). Anhand des Leitfadens achtete der Interviewer darauf, dass möglichst alle Teilaspekte des Themenbereichs vom Befragten erwähnt wurden (,Da gab es sicher Alternativen zum Gekauften. Was war ausschlaggebend für Ihre letztliche Entscheidung?“). Der Interviewer versuchte dabei zu erfahren, wie sich der Kauf angebahnt hatte, ob bzw. wie sich die Befragten vor dem Kauf informiert hatten, wie sich die Kaufentscheidung entwickelte, wo der Kauf stattfand und was letztendlich gekauft wurde. Die Intention der zugehörigen Fragen war es, unter anderem zu erfahren, welche externen Faktoren die Befragten während des Prozesses der Kaufentscheidung wahrnahmen und welche davon für sie letztlich relevant waren. Konkrete Fragen dieses Themenbereiches waren beispielsweise, ob verschiedene Qualitäten im Einzelhandel angeboten werden oder inwieweit der Preis eine wichtige Rolle bei der Kaufentscheidung spielte. Des Weiteren war von Interesse, ob vor dem Kauf ein Mangel an Blumen oder Pflanzen empfunden wurde oder ob der Kauf impulsiv im Sinne von ungeplant 
stattfand. Im Mittelpunkt dieser Interviewphase stand für den Interviewer die Frage, welche externen Faktoren die Käufer kennen, bewusst wahrnehmen und schätzen. Weiterhin sollte die Frage beantwortet werden, welche dieser externen Faktoren für die jeweiligen Befragten von besonderem Interesse waren und wovon sich die Befragten besonders angezogen fühlten bzw. welche externen Faktoren für die jeweils Befragten keine entscheidende Rolle spielten.

Neben den bereits beschriebenen Themenbereichen des Interviews sollten im Bereich „Motive“ noch weitere, bisher eventuell noch nicht angesprochene interne Faktoren der Befragten erkannt werden. So wurde versucht zu erörtern, inwieweit es für die Befragten eine Rolle spielt, ob eine Blume oder Pflanze einen konkreten Nutzen als Mehrwert aufweist (z. B. Topfkräuter: zur Dekoration und zum Kochen). Des Weiteren wurde als Kaufmotiv „Es ist ein Geschenk“ vorgegeben. Der Interviewer versuchte zu erfahren, ob sich Kaufmotive in Abhängigkeit vom künftigen Besitzer der Blume oder Pflanze wandeln (,Wie ändert sich Ihre Einstellung zum Nutzen, wenn Sie die Blume oder Pflanze als Geschenk kaufen?“). Die Intention dieser Fragen lag dabei nicht nur darin, eine Abwägung zwischen Ästhetik und Nutzen von Blumen und Pflanzen aufgezeigt zu bekommen. Vielmehr sollten auch Rückschlüsse gezogen werden, inwieweit sich interne Faktoren ändern, wenn die Befragten den Kauf nicht für den Eigenverbrauch tätigen, sondern die Blume oder Pflanze als Geschenk für eine andere Person kaufen.

Im letzten Themengebiet des Interviews, „Segmentierungskriterien“, wurde primär versucht, über Persönliches der Befragten zu sprechen. Die Fragen dieses Themenbereiches wurden nach Möglichkeit alle am Ende des Interviews gestellt. Einerseits bestand die Chance, dass sich zum Ende des Gesprächs eine Vertrauensbasis eingestellt hatte, die das Preisgeben persönlicher Informationen eventuell positiv beeinflussen könnte. Andererseits wäre bei einem früheren Zeitpunkt das Risiko gewesen, dass der Gesprächspartner das Interview abbricht, da er keinerlei intime Informationen teilen möchte. Ein Abbruch am Ende des Interviews wäre weniger dramatisch gewesen als zu Beginn, da so zumindest die Informationen aus den ersten vier Themenbereichen vorgelegen hätten. Der Themenbereich „Segmentierungskriterien“ enthielt einfache Fragen wie beispielsweise „Was denken Sie über Bioprodukte?“, aber auch intime Fragen, beispielsweise nach körperlichen Einschränkungen, die eventuelle Gartenarbeit erschweren könnten (z. B. Allergien), oder nach dem verfügbaren Haushaltseinkommen. Ziel dieses Themenbereiches war es, basierend auf den Antworten eine grobe Vorabsegmentierung der Befragten durchführen zu können, um so eventuell bereits im Vorfeld einige unscharf definierten Gruppen innerhalb aller Befragten identifizieren zu können. 


\subsection{Ergebnisse der Interviews}

Als qualitative Vorstudie zur empirischen Untersuchung dieser Forschungsarbeit wurden elf Interviews zum Bereich „Blumen und Pflanzen“ durchgeführt. Tabelle 3 charakterisiert die elf Probanden, die an der Befragung teilnahmen.

Tabelle 3: Charakterisierung Vorstudie

\begin{tabular}{|l|c|}
\hline & Charakteristika Teilnehmer \\
\hline Geschlecht & Tiefeninterviews für BuP \\
\hline weiblich & 9 \\
\hline männlich & 2 \\
\hline Durchschnittliches Alter & 51 \\
\hline Beschäftigung & \\
\hline Rentner & 4 \\
\hline Hausfrau/-mann & 2 \\
\hline arbeitet Teilzeit & 3 \\
\hline arbeitet Vollzeit & 2 \\
\hline $\begin{array}{l}\text { Durchschnittliche } \\
\text { Haushaltgröße in Pers. }\end{array}$ & 2,55 \\
\hline $\begin{array}{l}\text { Durchschnittliche } \\
\text { Gartengröße in qm }\end{array}$ & 206 \\
\hline
\end{tabular}

Quelle: Eigene Darstellung

Betrachtet man die Tabelle mit der Übersicht der einzelnen Interviews, so sind einige Aspekte bemerkenswert, die nicht unerwähnt bleiben sollen. Beispielsweise sind die Frauen deutlich in der Mehrzahl. Da die Forschungsfrage allerdings ganz konkret einen Bezug zu den Konsumenten hat, ist dieses Ungleichgewicht in der Geschlechterverteilung zu tolerieren, wenn nicht sogar zu begrüßen. Schließlich war es das Ziel der Vorstudie, mit denjenigen Personen ins Gespräch zu kommen, die für die Entscheidungen in einem Haushalt bezüglich Blumen und Pflanzen zuständig sind. Des Weiteren fällt die hohe Zahl der Rentner auf, die an der Befragung teilnahmen. $\mathrm{Ob}$ sich die überdurchschnittliche Beteiligung von Rentnern allein damit 
erklären lässt, dass Rentner „einfach“ mehr Zeit haben, lässt sich zu diesem Zeitpunkt noch nicht beantworten. Es ergab sich aber somit allein durch die Gruppenzusammensetzung die Frage, ob mit steigendem Alter auch die Affinität zum Thema „Blumen und Pflanzen“ steigt. Des Weiteren sorgt die hohe Zahl von Rentnern für den hohen Altersdurchschnitt der Gruppe, was wiederum ein Problem darstellen könnte, wenn dadurch jüngere Personen als Konsumenten von Blumen und Pflanzen zu wenig beachtet würden. Dass deren Meinungsbild völlig auBer Acht geblieben ist, kann allerdings ausgeschlossen werden, da die Bandbreite des Alters der Befragten zwischen 28 bis 63 Jahren lag.

Die durchschnittliche Haushaltsgröße ergibt sich aus einer relativ geringen Bandbreite von ein bis vier Personen pro Haushalt. Die Gartengröße unterscheidet sich wiederum sehr stark. Allerdings gilt es zu beachten, dass durchschnittliche Werte gerade bei Befragungen mit einer kleinen Stichprobe sehr anfällig für Ausreißer sind. Die Bandbreite der Angaben zur Gartengröße reichte von 0 bis $1.500 \mathrm{~m}^{2}$.

Ziel der geführten Interviews war es, einen ersten Einblick in die Gedankenwelt der Konsumenten zu erhalten. Auch ihr konkretes Verhalten, ihre Erwartungen und Wünsche in Zusammenhang mit Blumen und Pflanzen sollten zumindest in groben Zügen erfasst werden. Des Weiteren war für die Vorstudie die Frage von besonderem Interesse, inwieweit die internen Faktoren der Probanden eine Rolle beim Kaufentscheidungsprozess spielen. Im weiteren Verlauf werden die konkreten Ergebnisse der Vorstudie vorgestellt.

\subsubsection{Einfuihrungsphase}

Der Themenbereich „Kennenlernen“ dient nicht der Beantwortung einer konkreten Fragestellung. Dennoch ergab sich bereits zu diesem frühen Stadium in den Interviews oft die Situation, dass die Probanden ihre Tauglichkeit als Interviewpartner selbst in Frage stellten. Nachdem sich der Interviewer als Autor einer wissenschaftlichen Arbeit über Blumen und Pflanzen vorstellte, schien bei den Probanden oftmals der Eindruck aufzukommen, dass ihnen nun ein Expertengespräch über Blumen und Pflanzen bevorstehe, in dem sie als Laie nur einen schlechten Eindruck hinterlassen könnten (,... ich weiß nicht, ob Sie da bei mir richtig sind ...“, ,... oje, bei mir geht doch sowieso so gut wie alles kaputt ...“, „,.. ich kann Ihnen da aber einfach nur das sagen, was mir gerade dazu einfällt ..."). Es schien, als ob sich die Probanden bereits im Vorfeld absichern wollten, dass sie keine Experten für den Bereich Blumen und Pflanzen sind, sondern nur „einfache Konsumenten“. Diese Unsicherheit ver- 
suchte der Interviewer zu zerstreuen, nicht auch zuletzt mit dem Argument, dass für die Interviews Experten nicht geeignet seien und „einfache Konsumenten“ ausdrücklich gesucht würden. Anhand dieser ersten Reaktionen der Probanden lässt sich bereits das breite Feld des Themas „Blumen und Pflanzen“ erkennen. Obwohl sich alle Probanden freiwillig für ein Interview zum Thema „Haus und Garten“ gemeldet hatten, erkannte man bei vielen eine Verunsicherung bei der Konkretisierung des Themas zu „Blumen und Pflanzen“. Obwohl jeder Proband damit bereits Erfahrung gesammelt hatte, stellten viele ihre Geeignetheit als Interviewpartner selbst in Frage, da sie sich damit nach mehrfacher Aussage „nicht gut auskennen“. Dies wirft bereits die Frage auf, ob die Verunsicherung der Probanden verallgemeinert werden kann im Sinne von „Die Verbraucher entfremden sich von Blumen und Pflanzen“.

\subsubsection{Einstellung zu Blumen und Pflanzen}

Trotz der Bedenken mancher Probanden zu Beginn der Befragung zeigte sich am Ende der Interviews, dass im Bereich „Einstellungen zu Blumen und Pflanzen“ für zehn der elf Befragten Interviewpartner Blumen und Pflanzen eine große und wichtige Rolle spielen. Dies gilt sowohl für BuP im Haus als auch für den Garten und/oder Balkon. Durch BuP wird das persönliche Umfeld verschönert und aufgewertet (,... wenn mal Besuch kommt, muss es ja auch schön aussehen ..."). Des Weiteren zeichnete sich ab, dass viele Befragte aus dem Umgang mit BuP positive Energien für sich selbst beziehen (,... gehören zum Leben dazu ...“, „... sind so wunderschön ...“, „,.. schenken Lebensfreude ...“). Grundsätzlich konnte bei allen bis auf einen Probanden gleich zu Beginn eine grundsätzlich positive Verbundenheit zu BuP festgestellt werden. Dies ergab sich neben den positiven Aussagen auch nonverbal über die Mimik und die Art und Weise, wie die einzelnen Worte gesucht wurden, um die Schönheit und Wichtigkeit von BuP adäquat zu beschreiben.

Neben der meist positiven Grundeinstellung zu BuP konnten fünf verschiedene Schwerpunktthemen im Bereich „Einstellung zu Blumen und Pflanzen“ festgestellt werden. Diese waren:

1. Entspannung

$(, \ldots$ in der Natur sein ...“, ,... relaxen ...“, ,... Pflege macht Spaß, wenn man die Erfolge sieht ...“, ,... total abschalten ...“).

2. Lebensqualität

(,... Spaß daran, mit Pflanzen umzugehen ...“, ,... sich damit zu umgeben ...“, „... macht alles lebendiger ...“, ,... Erfolg gibt einem ein gutes Gefühl ...“) 


\section{Dekoration}

(,... machen das Heim wohnlich ...“, „,.. geben Charme ...“, „,.. verschönern die Wohnung ...")

4. Arbeit

$(, \ldots$ kostet Zeit ...“, ,.. bevorzuge pflegeleichte Pflanzen ...“, ,.. weil sie pflegeleicht sind ...", ,,.. mühsam, aber lohnenswert ...")

5. Raumklima

(,... Duft der Pflanzen ...“, „... Pflanzen entgiften die Luft ...“, „,. gutes Klima sorgt für bessere Lebensqualität ...“, „,.. sorgen für einen besseren Schlaf ...“).

Darüber hinaus konnten durch Fragen nach der Grabpflege die Erkenntnis gewonnen werden, dass den befragten Personen bepflanzte Gräber besser gefallen als Gräber ohne Bepflanzung. Dies gaben acht der elf Befragten an, und sie erwähnten im gleichen Zusammenhang, dass ihnen die damit verbundene Mehrarbeit zwar lästig, aber die Mühe wert ist. Auffällig war dabei, dass mehrere Probanden erwähnten, für ihre eigenen Gräber später einmal keine Bepflanzung zu wünschen, da sie die damit verbundene Arbeit ihren Kindern oder Angehörigen nicht zumuten wollen $(, \ldots$ Ich bevorzuge ein bepflanztes Grab. Für mein eigenes Grab will ich aber eine Platte. Ich will meinen Kindern die Arbeit mit einem bepflanzten Grab nicht zumuten ...").

Betrachtet man die bisherigen Ergebnisse zu den Einstellungen zu BuP, zeigt sich bereits hier, dass eine positive Grundstimmung bei fast allen Befragten zu beobachten war. Allerdings war auch den meisten die damit verbundene „Arbeit“ eine schnelle Assoziation. Weitere Assoziationen sollten in der Frage nach den ersten Gedanken erkannt werden. Die Antworten reichten von negativ besetzt bis hin zu nahezu euphorisch. Im Folgenden werden die Antworten in vier Bereiche eingeteilt, in die sich die Antworten nach Ansicht des Autors am besten einfügen lassen.

Negativ:

Wie erwähnt war nur eine der elf befragten Personen grundsätzlich nicht positiv gegenüber Blumen und Pflanzen eingestellt. Die Argumente dafür lauteten: „... machen Arbeit ...“, „... muss man Gießen ...“, ,... brauchen Pflege ...“, „,.. bei Schnittblumen ist der Tod ja schon eingeläutet ...". 
Leicht positiv:

Leicht positive Assoziationen wurden von drei der elf Befragten wahrgenommen. Sie erzählten in wohlwollender Stimmung von „... steigendem Wohlbefinden ...“, „... Lebensfreude ..." und von ,... Atmosphäre in Räumen ...", die durch BuP geschaffen wird.

Sehr positiv:

Die meisten (vier von elf) Personen berichteten sehr positiv über ihre Assoziationen mit Blumen und Pflanzen. Neben den ,... bunten Farben ... “ wurde berichtet über „,.. sehr lang anhaltende Freude...“, „das ganze Ambiente um einen herum wird verschönert ...“ und „... machen aus Anlässen etwas Besonderes ...".

\section{Euphorisch:}

Bei drei der elf Befragten konnte eine ganz besonders stark positiv ausgeprägte „Verbindung“ zu BuP festgestellt werden. Dabei gilt es zu erwähnen, dass die Antworten allein vom Wortlaut her auch in den Bereich „stark positiv“ passen würden. Die Kombination des Gesagten mit der beobachteten Körpersprache der Befragten würde eine derartige Einteilung allerdings nicht mehr sinnvoll machen. Typische Aussagen hier waren: „... sind einfach so wunderschön ...“, „... setzen bunte Akzente mit all ihren schönen Farben ...“, „... Pflanzen geben einem etwas zurück ...".

\subsubsection{Reale Kaufsituation}

Zum Themenbereich der realen Kaufsituation zeichnet sich nach Durchführung aller Interviews ab, dass es drei verschiedene Formen des Kaufes von Blumen und Pflanzen gibt:

\section{Geplanter Kauf}

2. Impulsiver Kauf

3. Mix aus geplantem und impulsivem Kauf.

Der geplante Kauf spielte bei den Probanden eine wichtige Rolle bei bedeutungsvollen Anschaffungen von BuP, beispielsweise wenn Pflanzen gesucht werden, die als Sichtschutz um den Garten dienen sollen, wenn es eine ganz besondere Solitärpflanze sein soll oder wenn die Blume oder Pflanze zum Verschenken gedacht ist. Bei den Probanden, die zuletzt einen geplanten Kauf von BuP angaben, ging vor dem Kauf stets ein Mangel voraus, der sich nicht „auf die Schnelle“ beheben ließ. Hohe Anschaffungskosten (,... wurde mir vom Fachmann so empfohlen ..."), die Suche nach einem ganz bestimmten Produkt (,... ich liebe Bambus ...") 
und die besondere Bedeutung der BuP waren die meistgenannten Gründe, warum die Probanden ganz gezielt und geplant ihren letzten Kauf von BuP tätigten.

Der impulsive Kauf zeichnete sich in den Interviews so ab, dass bei den Konsumenten kein Mangel an BuP herrschte, sie aber trotzdem „ganz spontan“ doch noch etwas kauften. Bei den entsprechenden Probanden wurde oft sinngemäß erwähnt, dass sie den Anblick einfach so schön fanden, ,weil es mir gleich gefallen hat“", „weil es so schön war“. Dabei war auffällig, dass alle Probanden, die von ihrem Spontankauf erzählten, auch gleich hinzufügten, dass das Gekaufte aber auch gar nicht teuer war. Für den Interviewer schien es dabei so, als ob sich die Probanden rechtfertigen wollten, weil sie etwas kauften, was sie eigentlich gar nicht brauchten. Dabei war wiederum auffällig, dass die Probanden den Preis als nebensächlich ansahen, da er absolut gesehen sehr gering war (,... Wie viel ich bezahlt habe, weiß ich nicht mehr, zwei bis drei Euro. Auf jeden Fall war es nicht viel ..."). Es entstand der Eindruck, dass Sachen, die gefallen, gerne spontan mitgenommen werden, ob ein Bedarf besteht oder nicht. Dabei kristallisierte sich eine Preisobergrenze von etwa drei Euro heraus, unterhalb derer der Preis die Kaufentscheidung recht gering zu beeinflussen scheint.

Ein Mix aus geplantem und impulsivem Kauf konnte bei solchen Probanden festgestellt werden, die zwar einen Mangel an BuP hatten und erkannten, aber nicht wussten, wie sie ihn beheben sollten. Konkret wollte eine Probandin beispielsweise eine Grünpflanze, wusste allerdings nicht, was es alles gibt und was für sie in Frage kommen könnte. Sie ging mit dem Plan, eine Grünpflanze zu kaufen, in ein Gartencenter und ließ sich vor Ort vom Angebot inspirieren. Letztendlich kaufte sie wie geplant eine Grünpflanze, für die sie sich allerdings erst spontan vor Ort entschied. Optik und Ästhetik waren dabei wiederum die Gründe, die letztendlich die spontane Auswahl begründeten.

Was das Informationsverhalten der Probanden betrifft, ergab sich die Konstellation, dass sieben von elf Befragten angaben, sich ,gar nicht“ zu informieren, bevor sie etwas kaufen. Vier von elf gaben an, sich vor Ort über Beratung zu informieren, und jeweils drei von elf gaben an, sich bei anderen Personen bzw. sich über die Werbung zu informieren (Mehrfachnennungen möglich). Die deutliche Mehrheit der Antwort „gar nicht“ weist darauf hin, dass diese Personen meist spontan und impulsiv kaufen, da bei einem geplanten Kauf konkurrierende Eigenschaften alternativer Produkte gegeneinander abgewogen werden, wozu eine Informationssammlung im Vorfeld nötig wäre. 
Bei der Frage nach der bevorzugten Wahl der Einkaufsstätte kristallisierte sich in der Vorstudie heraus, dass die Probanden das Angebot als gar nicht so differenziert wahrnehmen, wie es Kapitel 2.4 vermuten lässt. In den Augen der Befragten gab es nur zwei verschiedene Vertriebskanäle des Einzelhandels für BuP: den Fachhandel und den Nicht-Fachhandel. Die feinere Untergliederung dieser beiden Sparten, wie sie in Kapitel 2.4 vorgenommen wurde, war den Probanden zwar meist bekannt, wurde aber nicht angewandt. Das Kriterium zur Unterscheidung der beiden Vertriebskanäle war stets „mit Verkäufer“" und „ohne Verkäufer“. Der Fachhandel zeichnet sich dadurch aus, dass er fachkompetente Beratung anbietet und ein sowohl tiefes als auch breites Sortiment vorhält, das auch stets optisch ansprechend präsentiert wird (,... Musterbepflanzungen ...“). Probanden finden Inspiration beim „Spaziergang“ durch die Gewächshäuser oder Ausstellungsflächen. Des Weiteren zeigte sich in den Interviews, dass dem Fachhandel eine bessere Qualität als dem Nicht-Fachhandel zugesprochen wird - allerdings auch das höhere Preisniveau. Weitere Eigenschaften, die dem Fachhandel zugeschrieben wurden, sind beispielsweise der gute Service $(, \ldots$ da kriegt man dann genau das, was man möchte, weil die es dort noch individuell ausdekorieren ..."), die persönliche Ansprache, Verlässlichkeit und der richtige Ort, wenn man ein Geschenk sucht.

Entsprechend zeichneten die Probanden ein Bild des Nicht-Fachhandels, der in ihren Augen die Waren nur zum Verkauf stellt, aber nicht entsprechend präsentiert (,... lieblos ...“). Des Weiteren werden die BuP im Nicht-Fachhandel nicht gepflegt, so dass die lebende Ware bereits nach wenigen Tagen im Verkauf sehr unanschaulich aussieht $(, \ldots$ alt ...“, , ,. überständig ..."). Weiterhin wurde von den Probanden erwähnt, dass das Angebot des Nicht-Fachhandels oft nicht in die entsprechende Jahreszeit passt (,... Geranien schon im März ...") und man dort nichts Individuelles kaufen kann, da alles Massenware ist. Dabei wurde allerdings auch bedacht, dass die Pflanzen im Nicht-Fachhandel an dem Tag, an dem sie in den Verkauf kommen, noch ganz frisch sind. Dazu wurde dem Nicht-Fachhandel von nahezu allen Befragten ein günstiges Preis-Leistungsniveau bescheinigt, wenn die Ware frisch ist. Mehrere Probanden erzählten auch, dass sie ihre BuP für den häufigeren Eigenbedarf (,... Wochenendstrauß ...“, „.. zwei kleine Primelchen für den Tisch ...“) im Nicht-Fachhandel kaufen. Wenn es allerdings etwas Besonderes sein soll, bevorzugen die Probanden den Fachhandel.

Bezüglich der Preise für BuP wurde von den Probanden primär unterschieden, ob sie absolut gesehen hoch oder niedrig waren. Als „niedrig“ kristallisierte sich während der Interviews eine Preisschwelle von etwa drei bis vier Euro heraus. Dabei erzählten sieben der elf Befragten, dass bei niedrigen Preisen der Preis für sie nicht entscheidend ist, sondern vielmehr die Optik 
bzw. das Gefallen des Produktes, beispielsweise ein 10er Bund Schnitttulpen. Wenn ein Produkt schön ist und dazu wenig kostet, stellt dies eine Kombination dar, bei der die Probanden öfters „schwach“ werden in dem Sinne, dass sie das Produkt impulsiv bzw. ungeplant und spontan kaufen. Bei hohen Preisen spielt deren Höhe für die Probanden hingegen eine wichtige Rolle. Stehen hier Anschaffungen an, informieren sich die Befragten vor dem Kauf und vergleichen die Preise. Typische Anschaffungen, die in den hochpreisigen Bereich fallen, sind Pflanzen für eine neue Hecke im Garten oder ein Solitär für die Terrasse oder das Wohnzimmer.

Für den Interviewer schien es so, dass das Risiko des Kaufs umso geringer wahrgenommen wird, je geringer der finanzielle Aufwand ist. Entsprechend hoch ist das Risiko bei hohen Anschaffungskosten. So erklärt sich die unterschiedliche Herangehensweise bezüglich des Informierens im Vorfeld, des Preisvergleichs oder auch des geplanten bzw. des impulsiven Kaufs. Bei niedrigen Anschaffungskosten ist das Risiko eines Fehlkaufs sehr gering und somit für die Probanden überschaubar. Bei höherwertigen Anschaffungen versuchen die Probanden, durch das Sammeln von Informationen im Vorfeld das Risiko eines Fehlkaufs zu minimieren.

\subsubsection{Motive}

Der thematische Interviewbereich „Motive“ ist inhaltlich sehr eng mit dem Bereich „Einstellungen zu Blumen und Pflanzen“ verbunden, was sich auch während der einzelnen Interviews gezeigt hat. Durch diese Vermischung ist eine exakte Trennung der Themen „Einstellungen“ und „Motive“ nicht praktikabel. Somit sei hier auf die Ergebnisse aus dem Punkt „Einstellungen“" verwiesen, die auch Motive für den Kauf oder auch Nichtkauf von Blumen und Pflanzen darstellen. Die ausgearbeiteten Ergebnisse bzw. Motive waren „Entspannung“, „Lebensqualität“, „Dekoration“, „Raumklima“ und „Arbeit“. Erwähnt sei, dass „Arbeit“ als negativ besetzt angesehen wurde, da man sich um BuP auch kümmern muss.

Die erwähnten Ergebnisse stellen jeweils individuelle Einschätzungen der Probanden dar, die ihren internen Faktoren entsprechen. Um weitere Ideen für Motive für den Kauf oder Nichtkauf von Blumen und Pflanzen zu erhalten, wurde in den Interviews auch nach dem pragmatischen Nutzen von BuP gefragt. Dies wurde über eine direkte offene Frage nach Nutzpflanzen gehandhabt, beispielsweise nach dem Einsatz von Topfkräutern. Dabei gaben vier der elf Befragten an, Topfkräuter für den Eigenverbrauch bei sich zu Hause zu haben. Für sechs Pro- 
banden waren Nutzpflanzen ,wichtig“, für fünf Personen „,nicht so wichtig“. Dabei gilt es zu erwähnen, dass vier der fünf Befragten, die „nicht so wichtig“ angaben, dennoch erwähnten, Nutzpflanzen in ihrer Wohnung oder im Garten bzw. auf dem Balkon zu haben. Somit gaben letztendlich acht von elf Interviewten an, Nutzpflanzen zu Hause zu haben.

Der Interviewer gewann den Eindruck, dass die meisten Probanden erst im Gespräch über diesen Punkt bemerkten, dass sie überhaupt Nutzpflanzen zu Hause haben. Trotz des weit verbreiteten Einsatzes von Nutzpflanzen scheinen sich die Befragten darüber recht wenig Gedanken zu machen. Dies lässt die Vermutung aufkommen, dass die meisten Nutzpflanzen „ungeplant“ im Sinne von spontan gekauft werden. Ein festgestellter konkreter Mangel, beispielsweise an Oregano, scheint bei den Befragten zuvor nicht aufgetreten zu sein. Eine tiefe „Verbundenheit“" zur Idee der Nutzpflanzen konnte nur bei einer Person festgestellt werden: „... mache vieles zu Hause selbst ... ist dann sicher schadstofffrei ...“. Zusammengefasst scheint sich die Frage, ob der Nutzen einer Pflanze ein Kaufmotiv darstellt, nicht eindeutig beantworten zu lassen. Durch die Gespräche zeigte sich jedoch, dass Nutzpflanzen zwar weit verbreitet sind, aber nicht sehr gewürdigt werden. Dies lässt wiederum die Vermutung zu, dass der Nutzen eine unterschwellig größere Rolle spielt, als es den Anschein hat, da die Nutzpflanzen wohl nicht wegen ihrer schönen Optik gekauft wurden.

Dass der Nutzen beim Kauf von BuP für den Eigenbedarf vermutlich doch eine größere Rolle spielt, als von den Befragten erwähnt, lässt sich auch anhand der Reaktionen auf folgende Frage ableiten: „Wie ändert sich Ihre Einstellung (zum Nutzen) wenn Sie Blumen oder Pflanzen zum Verschenken kaufen“? Alle Befragten differenzierten bei dieser Frage sofort sehr stark die vorgegebene Situation von der des Kaufs für den Eigenbedarf. Bei keinem der Befragten spielt der Nutzen einer Blume oder Pflanze eine Rolle, wenn der Grund des Kaufs darin besteht, dass sie verschenkt werden soll (,... soll einfach nur schön sein ...“, ,... da ist der Nutzen dann nicht so wichtig ..."). Es zeigte sich sehr schnell, dass einzig die ansprechende Optik, die Schönheit und das „Besondere“ wichtige Kaufmotive sind, wenn es darum geht, ein Geschenk zu finden. Der Nutzen spielt demnach nur eine Rolle, wenn der Kauf für den Eigenbedarf gedacht ist.

\subsubsection{Segmentierungskriterien}

Als letzter Teil der Interviews folgte die Abfrage von Segmentierungskriterien. Dazu gehörten einerseits die typischen soziodemographischen Segmentierungskriterien der Interviewpartner. 
Die dazu abgefragten Parameter wurden in Form von geschlossenen Fragen gestellt, die ebenfalls im Anhang einsehbar sind. Die daraus ersichtlichen Gruppierungen wurden bereits im Kapitel „Ergebnisse“ tabellarisch dargestellt, so dass hier auf die entsprechende Stelle verwiesen und auf eine nochmalige Darstellung verzichtet wird. Andererseits wurde versucht, neben den typischen soziodemographischen Kriterien weitere Kriterien zur Segmentierung zu erfassen, darunter Kriterien zur Mediennutzung, des allgemeinen Kaufverhaltens, psychologische Kriterien, physiologische Kriterien (wie beispielsweise Krankheiten, die bei der Gartenarbeit hinderlich sein könnten) und Zeitkriterien. Fragen zu den letztgenannten Kriterien wurden nach Möglichkeit offen gestellt, so dass die Probanden sich nicht in vorgegebenen Antwortalternativen zurechtfinden mussten.

Da die Vielfalt der Antwortmöglichkeiten zur Nutzung von Medien sehr breit ist, ließ sich aus den Antworten der Befragten keinerlei Auffälligkeit ableiten. Jeder der Befragten gab zwar an, in welcher Art und Weise er die diversen Medien nutzt, allerdings stets nur in sehr allgemeiner Form $(, \ldots$ Zeitschriften lese ich, was gerade da ist ...“, „,.. im Fernsehen schaue ich nur, was interessant ist ...“, „,.. Radio meistens nur im Auto ...“).

Angesprochen auf die zunehmenden Möglichkeiten des One-Stop-Shoppings, zeichnete sich ein differenzierteres Bild: Den meisten Befragten gefällt es, viele verschiedene Geschäfte an einem Ort besuchen zu können. Besonders „das breite Warenangebot“, die „großzügigen Parkmöglichkeiten“, die „kurzen Wege“ und somit die „Zeitersparnis“ waren Punkte, die während der Interviews als positive Eigenschaften genannt wurden. Allerdings wurde von den gleichen Probanden auch abgewogen, dass in großen Einkaufszentren „das Persönliche“ fehle und oftmals viele Leute um einen herum seien, was als „unangenehm“ empfunden wurde. Weiter war allen Befürwortern bewusst, dass große Einkaufszentren für alteingesessene Einzelhändler meist schlecht sind. Für den Interviewer stellte sich die Situation oft so dar, dass die Befragten gerne die Vorzüge des One-Stop-Shoppings in Anspruch nehmen, dabei allerdings ein schlechtes Gewissen haben, da sie wissen, dass sie dadurch den regionalen, einheimischen Einzelhändlern schaden. Nur eine Befragte lehnte das Konzept des One-Stop-Shoppings generell ab. Dieses werde sich ,auf keinen Fall durchsetzen“, da es viel zu ,,anonym“ und „kalt“" sei. Die entsprechende Person kauft ganz bewusst bei jedem Facheinzelhändler ein. Sie verzichtet gezielt auf die Vorzüge des One-Stop-Shoppings und sieht dies als ihre Art an, den regionalen Einzelhandel zu unterstützen.

Mit Blick auf das psychologische Kriterium wurden die Probanden allgemein nach ihrer Meinung zu Bioprodukten gefragt. Dabei zeigte sich, dass das Thema Bio einerseits sehr ernst 
und andererseits auch sehr differenziert wahrgenommen wird. So befürworteten alle Befragten die grundsätzliche Idee, für die Bioprodukte im breiten Volksverständnis stehen. Allerdings wurde im gleichen Atemzug oftmals auch eine Verunsicherung gegenüber Bio deutlich (,... Wo fängt Bio an, wo hört Bio auf? ...“, ,, . da ist sicher nicht überall Bio drin ...“). Entscheidend für den Kauf von Bioprodukten ist für die Befragten oftmals nicht, ob „Bio“ auf der Verpackung steht, sondern vielmehr, ob sie darauf vertrauen können, dass auch wirklich Bio enthalten ist, wo mit Bio geworben wird. Sind die Probanden überzeugt, dass sie ein biologisch hergestelltes Produkt erhalten, sind sie auch gerne und aus Überzeugung bereit, Mehrkosten in Kauf zu nehmen. Begründet wurde dies meist mit „gesünderer Ware“ und teilweise auch damit, dass man durch die Unterstützung von Bio „den Tieren ein artgerechtes Leben“ ermöglichen kann. Eine der elf befragten Personen ist grundsätzlich von Bio überzeugt. Sie kauft wenn irgendwie möglich nur Bioprodukte und achtet auch sonst in ihrem Leben darauf, alles so umweltschonend wie möglich $\mathrm{zu}$ machen $(, \ldots$ komme gerade aus einem Bio-Hotel ...").

Als physiologische Kriterien wurden die Interviewten nach körperlichen Beschwerden allgemeiner Art gefragt, die sich eventuell auch auf ihre Arbeit mit BuP auswirken könnten. Dabei gaben die meisten der Befragten an, keinerlei spezielle Beschwerden zu haben, lediglich zwei Personen sprachen über gelegentliche Rückenbeschwerden. Da die Frage nach körperlichen Beschwerden sehr ins Privatleben der Probanden vordringt, verzichtete der Interviewer darauf, detailliertere Nachfragen zu stellen. Somit war die Frage für den weiteren Verlauf der Arbeit nicht sehr hilfreich.

Da der Einzelhandel von BuP sehr stark von saisonalen Schwankungen betroffen ist, wurden die Probanden danach gefragt, ob sie zu bestimmten Jahreszeiten oder zu bestimmten Anlässen BuP kaufen. Erwartungsgemäß kaufen viele der Probanden im Frühjahr besonders viele BuP, um sich für die warmen Sommermonate den Garten oder den Balkon zu verschönern. Für die meisten Probanden ist das Thema allerdings mit Beendigung der Erstbepflanzung bereits erledigt. Zwei der Probanden sagten, dass sie dann im Sommer weiter BuP für den Garten oder den Balkon kaufen, da sie deren Verschönerung als fortlaufenden Prozess betrachten. Die Befragten meinten überwiegend, dass sie meistens diejenigen Artikel kaufen, die gerade zur Jahreszeit passen (,... Narzissen zu Ostern ...“, „... Weihnachtsstern zu Weihnachten ..."). Unabhängig von der Jahreszeit gaben alle Interviewten an, auch für besondere Anlässe BuP zu kaufen, beispielsweise als Geschenk für andere Personen oder auch für den Eigenbedarf, etwa als Tischdekoration, wenn Besuch erwartet wird. 


\section{Methodik der quantitativen Untersuchung}

Das Ziel der Forschungsarbeit besteht darin, die Kaufmotive der Konsumenten von BuP zu erfassen und darauf basierend eine Kundensegmentierung durchzuführen. Eine solche Segmentierung und die daraus ableitbaren Erkenntnisse sind für sämtliche Marktakteure der grünen Branche von großem Interesse und können eine erfolgreiche Marktbearbeitung unterstützen.

Wie erwähnt sind solche Untersuchungen in der BuP-Branche selten. Die erste und gleichzeitig letzte große wissenschaftliche Arbeit zu diesem Thema wurde 1983 von Marianne Altmann als Dissertation an der Universität Hannover verfasst. In „Konsumententypologie auf dem Zierpflanzenmarkt" beschreibt sie verschiedene Segmente, die als Käufergruppen auf dem BuP-Markt aktiv sind (Altmann 1983). Da die Arbeit mehr als 30 Jahre zurückliegt, kann davon ausgegangen werden, dass die von ihr gefundenen Segmente heute in ihrer damaligen Ausprägung nicht mehr oder nur in sehr veränderter Form existent sind. Aktuelle Handlungsempfehlungen aus den damaligen Erkenntnissen abzuleiten ist demnach nicht zielfördernd.

Aus dem Verlagshaus BLOOM's mit Sitz in Ratingen stammt eine weitere Arbeit, die sich mit der Segmentierung von Konsumenten der BuP-Branche beschäftigt. 2008 veröffentlicht, hat sie in der grünen Branche weite Verbreitung gefunden. Sie segmentiert die Konsumenten anhand der sogenannten „BLOOM's Lebenswelten“. Die Bloom’schen Lebenswelten basieren vor allem auf der bereits vorgestellten Studie der Sinus-Milieus (Knapp 2008, S. 26). Um den Nutzen für die grüne Branche so groß wie möglich zu gestalten, hat BLOOM's die SinusMilieus vereinfacht und an die Gegebenheiten der grünen Branche angepasst. BLOOM's unterscheidet fünf verschiedene Lebenswelten, in denen sich Konsumenten von BuP befinden können. Für jede Lebenswelt bietet BLOOM's eine Beschreibung, anhand derer sich Marktakteure Handlungsempfehlungen ableiten können.

Kritisch zu bemerken ist allerdings, dass mit den Sinus-Milieus als Basis die Konsumenten allgemein und nicht spezifisch als BuP-Kunden in die Beschreibung der Bloom'schen Lebenswelten einfließen. Gerade die Besonderheiten an Kundenwünschen und -erwartungen im Bereich der BuP-Branche finden dabei keine angemessene Aufmerksamkeit. Des Weiteren erfolgt die Zuordnung der Konsumenten zu einer der fünf BLOOM'schen Lebenswelten heuristisch und nicht anhand empirischer Daten. Es besteht somit das Risiko, dass wichtige Kriterien der Konsumenten keine Beachtung finden und diese somit einer unpassenden Lebenswelt 
zugeordnet werden. Daraus ergibt sich die Gefahr, dass die Beschreibung der Lebenswelten unscharf und verzerrt ist und daher die Bloom'sche Beschreibung der Zielgruppen der grünen Branche nicht der Realität entspricht.

Im weiteren Verlauf dieses Kapitels wird die Methodik vorgestellt, mit deren Hilfe in dieser Arbeit eine Typologie erarbeitet wird, die sowohl wissenschaftlich belastbar ist als auch auf aktuellem Datenmaterial beruht.

\subsection{Anpassung des Segmentierungsschemas}

Eine Marktsegmentierung basiert auf verschiedenen Kriterien, nach welchen eine heterogene Gruppe segmentiert wird (vgl. Kap. 3). Abbildung 32 (s. oben S. 72) zeigt verschiedene gruppierte Segmentierungskriterien, die alle als grundsätzliche Segmentierungskriterien in Frage kommen. Zur näheren Beschreibung und Würdigung der einzelnen Kriterien sei hiermit auf Kapitel 3 verwiesen. Da hier für die Untersuchung allerdings eine „lebensstilbasierte“ Segmentierung angestrebt wird, gilt es, in einem ersten Schritt zu prüfen, welche der bereits vorgestellten Kriterien am besten zur Operationalisierung im Fragekatalog geeignet sind. Wie ebenfalls bereits beschrieben, basieren die Milieu-Forschung und die daraus entstandenen (Sinus)-Milieus auf der Theorie der Lebensstile, die wiederum in der Übersicht der Segmentierungskriterien in der Gruppe „Psychologische Kriterien“ enthalten sind.

Die im Kontext der hier zu beantwortenden Forschungsfrage anstehende Segmentierung anhand der vorhandenen und bereits in Kapitel 3 beschriebenen allgemeinen Lebensstiltypologien erweist sich allerdings als problematisch. Die komplexen Typen der bereits definierten Lebensstile oder Milieus lassen auf individueller Ebene nur sehr schwer eindeutige Vorhersagen darüber zu, wie sich ein Individuum in einer bestimmten Situation verhält. Dazu sind Lebensstile als solche empirisch nicht scharf genug zu erfassen, da sie sich aus vielen, wechselseitig abhängigen Variablen speisen. Als weiteres Problem ergibt sich, dass Lebensstil-Typologien nicht produktspezifisch sind. Es ist in Bezug zu BuP demnach sinnvoll, neue produktspezifische Typologien zu entwickeln, die aussagekräftigere Daten zur Erklärung des spezifischen Konsumentenverhaltens ermöglichen. Dabei ist kein vollständiger Lebensstilansatz als Basis nötig, vielmehr können zur Erstellung der entsprechenden Segmente wenige, aber effektive Prädikatoren ausreichen, um die Typologie dem Anspruch der Forschungsfrage gerecht werden zu lassen (Trommsdorff/Teichert 2011, S. 196; Lüdtke 1990, S. 452; Banning 1987, S. 155; Enneking/Franz 2005, S. 36). 
Basierend auf dem „Schema zur Rekonstruktion von Lebensstilen“ nach Lüdtke werden im Folgenden Elemente vorgestellt, die eine allgemeine Abfrage von Lebensstil-Konzeptionen ermöglichen. Abbildung 46 zeigt die den bereits bekannten Dimensionen untergeordneten Kriterien nach Lüdtke.
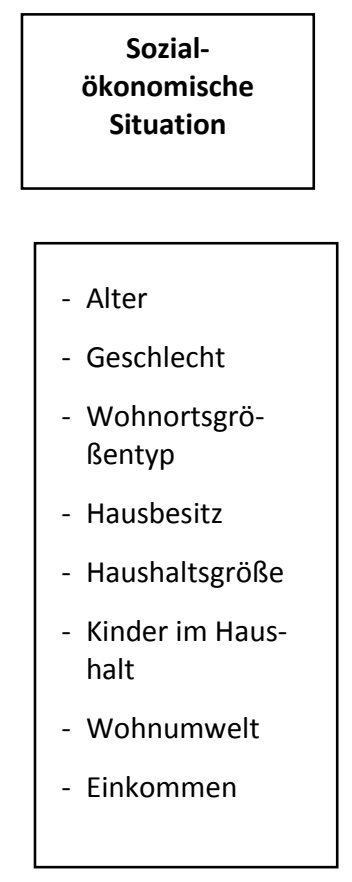
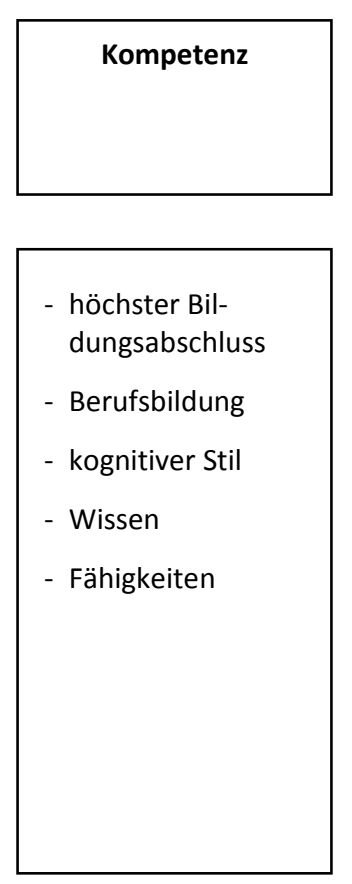
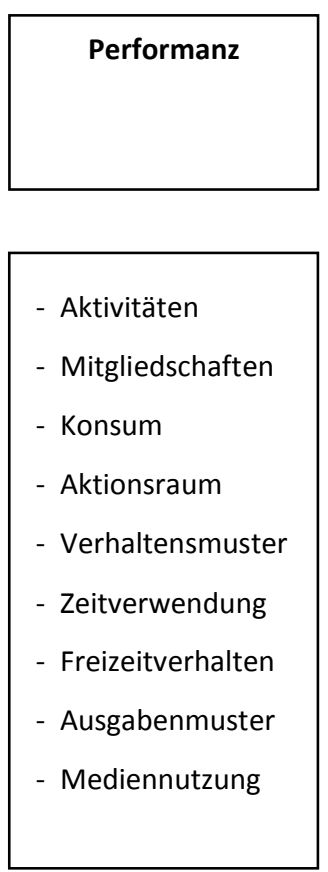

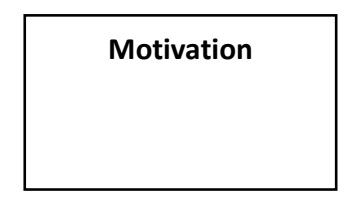

\begin{tabular}{|l|}
\hline - Ziele \\
- Präferenzen \\
- Sinn des Han- \\
delns \\
- Einstellungen \\
- Bedürfnis- \\
Wert-Orien- \\
tierung \\
- Selbstdarstel- \\
lung \\
- Repräsentation
\end{tabular}

\section{Abbildung 46: Dimensionen von Lebensstil-Konzeptionen}

Quelle: Eigene Darstellung in Anlehnung an Lüdtke 1989, S. 42; Freter 2008, S. 93

Die aufgeführten Dimensionen vereinnahmen die im allgemeinen Segmentierungsmodell vorgestellten demographischen, soziologischen und psychographischen Kriterien. Nicht berücksichtigt werden allerdings physiologische Kriterien, Zeitkriterien und Kriterien des Nutzens. Die Kriterien des beobachtbaren Kaufverhaltens deckt das Schema nach Lüdtke teilweise ab. Sie sind mit dem Kriterium „Konsum“ der Dimension „Performanz“ untergeordnet.

Dies führt zu der Problematik, dass das Lüdtke'sche Schema zwar eine lebensstilbasierte Segmentierung ermöglicht, die in gewissen Zügen produktspezifisch ausgestaltet werden kann. Allerdings berücksichtigt das Lüdtke'sche Schema die Segmentierungskriterien des beobachtbaren Kauferhaltens in den Augen des Autors nicht stark genug. Wie aus den geführten Interviews der qualitativen Vorstudie hervorgeht, sind ebenjene Kriterien sehr wichtige Quellen für produktspezifische Abfragen. Der Einsatz der Abfrage-Dimension „Kriterien des be- 
obachtbaren Kaufverhaltens“ ist im Kontext dieser Arbeit somit von entscheidender Bedeutung und wird daher im Folgenden besonders beachtet.

Das hier verwendete theoretische Schema wird dementsprechend vom Autor an die besonderen Anforderungen dieser Arbeit angepasst. Es basiert sowohl auf den vier vorgestellten theoriebasierten Dimensionen nach Lüdtke als auch auf der nun hinzugefügten Dimension „Beobachtbares Kaufverhalten“. Diese Dimension verleiht dem späteren Segmentierungsmodell die nötige Praxisnähe und erleichtert somit die schlussfolgernden Handlungsempfehlungen für die Praxis.

Die im Folgenden berücksichtigten fünf Dimensionen zur Erstellung der lebensstilbasierten Typologie sind demnach:

- Sozialökonomische Situation

- Kompetenz

- Performanz

- Motivation

- Beobachtbares Kaufverhalten

Die physiologischen Kriterien, die Zeitkriterien und die Nutzenkriterien werden für die Konstruktion des Schemas bewusst nicht mehr berücksichtigt. Sie wurden bereits in der qualitativen Vorstudie analysiert und werden anhand der gesammelten Antworten der Interviewten vom Autor als nahezu nicht relevant für die angestrebte Segmentierung befunden.

In der folgenden Abbildung 47 und im darauffolgenden Kapitel 6.2 werden die theorie- und literaturbasierten Segmentierungskriterien des 5-Dimensionen-Schemas vom Autor an die konkrete Fragestellung dieser Forschungsarbeit angepasst. Die Anpassung der nach Lüdtke allgemein gehaltenen Segmentierungskriterien an die Thematik „Blumen und Pflanzen“ basiert dabei sowohl auf den Erkenntnissen der qualitativen Vorstudie als auch auf der entsprechenden Literatur. Abbildung 47 zeigt das angepasste Schema und ist als direkte Weiterentwicklung von Abbildung 46 zu sehen. 

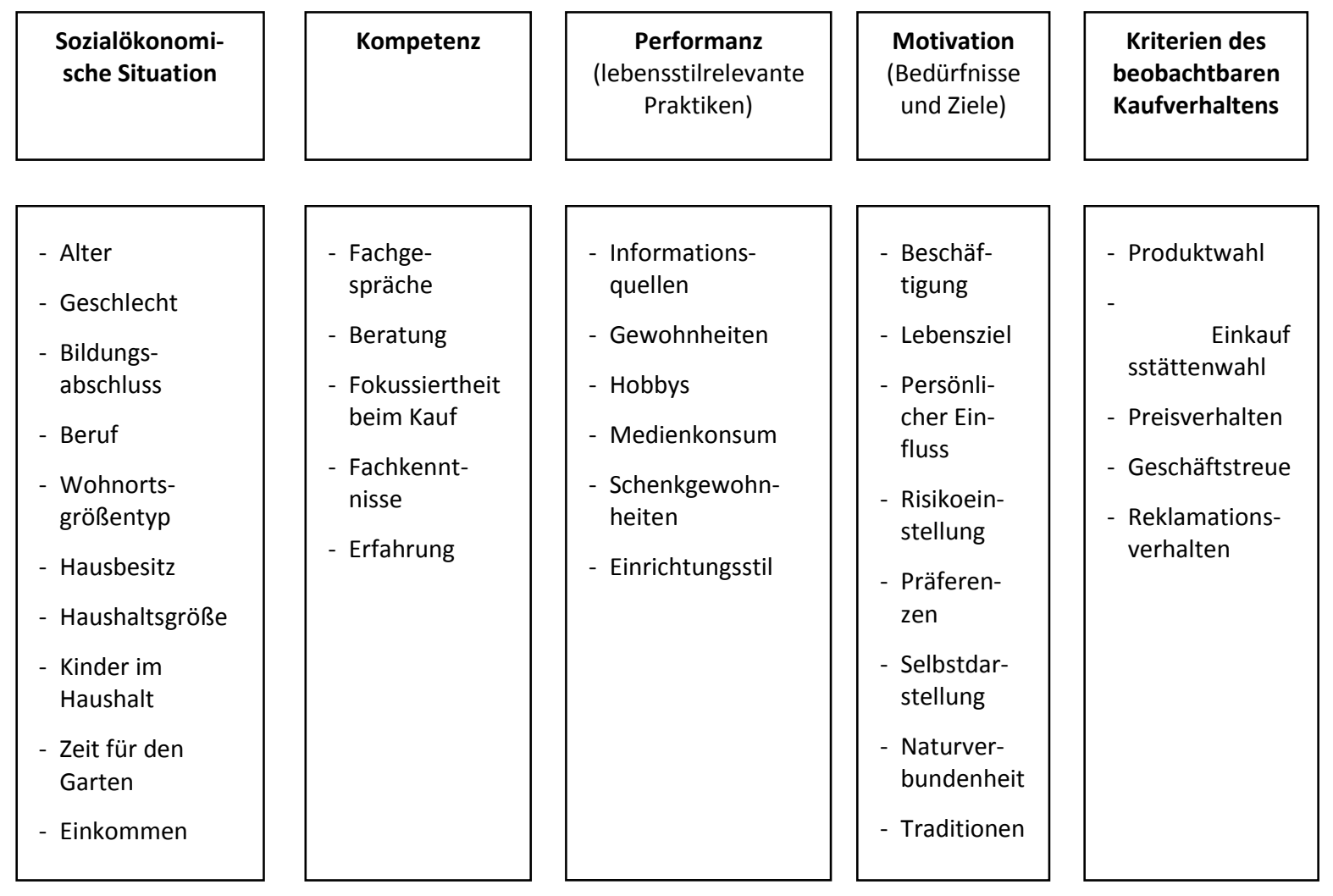

\section{Abbildung 47: Dimensionen von Lebensstil-Konzeptionen bezogen auf BuP}

Quelle: Eigene Darstellung in Anlehnung an Lüdtke 1989, S. 42; Freter 2008, S. 93

\subsection{Umsetzung der Forschungsfragen in den Fragekatalog}

Die Operationalisierung der fünf Dimensionen stellt einen wesentlichen Teil dieser Arbeit dar. Die aus der wissenschaftlichen Literatur bekannten allgemeinen Segmentierungskriterien werden an dieser Stelle den Gegebenheiten der BuP-Thematik angepasst. Diese Anpassung basiert auf den vom Autor geführten Interviews der qualitativen Vorstudie und der entsprechenden Literatur. Die gesammelten Interviews und deren Auswertung erlauben einen Einblick in die Motive, Einstellungen und Sichtweisen der Probanden, die sich in der Generierung der Items niederschlagen.

Im Folgenden werden die im Fragekatalog eingesetzten Items vorgestellt, wobei sie thematisch geordnet erläutert werden, nicht in der Reihenfolge, in der sie im Fragekatalog erscheinen. Zusätzlich sei auf die Tabelle 15 im Anhang verwiesen. Diese zeigt in komprimierter Form nochmals die Zuordnungen der Items zu den entsprechenden Dimensionen, allerdings sortiert nach ihrer Reihenfolge im Fragekatalog. 
Des Weiteren sei an dieser Stelle noch erwähnt, dass im Fragebogen Fragen enthalten sind, auf die bisher noch nicht eingegangen wurde. Durch eine Kooperation des Autors mit einem anderen wissenschaftlichen Projekt an der Hochschule Osnabrück wurden die zum Teil gemeinsam erhobenen Daten von beiden Kooperationspartnern unter verschiedenen Zielsetzungen analysiert. Durch das gemeinsame Entwickeln und die gemeinschaftliche Nutzung der Ergebnisse sind vier der Fragen direkt nur für den Kooperationspartner von Bedeutung, während die restlichen Fragen für beide Kooperationspartner interessant sind. Für die hier beschriebene Forschungsarbeit wäre eine Berücksichtigung von Fragen mit einer starken Fokussierung auf Gartenrosen zwar nicht nachteilig, aber auch nicht zielführend, weshalb auf sie in der weiteren Analyse verzichtet wurde.

\subsubsection{Methodische Vorüberlegungen}

Bevor das 5-Dimensionen-Modell zur Segmentierung operationalisiert werden kann, muss geklärt werden, anhand welcher statistischer Analysemethoden der Datensatz später untersucht werden soll. Entsprechende Überlegungen empfehlen sich an dieser Stelle, da die Fragestellung und die angebotenen Antwortoptionen für die Befragten oftmals die Möglichkeiten der Analyse beeinflussen.

Die in dieser Arbeit angestrebte lebensstilbasierte Typologie entspricht in ihren Grundzügen einer konventionellen, konsumentenseitigen Marktsegmentierung. Das sowohl in der Praxis als auch in der wissenschaftlichen Literatur am weitesten verbreitete Werkzeug zur empirischen Herausarbeitung einer Segmentierung ist die Clusteranalyse. Grundsätzlich kann eine Clusteranalyse mit ausgewählten Original-Items aus einem Fragekatalog erfolgen oder die ausgewählten Items aus dem Fragekatalog fließen gebündelt in die Clusteranalyse ein. In diesem Falle ist vor der Clusteranalyse eine Faktorenanalyse einzelner Items durchzuführen, so dass anstatt der einzelnen Original-Items die Faktorenwerte der extrahierten Faktoren in die Clusteranalyse eingehen.

Da nicht alle einzelnen Items eines Fragebogens bei der Clusteranalyse Berücksichtigung finden, teilen sie sich in die Gruppen der aktiven und der passiven Variablen auf. Aktive Variablen sind jene, die in die Clusteranalyse einfließen. Auf ihnen basiert die Bildung der Cluster. Dabei ist nicht entscheidend, ob die Variablen einzeln und direkt oder erst nach erfolgter Faktorenanalyse im Verbund als Faktor in die Clusteranalyse einfließen. Aktive Variablen sind von entscheidender Bedeutung für die inhaltliche Interpretation der gefundenen Cluster. Auf 
ihnen basieren die Cluster, sie spiegeln deren „Innenleben“ wider. Passive Variablen eines Fragekataloges sind hingegen jene Variablen, die nicht in die Clusteranalyse einfließen. Sie dienen der besseren ,äußeren“ Beschreibbarkeit der gefundenen Cluster, da sie oft soziodemographische Merkmale darstellen, wie beispielsweise Alter, Geschlecht oder Einkommen. Passive Variablen adressieren somit die Cluster, die aufgrund der aktiven Variablen gefunden wurden. Nur in der Kombination aktiver und passiver Variablen können Cluster sowohl erfolgreich gebildet als auch erfolgreich beschrieben werden. Eine Verknüpfung von aktiven und passiven Variablen ist somit Voraussetzung dafür, dass in einem weiteren Schritt absatzpolitische Maßnahmen zielgenau vorgenommen werden können (Hüttner/Schwarting 2002, S. 330 ff.; Bacher et al. 2010, S. 468).

Analog zur theoretisch beschriebenen Vorgehensweise wird im Folgenden in dieser Arbeit verfahren: Die Segmentierung erfolgt anhand einer Clusteranalyse, der eine Faktorenanalyse vorgeschaltet wird. Dabei dient das beschriebene 5-Dimensionen-Schema als Fundament der Clusterung. Innerhalb jeder Dimension werden für die jeweils zugehörigen Segmentierungskriterien entsprechende Items für den Fragekatalog generiert. Man spricht von der „Operationalisierung" der einzelnen Dimensionen. Da die Operationalisierung ein elementarer Teil des Brückenschlags von der Theorie zu der konkreten Bearbeitung der Forschungsfrage ist, wird im folgenden Kapitel detailliert auf sie eingegangen.

Da die angestrebte Typologie auf der Theorie der Lebensstile basiert, eignen sich für die Faktorenanalyse vorrangig diejenigen Items, die innerhalb der lebensstilnahen Dimensionen „Kompetenz“, „Performanz“ und „Motivation“ entwickelt wurden. Aus diesen drei Dimensionen ausgewählte Items fließen einzeln in die Faktorenanalyse ein und werden somit zu Faktorenwerten verdichtet (siehe dazu Kap. 7.1). Bezogen auf ihre inhaltliche Aussagekraft werden die auf diese Weise generierten Faktoren im Folgenden „Motivfaktoren“ genannt, da sie die Motive der Befragten in Bezug auf deren Handeln in der BuP-Welt schärfen und verdichten.

Diese extrahierten Motivfaktoren dienen im nächsten Analyseschritt als aktive Variablen der Clusteranalyse und gehen als solche in diese ein (siehe dazu Kap. 7.2). Die Bildung der Cluster basiert somit ausschließlich auf den Motivfaktoren, wodurch man von einer ,,motivbasierten“ oder „lebensstilbasierten“ Clusteranalyse sprechen kann. Innerhalb eines Clusters sind die Motive homogen, zwischen den Clustern sind sie heterogen. 
Für die Beschreibung der gefundenen Cluster dienen einerseits die eingegangenen Motivfaktoren, die das Innenleben der Cluster erklären. Andererseits werden zur genaueren Beschreibung der Cluster in einer erweiterten Analyse zusätzlich die passiven Variablen herangezogen, die im Falle dieser Arbeit primär den Dimensionen „Sozialökonomische Situation“ und „Kriterien des beobachtbaren Kaufverhaltens“ untergeordnet sind. Wie beschrieben, kann eine exakte und praxisrelevante Beschreibung der Cluster nur erfolgen, wenn sowohl die aktiven als auch die passiven Variablen in Kombination in die Beschreibung der Cluster einfließen. Für diese Arbeit bedeutet dies, dass alle fünf Dimensionen in die Beschreibung der Cluster einfließen müssen, um eine möglichst exakte Beschreibung der Cluster sicherzustellen.

\subsubsection{Operationalisierung der sozialökonomischen Situation}

Die abgefragten Kriterien der sozialökonomischen Situation können als „klassische“ Segmentierungskriterien angesehen werden, da sie zur Beschreibung von Segmenten oder Konsumenten am weitesten verbreitet sind. So werden auch im vorliegenden Fragekatalog das Geschlecht und das Alter der Probanden sowie die Größe des Haushalts, in dem sie leben, erhoben. Erfragt werden aber auch der höchste Bildungsabschluss, der aktuell ausgeübte Beruf und das Netto-Haushaltseinkommen der Probanden. Zu Letzterem sind im Fragekatalog acht verschiedene Intervalle des monatlichen Netto-Haushaltseinkommens angegeben, wovon das zutreffende angekreuzt werden soll. Die Intervalle beginnen betragsmäßig bei „,<500 €“" und

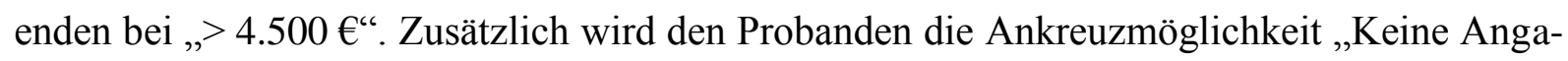
be“ angeboten. Da das Preisgeben des Einkommens eine private und relativ persönliche Information ist, wird im Fragekatalog auf die offene Frage nach dem Einkommen verzichtet. Die Möglichkeit, die Höhe des Einkommens durch Ankreuzen eines Intervalls preiszugeben, erscheint weniger heikel, da sich das Einkommen im ganzen Intervall bewegen kann und somit auch durch Ankreuzen nicht exakt taxiert werden muss. Ziel der Erhebung der Einkommen ist es zu erkennen, ob das Kaufverhalten mit dem Einkommen in Verbindung steht.

Eine Abfrage des Wohnortsgrößentyps erfolgt in Frage 16 („Wo wohnen Sie zur Zeit?“), wiederum durch Vorgabe von Intervallen, welche die Probanden ankreuzen sollen. Insgesamt werden vier Intervalle angeboten: von ,in einem Dorf“ bis zu ,in einer Stadt mit mehr als 100.000 Einwohnern“. Obwohl die Beantwortung der Frage nach der Wohnortsgröße weit weniger heikel ist als die der Frage nach dem Einkommen, wird auch in Frage 16 auf die offene Fragestellung verzichtet - allerdings mit Blick auf die bessere Analysierbarkeit von bereits kategorisierten Antworten, nicht, um die Hemmschwelle zur Antwort herabzusetzen. 
Ziel dieser Frage ist es, eventuelle Unterschiede im Kaufverhalten von Stadtbewohnern im Vergleich zu Bewohnern ländlicher Gegenden zu erkennen. Mit Frage 17 wird, basierend auf einer bestehenden Studie, die Wohnsituation abgefragt, d. h., ob in Eigentum oder zur Miete gewohnt wird, wiederum mit der Möglichkeit, das jeweils Zutreffend anzukreuzen (o. V. 2013). Mit Frage 18 und Frage 19 wird in Erfahrung gebracht, ob die Probanden eine Terrasse oder einen Garten haben; trifft dies zu, wird nach der jeweiligen Größe in Quadratmetern gefragt (o. V. 2013). Ziele dieser Abfragen sind wiederum Rückschlüsse auf Zusammenhänge von Wohnsituationen und Kaufverhalten. So ist beispielsweise zu erwarten, dass Personen, die im Eigenheim wohnen und einen Garten haben, mehr gärtnerische Aktivitäten an den Tag legen als solche, die zur Miete wohnen und keinen Garten haben.

\subsubsection{Operationalisierung der Kompetenz}

Die Dimension „Kompetenz“ steht für Wissen und Fähigkeiten. Die Kenntnis der Kompetenzen der Probanden in Bezug auf BuP bietet für die spätere Charakterisierung der Segmente wichtige Informationen, die im Fragebogen in verschiedenen Formen abgefragt werden.

Frage 8 gliedert sich in drei verschiedene Aussagen auf, welche die Probanden auf einer

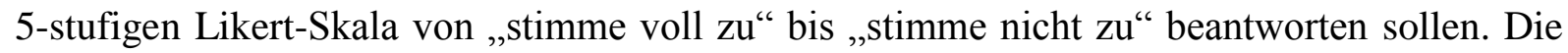
Intention dieser Frage ist es, Rückschlusse über das Involvement der Probanden im Hinblick auf BuP zu erhalten. Dabei wurde zur Messung des Involvements zwischen den Ansätzen „Single Score“ und „Multi Score“ abgewogen. Die Entscheidung fiel auf eine angepasste mehrdimensionale Messung - angepasst insofern, als die mehrdimensionale Messung von vier Dimensionen ausgeht, die jeweils anhand mehrerer Items mit einer Likert-Skala gemessen werden. Im Fragebogen wurde auf die vier einzelnen Dimensionen zugunsten einer realistischen Situation, die für die Probanden skizziert wird, verzichtet. Damit richtet sich der Fokus auf den Kaufprozess der Probanden, was letztlich eine zielgenauere, weil praxisnähere Analyse ermöglichen soll.

In Frage 8.1 wird der Proband gefragt, ob er vor dem Kauf einer Pflanze überlegt, wo diese später stehen könnte. Dadurch versucht der Autor die Fokussiertheit der Probanden beim Kauf zu erfragen, d. h. in Erfahrung zu bringen, ob die Probanden so sehr mit dem Thema BuP vertraut sind, dass sie im Vorfeld des Kaufs bereits Alternativen durchspielen und diese gegebenenfalls auch verwerfen können. Frage 8.2 bezieht sich auf den Austausch von im Bereich BuP gesammelten Erfahrungen mit Freunden und Bekannten. Die Beantwortung dieser 
Frage soll einerseits zeigen, wie sehr sich die Probanden vor dem Kauf mit der Materie auseinandersetzen. Andererseits soll sie Aufschluss darüber geben, wer ein Fachgespräch über BuP aktiv sucht und führen möchte. Frage 8.3 bezieht sich inhaltlich auf das Thema „Beratung“, um später herausarbeiten zu können, wer sich inhaltlich mit der Materie auseinandersetzt und wer grundsätzlich erkannt hat, dass er weitere Beratung benötigt. Mit dem Zusatz „nehme ich mir genügend Zeit“ soll verdeutlicht werden, ob die Probanden ganz bewusst und überlegt eine Pflanze kaufen oder ob sie „im Vorbeigehen“ etwas kaufen, das in ihren Augen passen könnte.

Die direkte Übertragung von Ausprägungen des Antwortverhaltens bei Frage 8 auf die Kompetenz ist dabei allerdings nicht ohne Weiteres möglich. So kann die Zustimmung zu den einzelnen Fragen isoliert betrachtet sowohl als niedrige als auch als hohe Kompetenz interpretiert werden. Beispielsweise kann eine niedrige Antwortausprägung bei Frage 8.3 so gedeutet werden, dass der Proband eine sehr hohe Kompetenz aufweist und daher sehr schnell und ohne Beratung kauft. Um die Ergebnisse der Frage 8 dennoch nutzen zu können, ist es daher nötig, die einzelnen Ergebnisse im Kontext der anderen Fragen zu bewerten und dementsprechend Rückschlüsse über die vorhandene Kompetenz zu erzielen.

In Frage 14 werden alternative Satzenden zur Aussage „Eine Gartenrose darf ruhig etwas mehr kosten, wenn ..." angeboten. Die Probanden bewerten die vorgegebenen Aussagen auf einer 5-stufigen Likert-Skala von „stimme voll zu“ bis „stimme nicht zu“. In Frage 14.1 bewerten die Probanden die Aussage „... sie in einem Container ist (und nicht wurzelnackt, also ohne Erde und Blätter)“. Bei einer Zustimmung zu dieser Aussage lässt sich erkennen, dass die Probanden die Kompetenz besitzen, die verschiedenen Verkaufsarten einer Gartenrose voneinander zu unterscheiden. Üblicherweise ist eine im Container kultivierte Gartenrose deutlich wertiger und hochpreisiger als eine Gartenrose, die wurzelnackt verkauft wird. Frage 14.2 misst, ob die Probanden neu gezüchtete Rosensorten von bestehenden Sorten unterscheiden können. Frage 14.3 fragt indirekt ab, ob die Probanden Besonderheiten bei Rosen von Standardware unterscheiden können. Ähnlich verhält es sich bei Frage 14.5, die abfragt, ob die Probanden erkennen können, wenn eine Rose „ganz besonders duftet“. Frage 14.8 schließt die Itembatterie der Frage 14 ab und zielt darauf, ob die Probanden die Kompetenz besitzen, eine „normale“ Gartenrose von einer Edelrose zu unterscheiden. Dabei gilt es zu bemerken, dass der Begriff „Edelrose“ nicht definiert ist. Vielmehr wird er als Verkaufsargument für normale Gartenrosen benutzt, die Besonderheiten aufweisen. Die Ablehnung einer 
Mehrpreisbereitschaft für „Edelrosen“ stellt in diesem Fall demnach einen Indikator für vorhandene Kompetenzen dar.

In Frage 20 werden die Probanden gefragt, wie viel Zeit sie im Sommer durchschnittlich pro Woche mit Gartenarbeit verbringen. Die Beantwortung der Frage mit einer hohen Stundenzahl dient als Indikator für eine vorhandene Kompetenz im Bereich BuP, da davon auszugehen ist, dass die Probanden einerseits entsprechende Erfahrung in der Materie haben und andererseits sich gerne mit der Materie auseinandersetzen, was zur Folge hat, dass die Probanden relativ BuP-affin sind.

\subsubsection{Operationalisierung der Performanz}

Die Dimension „Performanz“ steht für Handlungen und Interaktionen der Probanden, die für deren Lebensstil im Hinblick auf BuP relevant sind. Um sie zu messen, werden wiederum verschiedene Items durch den Fragebogen abgefragt.

In Frage 3 findet sich eine Itembatterie über die Schenkgewohnheiten der Probanden bezogen auf BuP, auch hier mit einer 5-stufigen Likert-Skala versehen. Zur Basisaussage „Ich kaufe gerne Blumen und Pflanzen als Geschenk, weil ..." werden drei verschiedene Gründe aufge-

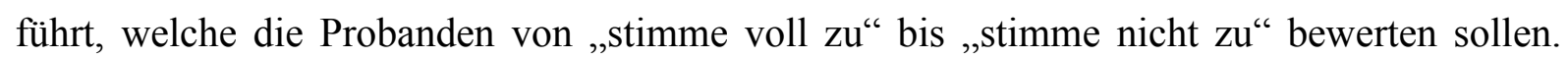
Thematisch schließt sich Frage 14.7 an, bei der die Probanden die Aussage „Eine Gartenrose darf ruhig etwas mehr kosten, wenn ich eine Rose als Geschenk kaufen möchte“ auf einer Skala von „stimme voll zu“ bis „stimme nicht zu“ bewerten sollen. Um das Informationsverhalten der Probanden zu erfragen, dient Frage 7. Dort werden sie nach den Informationsquellen befragt, die sie heranziehen, wenn sie etwas Bestimmtes über BuP wissen möchten. Als Antwortmöglichkeit werden fünf verschiedene Alternativen angeboten (von „Internet“ bis „Züchter“), die auf einer 3-stufigen Skala (von „häufig“ bis „nie“) bewertet werden.

Mit Frage 23 wird erörtert, welche individuelle Einstellung die Probanden Pflanzenschutzmitteln gegenüber haben. Es werden drei verschiedene Aussagen über Pflanzenschutzmittel und deren Einsatz vorgegeben, die sie jeweils auf einer 5-stufigen Skala (von „stimme voll zu“ bis „stimme nicht $\mathrm{zu}^{\text {“) }}$ bewerten sollen. In Frage 24.2 wird wiederum eine Aussage vorgegeben; die Bewertung von „Pflanzen sind mein wichtigstes Hobby“ dient der Messung, inwieweit das Thema BuP in der persönlichen Lebensführung der Probanden verankert ist. In Frage 26 wird erfragt, wie der persönliche Einrichtungsstil der Wohnzimmer der Probanden aussieht. Dazu werden ihnen fünf Bilder von fünf verschiedenen Wohnzimmern gezeigt, bei denen sie 
mithilfe von Zahlen $(1=$ bestes, 2 = zweitbestes, ...) eine Abstufung der gezeigten Wohnzimmer durchführen sollen, und zwar von dem Wohnzimmer, das ihnen am besten gefällt, bis zu dem, das ihnen am wenigsten gefällt. Die gezeigten Bilder entstammen einer bestehenden Lebensstilsegmentierung, so dass die Antwort der Probanden Rückschlüsse darauf zulässt, welchem Segment sie angehören. Den einzelnen Bildern wurden in der bestehenden Studie folgende Bezeichnungen und Beschreibungen zugeordnet (Knapp 2008):

Bild 1: „Prestige“: anspruchsvolle Genießer, extrovertiert, Besonderheiten, Style

Bild 2: „Easy“: unkomplizierte Optimisten, ökologisch, naturverbunden, sparsam, pragmatisch

Bild 3: „Tradition“: liebevolle Bewahrer, ordnungsliebend, Kontinuität, arbeitsam, sparsam

Bild 4: „Joy“: unkonventionelle Stilmixer, kreativ, introvertiert, Genießer

Bild 5: „Modern“: ausdrucksvolle Individualisten; Schönheiten, Selbstverwirklichung, Authentizität

\subsubsection{Operationalisierung der Motivation}

Die Dimension „Motivation“ steht verallgemeinert für den Sinn und die Gründe des Handelns der Probanden in Bezug auf BuP. Um diese messbar zu machen, werden in Frage 10 Gründe erfragt, welche dazu führten, dass sich die Probanden eine Gartenrose kauften. Zur Beantwortung dieser Frage wird den Probanden eine Itembatterie mit vier verschiedenen Kaufgründen angeboten, die jeweils mithilfe einer 3-stufigen Skala bewertet werden sollen. Die Bewertungsalternativen der angebotenen Gründe sind „ein Hauptgrund“, „ein weniger wichtiger Grund“ und „kein Grund“. Ziel der Frage 10 ist neben dem Erkennen der direkten Beweggründe des Kaufes auch die Möglichkeit, Aufschluss darüber zu erhalten, durch welche Eigenschaften die Preisbereitschaft der Konsumenten erhöht werden kann. In Frage 14.4 werden die Probanden gebeten, die Aussage „Eine Gartenrose darf ruhig etwas mehr kosten, wenn sie ein Qualitätssiegel trägt“ auf einer 5-stufigen Skala von „stimme voll zu“ bis „stimme nicht zu“ zu bewerten. Die Einordnung der Probanden anhand ihres Antwortverhaltens kann als Indikator für ihre Risikoeinstellung verstanden werden, da eine besonders gute Qualität das subjektiv empfundene Risiko eines Nicht-Anwachsens der Rose minimiert.

Mit Frage 22 wird erfragt, welche individuellen Aspekte beim Gärtnern für die Probanden im Vordergrund stehen. Den Probanden wird der Satz „Mit dem Thema Garten und Gärtnern as- 
soziiere ich besonders ..." vorgeben. Dazu folgen sechs alternative Satzenden, beispielsweise „Ausleben von Kreativität“ oder „Arbeit“. Jede der sechs Alternativen ist auf einer 5-poligen Skala zu bewerten, wobei die Skala von „stimme voll zu“ bis „stimme nicht zu“ reicht. Durch das Antwortverhalten der Probanden lassen sich Rückschlüsse über deren Präferenzen und Beschäftigungen ebenso wie über deren Naturverbundenheit ziehen. Mit Frage 24 wird unter anderem abgefragt, welche emotionale Rolle Blumen und Pflanzen für die Konsumenten spielen. Dazu wird den Probanden eine Itembatterie mit elf Statements vorgelegt, die diese auf einer 5-poligen Skala (von „stimme voll zu“ bis „stimme nicht zu“) bewerten sollen. Einzelne Items der Frage 24 wurden dabei der Literatur entnommen und adaptiert (Gabriel 2011; o. V. 2012). Von den elf zu bewertenden Statements aus Frage 24 sind neun Statements der Dimension „Motivation“ zuzurechnen. Anhand der Beantwortung der „Motivation“-Items aus Frage 24 lassen sich Rückschlüsse auf die Selbstdarstellung der Probanden in Bezug auf BuP (Fragen 24.1, 24.2, 24.11), ihre Präferenzen (Fragen 24.6 und 24.9), ihre Beschäftigung (Frage 24.4) und ihre Naturverbundenheit (Fragen 24.5, 24.8 und 24.10) ziehen.

Anhand von Frage 25 werden allgemeine Wertvorstellungen der Probanden erfragt. Mitsamt ihren Items ist sie ohne weitere Anpassungen der wissenschaftlichen Literatur entnommen (Schmidt et al. 2007), da sie sehr gut in den Kontext des Fragebogens passt. Analog zu Frage 24 sind auch in Frage 25 einzelne Statements auf einer 5-poligen Skala von „stimme voll

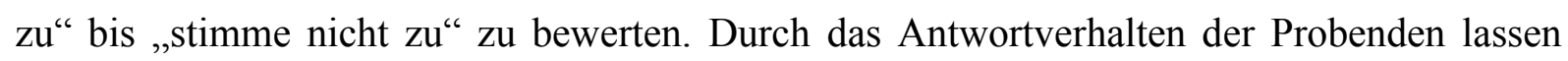
sich Rückschlüsse auf deren Risikoeinstellung (Frage 25.1), ihre Wertschätzung von Traditionen (Frage 25.2) und ihr Lebensziel (Frage 25.3) ziehen.

\subsubsection{Operationalisierung des beobachtbaren Kaufverhaltens}

Die Kriterien des beobachtbaren Kaufverhaltens hat der Autor dem Schema zur Rekonstruktion von Lebensstilen hinzugefügt. Wie erwähnt, haben sich diese Kriterien sowohl aus theoretischer Sicht als auch während der Interviews der qualitativen Vorstudie als äußerst relevant herausgestellt. Dementsprechend stellen sie eine sehr wichtige Dimension für diese Arbeit dar.

Mit Beantwortung der Frage 1 geben die Probanden an, ob sie während der letzten 12 Monate Blumen oder Pflanzen gekauft haben. Die Befragung wird nur mit denjenigen Personen weitergeführt, welche diese Frage mit ,ja“ beantworten. In Frage 2 werden die Probanden nach dem bei ihnen vorherrschenden Kaufgrund für BuP gefragt, d. h., ob sie BuP primär für sich 
selbst oder zum Verschenken kaufen. Zur Beantwortung dieser Frage wird eine 5-stufige Antwortskala vorgegeben, die von ,deutlich häufiger für mich selbst“ bis „,deutlich häufiger als Geschenk" reicht. In Frage 4 wird abgefragt, wo die Probanden in den letzten zwölf Monaten ihre Pflanzenkäufe getätigt haben. Zur Beantwortung werden acht verschiedene Einkaufsstätten (von „Discounter“ bis „Blumenfachgeschäft“) genannt, welche die Probanden ankreuzen können. Dabei basiert Frage 4 auf einer bereits erfolgreich getätigten Umfrage und auf spezieller Fachliteratur (Gabriel 2011; o. V. 2013). Anhand von Frage 9 wird abgefragt, ob es sich beim letzten Kauf der Probanden um einen geplanten Kauf oder um einen Spontankauf gehandelt hat. Die Antwort auf diese Frage ist bereits für sich sehr relevant für den weiteren Verlauf dieser Arbeit, zudem bietet Frage 9 aber auch viele Anknüpfungspunkte für weitere Analysen, beispielsweise bei Untersuchungen zur Preisbereitschaft oder bei der Frage, welche Erwartungen die Probanden an bestimmte Vertriebskanäle haben. Frage 9 erfasst eine konkrete, real erlebte Situation, was im ersten Moment zur Analyse weniger geeignet erscheint. Diese Art der Abfrage ist allerdings bewusst gewählt, da Konsumenten hybride Tendenzen aufweisen. Das bedeutet, dass ein einzelner Konsument sowohl geplant als auch spontan kauft. Die Festlegung auf nur eine Kaufart würde somit die andere ausschließen, was nicht der Realität entspricht. Die Abfrage soll somit ein Ergebnis liefern, das für konkrete, real durchlaufene Kaufentscheidungsprozesse steht, womit das Ergebnis möglichst praxisnah werden soll.

Mit Frage 5 wird abgefragt, welche Erwartungen die Probanden an ein Einzelhandelsgeschäft für BuP haben. Zur Auswahl werden den Probanden acht verschiedene Merkmale eines Geschäftes angeboten (z. B. „guter Service“ oder „unkomplizierter schneller Einkauf“), wovon die Probanden die drei Ausprägungen ankreuzen sollen, die ihnen am wichtigsten sind. Das Antwortverhalten lässt dabei Rückschlüsse über die Einkaufsstättenwahl zu. In Frage 6 werden die Probanden danach gefragt, in welchem Vertriebskanal für BuP ihre Erwartungen hinsichtlich der Dimensionen „Qualität“, „Service“ und „Preisniveau“ am besten erfüllt werden. Dazu werden drei verschiedene 3x8-Matrizen abgebildet. Jeden der acht verschiedenen Vertriebskanäle (von „Blumenfachgeschäft“ bis „Discounter“) sollen die Probanden in Bezug auf Qualität, Service und Preisniveau einschätzen. Jede Dimension wird dabei pro Vertriebskanal mit einer 3-stufigen Skala von „schlecht“ bis ,hoch“ bewertet. Eine mögliche Antwortkombination stellt somit beispielsweise die ausformulierte Antwort „Der Service im Baumarkt ist in der Regel hoch“ dar. Mit den acht Vertriebskanälen, den drei Dimensionen und der 3-stufigen Antwortskala entsteht in Frage 6 somit ein Block von insgesamt 72 Items, der den Probanden 
vorgelegt wird. Die Vorgabe einer nur 3-stufigen Likert-Skala als Antwortoption ist darin begründet, dass eine detailliertere Skala die entsprechenden Itembatterien den Probanden als zu komplex im Sinne von unübersichtlich erscheinen lassen könnte. Die Probanden sollen von der Frage nicht abgeschreckt werden. Mit der Festlegung auf eine 3-stufige anstatt eine 5-stufige Skala ist Frage 6 in den Augen des Autors für die Probanden zumutbar und wird folglich so im Fragebogen verwendet.

Mit Frage 21 wird durch eine offene Fragestellung erhoben, wie viel Geld die Probanden in etwa pro Jahr für Blumen und Pflanzen ausgeben. Mit der direkt anschließenden Folgefrage 21a wird ebenfalls offen gefragt, wie viel Geld vom angegebenen Betrag aus Frage 21 für entsprechende BuP-Geschenke in etwa pro Jahr ausgegeben wird. Mit Frage 13 und Frage 14.6 wird die Zahlungsbereitschaft der Probanden eruiert. Frage 13 erkundigt sich mithilfe einer offenen Frage nach dem maximalen Betrag, den die Probanden für „,eine perfekte Gartenrose im Plastiktopf (Durchmesser $18 \mathrm{~cm}$ )“ zu bezahlen bereit sind. Frage 14 bringt eine Itembatterie mit einer 5-stufigen Likert-Skala, bei der die Probanden angeben können, welche Eigenschaften einer Gartenrose sie wie stark dazu bewegt, etwas mehr Geld für sie auszugeben. Frage 14.6 zielt darauf, inwieweit es für die Probanden beim Kauf einer Gartenrose eine Rolle spielt, ob sie „gerade etwas mehr Geld im Portemonnaie“ haben.

\subsection{Durchführung der Studie}

Um die dieser Arbeit zu Grunde liegende Forschungsfrage adäquat beantworten zu können, ist es nötig, die empirischen Daten der Hauptstudie mit verschiedenen Methoden zu analysieren. Dabei gilt es zu beachten, dass die Qualität der Ergebnisse maßgeblich von der Qualität des gewählten Forschungsdesigns bestimmt wird. Um diese Qualität zu gewährleisten, wurde der Fragekatalog vor seiner endgültigen Fassung mehrfach überarbeitet. Bevor der erste quantitative Test des Fragebogens durchgeführt wurde, begutachteten ihn sieben Akademiker, die mit der Materie „Fragekatalog erstellen“ vertraut sind, kritisch. Als Ergebnis dieser ersten qualitativen Prüfung wurden mehrere kleinere Änderungen am Fragekatalog durchgeführt. Besonderes Augenmerk lag dabei auf dem logischen Aufbau des Fragebogens und auf sprachlichen Feinheiten. Ziel der qualitativen Prüfung war es, den Fragekatalog für die Probanden so verständlich wie möglich zu machen.

Nach diesen qualitativen Anpassungen des Fragebogens wurde die aktualisierte Fragebogenversion einem quantitativen Pretest unterzogen. Zu diesem Zweck wurden 50 Probanden an- 
hand des Fragebogens befragt. Während der Befragung wurde von Seiten des Interviewers verstärkt darauf geachtet, Verständnisprobleme der Befragten aufzudecken. Die Antworten zu den Fragebögen des quantitativen Pretests wurden unter besonderer Sorgfalt in das Statistiksoftwareprogramm „SPSS“ übertragen. Dieses wurde dazu benutzt, den Pretest-Datensatz detailliert auf Schwachstellen zu analysieren. Die erste offensichtliche Schwachstelle zeigte sich bei der Bearbeitungszeit des Fragebogens, die im Durchschnitt bei ca. 15 Minuten lag. Ziel war, die Probanden mit dem Fragekatalog etwa 10 Minuten, maximal aber 12 Minuten zu beanspruchen. Um Zeit einzusparen, war eine weitere Anpassung erforderlich. So wurde der Begrüßungstext bzw. die Ansprache der Probanden verkürzt. Aus Frage 3 wurde ein Item entfernt. Es gab „...weil sich jeder über Blumen und Pflanzen freut““als Antwortalternative. Die Datenanalyse zeigte, dass nahezu alle Probanden im zustimmenden Bereich lagen und dass diese Frage somit keine hinreichende Differenzierungsmöglichkeit bot. In Frage 5 wurde die Antwortalternative „Sonstiges und zwar “ gestrichen, da während des Pretests niemand diese Ausweichoption genutzt hat. In Frage 6 wurde für alle drei abgefragten Ausprägungen die Antwortalternative ,weiß nicht“ gestrichen. Dies lag zum einen daran, dass Frage 6 insgesamt 96 Optionen zum Ankreuzen hatte, was die Probanden abschreckte. Durch die Reduzierung auf 72 Kästchen sollte die optische Abschreckung der Frage reduziert werden. Zum anderen wurde ,weiß nicht“ gestrichen, um die Probanden ohne diese Ausweichoption zu einer Bewertung zu ,zwingen“. Da die Probanden keine Profis sind, zeigte sich beim Pretest, dass sehr viele diese Ausweichoption in Anspruch nahmen, was der späteren Analyse nicht dienlich sein konnte. Ohne die Antwortmöglichkeit „weiß nicht“ geben die Probanden zumindest ihr subjektives Empfinden im Hinblick auf die drei abgefragten Parameter kund. Es zeigt somit das Image, das die Vertriebskanäle auf die Probanden haben.

Aus Frage 8 und aus Frage 10 wurde ebenfalls jeweils ein Item gestrichen, das keine guten Ergebnisse geliefert hatte. In Frage 21 wurden drei Unterfragen entfernt. Eine Unterfrage zielte auf die Ausgaben für Beet- und Balkonpflanzen pro Jahr. Die Befragung zeigte, dass die Probanden ihre Ausgaben für Beet- und Balkonpflanzen nur schwer oder gar nicht von ihren Gesamtausgaben für BuP trennen konnten. Die Unterfragen „Wie viel Geld geben Sie ungefähr im Jahr für Blumen und Pflanzen aus, die sie verschenken?““ und „Wie viel Geld geben Sie ungefähr im Jahr für Blumen und Pflanzen aus, die Sie für sich selbst kaufen?“" wurden ebenfalls gestrichen. Sie erzeugten Verwirrung, da in der gleichen Frage schon die Ausgaben für BuP insgesamt erfragt wurden. Zusätzlich stiftete Verwirrung, dass den Probanden unklar war, ob die Ausgaben für verschenkte Beet- und Balkonpflanzen berücksichtigt werden sollen 
oder nicht. Die drei gestrichenen Unterfragen wurden anhand der separaten Folgefrage 21a „davon als Geschenk“ ersetzt. In Frage 22 wurde wiederum ein Item gestrichen, das nur unbefriedigende Ergebnisse lieferte. Bei Frage 24 entfielen vier Items; zwar hatten sie zum Teil verwertbare Ergebnisse geliefert, allerdings war die Itembatterie aus Frage 24 sehr lang und beanspruchte viel Bearbeitungszeit. Die Auswahl der zu streichenden Items wurde daher aufgrund einer Faktorenanalyse getroffen. Diese ergab für Frage 24 vier Faktoren, von denen auf einen Faktor ganz besonders viele Items hochluden. Von Faktor 1 wurden vier Items ausgewählt, die gestrichen wurden, da sie innerhalb eines Faktors ohnehin das „Gleiche“ messen. Zusammengefasst handelte es sich somit um Fragen, deren Ergebnisse nicht gut differenzieren, die das Gleiche messen, auf die keiner geantwortet hat und die von den Probanden als zu kompliziert eingeschätzt wurden.

Neben den genannten Änderungen wurde der Fragebogen in weiteren Detailpunkten bearbeitet. Eine detaillierte Auflistung aller Änderungen des Fragekatalogs sowohl nach dem qualitativen als auch nach dem quantitativen Pretest findet sich im Anhang unter „Anmerkungen und Ergebnisse der Pretests“ (Anhang 2). Weiterhin sei erwähnt, dass bei der Erstellung der verschiedenen Items des Fragekatalogs versucht wurde, auf bereits publizierte „Marketing Scales“ zurückzugreifen. Da für diese spezielle Art der Fragestellung im Kontext von BuP allerdings die entsprechenden Marketing Scales fehlen, wurden die meisten Fragen selbst entwickelt. Bei der Übertragung der Ergebnisse von den Fragebögen in die statistische Auswertungssoftware SPSS wurde besonders sorgfältig und ohne Zeitdruck gearbeitet, wobei die eingegebenen Werte stets einer Plausibilitätskontrolle unterzogen wurden. So sollten Tippund Übertragungsfehler ausgeschlossen werden, um einen qualitativ hochwertigen Datensatz er erhalten.

Zur Beantwortung der dieser Arbeit zu Grunde liegenden Fragestellung fand die Datenerhebung im Zeitraum Mitte April 2014 bis Mitte Juli 2014 statt. Die Befragung der Konsumenten ( $n=881)$ erfolgte im Raum Osnabrück. Die Orte, an denen Konsumenten angesprochen wurden, waren ein Gartencenter, eine Baumschule und ein Baumarkt. Diese drei Orte wurden ausgewählt, weil sich Leute, die aus den Geschäften kommen, kurze Zeit zuvor höchstwahrscheinlich mit dem Thema BuP beschäftigt haben und so an dem Thema des Fragebogens wohl ein größeres Interesse haben als beispielsweise wahllos angesprochene Passanten in der Innenstadt. Sämtliche für diese Arbeit verwendeten Daten wurden anhand eines Fragebogens ermittelt, der den Probanden vor Ort ausgehändigt wurde. Die Probanden füllten den Frage- 
bogen selbständig aus, wobei stets der Interviewende in der Nähe war, falls es Klärungsbedarf gab.

Tabelle 4: Charakterisierung Hauptstudie

\begin{tabular}{|c|c|c|c|}
\hline & $\mathrm{n}=880$ & in $\%$ & $\begin{array}{l}\text { Vergleich } \\
\text { BRD in \% }\end{array}$ \\
\hline \multicolumn{4}{|l|}{ Geschlecht $(n=869)$} \\
\hline weiblich & 591 & 68,0 & 51,0 \\
\hline männlich & 278 & 32,0 & 49,0 \\
\hline Durchschnittliches Alter $(n=844)$ & 53,0 Jahre & & 44,1 Jahre \\
\hline \multicolumn{4}{|l|}{ Beruf $(n=868)$} \\
\hline Keine Angabe & 18 & 2,1 & k. A. \\
\hline Erwerbslos & 3 & 0,3 & k. A. \\
\hline Hausfrau/Hausmann & 63 & 7,3 & k. A. \\
\hline Rentner/Pensionär & 212 & 24,4 & k. A. \\
\hline In Ausbildung/Studium & 34 & 3,9 & k. A. \\
\hline Teilzeiterwerbstätig & 182 & 21,0 & k. A. \\
\hline Vollerwerbstätig & 356 & 41,0 & k. A. \\
\hline $\begin{array}{l}\text { Durchschnittliche } \\
\text { Haushaltgröße in Pers. }\end{array}$ & 2,64 Personen & & 2,01 Personen \\
\hline \multicolumn{4}{|l|}{$\begin{array}{l}\text { Durchschnittliches }(\mathrm{n}=848) \\
\text { Haushaltseinkommen }\end{array}$} \\
\hline keine Angabe & 252 & 29,7 & k. A. \\
\hline$<500 €$ & 10 & 1,2 & k. A. \\
\hline $500-900 €$ & 13 & 1,5 & k. A. \\
\hline $900-1.300 €$ & 44 & 5,2 & k. A. \\
\hline $1.300-1.700 €$ & 60 & 7,1 & k. A. \\
\hline $1.700-2.000 €$ & 57 & 6,7 & k. A. \\
\hline $2.000-2.500 €$ & 122 & 14,4 & k. A. \\
\hline $2.600-4.500 €$ & 196 & 23,1 & k. A. \\
\hline$>4.500 €$ & 94 & 11,1 & k. A. \\
\hline
\end{tabular}

Quelle: Eigene Darstellung in Anlehnung an Bundesinstitut für Bevölkerungsforschung 2015 
Tabelle 4 zeigt die soziodemographische Zusammensetzung der befragten Personen und die entsprechenden Vergleichsdaten der Bundesrepublik Deutschland, sofern diese verfügbar waren. Betrachtet man die Beschreibung der Befragten, fällt auf, dass Frauen in der Stichprobe und auch im Vergleich zum bundesdeutschen Durchschnitt deutlich überrepräsentiert sind. Dies könnte daran liegen, dass sich die Befragung nur an eine bestimmte Zielgruppe richtete: an Personen, die während der letzten 12 Monate Blumen oder Pflanzen gekauft haben. Es liegt daher der Verdacht nahe, dass manche befragte Männer somit an der Befragung gar nicht teilnehmen konnten. Des Weiteren muss man davon ausgehen, dass deutlich mehr Frauen als Männer von den Interviewern angesprochen wurden, was daran liegt, dass in Deutschland die Erledigung von Einkäufen im Wesentlichen die Aufgabe der Frauen ist. Unabhängig von den Gründen für das Ungleichgewicht zwischen den Geschlechtern in der Stichprobe ist dieses Ungleichgewicht zugunsten der Frauen für die Studie allerdings wünschenswert. Ziel der Befragung ist es, Empfehlungen für ein besseres Angebot an Blumen und Pflanzen für diejenigen Personen zu formulieren, die bei Anschaffungen für die Familie bzw. den Haushalt hauptsächlich die Kaufentscheidungen treffen - und dies sind die Frauen. Daher ist deren Übergewicht in der Stichprobe dem Ziel dienlich.

Weiterhin zeigt sich, dass das durchschnittliche Alter der Stichprobe deutlich über dem bundesdeutschen Durchschnitt liegt. Eine Erklärung hierfür könnte sein, dass die Befragung tagsüber während normalen Arbeitstagen durchgeführt wurde. Es ist somit zu vermuten, dass zu diesen Zeiten überdurchschnittlich viele Personen angesprochen wurden, welche bereits im Ruhestand sind und nicht mehr im Arbeitsleben stehen. Die deutliche Abweichung der durchschnittlichen Haushaltsgröße in der Stichprobe vom bundesdeutschen Durchschnitt überrascht. Gerade die getroffene Überlegung, dass in der Stichprobe Rentner und Pensionäre überrepräsentiert sind, würde vermuten lassen, dass die durchschnittliche Haushaltsgröße der Stichprobe relativ klein ist, tatsächlich ist sie aber größer als der bundesdeutsche Durchschnitt. Diese Abweichung scheint jedoch für die weiteren Analysen nicht nachteilig zu sein. Weiterhin konnten keine weiteren direkt vergleichbaren Daten für die Bundesrepublik Deutschland ausfindig gemacht werden. Gründe hierfür sind einerseits, dass gängige Statistiken das Haushaltseinkommen mit der Haushaltsgröße in Verbindung setzen, und andererseits die kombinierten Abfragen in der Stichprobe, beispielsweise „Ausbildung/Studium“ oder „Rentner/Pensionär“،. 


\section{Ergebnisse}

\subsection{Ergebnisse der Faktorenanalyse}

Der Aufbau dieser Arbeit orientiert sich stark an der Tatsache, dass das hier bearbeitete Themengebiet in der wissenschaftlichen Literatur bisher nur wenig Beachtung gefunden hat. Dementsprechend waren bisher sämtliche theoretischen und praktischen Vorarbeiten zur Hauptstudie explorativ ausgelegt. Diesem Gedanken folgend wird auch bei der Analyse der Ergebnisse explorativ und nicht hypothesengeleitet verfahren. Die Arbeit versteht sich als Grundlagenforschung; vor diesem Hintergrund dominiert der explorative Charakter.

Der Fragebogen besteht, wie gezeigt wurde, aus verschiedenen Items, welche die fünf verschiedenen Dimensionen messen, die wiederum darauf ausgerichtet, eine lebensstilbasierte Typologisierung zu ermöglichen (siehe Kap. 6). Der Idee des explorativen Charakters dieser Studie folgend wird in einem ersten Analyseschritt eine explorative Faktorenanalyse durchgeführt. Deren Zweck besteht darin, Items, denen sich der gleiche Faktor zuordnen lässt, zu gruppieren. Als manifeste Variablen gelten dabei die einzelnen Items im Umfeld der Faktorenanalyse, als Hintergrundvariablen oder latente Variablen die Faktoren selbst. Werden bei der explorativen Faktorenanalyse viele manifeste Variablen zu wenigen Faktoren zusammengefasst, die so im Vorfeld noch nicht bekannt waren, lassen sich eingehendere Einblicke in das Datenmaterial gewinnen. Zugleich können viele manifeste Variablen in einigen wenigen Faktoren abgebildet werden, was die Komplexität deutlich verringert (Hartmann 1999a, S. 505 ff.; Brosius 2006, S. 639).

Um in einem späteren Schritt verschiedene lebensstilbasierte Cluster beschreiben zu können, ist es zunächst notwendig zu verstehen, was die Befragten in Bezug auf BuP überhaupt bewegt. Es müssen also deren Motive in Bezug auf BuP sichtbar gemacht werden. Da komplexe Motive nicht direkt abfragbar und somit nicht direkt messbar sind, werden zunächst alle Items, die als geeignet erschienen, in eine Faktorenanalyse integriert, um verschiedene „motivbasierte“ latente Konstrukte zu erhalten. Zur deren Extraktion wird die Hauptkomponentenanalyse und die Varimax-Rotation genutzt. Es stellt sich zunächst ein Ergebnis ein, das aus sechs verschiedenen Faktoren besteht, die unterschiedlich gut zu interpretieren sind. Durch die mehrfache Variation der integrierten Items und der Unterdrückung von Faktorladungen, die kleiner als 0,5 sind, stellt sich letztendlich ein Ergebnis der Faktorenanalyse ein, das in Tabelle 5 dargestellt wird. 
Tabelle 5: Extrahierte Faktoren

\begin{tabular}{|c|c|}
\hline Faktor 1: Kultivationserfolg durch Pflanzenschutz & Ladung \\
\hline Pflanzenschutzmittel sind ein wichtiger Bestandteil meiner Arbeit mit Pflanzen & 0,780 \\
\hline Bevor eine Pflanze abstirbt, greife ich schon einmal zu Pflanzenschutzmitteln & 0,895 \\
\hline $\begin{array}{l}\text { Ich benutze grundsätzlich keine Pflanzenschutzmittel, weil diese schädlich für Menschen und die } \\
\text { Umwelt sind }\end{array}$ & $-0,876$ \\
\hline \multicolumn{2}{|l|}{ Faktor 2: Entspannung beim Gärtnern } \\
\hline Mit dem Thema „Garten“ und „Gärtnern“ assoziiere ich besonders Entspannung und Ruhe & 0,657 \\
\hline Pflanzen sind mein wichtigstes Hobby & 0,625 \\
\hline Ich empfinde es als entspannend, wenn ich mich mit Pflanzen beschäftige & 0,782 \\
\hline Für mich ist das Wichtigste bei einer Pflanze, dass sie pflegeleicht ist & $-0,569$ \\
\hline Mit dem Thema „Garten“ und „Gärtnern“ assoziiere ich besonders Arbeit & $-0,561$ \\
\hline \multicolumn{2}{|l|}{ Faktor 3: Individualität und Stil ausdrücken } \\
\hline $\begin{array}{l}\text { Ich kaufe gerne Blumen und Pflanzen als Geschenk, weil man die Möglichkeit hat, mit außerge- } \\
\text { wöhnlichen Produkten besonders zu beeindrucken }\end{array}$ & 0,532 \\
\hline $\begin{array}{l}\text { Für mich sind ausgefallene Farben oder Formen von Pflanzen wichtig, um so meine Individualität } \\
\text { auszudrücken }\end{array}$ & 0,705 \\
\hline Zuhause sind Blumen und Pflanzen das Highlight in meinem Wohnkonzept & 0,707 \\
\hline $\begin{array}{l}\text { Es macht mir Spaß, mit Pflanzen in meiner Wohnung / meinem Garten meinen eigenen Stil zu un- } \\
\text { terstreichen }\end{array}$ & 0,656 \\
\hline \multicolumn{2}{|l|}{ Faktor 4: Bequemes Verschenken } \\
\hline $\begin{array}{l}\text { Ich kaufe gerne Blumen und Pflanzen als Geschenk, weil ich ein schönes Geschenk habe, ohne je- } \\
\text { des Mal wieder kreativ sein zu müssen }\end{array}$ & 0,845 \\
\hline $\begin{array}{l}\text { Ich kaufe gerne Blumen und Pflanzen als Geschenk, weil man es noch auf die Schnelle besorgen } \\
\text { kann }\end{array}$ & 0,812 \\
\hline \multicolumn{2}{|l|}{ Faktor 5: Zahlungsbereitschaft bei besonderem Mehrwert } \\
\hline Eine Gartenrose darf ruhig etwas mehr kosten, wenn sie eine ganz besondere Blüte hat & 0,682 \\
\hline Eine Gartenrose darf ruhig etwas mehr kosten, wenn sie ganz besonders duftet & 0,683 \\
\hline Eine Gartenrose darf ruhig etwas mehr kosten, wenn ich eine Rose als Geschenk kaufen möchte & 0,598 \\
\hline $\begin{array}{l}\text { Wenn ich mich in eine Blume oder Pflanze verliebe, dann kaufe ich sie, ohne groß auf den Preis zu } \\
\text { achten }\end{array}$ & 0,500 \\
\hline \multicolumn{2}{|l|}{ Kaiser-Meyer-Olkin-Kriterium $(\mathrm{KMO})=0,719$, Bartlett-Test auf Sphärizität df $=171$} \\
\hline Lösung erklärt 57,4 \% der Gesamtvarianz & \\
\hline
\end{tabular}

Quelle: Eigene Darstellung 
Tabelle 5 verdeutlicht, welche verschiedenen Items einem Faktor zugeordnet werden können und in welcher Höhe die entsprechenden Items auf den zugehörigen Faktor laden. Als Ergebnis der Faktorenanalyse stellt sich heraus, dass sich die Kaufmotive der Befragten in fünf verschiedene Motive bzw. hier in fünf verschiedene Faktoren aufteilen lassen, auf deren Bezeichnung im Folgenden näher eingegangen wird.

Auf Faktor 1 laden ausschließlich die Items aus Frage 23, die sich mit der persönlichen Einstellung der Probanden zum Thema Pflanzenschutz befassen. Alle anderen Items der Faktorenanalyse gehen mit Ladungen kleiner als 0,5 in den Faktor 1 ein, was bedeutet, dass sie nicht zur Erklärung von Faktor 1 taugen, da ihre Ladung zu schwach ausgeprägt ist. Die hohen Faktorladungen der drei Items aus Frage 23 zeigen einerseits, dass deren Inhalt sehr stark den Charakter des Faktors prägen. Die geringen Faktorladungen bei allen anderen Items zeigen andererseits, dass der Fokus in Faktor 1 primär auf dem Kultivationserfolg liegt und zu diesem Zweck der Einsatz von Pflanzenschutzmitteln dazugehört. Durch den Einsatz von Pflanzenschutzmitteln soll der Prozess der Kultivation gesteuert und kontrolliert werden, was einer professionellen Art der Kultivation nahekommt. Daher wird Faktor 1 im Folgenden als Faktor „Kultivationserfolg durch Pflanzenschutz“ benannt.

Faktor 2 fasst Items zusammen, die sich auf die Freizeitqualität des Gärtnerns beziehen und dessen Entspannungsaspekte hervorheben. Pragmatische und arbeitsbezogene Aspekte treten dabei in den Hintergrund bzw. gehen sogar negativ in den Faktor ein. Besonders wichtig ist die Entspannung, die durch die Beschäftigung mit Pflanzen entsteht. Vor diesem Hintergrund wird Faktor 2 ,,Entspannung beim Gärtnern“ genannt.

Faktor 3 steht für eine Art nach außen gerichteten Zurschaustellens durch BuP. Ebenso steht dieser Faktor für Extrovertiertheit. Das Persönliche ist ein „Highlight“ und soll auch gerne anderen Personen, also „Externen“, gezeigt werden. Faktor 3 wird demnach als „Individualität und Stil ausdrücken “ benannt.

Obwohl im Faktor 4 nur zwei Items hochladen, ist dieser aufgrund der Faktorladungen bemerkenswert. Die Items betonen die Einfachheit und die damit verbundene Schnelligkeit eines Blumengeschenkes. Parallel betonen sie den Effekt, dass man trotz dieser Eigenschaften mit BuP als Geschenk die Möglichkeit hat, ein ausgefallenes Geschenk zu überreichen. Faktor 4 steht für die Kombination aus Pragmatismus und der trotzdem vorhandenen Möglichkeit, mit einem schnell besorgten Geschenk ein kreativ (wirkendes) Geschenk überreichen zu 
können. Aufgrund dieser Zusammensetzung wird Faktor 4 im Folgenden als ,, bequemes Verschenken " bezeichnet.

In Faktor 5 sind diejenigen Items zusammengefasst, die einen positiven Einfluss auf die Zahlungsbereitschaft der Befragten bei Gartenrosen haben. Dadurch, dass die Items für „etwas Besonderes“" stehen, stellen sie für die Befragten einen Mehrwert dar, für den sie eine erhöhte Zahlungsbereitschaft aufweisen. Alternativ entsteht diese, wenn eine Rose als besonderes Geschenk gekauft werden soll. Durch die Kombination dieser Gründe für eine erhöhte Zahlungsbereitschaft wird Faktor 5 demnach mit „Zahlungsbereitschaft bei besonderem Mehrwert" benannt.

\subsection{Ergebnisse Clusteranalyse}

Das angestrebte Ergebnis der vorliegenden Arbeit ist eine lebensstilbasierte Typologie. Um diese zu erreichen, wird eine Clusteranalyse durchgeführt. Dem starken explorativen Charakter dieser Arbeit folgend wird als Clustermethode die Ward-Methode gewählt. Im Gegensatz zu partitionierenden Methoden erfordert sie keine Festlegung einer Startpartition, die wiederum auf theoretischen Vorkenntnissen basieren sollte. Die Clusterung erfolgt nicht anhand von zu prüfenden Hypothesen. Primäres Ziel der Clusteranalyse ist das Erkennen von Mustern und Strukturen. Die aktiven Variablen der Clusteranalyse stellen die fünf extrahierten Faktoren der Faktorenanalyse dar. Dadurch, dass die einzelnen Faktoren gebündelte Motive zusammenfassen, kann man von einer motivbasierten Clusteranalyse sprechen, welche die Basis für die angestrebte lebensstilbasierte Typologie darstellt.

In die Clusteranalyse gingen $\mathrm{n}=806$ Datensätze ein. Als Vorgabe für die Anzahl an auszugebenden Clustern wurde basierend auf dem Elbow-Diagramm das Intervall vier bis sechs Cluster gewählt, so dass verschiedene Lösungsmöglichkeiten bewertet werden konnten. Lösungen mit weniger als vier bzw. mehr als sechs Clustern wurden als nicht sinnvoll angesehen und somit bereits im Vorfeld ausgeschlossen, was am Trade-off zwischen Komplexitätsreduktion und Informationsverlust liegt. Bei zu wenigen Clustern ist der Informationsverlust zu groß, bei zu vielen Clustern die dadurch entstehende Komplexität hinderlich (Brosius 2008, S. 753; Freter 2008, S. 210). Bei der Festlegung auf die Clusterlösung, die dem Autor als passendste erscheint, steht in erster Linie die gute Beschreibbarkeit der gefundenen Cluster und die theoretische Plausibilität im Vordergrund. 
Die ausgegebene 4-Cluster-Lösung stellte sich als schwierig zu interpretieren dar, da das erste Cluster mit $n=357$ deutlich zu groß war. Eine möglichst exakte Beschreibung dieses Clusters war sehr problematisch, da die Beschränkung auf vier Cluster einen zu großen Informationsverlust verursachte. Teilweise fanden sich in diesem Cluster auch sehr widersprüchliche Werte wieder, was eine sinnvolle Interpretation unmöglich machte. Die 5-Cluster Lösung erwies sich im Vergleich dazu als sinnvoll. Von den zuvor vier Clustern hat sich das größte Cluster gesplittet, wobei die anderen drei Cluster Bestand hatten und sich im Vergleich zur 4-ClusterLösung nicht veränderten. Aus dem großen Cluster der 4-Cluster-Lösung mit $n=357$ ergaben sich nun ein Cluster mit $n=171$ und ein Cluster mit $n=186$. Dies erleichterte die Interpretierbarkeit, was die 5-Cluster-Lösung zu einer möglichen Lösung machte. Allerdings ergaben sich Probleme bei der Interpretation des ersten Clusters. Bei Betrachtung der 6Cluster-Lösung zeigte sich, dass sich das problematische Cluster splittete, was die Chance einer besseren Interpretierbarkeit erhöhte. Die Größe der Cluster und die Mittelwerte der aktiven Variablen ergeben sich für die 6-Cluster-Lösung gemäß Tabelle 6 und 7. Dabei sind die Größen der Cluster aus Tabelle 6 gleichzeitig als Segmentgröße bzw. potenzieller Marktanteil anzusehen. So hat das Cluster „Kultivator“ beispielsweise eine Segmentgröße von $11 \%$ des relevanten Marktes $(\mathrm{n}=806)$.

Tabelle 6: Zusammensetzung der Cluster

\begin{tabular}{|l|l|c|c|c|}
\hline \multicolumn{2}{|l|}{} & Häufigkeit & Prozent & $\begin{array}{c}\text { Kumulierte } \\
\text { Prozente }\end{array}$ \\
\hline Gültig & Kultivator & 97 & 11 & 12 \\
\hline & Durchschnittlicher Gartenfreund & 186 & 21,1 & 35,1 \\
\hline & Pflanzengenießer & 140 & 15,9 & 52,5 \\
\hline & Desinteressierter Pragmatiker & 225 & 25,6 & 80,4 \\
\hline & Extrovertierter Naturbewahrer & 74 & 8,4 & 89,6 \\
\hline Fehlend & & 84 & 9,5 & 100 \\
\hline Gesamt & & 806 & 91,6 & \\
\hline
\end{tabular}

Quelle: Eigene Darstellung 
Tabelle 7: Stärke der Einflüsse der Motivfaktoren auf die gefundenen Cluster

\begin{tabular}{|l|c|c|c|c|c|}
\hline & $\begin{array}{l}\text { Kultivations- } \\
\text { erfolg }\end{array}$ & $\begin{array}{c}\text { Entspannung } \\
\text { beim Gärtnern }\end{array}$ & $\begin{array}{c}\text { Individ. u. } \\
\text { Stil ausdr. }\end{array}$ & $\begin{array}{c}\text { Bequemes } \\
\text { Verschenken }\end{array}$ & $\begin{array}{c}\text { Zahlungsbereit- } \\
\text { schaft bei } \\
\text { Mehrwert }\end{array}$ \\
\hline Kultivator & 0,7531471 & 0,4240892 & 0,1951149 & 0,2426561 & $-1,2805861$ \\
\hline $\begin{array}{l}\text { Durchschnittlicher } \\
\text { Gartenfreund }\end{array}$ & 0,5565506 & 0,3529572 & 0,0069762 & 0,687556 & 0,5843639 \\
\hline Pflanzengenießer & $-0,612508$ & 0,5219004 & 0,5664383 & $-0,8006414$ & 0,4892023 \\
\hline $\begin{array}{l}\text { Desinteressierter } \\
\text { Pragmatiker }\end{array}$ & $-0,4776905$ & $-1,0460332$ & $-0,30086$ & $-0,0200712$ & $-0,1108508$ \\
\hline $\begin{array}{l}\text { Extrovertierter Na- } \\
\text { turbewahrer }\end{array}$ & $-0,6460814$ & 0,3396824 & 0,6424734 & 0,8285677 & $-0,395356$ \\
\hline $\begin{array}{l}\text { Konservativer } \\
\text { Kleingärtner }\end{array}$ & 0,7674742 & 0,3615266 & $-0,944937$ & $-1,1444199$ & 0,0146979 \\
\hline
\end{tabular}

Quelle: Eigene Darstellung

Obwohl die einzelnen Cluster der Clusteranalyse noch nicht näher vorgestellt worden sind, sind sie in Tabelle 6 und 7 bereits benannt, um das Verständnis der Tabellen zu erleichtern; auf die Bezeichnungen wird im Folgenden näher eingegangen. Wie beschrieben gehen als aktive Variable die fünf extrahierten Faktoren der Faktorenanalyse in die Clusteranalyse ein. Dementsprechend lassen sich die extrahierten Cluster anhand ihrer immanenten Motive beschreiben, wozu die Mittelwerte der Cluster aus Tabelle 7 interpretiert werden.

\subsubsection{Kultivator}

Bei der Betrachtung des Clusters „Kultivator“ fällt auf, dass hier der Faktor „Zahlungsbereitschaft bei besonderem Mehrwert" die wichtigste Rolle spielt. Personen, die in Cluster $1 \mathrm{zu}$ sammengefasst werden, sind dem Kaufmotiv „Zahlungsbereitschaft bei besonderem Mehrwert" nicht zugänglich. Durch das negative Vorzeichen lehnen Personen dieses Clusters diese Idee ab. Bezieht man direkt die einzelnen Items, die in den Faktor „Zahlungsbereitschaft bei besonderem Mehrwert" einfließen, in die Analyse mit ein, zeigt sich deutlich, dass Personen in Cluster 1 sehr preissensibel sind. Auch für begründete Mehrkosten sind sie nicht bereit, mehr Geld für eine Gartenrose oder eine Pflanze auszugeben. Als stärkster positiver Faktor geht „Kultivationserfolg durch Pflanzenschutz“ in Cluster 1 ein. Es steht das Motiv „Kultivationserfolg“ im Vordergrund, wozu auch deutlich erkennbar der Einsatz von Pflanzenschutz- 
mitteln gehört. Eventuelle Bedenken über Unverträglichkeiten der Pflanzenschutzmittel gegenüber Mensch und Natur hegen Personen dieses Clusters nicht. Pflanzenschutzmittel gehören für sie zu ihrer Arbeit mit Pflanzen ganz selbstverständlich dazu. Sie kontrollieren und steuern damit die Kultivation der Pflanzen.

Der Faktor „Entspannung beim Gärtnern“ ist für Personen des ersten Clusters der zweite ausschlaggebende Aspekt. Er sagt aus, dass der Umgang mit BuP als wichtiges Hobby angesehen wird. Dabei finden die Personen innere Ruhe und Entspannung. Sie genießen die Beschäftigung mit Pflanzen und sehen diese nicht als Arbeit im Sinne eines „notwendigen Übels“ an. In Bezug auf Blumengeschenke zeigt sich, dass für Cluster 1 der pragmatische Aspekt eine Rolle spielt. Die Personen in diesem Cluster schätzen die Möglichkeit, ohne großen Aufwand ein schönes Geschenk machen zu können. Es lässt sich daher vermuten, dass sie sehr zielorientiert sind und ihr Ziel mit möglichst geringem Aufwand zu erreichen suchen.

Letztlich fließt noch der Faktor „Individualität und Stil ausdrücken“ positiv in Cluster 1 ein, wenn auch mit dem geringsten positiven Mittelwert. Die Extrovertiertheit, die dem Faktor zu Grunde liegt, ist Personen aus Cluster 1 nur bedingt wichtig. Es gefällt ihnen, wenn sie ihren Kultivationserfolg nach außen hin präsentieren können, jedoch spielt dies für sie nur eine untergeordnete Rolle. Der Fokus liegt für Personen dieses Clusters auf dem Prozess, der vor der Präsentation liegt - der Phase der Aufzucht, Pflege und Kultivation. Es ist zu vermuten, dass Personen dieses Clusters bei der Zurschaustellung ihrer Pflanzen weniger die Schönheit ihres „Produktes“ in den Vordergrund stellen, sondern vielmehr die erfolgreiche Arbeit, die sie in die Pflanze investiert haben und auf die sie stolz sind.

Angesichts dieser Aspekte, die alle gleichzeitig zu berücksichtigen sind, wird eine Person, die Cluster 1 zugehört, als „Kultivator“ bezeichnet. Mit n=97 bzw. $11 \%$ des relevanten Marktes stellt dieses Cluster das viertgrößte von sechs dar.

\subsubsection{Durchschnittlicher Gartenfreund}

Bei der Betrachtung der Faktoren, die in Cluster 2 einfließen, fällt auf den ersten Blick auf, dass alle ausgewiesenen Mittelwerte mit einem positiven Vorzeichen versehen sind. Das bedeutet, dass alle Faktoren von Personen aus Cluster 2 gutgeheißen werden und kein Faktor abgelehnt wird. Dabei weisen die Faktoren „Bequemes Verschenken“, „Zahlungsbereitschaft bei besonderem Mehrwert“ und „Kultivationserfolg durch Pflanzenschutz“ eine annähernd gleiche Ausgeprägtheit innerhalb des Clusters auf, wobei der erstgenannte Faktor am ausge- 
prägtesten ist. Personen aus Cluster 2 schätzen die Vorzüge eines Blumengeschenkes in der Art, dass sie ohne großen Aufwand ein kreatives und schönes Geschenk machen können. Sie sind pragmatisch veranlagt, schätzen aber den Stellenwert von Blumen und Pflanzen als Geschenk nicht als niedrig, sondern als relativ hoch ein. Dies zeigt sich auch daran, dass der Faktor „Zahlungsbereitschaft bei besonderem Mehrwert“ als zweitwichtigster Faktor in das Cluster einfließt. Er signalisiert, dass Personen aus Cluster 2 für Blumengeschenke eine erhöhte Preisbereitschaft haben. Des Weiteren zeigt er an, dass BuP, die Personen aus Cluster 2 besonders ansprechen, ebenfalls etwas mehr kosten dürfen. Es zeichnet diese Personen somit aus, dass es ihnen wichtig ist, etwas Schönes zu verschenken oder selbst zu haben, um sich daran zu erfreuen. Dabei spielt der Preis eine untergeordnete Rolle. Etwas besonders Schönes darf für Personen aus Cluster 2 auch etwas kosten. Für sie ist das Geld gut ausgegeben, denn man macht damit jemand anders oder sich selbst eine Freude.

Als letzter der drei nahezu gleich wichtigen Faktoren fließt „Kultivationserfolg durch Pflanzenschutz" ein. Personen aus Cluster 2 ist es wichtig, die Kultivation ihrer Pflanzen zum Erfolg zu bringen. Dabei gehört für sie auch der Einsatz von Pflanzenschutzmitteln. Sie erfreuen sich selbst daran, wenn das Ergebnis ihrer Arbeit eine Pflanze ist, die besonders duftet oder besonders schön blüht. Der Umgang und die Arbeit mit Pflanzen sind für sie nicht unangenehm. Vielmehr sehen sie diese Arbeit als ihr Hobby an, bei dem sie sich entspannen können. Annähernd keine Rolle spielt dabei für Personen aus Cluster 2 die Tatsache, dass sie sich in ihren kultivierten „Schönheiten“ selbst verwirklichen, da „Individualität und Stil ausdrücken“ für sie praktisch keine Rolle spielt. Sie sehen ihre Pflanzen nicht als Mittel, um sich selbst darzustellen, sondern gärtnern nur für sich selbst. Da sie allerdings auch die Ergebnisse ihrer Arbeit besonders genießen, ist die Bezeichnung „Gartenfreund“ treffend. Sie drückt die Affinität zum Gärtnern und zum Garten an sich aus.

Da das Gesamtbild des „Gartenfreundes“ keinerlei Extreme aufweist, scheint es sich um ein „normales“ Cluster zu handeln, weshalb im weiteren Verlauf dieser Arbeit eine Person aus Cluster 2 als „Durchschnittlicher Gartenfreund“ bezeichnet wird. Es handelt sich dabei mit $\mathrm{n}=186$ bzw. 21,2 \% des relevanten Marktes um das zweitgrößte der sechs Cluster.

\subsubsection{Pflanzengenießer}

Für Cluster 3 zeigt sich, der Mittelwert des Faktors 4 am ausgeprägtesten ist, wobei er mit einem negativen Vorzeichen versehen ist. Personen des Clusters 3 lehnen demnach die Motiv- 
aussagen, welche der Faktor „Bequemes Verschenken“ bündelt, ab. Der Faktor „Bequemes Verschenken“ zeichnet sich dadurch aus, dass er die pragmatische, schnelle und somit unkomplizierte Art beschreibt, die ein Blumengeschenk als Vorteil bietet. Durch die Ablehnung dieser Vorteile zeigt sich, dass Personen aus Cluster 3 sich gerne mit dem Thema BuP beschäftigen. Sie lehnen es ab, ein Blumengeschenk ,,auf die Schnelle“ zu besorgen. Sie wollen sich damit Zeit lassen. Sie suchen sich in Ruhe ein besonderes Geschenk aus, einerseits, weil sie gerne mit außergewöhnlichen Geschenken beeindrucken, andererseits, weil sie Besonderes auch selbst zu schätzen wissen. Bestätigt wird dies anhand der Faktoren „Individualität und Stil ausdrücken“ und „Zahlungsbereitschaft bei besonderem Mehrwert“.

Personen aus Cluster 3 sind nach den Personen aus Cluster 5 diejenigen, welche am liebsten sich und ihre Persönlichkeit zur Schau stellen. Sie sind diesbezüglich extrovertiert. Dazu nutzen sie auch Geschenke. Sie wollen andere damit beeindrucken, welches außergewöhnliche Geschenk sie gefunden haben. Sie drücken dadurch ihre Individualität und ihren eigenen Stil aus, den sie nach außen präsentieren wollen. Dabei geht es ihnen allerdings nicht nur allein um das Zurschaustellen. Vielmehr genießen sie für sich selbst auch den Umgang mit den Besonderheiten von Blumen und Pflanzen, wie der Faktor „Zahlungsbereitschaft bei besonderem Mehrwert“ zeigt. Sie schätzen besondere Merkmale wie beispielsweise eine spezielle Blüte oder einen besonderen Duft. Dabei zeigen diese Personen für besonders ansprechende Pflanzen eine erhöhte Zahlungsbereitschaft, was sowohl für den Kauf für den Eigenbedarf als auch für den Kauf als Geschenk gilt.

Personen aus Cluster 3 ziehen ihren Nutzen nicht nur aus der Schönheit von BuP. Auch „Entspannung beim Gärtnern“ spielt eine positive Rolle. Das Gärtnern und der Umgang mit Pflanzen werden nicht als Arbeit empfunden, vielmehr als innerer Ausgleich und Entspannung. Dabei spielt es keine Rolle, dass eine Pflanze pflegeleicht ist. Sich um sie zu kümmern wird als Hobby angesehen. Trotz der Affinität zu schönen und besonderen BuP lehnen Personen aus Cluster 3 den Einsatz von Pflanzenschutzmitteln ab, um zum Kultivationserfolg zu kommen. Sie halten diese für schädlich für die Umwelt, welche sie genießen wollen.

Vor dem Hintergrund dieser erkannten Merkmale erhält Cluster 3 im Folgenden die Bezeichnung „Pflanzengenießer“. Das Cluster stellt mit $\mathrm{n}=140$ bzw. 15,9\% des relevanten Marktes das drittgrößte des sechs Cluster dar. 


\subsubsection{Desinteressierter Pragmatiker}

Für Cluster 4 ist auffällig, dass sämtliche Mittelwerte des Clusters mit einem negativen Vorzeichen versehen sind, was bereits den ersten Verdacht aufkommen lässt, dass Personen aus Cluster 4 keine Affinität zu BuP haben. Untermauert wird der Anfangsverdacht durch die starke Ablehnung des Faktors „Entspannung beim Gärtnern“. Von allen sechs Clustern ist Cluster 4 das einzige, in das dieser Faktor negativ eingeht, wobei er die stärkste Ausprägung im Cluster hat. Dies bedeutet, dass Personen aus Cluster 4 die Arbeit mit BuP als Arbeit empfinden. Beim Gärtnern finden sie weder innere Ruhe noch Entspannung. Für sie ist es daher wichtig, dass Pflanzen pflegeleicht sind und sie damit möglichst wenig zu tun haben. Gärtnerische Arbeit wird nicht als Hobby aufgefasst, sondern scheint vielmehr als notwendiges Übel angesehen zu werden. Dabei spielt bei diesem notwendigen Übel der Kultivationserfolg für Personen aus Cluster 4 keine entscheidende Rolle - zumindest nicht in der Art, dass sie es für notwendig erachten, dem Kultivationserfolg mit Pflanzenschutzmitteln nachzuhelfen. Vielmehr wird deren Einsatz abgelehnt, da Schäden für Mensch und Natur befürchtet werden.

Das Zurschaustellen des eigenen Stils oder der Persönlichkeit anhand von Pflanzen ist für Personen aus Cluster 4 ebenfalls kein Antrieb. Sie lehnen Ideen dieser Art ab, ebenso wie das Stilisieren von BuP zu „Highlights eines Wohnkonzepts“ oder Ähnlichem. Passend dazu ist, dass Personen aus Cluster 4 mit schönen Blumengeschenken andere nicht beeindrucken wollen. Sie sind nicht extrovertiert. „Zahlungsbereitschaft bei besonderem Mehrwert“ ist für sie ebenfalls keine Motivation für den Umgang mit BuP. Besondere Blüten oder besondere Düfte sind ihnen nicht wichtig. Dies zeigt sich auch in der fehlenden Mehrpreisbereitschaft für solche Besonderheiten. Sie haben kein Interesse daran, sich selbst etwas Gutes zu tun, und sind nicht introvertiert.

„Bequemes Verschenken“ ist der Faktor, der am wenigsten negativ in die Clusterbeschreibung einfließt. Das bedeutet, dass die Personen aus Cluster 4 sich im Vergleich zu den anderen Faktoren am besten mit den Motiven des Faktors 4 identifizieren können. Eine pragmatische, schnelle, einfache und unkomplizierte Art der Geschenkbeschaffung ist ihnen wichtig. Personen aus Cluster 4 sind froh, wenn sie ein Geschenk haben. Ihnen ist nicht wichtig, was ihr Geschenk über sie aussagt, noch wollen sie selbst damit etwas aussagen.

Vor diesem Hintergrund bestätigt sich der Anfangsverdacht. Personen aus Cluster 4 beschäftigen sich nicht aus Überzeugung mit Blumen und Pflanzen; sie gehören für sie einfach dazu. Sie sehen darin keinen tieferen Sinn, sondern machen es einfach. Wer Cluster 4 zugehört, 
lässt sich daher treffend als „Desinteressierter Pragmatiker“ bezeichnen. Wie aus Tabelle 6 ersichtlich ist, handelt es sich dabei mit $n=225$ bzw. 25,6\% des relevanten Marktes um das größte Cluster.

\subsubsection{Extrovertierter Naturbewahrer}

Bei Betrachtung des Clusters 5 ist besonders auf Parallelen zum „Kultivator“, dem Vertreter des Clusters 1, zu achten bzw. diejenigen Parameter zu suchen, anhand derer die beiden Cluster sich voneinander abgrenzen lassen, da sie sich aus dem als zu groß erachteten Cluster 1 der 5-Cluster-Lösung ergeben haben. Betrachtet man die gesamten Mittelwerte der eingehenden Faktoren für Cluster 1 und Cluster 5, fällt auf, dass sich alle Vorzeichen der Faktoren entsprechen außer für Faktor 1. Während dieser positiv in das Cluster „Kultivator“ einfließt, geht er negativ in Cluster 5 ein. Dies scheint ein Anhaltspunkt für eine spätere Differenzierung zu sein. Personen aus Cluster 5 lehnen die Motive, die in „Zahlungsbereitschaft bei besonderem Mehrwert" gebündelt sind, ab. Sie haben für sich kein Interesse an besonders schönen oder besonders duftenden Pflanzen. Daher zeigen sie hier auch keine erhöhte Preisbereitschaft. Auch wenn sie sich spontan in eine Pflanze „verlieben“, spielt der Preis eine wichtige Rolle.

Sich selbst mit BuP etwas Gutes zu tun, ist für Personen aus Cluster 5 nicht wichtig, während der Faktor „Bequemes Verschenken“ eine große Rolle spielt: Personen aus Cluster 5 schätzen sehr die Möglichkeit, mit einem Blumengeschenk schnell und unkompliziert ein ausgefallenes Geschenk präsentieren und ohne großen Aufwand bei anderen Leute den Eindruck erwecken zu können, kreativ zu sein. Bestätigt wird dies durch die parallele Berücksichtigung des Faktors „Individualität und Stil ausdrücken“. Dieser zeigt explizit, dass Personen aus Cluster 5 sehr extrovertiert sind. Bezogen auf das Blumengeschenk zeigt sich dies in der positiv einflieBenden Aussage „Ich kaufe gerne BuP als Geschenk, weil man damit die Möglichkeit hat, mit außergewöhnlichen Produkten zu beeindrucken“. Weiterhin zeigt „Individualität und Stil ausdrücken“, dass Personen aus Cluster 5 mit ihren Pflanzen ihre Individualität und ihren eigenen Stil unterstreichen - Eigenschaften, die sie als extrovertierte Personen auch anhand ihrer Pflanzen sehr gerne nach außen hin zur Schau stellen. Sie sind Außendarsteller. Bestätigt wird diese Aussage dadurch, dass es für Personen aus Cluster 5 am unwichtigsten ist, beim Gärtnern etwas für sich selbst zu tun, was wiederum die Vermutungen für Faktor ,Zahlungsbereitschaft bei besonderem Mehrwert“ bestätigt. „Entspannung beim Gärtnern“ wird nicht abgelehnt, dennoch sind Ruhe und Entspannung nicht die ausschlaggebenden Motive für Personen aus Cluster 5. Sie beschäftigen sich nicht ungerne mit BuP, tun dies aber nicht aus dem 
Grund, weil es ihr wichtigstes Hobby ist und sie dadurch sich selbst etwas Gutes tun. Sie beschäftigen sich primär mit BuP, weil sie damit sich und ihre Pflanzen zeigen wollen.

Überraschend ist ihre Einstellung zum Faktor „Kultivationserfolg durch Pflanzenschutzmittel“. Für Personen, die bisher als extrovertierte Außendarsteller beschrieben wurden, wäre eine stark ausgeprägte Affinität zu diesem Faktor zu erwarten gewesen. Die Ergebnisse der Clusteranalyse zeigen aber, dass Personen aus Cluster 5 diesen Faktor sehr stark ablehnen. Das bedeutet, dass sie zwar in ihrer Art sehr stark nach außen orientiert sind, dabei aber ihr Bild, das sie anhand ihrer Pflanzen darstellen, nicht mit chemischen Mitteln aufbessern wollen. Personen aus Cluster 5 lehnen den Einsatz von Pflanzenschutzmitteln zugunsten eines besseren Kultivationserfolgs ab. Sie fürchten Schäden für den Mensch und die Natur, die sie bewahren wollen. Insofern zeigen sich weniger Parallelen mit dem Cluster „Kultivator“ als im Vorfeld vermutet. Personen aus Cluster 5 sind extrovertierter; für sie steht nicht der Kultivationserfolg im Vordergrund, sondern ihre Außendarstellung. Dabei lehnen Personen aus Cluster 5 im Gegensatz zu denen aus Cluster 1 den Einsatz von Pflanzenschutzmitteln deutlich ab und erscheinen damit als weniger ,,professionell““.

Aufgrund der ausgeprägten Extrovertiertheit in Kombination mit der strikten Ablehnung von chemischen Hilfsmitteln wird die Bezeichnung „Extrovertierter Naturbewahrer“ für Cluster 5 gewählt. Dabei weist dieser Cluster mit $\mathrm{n}=74$ bzw. 8,4 \% des relevanten Marktes den kleinsten Anteil aller Cluster am relevanten Markt auf.

\subsubsection{Konservativer Kleingärtner}

Für Cluster 6 zeigt sich eine deutliche Ablehnung der Faktoren „,bequemes Verschenken“ und „Individualität und Stil ausdrücken“ sowie eine deutliche Affinität zum Faktor „Kultivationserfolg durch Pflanzenschutz“. Auf den ersten Blick lässt sich somit vermuten, dass Personen aus Cluster 6 sich deutlich von denen aus Cluster 5 unterscheiden. Durch die starke Ablehnung der Motive „Bequemes Verschenken“ und „Individualität und Stil ausdrücken“ zeigt sich, dass Personen aus Cluster 6 keine extrovertierten Personen sind. Sie sehen in Pflanzen das Pragmatische: das, was sie sind, und nicht das, was sie in den Augen von manchen Personen sein sollen. Diese Einschätzung zeigt sich auch bei ihrer Einstellung zu Blumengeschenken: Personen aus Cluster 6 haben nicht die Intention, mit ihrem Blumengeschenk andere Leute zu beeindrucken. Sie sehen es viel mehr als „richtiges“ Geschenk an, nicht als Transportmittel von Eitelkeiten. Daher erklärt sich auch die Ablehnung der Idee, dass man ein 
Blumengeschenk ,,auf die Schnelle“ besorgen kann. Personen aus Cluster 6 stehen dem Wesen der Pflanzen sehr seriös gegenüber. Sie sehen darin kein „Spielzeug“.

Für den Kultivationserfolg sind Personen aus Cluster 6 bereit, chemische Mittel einzusetzen. Unter allen Clustern zeigt sich bei ihnen die größte Affinität. Für sie gehört zum Prozess der Kultivation, dass Pflanzen chemisch behandelt werden. So können sie selbst in die Entwicklung ihrer Pflanzen eingreifen und diese gegebenenfalls in die gewünschte Richtung lenken. Sie sind in dieser Beziehung den Vertretern aus Cluster 1 sehr ähnlich. Ihre Art, mit Pflanzen umzugehen, gleicht der eines Profis. Dies zeigt sich auch daran, dass die Motive „Entspannung“ und „Ruhe finden“ für sie zwar eine gewisse, aber nicht die entscheidende Rolle spielen. Sie sehen den Umgang mit Pflanzen durchaus als ihr Hobby an, bei dem sie sich entspannen können, wissen aber auch um die Arbeit, die damit verbunden ist. Man könnte sie daher als „seriöse, sachliche Hobbygärtner“ bezeichnen. Dies zeigt sich auch daran, dass es für sie bei der Pflanzenwahl nicht entscheidend ist, dass eine Pflanze pflegeleicht ist. Ihren Nutzen ziehen sie vielmehr gerade daraus, eine Pflanze zu pflegen und sich um sie zu kümmern.

Im Gegensatz dazu hat der Faktor „Zahlungsbereitschaft bei besonderem Mehrwert“ für die Personen aus Cluster 6 die geringste Ausprägung, was bedeutet, dass sie nicht für sich selbst und zum Verwöhnen ihrer Sinne kultivieren. Weiterhin zeigt sich, dass sie für besondere Merkmale bei Pflanzen keine entsprechende Wertschätzung haben, weder persönlich noch in Form einer gesteigerten Preisbereitschaft. Insofern zeigt sich, dass die Bezeichnung „Hobbygärtner" nicht passend ist, da man einerseits ein Hobby für sich selbst betreibt und andererseits normalerweise auch bereit ist, für sein Hobby gewisse Mehrkosten in Kauf zu nehmen. Vielmehr scheint es sich um Personen zu handeln, die gerne für sich sind und keinen Drang haben, sich nach außen zu präsentieren. Sie scheinen diesbezüglich sehr konservativ zu sein. Weitere Ergebnisse weisen darauf hin, dass Personen aus Cluster 6 einen nahezu professionellen Umgang mit Pflanzen pflegen und dabei keine Freizeit- oder Hobbygärtner sind. Allerdings kann auch nicht davon ausgegangen werden, dass es sich bei ihnen tatsächlich um professionelle Gärtner handelt.

Angesichts dieser Ergebnisse liegt die Bezeichnung „Konservativer Kleingärtner“ für diesen Cluster nahe. Mit $\mathrm{n}=84$ bzw. 9,5\% des relevanten Marktes ist Cluster 6 des zweitkleinste aller sechs untersuchten Cluster. 


\subsection{Clusterzuordnung unter Berücksichtigung der Lebensstildimensionen}

Die Analyse und die daraus folgende Beschreibung der sechs Cluster basiert bisher ausschließlich auf den aktiven Variablen, also denjenigen Items des Fragebogens, die in den Motivfaktoren gebündelt und in die anschließende Clusteranalyse integriert worden sind. In diesem Kapitel erfolgt eine erweiterte Analyse der ermittelten Cluster unter Berücksichtigung des Einflusses der theoretischen Lebensstildimensionen. Dabei kommen die passiven Variablen zum Einsatz, also diejenigen, die bisher nicht in der Clusteranalyse berücksichtigt wurden.

Wie in Kapitel 6 beschrieben, ist jedes Item einer Dimension zugeordnet worden (siehe dazu auch Anhang 3, „Dimension der Items“). Im Folgenden werden die einzelnen Cluster in der Reihenfolge der einzelnen Dimensionen beschrieben. Dies erleichtert die Vergleichbarkeit und Unterscheidbarkeit zwischen den Clustern und vermeidet zugleich Wiederholungen. Indem der Einfluss der fünf Lebensstildimensionen auf die Clusterzugehörigkeit berücksichtigt wird, setzt sich die Gesamtbeschreibung eines Clusters aus mehreren Teilen zusammen. Wie in Kapitel 6 auf theoretischer Basis gezeigt, kann nur die kombinierte Interpretation von aktiven Variablen (Beschreibung der Cluster anhand der Motivfaktoren) und passiven Variablen (Berücksichtigung des Einflusses der fünf Lebensstildimensionen auf die Clusterzugehörigkeit) die gefundenen Cluster möglichst exakt und realitätsnah beschreiben.

Um dies zu erreichen, liegt der folgenden Analyse der passiven Variablen die Methode der multinominalen logistischen Regressionsanalyse zu Grunde. Diese ermöglicht eine Prognose darüber, mit welcher Wahrscheinlichkeit eine Person einem bestimmten Cluster zugeordnet wird, sowie über die Stärke, mit der einzelne passive Items diese Wahrscheinlichkeit beeinflussen. Da der Reaktionskoeffizient einer Variablen allerdings sehr stark von der Konstruktion der jeweiligen Messskala abhängt, wird er im Folgenden nur anhand seiner Wirkungsrichtung über das Vorzeichen Beachtung finden. Die Vergleiche zwischen den gefundenen Clustern erfolgen anhand der Wald-Statistik, die vom Skalenniveau unabhängig ist und dem t-Wert entspricht (Backhaus et al. 2003, S. 453 ff.; Diaz-Bone/Künemund 2003, S. 14; Urban 1993, S. 39).

Zu diesem Zweck wird in der multinominalen logistischen Regression das Cluster „Desinteressierter Pragmatiker“، als Referenzcluster definiert. Die Auswahl dieses Clusters liegt darin begründet, dass sich der „desinteressierte Pragmatiker“ bisher sehr deutlich von den anderen fünf Clustern unterscheidet (siehe Kap. 7.2). Durch diese Auswahl soll die weitere Beschreibung bzw. die Differenzierung der restlichen, bisher nur anhand der Motivfaktoren beschrie- 
benen Cluster erleichtert werden. Die weiteren Ausführungen über die entsprechenden Cluster basieren dabei ausschließlich auf Items, die mindestens signifikant sind ( $p<0,1)$, da sie die Cluster besonders stark charakterisieren. Die Untersuchung des Einflusses der theoretischen Lebensstildimensionen auf die Clusterzugehörigkeit erfolgt in der Reihenfolge der beschriebenen fünf Dimensionen. Die Interpretation der Ergebnisse wird separat im nachfolgenden Kapitel vorgenommen.

\subsubsection{Sozioökonomische Situation}

In die erweiterte Analyse der sozioökonomischen Dimension fließen alle Items dieser Dimension ein, da sie bisher noch nicht als aktive Variable eingesetzt worden sind. Um die Analysemethode der multinominalen logistischen Regression anwenden zu können, müssen im Vorfeld einige Variablen verdichtet und dichotomisiert werden. Dies betrifft die Variablen der Fragen 16, 17, 28, 29 und 32 des Fragekataloges. Beim Verdichten werden viele einzelne, verschiedene Antworten in Antwortklassen bzw. Intervallen zusammengefasst. Dies hat zwar einen Informationsverlust zur Folge, jedoch wird dieser durch die reduzierte Komplexität der Antworten wieder ausgeglichen. Beim Dichotomisieren wird die Gesamtheit von Antworten durch mehrere Teilgesamtheiten ersetzt. Dabei stellt jede Teilgesamtheit ein Merkmal dar, das nur zwei verschiedene Ausprägungen annehmen kann, im einfachsten Falle ,ja“ und „nein“. Beim Dichotomisieren werden Mehrfachantworten demnach ohne Informationsverlust in einen binären Code zerlegt. Die entstehenden Teilgesamtheiten werden auch „Dummy-Variablen“"genannt.

Verdichtet werden die verschiedenen Antworten aus Frage 16 „Wo leben Sie zur Zeit?“ durch die Klassen ,in einer Stadt“ und „,in einem Dorf“. Frage 17 wird zusammengefasst in die Antworten „,in Eigentum“ und „zur Miete“. Die Antworten zu Frage 28 nach dem höchsten Schulabschluss werden verdichtet zu „bis Abitur oder Vergleichbares“ und „Abitur oder mehr“. Die Antworten zum aktuellen Beruf aus Frage 29 sind klassifiziert als „Rentner“, „teilzeitbeschäftigt“, „vollzeitbeschäftigt“ und „,anderes“. Frage 32 erfasst das Netto-Haushaltseinkommen. Dieses wird zusammengefasst zu den Klassen „0-1.300 €““, „1.301-2.500 €“ und „über $2.500 €^{“}$. Nach dem Generieren dieser neuen, verdichteten Variablen werden sie dichotomisiert und gehen anschließend als Dummy-Variablen in die multinominale logistische Regressionsanalyse ein. Dabei ist allerdings zu beachten, dass von den generierten Dummy-Variablen jeweils eine Dummy-Variable nicht in das Regressionsmodell eingehen darf. Sie dient den jeweils einfließenden Dummy-Variablen als Referenzkategorie (Bühl 
2008, S. 383). Für die erweiterte Analyse werden die folgenden Items der oben genannten Fragen jeweils als Referenzkategorie ausgewählt: Frage 16 „,in einem Dorf“, Frage 17 „zur Miete“, Frage 28 „Abitur oder mehr“, Frage 29 ,anderes“ und Frage 32 ,über $2.500 €$ “河

Tabelle 8: Einflussvariablen sozioökonomische Dimension

\begin{tabular}{|c|c|c|c|c|c|}
\hline Item & $\begin{array}{l}\text { Kulti- } \\
\text { vator }\end{array}$ & $\begin{array}{l}\text { Durchsch. } \\
\text { Garten- } \\
\text { freund }\end{array}$ & $\begin{array}{c}\text { Pflanzen- } \\
\text { genießer }\end{array}$ & $\begin{array}{c}\text { Extrov. } \\
\text { Naturbe- } \\
\text { wahrer }\end{array}$ & $\begin{array}{c}\text { Konserv. } \\
\text { Klein- } \\
\text { gärtner }\end{array}$ \\
\hline Konstanter Term & $\begin{array}{l}-1,648 \\
(2,109)\end{array}$ & $\begin{array}{c}-2,178 * * \\
(5,601)\end{array}$ & $\begin{array}{c}-1,927 * \\
(3,537)\end{array}$ & $\begin{array}{l}-1,657 \\
(2,202)\end{array}$ & $\begin{array}{c}-3,850 * * * \\
(10,270)\end{array}$ \\
\hline F17 Wohnen Sie in Wohneigentum? & $\begin{array}{c}0,964 * * \\
(6,558)\end{array}$ & $\begin{array}{l}0,947 * * \\
(10,457)\end{array}$ & & & $\begin{array}{l}1,148 * * \\
(7,090)\end{array}$ \\
\hline F27 Alter & $\begin{array}{c}0,036 * * \\
(6,134)\end{array}$ & $\begin{array}{c}0,023^{* *} \\
(3,889)\end{array}$ & $\begin{array}{c}0,051 * * * \\
(15,655)\end{array}$ & & $\begin{array}{l}0,051^{* *} \\
(10,994)\end{array}$ \\
\hline $\begin{array}{l}\text { F28 Welchen höchsten Schulabschluss haben Sie? } \\
\text { Bis Abitur oder Vergleichbares }\end{array}$ & & & & & $\begin{array}{c}0,677 * * \\
(4,510)\end{array}$ \\
\hline F29.1 Sind Sie zurzeit Rentner? & $\begin{array}{c}-1,053^{*} \\
(3,268)\end{array}$ & $\begin{array}{c}-1,545^{* *} \\
(9,278)\end{array}$ & & & \\
\hline F29.2 Sind Sie zurzeit Vollzeit berufstätig? & $\begin{array}{c}-0,798 * \\
(3,206)\end{array}$ & & $\begin{array}{c}-1,527 * * * \\
(15,250)\end{array}$ & $\begin{array}{c}-0,981^{*} \\
(4,560)\end{array}$ & \\
\hline F29.2 Sind Sie zurzeit Teilzeit berufstätig? & & & $\begin{array}{c}-1,123 * * \\
(7,751)\end{array}$ & $\begin{array}{c}-0,990 * \\
(3,855)\end{array}$ & $\begin{array}{c}-0,909 * \\
(2,841)\end{array}$ \\
\hline F30 Wie viele Personen gehören zu Ihrem Haushalt? & $\begin{array}{c}-0,351 * * \\
(5,480)\end{array}$ & & & & \\
\hline $\begin{array}{l}\text { F32 Wie hoch ist Ihr Netto-Haushaltseinkommen? } \\
0-1.300 € .\end{array}$ & $\begin{array}{c}-0,710^{*} \\
(2,859)\end{array}$ & & & & \\
\hline F33 Geschlecht & & $\begin{array}{l}0,449 * \\
(3,236)\end{array}$ & $\begin{array}{c}0,570 * * \\
(3,969)\end{array}$ & & \\
\hline \multicolumn{6}{|c|}{ Pseudo-R2: Cox/Snell $=0,178$, Nagelkerke $=0,184$, McFadden $=0,057$} \\
\hline \multicolumn{6}{|c|}{ Parameterschätzer + Wald-Test in Klammern, $*=\mathrm{p}<0,1 ; * *=\mathrm{p}<0,05 ; * * *=\mathrm{p}<0,001$} \\
\hline Referenzkategorie: Desinteressierter & & & & & \\
\hline
\end{tabular}

Quelle: Eigene Berechnungen

Tabelle 8 zeigt die signifikanten Items der sozioökonomischen Dimension. Zu besseren Verständnis dieser Items und der folgenden Tabellen sei auf die Erläuterungen über das Ausschlusskriterium von Items, die Wirkungsrichtung eines Effektes und dessen Stärke in Kapitel 7.3 verwiesen. Des Weiteren soll nochmals daran erinnert werden, dass das Cluster „Desinteressierter Pragmatiker“ das Referenzcluster darstellt. In jeder der folgenden Tabellen der 
Einflussvariablen kann man sich dieses Cluster als eigene Spalte vorstellen, in der jedes Element den Wert 0 aufweist.

Für den „Kultivator“ zeigt sich bezüglich der Wohnsituation, dass in diesem Cluster Wohneigentum signifikant häufiger verbreitet ist als im Referenzcluster „Desinteressierter Pragmatiker“. Dabei weist allerdings das Cluster „Kultivator“ von allen Clustern, die eine höhere Wohneigentumsquote als das Referenzcluster haben, die schwächste Ausprägung auf $(6,558)$. Dies bedeutet im Umkehrschluss, dass die Cluster „Durchschnittlicher Gartenfreund“ und „Konservativer Kleingärtner“ ebenfalls eine höhere Wohneigentumsquote haben als das Referenzcluster und zusätzlich beide jeweils eine höhere Wohneigentumsquote als das Cluster „Kultivator“. Bezüglich des Alters lässt sich feststellen, dass innerhalb der Stichprobe das durchschnittliche Alter des Referenzclusters bei 48,1 Jahre liegt. Für den „Kultivator“ zeigt sich anhand des positiven Vorzeichens des Beta-Wertes, dass dieses Cluster ein signifikant höheres Alter hat (6,134). Dabei ähnelt der „Kultivator“ im Hinblick auf das Alter dem Cluster „Durchschnittlicher Gartenfreund“, während die Cluster „Pflanzengenießer“ und „Konservativer Kleingärtner“ deutlich älter sind als der „Kultivator“ und der „Pflanzengenießer“.

Die Analyse der beruflichen Situation zeigt, dass trotz des höheren Alters im Cluster „Kultivator“" sowohl leicht weniger Rentner $(3,268)$ als auch leicht weniger Vollzeitbeschäftigte $(3,206)$ zusammengefasst sind. Für F30 zeigt sich, dass innerhalb der Stichprobe im Referenzcluster durchschnittlich 2,8 Personen zum Haushalt gehören, während im Cluster „Kultivator“" auf Grund des negativen Vorzeichens signifikant weniger Personen zum Haushalt gehören. Diesbezüglich ist es das einzige Cluster, das sich vom Referenzcluster signifikant unterscheidet. Bei der Frage nach dem Einkommen wird deutlich, dass der „Kultivator“ im untersten Einkommensbereich weniger vertreten ist als das Referenzcluster, wobei er wiederum das einzige aller Cluster bildet, das sich in dieser Frage signifikant vom Referenzcluster unterscheidet.

Das Cluster „Durchschnittlicher Gartenfreund“ unterscheidet in der Frage nach Wohneigentum in der Art, dass in ihm Wohneigentum sowohl häufiger als im Referenzcluster als auch häufiger als in allen anderen Clustern verbreitet ist $(10,457)$. Bezüglich des Alters zeigt sich, dass der „durchschnittliche Gartenfreund“ ein leicht höheres Alter aufweist als der „desinteressierte Pragmatiker“, wobei die Stärke dieser Ausprägung von allen Clustern die geringste ist $(3,889)$, was bedeutet, dass das Cluster das jüngste aller Cluster darstellt. Die Analyse der beruflichen Situation zeigt, dass im Cluster „Durchschnittlicher Gartenfreund“ die wenigsten Rentner vertreten sind $(9,278)$. In der Frage des Geschlechts zeigt sich anhand des 
positiven Vorzeichens, dass im Vergleich zum Referenzcluster hier signifikant mehr Frauen vertreten sind.

Das Cluster „Pflanzengenießer“ unterscheidet sich in der Frage nach dem Alter von allen Clustern am deutlichsten vom Cluster „Desinteressierter Pragmatiker“ (15,665). Der „Pflanzengenießer“ ist demnach deutlich älter als das Referenzcluster und stellt das älteste aller Cluster dar. Für die aktuelle berufliche Situation zeigt sich, dass der „Pflanzengenießer“ deutlich seltener voll berufstätig ist, sowohl im Vergleich mit dem Referenzcluster als auch im Vergleich mit allen anderen Clustern (15,250). Parallel ist auch zu erkennen, dass Gleiches für „,in Teilzeit berufstätig“ gilt. Unter allen Clustern weist der „Pflanzengenießer“ den stärksten Zusammenhang bei negativem Vorzeichen auf (7,751). Neben dem „durchschnittlichen Gartenfreund“ bildet der „Pflanzengenießer“ das einzige Cluster, das sich beim Geschlecht signifikant vom Referenzcluster unterscheidet. Wie schon das Cluster „Durchschnittlicher Gartenfreund“ beinhaltet das Cluster „Pflanzengenießer“ signifikant mehr Frauen $(3,969)$.

Das Cluster „Extrovertierter Naturbewahrer“ unterscheidet sich ausschließlich anhand der beruflichen Situation vom Referenzcluster. Die Analyse zeigt einerseits, dass der „extrovertierte Naturbewahrer“ weniger in Vollzeit berufstätig ist (4,560), andererseits aber auch, dass er weniger in Teilzeit berufstätig ist als die Vertreter des Referenzclusters. Bei allen anderen Variablen konnten keine signifikanten Unterschiede zum Referenzcluster festgestellt werden. Das Nicht-Vorhandensein von signifikanten Unterschieden bedeutet, dass die Ausprägungen der Items beim „extrovertierten Naturbewahrer“ denen beim „desinteressierten Pragmatiker“ sehr ähnlich sind. Das bedeutet beispielsweise unter anderem, dass der „extrovertierte Naturbewahrer" jünger ist als die typischen Vertreter der anderen Cluster, da diese alle älter sind als das Referenzcluster.

Das Cluster „Konservativer Kleingärtner“ unterscheidet sich anhand des Wohneigentums vom Referenzcluster, insofern es beim „konservativen Kleingärtner“ häufiger vorkommt (7,090). Es ähnelt dabei sehr dem Cluster „Kultivator“. Bei der Frage nach dem Alter zeigt sich, dass auch der „,konservative Kleingärtner“ ein höheres Alter hat als der „,desinteressierte Pragmatiker“. Gemeinsam mit dem Cluster „Pflanzengenießer“ stellen diese beiden Cluster die Gruppe der „Älteren“ dar. Als einziges Cluster unterscheidet sich das des „,konservativen Kleingärtners“ in der Frage nach dem höchsten Schulabschluss vom Referenzcluster und somit auch von allen anderen Clustern. Im Bereich „Bis Abitur oder Vergleichbares“ ist das Cluster signifikant häufiger vertreten als alle anderen Cluster. Für die aktuelle berufliche Si- 
tuation zeigt sich, dass der „konservative Kleingärtner“ seltener Teilzeit beschäftigt ist als die Vertreter des Referenzclusters und auch seltener als alle anderen Cluster $(2,841)$.

\subsubsection{Kompetenz}

Zur Analyse der Dimension „Kompetenz“ sind bereits Items als aktive Variable in die Clusteranalyse eingeflossen. Tabelle 9 zeigt daher ausschließlich die passiven Items dieser Dimension.

Tabelle 9: Einflussvariablen Dimension Kompetenz

\begin{tabular}{|c|c|c|c|c|c|}
\hline Item & $\begin{array}{l}\text { Kulti- } \\
\text { vator }\end{array}$ & $\begin{array}{l}\text { Durchschn. } \\
\text { Garten- } \\
\text { freund }\end{array}$ & $\begin{array}{l}\text { Pflanzen- } \\
\text { genießer }\end{array}$ & $\begin{array}{l}\text { Extrov. } \\
\text { Natur- } \\
\text { bewahrer }\end{array}$ & $\begin{array}{l}\text { Konserv. } \\
\text { Klein- } \\
\text { gärtner }\end{array}$ \\
\hline Konstanter Term & $\begin{array}{c}-2,103 * * \\
(7,413)\end{array}$ & $\begin{array}{c}-2,123 * * * \\
(10,221)\end{array}$ & $\begin{array}{c}-2,830 * * * \\
(14,937)\end{array}$ & $\begin{array}{l}-4,614 * * * \\
(19,965)\end{array}$ & $\begin{array}{c}-1,327 * \\
(3,178)\end{array}$ \\
\hline $\begin{array}{l}\text { F 8.2 Um sicher zu sein, die richtige Wahl beim Kauf } \\
\text { einer Blume oder Pflanze zu treffen, frage ich vor dem } \\
\text { Kauf meine Freunde oder Bekannte nach ihren Erfah- } \\
\text { rungen. }\end{array}$ & $\begin{array}{l}-0,196 * \\
(3,486)\end{array}$ & & $\begin{array}{c}-0,156 * \\
(2,831)\end{array}$ & & $\begin{array}{c}-0,200 * \\
(3,406)\end{array}$ \\
\hline $\begin{array}{l}\text { F } 8.3 \text { Wenn ich eine Blume oder Pflanze kaufe, nehme } \\
\text { ich mir genügend Zeit und lasse mich beraten. }\end{array}$ & & $\begin{array}{c}0,297 * * \\
(8,348)\end{array}$ & $\begin{array}{l}0,375 * * \\
(11,489)\end{array}$ & $\begin{array}{c}0,376 * * \\
(7,660)\end{array}$ & \\
\hline $\begin{array}{l}\text { F 14.1 Eine Gartenrose darf ruhig etwas mehr kosten, } \\
\text { wenn sie in einem Container ist (und nicht wurzel- } \\
\text { nackt, also ohne Erde und Blätter). }\end{array}$ & $\begin{array}{c}0,237 * * \\
(4,317)\end{array}$ & $\begin{array}{c}0,495 * * * \\
(22,071)\end{array}$ & $\begin{array}{c}0,300 * * \\
(7,778)\end{array}$ & $\begin{array}{c}0,294 * * \\
(5,227)\end{array}$ & $\begin{array}{c}0,332 * * \\
(7,654)\end{array}$ \\
\hline $\begin{array}{l}\text { F 14.2 Eine Gartenrose darf ruhig etwas mehr kosten, } \\
\text { wenn es sich um eine neue Sorte handelt. }\end{array}$ & & $\begin{array}{c}0,380 * * * \\
(16,385)\end{array}$ & $\begin{array}{c}0,413 * * * \\
(16,573)\end{array}$ & & \\
\hline $\begin{array}{l}\text { F 14.8 Eine Gartenrose darf ruhig etwas mehr kosten, } \\
\text { wenn sie eine Edelrose ist. }\end{array}$ & $\begin{array}{c}-0,337 * * \\
(9,911)\end{array}$ & & & & $\begin{array}{l}-0,321 * * \\
(8,399)\end{array}$ \\
\hline $\begin{array}{l}\text { F } 20 \text { Wie viel Zeit verbringen Sie im Sommer durch- } \\
\text { schnittlich pro Woche mit Gartenarbeit? }\end{array}$ & $\begin{array}{c}0,133 * * * \\
(34,685)\end{array}$ & $\begin{array}{c}0,088 * * * \\
(16,838)\end{array}$ & $\begin{array}{c}0,126 * * * \\
(34,570)\end{array}$ & $\begin{array}{c}0,090 * * * \\
(12,829)\end{array}$ & $\begin{array}{c}0,114 * * * \\
(22,559)\end{array}$ \\
\hline \multicolumn{6}{|c|}{ Pseudo-R2: Cox/Snell $=0,256$, Nagelkerke $=0,265$, McFadden $=0,087$} \\
\hline \multicolumn{6}{|c|}{ Parameterschätzer + Wald-Test in Klammern, $*=\mathrm{p}<0,1 ; * *=\mathrm{p}<0,05 ; * * *=\mathrm{p}<0,001$} \\
\hline
\end{tabular}

Quelle: Eigene Berechnungen

Dabei werden In Tabelle 9 auch nur diejenigen Items dargestellt, die ein ausreichendes Signifikanzniveau aufweisen. Zum besseren Verständnis des Ursprungs der Items sei hiermit auf Abbildung 47 verwiesen. 
Das Cluster „Kultivator““ lässt sich im Vergleich zum Referenzcluster anhand der Mehrpreisbereitschaft für eine Edelrose hoch signifikant unterscheiden. Da der Beta-Wert allerdings negativ eingeht, bedeutet dies, dass der „Kultivator“ nicht bereit ist, für eine Edelrose mehr zu bezahlen als für eine Gartenrose. Die Stärke des Zusammenhangs zwischen dieser unabhängigen Variable und dem Cluster ist mit 9,911 relativ stark ausgeprägt. Hierbei weist der „Kultivator“ sehr große Ähnlichkeit mit dem „,konservativen Kleingärtner“ auf. Für den „Kultivator“ ist allerdings eine erhöhte Preisbereitschaft festzustellen für den Fall, dass die Gartenrose in einem Container verkauft wird anstatt wurzelnackt. Dabei fällt die Stärke der Mehrpreisbereitschaft von allen Clustern am geringsten aus (4,317). Er ähnelt dabei dem ,extrovertierten Naturbewahrer“. Der „Kultivator“ fragt vor dem Kauf einer Blume oder Pflanze nicht im Bekanntenkreis nach bereits gemachten Erfahrungen. Dabei unterscheidet er sich allerdings nahezu nicht vom „Pflanzengenießer“ und vom „konservativen Kleingärtner“. Im Vergleich zum Referenzcluster zeigt sich, dass der „Kultivator“ im Sommer deutlich mehr Zeit pro Woche für Gartenarbeit aufwendet. Gemeinsam mit dem „Pflanzengenießer“ zeigt der „Kultivator“ diesbezüglich die stärksten Einflussgrößen aller Cluster $(34,685)$ auf.

Das Cluster „Durchschnittlicher Gartenfreund“ unterscheidet sich am deutlichsten anhand der Mehrpreisbereitschaft für eine Containerrose vom Referenzcluster, wobei der „durchschnittliche Gartenfreund“ diesbezüglich auch deutlich die ausgeprägteste Mehrpreisbereitschaft unter allen Clustern aufweist (22,071). Ebenso zeigt sich bei ihm eine erhöhte Preisbereitschaft für neue Sorten von Gartenrosen $(16,385)$, wobei dieser Wert nahezu gleich ist wie beim „Pflanzengenießer“ (16,573). Sie bilden die beiden einzigen Cluster, die für neue Sorten eine signifikante Mehrpreisbereitschaft erkennen lassen. Bezogen auf die Abfrage nach dem Verhalten während des Kaufes zeigt sich, dass sich der „durchschnittliche Gartenfreund“ mehr Zeit lässt und sich auch mehr beraten lässt, wobei die Stärke dieses Zusammenhangs nicht sehr ausgeprägt ist (8,348). Im Vergleich zum Referenzcluster verbringt der „durchschnittliche Gartenfreund“ im Sommer mehr Zeit mit Gartenarbeit (16,838). Verglichen mit allen anderen Clustern weist er jedoch gemeinsam mit dem „extrovertierten Naturbewahrer“ die geringste Zeit im Garten auf.

Ein deutliches Unterscheidungsmerkmal vom Referenzcluster ist für das Cluster „Pflanzengenießer“" die eben erwähnte Mehrpreisbereitschaft bei einer neuen Sorte von Gartenrosen (16,573). Bei Gartenrosen im Container zeigt sich für den „Pflanzengenießer“ ebenfalls eine Mehrpreisbereitschaft (7,778), die der des „,konservativen Kleingärtners“ ähnelt. Beim Verhalten vor dem Kauf einer Blume oder Pflanze holt der „Pflanzengenießer“ weniger Informa- 
tionen aus dem Bekanntenkreis ein als ein Vertreter des Referenzclusters, da dieser Wert negativ ausgewiesen ist. Allerdings ist die Stärke dieses Zusammenhangs recht gering $(2,831)$. Gegensätzlich verhält es sich beim Verhalten während des Kaufes. Hierbei lässt sich der „Pflanzengenießer“ deutlich mehr Zeit und lässt sich auch mehr beraten $(11,489)$. Von allen Clustern weist „Pflanzengenießer“ hierbei die deutlichste Ausprägung auf. Mit Blick auf die Arbeitsstunden verbringt der „Pflanzengenießer“ deutlich mehr Zeit im Garten als ein Vertreter des Referenzclusters (34,570). Gemeinsam mit dem „Kultivator“ bildet der „Pflanzengenießer“ in diesem Punkt die Spitzengruppe.

Das Cluster „Extrovertierter Naturbewahrer“ unterscheidet sich unter anderem durch das Verhalten während des Kaufs vom Referenzcluster. Der „extrovertierte Naturbewahrer“ nimmt sich vergleichsweise mehr Zeit und lässt sich auch vergleichsweise mehr beraten. Von allen Clustern, auf die dieser Zusammenhang zutrifft, ist er beim „extrovertierten Naturbewahrer“ allerdings am schwächsten ausgeprägt (7,660). Parallel weist dieses Cluster eine leicht erhöhte Preisbereitschaft für Gartenrosen auf, die im Container verkauft werden anstatt wurzelnackt (5,227). Ebenfalls wendet der „extrovertierte Naturbewahrer“ mehr Zeit für Gartenarbeit auf als der „desinteressierte Pragmatiker“, wobei die Stärke dieses Zusammenhangs bei beim „extrovertierten Naturbewahrer“ am geringsten von allen ausgeprägt ist $(12,829)$. Gemeinsam mit dem ,durchschnittlichen Gartenfreund“ bildet er die Gruppe mit den geringsten Stunden an Gartenarbeit.

Das Cluster „Konservativer Kleingärtner“ unterscheidet sich unter anderem anhand der Mehrpreisbereitschaft vom Referenzcluster. Es zeigt sich einerseits, dass der „konservative Kleingärtner“ eine erhöhte Preisbereitschaft für Gartenrosen im Container $(7,654)$ aufweist. Andererseits ist bei ihm aufgrund des negativen Vorzeichens gemeinsam mit dem Cluster „Kultivator“ auch eine negative Mehrpreisbereitschaft für Edelrosen im Vergleich zu Gartenrosen zu erkennen (8,399). Beim Verhalten vor dem Kauf sucht der „konservative Kleingärtner“ weniger Rat bei Bekannten und Freunden als die Vertreter des Referenzclusters, da dieser Wert mit einem negativen Vorzeichen versehen ist. Dabei ist die Stärke des Zusammenhangs allerdings relativ gering $(3,406)$ und entspricht in etwa den Ausprägungen beim „Kultivator“ und beim „Pflanzengenießer“. Der „konservative Kleingärtner“ verbringt mehr Zeit mit Gartenarbeit als Vertreter des Referenzclusters (22,559), aber auch deutlich weniger als der „Kultivator“ und der „Pflanzengenießer“. 


\subsubsection{Performanz}

Da bereits viele Items der Dimension „Performanz“ als aktive Variablen herangezogen worden sind, basiert die erweiterte Analyse dieser Dimension lediglich auf den Fragen 7 und 26. Tabelle 10 zeigt deren Ergebnisse.

Tabelle 10: Einflussvariablen Dimension Performanz

\begin{tabular}{|c|c|c|c|c|c|}
\hline Item & $\begin{array}{l}\text { Kulti- } \\
\text { vator }\end{array}$ & $\begin{array}{l}\text { Durchschn. } \\
\text { Garten- } \\
\text { freund }\end{array}$ & $\begin{array}{l}\text { Pflanzen- } \\
\text { genießer }\end{array}$ & $\begin{array}{l}\text { Extrov. } \\
\text { Natur- } \\
\text { bewahrer }\end{array}$ & $\begin{array}{l}\text { Konserv. } \\
\text { Klein- } \\
\text { gärtner }\end{array}$ \\
\hline Konstanter Term & $\begin{array}{l}2,354 * \\
(2,907)\end{array}$ & $\begin{array}{c}0,032 \\
(0,001)\end{array}$ & $\begin{array}{c}0,719 \\
(0,319)\end{array}$ & $\begin{array}{c}1,013 \\
(0,459)\end{array}$ & $\begin{array}{c}-0,237 \\
(0,025)\end{array}$ \\
\hline $\begin{array}{l}\text { F7.1 Welche Informationsquellen ziehen Sie heran, } \\
\text { wenn Sie etwas Bestimmtes über BuP wissen möchten? } \\
\text { Prospekte. }\end{array}$ & $\begin{array}{c}0,387 * * \\
(4,462)\end{array}$ & & & & \\
\hline $\begin{array}{l}\text { F7.2 Welche Informationsquellen ziehen Sie heran, } \\
\text { wenn Sie etwas Bestimmtes über BuP wissen möchten? } \\
\text { Zeitschriften/Bücher }\end{array}$ & $\begin{array}{c}0,505 * * \\
(8,528)\end{array}$ & $\begin{array}{c}0,817 * * * \\
(31,158)\end{array}$ & $\begin{array}{c}0,855^{* * *} \\
(28,523)\end{array}$ & $\begin{array}{c}0,508^{* *} \\
(7,192)\end{array}$ & $\begin{array}{c}0,963 * * * \\
(25,395)\end{array}$ \\
\hline $\begin{array}{l}\text { F7.3 Welche Informationsquellen ziehen Sie heran, } \\
\text { wenn Sie etwas Bestimmtes über BuP wissen möchten? } \\
\text { Internet. }\end{array}$ & $\begin{array}{c}0,398 * * \\
(6,060)\end{array}$ & $\begin{array}{c}0,337 * * \\
(6,453)\end{array}$ & & & \\
\hline $\begin{array}{l}\text { F7.4 Welche Informationsquellen ziehen Sie heran, } \\
\text { wenn Sie etwas Bestimmtes über BuP wissen möchten? } \\
\text { Freunde/Bekannte/Familie. }\end{array}$ & $\begin{array}{c}-0,358^{*} \\
(3,774)\end{array}$ & & & & \\
\hline $\begin{array}{l}\text { F7.5 Welche Informationsquellen ziehen Sie heran, } \\
\text { wenn Sie etwas Bestimmtes über BuP wissen möchten? } \\
\text { Züchter }\end{array}$ & & $\begin{array}{c}0,390 * * \\
(6,976)\end{array}$ & $\begin{array}{c}0,579 * * * \\
(13,861)\end{array}$ & & \\
\hline $\begin{array}{l}\text { F26.1 In welchem Wohnzimmer würden Sie sich bei } \\
\text { sich zu Hause am wohlsten fühlen? Bild } 1\end{array}$ & $\begin{array}{r}0,468 * * * \\
(13,240)\end{array}$ & $\begin{array}{c}0,239 * * \\
(4,533)\end{array}$ & $\begin{array}{c}0,439 * * * \\
(14,201)\end{array}$ & & $\begin{array}{r}0,291 * * \\
(4,516)\end{array}$ \\
\hline $\begin{array}{l}\text { F26.3 In welchem Wohnzimmer würden Sie sich bei } \\
\text { sich zu Hause am wohlsten fühlen? Bild } 3\end{array}$ & $\begin{array}{l}0,476^{* *} \\
(10,749)\end{array}$ & & & $\begin{array}{c}0,367 * * \\
(5,579)\end{array}$ & $\begin{array}{l}0,290^{*} \\
(3,518)\end{array}$ \\
\hline \multicolumn{6}{|c|}{ Pseudo-R2: Cox/Snell $=0,208$, Nagelkerke $=0,215, \mathrm{McFadden}=0,068$} \\
\hline \multicolumn{6}{|c|}{ Parameterschätzer + Wald-Test in Klammern, $*=p<0,1 ; * *=p<0,05 ; * * *=p<0,001$} \\
\hline Referenzkategorie: Desinteressierter Pragmatiker & & & & & \\
\hline
\end{tabular}

Quelle: Eigene Berechnungen

Dabei bedürfen die Ergebnisse der Frage 26 näherer Betrachtung. Da bei der Bewertung der Wohnzimmerbilder durch die Probanden das Schulnotenprinzip angewandt wurde, entsprechen kleine Werte (z. B. Note 1) einer großen Zustimmung. Dementsprechend wären negative Vorzeichen in diesem Falle als Zustimmung zu werten und nicht als Ablehnung. Da der Zu- 
sammenhang von negativem Vorzeichen mit positivem Eindruck allerdings stets zum „Umdenken“ zwingt, wurden die Ergebnisse der Frage 26 umkodiert. Konkret bedeutet dies, dass das Vorzeichen geändert dargestellt wird und somit große Werte repräsentativ sind für eine große Zustimmung. Durch die Umkodierung soll das intuitive Verständnis der Tabelle erleichtert werden. Weiterhin sei bezüglich Frage 26 erwähnt, dass zwei der vier im Fragekatalog vorgestellten Bilder nicht in der Tabelle aufgeführt sind. Dies liegt daran, dass die Items der entsprechenden Bilder nicht ausreichend signifikant waren. Wie alle anderen Items, die kein ausreichendes Signifikanzniveau aufweisen, werden daher auch die entsprechenden Bilder nicht näher betrachtet. Zum besseren Verständnis über den Ursprung der Items der Dimension „Performanz“ sei hiermit auf Abbildung 47 bzw. auf Kapitel 6.2.4 verwiesen.

Das Cluster „Kultivator“ unterscheidet sich in seinem Informationsverhalten vom Referenzcluster und von allen anderen Clustern in der Art, dass es das einzige Cluster ist, das Freunde/ Bekannte/Familie explizit nicht als Informationsquelle heranzieht, wenn es etwas Bestimmtes über BuP wissen möchte $(3,774)$. Innerhalb der Dimension „Performanz“ stellt diese Ablehnung ein Alleinstellungsmerkmal des „Kultivators“ dar. Bezüglich des Informationsverhaltens zeigt sich, dass der „Kultivator“ wiederum der Einzige ist, der Prospekte zur Informationsbeschaffung heranzieht. Über das Internet informiert er sich auch, wobei er hier aber kein Alleinstellungsmerkmal aufweist - der „durchschnittliche Gartenfreund“ informiert sich ebenfalls online, auch in der nahezu gleichen Stärke der Ausprägung. Bücher und Zeitschriften sind für den „Kultivator“ wie für alle anderen Cluster auch eine mögliche Informationsquelle. Dabei ist zu bemerken, dass der „Kultivator“ und der „extrovertierte Naturbewahrer“ diejenigen sind, die bei Bücher und Zeitschriften die Gruppe mit der geringsten Ausprägung darstellen. Bezogen auf den Einrichtungsstil zeigt sich, dass dem „Kultivator“ das Bild 1 „Prestige“ am besten gefällt (13,240), gefolgt von Bild 3 „Tradition“ (10,749). In der Reihung der Bilder entspricht die Ausprägung beim „Kultivator“ der des „konservativen Kleingärtners“, auch wenn diese deutlich schwächer ausfällt.

Beim Informationsverhalten im Cluster „Durchschnittlicher Gartenfreund“ zeigt sich, dass dieser eine sehr starke Neigung zu Zeitschriften und Bücher hat. Unter allen Clustern ist diese hier am ausgeprägtesten $(31,158)$. Dabei gruppieren sich mit ebenfalls stark ausgeprägten Werten für Bücher und Zeitschriften noch die Cluster „Pflanzengenießer“ und „Konservativer Kleingärtner“ zur Gruppe der Bücher- und Zeitschriftenfreunde. Abgesehen davon informiert sich dieses Cluster im Vergleich zum Referenzcluster auch mehr im Internet. „Kultivator“ ist neben „Durchschnittlicher Gartenfreund“ das einzige Cluster, das sich im Internet informiert, 
wobei die Stärke des Zusammenhangs nahezu gleich ist (6,453). Zusammen mit dem Cluster „Pflanzengenießer“ ist „Durchschnittlicher Gartenfreund“ auch das einzige Cluster, das sich bei Züchtern informiert, die Stärke ist beim „durchschnittlichen Gartenfreund“ allerdings geringer $(6,976)$. Bezogen auf die Frage des Einrichtungsstils zeigt sich, dass Bild 1 „Prestige“ für den „,durchschnittlichen Gartenfreund“ ein Trennungsmerkmal gegenüber dem Referenzcluster ist: Ihm gefällt Bild 1 signifikant besser als dem „desinteressierten Pragmatiker“ $(4,533)$.

Das Cluster „Pflanzengenießer“ zeigt ähnlich wie das Cluster „Durchschnittlicher Gartenfreund" beim Informationsverhalten eine deutliche Neigung zu Zeitschriften und Büchern. Diese ist stark ausgeprägt $(28,523)$, wenn auch nicht ganz so stark wie beim „durchschnittlichen Gartenfreund“. Neben Zeitschriften und Büchern informiert sich der „Pflanzengenießer“ signifikant mehr beim Züchter als das Referenzcluster (13,861). Wie bereits erwähnt, ist er neben dem „durchschnittlichen Gartenfreund“ das einzige Cluster, das sich beim Züchter direkt informiert. Für ihn gilt dies sogar noch mehr als für den „durchschnittlichen Gartenfreund“. Bei der Frage nach dem Einrichtungsstil zeigt sich, dass auch der „Pflanzengenießer“ Bild 1 „Prestige“ als schön ansieht. Von allen Clustern hat er hierbei die stärkste Ausprägung $(14,201)$.

Für das Cluster „Extrovertierter Naturbewahrer“ zeigt sich bezüglich des Informationsverhaltens, dass es sich einzig bei der Nutzung von Büchern und Zeitschriften zu Informationszwecken vom Referenzcluster unterscheidet. Der „extrovertierte Naturbewahrer“ nutzt diese Medien zwar mehr als das Referenzcluster, jedoch weniger als alle anderen Cluster (7,192). Zudem bildet er das einzige Cluster, das sich bei Bild 1 „Prestige“ nicht signifikant vom Referenzcluster unterscheidet. Die Unterscheidung bezüglich des Einrichtungsstils findet anhand von Bild 3 ,Tradition“ statt; dem „extrovertierten Naturbewahrer“ sagt dieser Einrichtungsstil signifikant mehr zu als dem Referenzcluster (5,579), allerding auch deutlich weniger als dem „Kultivator““

Das Cluster „Konservativer Kleingärtner“ unterscheidet sich beim Informationsverhalten deutlich vom Referenzcluster. Wie alle anderen Cluster auch nutzt der „konservative Kleingärtner" die Medien Zeitschriften und Bücher signifikant mehr $(25,395)$. Wie bereits oben beschrieben, gehört er bei der Nutzung von Büchern und Zeitschriften gemeinsam mit dem „durchschnittlichen Gartenfreund“ und „Pflanzengenießer“ zur Spitzengruppe, während der „Kultivator“ und der „extrovertierte Naturbewahrer“ hier eine deutlich geringere Affinität aufweisen. Anhand des Antwortverhaltens zum Einrichtungsstil zeigt sich, dass der „konser- 
vative Kleingärtner“ tendenziell den gleichen Geschmack hat wie der „Kultivator“. Beide finden jeweils Bild 1 „Prestige“ $(4,516)$ und Bild 3 „Tradition“ $(3,518)$ signifikant besser als das Referenzcluster. Allerdings sind die jeweiligen Zusammenhangsstärken beim „Kultivator“ noch ausgeprägter als beim „konservativen Kleingärtner“.

\subsubsection{Motivation}

Analog zur vorhergehenden Vorgehensweise zeigt Tabelle 11 die relevanten Items der Dimension „Motivation“. Dabei ist zu erwähnen, dass viele Items dieser Dimension aus dem Fragekatalog bereits als aktive Variablen in den Motivfaktoren enthalten sind. Tabelle 11 zeigt daher ausschließlich passive Variablen. Dass nicht alle Items der Dimension „Motivation" als aktive Variablen in die Generierung der Motivfaktoren einfließen, liegt daran, dass in den Augen des Autors nicht alle Items der Dimension gleich gut geeignet sind, lebensstilbasierte Motivfaktoren zu generieren. Da zur genauen Beschreibung von Clustern sowohl aktive als auch passive Variablen notwendig sind (siehe Kap. 6.2.1), fließen die übrigen Items der Dimension „Motivation“ in die folgende Analyse ein. Zum besseren Verständnis über den Ursprung der folgenden Items sei hiermit nochmals auf Abbildung 47 verwiesen.

Das Cluster „Kultivator“ lässt sich anhand des Kaufgrundes vom Referenzcluster differenzieren. Für Frage 10.3 zeigt sich, dass der „Kultivator“ signifikant öfter den Kaufgrund „Blütenfarbe“ angibt als der „desinteressierte Pragmatiker“, wobei die Stärke dieses Zusammenhangs relativ gering ist $(6,646)$. Im Hinblick auf die Assoziationen von „Garten und Gärtnern“ weist der „Kultivator“ einen negativen Zusammenhang mit dem Thema „Geselligkeit und Feiern“ auf $(3,208)$. Mit dem „konservativen Kleingärtner“ ist der „Kultivator“ das einzige Cluster, das sich diesbezüglich vom Referenzcluster unterscheidet. Einen stärkeren und dabei auch jeweils positiven Zusammenhang zeigt der „Kultivator“ bei den Assoziationen „Körperlicher Ausgleich“ $(3,795)$ und „Ausleben von Kreativität“ $(8,090)$. Bei Frage 22.6 ergibt sich für den „Kultivator“ wiederum ein negativer Wert, was bedeutet, dass er im Vergleich zum Referenzcluster weniger ökologische Aspekte mit dem Thema „Garten und Gärtnern“ verbinden. 
Tabelle 11: Einflussvariablen Dimension Motivation

\begin{tabular}{|c|c|c|c|c|c|}
\hline Item & $\begin{array}{l}\text { Kulti- } \\
\text { vator }\end{array}$ & $\begin{array}{l}\text { Durchschn. } \\
\text { Garten- } \\
\text { freund }\end{array}$ & $\begin{array}{l}\text { Pflanzen- } \\
\text { genießer }\end{array}$ & $\begin{array}{l}\text { Extrov. } \\
\text { Natur- } \\
\text { bewahrer }\end{array}$ & $\begin{array}{l}\text { Konserv. } \\
\text { Klein- } \\
\text { gärtner }\end{array}$ \\
\hline Konstanter Term & $\begin{array}{c}-2,103 * * \\
(7,413)\end{array}$ & $\begin{array}{c}-2,123 * * \\
(10,271)\end{array}$ & $\begin{array}{c}-2,830 * * * \\
(14,937)\end{array}$ & $\begin{array}{c}-4,614 * * * \\
(19,965)\end{array}$ & $\begin{array}{c}-1,327 * \\
(3,178)\end{array}$ \\
\hline $\begin{array}{l}\text { F10.3 Warum haben Sie sich entschieden, diese letzte } \\
\text { Gartenrose zu kaufen? Wegen der Blütenfarbe. }\end{array}$ & $\begin{array}{c}0,722 * * \\
(6,646)\end{array}$ & $\begin{array}{c}0,633 * * \\
(7,532)\end{array}$ & & $\begin{array}{c}0,619 * * \\
(3,440)\end{array}$ & \\
\hline $\begin{array}{l}\text { F10.4 Warum haben Sie sich entschieden, diese letzte } \\
\text { Gartenrose zu kaufen? Weil sie im Angebot war. }\end{array}$ & & & & $\begin{array}{l}0,464^{*} \\
(3,166)\end{array}$ & \\
\hline $\begin{array}{l}\text { F14.4 Eine Gartenrose darf ruhig etwas mehr kosten, } \\
\text { wenn sie ein Qualitätssiegel trägt. }\end{array}$ & & $\begin{array}{r}0,525 * * * \\
(15,725)\end{array}$ & $\begin{array}{c}0,476 * * * \\
(11,262)\end{array}$ & & $\begin{array}{l}0,261 * \\
(3,222)\end{array}$ \\
\hline $\begin{array}{l}\text { F22.2 Mit dem Thema „Garten“ und „Gärtnern“ asso- } \\
\text { ziiere ich besonders Geselligkeit und Feiern. }\end{array}$ & $\begin{array}{c}-0,241^{*} \\
(3,208)\end{array}$ & & & & $\begin{array}{c}-0,331 * * \\
(6,169)\end{array}$ \\
\hline $\begin{array}{l}\text { F22.3 Mit dem Thema „Garten“ und „Gärtnern“ asso- } \\
\text { ziiere ich besonders körperlichen Ausgleich. }\end{array}$ & $\begin{array}{c}0,293 * * \\
(3,795)\end{array}$ & $\begin{array}{c}0,281 * * \\
(4,913)\end{array}$ & & $\begin{array}{l}0,299 * \\
(2,943)\end{array}$ & \\
\hline $\begin{array}{l}\text { F22.5 Mit dem Thema „Garten“ und „Gärtnern“ asso- } \\
\text { ziiere ich besonders Ausleben von Kreativität. }\end{array}$ & $\begin{array}{c}0,480 * * \\
(8,090)\end{array}$ & $\begin{array}{r}0,526 * * * \\
(13,092)\end{array}$ & $\begin{array}{l}1,013 * * * \\
(31,034)\end{array}$ & & \\
\hline $\begin{array}{l}\text { F22.6 Mit dem Thema „Garten“ und „Gärtnern“ asso- } \\
\text { ziiere ich besonders ökologische Aspekte (Respekt vor } \\
\text { der Natur, eigener Anbau von Lebensmitteln). }\end{array}$ & $\begin{array}{c}-0,236^{*} \\
(3,097)\end{array}$ & & & & \\
\hline $\begin{array}{l}\text { F24.5 Durch Blumen und Pflanzen im Haus kann man } \\
\text { die Kinder gut an naturbezogene Themen heranführen. }\end{array}$ & $\begin{array}{l}0,260^{*} \\
(3,130)\end{array}$ & $\begin{array}{c}0,389 * * \\
(9,067)\end{array}$ & $\begin{array}{l}0,266^{*} \\
(3,779)\end{array}$ & & \\
\hline $\begin{array}{l}\text { F24.6 Ich kann es nicht gut ertragen, zu sehen, wenn } \\
\text { es einer Pflanze schlecht geht. }\end{array}$ & $\begin{array}{c}0,458^{* *} \\
(8,461)\end{array}$ & $\begin{array}{c}0,338 * * \\
(6,855)\end{array}$ & $\begin{array}{c}0,426 * * \\
(8,930)\end{array}$ & $\begin{array}{c}0,392 * * \\
(4,698)\end{array}$ & \\
\hline $\begin{array}{l}\text { F25.3 Ich mag Überraschungen. Es ist mir wichtig, ein } \\
\text { aufregendes Leben zu führen. }\end{array}$ & $\begin{array}{c}-0,297 * \\
(3,041)\end{array}$ & & & & \\
\hline \multicolumn{6}{|c|}{ Pseudo-R2: Cox/Snell $=0,352$, Nagelkerke $=0,364$, McFadden $=0,126$} \\
\hline \multicolumn{6}{|c|}{ Parameterschätzer + Wald-Test in Klammern, $*=\mathrm{p}<0,1 ; * *=\mathrm{p}<0,05 ; * * *=\mathrm{p}<0,001$} \\
\hline Referenzkategorie: Desinteressierter Pragmatiker & & & & & \\
\hline
\end{tabular}

Quelle: Eigene Berechnungen

Der „Kultivator“ bildet das einzige Cluster, das sich in dieser Hinsicht zwar nur schwach (3,097), aber dennoch signifikant vom Referenzcluster unterscheidet. Anhand der Ergebnisse zu Frage 24.5 zeigt sich, dass der „Kultivator“ mehr als der „,desinteressierte Pragmatiker“ der Aussage zustimmt, dass man Kinder durch BuP im Haus gut an naturbezogene Themen heranführen kann. Dies lässt für das Cluster eine gewisse Naturverbundenheit vermuten, auch wenn sie von allen Clustern am geringsten ausgeprägt ist $(3,130)$. Am deutlichsten unterscheidet sich der „Kultivator“ vom Referenzcluster anhand der auf Frage 24.6 bezogenen Aussage „Ich kann es nicht gut ertragen, zu sehen, wenn es einer Pflanze schlecht geht“. BuP 
scheinen demnach eine deutlich dominantere emotionale Rolle für den „Kultivator“ zu spielen als für den „desinteressierten Pragmatiker“ $(8,461)$. Im Hinblick auf das Lebensziel in Frage 25.3 zeigt sich, dass der „Kultivator“ das einzige Cluster bildet, das sich signifikant vom Referenzcluster abhebt. Da der entsprechende Wert negativ eingeht, ist es für den „Kultivator“ dementsprechend weniger wichtig, ein aufregendes Leben zu führen $(3,041)$.

Beim Cluster „Durchschnittlicher Gartenfreund“ ist das deutlichste Unterscheidungsmerkmal vom Referenzcluster das subjektive Risikoempfinden beim BuP-Kauf (Frage 14.4). Hier zeigt sich der für das Cluster stärkste gemessene Zusammenhang (15,725), was auf eine Risikoaversion des „durchschnittlichen Gartenfreundes“ beim BuP-Kauf hinweist. Bei den Assoziationen zum Thema „Garten und Gärtnern“ unterscheidet er sich besonders stark anhand der Assoziation „Ausleben von Kreativität" vom Referenzcluster (13,092), aber auch anhand von „Körperlicher Ausgleich“ $(4,913)$. Obwohl das Ausleben von Kreativität für den „durchschnittlichen Gartenfreund“ eine größere Rolle spielt als der körperliche Ausgleich, ist es ihm weit weniger wichtig als dem „Pflanzenfreund“, der hier die Spitzenposition vertritt. Bei der Analyse des Ergebnisses zu Frage 10.3 zeigt sich, dass „Blütenfarbe“ für den „durchschnittlichen Gartenfreund" ein Kaufgrund ist. Dabei weist er bei dieser Alternative den stärksten Zusammenhang aller Cluster auf (7,532). Dies trifft ebenfalls beim Thema „Naturverbundenheit“ in Zusammenhang mit Frage 24.5 zu (9,067). Im Vergleich zum Referenzcluster lässt der „durchschnittliche Gartenfreund“ eine ausgeprägtere Verbundenheit mit Blumen und Pflanzen erkennen (Frage 24.6), auch wenn die Stärke dieses Zusammenhangs nicht sonderlich ausgeprägt ist $(6,855)$.

Den mit Abstand stärksten Zusammenhang zwischen unabhängiger und abhängiger Variable innerhalb der Dimension „Motivation“ weist das Cluster „Pflanzengenießer“ bei Frage 22.5 auf. Es unterscheidet sich hier von allen Clustern am deutlichsten vom Referenzcluster. Der „Pflanzengenießer“ assoziiert mit dem Thema „Garten und Gärtnern“ von allen Clustern am stärksten das Thema „Ausleben von Kreativität““ $(31,034)$. Weiterhin kann er anhand des subjektiven Risikoempfindens differenziert werden (Frage 14.4). Der „Pflanzengenießer“ zeigt, ähnlich wie der „durchschnittliche Gartenfreund“, im Vergleich zum Referenzcluster eine deutlich höhere Zahlungsbereitschaft für Gartenrosen mit Qualitätssiegel (11,262). Die Naturverbundenheit ist gemäß Frage 24.5 im Cluster „Pflanzengenießer“ leicht ausgeprägter als im Referenzcluster (3,779), ebenso die emotionale Rolle, die BuP für das Cluster spielen $(8,930)$. 
Das Cluster „Extrovertierter Naturbewahrer“ unterscheidet sich innerhalb der Dimension „Motivation“ in mehreren Eigenschaften signifikant vom Referenzcluster, wobei alle Unterschiede recht schwach ausgeprägt sind. Am deutlichsten fällt der Unterschied in Frage 24.6 aus, der emotionalen Rolle von BuP. Diese ist für den „extrovertierten Naturbewahrer“ größer als für den „desinteressierten Pragmatiker“ (4,698), gleichzeitig aber auch geringer als bei allen anderen Clustern. Kauft der „extrovertierte Naturbewahrer“ eine Rose, entscheidet er sich sowohl öfters anhand der Blütenfarbe $(3,440)$ als auch anhand der Tatsache, dass die Gartenrose im Angebot war, für einen Kauf $(3,166)$. Weiterhin unterscheidet sich der „extrovertierte Naturbewahrer“ anhand der Assoziation „Garten und Gärtnern“ mit dem Thema „Körperlicher Ausgleich“ signifikant, wobei er von allen Clustern für diese Assoziation die schwächste aller signifikanten Ausprägungen aufweist $(2,943)$.

Das Cluster „Konservativer Kleingärtner““ hebt sich vom Referenzcluster besonders anhand der Assoziation „Garten und Gärtnern“ mit dem Thema „Geselligkeit und Feiern“ ab. Ebenso wie beim „Kultivator“ ist diese Assoziation negativ besetzt; beide weisen eine geringere Verbundenheit auf als das Referenzcluster, wobei die „Ablehnung“ dieser Assoziation beim „konservativen Kleingärtner“ deutlich ausgeprägter ist $(6,169)$. Ein weiteres Differenzierungsmerkmal ist die Risikoneigung beim BuP-Kauf (Frage 14.4). Sie ist beim „konservativen Kleingärtner“ leicht ausgeprägter als beim Referenzcluster $(3,222)$, allerdings auch weit weniger ausgeprägt als beim „,durchschnittlichen Gartenfreund“ und beim „Pflanzenfreund“.

\subsubsection{Beobachtbares Kaufverhalten}

Analog zur erweiterten Analyse der sozioökonomischen Situation sind bisher noch keine Items der Dimension „Beobachtbares Kaufverhalten“ als aktive Variable in Erscheinung getreten. Daher fließen alle Items dieser Dimension in die folgende Analyse ein, was sich im Umfang von Tabelle 12 zeigt. Zu erwähnen ist die Besonderheit der Items 6.10 bis 6.15., die sich um das Preisniveau der verschiedenen Vertriebskanäle drehen. Durch die Fragestellung und die entsprechende Codierung im Fragekatalog stehen negative Vorzeichen in der Analyse für das Attribut „günstige Preise“ und positive Vorzeichen demnach für „hohe Preise“. Zur konkreten Darstellungsform dieser Abfrage sei auf den Fragekatalog im Anhang verwiesen. 
Tabelle 12: Einflussvariablen Dimension Beobachtbares Kaufverhalten

\begin{tabular}{|c|c|c|c|c|c|}
\hline Item & $\begin{array}{l}\text { Kulti- } \\
\text { vator }\end{array}$ & $\begin{array}{l}\text { Durchsch. } \\
\text { Garten- } \\
\text { freund }\end{array}$ & $\begin{array}{l}\text { Pflanzen- } \\
\text { genießer }\end{array}$ & $\begin{array}{l}\text { Extrov. } \\
\text { Natur- } \\
\text { bewahrer }\end{array}$ & $\begin{array}{l}\text { Konserv. } \\
\text { Klein- } \\
\text { gärtner }\end{array}$ \\
\hline Konstanter Term & $\begin{array}{l}-5,842 \\
(0,448)\end{array}$ & $\begin{array}{r}-0,881 \\
(0,059)\end{array}$ & $\begin{array}{l}-1,238 \\
(0,079)\end{array}$ & $\begin{array}{l}-4,784 \\
(0,712)\end{array}$ & $\begin{array}{l}-5,803 \\
(1,077)\end{array}$ \\
\hline $\begin{array}{l}\text { F2 Blumen und Pflanzen kann man sich selbst kaufen, } \\
\text { aber auch verschenken. Bitte geben Sie an, was bei Ihnen } \\
\text { mehrheitlich zutrifft! }\end{array}$ & $\begin{array}{c}-0,640 * \\
(2,750)\end{array}$ & & & $\begin{array}{l}0,679^{*} \\
(2,780)\end{array}$ & \\
\hline $\begin{array}{l}\text { F4.1 Wo haben Sie persönlich in den letzten zwölf Mona- } \\
\text { ten Pflanzen und Gartenbedarf gekauft? Baumarkt }\end{array}$ & & & $\begin{array}{c}-1,018^{*} \\
(3,239)\end{array}$ & & \\
\hline F4.2 dito. Antwort: Gartencenter & & $\begin{array}{l}1,011 * * \\
(4,080)\end{array}$ & & & $\begin{array}{l}1,331 * \\
(3,536)\end{array}$ \\
\hline F4.3 dito. Antwort: Supermarkt & & & & $\begin{array}{l}1,982 * * \\
(4,479)\end{array}$ & \\
\hline F4.4 dito. Antwort: Gärtnerei & & & & & $\begin{array}{l}1,554 * \\
(, 294)\end{array}$ \\
\hline F4.5 dito. Antwort: Blumenfachgeschäft & & & $\begin{array}{l}1,155^{* *} \\
(5,183)\end{array}$ & & \\
\hline F4.6 dito. Antwort: Baumschule & $\begin{array}{l}1,922 * * \\
(4,966)\end{array}$ & $\begin{array}{l}1,720 * * * \\
(12,189)\end{array}$ & $\begin{array}{l}1,377 * * \\
(5,929)\end{array}$ & $\begin{array}{l}3,632 * * * \\
(15,639)\end{array}$ & $\begin{array}{l}1,875 * * \\
(6,556)\end{array}$ \\
\hline $\begin{array}{l}\text { F5.1 Welche drei Aspekte sind bei der Wahl der Ein- } \\
\text { kaufsstätte für Sie am wichtigsten, wenn Sie BuP kaufen } \\
\text { möchten? Freundliche Mitarbeiter }\end{array}$ & $\begin{array}{l}2,048^{*} \\
(3,714)\end{array}$ & & & $\begin{array}{l}1,926^{*} \\
(3,057)\end{array}$ & \\
\hline F5.2 dito. Antwort: Wo ich mich auskenne. & & $\begin{array}{l}1,659^{*} \\
(3,479)\end{array}$ & $\begin{array}{l}1,767 * \\
(3,374)\end{array}$ & $\begin{array}{c}2,954 * * \\
(3,907)\end{array}$ & $\begin{array}{l}2,723^{*} \\
(3,620)\end{array}$ \\
\hline F5.3 dito. Antwort: Nähe zum Wohnort & & & $\begin{array}{c}-1,702 * * \\
(4,730)\end{array}$ & & \\
\hline F5.4 dito. Antwort: Große Auswahl & & & & $\begin{array}{c}2,751 * * \\
(6,211)\end{array}$ & \\
\hline F5.6 dito. Antwort: Günstige Preise & & & & $\begin{array}{l}2,371^{*} \\
(3,709)\end{array}$ & \\
\hline $\begin{array}{l}\text { F6.1 Wo werden Ihre Erwartungen hinsichtlich Produkt- } \\
\text { qualität beim Kauf von Blumen und Pflanzen am besten } \\
\text { erfüllt? Baumarkt }\end{array}$ & $\begin{array}{l}1,954 * * \\
(5,730)\end{array}$ & & & & \\
\hline F6.2 dito. Antwort: Discounter & $\begin{array}{c}-3,109 * * \\
(4,406)\end{array}$ & $\begin{array}{c}-1,252 * * \\
(4,354)\end{array}$ & & & \\
\hline F6.3 dito. Antwort: Wochenmarkt & & & $\begin{array}{c}-1,366 * * \\
(5,376)\end{array}$ & $\begin{array}{c}-2,038 * * \\
(6,305)\end{array}$ & \\
\hline F6.4 dito. Antwort: Gärtnerei & & & & & $\begin{array}{c}-1,829^{*} \\
(3,169)\end{array}$ \\
\hline $\begin{array}{l}\text { F6.5 Wo werden Ihre Erwartungen hinsichtlich Service } \\
\text { beim Kauf von Blumen und Pflanzen am besten erfüllt? } \\
\text { Gartencenter }\end{array}$ & $\begin{array}{l}1,277 * \\
(3,326)\end{array}$ & & $\begin{array}{l}1,184 * * \\
(5,022)\end{array}$ & & \\
\hline F6.6 dito. Antwort: Discounter & & $\begin{array}{l}1,987^{*} \\
(3,166)\end{array}$ & & & \\
\hline
\end{tabular}




\begin{tabular}{|c|c|c|c|c|c|}
\hline F6.7 dito. Antwort: Supermarkt & & & $\begin{array}{c}-1,847 * \\
(2,725)\end{array}$ & & \\
\hline F6.8 dito. Antwort: Wochenmarkt & & & & $\begin{array}{c}2,909 * * * \\
(10,479)\end{array}$ & \\
\hline F6.9 dito. Antwort: Gärtnerei & & & & & $\begin{array}{l}2,362^{*} \\
(3,480)\end{array}$ \\
\hline $\begin{array}{l}\text { F6.10 Wo werden Ihre Erwartungen hinsichtlich Preise } \\
\text { beim Kauf von Blumen und Pflanzen am besten erfüllt? } \\
\text { Baumarkt }\end{array}$ & $\begin{array}{c}-1,947 * * \\
(6,471)\end{array}$ & & & & $\begin{array}{r}-1,209^{*} \\
(2,948)\end{array}$ \\
\hline F6.11 dito. Antwort: Supermarkt & $\begin{array}{c}4,438 * * * \\
(13,933)\end{array}$ & $\begin{array}{l}1,425^{* *} \\
(4,096)\end{array}$ & $\begin{array}{l}1,560 * \\
(3,423)\end{array}$ & & \\
\hline F6.12 dito. Antwort: Baumschule & $\begin{array}{c}2,071 * * \\
(5,827)\end{array}$ & & & & \\
\hline F6.13 dito. Antwort: Gärtnerei & & $\begin{array}{c}-1,305^{* *} \\
(6,380)\end{array}$ & & $\begin{array}{c}-1,954 * * \\
(5,455)\end{array}$ & $\begin{array}{l}1,376^{*} \\
(3,415)\end{array}$ \\
\hline F6.14 dito. Antwort: Wochenmarkt & & $\begin{array}{c}-0,940^{*} \\
(4,832)\end{array}$ & $\begin{array}{c}-1,298 * * \\
(7,256)\end{array}$ & & \\
\hline F6.15 dito. Antwort: Gartencenter & & & & $\begin{array}{c}-1,536^{*} \\
(3,594)\end{array}$ & $\begin{array}{l}1,234^{*} \\
(2,770)\end{array}$ \\
\hline $\begin{array}{l}\text { F9 Bitte erinnern Sie sich an Ihren letzten Kauf einer Gar- } \\
\text { tenrose zurück! Hatten Sie den Kauf der Gartenrose ge- } \\
\text { plant oder ist die Entscheidung spontan im Geschäft gefal- } \\
\text { len? }\end{array}$ & $\begin{array}{c}-4,858 * * * \\
(15,146)\end{array}$ & & & & \\
\hline $\begin{array}{l}\text { F14 Eine Gartenrose darf ruhig etwas mehr kosten, wenn } \\
\text { ich gerade etwas mehr Geld im Portemonnaie habe. }\end{array}$ & $\begin{array}{c}-1,105 * * \\
(8,371)\end{array}$ & & & & \\
\hline $\begin{array}{l}\text { F21 Wie viel Geld geben Sie ungefähr im Jahr für Blumen } \\
\text { und Pflanzen aus? }\end{array}$ & & & $\begin{array}{c}0,004 * * \\
(5,981)\end{array}$ & & $\begin{array}{l}0,003^{*} \\
(2,989)\end{array}$ \\
\hline F21a ... davon als Geschenk? & & & $\begin{array}{c}-0,009 * \\
(2,993)\end{array}$ & & $\begin{array}{c}-0,016^{* *} \\
(4,836)\end{array}$ \\
\hline \multicolumn{6}{|c|}{ Pseudo-R2: Cox $/$ Snell $=0,692$, Nagelkerke $=0,717$, McFadden $=0,351$} \\
\hline \multicolumn{6}{|c|}{ Parameterschätzer + Wald-Test in Klammern, $*=\mathrm{p}<0,1 ; * *=\mathrm{p}<0,05 ; * * *=\mathrm{p}<0,001$} \\
\hline Referenzkategorie: Desinteressierter Pragmatiker & & & & & \\
\hline
\end{tabular}

Quelle: Eigene Berechnungen

Das Cluster „Kultivator“ unterscheidet sich im Hinblick auf den Kaufgrund vom Referenzcluster. Wie aus Frage 2 ersichtlich wird, kauft der „Kultivator“ BuP häufiger zum Verschenken als der „desinteressierte Pragmatiker“. Weiterhin hebt er sich anhand der bevorzugten Einkaufsstätten ab. Wie alle anderen Cluster auch kauft auch er häufiger in einer Baumschule ein als das Referenzcluster, wobei dieser Zusammenhang beim „Kultivator“ den schwächsten aller Werte aufweist $(4,966)$. Besonderen Wert legt er beim Einkauf auf freundliche Mitarbeiter $(3,714)$ - zusammen mit dem „extrovertierten Naturbewahrer“ ist er der Einzige, für den 
der Aspekt der Freundlichkeit relevant ist. Im Gegensatz zu allen anderen Clustern unterscheidet sich der Aspekt einer vertrauten Einkaufsumgebung (F5.2) beim „Kultivator“ nicht signifikant vom Referenzcluster. Er spricht zudem als einziges Cluster dem Baumarkt als Einkaufsstätte eine hohe Qualität zu (5,730), dem Discounter hingegen eine schlechte $(4,406)$. Bei den Erwartungen an den Service unterscheidet sich der „Kultivator“ ausschließlich bei der Kategorie „Gartencenter“ vom Referenzcluster. In seinen Augen ist der Service in Gartencentern signifikant besser $(3,326)$. Hinsichtlich der Preiserwartungen spricht der „Kultivator“ im Gegensatz zum Supermarkt dem Baumarkt günstige Preise zu $(6,471)$, wobei der Wert der Stärke besonders ausgeprägt ist $(13,933)$. Ebenso schätzt der „Kultivator“ das Preisniveau von Baumschulen höher ein als das Referenzcluster, wobei die Stärke deutlich geringer ausfällt als bei der Kategorie „Supermarkt“ $(5,827)$. Das Cluster „Kultivator“ ist das einzige, das sich hier von den Preiserwartungen des Referenzclusters signifikant unterscheidet. Am deutlichsten grenzt es sich vom Referenzcluster und von allen anderen Clustern aber bei Frage 9 ab: Der „Kultivator“ ist derjenige, der am ehesten BuP geplant kauft $(15,146)$. Alle anderen Cluster unterscheiden sich in dieser Frage nicht signifikant vom „desinteressierten Pragmatiker“. Eine entspannte finanzielle Situation (F14) hat hingegen beim „Kultivator“ keinen Einfluss auf eine eventuelle Mehrpreisbereitschaft für eine Gartenrose $(8,371)$, in diesem Punkt ist er das einzige Cluster, das sich vom Referenzcluster unterscheidet.

Das Cluster „Durchschnittlicher Gartenfreund“ unterscheidet sich in zwei Punkten der Einkaufsstättenwahl vom Referenzcluster. So findet der Kauf bei ihm häufiger im Gartencenter $(4,080)$ und häufiger in der Baumschule $(12,189)$ statt. Bei der Wahl der Einkaufsstätte legt der „durchschnittliche Gartenfreund“ darauf Wert, dass er sich in dieser auskennt $(3,479)$. Bei den Qualitätserwartungen kommt der Discounter beim ihm nicht gut weg. Neben dem „Kultivator“ bildet er das einzige Cluster, das die Kategorie „Discounter“ in dieser Frage als schlecht bewertet $(4,354)$. Allerdings spricht der „durchschnittliche Gartenfreund“ als einziger Cluster Discountern einen guten Service zu $(3,166)$. Hohe Preise erwartet er im Supermarkt (4,096), hingegen im Vergleich zum Referenzcluster niedrigere Preise auf dem Wochenmarkt $(4,832)$ und in einer Gärtnerei $(6,380)$. Die Erwartung günstiger Preise in der Gärtnerei ist beim „durchschnittlichen Gartenfreund“ unter allen Clustern am ausgeprägtesten.

Das Cluster „Pflanzengenießer““ unterscheidet sich bei der Wahl der bevorzugten Einkaufsstätte in mehreren Ausprägungen vom Referenzcluster und den anderen Clustern. Als einziges unterscheidet es sich signifikant bei der Wahl der Kategorie „Baumarkt“ vom „desinteressierten Pragmatiker“. Da das negative Vorzeichen die Wirkungsrichtung bestimmt, bedeutet dies, 
dass der „Pflanzengenießer“ während der letzten 12 Monate signifikant weniger im Baumarkt eingekauft hat als der „desinteressierte Pragmatiker“ und somit auch weniger als alle anderen Cluster (3,239). Mehr als das Referenzcluster und auch mehr als alle anderen Cluster hat der „Pflanzengenießer“ hingegen im Blumenfachgeschäft eingekauft $(5,183)$. Dies trifft auch für den Kauf in einer Baumschule zu (5,929), allerdings haben alle Cluster dort mehr als das Referenzcluster eingekauft, so dass die „Baumschule“ das Cluster „Pflanzengenießer“ weniger charakterisiert als die beiden Ausprägungen „Baumarkt“ und „Blumenfachgeschäft“. Das bevorzugteste Kriterium für die Wahl der Einkaufsstätte ist für den „Pflanzengenießer“ die Vertrautheit der Einkaufsstätte (3,374). Von allen Clustern ist dabei der Aspekt „Nähe zum Wohnort“ am unwichtigsten (4,730); der „Pflanzengenießer“ bildet das einzige Cluster, das sich hier signifikant vom Referenzcluster unterscheidet. Keine gute Qualität erwartet der „Pflanzengenießer“ auf dem Wochenmarkt $(5,376)$. Nur der „extrovertierte Naturbewahrer“ weist hier die gleiche negative Tendenz der Qualitätserwartung auf, während sich alle anderen Cluster diesbezüglich nicht unterscheiden. Einen guten Service erwartet der „PflanzengenieBer“ im Gartencenter (5,022), im Supermarkt hingegen einen schlechten $(2,725)$. Dabei ist er der Einzige, der sich mit seinen Serviceerwartungen gegenüber dem Supermarkt signifikant vom Referenzcluster unterscheidet. Zudem erwartet er im Supermarkt höhere Preise $(3,423)$, auf dem Wochenmarkt hingegen günstige $(7,256)$. Für die Fragen 21 und 21a zeigt sich, dass der „Pflanzengenießer“ mehr Geld für BuP ausgibt als das Referenzcluster (5,981), dabei aber anteilsmäßig weniger für Geschenke $(2,993)$.

Das Cluster „Extrovertierter Naturbewahrer“ unterscheidet sich neben dem „Kultivator“ als einziges im Hinblick auf den Kaufgrund von BuP. Allerdings kauft der „extrovertierte Naturbewahrer" mehr für den Eigenbedarf als zum Verschenken (2,780). Was die Wahl der Einkaufsstätte betrifft, kauft er mehr als andere im Supermarkt ein (4,479), am meisten von allen Clustern aber in der Baumschule (15,639). Dabei sind ihm verschiedene Aspekte der Einkaufsstättenwahl wichtig. Eine große Auswahl ist das wichtigste Kriterium $(6,211)$, gefolgt von den drei nahezu gleich wichtigen Aspekten „Auskennen in der Einkaufsstätte“ $(3,907)$, „Günstige Preise“ $(3,709)$ und „Freundliche Mitarbeiter“ $(3,057)$. Hinsichtlich der Qualitätserwartungen unterscheidet der ,extrovertierte Naturbewahrer“ sich nur mit Blick auf den Wochenmarkt signifikant vom Referenzcluster. Er erwartet dort ebenso wie der „Pflanzengenießer“ eine schlechte Qualität, wobei dies bei ihm noch ausgeprägter ist $(6,305)$. Andererseits spricht er dem Wochenmarkt einen guten Service zu $(10,479)$ und unterscheidet sich 
hier als einziges Cluster vom Referenzcluster. Von einer Gärtnerei erwartet er ein günstiges Preisniveau $(5,455)$, ebenso vom Gartencenter, wenngleich weniger ausgeprägt $(3,594)$.

Das Cluster „Konservativer Kleingärtner““ unterscheidet sich bei der Einkaufsstättenwahl in mehreren Punkten signifikant vom Referenzcluster. Neben dem „durchschnittlichen Gartenfreund“ ist es das einzige Cluster, das mehr im Gartencenter einkauft $(3,536)$, und als einziges überhaupt kauft der „konservative Kleingärtner“ in einer Gärtnerei ein, wenn auch nicht sehr ausgeprägt $(0,294)$. Wie alle anderen Cluster auch kauft er auch mehr in einer Baumschule (6,556). Der wichtigste Aspekt für die Wahl einer bestimmten Einkaufsstätte ist für ihn die Vertrautheit mit dieser $(3,620)$. In einer Gärtnerei erwartet er schlechte Qualität $(3,169)$, womit er sich von allen anderen Clustern unterscheidet, zugleich spricht er der Gärtnerei jedoch einen guten Service zu $(3,480)$ - auch darin unterscheidet er sich wiederum von allen anderen Clustern. Von der Gärtnerei erwartet er ein hohes Preisniveau $(3,415)$, ebenso vom Gartencenter, wenngleich weniger ausgeprägt $(2,770)$. Schließlich gibt der „konservative Kleingärtner“ mehr Geld für BuP aus als das Referenzcluster (2,989), dabei aber anteilsmäßig weniger für Geschenke $(4,836)$. In seinem Ausgabenmuster entspricht er damit dem „PflanzengenieBer".

\subsection{Segmentbeschreibung anhand der Lebensstildimensionen}

Nachdem die Ergebnisse der erweiterten Analyse beschrieben sind, folgt in diesem Abschnitt deren Interpretation. Ziel ist es, die Cluster so zu beschreiben, dass sie als Segmente im Sinne des Marketings angesehen werden können. Die zugehörigen Handlungsempfehlungen für die Praxis folgen separat in Kapitel 7.5.

Um die Beschreibung möglichst ganzheitlich zu präsentieren, sei auf den Theorieteil und die dieser Arbeit zu Grunde liegende Methodik verwiesen (s. Kap. 6.2.1). Nur unter Berücksichtigung und Verknüpfung von sowohl aktiven als auch passiven Variablen lässt sich demnach ein möglichst vollständiges Bild der Segmente darstellen. Hierzu sei auf die bereits erfolgte Beschreibung der Cluster anhand der aktiven Variablen hingewiesen. Sie basiert bislang ausschließlich auf den extrahierten Motivfaktoren (s. Kap. 7.2). Die Beschreibung der Cluster wird ausgehend davon im Folgenden durch die Interpretation der zugehörigen passiven Variablen komplettiert.

Da es sich um sehr viele passive Items handelt, wird für die Darstellungsform auf zwei verschiedene Item-Tabellen zurückgegriffen. Eine Item-Tabelle zeigt diejenigen passiven Items, 
die sich in der multinominalen logistischen Regressionsanalyse (Kap. 7.3.) als für die Differenzierung zwischen den Clustern nicht sehr geeignet erwiesen haben. Sie stellen homogene Items dar und sind daher nicht genügend für den späteren Einsatz von segmentspezifischen Marketing-Tools geeignet. Im weiteren Verlauf dieser Arbeit wird daher auf diese homogenen Items nicht weiter eingegangen. Zur Vollständigkeit sind sie aber im Anhang in Tabelle 14 einzusehen.

Tabelle 13 stellt die zweite Item-Tabelle dar. Sie umfasst die restlichen passiven Items, also diejenigen, die sich als starke Differenzierungsmerkmale zwischen den Clustern erwiesen haben. Sie werden im Folgenden als „heterogene Items“ bezeichnet. Um eine Unterscheidung zwischen heterogenen und homogenen Items treffen zu können, werden alle Items, die in allen Clustern einen Wald-Wert $<4$ aufweisen, den homogenen Items der Tabelle 14 zugewiesen. Demgegenüber werden alle Items, die in mindestens einem Cluster einen Wald-Wert $>4$ aufweisen, den heterogenen Items der Tabelle 13 zugeschrieben. $\mathrm{Zu}$ erwähnen ist, dass die Items aus Frage 33 zur sozioökonomischen Dimension („Geschlecht“) trotz jeweiliger WaldWerte $<4$ in die Gruppe der heterogenen Items aufgenommen wurden. Dies liegt darin begründet, dass trotz der relativ schwachen Wald-Werte zumindest Tendenzen erkannt werden können, welche Segmente mehr weiblich oder mehr männlich ausgeprägt sind. Auch gilt es zu beachten, dass das Cluster „Desinteressierter Pragmatiker“ das Referenzcluster der hier zu Grunde liegenden Regressionsanalyse darstellt; alle Ergebnisse der hier analysierten Cluster sind in Relation zu diesem zu sehen. Zur Beschreibung des Referenzclusters sei hiermit auf Kapitel 7.2.4 verwiesen. Die Bilder zu den Fragen 26.1 und 26.3 im Fragekatalog sind im Anhang abgebildet.

Betrachtet man in Tabelle 13 das Cluster „Kultivator“, zeigt sich, dass es neben den Clustern „Durchschnittlicher Gartenfreund“ und „Konservativer Kleingärtner“ häufiger eine eigene Wohnung oder ein eigenes Haus bewohnt als das Referenzcluster. Auch ist der „Kultivator“ zwar älter als das Referenzcluster, aber jünger als der „Pflanzengenießer“ und der „,konservative Kleingärtner“ und darf somit im mittleren Altersbereich aller Cluster vermutet werden. Bezogen auf die Haushaltsgröße unterscheidet sich das Cluster „Kultivator“ als einziges signifikant vom Referenzcluster, was bedeutet, dass in ihm von allen Clustern die wenigsten Personen im Haushalt leben. 
Tabelle 13: Heterogene Items

\begin{tabular}{|c|c|c|c|c|c|}
\hline Item & $\begin{array}{l}\text { Kulti- } \\
\text { vator }\end{array}$ & $\begin{array}{l}\text { Durchsch. } \\
\text { Garten- } \\
\text { freund }\end{array}$ & $\begin{array}{l}\text { Pflanzen- } \\
\text { genießer }\end{array}$ & $\begin{array}{l}\text { Extrov. } \\
\text { Natur- } \\
\text { bewahrer }\end{array}$ & $\begin{array}{l}\text { Konserv. } \\
\text { Klein- } \\
\text { gärtner }\end{array}$ \\
\hline \multicolumn{6}{|l|}{ Sozioökonomische Dimension } \\
\hline F17 Wohnen Sie in Wohneigentum? & ++ & +++ & & & ++ \\
\hline F27 Alter & ++ & & ++++ & & +++ \\
\hline $\begin{array}{l}\text { F28 Welchen höchsten Schulabschluss haben Sie? } \\
\text { Bis Abitur oder Vergleichbares }\end{array}$ & & & & & + \\
\hline F29.1 Sind Sie zurzeit Rentner? & & --- & & & \\
\hline F29.2 Sind Sie zurzeit Vollzeit berufstätig? & & & ---- & -- & \\
\hline F29.2 Sind Sie zurzeit Teilzeit berufstätig? & & & -- & & \\
\hline F30 Wie viele Personen gehören zu Ihrem Haushalt? & -- & & & & \\
\hline F33 Geschlecht & & + & + & & \\
\hline \multicolumn{6}{|l|}{ Dimension Kompetenz } \\
\hline $\begin{array}{l}\text { F } 8.3 \text { Wenn ich eine Blume oder Pflanze kaufe, nehme ich } \\
\text { mir genügend Zeit und lasse mich beraten. }\end{array}$ & & +++ & +++ & ++ & \\
\hline $\begin{array}{l}\text { F 14.1 Eine Gartenrose darf ruhig etwas mehr kosten, wenn } \\
\text { sie in einem Container ist (und nicht wurzelnackt, also ohne } \\
\text { Erde und Blätter). }\end{array}$ & ++ & ++++ & ++ & ++ & ++ \\
\hline $\begin{array}{l}\text { F 14.2 Eine Gartenrose darf ruhig etwas mehr kosten, wenn } \\
\text { es sich um eine neue Sorte handelt. }\end{array}$ & & ++++ & ++++ & & \\
\hline $\begin{array}{l}\text { F } 14.8 \text { Eine Gartenrose darf ruhig etwas mehr kosten, wenn } \\
\text { sie eine Edelrose ist. }\end{array}$ & --- & & & & --- \\
\hline $\begin{array}{l}\text { F } 20 \text { Wie viel Zeit verbringen Sie im Sommer durchschnitt- } \\
\text { lich pro Woche mit Gartenarbeit? }\end{array}$ & ++++ & ++++ & ++++ & +++ & ++++ \\
\hline \multicolumn{6}{|l|}{ Dimension Performanz } \\
\hline $\begin{array}{l}\text { F7.1 Welche Informationsquellen ziehen Sie heran, wenn } \\
\text { Sie etwas Bestimmtes über BuP wissen möchten? Prospekte }\end{array}$ & ++ & & & & \\
\hline F7.2 dito. Antwort: Zeitschriften/Bücher & +++ & ++++ & ++++ & ++ & ++++ \\
\hline F7.3 dito. Antwort: Internet & ++ & ++ & & & \\
\hline F7.5 dito. Antwort: Züchter & & ++ & ++++ & & \\
\hline $\begin{array}{l}\text { F26.1 In welchem Wohnzimmer würden Sie sich bei sich zu } \\
\text { Hause am wohlsten fühlen? Bild } 1\end{array}$ & ++++ & ++ & ++++ & & ++ \\
\hline $\begin{array}{l}\text { F26.3 In welchem Wohnzimmer würden Sie sich bei sich zu } \\
\text { Hause am wohlsten fühlen? Bild } 3\end{array}$ & +++ & & & ++ & \\
\hline \multicolumn{6}{|l|}{ Dimension Motivation } \\
\hline $\begin{array}{l}\text { F10.3 Warum haben Sie sich entschieden, diese letzte Gar- } \\
\text { tenrose zu kaufen? Wegen der Blütenfarbe }\end{array}$ & ++ & ++ & & & \\
\hline $\begin{array}{l}\text { F14.4 Eine Gartenrose darf ruhig etwas mehr kosten, wenn } \\
\text { sie ein Qualitätssiegel trägt. }\end{array}$ & & ++++ & +++ & & \\
\hline $\begin{array}{l}\text { F22.2 Mit dem Thema „Garten“ und „Gärtnern“ assoziiere } \\
\text { ich besonders Geselligkeit und Feiern. }\end{array}$ & & & & & -- \\
\hline F22.3 dito. Antwort: körperlichen Ausgleich & & ++ & & & \\
\hline F22.5 dito. Antwort: Ausleben von Kreativität & +++ & ++++ & ++++ & & \\
\hline
\end{tabular}




\begin{tabular}{|c|c|c|c|c|c|}
\hline $\begin{array}{l}\text { F24.5 Durch Blumen und Pflanzen im Haus kann man die } \\
\text { Kinder gut an naturbezogene Themen heranführen }\end{array}$ & & +++ & & & \\
\hline $\begin{array}{l}\text { F24.6 Ich kann es nicht gut ertragen, zu sehen, wenn es einer } \\
\text { Pflanze schlecht geht. }\end{array}$ & +++ & ++ & +++ & ++ & \\
\hline \multicolumn{6}{|l|}{ Dimension Beobachtbares Kaufverhalten } \\
\hline $\begin{array}{l}\text { F4.2 Wo haben Sie persönlich in den letzten } 12 \text { Monaten } \\
\text { Pflanzen und Gartenbedarf gekauft? Gartencenter }\end{array}$ & & ++ & & & \\
\hline F4.3 dito. Antwort: Supermarkt & & & & ++ & \\
\hline F4.5 dito. Antwort: Blumenfachgeschäft & & & ++ & & \\
\hline F4.6 dito. Antwort: Baumschule & ++ & ++++ & ++ & ++++ & ++ \\
\hline $\begin{array}{l}\text { F5.3 Welcher Aspekt ist für Sie bei der Wahl der Einkaufs- } \\
\text { stätte am wichtigsten, wenn Sie BuP kaufen möchten? Nähe } \\
\text { zum Wohnort }\end{array}$ & & & -- & & \\
\hline F5.4 dito. Antwort: Große Auswahl & & & & ++ & \\
\hline $\begin{array}{l}\text { F6.1 Wo werden Ihre Erwartungen hinsichtlich Produktqua- } \\
\text { lität beim Kauf von Blumen und Pflanzen am besten erfüllt? } \\
\text { Baumarkt }\end{array}$ & ++ & & & & \\
\hline F6.2 dito. Antwort: Discounter & -- & -- & & & \\
\hline F6.3 dito. Antwort: Wochenmarkt & & & -- & -- & \\
\hline $\begin{array}{l}\text { F6.5 Wo werden Ihre Erwartungen hinsichtlich Service beim } \\
\text { Kauf von Blumen und Pflanzen am besten erfüllt? Garten- } \\
\text { center }\end{array}$ & & & ++ & & \\
\hline F6.8 dito. Antwort: Wochenmarkt & & & & +++ & \\
\hline $\begin{array}{l}\text { F6.10 Wo werden Ihre Erwartungen hinsichtlich Preise beim } \\
\text { Kauf von Blumen und Pflanzen am besten erfüllt? Baumarkt }\end{array}$ & -- & & & & \\
\hline F6.11 dito. Antwort: Supermarkt & ++++ & ++ & & & \\
\hline F6.12 dito. Antwort: Baumschule & ++ & & & & \\
\hline F6.13 dito. Antwort: Gärtnerei & & -- & & -- & \\
\hline F6.14 dito. Antwort: Wochenmarkt & & -- & -- & & \\
\hline $\begin{array}{l}\text { F9 Bitte erinnern Sie sich an Ihren letzten Kauf einer Gar- } \\
\text { tenrose zurück! Hatten Sie den Kauf der Gartenrose geplant } \\
\text { oder ist die Entscheidung spontan im Geschäft gefallen? }\end{array}$ & ---- & & & & \\
\hline $\begin{array}{l}\text { F14 Eine Gartenrose darf ruhig etwas mehr kosten, wenn ich } \\
\text { gerade etwas mehr Geld im Portemonnaie habe. }\end{array}$ & -- & & & & \\
\hline $\begin{array}{l}\text { F21 Wie viel Geld geben Sie ungefähr im Jahr für Blumen } \\
\text { und Pflanzen aus? }\end{array}$ & & & ++ & & \\
\hline F21a ... davon als Geschenk? & & & & & -- \\
\hline \multicolumn{6}{|l|}{ Referenzcluster $=$ Desinteressierter Pragmatiker } \\
\hline \multicolumn{6}{|c|}{ Alle Ergebnisse dieser Tabelle beziehen sich auf das Referenzcluster. } \\
\hline \multicolumn{6}{|c|}{ Bei Beta $>$ 0: Wald-Werte: $[0 ; 4]=+;[4 ; 8]=++;[8 ; 12]=+++>12=++++$} \\
\hline \multicolumn{6}{|c|}{ Bei Beta $<0:$ Wald-Werte $:[0 ; 4]=-;[4 ; 8]=--;[8 ; 12]=---;>12=----$} \\
\hline
\end{tabular}

\section{Quelle: Eigene Berechnungen}


Eine Mehrpreisbereitschaft bei besserer Qualität (F14.1) ist beim „Kultivator“ zwar im Vergleich zum Referenzcluster gegeben, im Vergleich zu allen anderen Clustern findet sich bei ihm jedoch die geringste Mehrpreisbereitschaft für eine bessere Qualität. Keine Mehrpreisbereitschaft besteht für eine Edelrose im Vergleich zu einer normalen Gartenrose. Dies verdeutlicht einerseits, dass der „Kultivator“ die Kompetenz hat zu unterscheiden, welche Merkmalsausprägungen aus fachlicher Sicht „,ihr Geld wert sind“. Andererseits bestätigen diese Ergebnisse auch die bereits erfolgte Beschreibung des Clusters anhand der aktiven Variablen: Dort wurde der „Kultivator“ als „sehr preissensibel“ charakterisiert (vgl. Kap. 7.2.1). Weiterhin lässt sich vermuten, dass er Mehrausgaben für eine Edelrose ablehnt, weil er weiß, dass er eine Edelrose leicht selbst kultivieren kann. Dies lässt wiederum auf eine ausgeprägte BuPKompetenz schließen, für die sich als weiterer Indikator die durchschnittliche Gartenarbeitszeit (F20) anführen lässt: Von allen Clustern verbringt der „Kultivator“ durchschnittlich die meiste Zeit im Sommer mit Gartenarbeit. Dies lässt vermuten, dass er einerseits Kompetenz auch aus gesammelter Erfahrung hat und andererseits die bisherige Beschreibung, dass „BuP als wichtiges Hobby angesehen“ werden, für den „Kultivator“ zutreffend ist. Bei der Frage, wie Informationen zu BuP gewonnen werden, nutzt der „Kultivator“ von allen Clustern die breiteste Palette an Informationsquellen. Als Einziger zieht er Prospekte zur Informationsgewinnung heran. Zeitschriften und Bücher werden ebenfalls mehr als im Referenzcluster genutzt, im Vergleich mit den anderen Clustern allerdings nicht so intensiv. Zusätzlich spielt für ihn das Internet eine Rolle; neben dem „durchschnittlichen Gartenfreund“ ist der Einzige, der das Internet als Informationsquelle heranzieht.

Im Hinblick auf den Einrichtungsstil bewertet der „Kultivator“ sowohl Bild 1 „Prestige“ als auch Bild 2 „Tradition“ sehr positiv. Legt man die Beschreibung der Bilder zu Grunde (Kap. 6.2.4), erscheint der „Kultivator“ als Kenner, der Besonderheiten zu schätzen weiß und diese bewahren will. Er ist ordnungsliebend, arbeitsam und sparsam. Dies bestätigt wiederum die bereits beschriebene Preissensibilität. Weiterhin zeigt sich, dass bei Blumen und Pflanzen für den „Kultivator“ auch Pflanzenschutzmittel dazugehören, um die Schönheit zu bewahren. Die Eigenschaften „ordnungsliebend“ und ,,arbeitsam“ unterstreichen die bereits erkannte Eigenschaft, dass „für Personen dieses Clusters (...) der Fokus auf der Phase der Aufzucht, Kultivation und Pflege liegt“" (s. Kap. 7.2.1). Wird nach dem Kaufgrund für die letzte Gartenrose gefragt, so ist die Blütenfarbe für den „Kultivator“ das einzige Kriterium, das ihm deutlich wichtiger ist als dem Referenzcluster. Es kann somit vermutet werden, dass eine gewisse Mehrpreisbereitschaft für besondere Blüten bzw. eine besondere Optik vorhanden ist. Weiter- 
hin zeigt sich, dass der „Kultivator“ mit dem Thema „Garten und Gärtnern“ das Ausleben von Kreativität verbindet, was zu einer eventuellen Mehrpreisbereitschaft für „Optik“ passt. Anhand der Antworten zu F24.6 lässt sich feststellen, dass der „Kultivator“ auch eine vergleichsweise stark naturverbunden ist. Es weist hierbei gemeinsam mit dem „PflanzengenieBer“ die deutlichste Ausprägung auf.

Innerhalb der Kriterien des beobachtbaren Kaufverhaltens ist zu erkennen, dass der „Kultivator“" während der letzten 12 Monate wie alle anderen Cluster auch in einer Baumschule signifikant mehr eingekauft hat als das Referenzcluster. Dabei weist er allerdings von allen Clustern die schwächste Ausprägung für „Baumschule“ auf. Des Weiteren ist er das einzige Cluster, das innerhalb von Frage 4 nur eine signifikante Ausprägung aufweist, nämlich „Baumschule“. Das bedeutet im Umkehrschluss, dass alle anderen möglichen Einkaufsstätten für den „Kultivator“ nicht besonders interessant sind. Als einziges Cluster sieht er in der Preispolitik der Baumschulen eine negative Eigenschaft (F6.12), wobei dies auch in ganz besonders ausgeprägter Form für Supermärkte gilt (F6.11). Anders bei den Baumärkten: Der „Kultivator“ sieht dort als einziges Cluster eine gute Qualität beim Angebot von BuP (F6.1) und empfindet das Preisniveau von Baumärkten auch als einziges aller Cluster als passend (F6.10). Dass er trotz dieser positiven Kombination nicht signifikant häufiger in Baumärkten einkauft, überrascht. Discountern, die BuP verkaufen, schreibt der „Kultivator“ eine schlechte Produktqualität (F6.2). Anhand von Frage 9 zeigt sich sehr deutlich, dass er sich in der Art des Kaufes sehr stark sowohl vom Referenzcluster als auch von allen anderen Clustern unterscheidet. Der „Kultivator“ kauft BuP sehr stark geplant ein. Im Umkehrschluss bedeutet dies, dass er keine Affinität zu spontanen Impulskäufen hat. Bestätigt wird diese deutliche Aussage anhand der Erkenntnis, dass auch eine entspannte finanzielle Situation den Kaufentscheidungsprozess nicht beeinflusst (F14). Gekauft wird lediglich, was benötigt bzw. geplant ist.

Für das Cluster „Durchschnittlicher Gartenfreund“ stellt sich die Situation der sozioökonomischen Dimension in der Art dar, dass es von allen Clustern am häufigsten in den eigenen vier Wänden wohnt. Dazu kommt, dass bei ihm im Vergleich zu allen anderen Clustern die mit Abstand geringste Rentnerquote vorliegt. Im Hinblick auf die Geschlechterverteilung ist der „durchschnittliche Gartenfreund“ mehr als weiblich zu charakterisieren als das Referenzcluster. Dabei ist allerdings zu bemerken, dass dieser Unterschied zwar messbar, aber nur sehr schwach ausgeprägt ist (F33). Für die weiteren Überlegungen soll für diesen Punkt daher weniger von einer Erkenntnis als von einer Tendenz die Rede sein. 
Was die BuP-Kompetenz betrifft, so weist der „,durchschnittliche Gartenfreund“ mit großem Abstand von allen Clustern die deutlichste Zustimmung zu Frage 14.1 auf. Dies lässt vermuten, dass bei ihm eine gewisse BuP-Kompetenz vorhanden ist. Dazu passt, dass er auch deutlich mehr Zeit im Garten verbringt als das Referenzcluster. Verglichen mit den anderen Clustern liegt dieser Wert zwar nur im mittleren Bereich, dennoch unterstreicht er tendenziell die obige Aussage. Passend scheint auch die Erkenntnis aus Frage 14.2, wo der „durchschnittliche Gartenfreund“" sehr deutlich der Aussage zustimmt, dass neue Sorten von Gartenrosen ruhig etwas mehr kosten dürfen. Er scheint demnach unterscheiden zu können, was bereits auf dem Markt und was neu ist. Zusätzlich zeigt die erhöhte Zahlungsbereitschaft für neue Sorten, dass er den Marktwert einer neuen Sorte abschätzen kann. Diese Erkenntnis bestätigt, was sich bereits früher herausgestellt hat: eine Affinität zu Neuheiten bzw. Besonderheiten und der Umstand, dass der Preis dabei eine untergeordnete Rolle spielt (Kap. 7.2.2). Weiterhin lässt sich erkennen, dass der „durchschnittliche Gartenfreund“ sich beim Kauf von BuP deutlich mehr Zeit lässt als das Referenzcluster und dass er auch eher bereit ist, sich beraten zu lassen (F8.3). Vor dem Hintergrund der bereits beschriebenen Erkenntnisse kann dies als Bestätigung der vermuteten BuP-Kompetenz gesehen werden. Die Bereitschaft, sich Zeit zu lassen und Beratung in Anspruch zu nehmen, dürfte dem Wunsch entsprechen, tiefer in die Materie einzutauchen und sich mit ihr aus eigenem Interesse genauer auseinanderzusetzen.

Alternativ zeigen die Ergebnisse der Analyse, dass der „durchschnittliche Gartenfreund“ von allen Clustern mit Abstand am häufigsten Bücher und Zeitschriften zur Informationsbeschaffung nutzt. Ebenfalls am häufigsten zieht er das Internet heran, wenn auch weniger stark als Bücher und Zeitschriften. Dabei ist er zusammen mit dem „Kultivator“ das einzige Cluster, das auf das Internet als Informationsquelle zurückgreift. Zusätzlich sucht der „durchschnittliche Gartenfreund“ das direkte Gespräch mit dem Züchter bzw. Gärtner und ist damit neben dem „Pflanzengenießer“ das einzige Cluster, das diese Informationsquelle nutzt, wobei dies beim „Pflanzengenießer“ häufiger der Fall ist. Bei der Analyse des bevorzugten Einrichtungsstils findet das Bild 1 „Prestige“ beim „,durchschnittlichen Gartenfreund“ die größte Zustimmung. Dabei ist allerdings zu beachten, dass diese Zustimmung im Vergleich mit allen anderen Clustern am geringsten ausfällt. Wie bereits in Kap. 6.2.4 beschrieben steht der Einrichtungsstil „Prestige“ für „Anspruchsvoller Genießer“, „Besonderheiten“ und „Style“. Dies unterstützt einerseits die bereits erfolgte Beschreibung, dass sich der „durchschnittliche Gartenfreund“ daran erfreut, etwas Schönes zu haben (Kap. 7.2.2), andererseits passen diese Attribute zu den obigen Erkenntnissen. Dem entspricht auch das Ergebnis zu Frage 10.3, bei welcher 
der „durchschnittliche Gartenfreund“ unter allen Clustern die größte Zustimmung aufweist. Als Kaufgrund für die letzte Gartenrose gibt er die Blütenfarbe an, was wiederum das Suchen nach Besonderem bzw. Schönem unterstreicht. Ebenfalls die größte Zustimmung aller Cluster zeigt sich bei der Frage, ob ein Qualitätssiegel einen höheren Preis von Gartenrosen rechtfertigt (F14.4). Dies lässt vermuten, dass das Cluster eine relativ ausgeprägte Risikoaversion hat, da ein Qualitätssiegel das subjektiv empfundene Risiko eines Ausfalls der Pflanze (z. B. durch Nicht-Anwachsen) verringert.

Der „durchschnittlicher Gartenfreund“ assoziiert mit dem Thema „Garten und Gärtnern“ als einziges aller Cluster „körperlichen Ausgleich“, wenn auch in relativ schwacher Ausgeprägtheit. Viel deutlicher fällt die Assoziation bei „Ausleben von Kreativität“ aus, wobei das Cluster hier im Vergleich mit allen anderen Clustern im mittleren Bereich liegt. Diese beiden Assoziationen zeigen, dass der „durchschnittliche Gartenfreund“ für sich selbst aktiv ist und nicht, um etwas zur Schau zu stellen. Dies deckt sich mit der bereits erfolgten Beschreibung, dass er „für sich selbst gärtnert“ und „beim Umgang mit BuP entspannt“ (Kap. 7.2.2). Weiterhin lässt sich eine deutliche Naturverbundenheit feststellen (F24.5). Hier unterscheidet sich der „durchschnittliche Gartenfreund“ als einziges Cluster vom Referenzcluster. Dazu passt, dass er es nicht gut ertragen kann, wenn er sieht, dass eine Pflanze leiden muss.

Bei den letzten Einkaufsstätten für BuP während der letzten 12 Monate zeigt sich, dass der „durchschnittliche Gartenfreund“ als einziges Cluster signifikant öfter im Gartencenter gekauft hat als das Referenzcluster. Obwohl dieser Wert nicht sehr ausgeprägt ist, lässt sich dennoch eine Affinität zu Gartencentern vermuten. Ein deutlich höherer Wert zeigt sich bei Baumschulen; neben dem Cluster „Extrovertierter Naturbewahrer“ hat der „durchschnittliche Gartenfreund" während der letzten 12 Monate dort am häufigsten eingekauft. Im Hinblick auf die Einschätzung der Produktqualität unterscheidet sich das Cluster nur bei Discountern signifikant vom Referenzcluster. Diesen weist der „durchschnittliche Gartenfreund“ eine schlechte Qualität bei BuP zu (F6.2). In Supermärkten erwartet er relativ hohe Preise, eine Einschätzung, die er nur mit dem Cluster „Kultivator“ teils, wo sie aber noch viel deutlicher zum Ausdruck kommt. Von allen Clustern geht er bei Gärtnereien von den günstigsten Preisen aus, und auch auf dem Wochenmarkt empfindet er die Preise günstiger als das Referenzcluster, wobei er sich diese Einschätzung nur mit einem weiteren Cluster teilt.

Das Cluster „Pflanzengenießer“ ist im Vergleich zum Referenzcluster und auch im Vergleich zu allen anderen Clustern dadurch charakterisiert, dass es das älteste aller Cluster ist. 
Dazu passt, dass der „Pflanzengenießer“ vergleichsweise am seltensten sowohl in Vollzeit als auch in Teilzeit berufstätig ist. Wie beim „durchschnittlichen Gartenfreund“ lässt der „Pflanzengenießer“ die Tendenz erkennen, eher weiblich zu sein als das Referenzcluster. Was die Kompetenz betrifft, verbringt er neben dem „Kultivator“ im Vergleich zu allen anderen Clustern die mit Abstand meiste Zeit im Garten. Dies bestätigt einerseits, dass sich das Cluster „gerne mit dem Thema BuP beschäftigt“ (Kap. 7.2.3), andererseits lässt sich dadurch auf Kompetenz durch Erfahrung schließen. Unterstützt wird diese These durch die Erkenntnisse aus Frage 14.1 und 14.2, wo die Gründe für eine eventuelle Mehrpreisbereitschaft eruiert werden. Neben dem „durchschnittlichen Gartenfreund“ weist der „Pflanzengenießer“ als einziges Cluster eine erhöhte Zahlungsbereitschaft für eine neue Sorte von Gartenrosen auf. Das lässt darauf schließen, dass er zwischen bestehenden und neuen Sorten unterscheiden und auch den Marktwert einer neuen Sorte richtig einschätzen kann. Parallel dazu ist er auch bereit, für eine Containerrose mehr Geld auszugeben als für eine wurzelnackte Rose, was neben einer relativ ausgeprägten Preisbereitschaft ebenfalls eine gewisse BuP-Kompetenz vermuten lässt. Der „Pflanzengenießer“ nimmt sich zudem von allen Clustern beim Kauf von BuP am meisten Zeit und lässt sich am meisten beraten (F8.3). Unter Beachtung der bisherigen Erkenntnisse ist dabei nicht davon auszugehen, dass mit der höchsten Ausprägung in F8.3 die höchste Ausprägung an Nicht-Kompetenz einhergeht. Vielmehr steht zu vermuten, dass der „Pflanzengenießer“ eine relativ ausgeprägte BuP-Kompetenz besitzt. Beim Kauf braucht er Zeit und Beratung, da er den „Umgang mit BuP genießt“ (Kap. 7.2.3). Zur Informationsgewinnung nutzt der „Pflanzengenießer“ primär Bücher und Zeitschriften. Hierbei weist er einen sehr hohen Wert auf, wenn auch nicht den höchsten. Letzteres ist allerdings bei der Informationsbeschaffung vom Züchter bzw. vom Gärtner der Fall: Neben dem „durchschnittlichen Gartenfreund“ ist der „Pflanzengenießer“ das einzige Cluster, das sich direkt beim Produzenten informiert, und bei ihm ist diese Eigenschaft deutlich ausgeprägter.

Was den Einrichtungsstil angeht, gefällt dem „Pflanzengenießer“ Bild 1 von allen Clustern am besten. Wie bereits beschrieben (Kap. 6.2.4), steht der Einrichtungsstil von Bild 1 für „Anspruchsvoller Genießer“, „Extrovertiertheit“, „Besonderheiten“ und „Style“. Dies deckt sich nahezu vollständig mit der Beschreibung des Clusters in Kap. 7.2.3, der zufolge es das Besonderes zu schätzen weiß, extrovertiert ist und durch BuP seinen individuellen Stil ausdrückt. Dazu passt auch, dass der „Pflanzengenießer“ mit dem Thema „Gärtnern und Garten“ sehr stark das Ausleben seiner Kreativität verbindet - hier weist er deutlich die stärkste Ausprägung aller Cluster auf. Zusätzlich kann vermutet werden, dass er in einem bestimmten 
Maße risikoavers ist, da er sich durch die erhöhte Preisbereitschaft für ein Qualitätssiegel wohl eine Art Garantie auf den Pflanzerfolg kaufen möchte. In diesem Punkt ist er zusammen mit dem „durchschnittlichen Gartenfreund“ das einzige Cluster, das sich auf diese Weise absichert. Weiterhin zeigt Frage 24.6, dass er sehr naturverbunden ist, zumal er hier die stärkste Ausprägung aller Cluster aufweist.

Während der letzten 12 Monate hat der Pflanzengenießer als einziges aller Cluster signifikant mehr im Blumenfachgeschäft gekauft als das Referenzcluster und somit auch mehr als alle anderen Cluster. Er hat während der letzten 12 Monate parallel auch in Baumschulen eingekauft, allerdings relativ wenig im Vergleich zu den anderen Clustern. Unwichtig ist ihm bei der Wahl der Einkaufsstätte für BuP die geographische Nähe (F5.3); diesbezüglich unterscheidet er sich als einziges Cluster vom Referenzcluster. Bei der Einschätzung der Qualität von verschiedenen Vertriebsschienen geht der „Pflanzengenießer“ von einer relativ schlechten Qualität bei BuP auf dem Wochenmarkt aus. Dementsprechend erwartet er auf dem Wochenmarkt auch günstige Preise. Als positiv bewertet er den Service in Gartencentern - er ist das einzige Cluster, das diese Einschätzung vertritt. Zudem gibt er von allen Clustern durchschnittlich am meisten Geld pro Jahr für BuP aus, was wiederum die Vermutung einer relativ ausgeprägten Zahlungsbereitschaft für dieses Cluster unterstreicht.

Betrachtet man die sozioökonomische Dimension, so entspricht das Cluster „Extrovertierter Naturbewahrer“ ziemlich exakt dem Referenzcluster. Einzig bei der Frage nach einer beruflichen Tätigkeit in Vollzeit zeigt sich, dass dies auf ihn seltener zutrifft als auf den „,desinteressierten Pragmatiker“. Mit Blick auf die Dimension „Kompetenz“ ist hervorzuheben, dass der „extrovertierte Naturbewahrer“ die mit Abstand geringste Zeit mit Gartenarbeit verbringt, was eine distanzierte Beziehung zum Thema Garten und somit auch eine gering ausgeprägte BuP-Kompetenz vermuten lässt. Verstärkt wird dieser Eindruck, wenn er bei Frage 14.1 wiederum von allen Clustern die schwächste Ausprägung aufweist. So erkennt er zwar deutlich häufiger als das Referenzcluster, dass eine Containerrose wertiger ist als eine wurzelnackte Rose, jedoch seltener als alle anderen Cluster. Im Wissen um diese beiden Tendenzen ist das Ergebnis zu Frage 8.3 zu verstehen: Der „extrovertierte Naturbewahrer“ nimmt sich beim Kauf von BuP deutlich mehr Zeit als das Referenzcluster, aber weniger als der „Pflanzengenießer“ und der „durchschnittliche Gartenfreund“; auch lässt er sich am seltensten beraten.

Mit Hilfe dieses Ergebnisses lässt sich in Verbindung mit der anhand der Motivfaktoren bereits erfolgten Beschreibung des Clusters als „Außendarsteller“ (Kap. 7.2.5) vermuten, dass 
der „extrovertierte Naturbewahrer“ weniger Interesse am fachlichen Thema BuP hat und daher wohl auch weniger kompetent ist als der „Pflanzengenießer“ und der „durchschnittliche Gartenfreund“. Da dem „Kultivator“ bereits eine ausgeprägte BuP-Kompetenz zugesprochen worden ist, ist davon auszugehen, dass der „extrovertierte Naturbewahrer“ auch weniger kompetent ist als dieser. Unterstützung für diese These findet sich beim Informationsverhalten. Einzig bei Zeitschriften und Büchern als Informationsquelle lässt sich ein signifikanter Unterschied zum Referenzcluster feststellen. Dabei fällt auf, dass der „extrovertierte Naturbewahrer“ bei seiner „einzigen“ Informationsquelle wiederum unter allen Clustern die schwächste Ausprägung aufweist.

Mit Blick auf den Einrichtungsstil fällt auf, dass der „extrovertierte Naturbewahrer“ als einziges Cluster sich bei Bild 1 nicht signifikant vom Referenzcluster unterscheidet. Allerdings lässt sich ein Unterschied bei Bild 3 „Tradition“ feststellen, das ihm besser gefällt als dem Referenzcluster. Legt man die Attribute dieses Einrichtungsstils zu Grunde (Kap. 6.2.4), scheint dies auf den ersten Blick widersprüchlich zu sein. „Liebevoller Bewahrer“, „Kontinuität“, „Tradition“ und „sparsam“ scheinen nicht zu dem Cluster zu passen, das bereits als „extrovertiert" beschrieben und als „Außendarsteller“ bezeichnet wurde. Bezieht man die Attribute allerdings weniger auf den spezifischen BuP-Lebensstil, sondern mehr auf den allgemeinen Lebensstil, zeigt sich, dass sie zu einem ökologisch geprägten Lebensstil passen. Daher scheint die getroffene Clusterbezeichnung „Extrovertierter Naturbewahrer“ als sehr passend. Bestätigt wird die These der ökologischen Grundhaltung des Clusters auch anhand der Aussage, dass der „extrovertierte Naturbewahrer“ es nicht gut ertragen kann, wenn er eine Pflanze leiden sieht (F24.6). Obwohl er in diesem Punkt eine vergleichsweise geringe Ausprägung aufweist, lässt sich eine Tendenz zur Naturverbundenheit vermuten.

Während der letzten 12 Monate hat der „extrovertierte Naturbewahrer“ von allen Clustern am häufigsten in Baumschulen eingekauft. Seine BuP kauft er jedoch auch in Supermärkten ein, worin er sich als Einziger vom Referenzcluster unterscheidet. Bei der Wahl der Einkaufsstätte ist es dem „extrovertierten Naturbewahrer“ besonders wichtig, eine große Auswahl zu haben. Er ist das einzige Cluster, das diesen Aspekt als wichtig ansieht. Eine Erklärung hierfür könnte der „Außendarsteller-Effekt" sein: Er sucht besonders schöne Pflanzen, um diese „nach auBen hin zur Schau zu stellen“ (Kap. 7.2.5). Da es ihm nicht um eine bestimmte Sorte von Pflanzen geht, sondern „nur“ um eine besonders schöne, ist eine große Auswahl sehr hilfreich. Was das Preisniveau betrifft, bescheinigt der „extrovertierte Naturbewahrer“ ausschließlich Gärtnereien günstige Preise. Überraschend ist, dass er dem Wochenmarkt einer- 
seits eine schlechte Qualität bei BuP und andererseits einen ausgesprochen guten Service bescheinigt. Wenn er aber dem Wochenmarkt als Einziger einen guten Service zuspricht, zugleich aber von einer schlechten Qualität ausgeht, könnte die Erklärung darin zu sehen sein, dass er auf dem Wochenmarkt nicht das Sortiment vorfindet, das er sucht. Tatsächlich ist die Auswahl hier oft sehr beschränkt, und ebenso wenig finden sich hier „spektakuläre“ Pflanzen im Angebot. Wochenmärkte scheinen eher „bodenständig“ zu sein und somit weniger für den Außendarsteller-Effekt geeignet. Dieser Widerspruch beschreibt das Cluster sehr gut: einerseits eine bodenständige, ökologische Grundhaltung, andererseits der Wunsch nach Ausgefallenem. Vor diesem Hintergrund dürfte die Bezeichnung des Clusters durchaus treffend sein.

Der „konservative Kleingärtner“ unterscheidet sich besonders bezüglich des Alters vom Referenzcluster. Nach dem „Pflanzengenießer“ bildet er das zweitälteste aller Cluster. Auch wohnt er häufiger im Eigenheim. Beim Schulabschluss fällt auf, dass es sich um das einzige Cluster handelt, das sich hier vom Referenzcluster unterscheidet: Der „konservative Kleingärtner“ weist signifikant häufiger den Abschluss „bis Abitur oder Vergleichbares“ auf, wenn auch nur in relativ geringem Ausmaß.

Im Hinblick auf die Kompetenz zeigt sich, dass der „konservative Kleingärtner“ relativ viel Zeit im Garten verbringt, was eine vorhandene BuP-Kompetenz infolge von Erfahrung vermuten lässt. Unterstrichen wird die These dadurch, dass er erkennt, dass eine Containerrose wertiger ist als eine wurzelnackte Rose. Wenn er die Aussage, dass eine Edelrose mehr kosten darf als eine Gartenrose, ablehnt, kann dies als Hinweis gelten, dass bei ihm vermutlich mehr als nur eine „normale“ BuP-Kompetenz vorhanden ist. Es ist anzunehmen, dass er über eine ausgeprägte BuP-Kompetenz verfügt. Neben dem „Kultivator“ ist er der Einzige, der eine Mehrpreisbereitschaft für eine Edelrose zurückweist. Da der Zusatz „edel“ keinerlei Auswirkung auf die Qualität der Pflanze hat, ist das für einen Kenner der Materie nur folgerichtig. Eine kompetente Person kann die Eigenschaften, die als ,edel“ verkauft werden, durch einfache gärtnerische Tätigkeiten wie Kreuzung oder Rückschnitt relativ einfach selbst hervorrufen. Dieses Bild wird durch die bereits getroffene Beschreibung bestätigt, dass es für dieses Cluster „nicht entscheidend ist, dass eine Pflanze pflegeleicht ist“ (Kap. 7.2.6). Vor diesem Hintergrund ist zu vermuten, dass der „konservative Kleingärtner“ gemeinsam mit dem „Kultivator“" von allen Clustern die ausgeprägteste BuP-Kompetenz aufweist. Unterstützung könnte diese These durch das Informationsverhalten finden. Der „konservative Kleingärtner“ nutzt dafür Zeitschriften und Bücher in besonderem Ausmaß. Da allerdings nicht festgestellt wer- 
den kann, ob es sich dabei um spezielle Fachliteratur handelt, kann die These nicht belegt, sondern lediglich vermutet werden.

Beim Einrichtungsstil zeigt sich, dass Bild 1 „Prestige“ in schwacher Ausprägung dem „konservativen Kleingärtner" mehr zusagt als dem Referenzcluster. Vergleicht man die Attribute dieses Einrichtungsstils („Anspruchsvoller Genießer“, „extrovertiert“, „Style“, s. Kap. 6.2.4) mit dem, was bisher als charakteristisch für den „konservativen Kleingärtner“ herausgearbeitet wurde, zeigen sich nahezu keine Übereinstimmungen. Vielmehr wäre zu vermuten gewesen, dass ihm Bild 3 „Tradition“ zusagt. Wie aus Tabelle 11 ersichtlich, gefällt ihm der Einrichtungsstil auf Bild 3 zwar besser als dem Referenzcluster. Da diese Ausprägung allerdings einen Wald-Wert kleiner 4 aufweist, wird sie im Folgenden nicht weiter thematisiert. Was mögliche Assoziationen mit dem Thema „Gärtnern und Garten“ betrifft, lassen sich beim „konservativen Kleingärtner“ keine Verbindung zu „Geselligkeit und Feiern“ erkennen (F22.2). Er stellt das einzige Cluster, das diese gedankliche Verknüpfung messbar ablehnt. Dabei bestätigen sich allerdings die bereits vorgenommenen Charakterisierungen als „nicht extrovertiert“ und „seriös“ (Kap. 7.2.6). Bei der Frage nach den Einkaufsstätten von BuP während der letzten 12 Monate weist das Cluster nur einen Vertriebskanal auf, den der Baumschule. Da diese Ausprägung im Vergleich aller Cluster nur im mittleren Bereich liegt, sind weiterführende Aussagen diesbezüglich nicht möglich.

\subsection{Normstrategien für die Praxis}

Nachdem alle sechs Segmente bekannt und beschrieben sind, folgt vor der Segmentbearbeitung die Identifizierung des Zielsegments bzw. der Zielsegmente. Zur methodischen Vorgehensweise der Identifikation eines oder mehrerer Zielsegmente sei auf Kapitel 3.2.4 verwiesen, zur allgemeinen Segmentbearbeitung auf Kapitel 3.2.5. Im Folgenden stehen die konkreten, segmentspezifischen Ergebnisse der Segmentbeschreibungen und deren konkrete Auswirkungen auf die Segmentbearbeitung im Vordergrund. Dabei wird für jedes der sechs Segmente geprüft, welches der sieben vorgestellten Marketinginstrumente als am geeignetsten erscheint und welche Optionen für konkrete Maßnahmen dadurch geschaffen werden. Die im Folgenden vorgestellten Maßnahmen stellen keinen Anspruch auf Vollständigkeit dar. Sie

sollen jedoch an konkreten Beispielen zeigen, welche Auswirkungen eine Orientierung an Segmenten haben kann. Die letztliche Entscheidung über die Wahl des oder der Zielsegmente und die entsprechende Ausgestaltung der segmentspezifischen Kundenansprache liegt dabei bei denjenigen, die über die Unternehmensstrategie entscheiden. Für weiterführende Informa- 
tionen der hier zum Einsatz kommenden sieben Marketinginstrumente sei auf Kapitel 4 verwiesen, im Besonderen auf Abbildung 43 und Abbildung 44.

Das Cluster „Kultivator“ stellt mit einer Segmentgröße von $11 \%$ des relevanten Marktes das drittgrößte aller untersuchten Segmente dar. Im Hinblick auf den Instrumenteneinsatz des Marketingmix zeigt sich für das Instrumentarium Product, dass der „Kultivator“ keine Mehrpreisbereitschaft und somit kein gesteigertes Interesse an einer ausgewiesen guten Qualität bei BuP aufweist. Eine Fokussierung des Sortiments auf „Premiumware“ oder „Gärtnerqualität“ wäre bei der segmentspezifischen Sortimentsausrichtung demnach kontraproduktiv. Da der „Kultivator“ lediglich das kauft, was er geplant hat, und nicht zu Impulskäufen neigt, ist parallel ein breites Sortiment erstrebenswert. Es ist nicht davon auszugehen, dass er spontan etwas Ähnliches als Ersatz kauft, wenn er nicht das findet, was er explizit sucht. Da er über eine ausgeprägte BuP-Kompetenz verfügt und sein BuP-Fokus ,,auf der Phase der Aufzucht, Kultivation und Pflege“ liegt, scheint unter den gegebenen Umständen eine Erweiterung des Angebots um Halbfertigprodukte sinnvoll. Dies würde auch das Instrumentarium Price positiv beeinflussen, da Halbfertigprodukte deutlich günstiger sind als fertige Produkte und der „Kultivator“ sehr preissensibel ist. Weiterhin scheint für eine segmentspezifische Sortimentspolitik auch die Aufnahme von Pflanzenschutzmittel sehr wichtig. Denkbar wäre hier beispielsweise eine Themeninsel mit verschiedenen Halbfertigprodukten, Erden und den passenden Düngebzw. Spritzmitteln dazu. Dies bietet zudem die Option für günstige Bündelangebote, beispielsweise „1 Sack Hortensienerde gratis bei Abnahme von 10 Stück Hortensien“, was wiederum positiven Einfluss auf die Wahrnehmung der Preise hätte. Weitere Möglichkeiten, um dem preissensiblen „Kultivator“ in puncto Preispolitik entgegenzukommen, sind die Instrumente der Preisdifferenzierung. So wären beispielsweise an Treuepunkte gekoppelte Vergünstigungen ein Instrument, um ihn segmentspezifisch anzusprechen. Weitere Möglichkeiten wären Staffelpreise, z. B. „Kauf 3, zahl 2“ oder die bereits angesprochene Bündelung von Preisen.

Was das Instrumentarium Promotion betrifft, ist es von Interesse, dass der „Kultivator“ sehr gut über Prospekte erreichbar ist, aber auch über Zeitschriften und Bücher. Da dies allerdings für alle Segmente gilt, drohen bei Büchern und Zeitschriften bei einer segmentspezifischen Ansprache hohe Streuverluste. Geeigneter erscheint das Internet: Der „Kultivator“ bildet zusammen mit dem „durchschnittlichen Gartenfreund“ das einzige Segment, das online auf Informationssuche geht. Als weitere Möglichkeit der Kommunikation bieten sich entsprechend gestaltete Etiketten oder Informationsschilder an. Angesichts der festgestellten Kompetenz 
des „Kultivators“ könnte eine Bezeichnung der Pflanze mit ihrem botanischen Namen für das Segment ansprechend. Weiterhin bestünde die Möglichkeit, neben den üblichen Pflegetipps auch gärtnerisch interessante Hintergrundinformationen auf das Etikett zu drucken. Diese Option stellt eine äußerst preiswerte Möglichkeit dar, ein naturverbundenes Segment wie den „Kultivator“ noch näher an das Produkt heranzuführen und das Interesse daran zu wecken bzw. zu steigern.

Obwohl der preissensible „Kultivator“ mit Baumschulen hohe Preise assoziiert, sind Baumschulen die einzigen Verkaufsstätten, die er besucht. Dies muss zu großen Teilen an deren Sortiment liegen, da er den Service von Baumschulen nicht als besonders positiv wahrnimmt. Auch eine gute Beratung in Baumschulen ist kein ausschlaggebender Punkt, da er darauf relativ wenig Wert legt. Baumärkte bieten in seinen Augen sowohl gute Qualität als auch relativ günstige Preise. Entsprechend wäre für diese Form der Einkaufsstätte der wohl wichtigste Ansatzpunkt für eine segmentspezifische Ansprache des „Kultivators“ die Überarbeitung des Sortiments. Damit könnten Baumärkte für ihn letztendlich so interessant werden, dass er dort auch einkauft. Für Discounter und Supermärkte hingegen scheint es sehr schwer, den „Kultivator" als Kunden zu gewinnen - in seinen Augen haben diese beiden Vertriebskanäle eine schlechte Qualität bzw. sind mit ihrem Angebot deutlich zu teuer.

Der „durchschnittliche Gartenfreund“ bildet mit ca. $21 \%$ Marktpotenzial das größte aller untersuchten Segmente und stellt daher bereits auf Grund seiner Größe ein sehr interessantes Potenzial dar. Da er sich bei BuP sehr für Schönes und Besonders interessiert, sollte die angebotene Produktpalette dementsprechend bestückt sein. Blühende Pflanzen, die ein farbenfrohes Bild zeichnen, unterstreichen dies mit ihrer optischen Wirkung. Dabei bietet ein tiefes Sortiment kein ausgesprochenes Potenzial für eine segmentspezifische Produktpolitik. Der Fokus der Produktpalette sollte primär auf die optische Wirkung ausgerichtet sein, unterstrichen durch die Betonung von Neuheiten und Raritäten. Da der „durchschnittliche Gartenfreund“"von allen Segmenten die wenigsten Rentner aufweist, bieten sich für die Vertriebspolitik angepasste Ladenöffnungszeiten an, beispielsweise durch längere Verkaufszeiten am Abend oder während des Wochenendes zu Lasten von frühen Verkaufszeiten während der Arbeitswoche. Die Preispolitik muss berücksichtigen, dass Preise für ihn eine untergeordnete Rolle spielen. Vor diesem Hintergrund sind freiwillige Preisnachlässe jeder Art für eine segmentspezifische Kundenansprache nicht zielführend. Vielmehr darf für dieses Segment Schönes auch etwas kosten und die Preise können dementsprechend angepasst werden. 
Bei der Kommunikationspolitik wird deutlich, dass Prospekte kein Medium sind, mit dem das Segment gezielt angesprochen werden kann. Vielmehr ist dies möglich anhand von Büchern und Zeitschriften, die der „durchschnittliche Gartenfreund“ von allen Segmenten am häufigsten liest. Allerdings weist er auch eine Affinität zum Internet und zu Gesprächen mit dem Züchter auf, wenngleich in jeweils deutlich schwächerer Ausprägung. Als Ansatzpunkte für eine gelungene Kundenansprache bieten sich neben dem Thema „Optik“ weitere Themen an. So wäre die erkannte Risikoaversion des „durchschnittlichen Gartenfreundes“ eine Möglichkeit, Personal- und Kommunikationspolitik segmentspezifisch zu kombinieren. Um das Risiko eines Fehlkaufes für ihn zu senken, bieten sich beispielsweise neben „Geld-zurückGarantie“, einer „Anwachsgarantie“ oder der Betonung von Qualitätssiegeln auch Beratungsgespräche vor Ort an. Der „durchschnittliche Gartenfreund“ legt auf Beratung großen Wert und nimmt sich dafür entsprechend Zeit. Weiterhin spielt das Thema „Kreativität“ für ihn eine überdurchschnittlich große Rolle. Musterbepflanzungen oder ausgefallene Vorschläge für die Kombination von verschiedenen Pflanzen zeigen Möglichkeiten auf, kreativ zu werden. Zusätzlich wird ihm durch den Umgang mit BuP eine Möglichkeit gezeigt, auf kreative und spielerische Art Naturverbundenheit zu erleben. Um diese Instrumente für die Kundenansprache nutzen zu können, ist es allerdings notwendig, dass die betroffenen Mitarbeiter die entsprechenden Qualifikationen aufweisen. Schulungen und Weiterbildungen sind daher notwendig, um mit dem Angebot stets am Puls der Zeit und somit möglichst nahe am Kunden zu sein. Da der „durchschnittliche Gartenfreund“ sich beim Einkauf sehr viel Zeit lässt, ist zu vermuten, dass das Segment sich das gesamte Angebot ansieht, um sich dann für das Schönste zu entscheiden. Vor diesem Hintergrund wäre mit Blick auf die Ausstattungspolitik überlegenswert, beschilderte „Spazierwege“ durch das gesamte Sortiment anzulegen, damit sich die Kunden inspirieren lassen können. Dabei scheint es wichtig, das gezeigte Sortiment entlang des Spazierweges immer wieder neu in Szene zu setzen, um so dem Eindruck vorzubeugen, dass das Sortiment auf Dauer langweilig wird. Nur so können Besonderheiten immer wieder neu zur Geltung gebracht werden.

Der „durchschnittliche Gartenfreund“ kauft von allen Segmenten am zweithäufigsten in Baumschulen ein. Parallel dazu bildet er das einzige Segment, das in Gartencentern einkauft, wenn auch deutlich seltener als in Baumschulen. Dies lässt einen Hang zum Kauf beim Fachhandel vermuten, was unterstrichen wird durch die schlechte Qualitätserwartung bei Discountern. Supermärkte werden von ihm als teuer, Gärtnereien und Wochenmärkte hingegen als günstig angesehen. Da in diesem Segment Preise allerdings keine signifikante Rolle spielen, 
dürfte dieser Parameter für die Einkaufsstättenwahl nicht entscheidend zu sein. Vielmehr scheint es Gartencentern und besonders Baumschulen besser als den Alternativen des Fachhandels zu gelingen, die Erwartungen des Segments zu treffen. Für Gärtnereien bietet es sich zur segmentspezifischen Ansprache an, die Produktpalette mehr am Segment auszurichten und dabei gleichzeitig die Preispolitik zu überdenken. Obwohl der ,durchschnittliche Gartenfreund“ nicht preissensibel ist, empfindet er Gärtnereien als preislich günstig. Dies zeigt für Gärtnereien einen preislichen Anpassungsspielraum nach oben.

Mit ca. $16 \%$ Marktpotenzial stellt der „Pflanzengenießer“ das zweitgrößte aller untersuchten Segmente dar. Die Ergebnisse der Analyse zeigen, dass das Segment einerseits deutlich die ältesten Personen, andererseits die wenigsten Berufstätigen aufweist, was sowohl Vollzeitals auch Teilzeitberufstätige umfasst. Vor diesem Hintergrund scheint eine Änderung der Ladenöffnungszeiten - wie etwa für den „durchschnittlichen Gartenfreund“ empfohlen - beim „Pflanzengenießer“ wenig hilfreich. Vielmehr kann davon ausgegangen werden, dass er tagsüber Zeit hat und die gängigen Öffnungszeiten für ihn ausreichen. Als Option für segmentspezifische Öffnungszeiten könnten sich bestimmte „Early-Bird-Angebote“ anbieten, beispielsweise durch Vergünstigungen während des Einkaufs zwischen 8 und 10 Uhr an Werktagen. So könnten Einzelhändler ihre umsatzschwache Verkaufszeit nutzen, um sich für Pflanzengenießer zu präsentieren. Dabei ist bei der Wahl der Produktpalette ein Fokus auf Optik im Sinne von Schönheiten und Neuheiten bzw. Besonderheiten zielführend, da das Segment „Besonderes zu schätzen weiß“. Da für den „Pflanzengenießer“ zusätzlich eine tendenzielle Risikoaversion festgestellt wurde, bieten sich Erweiterungen des Sortiments durch Produkte mit „Geld-zurück-Garantie“, „Anwachsgarantie“ oder ausgewiesenen Qualitätssiegeln an. Dabei spielt der monetäre Aspekt für den „Pflanzengenießer“ eine untergeordnete Rolle. Da er für schöne BuP eine erhöhte Zahlungsbereitschaft aufweist, bietet sich für Unternehmen eine segmentspezifische Anpassung ihrer Preispolitik an.

Bei der Standortwahl zeigt sich, dass der „Pflanzengenießer“ auch längere Wege in Kauf nimmt. Dementsprechend kann der als relevant angesehene Markt für dieses Segment geographisch ausgedehnt werden, was wiederum Auswirkungen auf die Weite der Kommunikationspolitik hat. Der „Pflanzengenießer“ ist einerseits sehr gut über Anzeigen in Büchern und Zeitschriften zu erreichen, andererseits sucht er auch gezielt das Gespräch mit dem Züchter. In Kombination mit der Kenntnis, dass er sich sehr gerne beraten lässt, bietet dies wiederum eine sehr gute Möglichkeit, um mit ihm ins Gespräch zu kommen. Weiterhin spielt das Thema Kreativität im Umgang mit BuP für ihn eine dominante Rolle, was einen guten Anknüpfungs- 
punkt bietet - hier können sich kleine Einzelhändler besonders gut segmentspezifisch profilieren, da sie meist individueller sind als große, zentralistisch gesteuerte Verkaufsstätten wie Baumärkte oder Gartencenter. Musterbepflanzungen von Balkonkästen oder Mustergräbern oder auch moderne Vorschläge für die Kombination von verschiedenen Pflanzen zeigen dem „Pflanzengenießer“ Möglichkeiten auf, seine Kreativität auszuleben und in Szene zu setzen. Zusätzlich wird ihm durch den Umgang mit BuP eine Möglichkeit gezeigt, auf kreative Art seiner Naturverbundenheit Ausdruck zu verleihen. Um diese Instrumente für die Kundenansprache nutzen zu können, ist es allerdings erforderlich, dass die betreffenden Mitarbeiter die entsprechenden Qualifikationen aufweisen. Schulungen und Weiterbildungen sind daher notwendig, um mit dem Angebot stets am Puls der Zeit und somit möglichst nahe am Kunden zu sein. Da sich der „Pflanzengenießer“ beim Einkauf sehr viel Zeit lässt und „den Umgang mit BuP genießt“", ist zu vermuten, dass er sich gerne das gesamte Angebot ansieht und sich dabei inspirieren lässt, um sich dann für das Schönste zu entscheiden. Vor diesem Hintergrund wäre im Rahmen der Ausstattungspolitik überlegenswert, beschilderte „Spazierwege“ oder „Themeninseln" durch das gesamte Sortiment anzulegen. Dabei scheint es wichtig, das gezeigte Sortiment entlang des Spazierweges immer wieder neu in Szene zu setzen, um so dem Eindruck vorzubeugen, dass das Sortiment auf Dauer langweilig wird. Nur so können Besonderheiten immer wieder neu in Szene gesetzt werden und sich die Kunden inspirieren lassen. Gleichzeitig unterstreicht eine wechselnde Präsentationsweise die eigene Kreativität, was dem „Pflanzengenießer“ zeigt, dass man auf einer Wellenlänge liegt.

Die Analyse des Einkaufsverhaltens hat ergeben, dass der „Pflanzengenießer“ wie alle anderen Segmente auch in Baumschulen einkauft. Verglichen mit den anderen Segmenten ist sein Hang zu Baumschulen allerdings recht wenig ausgeprägt. Vielmehr ist er das einzige Segment, das in Blumenfachgeschäften einkauft, was die bereits angesprochene Vermutung bestätigt, dass in puncto Kreativität kleine und dadurch „,persönlichere“ Einkaufsstätten mehr der Vorstellung des „Pflanzengenießers“ entsprechen als große Einkaufsstätten. Weiterhin zeigt sich, dass er dem Wochenmarkt sowohl günstige Preise, aber auch eine schlechte Qualität zuspricht. Hierbei zeigen sich allerdings die Potenziale, die dieses Segment dem Wochenmarkt bietet, der ja als kleine, individuelle Einkaufstätte im Fokus des „Pflanzengenießers“ liegt. Als primäre Stellschraube zur segmentspezifischen Kundenansprache erweist sich für den Wochenmarkt das angebotene Sortiment. Dies gilt es wie beschrieben anzupassen. Dabei empfiehlt es sich, parallel die Preise der erhöhten Zahlungsbereitschaft des Segments anzupassen. Es kann vermutet werden, dass Standardprodukte für den „Pflanzengenießer“ wenig 
interessant sind. Daher werden zentral gesteuerte Vertriebskanäle auf Grund ihrer Größe Schwierigkeiten haben, dieses Segment zu erreichen. Hierbei sind die Vorteile deutlich auf Seiten der kleineren, beispielsweise inhabergeführten Einkaufsstätten zu vermuten.

Der „extrovertierte Naturbewahrer“ ist mit einem Potenzial von ca. $8 \%$ des relevanten Marktes das kleinste aller Segmente. Da es relativ wenig Vollzeitberufstätige umfasst, kann wie beim „Pflanzengenießer“ davon ausgegangen werden, dass eine Ausdehnung der Ladenöffnungszeiten in den Abend zu Lasten von Verkaufszeit am Morgen wenig zielführend ist. Da der „extrovertierte Naturbewahrer“ sich wenig Zeit für seinen Kauf nehmen will, dabei keine Beratung wünscht und als extrovertiert geprägter Außendarsteller „keine bestimmte BuP sucht, sondern nur eine besonders schöne“, ergeben sich mehrfache Anforderungen an das Sortiment und dessen Präsentation. Durch den Außendarsteller-Effekt ist ein umfangreiches Sortiment sehr im Sinne des „extrovertierten Naturbewahrers“. Allerdings kollidiert das Ziel einer großen Auswahl in der Praxis mit den Zielen „schnell“ und „keine Beratung“. Da das Segment als nicht BuP-kompetent beschrieben wurde, besteht die große Gefahr, dass ein zu großes Angebot abschreckt. Um dennoch ein ansprechendes Sortiment zeigen zu können, bietet es sich an, eine Vorauswahl im Sinne des „extrovertierten Naturbewahrers“ zu treffen. Beispielsweise könnte das Sortiment pro Monat aus den zehn gängigsten Artikeln bestehen, allerdings jeweils mit verschiedenen, besonderen Ausprägungen, wie beispielsweise „zwergwüchsig“, „,schwarzstielig“ oder ,großblättrig“. So wäre ein Angebot geschaffen, das sowohl in kurzer Zeit überblickt werden kann als auch den Schwerpunkt segmentspezifisch nur auf Ausgefallenes und Besonderes legt. Gleichzeitig würde diese Art des fokussierten Angebots das Ziel „wenig Beratung“ unterstützen. Um dem „extrovertierten Naturbewahrer“ bei der Kaufentscheidung dennoch die wichtigsten Infos rund um die Pflanze verfügbar zu machen, bieten sich entsprechende Etikettierungen an, die sich auf das für die Pflanze Relevante konzentrieren. Durch die Kombination dieser Merkmale der Angebotspräsentation besteht die Möglichkeit, seine Personalkosten im Verkaufsbereich sehr gering zu halten, was wiederum die Option einer segmentspezifischen Preispolitik ermöglicht. Durch geringe Kosten besteht die Möglichkeit, Kostenvorteile an die Kunden weiterzugeben, was in den Augen des als sparsam beschriebenen „extrovertierten Naturbewahrers“ wünschenswert wäre. Weiterhin bieten sich zur segmentspezifischen Preispolitik die Vorteile der Preisdifferenzierung an, beispielsweise anhand von Treuepunkten. Im Hinblick auf die Kommunikationspolitik zeigt sich, dass das Segment am besten über Anzeigen in Büchern und Zeitschriften zu erreichen ist. 
Der „extrovertierte Naturbewahrer“ kauft - wie alle anderen Segmente auch - in Baumschulen ein, im Vergleich mit den anderen tut er dies jedoch am häufigsten. Gleichzeitig ist er das einzige Segment, das in Supermärkten BuP einkauft. Dieser Kombination aus typischem Fachhandel und typischem Nicht-Fachhandel könnte mit einem Selbstbedienungsgeschäft entsprochen werden. Mit den oben beschriebenen Ausprägungen eines solchen Geschäftes gelänge es, viele Vorteile von Baumschulen und Supermärkten zu vereinen. Dabei zeigt sich für Gärtnereien das größte Potenzial, den „extrovertierten Naturbewahrer“ von Baumschulen und Supermärkten abzuwerben. In seinen Augen gelten Gärtnereien als günstig, was diesem preissensiblen Segment entgegenkommen dürfte. Differenziert stellt sich die Situation für den Wochenmarkt dar. In den Augen des „extrovertierten Naturbewahrers“ bietet er zwar einen guten Service, allerdings auch eine schlechte Qualität bei BuP. Da für den „extrovertierten Naturbewahrer" das Thema BuP-Qualität allerdings nicht von entscheidender Bedeutung ist, stellt dieser Parameter wohl nicht den Hauptgrund dafür dar, wieso er seine BuP nicht auf Wochenmärkten kauft.

Der „konservative Kleingärtner“ stellt mit ca. $10 \%$ Marktpotenzial das zweitkleinste aller Segmente. Bedingt durch seine Altersstruktur kann davon ausgegangen werden, dass besonders ausgedehnte Ladenöffnungszeiten für eine segmentspezifische Ausrichtung nicht sehr zielführend sind. Vielmehr geben die vielen Parallelen zum „Kultivator“ Hinweise auf die Potenziale, die eine gezielte, segmentspezifische Kundenansprache ermöglichen. Der „,konservative Kleingärtner“" ist ausgesprochen kompetent, nicht extrovertiert, seriös, zeigt keine Wertschätzung für Besonderheiten und zieht seinen Nutzen daraus, dass er Pflanzen pflegt und sich um sie kümmert. Vor diesem Hintergrund scheint ein „seriöses“ Angebot dem Segment am besten zu entsprechen: ein Angebot, das die gängigsten Artikel bietet, ohne dabei jedoch spezielle, ausgefallene Merkmale in den Fokus zu rücken. Weiterhin ist aufgrund seiner Kompetenz denkbar, dass der „konservative Kleingärtner“ eine gewisse Affinität zur Idee des Verkaufs von Halbfertigprodukten aufweist. Dies würde ihm die Gelegenheit geben, sich in besonderem Maße um Pflanzen zu kümmern und sie zu pflegen. Zusätzlich dürfte die optisch meist nicht überzeugende Wirkung von Halbfertigprodukten für das Segment keine Kaufhemmschwelle darstellen, da es einerseits die notwendige Kompetenz besitzt zu wissen, was sich aus der Halbfertigware entwickeln wird, und andererseits beim Kauf keinen gesteigerten Wert auf Besonderheiten in Sinne von schöner Optik legt. Insofern der „konservative Kleingärtner“ ganz explizit soziale Aspekte wie Geselligkeit und Feiern mit anderen Leuten im Garten ablehnt, scheidet eine Kundenansprache auf dieser Grundidee aus. Vielmehr dürfte 
seine Kompetenz einen günstigen Ansatzpunkt bieten. Botanische Bezeichnungen und ausgeprägte Pflege- und Hintergrundinformationen eignen sich daher, um das Segment anzusprechen. Dabei stellen entsprechende Anzeigen in Büchern und Zeitschriften das einzige Medium dar, mit dem es sich gezielt erreichen lässt.

Der „,konservative Kleingärtner“ kauft ausschließlich in Baumschulen ein, und dies primär für den Eigenbedarf, was wiederum seine seriöse Grundhaltung unterstreicht. Vor diesem Hintergrund scheint er für große Marketingaktionen oder Ähnliches wenig offen. Vielmehr sollte dem Segment „seriös“ begegnet werden, was beispielsweise eine besondere Berücksichtigung von Saisonware bedeuten könnte. Während Mitbewerber die Saison für bestimmte Pflanzen immer mehr nach vorne ziehen, könnte dies ein Punkt sein, um den „,konservativen Kleingärtner“ gezielt anzusprechen. Da er im Hinblick auf BuP macht, was erforderlich ist, und da er ordnungsliebend ist, kann davon ausgegangen werden, dass er eine Affinität zu Saisonbepflanzungen hat: Bedingt durch die Jahreszeiten ist irgendwann jede Saisonbepflanzung nicht mehr zeitgemäß, sieht dementsprechend nicht mehr ordentlich aus und sollte somit ersetzt werden. Parallel zum Fokus auf Saisonware könnten sich kurzfristig angesetzte Schwerpunkttage als besonderes Mittel erweisen, den „konservativen Kleingärtner“ anzusprechen. Dies empfiehlt sich vor allem nach Ereignissen, die die „BuP-Ordnung“ in seinen Augen stören könnten, beispielsweise nach einer anhaltenden Schlecht-Wetter-Periode oder nach starkem Hagel. 


\section{Schlussbetrachtung}

Nachdem die Segmente anhand aller zur Verfügung stehenden Variablen beschrieben sind, folgt in diesem Kapitel die abschließende Betrachtung der Arbeit. Dabei werden zunächst die ihr zu Grunde liegenden Methoden diskutiert. Mit einer Einordnung der Arbeit in das große Themenfeld des Einzelhandels für Blumen und Pflanzen schließen die Ausführungen.

\subsection{Diskussion der Methoden}

Die eingehende Betrachtung des Marktes für Blumen und Pflanzen (Kap. 2) hat gezeigt, dass er sich in einer problematischen Schieflage befindet. Verschiebungen von Umsatzanteilen, reales Marktschrumpfen und Verschiebungen des nachgefragten Sortiments sind dabei nur einige der beschriebenen Probleme. Um dieser Entwicklung zu begegnen, versucht die vorliegende Arbeit, die Konsumenten anhand ihrer Kaufmotive für Blumen und Pflanzen zu segmentieren. Dabei ist die Wahl der zum Einsatz gekommenen Methoden sehr vom aktuellen Stand der Forschung beeinflusst. Wie bereits erwähnt, sind wissenschaftliche Publikationen zu der hier behandelten Problematik so gut wie nicht vorhanden. Angesichts dessen war es dem Autor nicht möglich, begründete theoriegeleitete Hypothesen darüber abzuleiten, welche Motive die Konsumenten von $\mathrm{BuP}$ in besonderem Maße beeinflussen. Vor diesem Hintergrund weist diese Arbeit einen ausgeprägten explorativen Charakter auf, was die Wahl der zum Einsatz gekommenen Methoden stark beeinflusst.

Basierend auf den theoriegeleiteten Ausführungen über Lebensstile (Kap. 3.1) und Marktsegmentierung (Kap. 3.2) wurden Lebensstile als geeignetes und relevantes Segmentierungskriterium für Konsumenten von BuP erkannt. Die Untersuchung ist somit sowohl problemorientiert als auch interdisziplinär. Die Lebensstilforschung ist ein Ansatz der Verhaltensforschung und somit auch zumindest in Teilen ein Ansatz der Konsumentenforschung und damit des verhaltensorientierten Marketings. Die hier vorgenommene Integration der Lebensstilforschung in das Feld des Marketings scheint somit nur folgerichtig. Ziel des interdisziplinären Einsatzes der Lebensstilforschung im Bereich des Marketings war es, mit Hilfe der vorhandenen Theorien, Methoden und Erkenntnissen der Lebensstilforschung ein besseres Verständnis über das Einkaufsverhalten der BuP-Konsumenten zu erlangen. Zu diesem Zweck wurde das „Schema zur Rekonstruktion von Lebensstilen“ nach Lüdtke (vgl. Abb. 37 und Abb. 46) an die Problemstellung dieser Arbeit angepasst. Die Kriterien der vier Dimensionen des Schemas 
„Sozioökonomische Dimension“, „Kompetenz“, „Performanz“ und „Motivation“ wurden dabei theoriebasiert an die Gegebenheiten des Marktes für BuP angepasst. Basierend auf diesen spezifischen Kriterien wurde ein Gesprächsleitfaden entwickelt, der in explorativen Einzelinterviews zum Einsatz kam. Diese Tiefeninterviews dienten dazu, das Verständnis über das Denken und Fühlen der Probanden zu erhöhen. Weiterhin sollten dadurch die internen Faktoren der Befragten, also deren Einstellungen, Werte, Erinnerungen und Erfahrungen besser bei der späteren Erstellung des Fragekatalogs berücksichtigt werden können.

Nach Durchführung und Reflexion der ersten Interviews zeigte sich, dass der Leitfaden überarbeitet werden musste. Neben den ausgearbeiteten Themen zu den vier Dimensionen nach Lüdtke waren während der Interviews konkrete Erlebnisse in den Einzelhandelsgeschäften immer ein wichtiges Thema. Vor diesem Hintergrund wurde deutlich, dass das Lüdtke'sche Schema zwar eine lebensstilbasierte Segmentierung ermöglicht, diese aber im Kontext der hier zu Grunde liegenden Forschungsfrage nur bedingt erfolgversprechend war. Da das Lüdtke'sche Schema die Segmentierungskriterien des beobachtbaren Kaufverhaltens nur unzureichend berücksichtigt, waren spezifische Ergebnisse in diesem als äußerst relevant erkannten Bereich nicht zu erwarten. Infolgedessen wurde das Lüdtke'sche Schema um die Dimension „Beobachtbares Kaufverhalten“ erweitert (s. Abb. 47). Basierend auf den folgenden explorativen Interviews und der Analyse ihrer Ergebnisse wurde ein erster Fragekatalog erstellt, der sowohl nach einem qualitativen als auch nach einem quantitativen Pretest mehrfach überarbeitet wurde (s. Kap. 6.3).

Die Befragung wurde an 880 Personen durchgeführt. Diese wurden im Raum Osnabrück vor einer Baumschule, einem Gartencenter und einem Baumarkt angesprochen. Die Auswahl der Befragungsorte scheint im Nachhinein nicht optimal gewählt zu sein. Die Intention der Auswahl der Befragungsorte lag darin, Personen zu finden, die einen Bezug zu BuP und somit eine geringere Hemmschwelle haben, an der Befragung teilzunehmen. Wie sich allerdings im weiteren Verlauf dieser Arbeit gezeigt hat, könnte diese Wahl der Befragungsorte die Generalisierbarkeit der Studie negativ beeinflussen. Beispielsweise wurde für jedes Segment erkannt, dass es während der letzten 12 Monate in Baumschulen eingekauft hat, wobei Baumschulen und Gärtnereien gemeinsam allerdings nur $10 \%$ des Jahresumsatzes der BuPBranche generieren. Es ist daher zu vermuten, dass Kunden der Vertriebskanäle Baumschule, Gartencenter und Baumarkt in der Stichprobe eventuell überrepräsentiert sein könnten. Eine Befragung wahllos angesprochener Personen, die nicht gerade aus einem BuP-Geschäft kommen, scheint daher für folgende wissenschaftliche Arbeiten vorteilhafter. 
Nach Sichtung und Eingabe der Befragungsergebnisse in die statistische Auswertungssoftware SPSS wurde in einem ersten Schritt eine motivbasierte Faktorenanalyse durchgeführt (s. Kap. 7.1). Diese hatte zum Ziel, die Ergebnisse zu verdichten, um so eine Komplexitätsreduktion zu erreichen, die schließlich tiefere Einblicke in die BuP-spezifischen Motive der Probanden ermöglichen sollte. Die letztlich erkannten fünf Motivfaktoren und deren Beschreibung (s. Tab. 5) haben sich im weiteren Verlauf dieser Arbeit mehrfach bestätigt bzw. wurden als zutreffend befunden. Vor diesem Hintergrund und den annehmbaren Gütemaßen der Faktorenanalyse scheint deren Einsatz als Analysemethode zur empirischen Ermittlung von motivbasierten latenten Konstrukten als gelungen.

Um die Forschungsfrage dieser Arbeit beantworten zu können, wurden die erkannten Motivfaktoren in eine motivbasierte Clusteranalyse überführt (s. Kap. 7.2). Bei der als am geeignetsten eingestuften 6-Cluster-Lösung konnten ca. 92 \% der Probanden einem der sechs Cluster zugeordnet werden. Die Beschreibung und Benennung der gefundenen Cluster erfolgte anhand der zugehörigen Mittelwerte der Motivfaktoren (s. Tab. 7). Diese ersten Informationen über die Cluster lieferten viele Erkenntnisse über die innenliegenden BuP-spezifischen Motive der Cluster bzw. der Probanden. Da diese Motive für das Verständnis von Kaufentscheidungsprozessen elementar sind, scheint die gewählte Methode der motivbasierten Clusteranalyse als geeignetes Instrument für die Gruppierung von großen Stichproben und auch für deren Beschreibung anhand der ihr zu Grunde liegenden Motive. Weiterhin haben sich die Beschreibungen der Cluster anhand der Motive im weiteren Verlauf dieser Arbeit mehrfach deutlich bestätigt, so dass davon ausgegangen werden kann, dass die Entscheidung zu einer motivbasierten Clusteranalyse zielführend im Sinne der Forschungsfrage war.

Um die gefundenen Cluster anhand der passiven Variablen des Fragekatalogs weiter beschreiben zu können (vgl. hierzu Kap. 6.2.1), wurde im Rahmen einer erweiterten Analyse (Kap. 7.3) eine multinominale logistische Regressionsanalyse durchgeführt. Dazu musste allerdings eines der sechs Cluster als Referenzcluster ,geopfert“ werden. Die Wahl fiel auf das Cluster „Desinteressierter Pragmatiker“ (s. Kap. 7.2.4). Als problematisch bei der Auswahl zeigte sich, dass das Referenzcluster das größte aller sechs Cluster war (s. Tab. 6). Es wurde dennoch als Referenzcluster ausgewählt, da es sich den Erkenntnissen zufolge, die bis zu diesem Punkt über das Cluster bereits gewonnen werden konnten, inhaltlich sehr stark von allen anderen Clustern abspaltete. Diese wiederum waren in ihren Beschreibungen alle ähnlich oder hatten zumindest inhaltliche Überschneidungen, so dass eine genauere Betrachtung dieser Cluster als notwendig erschien. Weiterhin wurde berücksichtigt, dass der „desinteressierte 
Pragmatiker“ kein „Musterkunde“ zu sein schien, während alle anderen Cluster in irgendeiner Weise einen Bezug zum Thema BuP aufwiesen. Für weitere wissenschaftliche Forschungen bietet sich der „desinteressierte Pragmatiker“ allerdings unter dem Gesichtspunkt der Clustergröße durchaus zur näheren Betrachtung an. Dies wäre auch dahingehend interessant, um zu erfahren, was das Desinteresse dieses Clusters genau ausmacht. So könnten eventuell Maßnahmen auf der Angebotsseite entwickelt werden, die gerade den „,desinteressierten Pragmatiker" näher an das Thema BuP heranführen.

Das Ergebnis der Regressionsanalyse hat gezeigt, dass viele Items der sozioökonomischen Dimension für die Clusterbildung nicht relevant im Sinne von signifikant sind. Dies überrascht einerseits, andererseits bestätigt es die Grundidee dieser Arbeit, nämlich dass Motive den Kaufentscheidungsprozess für BuP dominieren. Weiterhin wurde deutlich, dass sich die Cluster in ihren Eigenschaften teilweise überschneiden. Dies ist aus Marketingsicht nicht optimal, unterstreicht aber den Faktor Mensch, der in dieser Studie im Mittelpunkt steht. Diese Unschärfe der Clustergrenzen war im Vorfeld der Analyse durchaus erwartet worden und wurde daher bereits kritisch hinterfragt. Letztlich kann dieses Problem als besondere Stärke des hier zu Grunde liegenden Modells angesehen werden, da die Grenzen der Cluster als Bereiche zu verstehen sind, in denen auch Überschneidungen zwischen einzelnen Cluster möglich sind, was die Realitätsnähe der Modellierung enorm erhöht (Schulze 2000, S. 163 ff., 384).

Weiterhin zeigte sich im weiteren Verlauf anhand der Regressionsanalyse, dass die Fragestellung und die zugehörigen Antwortmöglichkeiten im Fragekatalog nicht immer optimal gewählt worden waren. So haben sich die Möglichkeiten zu Mehrfachnennungen in Frage 4 und 5 als problematisch im Sinne der Auswertung erwiesen. Eine jeweils eigene Frage pro Item wäre für die spätere Analyse nicht nachteilig gewesen und hätte zusätzlich beispielsweise die Option erlaubt, pro erkanntem Cluster die zugehörige favorisierte Einkaufsstätte zu nennen. Da dies allerdings auch unter forschungsökonomischen Gesichtspunkten zu bewerten ist (z. B. Mehraufwand bei Befragung), kann dieser Punkt nicht abschließend bewertet werden. Dennoch sei auf die Erkenntnis hingewiesen, dass der Aufwand, der bei der Vorbereitung einer Befragung betrieben wird, sich bei der Analyse sehr schnell amortisiert. Bei der Gestaltung des Fragebogens sollte stets daran gedacht werden, dass nach der erfolgten Befragung keine Nachfragen mehr möglich sind. Dementsprechend soll hier nochmals auf die Wichtigkeit einer exakten, genauen und vorausschauenden Planung der Befragung hingewiesen werden. 
Neben den Problemen zeigte sich aber auch, dass sich die gebildeten Cluster inhaltlich nicht nur überschnitten, sondern auch sehr eindeutig differenzieren ließen. Es gibt somit signifikante Unterschiede zwischen den Clustern, was sowohl für die clusterbildenden (hier: aktive) Variablen als auch für die clustererklärenden (hier: passiven) Variablen gilt. Da die Ergebnisse sowohl für den aktiven als auch für den passiven Bereich demnach als gelungen betrachtet werden können, kann auch die Gesamtlösung sowohl als robust als auch als methodisch gelungen angesehen werden (Byrd-Bredbenner/Maurer Abbot/Cussler 2008).

Die Gesamtlösung zeichnet sich weiterhin dadurch aus, dass sie eine praxisnahe und marketinggeprägte Perspektive auf die gewonnenen Erkenntnisse ermöglicht (s. Kap. 7.5). Unter Berücksichtigung der Fragestellung kann somit festgehalten werden, dass eine empirisch abgesicherte, lebensstilbasierte Segmentierung der Kunden der BuP-Branche erreicht wurde. Zudem lässt sich festhalten, dass die zum Einsatz gekommenen Methoden allesamt zielführend waren und letztlich das zentrale Forschungsziel dieser Arbeit als erreicht angesehen werden kann.

Nicht vergessen werden darf jedoch, dass die Mechanismen eines Kaufentscheidungsprozesses bei Konsumenten jeder Art meist unbewusst ablaufen. Diese Mechanismen wie auch ihre Auslöser sind daher quantitativ sehr schwer empirisch zu erfassen. Vor diesem Hintergrund möchte diese Arbeit als Fundament für weitere wissenschaftliche Arbeiten in diesem Bereich dienen. Die hier gewonnen Erkenntnisse sollen die Generierung weiterführender Theorien und Hypothesen unterstützen, um die Forschung in diesem noch schwach besetzten Bereich voranzubringen.

\subsection{Würdigung}

Die Branche für Blumen und Pflanzen befindet sich seit langem in einer Dauerstagnation. Der Markt ist gesättigt und generiert keinerlei dynamisches Wachstum. Während der letzten Jahre waren sogar Phasen des realen Branchenschrumpfens zu beobachten. Verteilungskämpfe zwischen den einzelnen Vertriebskanälen, die meist auf der preislichen Schiene ausgefochten werden, um die übrig gebliebene Kundschaft sind die Folge. Für viele Einzelhändler von Blumen und Pflanzen ist dieser Wettbewerb um den bestehenden Markt, in dem sich mittlerweile auch große, branchenfremde Einzelhandelskonzerne tummeln, teilweise ruinös. Um diesem Wettbewerb zu entgehen und um zusätzlich auch eigenes Marktwachstum anzukurbeln, müssen Einzelhändler umdenken: Während landauf, landab beispielsweise mit großen 
Rabattaktionen und Sonderpreisen um Kunden geworben wird, sollten sie sich fragen, ob das die eigene bzw. potenzielle Kundschaft auch von ihnen erwartet. Marketingaktionen, die am bestehenden Kundenstamm vorbeigehen und wenige bis gar keine neue Kunden akquirieren, sind auf Dauer nicht nur ruinös, sondern verwirren auch die Stammkundschaft, die so eventuell gar abgeschreckt werden könnte.

Um diesem Szenario zu entgehen, ist es nötig, seine Kundschaft möglichst detailliert kennenzulernen und sich als Einzelhändler genau an deren Erwartungen zu orientieren. Mit dieser Arbeit soll ein erster Schritt in diese Richtung gemacht werden. Da die Branche seit vielen Jahren keine wissenschaftliche Segmentierung der Konsumenten mehr durchgeführt hat, stellt sie den ersten Versuch in diesem Bereich dar. Durch das Segmentieren der Konsumenten anhand von Lebensstilen bietet sie ein neuartiges Fundament, das der Branche nun zur Verfügung steht. Die Orientierung anhand von Lebensstilen der Konsumenten ermöglicht es Einzelhändlern, ihre Marketingaktivitäten von den gängigen Marketinginstrumenten, die in ihrer Zielgenauigkeit der Kundenansprache meist sehr begrenzt und daher sehr teuer sind, abzukoppeln. Die hier durchgeführte Segmentierung bietet Einzelhändlern erstmals die Gelegenheit, eine empirisch nachgewiesene Zielgruppe zu definieren und sie genau und zielgerichtet zu bearbeiten. Eine exakte Ansprache der bestehenden Kunden und ein in der Außenwirkung geschärftes Unternehmensprofil sind die Folgen, die aus Kunden Stammkunden und aus Interessenten Neukunden machen können. Vor diesem Hintergrund stellt diese Arbeit für die Branche ein wichtiges Werkzeug dar, das die Palette der Handlungsoptionen der Einzelhändler im Kampf um Kunden maßgeblich erweitert.

Darüber hinaus wird allen Teilnehmern der Branche eine Anleitung an die Hand gegeben, wie jeder seine eigene, lebensstilbasierte Kundensegmentierung durchführen kann. Durch die offene, transparente und schrittweise Entwicklung des Segmentierungsmodells lässt es sich von jedem leicht nachvollziehen und anhand der Kriterien der Lebensstildimensionen individuell an die jeweiligen Gegebenheiten vor Ort anpassen. Die Generalisierbarkeit der Anwendung und ihre zugangsfreie Publikation dürfte für die Branche ein großer Schritt sein. Wäre diese Untersuchung privatwirtschaftlich durchgeführt worden, wären die Ergebnisse nicht öffentlich oder allenfalls gegen Entgelt zugänglich. Diese Arbeit versteht sich somit nicht nur als Darstellungsform einer wissenschaftlichen Untersuchung zu einem für die Branche relevanten Thema, sondern auch als wissenschaftlich fundiertes Fundament für weitere Erhebungen und Forschungen. 
Konkret wäre es wünschenswert, wenn möglichst viele Branchenteilnehmer durch diese Studie animiert werden, auf ihre Kunden zuzugehen und diese besser kennenzulernen. Für die weitere wissenschaftliche Forschung bietet sie als Grundlagenarbeit einen Pool an Erkenntnissen an, aus dem weiterführende Hypothesen generiert werden können. Forschungsbedarf besteht in den Augen des Autors vor allem in der Frage, wie die vielen Nichtkäufer näher an das Thema Blumen und Pflanzen herangeführt werden können. 


\section{Quellenverzeichnis}

Abell, D.: Defining the business. The starting point of strategic planning. Prentice-Hall, Englewood-Cliffs 1980.

Ahlert, D.; Kenning, P.: Handelsmarketing. Grundlage der marktorientierten Führung von Handelsbetrieben. Springer, Berlin 2007.

Ajzen, I.: Nature and opinion of attitudes. In: Annual Review of Psychology, 52 (2001), S. 27-58.

Albersmeier, F.; Spiller, A.: Das Ansehen der Fleischwirtschaft: Zur Bedeutung einer stufenübergreifenden Perspektive. In: Böhm, J., Albersmeier, F. und Spiller, A. (Hrsg.): Die Ernährungswirtschaft im Scheinwerferlicht der Öffentlichkeit. Reihe Agrarökonomie, EuI, Lohmar 2009.

Altmann, M.: Konsumententypologie auf dem Zierpflanzenmarkt. Dissertation, Fachbereich Gartenbau, Universität Hannover, 1983.

Altmann, M.; Stenger, M.: „Ein neuer Konsumtrend?“ - Verbrauchertrends von morgen. In: TASPO. Zeitung für den grünen Markt, 144 (2010), Nr. 1, S. 3.

Ascheberg, C.: Die Sigma-Milieus, das globale Zielgruppen- und Trendsystem. Internetabfrage vom 16.07.2010, http://www.sigma-online.com/de/Articles_and_Reports/zielgruppenfor schung.pdf

Bacher, J. et al.: Clusteranalyse. Anwendungsorientierte Einführung in Klassifikationsverfahren. 3. Aufl., Oldenbourg, München 2010.

Backhaus K. et al.: Multivariate Analysemethoden. Eine anwendungsorientierte Einführung. 10. Aufl., Springer, Berlin 2003.

Backhaus K. et al.: Multivariate Analysemethoden. Eine anwendungsorientierte Einführung. 11. Aufl., Springer, Berlin 2006.

Backhaus K. et al.: Multivariate Analysemethoden. Eine anwendungsorientierte Einführung. 12. Aufl., Springer, Berlin 2008.

Banning, T.: Lebensstilorientierte Marketing-Theorie. Physica, Heidelberg 1987.

Barth, K.: Standortpolitik. In: Hermanns, A. et al. (Hrsg.): Handbuch Mode-Marketing. Frankfurt a. M. 1991, S. 735-747.

Barth, K.: Betriebswirtschaftslehre des Handels. 4. Aufl., Gabler, Wiesbaden 1999.

Barth, K.; Hartmann, M.: Germany. In: Howe, S. (Hrsg.): Retailing in the European Union. Structure, Competition and Performance. Taylor and Francis, London 2003, S. 56-80 
Bea, F.; Haas, J.: Strategisches Management. 4. Aufl., Lucius \& Lucius, Stuttgart 2005.

Beck, U.: Risikogesellschaft. Auf dem Weg in eine andere Moderne. Suhrkamp, Frankfurt a. M. 1986.

Beck, U.; Beck-Gernsheim, E.: Individualisierung in modernen Gesellschaften - Perspektiven und Kontroversen einer subjektorientierten Soziologie. In: Beck, U. und Beck-Gernsheim, E. (Hrsg.): Riskante Freiheiten. Individualisierung in modernen Gesellschaften. Suhrkamp, Frankfurt 1994, S. 10-39.

Becker, J.: Marketing-Konzeption. Grundlagen des zielstrategischen und operativen Marketingmanagements. 8. Aufl., Vahlen, München 2006.

Bednarsky, I.: Individuell statt x-beliebig. In: Gestalten und Verkaufen. Das Ideenmagazin für Einzelhandelsgärtner und Floristen, 20 (2010), Nr. 2, S. 18.

Behr, H.: Produktions- und Wirtschaftstendenzen im Zierpflanzenbau. Spezialausgabe für Meldebetriebe. In: Agrarmarkt Informationsgesellschaft mbh (Hrsg.): Anbauerhebung Herbstausgabe, Heft Nr. 2, Bonn 2010.

Behr, H.: Produktions- und Wirtschaftstendenzen im Zierpflanzenbau. Spezialausgabe für Meldebetriebe. In: Agrarmarkt Informationsgesellschaft mbh (Hrsg.): Anbauerhebung Frühjahr 2011, Heft Nr. 1, Bonn 2011.

Behr, H.: Anbauerhebung Zierpflanzen Frühjahr 2015. Produktions- und Wirtschaftstendenzen im Zierpflanzenbau. Spezialausgabe für Meldebetriebe. In: Agrarmarkt Informationsgesellschaft mbh (Hrsg.): Markt Report, Heft Nr. 1/2015, Bonn 2015.

Behr, H.; Niehues, R.: Markt und Absatz. In: Dirksmeyer, W. (Hrsg.): Status quo und Perspektiven des deutschen Produktionsgartenbaus (Landbauforschung, Sonderheft 330). Braunschweig 2009.

Behringer, L.: Lebensführung als Identitätsarbeit: Der Mensch im Chaos des modernen Alltags. Campus, Frankfurt a. M. 1998.

Berekoven, L.: Erfolgreiches Einzelhandelsmarketing. Grundlagen und Entscheidungshilfen. 2. Aufl., Beck, München 1995.

Berekoven, L. et al: Marktforschung. Methodische Grundlagen und praktische Anwendung. 11. Aufl., Gabler, Wiesbaden 2006.

Bergener, B.: Die EKP-Checkliste: Wegweiser zur Überprüfung des eigenen Fachgeschäfts. In: Florist Ratgeber. Kennzahlen, Erfolg im Blumeneinzelhandel. Haymarket, Braunschweig 2008, S. 89-90.

Berndt, R. et al: Internationales Marketingmanagement. 4. Aufl., Springer, Heidelberg 2010. 
Bundesinstitut für Bevölkerungsforschung: Demografieportal des Bundes und der Länder. Internetabfrage vom 27.11.2015, http://www.demografieortal.de/DE/Informieren/_function/ suche/fakten_formular.html?nn=3182748\&cl2Categories_Format=01_statistiken

Blasius, J.; Winkler, J.: Gibt es die „feinen Unterschiede“? Eine empirische Überprüfung der Bourdieuschen Theorie. Kölner Zeitschrift für Soziologie und Sozialpsychologie, 41 (1998), S. 72-94.

Böhler, H.: Marktforschung. 3. Aufl., Kohlhammer, Stuttgart 2004.

Böhnlein, C.: Vorlesungsunterlagen. Unternehmensmodelle. SS 2005. Kapitel 10. Change Management. Würzburg 2005.

Brachinger, H.; Ost, F.: Modelle mit latenten Variablen. Faktorenanalyse, Latent-StructureAnalyse und LISREL-Analyse. In: Fahrmeir, L. et al. (Hrsg.): Multivariate statistische Verfahren. 2. Aufl., de Gruyter, Berlin 1996, S. 639-659.

Brosius, F.: SPSS 8. Professionelle Statistik unter Windows. mitp, REDLINE GmbH, Heidelberg 2006.

Brosius, F.: SPSS 16. Das mitp-Standardwerk. REDLINE GmbH, Heidelberg 2008.

Bruhn, M.: Internes Marketing als Forschungsgebiet in der Marketingwissenschaft. Eine Einführung in die theoretischen und praktischen Probleme. In: Bruhn, M. (Hrsg.): Internes Marketing. Integration der Kunden- und Mitarbeiterorientierung. Grundlagen, Implementierung, Praxisbeispiele. 2. Aufl., Gabler, Wiesbaden 1999, S. 537-576.

Bruhn, M.: Marketing. Grundlagen für Studium und Praxis. 7. Aufl., Gabler, Wiesbaden 2004.

Bruhn, M.: Kommunikationspolitik. Systematischer Einsatz der Kommunikation für Unternehmen. 3. Aufl., Vahlen, München 2005a.

Bruhn, M.: Unternehmens- und Marketingkommunikation. Handbuch für ein integriertes Kommunikationsmanagement, Vahlen, München 2005b.

Bruns, J.: Identifizierung und Messbarkeit von Marktsegmenten. In: Pepels, W. (Hrsg.) Marktsegmentierung. Erfolgsnischen finden und besetzen. 2. Aufl., Symposium, Düsseldorf 2007.

Bruns, J.: Direktmarketing. 2. Aufl., Kiehl, Ludwigshafen 2007.

Bühl, A.: PASW18. Einführung in die moderne Datenanalyse. 11. Aufl., Pearson, München 2008.

Bühl, A.: PASW18. Einführung in die moderne Datenanalyse. 12. Aufl., Pearson, München 2010. 
Burzan, N.: Soziale Ungleichheit. Eine Einführung in die zentralen Theorien. 2. Aufl., Verlag für Sozialwissenschaften, Wiesbaden 2005.

Byrd-Bredbenner, C.; Maurer Abbot, J.; Cussler, E.: Mothers of young children cluster into 4 groups based on psychographic food decision influencers. In: Nutrition Research, 28 (2008), S. 506-516.

Centrale Marketing-Gesellschaft der deutschen Agrarwirtschaft. Internetabfrage vom 05.12.2009, http://www.cma.de/b2b/business-service/index.html

Diaz-Bone, R.: Milieumodelle und Milieuinstrumente in der Marktforschung. In: Forum Qualitative Sozialforschung. Erstellungsdatum Mai 2004, http:// www.qualitative-research.net/ index.php/fqs/article/view/595/1292

Diaz-Bone, R.: Kulturwelt, Diskurs und Lebensstil. Eine diskurstheoretische Erweiterung der Bourdieuschen Distinktionstheorie. 2. Aufl., Verlag für Sozialwissenschaften, Wiesbaden 2010 .

Diaz-Bone, R.; Künemund, H.: Einführung in die binäre logistische Regression. Mitteilungen aus dem Schwerpunktbereich Methodenlehre, Heft Nr. 56, Berlin 2003.

Diez, W.: Automobilmarketing. Navigationssystem für neue Absatzstrategien. 5. Auflage, Mi-Fachverlag, Landsberg/Lech 2006.

Diller, H.: Preispolitik. 4. Aufl., Kohlhammer, Stuttgart 2008.

Drieseberg, T.: Lebensstil-Forschung. Theoretische Grundlagen und praktische Anwendungen. Physica, Heidelberg 1995.

Ebner, M.: Preispolitik in der Kompositversicherung. Verlag Versicherungswirtschaft, Karlsruhe 2010.

Eckey, H. et al.: Multivariate Statistik. Grundlagen, Methoden, Beispiele. Gabler, Wiesbaden 2002.

EHI Retail Institut e. V.: Bruttoumsatz je Quadratmeter Geschäftsfläche im deutschen Textilfachhandel in den Jahren 2002 bis 2008 in Euro. Internetabfrage 22.11.2010, http://www. handelsdaten.de/statistik/daten/studie/71995/umfrage/textilfachhandel-\%96-bruttoumsatzje-quadratmeter-geschaeftsflaeche-im-deutschen-textilfachhandel/

Elgner, N.: Zehn Methoden zur verträglichen Preisanpassung. In: TASPO. Zeitung für den grünen Markt, 143 (2009), Nr. 16, S. 18.

Enneking, U.; Franz, R.: Lebensstilkonzepte und Nachhaltigkeit. Stand der Forschung und Anwendungsbeispiele. In: TU München (Hrsg.): Consumer Science. Nachhaltiger Konsum und Verbraucherpolitik im 21. Jahrhundert. München 2005. 
Esch, F.-R. et al: Marketing. Eine managementorientierte Einführung. Vahlen, München 2006.

Fazio, R. et al.: Attitude accessibility, attitude behaviour consistency and the strength of the object evaluation. In: Journal of Experimental Social Psychology, 8 (1982), S. 339-357.

Fern, E.: Advanced Focus Group Research. Sage, Thousand Oaks 2001.

Flick, U.: Qualitative Forschung. Theorie, Methoden, Anwendung in Psychologie und Sozialwissenschaften. Ein Handbuch. Rowohlt, Reinbek 1999.

Foscht, T.; Swoboda, W.: Käuferverhalten: Grundlagen - Perspektiven - Anwendungen. 3. Aufl., Gabler, Wiesbaden 2007.

Freimuth, J.: Steuerungslogiken und Veränderungsmanagement. Vortrag am 28.01.2005 in der Vorlesung „Logistik Consulting“ an der Universität Nürnberg.

Freter, H.: Markt- und Kundensegmentierung. Kundenorientierte Markterfassung und bearbeitung. 2. Aufl., Kohlhammer, Stuttgart 2008.

Freud, J.; Götzer, K.: Vom Geschäftsprozess zum Workflow. Ein Leitfaden für die Praxis. Hanser, München 2008.

Frewer, L. et al.: The influence of initial attitudes on responses to communication about genetic engineering in food production. In: Agriculture and Human Values, 15 (1998), S. 1530 .

Friebertshäuser, B.: Interviewtechniken. Ein Überblick. In: Friebertshäuser, B. und Prengel, A. (Hrsg.): Handbuch qualitative Forschungsmethoden in der Erziehungswissenschaft. Juventa, Weinheim 1997.

Gabriel, A.: Kundenzufriedenheit und Kundenorientierung im gärtnerischen Einzelhandel. Marketingtag 2011, Landwirtschaftskammer Schleswig-Holstein, Ellerhoop, 26.06.2011.

Georg, W.: Soziale Lage und Lebensstil. Eine Typologie. Leske + Budrich, Opladen 1998.

Gläser, J.; Laudel, G.: Experteninterviews und qualitative Inhaltsanalyse. 2. Aufl., VS Verlag für Sozialwissenschaften, Wiesbaden 2006.

Götze, W. et al.: Statistik. Oldenbourg, München 2002.

Goldmann, A.; McDonald, S.: The Group Depth Interview. Principles and Practice. Prentice Hall, Englewood Cliffs, 1987.

Günther, H.; Tempelmeier, H.: Produktion und Logistik. 6. Aufl., Springer, Berlin 2005.

Haller, S.: Zielgruppenauswahl für Dienstleister. In: Pepels, W (Hrsg.) Marktsegmentierung. Erfolgsnischen finden und besetzen. 2. Aufl., Symposium, Düsseldorf 2007. 
Hamm, I.: Eine lebensstilistische Segmentierung des Jugendmarktes. Dissertation an Universität Mannheim, Mannheim 2003.

Hartmann, P.: Lebensstilforschung. Darstellung, Kritik und Weiterentwicklung. Leske + Budrich, Opladen 1999.

Hartung, J.; Elpelt, B.: Multivariate Statistik. Lehr- und Handbuch der angewandten Statistik. 6. Aufl., Oldenbourg, München 1999.

Heise, G.: Internationale Marktsegmentierung im Automobilmarketing. Deutscher Universitätsverlag, Wiesbaden 1997.

Hellmann, K.: Soziologie der Marke. Suhrkamp, Frankfurt a. M. 2003.

Herrmann, A.; Huber, F.: Nutzenorientierte Gestaltung der Distributionspolitik. In: Beisheim, O. (Hrsg.): Distribution im Aufbruch. Bestandsaufnahme und Perspektiven. Vahlen, München 1999.

Holland, H.: Direkt-Marketing. 2. Aufl., Hanser, München 2004.

Hölscher, B.: Lebensstile durch Werbung? Zur Soziologie der Life-Style-Werbung. VS Verlag für Sozialwissenschaften, Wiesbaden 1998.

Homburg, C.; Krohmer, H.: Marketingmanagement. Strategie, Instrumente, Umsetzung, Unternehmensführung. 2. Aufl., Gabler, Wiesbaden 2006.

Hradil, S.: Sozialstrukturanalyse in einer fortgeschrittenen Gesellschaft. Von Klassen und Schichten zu Lagen und Milieus. Leske + Budrich, Opladen 1987.

Hradil, S.: Soziale Ungleichheit in Deutschland. 7. Aufl., Leske + Budrich, Opladen 1999.

Hungenberg, H.: Strategisches Management in Unternehmen. Ziele, Prozesse, Verfahren. 3. Aufl., Gabler, Wiesbaden 2004.

Hüttner, M.; Schwarting, U.: Grundzüge der Marktforschung. 7. Aufl., Oldenbourg, München 2002.

IBH Retail Consults GmbH, Köln: Wareneinsatz, Materialbestand und Lagerumschlag im Einzelhandel nach Branchen. Informationsabfrage am 12.11.2010, http://www.handels wissen.de/data/almanach/Einzelhandelsstrukturen/Betriebswirtschaftliche_Kennziffern/Wa reneinsatz_Materialbestand_Lagerumschlag_Einzelhandel_Branchen.php

Institut für Handelsforschung, Köln: Begriffsdefinitionen aus der Handels- und Absatzwirtschaft, Jahrgang 1995. Informationsabfrage am 29.11.2010, http://www.ifhkoeln.de/ katalog_e_-_begriffsdefinitionen_aus_der_handels-_835901.php

Institut für Handelsforschung, Köln: Personalkosten im Facheinzelhandel - große Bandbreite zwischen Branchen und unterschiedliche Entwicklungen im Zeitablauf. Veröffentlichungs- 
datum: 12.03.2008, http://www.ifhkoeln.de/personalkosten_im_facheinzelhandel_eo_ grosse.php

Jensen, O.: Clusteranalyse. In: Herrmann A. et al. (Hrsg.): Handbuch Marktforschung. Methoden, Anwendungen, Praxisbeispiele. 3. Aufl., Gabler, Wiesbaden 2008, S. 335-372.

Karasu, M.: Marketing für Kundengewinnung und Kundenbindung. Am Beispiel eines Sozialunternehmens. Diplomica, Hamburg 2010.

Keegan, W. et al.: Globales Marketing Management. Eine europäische Perspektive. Oldenbourg, München 2002.

Klocke, A.; Luck D.: Lebensstile in der Familie. Staatsinstitut für Familienforschung an der Universität Bamberg, Bamberg 2001.

Knapp, N.: Konsumentenwünsche und Lebenswelten. Profilierungsstrategien für den grünen Markt. Bloom's, Ratingen 2008.

Koch, J.: Die Marktsegmentierung als Ansatz zur Modellierung von (unbeobachtetem) heterogenem Konsumentenverhalten unter Verwendung von finiten Mischungsmodellen. Empirisches Beispiel und Simulation. Peter Lang, Frankfurt 2006.

Köcher, R.: Konsum und Mediennutzen in der multioptionalen Gesellschaft, 2006. Internetabfrage am 09.01.2011, http://awa-online.de/

Köhler, R.: Beiträge zum Marketing-Management. Planung, Organisation, Controlling. 3. Aufl., Poeschel, Stuttgart 1993.

Kohrmann, O.: Mehrstufige Marktsegmentierung zur Neukundenakquisition am Beispiel der Telekommunikation. Gabler, Wiesbaden 2003.

Koschnick, W.: Von der Poesie der schönen Namensgebung. Glanz und Elend von LifestyleTypologien. In: Focus-Jahrbuch, München 2006, S. 43-96.

Kotler, P. et al.: Marketing-Management. Strategien für wertschaffendes Handeln. 10. Aufl., Pearson, München 2006.

Kotler, P. et al.: Marketing-Management. Strategien für wertschaffendes Handeln. 12. Aufl., Pearson, München 2007a.

Kotler, P. et al: Grundlagen des Marketing. 4. Aufl., Pearson, München 2007b.

Kotler, P. et al: Grundlagen des Marketing. 5. Aufl., Pearson, München 2011.

Kozlova, O.: Selbstkonzepttheorie. Interdependenz zwischen Persönlichkeit (vor allem Selbstbild), Lebensstil und Konsum. Grin, Norderstedt 2008.

Krais, B.; Gebauer, G.: Habitus. Themen der Soziologie. Transcript, Bielefeld 2002. 
Kreimer, T.; Gerling, M.: Status quo und Perspektiven im deutschen Lebensmitteleinzelhandel 2006. Hrsg.: KPMG AG, Köln. Internetabfrage am 19.11.2010, http://www.kpmg.de/ docs/status_quo_und_perspektiven_im_deutschen_Lebensmitteleinzelhandel_2006_de.pdf

Kroeber-Riel, W.; Weinberg, P.: Konsumentenverhalten. 6. Aufl., Pearson, München 1996.

Kroeber-Riel, W.; Weinberg, P.: Konsumentenverhalten. 8. Aufl., Pearson, München 2003.

Kuss, A.: Marktforschung. Grundlagen der Datenerhebung und Datenanalyse. 3. Aufl., Gabler, Wiesbaden 2010.

Kuß, A.; Tomczak, T.: Marketingplanung. Einführung in die marktorientierte Unternehmensund Geschäftsfeldplanung. Gabler, Wiesbaden 2002.

Kuß, A.; Tomczak, T.: Käuferverhalten. Eine marketingorientierte Einführung. 3. Aufl., UTB, Stuttgart 2004.

Lamnek, S.: Qualitative Sozialforschung. 4. Aufl., Beltz, Weinheim 2005.

Landgard, „Ländergesellschaften“. Internetabfrage am 03.05.2011, https://www.landgard.de/ index.php?id=409\&L=1

Lange, D.: Betriebswirtschaftliche Situation im Produktionsgartenbau. In: Dirksmeyer, W. (Hrsg.): Status quo und Perspektiven des deutschen Produktionsgartenbaus (Landbauforschung, Sonderheft 330). Braunschweig 2009.

Lenz, B.: Verkettete Orte: Filières in der Blumen- und Zierpflanzenproduktion. LIT, Münster 2005.

Lerchenmüller, M.: Handelsbetriebslehre. 4. Aufl., Kiehl, Ludwigshafen 2003.

Lüdtke, H.: Expressive Ungleichheit. Zur Soziologie der Lebensstile. Leske + Budrich, Opladen 1989.

Lüdtke, H.: Lebensstile als Dimension handlungsproduzierter Ungleichheit. In: Berger, P. und Hradil, S. (Hrsg.): Lebenslagen, Lebensläufe, Lebensstile. Schwartz, Göttingen 1990.

Mayer, H.: Interview und schriftliche Befragung. Entwicklung, Durchführung und Auswertung. 3. Aufl., Oldenbourg, München 2006.

Mayring, P.: Einführung in die qualitative Sozialforschung. Beltz, Weinheim 1999.

Meffert, H.: Marketing. Grundlagen marktorientierter Unternehmensführung. Konzepte, Instrumente, Praxisbeispiele. 9. Aufl., Gabler, Wiesbaden 2000.

Meffert, M.; Bruhn, M.: Dienstleistungsmarketing. Grundlagen, Konzepte, Methoden. Mit Fallstudien. 5. Aufl., Gabler, Wiesbaden 2006.

Meffert, H. et al.: Marketing. Grundlagen marktorientierter Unternehmensführung. Konzepte - Instrumente - Praxisbeispiele. 10. Aufl., Gabler, Wiesbaden 2008. 
Meuser, M.; Nagel, U.: Das Experteninterview. Wissenssoziologische Voraussetzungen und methodische Durchführung. In: Friebertshäuser, B. und Prengel, A. (Hrsg.): Handbuch qualitative Forschungsmethoden in der Erziehungswissenschaft. Juventa, Weinheim 1997, S. $481-491$.

Michaelis, M.: Internes Marketing in Dienstleistungsnetzwerken. Konzeption und Erfolgsmessung. Gabler, Wiesbaden 2009.

Michman, R.: Lifestyle Market Segmentation. Praeger Frederick, London 1991.

Mitchell, A.: The Nine American Lifestyles. Who we are and where we're going. Macmillian Pub Co., New York 1983.

Müller, H.: Sozialstruktur und Lebensstile. Der neuere theoretische Diskurs über soziale Ungleichheit. Suhrkamp, Frankfurt 1992.

Müller-Hagedorn, L.; Schuckel, M.: Einführung in das Marketing. 3. Aufl., SchäfferPoeschel, Stuttgart 2003.

Müller-Schneider, T.: Schichten und Erlebnismilieus. Der Wandel der Milieustruktur in der Bundesrepublik Deutschland. Deutscher Universitätsverlag, Wiesbaden 1994.

Niehues, R.: Aktuelle Daten im Blumen- und Pflanzenmarkt. Zukunft Marktdaten. Vortrag auf Beratertagung am 12.05.2009 bei ZMP GmbH, Abteilung Gartenbau, Rochusstraße 2, 53123 Bonn.

Nielsen Company, Frankfurt a. M.: Informationsabfrage am 09.01.2011, http://www.nielsenmedia.de/pages/template.aspx?level=2\&treeViewID=3.103.0.0.0

Nolte, K.: Lebensstil und Markenbild: eine konsumsoziologische Perspektive. Cuvillier, Göttingen 2005 .

Oelsnitz, D. von der: Marktorientierte Organisationsgestaltung. Eine Einführung. Kohlhammer, Stuttgart 2000.

Otte, G.: Auf der Suche nach „,neuen sozialen Formationen und Identitäten“ -Soziale Integration durch Klassen oder Lebensstile? In: Friedrichs, J. (Hrsg.): Die IndividualisierungsThese. Leske + Budrich, Opladen 1998, S. 181-220.

Otte, G.: Sozialstrukturanalysen mit Lebensstilen. Eine Studie zur theoretischen und methodischen Neuorientierung der Lebensstilforschung. 2. Aufl., Verlag für Sozialwissenschaften, Wiesbaden 2008.

o. V.: Gartenbau in der Bundesrepublik Deutschland. Informationsabfrage am 03.12.2010, http://www.g-net.de/content/branche/daten_zierpflanzenbau.php

o. V.: KommunikationsAnalyse 2012. Frauen in Deutschland: Einstellungen, Marken, Medien. Gruner + Jahr, Hamburg 2012. 
o. V.: Was konsumierst du? Basisinformationen für fundierte Mediaentscheidungen. Verbrauchs- und Medienanalyse 2013.

Papastefanou, G.: Variatio delectat? - Verbreitung und sozialstrukturelle Differenzierung der Konsumvariabilität. In: Jäckel, M. (Hrsg.): Ambivalenzen des Konsums und der werblichen Kommunikation. Verlag für Sozialwissenschaften, Wiesbaden 2007, S. 235-260.

Piller, F.: Mass Customization. Ein wettbewerbsstrategisches Konzept im Informationszeitalter. 4. Aufl., Gabler, Wiesbaden 2006.

Pirner, M.: Best Ager als Zielgruppe für den Lebensmitteleinzelhandel. Diplomica, Hamburg 2010.

Raab, G.; Unger, F.: Marktpsychologie. Gabler, Wiesbaden 2005.

Reeb, M.: Lebensstilanalysen mit der strategischen Marktforschung. Deutscher Universitätsverlag, Wiesbaden 1998.

Reinelt, J.; Fauconnier, C.: Mining for Meaning. Segmentierung mit Relevanz. In: Planung und Analyse, 32 (2005), Nr. 4, S. 40-44.

Rennhak, C.; Kesting, T.: Marktsegmentierung in der deutschen Unternehmenspraxis. Gabler, Wiesbaden 2008.

Reusswig, F.: Lebensstile und Naturorientierungen. Gesellschaftliche Naturbilder und Einstellungen zum Umweltschutz. In: Rink, D. (Hrsg.): Lebensstile und Nachhaltigkeit. Konzepte, Befunde und Potentiale. Leske + Budrich, Opladen 2002.

Reuter, M.; Blees, T.: Trends im Handel 2010. Hrsg.: KPMG AG, Köln. Veröffentlichungsdatum 12.02.2006, http://www.kpmg.de/Presse/3049.htm

Reutterer, T.: Bestandsaufnahme und aktuelle Entwicklungen bei der Segmentierungsanalyse von Produktmärkten. In: Journal für Betriebswirtschaft, 53 (2003), Nr. 2, S. 52-74.

Richter, R.: Die Lebensstilgesellschaft. Verlag für Sozialwissenschaften. Wiesbaden 2005.

Roslow, S. et al.: Impact of situational variables and demographic attributes in two seasons on purchase behavior. In: European Journal of Marketing, 34 (2000), Nr. 9, S. 1167-1180.

Salcher, E.: Psychologische Marktforschung. De Gruyter, Berlin 1995.

Scherer, C.; Cho, H.: A social network contagion theory of risk perception. In: Risk Analysis, 23 (2003), S. 261-267.

Schendra, C.: Clusteranalyse mit SPSS. Mit Faktorenanalyse. Oldenbourg, München 2010.

Scheer, B.: Nutzenbasierte Marktsegmentierung. Eine kaufprozessorientierte empirische Untersuchung zur Wirkungsmessung von Marketing-Aktivitäten. Gabler, Wiesbaden 2008. 
Schmidt, P. et al.: Die Messung von Werten mit dem „Portraits Value Questionaire“. In: Zeitschrift für Sozialpsychologie, 38 (2007), Nr. 4, S. 261-275.

Scholl, G.; Hage, M.: Lebensstile, Lebensführung und Nachhaltigkeit (Schriftenreihe des Instituts für ökologische Wirtschaftsforschung (IÖW), Band 176). IÖW, Berlin 2004.

Schulze, G.: Die Erlebnisgesellschaft. Kultursoziologie der Gegenwart. Campus, Frankfurt a. M. 2000.

Schulze, G.: Die Erlebnisgesellschaft. Kultursoziologie der Gegenwart. 2. Aufl., Campus, Frankfurt a. M. 2005.

Schwartz, S.: A theory of cultural values and some implications for work. In: Applied Psychology: An Internal Review, 48 (1999), S. 23-47.

Seyffert, R.: Wirtschaftslehre des Handels. Hrsg.: Sundhoff, E. 5. Aufl., Leske + Budrich, Opladen 1972.

Silberer, G.: Die Stimmung als Werbewirkungsfaktor. In: Marketing ZFP, 21 (1999), Nr. 2, S. 131-148.

Simmel, G.: Philosophie des Geldes. Internetabfrage vom 15.06.2010, http://www.digbib.org/ Georg_Simmel_1858/Philosophie_des_Geldes

Sinus Sociovision GmbH: Sinus Milieus - Deutschland. Internetabfrage vom 22.06.2010a, http://www.sinus-sociovision.de

Sinus Sociovision GmbH: Die Sinus-Milieus. Internetabfrage vom 14.06.2010b, http://www. sociovision.de/uploads/tx_mpdownloadcenter/infoblatt_d_2009_01.pdf

Skiera, B.: Preisdifferenzierung. In: Albers, S. et al. (Hrsg.): Marketing mit interaktiven Medien. Strategien zum Markterfolg. 3. Aufl., Frankfurter Allgemeine Buch, Frankfurt a. M. 2001, S. 267-281.

Solomon M. et. al.: Consumer Behaviour. A European Perspective. 3. Aufl., Prentice Hall, New Jersey 2006.

Statistisches Bundesamt Deutschland. Internetabfrage vom 20.07.2009, https://www.destatis. de/DE/ZahlenFakten/GesamtwirtschaftUmwelt/Preise/Verbraucherpreisindizes/Verbrauche rpreisindizes.html;jsessionid=70EF8D3D79FF338E09874C8A7C9B500C.cae4\#Tabellen

Statistisches Bundesamt Deutschland. Internetabfrage vom 10.06.2015, https://www-gensis. destatis.de/genesis/online;jsessionid=91A5970EBD99F1096EF6470E0C5F132A.tomcat_ GO_2_1?operation=previous \&levelindex $=3 \&$ levelid $=1433930542421 \&$ step $=3$

Steimer, I.: Milieuspezifisches Marketing für Biolebensmittel. Lebensstil als Grundlage der Marktsegmentierung. Dr. Müller Verlag, Frankfurt a. M. 2006. 
Stihler, A.: Ausgewählte Konzepte der Sozialpsychologie zur Erklärung des modernen Konsumverhaltens. In: Rosenkranz, D. (Hrsg.): Konsum. Soziologische, ökonomische und psychologische Aspekte. Leske + Budrich, Opladen 2000, S. 169-187.

Stöger, R.: Prozessmanagement. Qualität, Produktivität, Konkurrenzfähigkeit. 2. Aufl., Schäffer-Poeschel, Stuttgart 2009.

Stuhldreier, U.: Mehrstufige Marktsegmentierung im Bankmarketing. Ein Erfolgsfaktor für das Privatkundengeschäft. DUV, Wiesbaden 2002.

Tenbült, P. et al.: Intuitive and explict reactions towards new food technologies. Attitude strength and familiarity. In: British Food Journal, 110 (2008), S. 622-635.

Trommsdorff, V.: Konsumentenverhalten. 3. Aufl., Kohlhammer, Stuttgart 1998.

Trommsdorff, V.; Teichert, T.: Konsumentenverhalten. 8. Aufl., Kohlhammer, Stuttgart 2011.

Urban, D.: LOGIT-Analyse. Statistische Verfahren zur Analyse von Modellen mit qualitativen Response-Variablen. Gustav-Fischer-Verlag, Stuttgart 1993.

Vester, M. et al.: Soziale Milieus im gesellschaftlichen Strukturwandel. Zwischen Integration und Ausgrenzung. Suhrkamp, Frankfurt a. M. 2001.

Voeth, M.: Gruppengütermarketing (Vahlens Handbücher der Wirtschafts- und Sozialwissenschaften). Vahlen, München 2003.

Wahl, A.: Lebensstile im Kontext von Generationen, Lebenslauf- und Zeitgeisteinflüssen. Hochschulschrift, TU Berlin, Berlin 2000.

Wannenwetsch, H.; Nicolai, S.: E-Supply-Chain-Management. Grundlagen, Strategien, Praxisanwendungen. 2. Aufl., Gabler, Wiesbaden 2004.

Wedel, M.; Kamakura, W.: Market Segmentation - Conceptual and Methodological Foundations, 2. Aufl., Springer, Boston 2003.

Weinstein, A.: Handbook of Market Segmentation - Strategic Targeting for Business and Technology Firms. 3. Aufl., Routledge, New York 2004.

Weis, C.: Marktforschung. 7. Aufl., Kiehl, Ludwigshafen 2008.

Weis, C.: Marketing. 15. Aufl., Kiehl, Ludwigshafen 2009.

Weißbacher, R.: Nachfragebündelungen als Marketinginstrument. Gabler, Wiesbaden 2006.

Wells, W.; Tigert, D.: Activities, Interests and Opinions. In: Journal of Advertising Research, 11 (1971), Nr. 4, S. 27-35.

Wirtz, B.: Integriertes Direktmarketing. Grundlagen, Instrumente, Prozesse. Gabler, Wiesbaden 2005. 
Wiswede, G.: Konsumsoziologie - Eine vergessene Disziplin. In: Rosenkranz, D. (Hrsg.): Konsum. Soziologische, ökonomische und psychologische Aspekte. Leske + Budrich, Opladen 2000, S. 24-72.

Witt, H.: Forschungsstrategien bei quantitativer und qualitativer Sozialforschung [36 Absätze]. Forum Qualitative Sozialforschung / Forum Qualitative Social Research, 2 (1), Art. 8, 2001. Internetabfrage vom 06.04.2014, http://nbn-resolving.de/urn:nbn:de:0114-fqs010189

Wöhe, G.: Einführung in die allgemeine Betriebswirtschaftslehre. 19. Aufl., Vahlen, München 1996.

Wöhe, G.: Einführung in die allgemeine Betriebswirtschaftslehre. 22. Aufl., Vahlen, München 2005.

Zentrale Markt- und Preisberichtstelle für Land-, Forst und Ernährungswirtschaft. Internetabfrage vom 05.12.2009, http://www.zmp.de/info/orientierung.asp 


\section{Anhang}

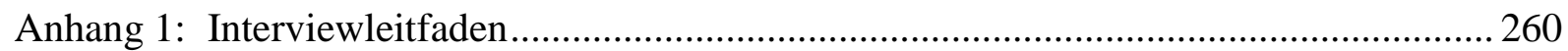

Anhang 2: Anmerkungen und Ergebnisse der Pretests ................................................ 265

Anhang 3: Alle für die Lebensstilanalyse berücksichtigten Dimensionen der Items im

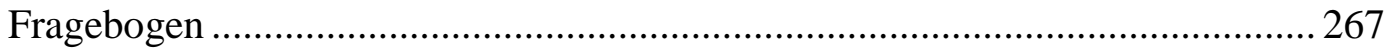

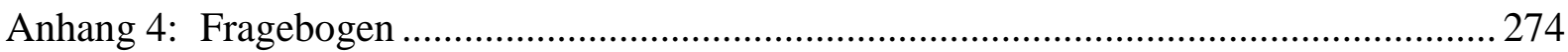

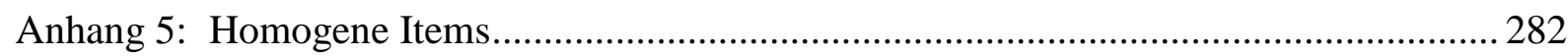




\title{
Anhang 1: Interviewleitfaden
}

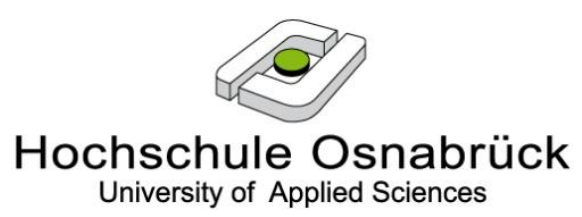

\author{
Dipl.-Kfm. Stefan von Peterffy \& Prof. Dr. Ulrich Enneking \\ Hochschule Osnabrück \\ Fakultät Agrarwissenschaften \& Landschaftsarchitektur \\ s.vonpeterffy@stud.uni-goettingen.de \\ Tel.: $0171 / 5788508$
}

\section{Lebensstilbasierte Kundensegmentierung im Blumen- und Pflanzeneinzelhandel \\ Leitfaden zum explorativen Interview mit Konsumenten und Konsumentinnen von Blumen und/oder Pflanzen}

Zielsetzung des Forschungsvorhabens ist es zu ermitteln, ob bzw. welche verschiedenen Käufergruppen es für Schnittblumen und/oder Topfpflanzen gibt. Aus diesen Erkenntnissen sollen MarketingEmpfehlungen für Einzelhändler von Blumen und/oder Pflanzen abgeleitet werden, an deren Ende ein besser auf den Kunden abgestimmtes Angebot steht.

Vorgehensweise im Rahmen des Interviews: Ich werde Ihnen im Laufe unseres Gespräches Fragen stellen und mir Notizen zu Ihren Antworten machen. Leider ist es mir nicht möglich, die Antworten vollständig mitzuschreiben. Aus diesem Grund möchte ich gerne ein Tonbandgerät mitlaufen lassen, das unser Gespräch aufzeichnet. Nach unserem Gespräch werde ich mich wahrscheinlich nicht mehr an jede Einzelheit erinnern können. Die Aufnahme ermöglicht es mir, im Zweifel noch einmal nachzuhören, ob ich alles richtig verstanden habe. Selbstverständlich werden der Inhalt unseres Gesprächs sowie alle anderen Informationen, die ich von Ihnen erhalte, vertraulich behandelt sowie ausschließlich zu Forschungszwecken und nur in anonymisierter Form verwandt. Stimmen Sie unter diesen Bedingungen der Aufzeichnung unseres Gespräches zu?

Für unser Interview unterscheide ich zwischen Schnittblumen und Topfpflanzen. Mit „Schnittblumen“ sollen auch sämtliche floristischen Arrangements wie Gestecke zu Allerheiligen oder Advent abgedeckt sein und auch Bundware, wie beispielsweise zehn Stück Tulpen- oder Rosenstiele ohne Dekoration, die typischerweise in vielen Supermärkten verkauft werden. Mit „Topfpflanzen“ sollen alle Pflanzen gemeint sein, die im Topf verkauft werden, also sämtliche blühenden und grünen Zimmerpflanzen als auch sämtliche Pflanzen für draußen bzw. für den Garten und/oder den Balkon. 
Einstieg: Wenn Sie an sich und Ihr zu Hause bzw. Ihren Garten/Balkon denken: Was für eine Rolle spielen da für Sie Blumen und Pflanzen? An welchen Blumen oder Pflanzen haben Sie die größte Freude? Wie pflegen Sie Ihre Schnittblumen oder Topfpflanzen?

Wenn Sie an Ihre Wohnung denken: Welche Rolle spielen Blumen oder Pflanzen bei deren Einrichtung für Sie? Wie spielt das Thema „Raumklima“ dabei eine Rolle dabei?

Eines Tages ist ja wirklich jeder Mensch davon betroffen: Wenn Sie an Gräber und deren Pflege denken, wie denken Sie über bepflanzte Gräber und über Gräber mit Steinplatte?

Emotionale Einstellung zu Blumen und Pflanzen: Wenn Sie an Blumen oder Topfpflanzen denken: Was sind die ersten Gedanken, die Ihnen da ganz spontan dazu einfallen? Was denken Sie, wieso sich manche Leute mehr an Schnittblumen und andere Leute mehr an Topfpflanzen erfreuen?

Reale Kaufsituation: Versuchen Sie sich doch an das letzte Mal zu erinnern, als Sie Schnittblumen oder Topfpflanzen gekauft haben. Erzählen Sie mir von Ihrem Kauf - wie lief der Kauf ab?

- Was haben Sie gekauft?

- Da gab es sicher mehrere Alternativen zur Auswahl. Wieso haben Sie sich für Ihre entschieden?

- Beschreiben Sie doch einmal die Art des Geschäftes.

- Haben Sie sich dabei beraten lassen?

- Für wen haben Sie das gekauft?

Informationsverhalten: Wie informieren Sie sich vor einem Kauf über das aktuelle Angebot von Schnittblumen oder Topfpflanzen?

Einkaufsstättenwahl: Wenn Sie einmal so darüber nachdenken, wo man überall Schnittblumen oder Pflanzen kaufen kann: Wie unterscheiden sich diese verschiedenen Einkaufsstätten voneinander?

- Wo kaufen Sie für gewöhnlich Ihre Schnittblumen oder Topfpflanzen?

- Wieso ist das für Sie ihre bevorzugte Einkaufsstätte für Schnittblumen oder Topfpflanzen?

- Gibt es verschiedene Qualitäten bei Schnittblumen oder Topfpflanzen?

Preis: Stellen Sie sich doch einmal vor, Sie sind unterwegs und haben gar nicht vor, eine Topfpflanze oder Schnittblumen zu kaufen, und trotzdem kommen Sie mit einer Topfpflanze oder mit Schnittblumen nach Hause. Was könnte Sie so spontan überzeugt haben, doch zuzugreifen?

- Wie beeinflusst der Preis Ihre Entscheidung, ob Sie kaufen oder nicht? 
Motive: Viele Schnittblumen oder Topfpflanzen haben eine konkrete Funktion, z. B. frische Topfkräuter in der Küche, Pflanzen mit Duftstoffen gegen Moskitos im Sommer, Lavendel schützen Rosen vor Läusen, Flieder lockt Schmetterlinge an, manche Menschen entspannen beim Bonsaischneiden.

- Inwieweit spielen für Sie Nutzen von Schnittblumen oder Topfpflanzen eine Rolle?

- Wie ändert sich diese Einstellung, wenn Sie Schnittblumen oder Topfpflanzen zum Verschenken kaufen?

In Supermärkten wie Edeka oder Rewe bekommt man nahezu alles für den täglichen Gebrauch. Heutzutage siedeln sich immer mehr Geschäfte in direkter Nachbarschaft solcher Supermärkte an, z. B. Fotogeschäfte, Reinigungen, Bäckereien, Schlüsseldienste usw.

- Wie gefällt Ihnen die Idee, dass Sie an einem Ort so viele verschiedene Dinge auf einmal erledigen können?

- Welche Fernsehsendungen schauen Sie regelmäßig?

- Welche Radiosender hören Sie?

- Welche Zeitschriften lesen Sie?

- Wozu nutzen Sie das Internet?

- Was denken Sie über Bio-Produkte?

- Gehen Sie gerne ins Theater / in die Oper / ins Kino / ins Museum / zu Konzerten?

Zeitkriterien: Gibt es besondere Jahreszeiten oder bestimmte Anlässe, zu denen Sie regelmäßig Schnittblumen oder Topfpflanzen kaufen (z. B zu bestimmten Feiertagen, zu Geburtstagen, für Besuche ...)?

Soziologische Kriterien / Kompetenz: Welchen Beruf haben Sie erlernt bzw. üben Sie aus? Welche Hobbys haben Sie?

Physiologische Kriterien: Haben Sie irgendwelche körperlichen Einschränkungen oder Beschwerden, die sich auf die Gartenarbeit auswirken, z. B. Allergien auf bestimmte Blumen o. Ä.?

Fragen von Seiten des Interviewpartners: Haben Sie noch eine Frage an mich? Gibt es noch etwas, das Sie in diesem Zusammenhang für wichtig erachten, worüber wir aber noch nicht gesprochen haben? 


\section{Kurzfragebogen:}

Im Anschluss an unser Gespräch möchte ich Sie um einige allgemeinere Angaben zu Ihrer Person bitten. Bitte füllen Sie möglichst alle Fragen aus! Auch diese Informationen werden wir lediglich in anonymisierter Form statistisch verwenden.

1. In welchem Jahr sind Sie geboren? (Jahr)

2. Welchen höchsten Schulabschluss haben Sie?

\section{$6 \square$ Noch Schüler}

$5 \square$ Volks-/Hauptschulabschluss

$4 \square$ Mittlere Reife / Realschulabschluss

$3 \square$ Abitur oder Vergleichbares

$2 \square$ Meister/Techniker/Fachschulabschluss

$1 \square$ Fachhochschul-/Hochschulabschluss

$0 \square$ Ohne Abschluss

Sonstiges und zwar:

3. Sie sind zurzeit?

$\begin{array}{ll}5 \square \text { Vollerwerbstätig } & 2 \square \text { Hausfrau/Hausmann } \\ 4 \square \text { Teilzeiterwerbstätig } & 1 \square \text { Rentner/Pensionär } \\ 3 \square \text { Student/Studentin } & 0 \square \text { Erwerbslos }\end{array}$

4. Wie viele Personen gehören insgesamt zu Ihrem Haushalt? Personen

5. Wie hoch ist Ihr verfügbares Haushaltseinkommen?

$\begin{array}{lll}0 \square \text { keine Angabe } & 3 \square 900-1.300 € & 6 \square 2.000-2.600 € \\ 1 \square<500 € & 4 \square 1.300-1.700 € & 7 \square 2.600-4.500 € \\ 2 \square 500-900 € & 5 \square 1.700-2.000 € & 8 \square>4.500 €\end{array}$

6. Wie groß ist Ihr Garten / Ihr Balkon (Nichtzutreffendes bitte streichen)? $\mathrm{m}^{2}$

7. Wie viel Zeit verbringen Sie durchschnittlich pro Woche mit Gartenarbeit? Stunden

8. Wie groß ist Ihre Wohnung / Ihr Haus (Nichtzutreffendes bitte streichen)? $\mathrm{m}^{2}$

9. Falls Sie ein Auto besitzen: Nennen Sie bitte die Automarke: 


\section{Postskriptum:}

Ort, Datum \& Uhrzeit:

Kleidungsstil:

Sonstige Anmerkungen / Eindrücke / nonverbale Reaktionen: 


\section{Anhang 2: Anmerkungen und Ergebnisse der Pretests}

\section{Änderungen im Fragebogen vor quantitativem Pretest (aus qualitativen Pretests):}

- Zeit: 10-10-13-14-16-13-14 min

- Frage 2: „häufiger“ statt „mehr“.

- Frage 4: Option 9 gestrichen, „Mehrfachnennungen“ eingefügt. Probanden konnten sich sonst nicht festlegen.

- Frage 9: „Gartenrose“ statt „Rose“; falls kein Kauf erfolgt, „,weiter zu Frage 11“ eingefügt.

- Frage 10: „Gartenrose“ statt „Rose“, Umformulierung in Warum-Frage.

- Frage 14: „Gartenrose“ statt „Rose“.

- Frage 25: Einleitungssatz hinzugefügt, um Probanden Thema zu umreißen.

- Frage 26: Kommafehler, „bitte vergeben Sie jede Zahl nur einmal“ eingefügt.

\section{Weitere Änderungen aus quantitativem Pretest:}

- Durchschnittszeit der Bearbeitung Fragebogen bei quantitativem Pretest: 15 Minuten. Ziel: 10-12 Minuten Bearbeitungszeit.

- Begrüßungsansprache verkürzt.

- Frage 3: Ein Item gestrichen. Ergebnis differenzierte nicht.

- Frage 5: „Sonstiges, und zwar " gestrichen.

- Frage 6: Antwortalternative „weiß nicht“" gestrichen.

- Frage 7: Aus zwei Items „Zeitschriften“ und „Bücher“ ein Item mit „Zeitschriften/Bücher“ gemacht.

- Frage 8: Ein Item gestrichen. Ergebnis differenzierte nicht.

- Frage 10: Ein Item gestrichen. Ergebnis differenzierte nicht.

- Frage 11: Als geschlossene Frage formuliert. War zuvor offen formuliert.

- Frage 13: „Plastiktopf“ anstatt Container.

- Frage 21: Abfrage nur auf BuP-Ausgaben allgemein bezogen. Abfrage nach Ausgaben für Beetund Balkonpflanzen gestrichen. Probanden können nicht unterscheiden.

- Frage 22: „Entspannung und Ruhe“ anstatt „Entspannung und Erholung“

- Frage 22: „Geselligkeit und Feiern“ anstatt „Geselligkeit und Freizeit“" 
- Frage 22: Ein Item gestrichen. Redundante Abfrage.

- Frage 24: „Die Rose ist die Königin der Blumen“ anstatt „Rosen sind für mich die Königin der Blumen“.

- Frage 24: Vier Items gestrichen. Differenzierten nicht. Gestrichene Items mit Faktorenanalyse über alle Items aus Frage 26 identifiziert.

- Frage 32: Item 4 Tausendertrennpunkt hinzugefügt. 


\section{Anhang 3: Alle für die Lebensstilanalyse berücksichtigten Dimensionen der Items im Fragebogen}

\begin{tabular}{|c|c|c|}
\hline Frage & Antwortskala & Dimension \\
\hline $\begin{array}{l}\text { F1: Haben Sie in den vergangenen } 12 \text { Monaten } \\
\text { Blumen oder Pflanzen gekauft? }\end{array}$ & Antwortmöglichkeit: ja oder nein & Beobachtbares Kaufverhalten \\
\hline $\begin{array}{l}\text { F2:Blumen und Pflanzen kann man sich selbst kau- } \\
\text { fen, aber auch verschenken. Bitte geben Sie an, was } \\
\text { bei Ihnen mehrheitlich zutrifft! }\end{array}$ & $\begin{array}{l}\text { 5-stufig: deutlich häufiger für } \\
\text { mich selbst - deutlich häufiger } \\
\text { als Geschenk }\end{array}$ & Beobachtbares Kaufverhalten \\
\hline \multicolumn{3}{|l|}{$\begin{array}{l}\text { F3: Bitte geben Sie an, wie Sie die folgenden Aussa- } \\
\text { gen bewerten! } \\
\text { Ich kaufe gerne Blumen und Pflanzen als Ge- } \\
\text { schenk, weil ... }\end{array}$} \\
\hline $\begin{array}{l}\text {... man die Möglichkeit hat, mit außergewöhnli- } \\
\text { chen Produkten besonders zu beeindrucken. }\end{array}$ & $\begin{array}{l}\text { 5-stufig: stimme voll zu - stim- } \\
\text { me nicht zu }\end{array}$ & Performanz \\
\hline $\begin{array}{l}\text {... ich ein schönes Geschenk habe, ohne jedes Mal } \\
\text { wieder kreativ sein zu müssen. }\end{array}$ & $\begin{array}{l}\text { 5-stufig: stimme voll } \mathrm{zu} \text { - stim- } \\
\text { me nicht } \mathrm{zu}\end{array}$ & Performanz \\
\hline ... man es noch auf die Schnelle besorgen kann. & $\begin{array}{l}\text { 5-stufig: stimme voll zu - stim- } \\
\text { me nicht } \mathrm{zu}\end{array}$ & Performanz \\
\hline \multicolumn{3}{|l|}{$\begin{array}{l}\text { F4: Wo haben Sie persönlich in den letzten zwölf } \\
\text { Monaten Pflanzen und Gartenbedarf gekauft? }\end{array}$} \\
\hline Baumarkt & Antwortmöglichkeit: ja oder nein & Beobachtbares Kaufverhalten \\
\hline Gartencenter & Antwortmöglichkeit: ja oder nein & Beobachtbares Kaufverhalten \\
\hline Supermarkt & Antwortmöglichkeit: ja oder nein & Beobachtbares Kaufverhalten \\
\hline Discounter & Antwortmöglichkeit: ja oder nein & Beobachtbares Kaufverhalten \\
\hline Gärtnerei & Antwortmöglichkeit: ja oder nein & Beobachtbares Kaufverhalten \\
\hline Blumenfachgeschäft & Antwortmöglichkeit: ja oder nein & Beobachtbares Kaufverhalten \\
\hline Wochenmarkt & Antwortmöglichkeit: ja oder nein & Beobachtbares Kaufverhalten \\
\hline Baumschule & Antwortmöglichkeit: ja oder nein & Beobachtbares Kaufverhalten \\
\hline \multicolumn{3}{|l|}{$\begin{array}{l}\text { F5: Welche drei Aspekte sind bei der Wahl der } \\
\text { Einkaufsstätte für Sie am wichtigsten, wenn Sie } \\
\text { BuP kaufen möchten? }\end{array}$} \\
\hline große Auswahl & Antwortmöglichkeit: ja oder nein & Beobachtbares Kaufverhalten \\
\hline Qualität der BuP & Antwortmöglichkeit: ja oder nein & Beobachtbares Kaufverhalten \\
\hline freundliche Mitarbeiter & Antwortmöglichkeit: ja oder nein & Beobachtbares Kaufverhalten \\
\hline guter Service & Antwortmöglichkeit: ja oder nein & Beobachtbares Kaufverhalten \\
\hline angenehme Atmosphäre & Antwortmöglichkeit: ja oder nein & Beobachtbares Kaufverhalten \\
\hline Nähe zum Wohnort & Antwortmöglichkeit: ja oder nein & Beobachtbares Kaufverhalten \\
\hline
\end{tabular}




\begin{tabular}{|c|c|c|}
\hline günstige Preise & Antwortmöglichkeit: ja oder nein & Beobachtbares Kaufverhalten \\
\hline unkomplizierter schneller Einkauf & Antwortmöglichkeit: ja oder nein & Beobachtbares Kaufverhalten \\
\hline \multicolumn{3}{|c|}{$\begin{array}{l}\text { F6: Wo werden Ihre Erwartungen hinsichtlich } \\
\text { Produktqualität, Service und Preis beim Kauf von } \\
\text { Blumen und Pflanzen am besten erfüllt? Bitte stu- } \\
\text { fen Sie Ihre Meinung auf der Skala ab. }\end{array}$} \\
\hline \multicolumn{3}{|l|}{ Qualität } \\
\hline Baumarkt & Antwortmöglichkeit: ja oder nein & Beobachtbares Kaufverhalten \\
\hline Gartencenter & Antwortmöglichkeit: ja oder nein & Beobachtbares Kaufverhalten \\
\hline Supermarkt & Antwortmöglichkeit: ja oder nein & Beobachtbares Kaufverhalten \\
\hline Discounter & Antwortmöglichkeit: ja oder nein & Beobachtbares Kaufverhalten \\
\hline Gärtnerei & Antwortmöglichkeit: ja oder nein & Beobachtbares Kaufverhalten \\
\hline Blumengeschäft & Antwortmöglichkeit: ja oder nein & Beobachtbares Kaufverhalten \\
\hline Wochenmarkt & Antwortmöglichkeit: ja oder nein & Beobachtbares Kaufverhalten \\
\hline Baumschule & Antwortmöglichkeit: ja oder nein & Beobachtbares Kaufverhalten \\
\hline \multicolumn{3}{|l|}{ Service } \\
\hline Baumarkt & Antwortmöglichkeit: ja oder nein & Beobachtbares Kaufverhalten \\
\hline Gartencenter & Antwortmöglichkeit: ja oder nein & Beobachtbares Kaufverhalten \\
\hline Supermarkt & Antwortmöglichkeit: ja oder nein & Beobachtbares Kaufverhalten \\
\hline Discounter & Antwortmöglichkeit: ja oder nein & Beobachtbares Kaufverhalten \\
\hline Gärtnerei & Antwortmöglichkeit: ja oder nein & Beobachtbares Kaufverhalten \\
\hline Blumengeschäft & Antwortmöglichkeit: ja oder nein & Beobachtbares Kaufverhalten \\
\hline Wochenmarkt & Antwortmöglichkeit: ja oder nein & Beobachtbares Kaufverhalten \\
\hline Baumschule & Antwortmöglichkeit: ja oder nein & Beobachtbares Kaufverhalten \\
\hline \multicolumn{3}{|l|}{ Preise } \\
\hline Baumarkt & Antwortmöglichkeit: ja oder nein & Beobachtbares Kaufverhalten \\
\hline Gartencenter & Antwortmöglichkeit: ja oder nein & Beobachtbares Kaufverhalten \\
\hline Supermarkt & Antwortmöglichkeit: ja oder nein & Beobachtbares Kaufverhalten \\
\hline Discounter & Antwortmöglichkeit: ja oder nein & Beobachtbares Kaufverhalten \\
\hline Gärtnerei & Antwortmöglichkeit: ja oder nein & Beobachtbares Kaufverhalten \\
\hline Blumengeschäft & Antwortmöglichkeit: ja oder nein & Beobachtbares Kaufverhalten \\
\hline Wochenmarkt & Antwortmöglichkeit: ja oder nein & Beobachtbares Kaufverhalten \\
\hline Baumschule & Antwortmöglichkeit: ja oder nein & Beobachtbares Kaufverhalten \\
\hline
\end{tabular}




\begin{tabular}{|c|c|c|}
\hline $\begin{array}{l}\text { F7: Welche Informationsquellen ziehen Sie heran, } \\
\text { wenn Sie etwas Bestimmtes über BuP wissen möch- } \\
\text { ten? }\end{array}$ & & \\
\hline Prospekte & 3-polig: häufig - nie & Performanz \\
\hline Zeitschriften/ Kataloge & 3-polig: häufig - nie & Performanz \\
\hline Internet & 3-polig: häufig-nie & Performanz \\
\hline Freunde/ Bekannte/ Familie & 3-polig: häufig - nie & Performanz \\
\hline Züchter & 3-polig: häufig - nie & Performanz \\
\hline \multicolumn{3}{|l|}{$\begin{array}{l}\text { F8: Bitte geben Sie an, welchen der folgenden Aus- } \\
\text { sagen Sie zustimmen! }\end{array}$} \\
\hline $\begin{array}{l}\text { Bevor ich mich entscheide, welche Pflanze ich kau- } \\
\text { fe, überlege ich, wo sie stehen könnte. }\end{array}$ & $\begin{array}{l}\text { 5-stufig: stimme voll } \mathrm{zu}-\text { stim- } \\
\text { me nicht } \mathrm{zu}\end{array}$ & Kompetenz \\
\hline $\begin{array}{l}\text { Um sicher zu sein, die richtige Wahl beim Kauf ei- } \\
\text { ner Blume oder Pflanze zu treffen, frage ich vor } \\
\text { dem Kauf meine Freunde oder Bekannte nach ih- } \\
\text { ren Erfahrungen. }\end{array}$ & $\begin{array}{l}\text { 5-stufig: stimme voll zu - stim- } \\
\text { me nicht } \mathrm{zu}\end{array}$ & Kompetenz \\
\hline $\begin{array}{l}\text { Wenn ich eine Blume oder Pflanze kaufe, nehme } \\
\text { ich mir genügend Zeit und lasse mich beraten. }\end{array}$ & $\begin{array}{l}\text { 5-stufig: stimme voll zu - stim- } \\
\text { me nicht zu }\end{array}$ & Kompetenz \\
\hline $\begin{array}{l}\text { F9: Bitte erinnern Sie sich an Ihren letzten Kauf } \\
\text { einer Gartenrose zurück! Hatten Sie den Kauf der } \\
\text { Gartenrose geplant oder ist die Entscheidung spon- } \\
\text { tan im Geschäft gefallen? }\end{array}$ & $\begin{array}{l}\text { Antwortmöglichkeit: spontan, } \\
\text { geplant, nie }\end{array}$ & Beobachtbares Kaufverhalten \\
\hline \multicolumn{3}{|l|}{$\begin{array}{l}\text { F10 Warum haben Sie sich entschieden, diese letzte } \\
\text { Gartenrose zu kaufen? }\end{array}$} \\
\hline Weil sie einfach schön war. & Ein Hauptgrund - kein Grund & Motivation \\
\hline $\begin{array}{l}\text { Weil sie an einen bestimmten Platz in meinem Gar- } \\
\text { ten / auf meinem Balkon passt. }\end{array}$ & Ein Hauptgrund - kein Grund & Motivation \\
\hline Wegen der Blütenfarbe. & Ein Hauptgrund - kein Grund & Motivation \\
\hline Weil sie im Angebot war. & Ein Hauptgrund - kein Grund & Motivation \\
\hline $\begin{array}{l}\text { F13: Welches ist der maximale Preis, den Sie bereit } \\
\text { wären, für die „perfekte Gartenrose“ im Plastik- } \\
\text { topf (Durchmesser } 18 \mathrm{~cm} \text { ) zu bezahlen? }\end{array}$ & $\begin{array}{l}\text { Antwortmöglichkeit: freier Be- } \\
\text { trag }\end{array}$ & Beobachtbares Kaufverhalten \\
\hline \multicolumn{3}{|l|}{$\begin{array}{l}\text { F14: Eine Gartenrose darf ruhig etwas mehr kos- } \\
\text { ten, wenn ... }\end{array}$} \\
\hline $\begin{array}{l}\text {... sie in einem Container ist (und nicht wurzel- } \\
\text { nackt, also ohne Erde und Blätter). }\end{array}$ & $\begin{array}{l}\text { 5-stufig: stimme voll } \mathrm{zu} \text { - stim- } \\
\text { me nicht } \mathrm{zu}\end{array}$ & Kompetenz \\
\hline ... es sich um eine neue Sorte handelt. & $\begin{array}{l}\text { 5-stufig: stimme voll zu - stim- } \\
\text { me nicht zu }\end{array}$ & Kompetenz \\
\hline ... sie eine ganz besondere Blüte hat. & $\begin{array}{l}\text { 5-stufig: stimme voll zu - stim- } \\
\text { me nicht zu }\end{array}$ & Kompetenz \\
\hline ... sie ein Qualitätssiegel trägt. & $\begin{array}{l}\text { 5-stufig: stimme voll zu - stim- } \\
\text { me nicht zu }\end{array}$ & Motivation \\
\hline
\end{tabular}




\begin{tabular}{|c|c|c|}
\hline ... sie ganz besonders duftet. & $\begin{array}{l}\text { 5-stufig: stimme voll zu - stim- } \\
\text { me nicht } \mathrm{zu}\end{array}$ & Kompetenz \\
\hline $\begin{array}{l}\text {... ich gerade etwas mehr Geld im Portemonnaie } \\
\text { habe. }\end{array}$ & $\begin{array}{l}\text { 5-stufig: stimme voll zu - stim- } \\
\text { me nicht zu }\end{array}$ & Beobachtbares Kaufverhalten \\
\hline ... ich eine Rose als Geschenk kaufen möchte. & $\begin{array}{l}\text { 5-stufig: stimme voll zu - stim- } \\
\text { me nicht } \mathrm{zu}\end{array}$ & Performanz \\
\hline ... sie eine Edelrose ist. & $\begin{array}{l}\text { 5-stufig: stimme voll zu - stim- } \\
\text { me nicht zu }\end{array}$ & Kompetenz \\
\hline \multicolumn{3}{|l|}{ F16 Wo leben Sie zurzeit? } \\
\hline In einer Stadt mit mehr als 100.000 Einwohnern. & Antwortmöglichkeit: ja oder nein & Sozioökonomisch \\
\hline $\begin{array}{l}\text { In einer Stadt mit etwa } 20.000 \text { bis } 100.000 \text { Einwoh- } \\
\text { nern. }\end{array}$ & Antwortmöglichkeit: ja oder nein & Sozioökonomisch \\
\hline In einer Stadt mit unter 20.000. & Antwortmöglichkeit: ja oder nein & Sozioökonomisch \\
\hline In einem Dorf. & Antwortmöglichkeit: ja oder nein & Sozioökonomisch \\
\hline \multicolumn{3}{|l|}{ F17: Wohnen Sie ... } \\
\hline ... im eigenen Haus? & Antwortmöglichkeit: ja oder nein & Sozioökonomisch \\
\hline ... in der eigenen Eigentumswohnung? & Antwortmöglichkeit: ja oder nein & Sozioökonomisch \\
\hline ... zur Miete? & Antwortmöglichkeit: ja oder nein & Sozioökonomisch \\
\hline F18: Haben Sie einen Balkon / eine Terrasse? & Antwortmöglichkeit: ja oder nein & Sozioökonomisch \\
\hline \multicolumn{3}{|l|}{$\begin{array}{l}\text { F18a: Wie groß ist Ihr Balkon / Ihre Terrasse un- } \\
\text { gefähr? }\end{array}$} \\
\hline Quadratmeterzahl & $\begin{array}{l}\text { Antwortmöglichkeit: freier Be- } \\
\text { trag }\end{array}$ & Sozioökonomisch \\
\hline F19: Haben Sie einen Garten? & Antwortmöglichkeit: ja oder nein & \\
\hline \multicolumn{3}{|l|}{ F19a: Wie groß ist Ihr Garten ungefähr? } \\
\hline Quadratmeterzahl & $\begin{array}{l}\text { Antwortmöglichkeit: freier Be- } \\
\text { trag }\end{array}$ & Sozioökonomisch \\
\hline \multicolumn{3}{|l|}{$\begin{array}{l}\text { F20: Wie viel Zeit verbringen Sie im Sommer } \\
\text { durchschnittlich pro Woche mit Gartenarbeit? }\end{array}$} \\
\hline Arbeitsstunden & $\begin{array}{l}\text { Antwortmöglichkeit: freier Be- } \\
\text { trag }\end{array}$ & Kompetenz \\
\hline \multicolumn{3}{|l|}{$\begin{array}{l}\text { F21: Wie viel Geld geben Sie ungefähr im Jahr für } \\
\text { Blumen und Pflanzen aus? }\end{array}$} \\
\hline Betrag & $\begin{array}{l}\text { Antwortmöglichkeit: freier Be- } \\
\text { trag }\end{array}$ & Beobachtbares Kaufverhalten \\
\hline \multicolumn{3}{|l|}{ F21a: Davon als Geschenk? } \\
\hline Betrag & $\begin{array}{l}\text { Antwortmöglichkeit: freier Be- } \\
\text { trag }\end{array}$ & Beobachtbares Kaufverhalten \\
\hline
\end{tabular}




\begin{tabular}{|c|c|c|}
\hline $\begin{array}{l}\text { F22: Mit dem Thema „Garten“ und „Gärtnern“" } \\
\text { assoziiere ich besonders ... }\end{array}$ & & \\
\hline ... Entspannung und Ruhe & $\begin{array}{l}\text { 5-stufig: stimme voll zu - stim- } \\
\text { me nicht } \mathrm{zu}\end{array}$ & Motivation \\
\hline ... Geselligkeit und Feiern & $\begin{array}{l}\text { 5-stufig: stimme voll zu - stim- } \\
\text { me nicht } \mathrm{zu}\end{array}$ & Motivation \\
\hline ... körperlichen Ausgleich & $\begin{array}{l}\text { 5-stufig: stimme voll zu - stim- } \\
\text { me nicht } \mathrm{zu}\end{array}$ & Motivation \\
\hline ... Arbeit & $\begin{array}{l}\text { 5-stufig: stimme voll zu - stim- } \\
\text { me nicht } \mathrm{zu}\end{array}$ & Motivation \\
\hline ... Ausleben von Kreativität & $\begin{array}{l}\text { 5-stufig: stimme voll zu - stim- } \\
\text { me nicht zu }\end{array}$ & Motivation \\
\hline $\begin{array}{l}\text {... ökologische Aspekte (Respekt vor der Natur, ei- } \\
\text { gener Anbau von Lebensmitteln) }\end{array}$ & $\begin{array}{l}\text { 5-stufig: stimme voll zu-stim- } \\
\text { me nicht } \mathrm{zu}\end{array}$ & Motivation \\
\hline \multicolumn{3}{|l|}{$\begin{array}{l}\text { F23: Bitte geben Sie an, welchen der folgenden } \\
\text { Aussagen zum Thema Pflanzenschutz Sie zustim- } \\
\text { men! }\end{array}$} \\
\hline $\begin{array}{l}\text { Pflanzenschutzmittel sind ein wichtiger Bestandteil } \\
\text { meiner Arbeit mit Pflanzen. }\end{array}$ & $\begin{array}{l}\text { 5-stufig: stimme voll zu - stim- } \\
\text { me nicht } \mathrm{zu}\end{array}$ & Performanz \\
\hline $\begin{array}{l}\text { Bevor eine Pflanze abstirbt, greife ich schon einmal } \\
\text { zu Pflanzenschutzmitteln. }\end{array}$ & $\begin{array}{l}\text { 5-stufig: stimme voll zu - stim- } \\
\text { me nicht } \mathrm{zu}\end{array}$ & Performanz \\
\hline $\begin{array}{l}\text { Ich benutze grundsätzlich keine Pflanzenschutzmit- } \\
\text { tel, weil diese schädlich für Menschen und die Um- } \\
\text { welt sind. }\end{array}$ & $\begin{array}{l}\text { 5-stufig: stimme voll } \mathrm{zu} \text { - stim- } \\
\text { me nicht } \mathrm{zu}\end{array}$ & Performanz \\
\hline \multicolumn{3}{|l|}{$\begin{array}{l}\text { F24: Bitte geben Sie an, welchen der folgenden } \\
\text { Aussagen zum Thema Pflanzen Sie zustimmen! }\end{array}$} \\
\hline $\begin{array}{l}\text { Für mich sind ausgefallene Farben oder Formen } \\
\text { von Pflanzen wichtig, um so meine Individualität } \\
\text { auszudrücken. }\end{array}$ & $\begin{array}{l}\text { 5-stufig: stimme voll } \mathrm{zu}-\text { stim- } \\
\text { me nicht } \mathrm{zu}\end{array}$ & Motivation \\
\hline $\begin{array}{l}\text { Zuhause sind Blumen und Pflanzen das Highlight } \\
\text { in meinem Wohnkonzept. }\end{array}$ & $\begin{array}{l}\text { 5-stufig: stimme voll } \mathrm{zu} \text { - stim- } \\
\text { me nicht } \mathrm{zu}\end{array}$ & Motivation \\
\hline Pflanzen sind mein wichtigstes Hobby. & $\begin{array}{l}\text { 5-stufig: stimme voll } \mathrm{zu} \text { - stim- } \\
\text { me nicht } \mathrm{zu}\end{array}$ & Performanz \\
\hline $\begin{array}{l}\text { Ich empfinde es als entspannend, wenn ich mich } \\
\text { mit Pflanzen beschäftige. }\end{array}$ & $\begin{array}{l}\text { 5-stufig: stimme voll zu - stim- } \\
\text { me nicht } \mathrm{zu}\end{array}$ & Motivation \\
\hline $\begin{array}{l}\text { Durch Blumen und Pflanzen im Haus kann man } \\
\text { die Kinder gut an naturbezogene Themen heran- } \\
\text { führen. }\end{array}$ & $\begin{array}{l}\text { 5-stufig: stimme voll zu - stim- } \\
\text { me nicht } \mathrm{zu}\end{array}$ & Motivation \\
\hline $\begin{array}{l}\text { Ich kann es nicht gut ertragen, zu sehen, wenn es } \\
\text { einer Pflanze schlecht geht. }\end{array}$ & $\begin{array}{l}\text { 5-stufig: stimme voll zu - stim- } \\
\text { me nicht } \mathrm{zu}\end{array}$ & Motivation \\
\hline $\begin{array}{l}\text { Auf ihre spezielle Art und Weise ist jede Blume } \\
\text { und jede Pflanze schön. }\end{array}$ & $\begin{array}{l}\text { 5-stufig: stimme voll zu - stim- } \\
\text { me nicht zu }\end{array}$ & Motivation \\
\hline $\begin{array}{l}\text { Für mich ist das Wichtigste bei einer Pflanze, dass } \\
\text { sie pflegeleicht ist. }\end{array}$ & $\begin{array}{l}\text { 5-stufig: stimme voll zu - stim- } \\
\text { me nicht zu }\end{array}$ & Motivation \\
\hline
\end{tabular}




\begin{tabular}{|c|c|c|}
\hline $\begin{array}{l}\text { Wenn ich mich in eine Blume oder Pflanze verliebe, } \\
\text { dann kaufe ich sie, ohne groß auf den Preis zu ach- } \\
\text { ten. }\end{array}$ & $\begin{array}{l}\text { 5-stufig: stimme voll zu - stim- } \\
\text { me nicht } \mathrm{zu}\end{array}$ & Motivation \\
\hline $\begin{array}{l}\text { Es macht mir Spaß, mit Pflanzen in meiner Woh- } \\
\text { nung / meinem Garten meinen eigenen Stil zu un- } \\
\text { terstreichen. }\end{array}$ & $\begin{array}{l}\text { 5-stufig: stimme voll } \mathrm{zu} \text { - stim- } \\
\text { me nicht } \mathrm{zu}\end{array}$ & Motivation \\
\hline \multicolumn{3}{|l|}{$\begin{array}{l}\text { F25: Im Folgenden geht es um allgemeine Wertvor- } \\
\text { stellungen. Bitte geben Sie an, welchen der folgen- } \\
\text { den Aussagen Sie zustimmen! }\end{array}$} \\
\hline $\begin{array}{l}\text { Es ist mir wichtig, in einem sicheren Umfeld zu le- } \\
\text { ben. Aktivitäten, die meine Sicherheit gefährden } \\
\text { könnten, vermeide ich. }\end{array}$ & $\begin{array}{l}\text { 5-stufig: stimme voll zu - stim- } \\
\text { me nicht zu }\end{array}$ & Motivation \\
\hline $\begin{array}{l}\text { Traditionen sind mir wichtig. An Sitten und Ge- } \\
\text { bräuche, die in meiner Familie überliefert sind, } \\
\text { versuche ich mich zu halten. }\end{array}$ & $\begin{array}{l}\text { 5-stufig: stimme voll zu - stim- } \\
\text { me nicht } \mathrm{zu}\end{array}$ & Motivation \\
\hline $\begin{array}{l}\text { Ich mag Überraschungen. Es ist mir wichtig, ein } \\
\text { aufregendes Leben zu führen. }\end{array}$ & $\begin{array}{l}\text { 5-stufig: stimme voll zu - stim- } \\
\text { me nicht zu }\end{array}$ & Motivation \\
\hline \multicolumn{3}{|l|}{$\begin{array}{l}\text { F26: In welchem Wohnzimmer würden Sie sich bei } \\
\text { sich zu Hause am wohlsten fühlen? }\end{array}$} \\
\hline Bild 1 & $\begin{array}{l}\text { Antwortmöglichkeit: bestes - } \\
\text { schlechtestes }\end{array}$ & Performanz \\
\hline Bild 2 & $\begin{array}{l}\text { Antwortmöglichkeit: bestes - } \\
\text { schlechtestes }\end{array}$ & Performanz \\
\hline Bild 3 & $\begin{array}{l}\text { Antwortmöglichkeit: bestes - } \\
\text { schlechtestes }\end{array}$ & Performanz \\
\hline Bild 4 & $\begin{array}{l}\text { Antwortmöglichkeit: bestes - } \\
\text { schlechtestes }\end{array}$ & Performanz \\
\hline Bild 5 & $\begin{array}{l}\text { Antwortmöglichkeit: bestes - } \\
\text { schlechtestes }\end{array}$ & Performanz \\
\hline \multicolumn{3}{|l|}{ F27 In welchem Jahr sind Sie geboren? } \\
\hline Geburtsjahr & $\begin{array}{l}\text { Antwortmöglichkeit: freier Be- } \\
\text { trag }\end{array}$ & Sozioökonomisch \\
\hline \multicolumn{3}{|l|}{ F28: Welchen höchsten Schulabschluss haben Sie? } \\
\hline Ohne Abschluss & Antwortmöglichkeit: ja oder nein & Sozioökonomisch \\
\hline Volks-/Hauptschulabschluss & Antwortmöglichkeit: ja oder nein & Sozioökonomisch \\
\hline Mittlere Reife / Realschulabschluss & Antwortmöglichkeit: ja oder nein & Sozioökonomisch \\
\hline Abitur oder Vergleichbares & Antwortmöglichkeit: ja oder nein & Sozioökonomisch \\
\hline Meister/Techniker/Fachschulabschluss & Antwortmöglichkeit: ja oder nein & Sozioökonomisch \\
\hline Fachhochschul-/Hochschulabschluss & Antwortmöglichkeit: ja oder nein & Sozioökonomisch \\
\hline \multicolumn{3}{|l|}{ F29: Sind Sie zurzeit ...? } \\
\hline Keine Angabe & Antwortmöglichkeit: ja oder nein & Sozioökonomisch \\
\hline
\end{tabular}




\begin{tabular}{|c|c|c|}
\hline Erwerbslos & Antwortmöglichkeit: ja oder nein & Sozioökonomisch \\
\hline Hausfrau/ Hausmann & Antwortmöglichkeit: ja oder nein & Sozioökonomisch \\
\hline Rentner/ Pensionär & Antwortmöglichkeit: ja oder nein & Sozioökonomisch \\
\hline In Ausbildung/ Studium & Antwortmöglichkeit: ja oder nein & Sozioökonomisch \\
\hline Teilzeiterwerbstätig & Antwortmöglichkeit: ja oder nein & Sozioökonomisch \\
\hline Vollzeiterwerbstätig & Antwortmöglichkeit: ja oder nein & Sozioökonomisch \\
\hline \multicolumn{3}{|c|}{$\begin{array}{l}\text { F30 Wie viele Personen gehören zu Ihrem Haus- } \\
\text { halt? }\end{array}$} \\
\hline Anzahl & $\begin{array}{l}\text { Antwortmöglichkeit: freier Be- } \\
\text { trag }\end{array}$ & Sozioökonomisch \\
\hline \multicolumn{3}{|c|}{ F31: Wie viele davon sind Kinder unter 12 Jahren? } \\
\hline Anzahl & $\begin{array}{l}\text { Antwortmöglichkeit: freier Be- } \\
\text { trag }\end{array}$ & Sozioökonomisch \\
\hline \multicolumn{3}{|c|}{ F32 Wie hoch ist Ihr Netto-Haushaltseinkommen? } \\
\hline Keine Angabe & Antwortmöglichkeit: ja oder nein & Sozioökonomisch \\
\hline$<500 €$ & Antwortmöglichkeit: ja oder nein & Sozioökonomisch \\
\hline $500-900 €$ & Antwortmöglichkeit: ja oder nein & Sozioökonomisch \\
\hline $900-1.300 €$ & Antwortmöglichkeit: ja oder nein & Sozioökonomisch \\
\hline $1.300-1.700 €$ & Antwortmöglichkeit: ja oder nein & Sozioökonomisch \\
\hline $1.700-2.000 €$ & Antwortmöglichkeit: ja oder nein & Sozioökonomisch \\
\hline $2.000-2.500 €$ & Antwortmöglichkeit: ja oder nein & Sozioökonomisch \\
\hline $2.500-4.500 €$ & Antwortmöglichkeit: ja oder nein & Sozioökonomisch \\
\hline$>4.500 €$ & Antwortmöglichkeit: ja oder nein & Sozioökonomisch \\
\hline \multicolumn{3}{|l|}{ F33 Sie sind ...? } \\
\hline Ein Mann & Antwortmöglichkeit: ja oder nein & Sozioökonomisch \\
\hline Eine Frau & Antwortmöglichkeit: ja oder nein & Sozioökonomisch \\
\hline
\end{tabular}




\section{Anhang 4: Fragebogen}

\section{Befragung zum Informations- und Kaufverhalten bei Blumen und Pflanzen Bearbeitungszeit ca. 10 Min.}

\section{Guten Tag!}

Ich bin Student an der Hochschule Osnabrück und arbeite gerade an einem Forschungsprojekt im Bereich Blumen und Pflanzen und Gartenrosen. Wir möchten damit erreichen, dass das Angebot noch besser auf Ihre Wünsche ausgerichtet wird. Hätten Sie 10 Minuten Zeit, um ein paar Fragen zu beantworten? Die Daten werden selbstverständlich nur in anonymisierter Form verwendet.

\section{Zunächst habe ich ein paar Fragen zum Thema Blumen und Pflanzen an Sie.}

1. Haben Sie in den vergangenen 12 Monaten Blumen oder Pflanzen gekauft?
$1 \square \mathrm{ja}$
$\circ \square$ nein $=>$ Abbruch

2. Blumen und Pflanzen kann man sich selbst kaufen, aber auch verschenken. Bitte geben Sie an, was bei Ihnen mehrheitlich zutrifft!

$\begin{array}{ccccc}\begin{array}{c}\text { Ich kaufe deutlich } \\ \text { häufiger für mich } \\ \text { selbst ein }\end{array} & \begin{array}{c}\text { Ich kaufe etwas } \\ \text { häufiger für mich } \\ \text { selbst ein }\end{array} & \text { Ausgeglichen } & \begin{array}{c}\text { Ich kaufe etwas } \\ \text { häufiger als Ge- } \\ \text { schenk ein }\end{array} & \begin{array}{c}\text { Ich kaufe deutlich } \\ \text { häufiger als Ge- } \\ \text { schenk ein }\end{array} \\ +2 \square & +1 \square & 0 \square & -1 \square & -2 \square\end{array}$

3. Bitte geben Sie an, wie Sie die folgenden Aussagen bewerten!

Ich kaufe gerne Blumen und Pflanzen als Stimme Stimme Teils / Stimme Stimme Geschenk, weil... $\quad$ voll zu $\quad \mathrm{zu} \quad$ Teils wenig zu nicht zu

...man die Möglichkeit hat, mit außergewöhnli- $\quad+2 \square \quad+1 \square \quad 0 \square \quad-1 \square \quad-2 \square$ chen Produkten besonders zu beeindrucken.
...ich ein schönes Geschenk habe, ohne jedes

$\begin{array}{llll}+2 & +1 \square \quad 0 \square & -1\end{array} \quad-2 \square$

Mal wieder kreativ sein zu müssen.

...man es noch auf die Schnelle besorgen kann.

$\begin{array}{llll}+2 & +1\end{array} \quad 0 \square \quad-1 \square \quad-2 \square$

4. Wo haben Sie persönlich in den letzten zwölf Monaten Pflanzen und Gartenbedarf gekauft? Mehrfachnennungen sind möglich!
$\square$ Baumarkt
$\square$ Gärtnerei
$\square$ Gartencenter
$\square$ Blumenfachgeschäft
$\square$ Supermarkt (z. B. EDEKA, REWE)
$\square$ Wochenmarkt
$\square$ Discounter (z. B. Aldi, Lidl)
$\square$ Baumschule 
5. Welche 3 Aspekte sind bei der Wahl der Einkaufsstätte für Sie am wichtigsten, wenn Sie Blumen und Pflanzen kaufen möchten? Es sind maximal 3 Nennungen möglich!

$\square$ große Auswahl

$\square$ Nähe zum Wohnort

$\square$ Qualität der Blumen und Pflanzen

$\square$ freundliche Mitarbeiter

$\square$ guter Service

$\square$ günstige Preise

$\square$ unkomplizierter, schneller Einkauf

$\square$ angenehme Atmosphäre

$\square$ wenn möglich immer eine Einkaufsstätte, wo ich mich auskenne

6. Wo werden Ihre Erwartungen hinsichtlich Produktqualität, Service und Preis beim Kauf von Blumen und Pflanzen am besten erfüllt? Bitte stufen Sie Ihre Meinung auf der Skala ab.

\begin{tabular}{|c|c|c|c|c|c|c|c|c|c|}
\hline & Die & $\begin{array}{c}\text { tät ist } i \\
\text { gel... }\end{array}$ & $\mathrm{Re}-$ & Der Se & ist in & Regel... & Die & $\begin{array}{l}\text { se sinc } \\
\text { egel... }\end{array}$ & der \\
\hline & hoch & mittel & schlecht & hoch & mittel & schlecht & hoch & mittel & günstig \\
\hline Baumarkt & $+3 \square$ & $+2 \square$ & $+1 \square$ & $+3 \square$ & $+2 \square$ & $+1 \square$ & $+3 \square$ & $+2 \square$ & $+1 \square$ \\
\hline Gartencenter & $+3 \square$ & $+2 \square$ & $+1 \square$ & $+3 \square$ & $+2 \square$ & $+1 \square$ & $+3 \square$ & $+2 \square$ & $+1 \square$ \\
\hline Supermarkt & $+3 \square$ & $+2 \square$ & $+1 \square$ & $+3 \square$ & $+2 \square$ & $+1 \square$ & $+3 \square$ & $+2 \square$ & $+1 \square$ \\
\hline Discounter & $+3 \square$ & $+2 \square$ & $+1 \square$ & $+3 \square$ & $+2 \square$ & $+1 \square$ & $+3 \square$ & $+2 \square$ & $+1 \square$ \\
\hline Gärtnerei & $+3 \square$ & $+2 \square$ & $+1 \square$ & $+3 \square$ & $+2 \square$ & $+1 \square$ & $+3 \square$ & $+2 \square$ & $+1 \square$ \\
\hline Blumengeschäft & $+3 \square$ & $+2 \square$ & $+1 \square$ & $+3 \square$ & $+2 \square$ & $+1 \square$ & $+3 \square$ & $+2 \square$ & $+1 \square$ \\
\hline Wochenmarkt & $+3 \square$ & $+2 \square$ & $+1 \square$ & $+3 \square$ & $+2 \square$ & $+1 \square$ & $+3 \square$ & $+2 \square$ & $+1 \square$ \\
\hline Baumschule & $+3 \square$ & $+2 \square$ & $+1 \square$ & $+3 \square$ & $+2 \square$ & $+1 \square$ & $+3 \square$ & $+2 \square$ & $+1 \square$ \\
\hline
\end{tabular}

7. Welche Informationsquellen ziehen Sie heran, wenn Sie etwas Bestimmtes über Blumen und Pflanzen wissen möchten?

$\begin{array}{lccc} & \text { Häufig } & \text { Selten } & \text { Nie } \\ \text { Prospekte / Kataloge } & +2 \square & +1 \square & 0 \square \\ \text { Zeitschriften / Bücher } & +2 \square & +1 \square & 0 \square \\ \text { Internet } & +2 \square & +1 \square & 0 \square \\ \text { Freunde / Bekannte / Familie } & +2 \square & +1 \square & 0 \square \\ \text { Züchter } & +2 \square & +1 \square & 0 \square\end{array}$

8. Bitte geben Sie an, welchen der folgenden Aussagen Sie zustimmen!

Bevor ich mich entscheide, welche Pflanze ich kaufe, überlege ich, wo sie stehen könnte.

Um sicher zu sein, die richtige Wahl beim Kauf einer Blume oder Pflanze zu treffen, frage ich vor dem Kauf meine Freunde oder Bekannte nach ihren Erfahrungen.

Wenn ich eine Blume oder Pflanze kaufe, nehme

$\begin{array}{ccccc}\begin{array}{c}\text { Stimme } \\ \text { voll zu }\end{array} & \begin{array}{c}\text { Stimme } \\ \text { zu }\end{array} & \begin{array}{c}\text { Teils / } \\ \text { Teils }\end{array} & \begin{array}{c}\text { Stimme } \\ \text { wenig zu }\end{array} & \begin{array}{c}\text { Stimme } \\ \text { nicht zu }\end{array} \\ +2 \square & +1 \square & 0 \square & -1 \square & -2 \square \\ +2 \square & +1 \square & 0 \square & -1 \square & -2 \square \\ +2 \square & +1 \square & 0 \square & -1 \square & -2 \square\end{array}$

ich mir genügend Zeit und lasse mich beraten. 
Nun habe ich noch einige Fragen zum Thema Gartenrosen.

9. Bitte erinnern Sie sich an Ihren letzten Kauf einer Gartenrose zurück! Hatten Sie den Kauf der Gartenrose geplant oder ist die Entscheidung spontan im Geschäft gefallen?
$1 \square$ geplanter Kauf
$2 \square$ spontaner Kauf
० $\square$ kein Kauf $=>$ weiter mit $F 11$

10. Warum haben Sie sich entschieden, diese letzte Gartenrose zu kaufen?

\section{Ein Hauptgrund}

Ein wenige wichtiger Grund

Kein Grund

Weil sie einfach schön war.

$+2 \square$

Weil sie an einen bestimmten Platz in mei-

nem Garten / auf meinem Balkon passt.

Wegen der Blütenfarbe.

Weil sie im Angebot war.
$+2 \square$

$+2 \square$

$+2 \square$
$+1 \square$

$+1 \square$

$+1 \square$

$+1 \square$ $\circ \square$

$\circ \square$

$\circ \square$

$\circ \square$

11. Welche Farbe finden Sie für eine Gartenrose am schönsten (nur 1 Nennung möglich)?

$\begin{array}{ll}{ }_{1} \square \text { Rot } & { }_{4} \square \text { Weiß } \\ { }_{2} \square \text { Rosa } & { }_{5} \square \text { Gelb } \\ { }_{3} \square \text { Orange } & \end{array}$

12. Welche der folgenden Wuchsformen von Gartenrosen finden Sie speziell für Ihren Garten bzw. Ihren Balkon schön?

$\begin{array}{lccc} & \text { Finde ich schön } & \text { Ich weiß nicht } & \begin{array}{c}\text { Finde ich nicht } \\ \text { schön }\end{array} \\ \text { Hochstämmchen } & +1 \square & 0 \square & -1 \square \\ \text { Bodendeckerrosen } & +1 \square & 0 \square & -1 \square \\ \text { Strauchrosen } & +1 \square & 0 \square & -1 \square \\ \text { Kletterrosen } & +1 \square & 0 \square & -1 \square\end{array}$

13. Welches ist der maximale Preis, den Sie bereit wären, für die „perfekte Gartenrose“ im Plastiktopf (Durchmesser $18 \mathrm{~cm}$ ) zu bezahlen? $€$

14. Bitte geben Sie an, wie Sie die folgenden Aussagen zum Preis einer Gartenrose bewerten!

\begin{tabular}{|c|c|c|c|c|c|}
\hline $\begin{array}{l}\text { Eine Gartenrose darf ruhig etwas mehr kos- } \\
\text { ten, wenn... }\end{array}$ & $\begin{array}{l}\text { Stimme } \\
\text { voll zu }\end{array}$ & $\begin{array}{l}\text { Stimme } \\
\text { zu }\end{array}$ & $\begin{array}{l}\text { Unent- } \\
\text { schieden }\end{array}$ & $\begin{array}{l}\text { Stimme } \\
\text { wenig zu }\end{array}$ & $\begin{array}{l}\text { Stimme } \\
\text { nicht zu }\end{array}$ \\
\hline $\begin{array}{l}\text {...sie in einem Container ist (und nicht wurzel- } \\
\text { nackt, also ohne Erde und Blätter). }\end{array}$ & $+2 \square$ & $+1 \square$ & $\circ \square$ & $-1 \square$ & $-2 \square$ \\
\hline ...es sich um eine neue Sorte handelt. & $+2 \square$ & $+1 \square$ & $0 \square$ & $-1 \square$ & $-2 \square$ \\
\hline ...sie eine ganz besondere Blüte hat. & $+2 \square$ & $+1 \square$ & $0 \square$ & $-1 \square$ & $-2 \square$ \\
\hline ...sie ein Qualitätssiegel trägt. & $+2 \square$ & $+1 \square$ & $\circ \square$ & $-1 \square$ & $-2 \square$ \\
\hline ...sie ganz besonders duftet. & $+2 \square$ & $+1 \square$ & $0 \square$ & $-1 \square$ & $-2 \square$ \\
\hline $\begin{array}{l}\text {...ich gerade etwas mehr Geld im Portemon- } \\
\text { naie habe. }\end{array}$ & $+2 \square$ & $+1 \square$ & $0 \square$ & $-1 \square$ & $-2 \square$ \\
\hline ...ich eine Rose als Geschenk kaufen möchte. & $+2 \square$ & $+1 \square$ & $0 \square$ & $-1 \square$ & $-2 \square$ \\
\hline ...sie eine Edelrose ist. & $+2 \square$ & $+1 \square$ & $0 \square$ & $-1 \square$ & $-2 \square$ \\
\hline
\end{tabular}


15. Bitte stellen Sie sich vor, dass Sie eine Gartenrose anhand des folgenden Etiketts gekauft haben. Nach einem Jahr sehen Sie ein nicht perfektes Resultat im Garten. Wie würden Sie in den folgenden Fällen reagieren? Mehrfachnennungen sind möglich!
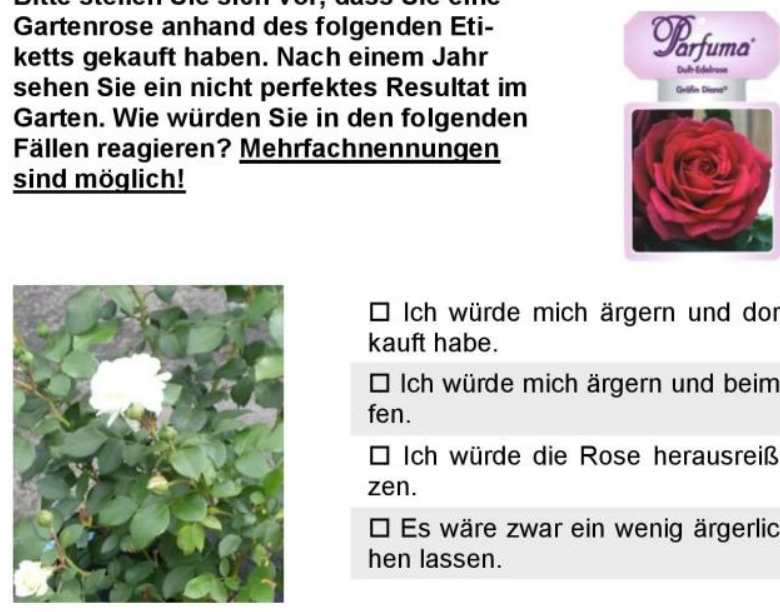

$\square$ Ich würde mich ärgern und dort beschweren, wo ich die Rose gekauft habe.

$\square$ Ich würde mich ärgern und beim nächsten Mal dort nicht wieder kaufen.

$\square$ Ich würde die Rose herausreißen und eine neue Pflanze einpflanzen.

$\square$ Es wäre zwar ein wenig ärgerlich, aber ich würde die Rose dort stehen lassen.

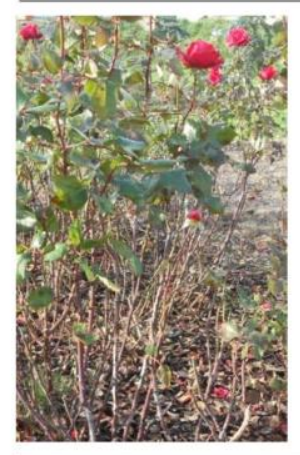

$\square$ Ich würde mich ärgern und dort beschweren, wo ich die Rose gekauft habe.

$\square$ Ich würde mich ärgern und beim nächsten Mal dort nicht wieder kaufen.

$\square$ Ich würde die Rose herausreißen und eine neue Pflanze einpflanzen

$\square$ Es wäre zwar ein wenig ärgerlich, aber ich würde die Rose dort stehen lassen.

$\square$ Mich würde es nicht besonders stören und ich würde die Krankheit behandeln.

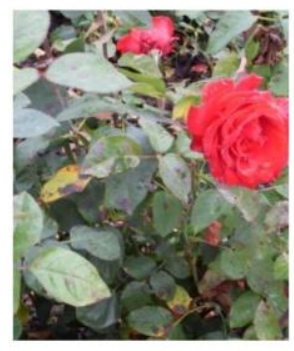

$\square$ Ich würde mich ärgern und dort beschweren, wo ich die Rose gekauft habe.

$\square$ Ich würde mich ärgern und beim nächsten Mal dort nicht wieder kaufen.

$\square$ Ich würde die Rose herausreißen und eine neue Pflanze einpflanzen.

$\square$ Es wäre zwar ein wenig ärgerlich, aber ich würde die Rose dort stehen lassen.

$\square$ Mich würde es nicht besonders stören und ich würde die Krankheit behandeln.

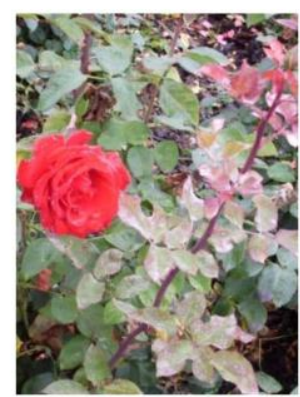

$\square$ Ich würde mich ärgern und dort beschweren, wo ich die Rose gekauft habe.

$\square$ Ich würde mich ärgern und beim nächsten Mal dort nicht wieder kaufen.

$\square$ Ich würde die Rose herausreißen und eine neue Pflanze einpflanzen.

$\square$ Es wäre zwar ein wenig ärgerlich, aber ich würde die Rose dort stehen lassen.

$\square$ Mich würde es nicht besonders stören und ich würde die Krankheit behandeln.

- bitte wenden - 
16. Wo leben Sie zurzeit?

${ }_{1} \square$ in einer Stadt mit mehr als 100.000 Einwohnern

${ }_{2} \square$ in einer Stadt mit etwa 20.000 bis 100.000 Einwohnern

${ }_{3} \square$ in einer Stadt mit unter 20.000 Einwohnern

${ }_{4} \square$ in einem Dorf

17. Wohnen Sie...
${ }_{1} \square \mathrm{im}$ eigenen Haus
$2 \square$ in der eigenen Eigentumswohnung
${ }_{3} \square$ zur Miete

18. Haben Sie einen Balkon / eine Terrasse?

${ }_{1} \square$ ja => Wie groß ist Ihr Balkon / Ihre Terrasse ungefähr?

$\circ \square$ nein

19. Haben Sie einen Garten?

${ }_{1} \square$ ja $\quad=>$ Wie groß ist Ihr Garten ungefähr?

$\circ \square$ nein

20. Wie viel Zeit verbringen Sie im Sommer durchschnittlich pro Woche mit Gartenarbeit?

Stunden

21. Wie viel Geld geben Sie ungefähr im Jahr für Blumen und Pflanzen aus?

$€ \quad=>$ davon als Geschenk $€$

22. Bitte geben Sie an, welchen der folgenden Aussagen zum Thema Garten und Gärtnern Sie zustimmen!

\begin{tabular}{|c|c|c|c|c|c|}
\hline $\begin{array}{l}\text { Mit dem Thema „Garten“ und „Gärtnern“" } \\
\text { assoziiere ich besonders... }\end{array}$ & $\begin{array}{l}\text { Stimme } \\
\text { voll zu }\end{array}$ & $\begin{array}{c}\text { Stimme } \\
\mathrm{zu}\end{array}$ & $\begin{array}{l}\text { Teils / } \\
\text { Teils }\end{array}$ & $\begin{array}{l}\text { Stimme } \\
\text { wenig zu }\end{array}$ & $\begin{array}{l}\text { Stimme } \\
\text { nicht zu }\end{array}$ \\
\hline ...Entspannung und Ruhe & $+2 \square$ & $+1 \square$ & $\circ \square$ & $-1 \square$ & $-2 \square$ \\
\hline ...Geselligkeit und Feiern & $+2 \square$ & $+1 \square$ & $0 \square$ & $-1 \square$ & $-2 \square$ \\
\hline ...körperlichen Ausgleich & $+2 \square$ & $+1 \square$ & $\circ \square$ & $-1 \square$ & $-2 \square$ \\
\hline ...Arbeit & $+2 \square$ & $+1 \square$ & ०ᄆ & $-1 \square$ & $-2 \square$ \\
\hline ...das Ausleben von Kreativität & $+2 \square$ & $+1 \square$ & $\circ \square$ & $-1 \square$ & $-2 \square$ \\
\hline $\begin{array}{l}\text {...̈̈kologische Aspekte (Respekt vor der } \\
\text { Natur, eigener Anbau von Lebensmitteln) }\end{array}$ & $+2 \square$ & $+1 \square$ & $\circ \square$ & $-1 \square$ & $-2 \square$ \\
\hline
\end{tabular}

23. Bitte geben Sie an, welchen der folgenden Aussagen zum Thema Pflanzenschutz Sie zustimmen!

\begin{tabular}{|c|c|c|c|c|c|}
\hline & $\begin{array}{l}\text { Stimme } \\
\text { voll zu }\end{array}$ & $\begin{array}{l}\text { Stimme } \\
\mathrm{zu}\end{array}$ & $\begin{array}{l}\text { Teils / } \\
\text { Teils }\end{array}$ & $\begin{array}{l}\text { Stimme } \\
\text { wenig zu }\end{array}$ & $\begin{array}{l}\text { Stimme } \\
\text { nicht zu }\end{array}$ \\
\hline $\begin{array}{l}\text { Pflanzenschutzmittel sind ein wichtiger } \\
\text { Bestandteil meiner Arbeit mit Pflanzen. }\end{array}$ & $+2 \square$ & $+1 \square$ & $\circ \square$ & $-1 \square$ & $-2 \square$ \\
\hline $\begin{array}{l}\text { Bevor eine Pflanze abstirbt, greife ich } \\
\text { schon einmal zu Pflanzenschutzmitteln. }\end{array}$ & $+2 \square$ & $+1 \square$ & $\circ \square$ & $-1 \square$ & $-2 \square$ \\
\hline Ich benutze grundsätzlich keine Pflanzen- & $+2 \square$ & $+1 \square$ & $\circ \square$ & $-1 \square$ & $-2 \square$ \\
\hline
\end{tabular}

Ich benutze grundsätzlich keine Pflanzenschutzmittel, weil diese schädlich für Menschen und die Umwelt sind. 
24. Bitte geben Sie an, welchen der folgenden Aussagen zum Thema Pflanzen Sie zustimmen!

\begin{tabular}{|c|c|c|c|c|c|}
\hline & $\begin{array}{l}\text { Stimme } \\
\text { voll zu }\end{array}$ & $\begin{array}{c}\text { Stimme } \\
\mathrm{zu}\end{array}$ & $\begin{array}{l}\text { Teils / } \\
\text { Teils }\end{array}$ & $\begin{array}{l}\text { Stimme } \\
\text { wenig zu }\end{array}$ & $\begin{array}{l}\text { Stimme } \\
\text { nicht zu }\end{array}$ \\
\hline $\begin{array}{l}\text { Für mich sind ausgefallene Farben oder } \\
\text { Formen von Pflanzen wichtig, um so meine } \\
\text { Individualität auszudrücken. }\end{array}$ & $+2 \square$ & $+1 \square$ & ㅁ & $-1 \square$ & $-2 \square$ \\
\hline $\begin{array}{l}\text { Zuhause sind Blumen und Pflanzen das } \\
\text { Highlight in meinem Wohnkonzept. }\end{array}$ & $+2 \square$ & $+1 \square$ & $0 \square$ & $-1 \square$ & $-2 \square$ \\
\hline Pflanzen sind mein wichtigstes Hobby. & $+2 \square$ & $+1 \square$ & ० ㅁ & $-1 \square$ & $-2 \square$ \\
\hline $\begin{array}{l}\text { Ich empfinde es als entspannend, wenn ich } \\
\text { mich mit Pflanzen beschäftige. }\end{array}$ & $+2 \square$ & $+1 \square$ & $0 \square$ & $-1 \square$ & $-2 \square$ \\
\hline $\begin{array}{l}\text { Durch Blumen und Pflanzen im Haus kann } \\
\text { man die Kinder gut an naturbezogene } \\
\text { Themen heranführen. }\end{array}$ & $+2 \square$ & $+1 \square$ & ० & $-1 \square$ & $-2 \square$ \\
\hline $\begin{array}{l}\text { Ich kann es nicht gut ertragen, zu sehen, } \\
\text { wenn es einer Pflanze schlecht geht. }\end{array}$ & $+2 \square$ & $+1 \square$ & $0 \square$ & $-1 \square$ & $-2 \square$ \\
\hline $\begin{array}{l}\text { Die Rose ist für mich die Königin der Blu- } \\
\text { men. }\end{array}$ & $+2 \square$ & $+1 \square$ & ० & $-1 \square$ & $-2 \square$ \\
\hline $\begin{array}{l}\text { Auf ihre spezielle Art und Weise ist jede } \\
\text { Blume und jede Pflanze schön. }\end{array}$ & $+2 \square$ & $+1 \square$ & $0 \square$ & $-1 \square$ & $-2 \square$ \\
\hline $\begin{array}{l}\text { Für mich ist das wichtigste bei einer Pflan- } \\
\text { ze, dass sie pflegeleicht ist. }\end{array}$ & $+2 \square$ & $+1 \square$ & $\circ \square$ & $-1 \square$ & $-2 \square$ \\
\hline $\begin{array}{l}\text { Wenn ich mich in eine Blume oder Pflanze } \\
\text { verliebe, dann kaufe ich sie, ohne groß auf } \\
\text { den Preis zu achten. }\end{array}$ & $+2 \square$ & $+1 \square$ & ० & $-1 \square$ & $-2 \square$ \\
\hline $\begin{array}{l}\text { Es macht mir Spaß mit Pflanzen in meiner } \\
\text { Wohnung / Garten meinen eigenen Stil zu } \\
\text { unterstreichen. }\end{array}$ & $+2 \square$ & +1 $\square$ & ० $\square$ & $-1 \square$ & $-2 \square$ \\
\hline
\end{tabular}

25. Im Folgenden geht es um allgemeine Wertvorstellungen. Bitte geben Sie an, welchen der folgenden Aussagen Sie zustimmen!

$\begin{array}{ccccc}\text { Stimme } & \text { Stimme } & \text { Teils / } & \text { Stimme } & \text { Stimme } \\ \text { voll zu } & \mathrm{zu} & \text { Teils } & \text { wenig zu } & \text { nicht zu }\end{array}$

Es ist mir wichtig, in einem sicheren Umfeld $\quad+2 \square \quad+1 \square \quad 0 \square \quad-1 \square \quad-2 \square$ zu leben. Aktivitäten, die meine Sicherheit gefährden könnten, vermeide ich.

Traditionen sind mir wichtig. An Sitten und Gebräuche, die in meiner Familie überliefert sind, versuche ich mich zu halten.

Ich mag Überraschungen. Es ist mir wichtig, ein aufregendes Leben zu führen.

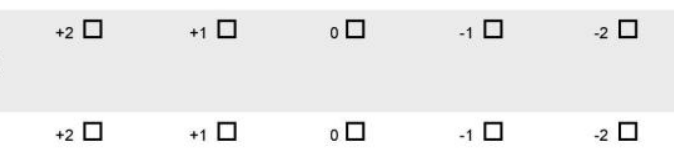


26. Im Folgenden finden Sie Bilder von fünf verschiedenen Wohnzimmern. Wir würden gerne wis sen, in welchem Wohnzimmer Sie sich bei sich zu Hause am wohlsten fühlen würden. Bitte bringen Sie die Bilder in eine Reihenfolge, indem Sie in das Kästchen rechts neben dem Bild eine Zahl schreiben. Dem Wohnzimmer, das Ihnen am besten gefällt, ordnen Sie eine $1 \mathrm{zu}$, dem Wohnzimmer, das Ihnen am zweitbesten gefällt, eine 2 usw. Bitte vergeben Sie jede Zahl nur einmal!
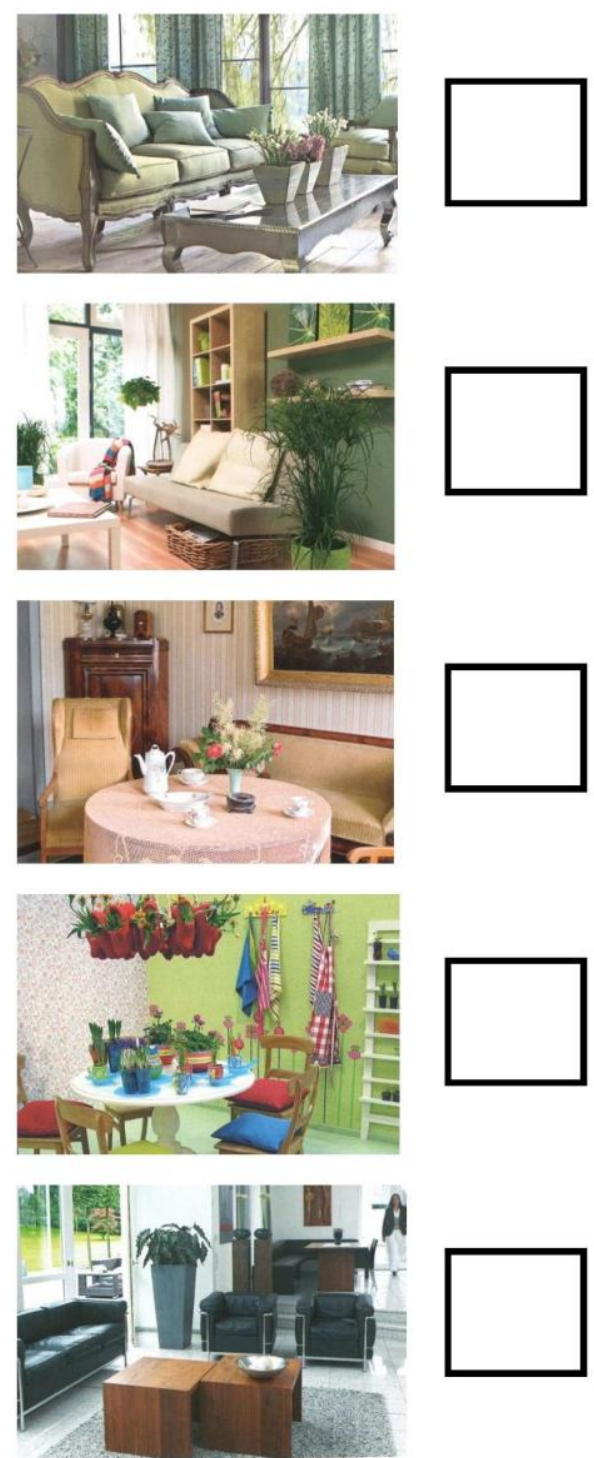

- bitte wenden - 
27. In welchem Jahr sind Sie geboren? 19 _ Jahr

28. Welchen höchsten Schulabschluss haben Sie?

${ }_{1} \square$ Volks-/ Hauptschulabschluss

${ }_{2} \square$ Mittlere Reife/ Realschulabschluss

$3 \square$ Abitur oder vergleichbares

${ }_{4} \square$ Meister/ Techniker/ Fach(ober)schulabschluss

${ }_{5} \square$ Fachhochschul-/ Hochschulabschluss

- $\square$ Ohne Abschluss

$\square$ Sonstiges und zwar

29. Sind Sie zurzeit?

$6 \square$ Vollerwerbstätig

${ }_{5} \square$ Teilzeiterwerbstätig

${ }_{4} \square$ In Ausbildung/Studium

$3 \square$ Rentner/Pensionär

$2 \square$ Hausfrau/Hausmann

$1 \square$ Erwerbslos

$\circ \square$ Keine Angabe

30. Wie viele Personen gehören zu Ihrem Haushalt?

Personen

31. Wie viele davon sind Kinder unter 12 Jahren? Personen

32. Wie hoch ist Ihr Netto-Haushaltseinkommen?

$\begin{array}{lll}0 \square \text { keine Angabe } & 3 \square 900-1.300 € & 6 \square 2.000-2.600 € \\ 1 \square<500 € & 4 \square 1.300-1.700 € & 7 \square 2.600-4.500 € \\ 2 \square 500-900 € & 5 \square 1.700-2.000 € & 8 \square>4.500 €\end{array}$

33. Sie sind ...?

$1 \square$ ein Mann

$2 \square$ eine Frau 


\section{Anhang 5: Homogene Items}

Tabelle 14: Homogene Items

\begin{tabular}{|c|c|c|c|c|c|}
\hline Item & $\begin{array}{l}\text { Kulti- } \\
\text { vator }\end{array}$ & $\begin{array}{l}\text { Durchsch. } \\
\text { Garten- } \\
\text { freund }\end{array}$ & $\begin{array}{l}\text { Pflanzen- } \\
\text { freund }\end{array}$ & $\begin{array}{c}\text { Extrov. } \\
\text { Natur- } \\
\text { bewahrer }\end{array}$ & $\begin{array}{l}\text { Konserv. } \\
\text { Klein- } \\
\text { gärtner }\end{array}$ \\
\hline \multicolumn{6}{|l|}{ Sozioökonomische Dimension } \\
\hline $\begin{array}{l}\text { F32 Wie hoch ist Ihr Netto-Haushaltseinkommen? } \\
0-1300 € .\end{array}$ & - & & & & \\
\hline \multicolumn{6}{|l|}{ Dimension Kompetenz } \\
\hline $\begin{array}{l}\text { F 8.2 Um sicher zu sein, die richtige Wahl beim Kauf einer } \\
\text { Blume oder Pflanze zu treffen, frage ich vor dem Kauf meine } \\
\text { Freunde oder Bekannte nach ihren Erfahrungen. }\end{array}$ & - & & - & & - \\
\hline \multicolumn{6}{|l|}{ Dimension Performanz } \\
\hline $\begin{array}{l}\text { F7.4 Welche Informationsquellen ziehen Sie heran, wenn Sie } \\
\text { etwas Bestimmtes über BuP wissen möchten? Freun- } \\
\text { de/Bekannte/Familie }\end{array}$ & - & & & & \\
\hline \multicolumn{6}{|l|}{ Dimension Motivation } \\
\hline $\begin{array}{l}\text { F10.4 Warum haben Sie sich entschieden, diese letzte Garten- } \\
\text { rose zu kaufen? Weil sie im Angebot war. }\end{array}$ & & & & + & \\
\hline $\begin{array}{l}\text { F22.6 Mit dem Thema „Garten“ und „Gärtnern“ assoziiere ich } \\
\text { besonders ökologische Aspekte (Respekt vor der Natur, eige- } \\
\text { ner Anbau von Lebensmitteln) }\end{array}$ & - & & & & \\
\hline $\begin{array}{l}\text { F25.3 Ich mag Überraschungen. Es ist mir wichtig, ein aufre- } \\
\text { gendes Leben zu führen. }\end{array}$ & - & & & & \\
\hline \multicolumn{6}{|l|}{ Dimension Beobachtbares Kaufverhalten } \\
\hline $\begin{array}{l}\text { F4.1 Wo haben Sie persönlich in den letzten zwölf Monaten } \\
\text { Pflanzen und Gartenbedarf gekauft? Baumarkt }\end{array}$ & & & - & & \\
\hline F4.2 dito. Antwort: Gartencenter & & + & & & + \\
\hline F4.4 dito. Antwort: Gärtnerei & & & & & + \\
\hline $\begin{array}{l}\text { F5.1 Welche drei Aspekte sind bei der Wahl der Einkaufsstätte } \\
\text { für Sie am wichtigsten, wenn Sie BuP kaufen möchten? } \\
\text { Freundliche Mitarbeiter }\end{array}$ & + & & & + & \\
\hline F5.2 dito. Antwort: Wo ich mich auskenne. & & + & + & + & + \\
\hline F5.6 dito. Antwort: Günstige Preise & & & & + & \\
\hline F6.4 dito. Antwort: Gärtnerei & & & & & - \\
\hline F6.6 dito. Antwort: Discounter & & + & & & \\
\hline F6.7 dito. Antwort: Supermarkt & & & - & & \\
\hline F6.9 dito. Antwort: Gärtnerei & & & & & + \\
\hline $\begin{array}{l}\text { Referenzcluster }=\text { Desinteressierter Pragmatiker } \\
\text { Alle Ergebnisse dieser Tabelle beziehen sich auf das Referenzcl } \\
\text { Bei Beta }>0: \text { Wald-Werte: }[0 ; 4]=+;[4 ; 8]=++;[8 ; 12]=+ \\
\text { Bei Beta }<0: \text { Wald-Werte }:[0 ; 4]=-;[4 ; 8]=-;[8 ; 12]=--\end{array}$ & $\begin{array}{l}\text { ster. } \\
+;>12 \\
-;>12\end{array}$ & $\begin{array}{l}++++ \\
----\end{array}$ & & & \\
\hline
\end{tabular}

Quelle: Eigene Berechnungen 